\title{
Ref.
}

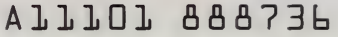

Polymer Degradation Mechanisms National Bureat of Standaxds 


UNITED STATES DEPARTMENT OF COMMERCE • Sinclair Weeks, Secretary NATIONAL BUREAU OF STANDARDS - A. Vi Astin, Director

\section{Polymer Degradation Mechanisms}

Proceedings of the NBS Semicentennial Symposium on Polymer Degradation Mechanisms Held at the NBS on September 24-26, 1951

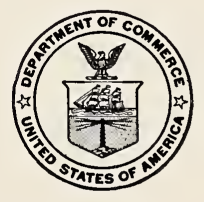

National Bureau of Standards Circular 525 Issued November 16, 1953 
National Bureau of Standards

Nov. 1, 1954

84712

QC

UE5: 


\section{Foreword}

Research on the durability of polymeric materials is of increasing significance in view of the growing importance of these synthetic products in our national economy and defense. A knowledge of the mechanisms by which polymers degrade is needed in order to predict more accurately their expected service life, to inhibit degradation more effectively, and to devise better methods of accelerated testing.

The papers in this volume record progress that has been made in elucidating the structure of the polymers and the mechanisms of their break-down. They were initially presented at the Symposium on Polymer Degradation Mechanisms, the eighth of a series of twelve symposia held by the National Bureau of Standards during its Semicentennial in 1951. The cooperation of the Office of Naval Research in making possible the symposia series is gratefully acknowledged.

The Symposium on Polymer Degradation Mechanisms was planned and conducted by the Bureau's Organic Plastics Section under the chairmanship of G. M. Kline. The following individuals served as session chairmen at the Symposium and gave encouragement and assistance at all stages: B. S. Biggs, Bell Telephone Laboratories; R. B. Mesrobian, Polytechnic Institute of Brooklyn; J. B. Nichols, E. I. du Pont de Nemours \& Co., Inc.; C. R. Stock, American Cyanamid Co.; Warren Stubblebine, Office of the Quartermaster General.

A. V. Astin, Director, National Bureau of Standards. 


\section{Contents}

1. Mechanisms of degradation of vinyl polymers with special reference to polystyrene, by H. H. G. Jellinek ...... 1

2. Mechanism and kinetics of thermal and photodegradation reactions, br Robert Simha

3. Photodegradation of polymethyl methacrylate, by P. R. E. J. Cowley and H. W. Melville

4. Effect of heat and light on polyvinyl chloride, by Donald Druesedow and C.F. Gibbs.

5. Photodegradation of polyvinyl chloride, by A. S. Kenyon --

6. Role of hydrogen chloride in polyvinyl chloride degradation, by Abb L. Scarbrough, W. L. Kellner, and P. W. Rizzo

7. Aging of vinyl chloride and vinylidene chloride polymers, by C. B. Havens.

Page

III

8. Alkaline degradation of polyacrylonitrile, by J. R. McCart-

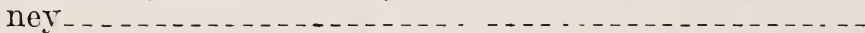

9. Aging of polyethylene, by B. S. Biggs

10. Photo-oxidation and stabilization of polythene, by A. R. Burgess

11. Oxygen-absorption studies on olefins with structures related to GR-S, by J. Reid Shelton

12. Break-down of cellulose esters by heat and light, by G. C. DeCroes and J. W. Tamblin

13. Oxidative degradation of ethyl cellulose, by L. F. McBurner . . . .

14. Study of degradation of polystrrene, using mass spectrometry, by B. G. Achhammer, M. J. Reiney, L. A. Wall, and F. W. Reinhart

15. Rates of thermal degradation of polystyrene and polyethylene in a racuum, by Samuel L. Madorsky.

16. Prrolysis of copolymers, by Leo A. Wal

17. Mechanism of the degradation of polyamides, by Bernard G. Achhammer, Frank W. Reinhart, and Gordon M. Kline 


\title{
1. Mechanisims of Degradation of Vinyl Polymers with Special Reference to Polystyrene
}

\author{
By H. H. G. Jellinek ${ }^{1}$
}

\section{Introduction}

Experimental data on the degradation of vinyl polymers are not yet very numerous, but as far as the bulk degradation of these polymers in vacuum is concerned, the experimental results suggest that degradation usually takes place by a reversal of the polymerization process. This "true" degradation, or depolymerization, is often obscured by reactions which, in the case of polystyrene, are due to the presence of a number of weak links; that is to say, small irregularities in the structure of these polymer chains can profoundly influence the degradation process.

A survey is given in this paper on possible mechanisms of degradation, stressing particularly those that have been verified by experiment. Thus a reverse polymerization mechanism is presented in this paper, and also a mechanism is given for simultaneous degradation and polymerization processes leading to an equilibrium state. Recently, Simha and coworkers [1] * developed a theory of degradation assuming a chain process. However, the assumptions and derivations in this paper differ in many respects from Simha's. Experimental results are discussed in the light of these theories, especially those obtained on polystyrene, which has been investigated more thoroughly than any other polymer. Subsequently, the effect of benzoyl peroxide and oxygen on the degradation is considered, and lastly, the degradation of vinyl polymers by ultrasonic waves is discussed.

No attempt is made to give a complete, detailed account of all degradation experiments or theories relevant to this field, but to show which course is followed by the degradation processes, and it is intended to give a critical appreciation of the main results rather than to enumerate all the experimental details.

\section{Theories of Degradation}

There are quite a number of possibilities as to how a long-chain molecule can be broken down into fragments: (1) Bonds in the chain may be broken at random, each bond being of equal strength and accessibility (random degradation) [2], (2) there may be a number of weak links distributed at random over the chain, which are more easily ruptured than the normal bonds (weak link degradation) [3], (3) the weak link or also the random degradation may be followed by a reaction whereby the chain ends, which are formed by the initial rupture of a link, split off a number of monomer units [4], (4) the degradation may consist in a reversal of the polymerization reaction, whereby the polymer chain ends become activated (initiation reaction), a rapid splitting off of monomer units from these activated ends

\footnotetext{
1 University of Adelaide, Adelaide, Australia.

${ }^{*}$ Figures in brackets indicate the literature references on p. 37 .
} 
follows (propagation reaction), until eventually the activation is lost by a termination reaction (chain theory) $[1,5]$.

The question arises as to how it can be ascertained which of these mechanisms is operative in any particular case. There are a number of quantities that are accessible to experiment: The number and weight average molecular weights or chain lengths and other molecular weight averages, size distributions, amount of monomer or small units formed during degradation. Obviously, a different functional relationship between these various quantities and time will exist for different mechanisms.

In the case of the random process there will be a rapid decrease of the weight average chain length. This also holds true for the weak link case. If the degradation were to take place from the chain ends only, a much slower decrease in the weight average chain length will occur. This will be demonstrated in more detail subsequently.

These statements will not apply to the number average chain length because, for the decrease in the number average chain length, it is of no consequence whether a link is ruptured at the end of the chain or whether the rupture takes place anywhere along the chain producing two relatively large fragments. However, if the number average chain length in the case of the chain theory is taken, not considering the monomer, then this number average chain length will show no decrease, or a slow one.

It may be pointed out here that for any degradation process, whatever the mechanism, also for degradation polymerization equilibria, the number average chain length is always given by

$$
P_{t} \equiv \frac{P_{n}}{s+1}
$$

where $P_{t}$ is the number average chain length at any time $t$ of the degradation process, $P_{n}$ the initial chain length, and $s$ the number of links ruptured on the average for each original chain.

Experimental data on the thermal degradation of vinyl polymers in vacuum indicate that in no case is the simple random mechanism operative. However, in the presence of benzoyl peroxide in solution, or in the presence of oxygen at moderate temperatures, the random degradation seems to be realized.

\section{Reverse polymerization}

As pointed out above, the true degradation of a vinyl long-chain molecule, is a reverse polymerization process. It consists mainly of three reactions, similarly as in the case of polymerization: 1 , Initiation; 2, propagation, that is, splitting off monomer units from the chain ends; 3 , termination.

The simplest case of the degradation of a homogeneous polymer sample may be considered.

An approximate treatment is given, assuming that the initiation reaction is slow, the propagation relatively fast, and that, on the average, $\gamma$ monomer units are broken off for one activated chain end. $\gamma$ is then the average kinetic chain length. It is further assumed that all links are of the same strength and that the initial chain length, $P_{n}$, is a multiple of $\gamma$, that is $P_{n}=a \gamma$. 
Then a reaction scheme is obtained as follows:

Initiation:

$$
\begin{aligned}
\frac{d m_{n}^{x}}{d t} & =k_{4} m_{n} \\
\frac{d m_{n-\gamma}^{x}}{d t} & =k_{4} m_{n-\gamma} \\
\ldots \ldots \ldots \ldots & \ldots \\
\frac{d m_{\gamma}^{x}}{d t} & =k_{4} m_{\gamma} .
\end{aligned}
$$

Propagation:

$$
\frac{d m_{1}}{d t}=\gamma \frac{d m_{n}^{x}}{d t}+\gamma \frac{d m_{n}^{x}-\gamma}{d t}+\ldots \gamma \frac{d m_{\gamma}^{x}}{d t}
$$

or

Termination:

$$
\frac{d m_{1}}{d t}=k_{5} m_{n}^{x}+k_{5} m_{n-\gamma}^{x}+\ldots k_{5} m_{\gamma}^{x} .
$$

$$
\begin{gathered}
-\frac{d m_{n}^{x}}{d t}=k_{6} m_{n}^{x} \\
-\frac{d m_{n-\gamma}^{x}}{d t}=k_{6} m_{n-\gamma}^{x} \\
\cdots \cdots \cdots \cdots \cdots \\
-\frac{d m_{\gamma}^{x}}{d t}=k_{6} m_{\gamma}^{x} .
\end{gathered}
$$

The kinetic chain length $\gamma$ will be given by $\gamma=k_{5} ! k_{6}$. Further,

and

$$
m_{n-(a-1) \gamma}=\frac{\left(k_{4} t\right)^{a-1}}{(a-1) !} \frac{m_{o}}{P_{n}} e^{-k_{4} t}=\frac{\left(k_{4} t\right)^{a-1}}{(a-1) !} m_{n}
$$

$$
m_{o}=m_{1}+m_{n} P_{n}+m_{n-\gamma}\left(P_{n}-\gamma\right)+\ldots m_{n-(a-1) \gamma}\left[P_{n}-(a-1)_{\gamma}\right],
$$

where $m_{n}^{x}$, etc., is the number of chains of length $P_{n}$ etc., having one activated chain end each; $k_{4}, k_{5}$, and $k_{6}$ are rate constants for initiation, propagation, and termination; $m_{1}$ the number of free monomer units in the system, and $m_{o}$ the total number of monomer units, free or bound, in the reaction mixture.

Two cases may be considered: (1) The kinetic chain length $\gamma$ is smaller than the original polymer chain length $P_{n}$; and (2) the kinetic chain length is equal or larger than the original polymer chain. Case 1: $\quad \gamma<P_{n}$.

The amount of monomer produced at any time $t$ is given by

$$
\frac{m_{1}}{m_{o}}=1-\frac{1}{P_{n}} e^{-k_{4} t} \sum_{a-1=0}^{a-1} \frac{\left(k_{4} t\right)^{a-1}}{(a-1) !}\left[P_{n}-(a-1)_{\gamma}\right] .
$$

The number average chain length at any time $t$ is approximately given by

$$
P_{t}=\frac{P_{n}}{s+1}=\frac{P_{n}}{P_{n}+e^{-k_{4} t}\left\{\sum_{a-1=0}^{a-1} \frac{\left(k_{4} t\right)^{a-1}}{(a-1) !}\left[1-P_{n}+(a-1)_{\gamma}\right]\right\}} .
$$


The number average chain length not considering the monomer is

$$
P_{t}^{\prime}=\frac{\left(m_{o}-m_{1}\right) P_{n}}{m_{o} e^{-k_{4} t} \sum_{a-1=0}^{a-1} \frac{\left(k_{4} t\right)^{a-1}}{(a-1) !}} .
$$

If only the initial stages for this case are considered, where the number of original chains that have been degraded completely is negligible, then

$$
d m_{1} / d t=k_{4} \gamma\left[m_{n}+m_{n-\gamma}+\ldots m_{\gamma}\right]
$$

or as the expression in the bracket must be equal to the initial number of chains $m_{o} / P_{n}$ in the system: $d m_{1} / d t=k_{4} \gamma\left(m_{o} / P_{n}\right)$. Finally,

$$
m_{1}=k_{4} \gamma \frac{m_{o}}{P_{n}} t .
$$

A zero-order reaction is thus obtained. ${ }^{2}$

Equation (5) provides a means to determine the number-average chain lengths, since the following relationship between two homogeneous polymer samples is valid at any time $t$ of the degradation process, provided $m_{o}$ is the same for both samples.

$$
\frac{m_{1, \mathrm{I}}}{m_{1, \mathrm{II}}}=\frac{P_{n, \mathrm{II}}}{P_{n, \mathrm{I}}} .
$$

Further, for the initial stages of the degradation

$$
P_{t}=\frac{P_{n}}{s+1}=\frac{m_{o}}{m_{1}+\frac{m_{o}}{P_{n}}}=\frac{P_{n}}{k_{4} \gamma t+1} .
$$

Therefore,

and

$$
s=k_{4} \gamma t
$$

$$
m_{1}=\frac{s m_{o}}{P_{n}}=\alpha m_{o}
$$

where $\alpha$ is usually called the degree of degradation.

Case 2: $\quad \gamma \geqq P_{n}$ or $a=1$.

In this case the number of monomer units at any time $t$ is given by

$$
\frac{m_{1}}{m_{o}}=1-e^{-k_{4} t}
$$

Equation (9) shows that the amount of monomer produced in this case is independent of the initial chain length. ${ }^{3}$

2 This result was obtained previously [5]. It was assumed then that $d m_{1} / d t=k . g$, where $g$ is the number of chain ends, which remains constant; therefore, $m_{1}=k \cdot g . t=k_{\text {exptl. }}$. .

3 This result was obtained previously [5]. We had then $-d g / d t=k . g$, where $g$ is the number of chain ends at time $t$. Because $g=g_{0}-2 m_{1} / P_{n}$, one obtains finally for 1 mole of polymer, $m_{1} / \mathrm{P}_{n}=1-e^{-k \ell}$, or for 1 base mole, $m_{1} / m_{0}=1-e^{-k t}$. 
The number average chain length is given by

$$
P_{t}=\frac{1}{1+e^{-k_{4} t}\left(\frac{1}{P_{n}}-1\right)},
$$

or

Therefore,

$$
P_{t} \cong \frac{1}{1-e^{-k_{4} l}} \cong \frac{m_{o}}{m_{1}} .
$$

$$
s \cong \frac{m_{1} P_{n}}{m_{0}} \cong P_{n}\left(1-e^{-k_{4} t}\right) .
$$

The number-average chain length and also the weight-average chain length, without considering the monomer units, must remain constant throughout the degradation process

$$
P_{t}^{\prime}=\frac{P_{n} \frac{m_{o}}{P_{n}} e^{-k_{4} t}}{\frac{m_{o}}{P_{n}} e^{-k_{4} t}}=P_{n} .
$$

Figure 1.1 shows the average number of broken links, $s$, for the chain theory $\left(P_{n}<\gamma\right.$ and $\left.P_{n}>\gamma\right)$ and the random process plotted against time $\left(P_{n}=10^{3}\right)$. It is seen that when $P_{n}>\gamma$ a straight-line relationship is obtained over a very considerable part of the reaction, whereas for $P_{n}<\gamma$, and for the random process, a departure from this straight line takes place. The two latter processes follow the same curve.

Figure 1.2 shows the amount of monomer in moles/base mole produced for these cases. Here again, for $P_{n}>\gamma$ a straight-line

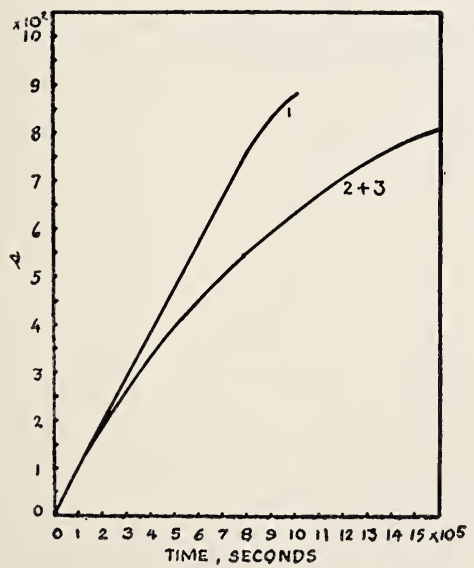

FIGURE 1.1. Number of average breaks, s, per original chain as a function of time.

$1, P_{n}>\gamma, k_{4}=10^{-5} \mathrm{sec}^{-1} ; 2, P_{n}<\gamma, k_{4}=$ $10^{-6}$ sec $^{-1} ; 3$, random case, $P_{n}=10^{3}$, $k=10^{-6} \mathrm{sec}^{-1}$.

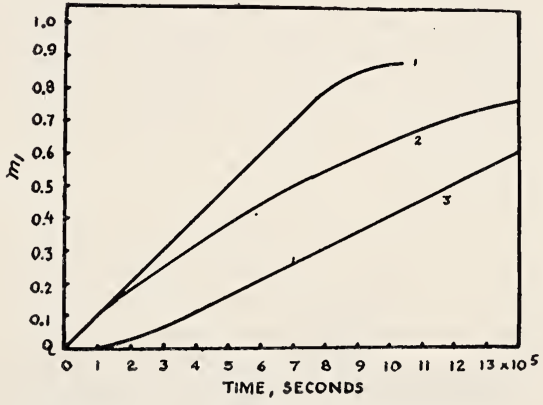

Figure 1.2. Monomer production as a function of time.

1 , Chain theory, $P_{n}>\gamma ; 2$, chain theory, $P_{n}<\gamma$; 3 , random theory. Rate constants and initial chain length as in figure 1.1 
relationship holds up to about 80 percent of the reaction; if $P_{n}<\gamma$, a departure from this straight line takes place. The random case shows an apparent induction period, but goes over eventually into a straight line.

Figure 1.3 shows the number-average chain length $P_{t}$ plotted against time for these cases. The random case and the case when $P_{n}<\gamma$ fall on the same curve.

The most significant differences for these three cases, however, are shown by plotting the $P_{t}^{\prime}$ values, that is, the number-average chain length, not considering the monomer, against time (fig. 1.4). A very rapid decrease in $P_{t}^{\prime}$ takes place for the random process. No decrease at all occurs when $P_{n}<\gamma$, and a moderate decrease takes place when $P_{n}>\gamma$. The same, of course, holds good for the respective weight average chain lengths, $P_{w}$.

The characteristic features of these types of degradation are summarized in table 1.1 .

TABLE 1.1. Summary of characteristics of various types of degradation

\begin{tabular}{|c|c|c|c|c|}
\hline $\begin{array}{l}\text { Type of degra- } \\
\text { dation }\end{array}$ & $s$ as function of time & $\begin{array}{l}m_{1} \text { as function of time } \\
\text { for given } m_{0}\end{array}$ & $P_{t}^{\prime}$ and $P_{w}$ & $P_{t}$ \\
\hline $\begin{array}{l}P_{n}<\gamma \\
\text { Random... } \\
\text { Weak link. }\end{array}$ & $\begin{array}{l}\text { Straight-line rela- } \\
\text { tionship over ap- } \\
\text { preciable part of } \\
\text { reaction. } \\
\text { Nonlinear relation- } \\
\text { ship; same as in } \\
\text { random case. } \\
\text { Nonlinear relation- } \\
\text { ship; same as } \\
P_{n}<\gamma \text {. } \\
\text { Nonlinear relation- } \\
\text { ship; stops when } \\
\text { all weak links are } \\
\text { broken. }\end{array}$ & $\begin{array}{l}\text { Straight-line rela- } \\
\text { tionship over ap- } \\
\text { preciable part of } \\
\text { reaction, depend- } \\
\text { ent on } P_{n} \text {. } \\
\text { Nonlinear relation- } \\
\text { ship, independent } \\
\text { of } P_{n} \text {. } \\
\text { Apparent induction } \\
\text { period; then al- } \\
\text { most linear. } \\
\text { Negligible...- }\end{array}$ & $\begin{array}{l}\text { No decrease } \\
\text { Rapid decrease...- } \\
\text { Fairly rapid de- } \\
\text { c re as e; stops } \\
\text { when all weak } \\
\text { links are broken. }\end{array}$ & $\begin{array}{l}\text { No significant } \\
\text { d ifference. } \\
P_{n}<\gamma \text { and ran- } \\
\text { dom case give } \\
\text { same curve. } \\
\\
\text { Stops at definite } \\
P_{\ell} \text { when all } \\
\text { weak links are } \\
\text { broken. }\end{array}$ \\
\hline
\end{tabular}

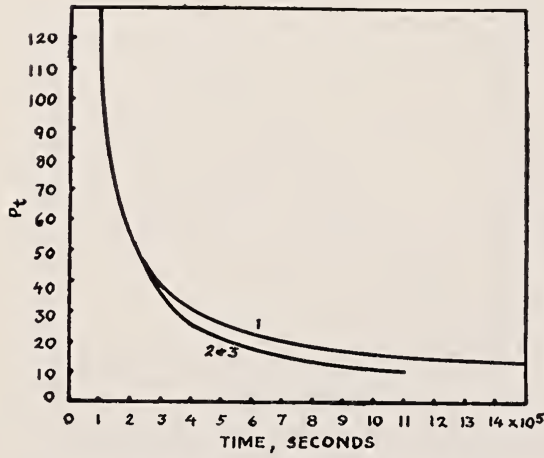

Figure 1.3. Number-averiage chain length as a function of time.

$1, P_{n}>\gamma ; 2, P_{n}<\gamma ; 3$, random theory. Compare figures 1.1 and 1.2 .

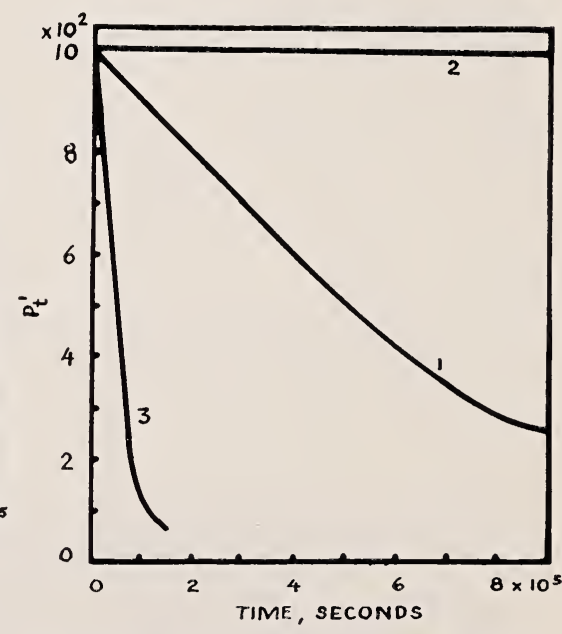

FIGURE 1.4. Number-average chain length without monomer as a function of time.

$1, P_{n}>\gamma ; 2, P_{n}<\gamma ; 3$, random theory. 
FigOre 1.5. Intrinsic viscosily as a function of number-average molecular weight of fraclionaled polystyrene samples.

1, Aecording to Alfrey et al. [7]; sample polymerized at $180^{\circ} \mathrm{C}$ 2 , aeeording to Bawn et al. [8] sample polymerized at $120^{\circ} \mathrm{C}$; 3 , aeeording to Alfrey et al. [7]; sample polymerized at $180^{\circ} \mathrm{C}$.

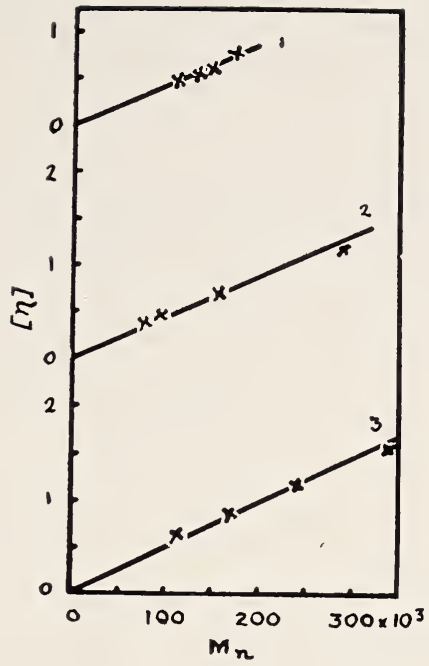

The question arises as to which relationship has to be chosen between intrinsic viscosity and molecular weight in order to evaluate intrinsic viscosity measurements made at various stages of the degradation process. It is not intended to enter into a detailed discussion as to which relationship is the correct one, but a purely utilitarian attitude will be adopted; only polystyrene is considered. G. V. Schulz [6] maintains, on the basis of numerous experimental data, that just in the case of polystyrene can the Staudinger rule be applied, whereas other authors hold that a relationship of the following type is obeyed, $[\eta]=K M^{\alpha}$.

If molecular weights up to about 300,000 to 400,000 are considered, it emerges that the Staudinger rule is sufficiently accurate to be used to evaluate degradation data. Results obtained by Mark and coworkers [7], and Bawn and coworkers [8] are shown in figure 1.5, where intrinsic viscosities are plotted against the corresponding molecular weights obtained by osmotic measurement. It is seen that the straight line relationship is fairly well obeyed; above a molecular weight of about 400,000 , however, the deviations from the straight line become appreciable.

\section{Degradation-polymerization equilibria}

It is assumed that the reaction proceeds in a closed system, that is, no monomer is allowed to escape from the system; then a degradation-polymerization process can be worked out, where the monomer polymerizes again to a number average chain length, $P_{e}$. A homogeneous polymer sample may be considered. The degradation reaction proceeds as before:

Case 1: $P_{n}=a \gamma, P_{e}<\gamma, P_{n}>\gamma$. 


\section{DEGRADATION}

Initiation:

$$
\begin{gathered}
\frac{d m_{n, d}^{x}}{d t}=k_{4} m_{n} \\
\frac{d m_{n-\gamma, d}^{x}}{d t}=k_{4} m_{n-\gamma} \\
\cdots \cdots \cdots \cdots \\
\frac{d m_{n-(a-1) \gamma, d}^{x}}{d t}=k_{4} m_{n-(a-1) \gamma} \\
\frac{d m_{e}^{x}}{d t}=k_{4} m_{\varrho .}
\end{gathered}
$$

Propagation:

$$
\left[\frac{d m_{1}}{d t}\right]_{D}=\gamma\left[\frac{d m_{n, d}^{x}}{d t}+\frac{d m_{n-\gamma, d}^{x}}{d t}+\cdots \frac{d m_{n-(a-1) \gamma, d}^{x}}{d t}\right]+P_{e} \frac{d m_{e}^{x}}{d t}
$$

or

$$
\left[\frac{d m_{1}}{d t}\right]_{D}=k_{5} m_{n, d}^{x}+k_{5} m_{n-\gamma_{, d}}^{x}+\ldots k_{5} m_{n-(a-1) \gamma, d}+k_{5} m_{e, d}^{x} .
$$

Termination:

$$
\begin{gathered}
-\frac{d m_{n, d}^{x}}{d t}=k_{6} m_{n, d}^{x} \\
\cdots \cdots \cdots \cdots \\
-\frac{d m_{n}^{x}-(a-1) \gamma, d}{d t}=k_{6} m_{n-(a-1) \gamma, d}^{x} \\
-\frac{d m_{e, d}^{x}}{d t}=k_{6} m_{e, d}^{x} \\
\gamma=\frac{k_{5}}{k_{6}}, \text { as before. }
\end{gathered}
$$

Polymerization may be considered to proceed as follows: Initiation:

$$
\frac{d m_{p}^{x}}{d t}=k_{1} m_{1}
$$

Propagation:

Termination:

$$
-\left[\frac{d m_{1}}{d t}\right]_{p}=k_{2} m_{p}^{x} m_{1}=\frac{d m_{p}^{x}}{d t} P_{e}
$$

$$
-\frac{d m_{p}^{x}}{d t}=k_{3} m_{p}^{x} m_{1}
$$


This polymerization process is, of course, assumed to be very simple. However, the main features of the combined process will be well brought out.

The total rate of monomer production is then

$$
\frac{d m_{1}}{d t}=k_{4} \gamma\left(m_{n}+m_{n-\gamma}+\ldots . m_{n-(a-1) \gamma}\right)+P_{e} k_{4} m_{e}-P_{e} k_{1} m_{1} .
$$

Further,

$$
\begin{gathered}
m_{n}=\frac{m_{o}}{P_{n}} e^{-k_{4} t} \\
\ldots \ldots \ldots \ldots \ldots \\
m_{n-(a-1) \gamma}=\frac{\left(k_{4} t\right)^{a-1}}{(a-1) !} \frac{m_{o}}{P_{n}} e^{-k_{4} t}=\frac{\left(k_{4} t\right)^{a-1}}{(a-1) !} m_{n}
\end{gathered}
$$

and

$m_{o}=m_{1}+m_{n} P_{n}+m_{n-\gamma}\left(P_{n}-\gamma\right)+\ldots+m_{n-(a-1) \gamma}\left[P_{n}-(a-1) \gamma\right]+m_{e} P_{e}$.

Therefore,

$$
m_{e} P_{e}=m_{o}-m_{1}-\frac{m_{o}}{P_{n}} e^{-k_{4} t} \sum_{a-1=0}^{a-1} \frac{\left(k_{4} t\right)^{a-1}}{(a-1) !}\left[P_{n}-(a-1) \gamma\right] .
$$

Hence,

$\frac{d m_{1}}{d t}=k_{4} \frac{m_{o}}{P_{n}} e^{-k_{4} t} \sum_{a=1=0}^{a-1} \frac{\left(k_{4} t\right)^{a-1}}{(a-1) !}\left[\gamma-P_{n}+(a-1) \gamma\right]+k_{4} m_{o}-m_{1}\left(k_{1} P_{e}+k_{4}\right)$, which yields on integration,

$$
\begin{aligned}
\frac{m_{1}}{m_{o}}= & e^{-\left(k_{4}+k_{1} P_{e}\right) t} \frac{1}{P_{n}}\left\{\sum _ { a - 1 = 0 } ^ { a - 1 } \frac { k _ { 4 } ^ { a } e ^ { k _ { 1 } P _ { e } t } } { k _ { 1 } P _ { e } ( a - 1 ) } \left[t^{a-1}-\frac{a-1}{k_{1} P_{e}} t^{a-2}\right.\right. \\
& \left.\left.\ldots+(-1)^{a-1} \frac{(a-1) !}{\left(k_{1} P_{e}\right)^{a-1}}\right]\left[\gamma-P_{n}+(a-1) \gamma\right]\right\} \\
& -\frac{1}{P_{n}} e^{-\left(k_{4}+k_{1} P_{e}\right) t} \sum_{a-1=0}^{a-1} \frac{k_{4}^{a}(-1)^{a-1}}{\left(k_{1} P_{e}\right)^{a}(a-1)}\left[\gamma-P_{n}+(a-1) \gamma\right] \\
& +\frac{k_{4}}{k_{4}+k_{1} P_{e}}\left(1-e^{-\left(k_{4}+k_{1} P_{e}\right) t}\right) .
\end{aligned}
$$

The number average chain length is given by

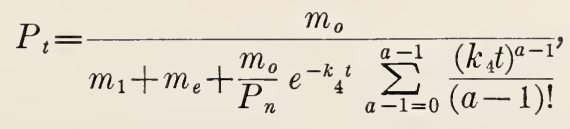

and the number average chain length, not considering the monomer, by

$$
P_{t}^{\prime}=\frac{m_{o}-m_{1}}{m_{e}+\frac{m_{o}}{P_{n}} e^{-k_{4} t} \sum_{a-1}^{a-1} \frac{\left(k_{4} t\right)^{a-1}}{(a-1) !}} .
$$


At $t=\infty$ or at equilibrium, the amount of monomer is given by

$$
\frac{m_{1, \infty}}{m_{o}}=\frac{k_{4}}{k_{4}+k_{1} P_{e}} .
$$

Further,

$$
\frac{m_{e, \infty}}{m_{o}}=1-\frac{k_{1}}{k_{4}+k_{1} P_{e}}=\frac{k_{1}}{k_{4}+k_{1} P_{e}} .
$$

Therefore

$$
\frac{m_{1, \infty}}{m_{e, \infty}}=\frac{k_{4}}{k_{1}}
$$

It remains now to consider the process starting with monomer. In this case the polymerization reaction is the same as before. The degradation reaction is given by

Initiation:

$$
\frac{d m_{e, d}^{x}}{d t}=k_{4} m_{e}
$$

Propagation:

$$
+\left[\frac{d m_{1}}{d t}\right]_{D}=\frac{d m_{e, d}^{x}}{d t} P_{e}=k_{4} P_{e} m_{e}=k_{5} m_{e, d}^{x}
$$

Termination:

$$
-\frac{d m_{e, d}^{x}}{d t}=k_{6} m_{e, d}^{x}
$$

Therefore, the total rate of disappearance of monomer is

$$
-\frac{d m_{1}}{d t}=k_{1} P_{e} m_{1}-k_{4} P_{e} m_{e}
$$

Further,

$$
m_{e} P_{e}=m_{o}-m_{1}
$$

Therefore,

$$
\frac{m_{1}}{m_{0}}=\frac{k_{4}+k_{1} P_{e} e^{-\left(k_{1} P_{e}+k_{4}\right) t}}{k_{1} P_{e}+k_{4}} .
$$

At equilibrium, that is, $t=\infty$,

$$
\frac{m_{1, \infty}}{m_{o}}=\frac{k_{4}}{k_{1} P_{e}+k_{4}} .
$$

Further,

$$
\frac{m_{e}}{m_{o}}=\frac{k_{1}\left(1-e^{-\left(k_{1} P_{e}+k_{4}\right) t}\right)}{k_{1} P_{e}+k_{4}} .
$$

Therefore,

$$
\frac{m_{e, \infty}}{m_{o}}=\frac{k_{1}}{k_{1} P_{e}+k_{4}} .
$$


Finally,

$$
\frac{m_{1, \infty}}{m_{e, \infty}}=\frac{k_{4}}{k_{1}}, \text { as before. }
$$

The number average chain length is given by

$$
P_{t}=\frac{k_{1} P_{e}+k_{4}}{k_{1} e^{-\left(k_{1} P_{e}+k_{4}\right) t}\left(P_{e}-1\right)+k_{4}+k_{1}},
$$

and at equilibrium,

$$
P_{t, \infty}=\frac{k_{1} P_{e}+k_{4}}{k_{4}+k_{1}}
$$

Of course, the chain length, not considering the monomer, remains constant:

Case 2: $P_{n} \leqq \gamma$ or $a=1$.

$$
P_{t}^{\prime}=P_{e}
$$

Equation (15) reduces then to

Further,

$$
\frac{m_{1}}{m_{0}}=\frac{k_{4}}{k_{4}+k_{1} P_{e}}\left(1-e^{-\left(k_{4}+k_{1} P_{e}\right) t}\right) .
$$

$$
\frac{m_{e}}{m_{0}}=\frac{k_{4}+k_{1} P_{e}-k_{4}\left(1-e^{-\left(k_{4}+k_{1} P_{e}\right) t}\right)-\left(k_{4}+k_{1} P_{e}\right) e^{-k_{4} t}}{P_{e}\left(k_{4}+k_{1} P_{e}\right)} .
$$

At equilibrium we have

and

$$
\frac{m_{1, \infty}}{m_{0}}=\frac{k_{4}}{k_{4}+k_{1} P_{e}}
$$

Finally,

$$
\frac{m_{e, \infty}}{m_{0}}=\frac{k_{1}}{k_{4}+k_{1} P_{e}} \text {. }
$$

$$
\frac{m_{1, \infty}}{m_{e, \infty}}=\frac{k_{4}}{k_{1}}
$$

The number average chain length is given by,

$$
P_{t}=\frac{P_{n} P_{e}}{e^{-k_{4} t}\left(P_{e}-P_{n}\right)+\frac{k_{4} P_{n} e^{-\left(k_{4}+k_{1} P_{e}\right) t}\left(1-P_{e}\right)}{k_{4}+k_{1} P_{e}}+\frac{P_{n} P_{e}\left(k_{4}+k_{1}\right.}{k_{4}+k_{1} P_{e}}} .
$$

And without monomer,

$$
P_{t}^{\prime}=\frac{P_{n} P_{e}\left(k_{1} P_{e}+k_{4} e^{-\left(k_{4}+k_{1} P_{e}\right) t}\right)}{k_{1} P_{e} P_{n}\left(1-e^{-k_{4} t}\right)+k_{4} P_{n}\left(e^{-\left(k_{4}+k_{1} P_{e}\right) t}-e^{-k_{4} t}\right)+P_{e} e^{-k_{4} t}\left(k_{4}+k_{1} P_{e}\right)} \cdot
$$

It will be of interest to prove that also for degradation polymerization processes of this type, the following relationship always holds:

$$
P_{t} \equiv \frac{P_{n}}{s+1}
$$


The number of broken links in the whole system is given by

$$
\frac{m_{o}}{P_{n}} s=\left(\frac{m_{o}}{P_{n}}-m_{n}\right)\left(P_{n}-1\right)-m_{e}\left(P_{e}-1\right) .
$$

Putting the relevant values for $m_{n}=m_{o} / P_{n} e^{-k_{4} t}$ and $m_{e}$ (eq 30) into the above equation, solving for $s$, and putting the expression for $s$ into eq (36), an expression identical with that of eq (34) is obtained.

Figure 1.6 shows the amount of monomer $m_{1}$ and monomer bound in $P_{e}$ chains $\left(m_{e} P_{e}\right)$ at any time $t$ of the reaction $\left(P_{n}=10^{3}\right)$, starting from pure polymer and pure monomer; $m_{1}$ produced, coming from the polymer side, is at any time equal to $m_{e} P_{e}$, starting with pure monomer.

Figure 1.7 shows the decrease of $P_{t}^{\prime}$, starting with pure polymer; $P_{t}^{\prime}$, starting with pure monomer, remains constant and equal to $P_{e}$.

The calculations, starting with pure monomer, are the same as before, and can also be carried through for $P_{n}<\gamma$, assuming a secondorder initiation reaction for the polymerization process.

In that case, the rate of production of monomer is $d m_{1} / d t=k_{4} P_{n} m_{n}+$ $k_{4} P_{e} m_{e}-k_{1} P_{e} m_{1}^{2}$. Further, $m_{o}=m_{1}+m_{e} P_{e}+m_{n} P_{n}$. Therefore,

$$
\frac{m_{1}}{m_{o}}=\frac{2 k_{4}\left(1-e^{-\left(k_{4}^{2}+4 k_{1} P_{e} k_{4} m_{o}\right)^{1 / 2}}\right)}{k_{4}+\left(k_{4}^{2}+4 k_{1} P_{e} k_{4} m_{o}\right)^{1 / 2}-\left[k_{1}-\left(k_{4}^{2}+4 k_{1} P_{e} k_{4} m_{o}\right)^{1 / 2}\right] e^{-\left(k_{4}^{2}+4 k_{1} P_{e} k_{4} m_{o}\right)^{1 / 2}}} .
$$

At equilibrium,

and

$$
\frac{m_{1, \infty}}{m_{o}}=\frac{2 k_{4}}{k_{4}+\left(k_{4}^{2}+4 k_{1} P_{e} k_{4} m_{o}\right)^{1 / 2}}
$$

At equilibrium,

$$
\frac{m_{e}}{m_{o}}=\frac{1}{P_{e}}\left(1-e^{-k_{4} t}\right)-\frac{m_{1}}{P_{e}}
$$

$$
\frac{m_{e, \infty}}{m_{o}}=\frac{\left(k_{4}^{2}+4 k_{1} P_{e} k_{4} m_{o}\right)^{1 / 2}}{P_{e}\left[k_{4}+\left(k_{4}^{2}+4 k_{1} P_{e} k_{4} m_{o}\right)^{1 / 2}\right]} .
$$

Therefore

$$
\frac{m_{1, \infty}}{m_{e, \infty}}=\frac{2 k_{4} P_{e}}{\left(k_{4}^{2}+4 k_{1} P_{e} k_{4} m_{o}\right)^{1 / 2}-k_{4}}=\frac{k_{4}+\left(k_{4}^{2}+4 k_{1} P_{e} k_{4} m_{o}\right)^{1 / 2}}{2 k_{1} m_{o}} .
$$

Finally, as

$$
\begin{gathered}
m_{1}^{2}=\frac{4 k_{4}^{2} m_{o}^{2}}{\left[k_{4}+\left(k_{4}+4 k_{1} P_{e} k_{4} m_{o}\right)^{1 / 2}\right]^{2}} \\
\frac{m_{1, \infty}^{2}}{m_{e, \infty}}=\frac{k_{4}}{k_{1}} .
\end{gathered}
$$

The number average chain length is given by $P_{t}=m_{o} /\left(m_{1}+m_{e}+m_{n}\right)$, where $m_{1}$ and $m_{e}$ are given by eq. (37) and (39), respectively, and $m_{n}=\frac{m_{o n}}{P_{n}} e^{-k_{4}{ }^{t}}$. At equilibrium, this reduces to

$$
P_{t, \infty}=\frac{P_{e}\left[k_{4}+\left(k_{4}^{2}+4 k_{1} P_{e} k_{4} m_{o}\right)^{1 / 2}\right]}{2 k_{4}\left(P_{e}-1\right)+k_{4}+\left(k_{4}^{2}+4 k_{1} P_{e} k_{4} m_{o}\right)^{1 / 2}} .
$$




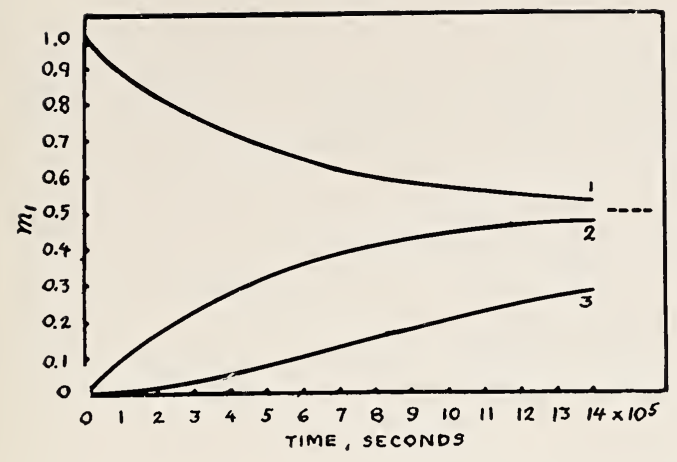

Figure 1.6. Degradation polymerization processes.

$P_{n}=10^{3}, P_{e}=10^{2}, P_{n}<\gamma, k_{4}=10^{-6} \mathrm{sec}^{-1}$, $k_{1}=10^{-8} \mathrm{sec}^{-1}$. 1, Decrease of monomer with time, starting with pure monomer; 2 , increase of monomer with time, starting with pure polymer, and increase in $P_{\theta} m_{\theta}$, starting with pure monomer; 3 , increase of with pure monomer; 3 , increase of
$P_{e} m_{\epsilon}$, starting with pure polymer.

Figure 1.7. Numberaverage chain length without monomer as a function of time.

$P_{n}=10^{3}, P_{n}<\gamma, P_{e}=10^{2}$, $k_{4}=10^{-6} \mathrm{Sec}^{-1}, \quad k_{1}=10^{-8} \mathrm{sec}^{-1}$.

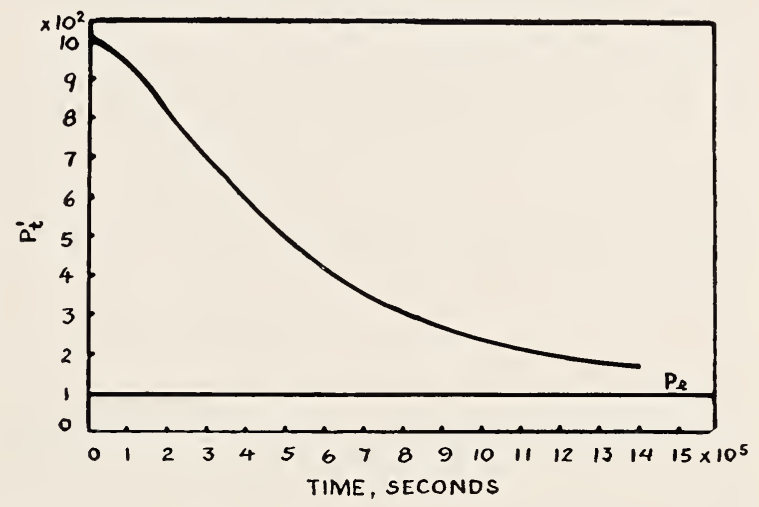

The number average chain length without monomer is given by

$$
P_{t}^{\prime}=\frac{P_{e}\left[k_{4}+A-\left(k_{4}-A\right) e^{-A t}-2 k_{4}\left(1-e^{-A t}\right)\right]}{\left(1-e^{-k_{4} t}\right)\left[k_{4}+A-\left(k_{4}-A\right) e^{-A t}\right]-2 k_{4}\left(1-e^{-A t}\right)},
$$

where $A=\left(k_{4}^{2}+4 k_{1} P_{e} k_{4} m_{o}\right)^{1 / 2}$, which reduces for $t=\alpha$ to

$$
P_{t, \infty}^{\prime}=P_{e} \text {. }
$$

If the process is considered from the monomer side, then the following relationships are obtained:

$$
\begin{aligned}
\frac{d m_{1}}{d t} & =k_{4} P_{e} m_{e}-k_{1} P_{e} m_{1}^{2}, \\
m_{o} & =m_{1}+P_{e} m_{o}, \\
\frac{m_{1}}{m_{o}} & \left.=\frac{\left(A-k_{4}\right) e^{-A t}+A+k_{4}}{2 k_{1} P_{e} m_{o}+k_{4}+A-\left(2 k_{1} P_{e} m_{o}+k_{4}-A\right) e^{-A t}}\right) .
\end{aligned}
$$

Therefore,

$$
\frac{m_{1, \infty}}{m_{n}}=\frac{k_{4}+A}{2 k_{1} P_{e} m_{o}+k_{4}+A}=\frac{2 k_{4}}{k_{4}+A} .
$$


Further,

$$
\frac{m_{e}}{m_{o}}=\frac{1}{P_{e}}-\frac{\left(A-k_{4}\right) e^{-A t}+A+k_{4}}{P_{e}\left[2 k_{1} P_{e} m_{o}+k_{4}+A-\left(2 k_{1} P_{e} m_{o}+k_{4}-A\right) e^{-A t}\right.},
$$

and

Therefore,

$$
\frac{m_{e, \infty}}{m_{o}}=\frac{1}{P_{e}}-\frac{A+k_{4}}{P_{e}\left[2 k_{1} P_{e} m_{o}+k_{4}+A\right]} .
$$

and

$$
\frac{m_{1, \infty}}{m_{e, \infty}}=\frac{k_{4}+A}{2 k_{1} m_{o}}
$$

$$
\frac{m_{1, \infty}^{2}}{m_{e, \infty}}=\frac{k_{4}}{k_{1}}, \text { as before. }
$$

The number average chain length is given by

$$
P_{t}=\frac{m_{o}}{m_{e}+m_{1}}
$$

or, not considering the monomer, by

$$
P_{t}^{\prime}=\frac{m_{e} P_{e}}{m_{e}}=P_{e} .
$$

If the initiation reaction for the polymerization is of the second order and the termination reaction is proportional to $m_{p}^{x 2}$, the same equations hold as in the previous case, only then $P_{e}$ is given by $k_{2}\left(k_{3} / k_{1}\right)^{-\frac{1}{2}}$ instead of $k_{2} / k_{3}$.

The cases where $P_{e}$ does not remain constant become much more complicated.

\section{Discussion of Experimental Results}

\section{Thermal Degradation in Bulk at Moderate Temperatures}

As was pointed out in the introduction, it is not intended to go into all the details of the degradation of vinyl polymers, but to discuss the principal reactions of the degradation process.

The thermal bulk degradation of polystyrene in vacuum over a temperature range of $248^{\circ}$ to $340^{\circ} \mathrm{C}$ may be considered first [9].

Figure 1.8 shows the decrease of the intrinsic viscosity with time for two fractionated samples at a number of temperatures [9]. It is seen that the intrinsic viscosities decrease rapidly at first, then the rate slows down, until degradation comes to an apparent standstill. According to the random theory, the average number of breaks in each original chain should be a linear function of the time of reaction as long as $s$, the average number of breaks, remains small. This is not the case. A rapid departure from linearity takes place. Further, the energy of activation derived from the rate constants of the initial part of the degradation has a very low value of $21.9 \mathrm{kcal}$.

There are a number of possibilities that might explain the observed deviation from the random theory: 1, Inhibitors might be formed dur- 
ing the degradation. 2, The walls of the reaction vessel might act catalytically. 3, The rate constants might depend on chain length. 4, An equilibrium between degradation and polymerization might be operative. 5, A number of weak links might be distributed along the polymer chains, and once these weak links have been broken the degradation stops.

It was shown by experiment that the walls of the reaction vessel did not influence the reaction, that the rate constants were independent of chain length, and that an equilibrium was not involved. As far as inhibitors are concerned, these would explain the slowing down of the reaction, but not its low energy of activation, which is quite insufficient to account for breaking a $\mathrm{C}-\mathrm{C}$ link.

It was thought most reasonable to assume weak links to be randomly distributed along the polymer chains, and such a mechanism was worked out in detail. Moreover, as it is well known that oxygen has a profound effect on the degradation as well as on the polymerization, it was likely that oxygen is somehow connected with these weak links. This assumption is supported by the findings of Jackson and Forsyth [10] who made an investigation of the power factor of polystyrene, which led to the conclusion that oxygen groups are incorporated in the polymer. Further Madorsky and coworkers [11] always found some carbon monoxide evolved during degradation of polystyrene, the amount of the gas being of such a magnitude that for each polymer chain a few atoms of oxygen would be available. The number of oxygen atoms found in this way agrees quite well with the number that was deduced from the kinetic experiments under discussion. It was also shown experimentally that these oxygen groups must be chemically bound to the polymer chains.

The kinetic results led further to the conclusion that not all of these oxygen groups lead to chain scission, but that some of these groups decompose without leading to rupture of the chain. Further, it had to be assumed that inhibitors are formed during the reaction. A re-

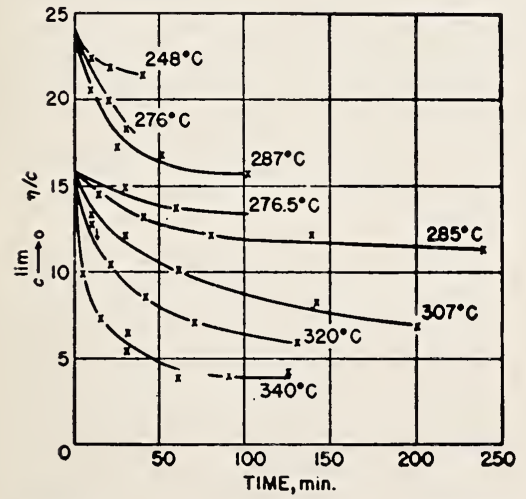

Figure 1.8. Decrease of limiting viscosity with time of two fractionated polystyrene samples [9].

Thermal degradation in vacuum.

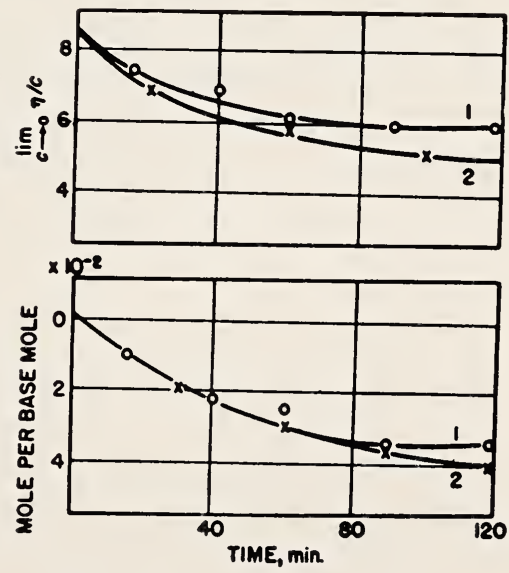

Figure 1.9. Decrease of limiting viscosity and simultaneous production of monomer during thermal degradation of fractionated polystyrene in vacuum [4]. 
action scheme was then obtained as follows [9]:

\section{Chain rupture.}

Weak links $\rightarrow$ Disappearance of weak links without leading to rupture.

Inhibitors.

An Arrhenius equation for the chain scission reaction gives an expression $k=1.33 \times 10^{5} e^{-24500 / R T} \mathrm{sec}^{-1}$.

The reaction scheme accounts satisfactorily for the low energy of actiration and the slowing down of the reaction. The small collision factor might have something to do with the small number of weak links in the whole system.

Howerer, the degradation reaction was found to be still more complicated. It was observed that an appreciable amount of monomer was produced during the reaction that could not be accounted for by a simple random degradation of links or weak links [4]. This production of monomer units ran strictly parallel to the number of weak links breaking as shown in table 1.2 and figure 1.9 [4].

\section{TABLE 1.2. Comparison of moles of monomer formed with number of chain breaks}

\begin{tabular}{|c|c|c|}
\hline \multirow{2}{*}{$\begin{array}{l}\text { A rerage num- } \\
\text { ber of breaks } \\
\text { per chain }\end{array}$} & \multicolumn{2}{|c|}{ Moles of monomer } \\
\hline & $\begin{array}{c}\text { Theoretical } \\
\text { (random) } \\
\text { per base mole }\end{array}$ & $\begin{array}{l}\text { Experimental } \\
\text { per base mole }\end{array}$ \\
\hline $\begin{array}{l}0.5 \\
1.0 \\
2.0\end{array}$ & $\begin{array}{l}1.3 \times 10^{-6} \\
3 \\
8\end{array}$ & $\begin{array}{l}0.8 \times 10^{-2} \\
1.5 \\
4.0\end{array}$ \\
\hline
\end{tabular}

This monomer production can be readily explained by the following mechanism: 1, Initiation: the rupture of a weak link whereby active chain ends are formed. 2, Propagation: A number of monomer units are split off successively from the newly formed chain ends. 3, Termination: The loss of actiration of the chain ends. Such a mechanism requires that the production of monomer units is proportional to the number of weak links broken. That this is the case is shown in figure 1.10 [4]. The number of monomer units per weak link ruptured could thus be calculated: 19.7 units were produced at $280^{\circ} \mathrm{C}$ and 12.6 units at $307^{\circ} \mathrm{C}$.

The work of Oakes and Richards [12] on polyethylene may be considered briefly. They also found, similarly as in the case of polystyrene, that the simple random theory could not account for their experimental results. A plot of the number of broken links against time departed rapidly from a straight line. At temperatures around $300^{\circ} \mathrm{C}$, they could explain their experimental results by assuming one weak link for every four polymer molecules. However, as the temperature was increased to about $360^{\circ} \mathrm{C}$, the assumption of one weak link in four polymer molecules no longer represented the experimental results; but it had to be assumed that different types of weak links are present, some of them rupturing at about $300^{\circ} \mathrm{C}$, others only rupturing at a temperature of about $360^{\circ} \mathrm{C}$. This is an unlikely assumption, and these authors preferred to assume a chain mechanism 
Figure 1.10. Moles of monomer produced as function of average number of broken bonds for the thermal degradation of fractionated polystyrene in vacuum [4].

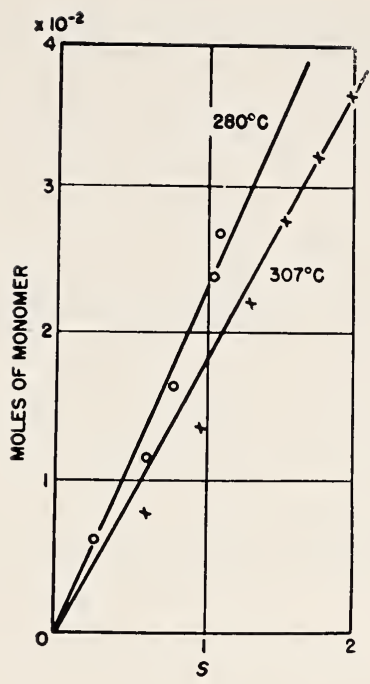

that is initiated by the rupture of a weak link. However, the apparent increase in the number of weak links can be explained easily by considering experiments carried out by Jellinek [5] in a vacuumbalance apparatus. It can be seen from these experiments that the production of small molecules, not necessarily ethylene, is quite appreciable. For instance, after about $2 \mathrm{hr}$, polyethylene of initial molecular weight 16,000 loses 10 percent of its original weight following a zeroorder reaction at $376^{\circ} \mathrm{C}$. The experiments of Oakes and Richards extended for about $40 \mathrm{hr}$ at $360^{\circ} \mathrm{C}$. Hence, the decrease of molecular weight after the initial rapid decrease is mainly due to a true depolymerization reaction, proceeding from the polymer chain ends. The high energy of activation of 60 to 70 kcal found by Richards and Oakes most likely pertains to the splitting off of small units from the polymer chain ends. From his zero-order reactions Jellinek found energies of activation increasing with molecular weight from 46 to 66 kcal. More molecular-weight determinations will have to be carried out on degradation products of polyethylene to establish definitely the mechanism of degradation for polyethylene.

\section{Elementary Steps}

The elementary steps for this mechanism of degradation of polystyrene are, of course, speculative, but some tentative suggestions may be presented.

It is most likely that the weak spots in polystyrene are formed during polymerization. The initiation reaction for this polymerization, provided small amounts of oxygen are present, can be postulated as follows:<smiles></smiles> 
Cyclization could occur readily, as this reaction would be strongly exothermic, $\Delta \mathrm{H}=-70 \mathrm{kcal}$ :<smiles>[R]C(CCC)COC</smiles>

The next step might consist in the formation of a radical:<smiles>[R]C1(C)COOC1([R])O</smiles>

Taking the values for the bond energies from the paper by Bolland and Gee [13], the following values for the heat of reaction are obtained:

$$
\Delta H=66+2 \times 99-2 \times 110-19=25 \mathrm{kcal} .
$$

Nineteen kcal are accounted for by the resonance energy for stabilizing the radical.

Objections may be raised against reaction (c) on the grounds that a reaction of the following type is energetically more favorable:<smiles>[R]C=CCCC=O</smiles>

The heat of this reaction is about $27 \mathrm{kcal}$ exothermic. However, it must be remembered that in order to get the polymerization going, only a relatively small amount of active nuclei has to be formed; that is, only a small fraction of the peroxide need react according to (c). Moreover, it is known that benzaldehyde is formed during the polymerization of styrene in the presence of oxygen and that conditions can be varied such that either polymerization or aldehyde formation is favored.

The nucleus formed in reaction (c) can then produce polymers by growing into a chain by propagation, termination, etc.

The degradation of polymer chains containing such hydroxyl groups can be pictured as follows. ${ }^{4}$ The points where the hydroxyl groups are attached to the chain constitute the weak points. The chain scission may proceed by formation of a keto and hydroxyl group, the chain ends so produced being activated:<smiles>[R]C(=O)C(C)O</smiles>

1 Compare in this connection a paper by L. H. Cross, R. B. Richards, and H. A. W illis [14], in which oxygen groups in ethylene polymers as found by infrared spectroscopy are discussed. These authors bring evidence that the following groups are present,<smiles>CCC(=O)C(O)CC</smiles> 
The heat of reaction for (e) amounts to $\Delta H=(81-19)+110-(87-19)$ $-99=5 \mathrm{kcal}$.

The next step may consist in the splitting off of monomer units from the chain ends:

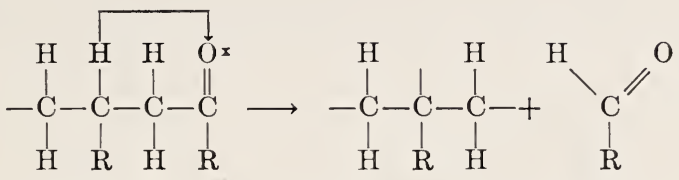

The heat of reaction for (f) is $\Delta H=(81-19)+(99-19)-(99-19)=$ $62 \mathrm{kcal}$. This heat of reaction is rather high, but this reaction could be formulated somewhat differently decreasing the heat of reaction to $43 \mathrm{kcal}$.

The next step can then consist in the production of monomer units:

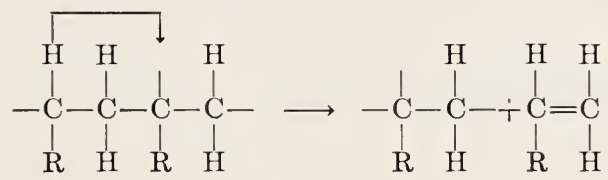

The heat of reaction for $(\mathrm{g})$ is $\Delta H=(81-19)+(99-19)-(99-19)-$ $64=-2 \mathrm{kcal}$. The termination reaction may be effected by the formation of a double bond, for example:<smiles>[R]/C=C\CC([R])C</smiles>

The mechanism for the chain end possessing the hydroxyl group is analogous to that outlined above.

The experimental results suggested further that not all of the weak points lead to chain scission; they may undergo other reaction, for example, dehydration:<smiles>[R]C(C)(O)C(O)C1([R])O[C@H]1C=O</smiles>

or perhaps even<smiles>[R]/C(=C/[CH2+][O-])C(O)C([R])(C)O</smiles> 


\section{Production of Monomer During Degradation}

The experiments on polystyrene were extended to a temperature region where the amount of monomer produced was quite appreciable. The degradation was studied in a vacuum spring-balance apparatus [5]; a typical set of curres for one fractionated polystyrene sample is given in figure 1.11. It can be seen that a zero order reaction is followed over quite an appreciable range of the reaction.

This reaction represents, then, what was termed above the "true" degradation or depolymerization reaction. Chain ends are activated; propagation consists in splitting off of monomer units until the chain ends are deactivated by the termination reaction. If the number of chain ends remains constant over an appreciable part of the reaction, then, as was shown above, a zero order reaction should result. The rate of monomer production is then given by eq (5),

$$
m_{1}=k_{4} \gamma \frac{m_{o}}{P_{n}} t,
$$

or the experimental rate constant by

$$
k_{\text {exptl. }}=k_{4} \gamma \frac{m_{o}}{P_{n}}
$$

The kinetic chain length can be estimated in the case of polymethyl methacrvlate, where it was shown by Melville and Grassie [15] that below a certain number average chain length the whole molecule degrades completely, once it has been activated.

The case of polystyrene is complicated by the weak links present in the polymer chains. This explains the initial rapid production of monomer as indicated by the experimental curves. Then an induction period follows, which can be explained by assuming that inhibitors are formed during the initial degradation. And eventually the true degradation takes its course.

It is possible to estimate the number of weak links in a polystyrene molecule, as the rate of degradation is proportional to the number of chain ends or chains in the system. If there are $X$ weak points on the arerage in one chain molecule, then, after all these weak links

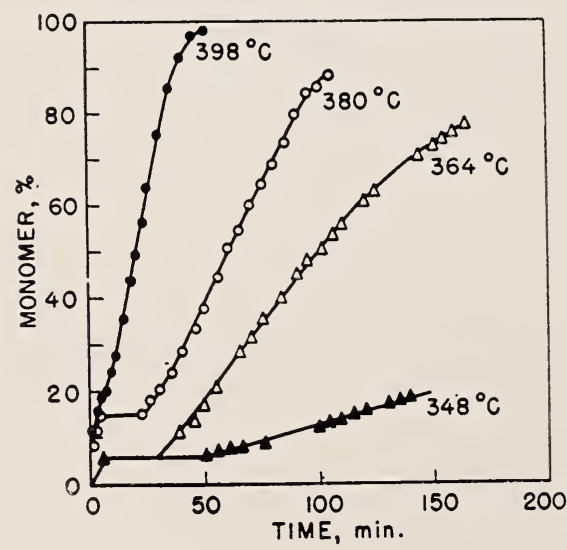

Figure 1.11. Percentage of monomer produced during thermal degradation of fractionated polystyrene in a vacuum spring-balance apparatus [5]. 
have been ruptured, the original chain length, $P_{n, \mathrm{I}}$, has decreased to a number a verage chain length: $P_{t}=P_{n, \mathrm{I}} /(X+1)$, and $k_{\text {expt1. }}$ is given by $k_{\text {expt1., I }}=k_{4} \gamma m_{o} /\left(P_{t, \mathrm{I}}\right)$.

Considering a second sample, similar relationships are valid: $k_{\text {expt1. }}$ $=k \gamma m_{o} /\left(P_{t, \mathrm{II}}\right)$. Therefore, $k_{\text {expt1., I }} / k_{\text {expt1., II }}=P_{t, \mathrm{II}} / P_{t, \mathrm{I}}$. Making the reasonable assumption that the number of weak links in a chain is proportional to its chain length, then $X_{\mathrm{I}} / X_{\mathrm{II}}=P_{n, \mathrm{I}} / P_{n, \mathrm{II}}$. From these equations it follows that

Therefore,

$$
\frac{k_{\text {expt1., I }}}{k_{\text {expt1..II }}}=\frac{P_{n, \mathrm{II}}}{P_{n, \mathrm{I}}} \frac{X_{\mathrm{I}}+1}{X_{\mathrm{I}} \frac{P_{n, \mathrm{II}}}{P_{n, \mathrm{I}}}+1}=\frac{X_{\mathrm{I}}+1}{X_{\mathrm{I}}+\frac{P_{n, \mathrm{I}}}{P_{n, \mathrm{II}}}} .
$$

$$
\frac{k_{\text {expt1., II }}}{k_{\text {expt1., I }}}=\frac{X_{\mathrm{I}}+\frac{P_{n, \mathrm{I}}}{P_{n, \mathrm{II}}}}{X_{\mathrm{I}}+1} .
$$

In this way the number of weak links could be calculated. They agreed satisfactorily with the number of weak links obtained from the intrinsic viscosity measurements of samples degraded at lower temperatures.

It is also possible to estimate roughly the upper limit of $\gamma$, the kinetic chain length, for polystrrene.

A fractionated sample may be considered of initial chain length of about 512. Such a chain was found to have about three weak links. If all weak links are ruptured, a number a verage chain length of about 130 is obtained. This sample follows a zero order reaction up to about 80 percent loss of weight at a temperature of $398^{\circ} \mathrm{C}$. The first 10-percent loss in weight is due to the monomer produced when weak links are ruptured, hence about eight monomer units are produced in this way for each newly formed chain end. Actually, the number of monomer units produced for each newly formed chain end will still be smaller, as at the same time monomer is split off the chain ends originally present. Further, the zero-order reaction is obeyed until about 30 percent of the chain length of 130 units is left, that is, the kinetic chain length due to this reaction must be smaller than about 40 monomer units.

The energy of activation obtained from the experimental rate constants was found to be $44.7 \mathrm{kcal}$ for all samples, irrespective of chain length, whereas the collision factor in the Arrhenius equation varied with the chain length, as was to be expected.

However, if the $k_{\text {expt1 }}$. rate constants are multiplied by the number average chain length after all weak links have been broken, then the rate constants can be expressed for all the different samples approximately by one Arrhenius equation

$$
k_{\text {exptl. }}^{\prime}=10^{15.55} e^{-44700 / R T}(\mathrm{~g} / \mathrm{sec}) / 100 \mathrm{~g} .
$$

As $k_{\text {exptl. }}^{\prime}=k_{4} \gamma m_{o}$, the rate constants can also be expressed in moles $\mathrm{sec}^{-1} /$ base mole.

Therefore, $k_{4} \gamma=10^{13.55} e^{-44700 / R T}(\mathrm{~g} / \mathrm{sec}) / \mathrm{g}$ or $(\mathrm{moles} / \mathrm{sec}) / \mathrm{b}$ ase mole.

Results obtained by Melville and Grassie [15] on polymethyl 
methacrylate can also be explained on the assumption that degradation takes place from the chain ends by initiation, propagation, and termination. In this case the kinetic chain length is very much longer than in the case of polystyrene. An upper estimate leads here to a kinetic chain length of about 2,000 monomer units. This has probably something to do with the fact that in the polymethyl methacrylate molecule the mobile $\alpha$-hydrogen has been replaced by a less reactive methyl group, for example:<smiles>[R]C(CCCC(C)C([R])CC)C(C)C</smiles>

First, the carbon-carbon link will be weaker in this case than in the case of polystyrene, and second, the mobile $\alpha$-hydrogen in polystyrene will probably facilitate the termination reaction by migrating to the monomer unit, which is in the process of being split off:<smiles>[R]C(=C)C([R])C([2H])(C)CC</smiles>

forming thus a diradical, which can easily form a double bond, thereby terminating the reaction before many monomer units have a chance to break off.

In the case of polymethyl methacrylate the process cannot be formulated in this way. It might be imagined that the following reaction takes place:<smiles>[R]/C=C\C([2H])C([R])C([R])(C)C([2H])CC</smiles>

A monoradical is formed and this has a much better chance to survive for a sufficiently long period than the diradical in the case of polystrrene. These suggestions are only tentative, and more experimental data will have to come forward before more definite conclusions can be drawn.

This long kinetic chain length in the case of polymethyl methacrylate means that polymer chains of that length, or smaller, degrade completely once they are activated. The number average chain length and also the weight average chain length, not considering the monomer, for a homogeneous polymer sample remains then constant during the degradation, as was shown experimentally. If the activation occurs at the chain ends, then the rate of monomer production should be independent of the initial chain length as long as $P_{n}<\gamma$ in agreement with eq (9). This was found to be the case, which constitutes a direct proof that activation takes place at the chain ends. If activation would take place anywhere along the chain, the rate of 
monomer production should be proportional to the initial chain length for equal weights of polymer samples. 'This is the result of the nature of the processes. In the first case, the rate of activation is proportional to the number of chain ends or chain molecules present. This means that if the chain length increases, the number of chain ends decreases in proportion, but for each activated chain end the number of monomer molecules produced increases in proportion to the chain length. In the second case, the rate of activation is proportional to the number of links in the whole system. However, the number of monomer molecules for each activation increases in proportion to the chain length.

Further, if $P_{n}>\gamma$, the monomer production should be proportional to $1 / P_{n}$, at least for quite an appreciable part of the reaction in accordance with eq (5). 'That this is the case is shown in figure 1.12, where the experimental initial rates of monomer production found by Melville and Grassie have been plotted against the reciprocal initial number average molecular weight. 'The position of the kink in the curve gives the approximate kinetic chain length. 'The slope of the straight line should be equal to $k_{4} \gamma m_{o}$. As always $0.2 \mathrm{~g}$ were taken, the rate constant for the initiation reaction at $220^{\circ} \mathrm{C}$ can be evaluated. If for the kinetic chain length $\gamma=1,670$ is chosen, $k_{4}$ can be derived from the slope. As molecular weights for the calculations are used, $\gamma$ must also be expressed in the same units, hence $k_{4}=5.36 \times 10^{-5} \mathrm{ml} \mathrm{sec}-1 \mathrm{~g}^{-1}$.

From the polymer samples with a number average chain length smaller than $\gamma, k_{4}$ may also be derived from the initial rate, as

$$
\frac{d m_{1}}{d t}=k_{1} m_{o}, \quad k_{4}=6.12 \times 10^{-5} \mathrm{ml} \mathrm{sec}{ }^{-1} \mathrm{~g}^{-1}
$$

If $\gamma=1,460$ were chosen, the $k_{4}$ value derived from the slope becomes also $6.12 \times 10^{-5} \mathrm{ml} \mathrm{sec}-1 \mathrm{~g}^{-1}$. It is thus seen that $\gamma$ values can be derived in this way.

Melville and Grassie also determined the energy of activation for the degradation process. A polymer sample of molecular weight 179,000 was degraded over a range of temperatures from $473^{\circ}$ to $503^{\circ}$ $\mathrm{K}$. The collision factor in the Arrhenius equation for this case is here calculated, using kinetic equations derived in this paper for the initial stage of degradation. As $P_{n}<\gamma$, the initial rate of monomer production is given by $d m_{1} / d t=k_{4} m_{o}$. An Arrhenius equation is then ob-

FIgURE 1.12. Initial rate of monomer production for polymethyl methacrylate as a function of the reciprocal numberaverage molecular weight.

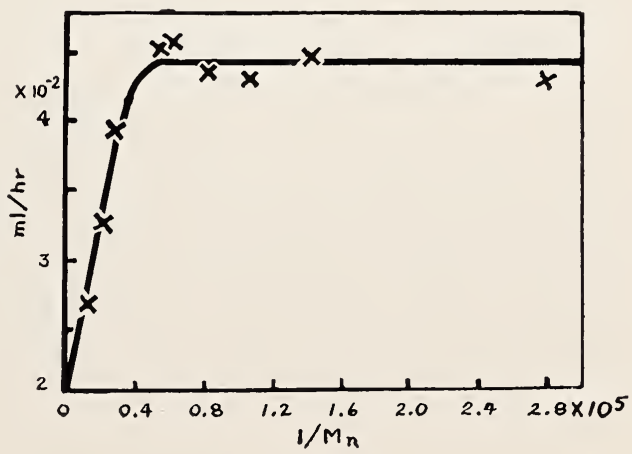


tained for this sample as follows: $k_{4}=2.69 \times 10^{9} e^{-31000 / R T}$ (moles of monomer/base mole)/sec.

Melville and Grassie also showed that the rate of monomer production is influenced by the kind of end group of the polymer molecule. For instance, a polymer obtained by photopolymerisation showed a faster rate of monomer production than one obtained with benzoyl peroxide as catalyst, and the latter in turn showed a faster rate than a polymer containing diphenylcyanomethyl as end groups.

The energy of activation changed with the extent of degradation and the type of end group, as shown below:

\begin{tabular}{|c|c|c|}
\hline Polymer & $\begin{array}{l}\text { Molecular } \\
\text { weight }\end{array}$ & $\begin{array}{l}\text { Energy of } \\
\text { activation }\end{array}$ \\
\hline 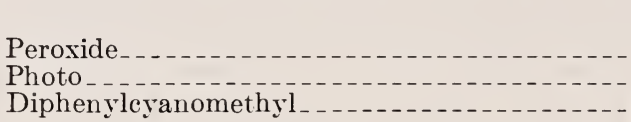 & $\begin{array}{l}36,000 \\
36,000 \\
-\end{array}$ & $\begin{array}{c}\text { kcal } \\
32 \\
40 \\
47\end{array}$ \\
\hline
\end{tabular}

Experiments on polytetrafluoroethylene [16] polymers also agree with a mechanism where monomer is split off from the chain ends.

Poly- $\alpha$-methylstyrene [5] shows quite different monomer production curres than polystyrene. However, this sample was unfractionated. It may be the case that this polymer has a longer kinetic chain length than polystyrene, as it has a methyl-group in $\alpha$-position instead of a reactive $\alpha$-hydrogen atom.

Polyethylene was also investigated in the vacuum balance [5]. This substance shows a zero-order reaction, also indicating that in this case small units are coming off the chain ends. The energy of activation decreases with chain length from 66 to $46 \mathrm{kcal}$. Until more data are available on the change of molecular weight with degradation, it cannot be stated with certainty which degradation mechanism is followed by this polymer. However, it is clear that the break-down does not take place according to a random process. This is shown by the fact that even after 40-percent loss of weight has been suffered by the polymer, there are still polymer chains of appreciable length left in the residue. If a random degradation were operative, then the a verage chain length would, at such an extent of degradation, be so small that all the material should raporize almost immediately. As a matter of fact, the zero reaction continues for about another hour.

Degradation of cross-linked polystyrene shows its own peculiarities [5]. Cross-linking was effected by varying amounts of $m$-divinylbenzene. Some samples were polymerized without catalyst, others with benzoyl peroxide as catalyst. An induction period was observed before degradation set in, which increased with the amount of crosslinking and could extend for over $2 \mathrm{hr}$. The samples containing equal amounts of $m$-divinylbenzene, but polymerized with benzoyl peroxide, showed a very much shortened induction period. At the end of the induction period, monomer was obtained similarly as in the case of normal polystyrene. Cross links seem to have to be ruptured first 
to obtain sufficient mobility of the chains, then the normal degradation from the chain ends sets in. Also Melville and Grassie [15] found induction periods for cross-linked polymethyl methacrylate before the normal degradation reaction started.

It remains now to discuss briefly the considerable amount of work on degradation of polymers where the reaction products have been analyzed [17]. The most extensive work of this kind has been carried out by Madorsky and coworkers $[11,18]$, who pyrolyzed polystyrene, polyisobutene, polyisoprene, polybutadiene, GR-S polymer, polyethylene, and polymethyl methacrylate. Several fractions during different stages of the degradation were collected: (1) a gaseous fraction, (2) a fraction which was volatile at room temperature, (3) a fraction that was not volatile at room temperature, and (4) a residue. The fractions could still be subdivided; reference should be made to the original papers. The volatile fraction was analyzed in a mass spectrometer. The experimental results agree, on the whole, with the kinetic experiments of other authors. For polystyrene, for example, it was found that about 64.6 percent of links ruptured yielded monomeric molecules and 34.6 percent of dimers; further, some trimer and tetramer were also obtained. This result is in agreement with the zero-order reaction found by Jellinek [5]. It agrees with the mechanism where small units are split off from the chain ends. That dimer and some trimer and tetramer are found is easily understandable, as there will be a certain probability for the rupture of links at the ends of the chains yielding monomers, a smaller probability for obtaining dimers, and still smaller probabilities for the production of trimer and tetramer. This in no way would alter the kinetic expressions for the process as long as these molecules are split off from the chain ends.

In the case of polymethyl methacrylate, Madorsky [18] found that 90 percent of all ruptures lead to monomer units. It should be possible by energetic considerations of the bond strengths, etc. involved, to get an estimate of the relative probabilities of breaking off monomer, dimer, etc. for various polymers. Polybutadiene, polyisoprene, and GR-S show a higher proportion of ruptures yielding molecules larger than monomers. But here again, random breaking of links cannot account for the experimental results. However, kinetic experiments should give a deeper insight into the mechanism of degradation of these polymers.

Lastly, there is polyethylene. The percentage ruptures leading in this case to monomeric molecules is quite small, about 12 percent, and about 80 percent of ruptures lead to molecules of a chain length up to about 25 monomer units. But, as pointed out above, polyethylene cannot be assumed to degrade according to a random process. The detailed mechanism can only be ascertained if measurements are made of the change of molecular weights of the residue with time.

It may be useful and of interest to summarize the main results which have been obtained for a number of vinyl polymers. This has been done in table 1.3 .

The relative stabilities or the various polymers, starting with the most stable polymer, are as follows: 


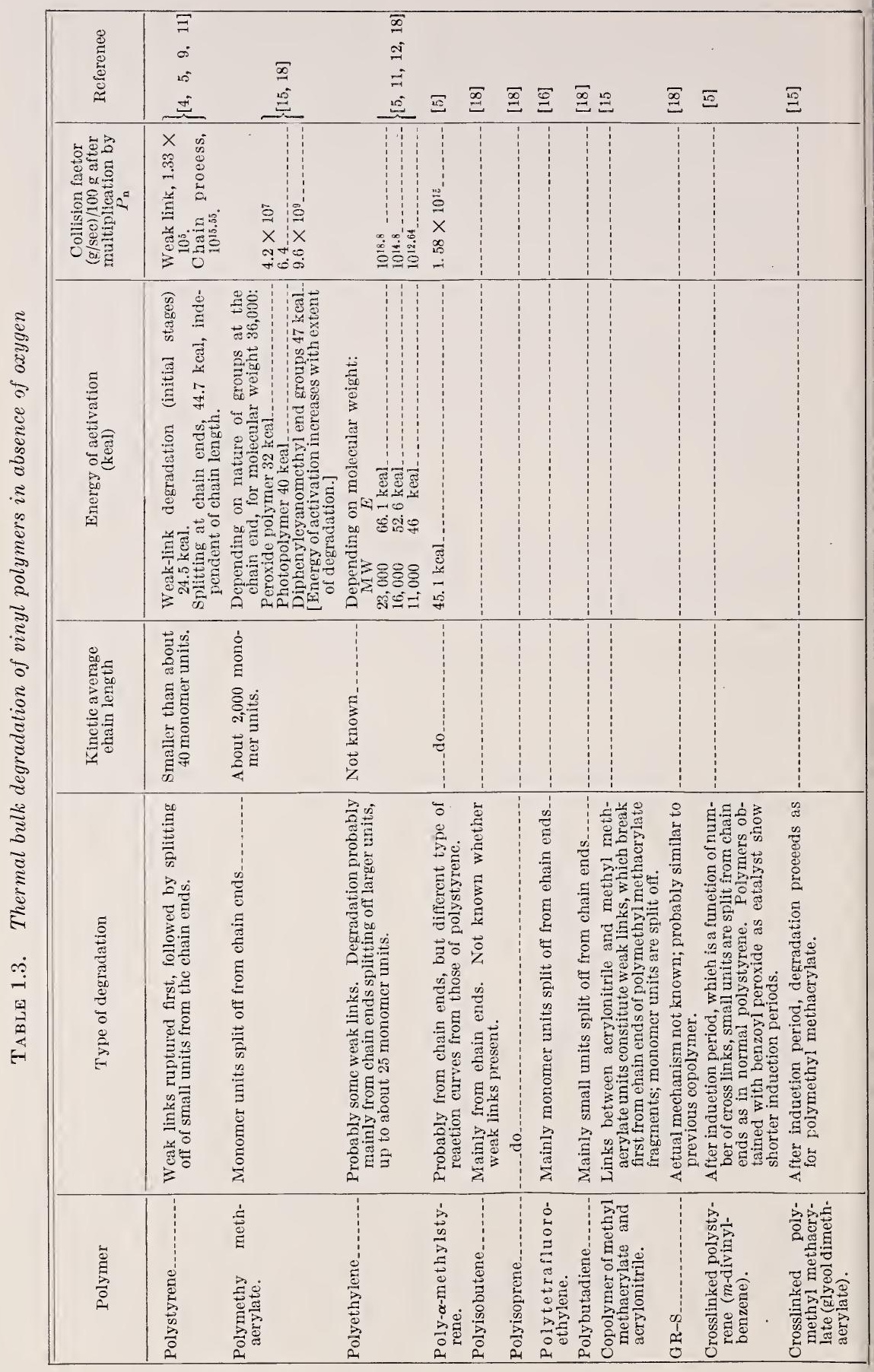


(1) According to Madorsky $[11,18]^{5}$ : polyethylene $>$ polybutadiene $>$ GR $-\mathrm{S}>$ polystyrene $>$ polyisobutene $>$ polyisoprene. Polymethyl methacrylate should probably come just after polystyrene.

(2) Seymour [17] found a similar order.

(3) Jellinek [5] found: polyethylene $>$ polystyrene $>$ poly- $\alpha$-methylstyrene.

\section{Polymerization-Degradation Processes}

Tobolsky, Mesrobian, and coworkers [19] attempted to find polymerization-depolymerization equilibria by polymerizing styrene and degrading polystyrene in solutions at $100^{\circ} \mathrm{C}$ in the presence of benzoyl peroxide and ultraviolet light. Apparent equilibrium states were found. However, it has to be [remembered at the very start that, especially for advanced stages of degradation, changes in viscosity due to chain scission are difficult to detect when measuring the viscosity in fairly concentrated solutions.

Moreover, the interesting work of Thompson, et al. [20], and especially that of Winkler and Montgomery [21], has shown that no equilibrium states are involved. A random process of chain scission caused by benzoyl peroxide and aided by any air present seems to be operative. This random scission reaction is independent of the initial chain length of the polystyrene sample, but the extent of scission is dependent on the amount of benzoyl peroxide added to the reaction mixture. For example, it was found that at $100^{\circ} \mathrm{C}$ in toluene solution, the number of scission points per monomer unit in the polymer chain is a linear function of the number of molecules of benzoyl peroxide added per monomer unit in the chain. The scission efficiency of benzoyl peroxide was found to be quite low. Winkler and Mont-

\footnotetext{
5 Madorsky's expression $\left.P=N \Sigma Y_{f} / M, j 18\right]$ is an approximate one, where $P$ is the number of ruptured links in $100 \mathrm{~g}$ of polymer, $Y_{f}$ the yield of any given fraction in weight percent of the total volatilized part, and $M_{f}$ its a verage molecular-weight.

The following relationship is valid for any degradation process and size distribution:
}

$$
P^{\prime}=B-A \text {, }
$$

where $P^{\prime}$ is the number of ruptured links in a polymer sample at a definite stage of the degradation process, $B$ is the number of chains in the total sample at this stage of the degradation process, and $A$ is the initial number of chains. This can also be written in the form:

$$
P^{\prime}=N\left[\Sigma \frac{b_{f}}{M_{f}}-\Sigma \frac{a_{n}}{M_{n}^{-}}\right] \text {, }
$$

where $N$ is A vogadro's number, $b_{f}$ is any one fraction in grams of number average molecular weight $M_{f}$ in the degraded polymer sample, and $a_{n}$ is any one fraction of number average molecular weight $M_{n}$ in the initial polymer sample.

If the number of ruptured links is large, then the second term can be neglected. Hence, if the $b_{f}$ fractions are expressed in weight percent, the following expression is obtained for $100 \mathrm{~g}$ of polymer sample:

$$
P^{\prime \prime}=N \Sigma \frac{b_{f}^{\prime}}{M_{f}}
$$

which is similar to Madorsky's expression, with the difference that in Madorsky's equation the weight fractions of the volatilized part only are considered.

If the polymer is only partly degraded, for example, by splitting off monomer units (or small units) from the ends, then, as long as the zero order reaction holds, $P^{\prime}=m_{1}$ (see eq (8)) or for $100 \mathrm{~g}$ of polymer $P^{\prime \prime}=$ $N \Sigma Y_{f} / M_{f}=m_{1}$, where $Y_{f}$ is a volatilized fraction in weight percent of the whole polymer.

However, if the degradation has passed the zero order stage, this expression is not strictly valid any more. If the polymer has been degraded and volatilized completely, then eq (b) is striclty valid, but as the number of broken links is then large Madorsky's expression is a very good approximation especially if the initial chain length is long.

For a random process of degradation. Madorsky's expression is not strictly valid for the initial stages of the degradation as there are a number of links ruptured in the chains that are not volatilized.

Equation (b) can also be expressed for $1 \mathrm{~g}$ of the total polymer as follows: $P=N\left[\left(1 / M_{t}\right)-\left(1 / M_{n}\right)\right]$, where $M_{t}$ is the number a verage molecular weight of the total polymer sample at any time $t$ of the degradation process, and $M_{n}$ is the initital number average molecular weight of the polymer. Such an expression was given by Oakes and Richards [12]. 
gomery give for $100^{\circ} \mathrm{C}$ : 1 scission point for every 1,000 benzoyl peroxide molecules. Scission efficiency is increased by the presence of air.

Jellinek [9] found that if a sample was degraded in bulk in racuum at a certain temperature and then transferred and kept at a lower temperature for a period of time, an increase in chain length did not take place. Hence, the polymer fragments-the monomer was continuously removed-did not recombine to longer chains. Similar results were also found by Winkler and Montgomery, who polymerized styrene in toluene solution in presence of benzoyl peroxide at $140^{\circ} \mathrm{C}$. After precipitation, washing, and drying of the polymer, the sample was dissolved in toluene solution and heated at $100^{\circ} \mathrm{C}$ for a considerable length of time. No change in viscosity could be detected.

All these experimental results are to be expected, according to what is known now about the mechanism of degradation. At a temperature of about $100^{\circ} \mathrm{C}$ the benzoyl peroxide is able to effect chain scission, but the temperature is not high enough to obtain activated chain ends giving rise to the production of monomer, which in turn could polymerize. Moreover, free polvmer radicals, if there are any in the mixture, do not recombine. It is more likely, however, that these radicals are not produced, but that the new chain ends have fragments of the benzoyl peroxide incorporated. The benzoyl peroxide is slowly used up in the process of chain scission, as it is not a true catalyst. This statement also holds good for the polymerization reaction, during which benzoyl peroxide is incorporated into the polymer chain. Moreover, it seems to be the case that inhibitors are formed during the degradation in the presence of benzoyl peroxide, slowing down the reaction. That such inhibitors are formed during degradation was shown by Jellinek [4] in his experiments on degradation of polystyrene in presence of oxygen. Votinov, Kobeko, and Marey [22] also came to the conclusion that inhibitors are produced during the degradation of polystyrene.

\section{Degradation in Presence of Oxygen}

If the bulk degradation of polystyrene is carried out in presence of oxygen at temperatures at which the degradation is moderate, the experiments can be described by a mechanism as follows [4]:

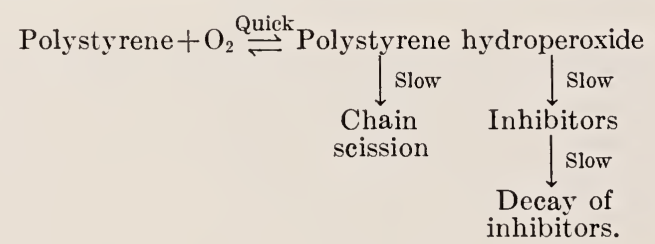

The chain scission in this case seems to be a random process. The energy of activation for the initial stages of the degradation was found to be 25.2 kcal over a range of temperatures from $183^{\circ}$ to $220^{\circ} \mathrm{C}$.

Lacau and Magat [23] studied the oxidative degradation of GR-S in toluene solution over a range of temperatures from $70^{\circ}$ to $140^{\circ} \mathrm{C}$. The rate of degradation is independent of chain length, and the energy of activation from the initial rates is 20 to $25 \mathrm{kcal}$. The results are in agreement with the conception that chain scission is brought about by peroxides chemically bound to the polymer chain. 
It would lead too far to discuss here experiments on the photochemical degradation of polystyrene [24], or degradation brought about by a number of compounds [25], or by milling and turbulent flow [26].

\section{Ultrasonic Degradation}

Schmid and coworkers [27] carried out extensive investigations on the ultrasonic degradation of synthetic high polymers. Schmid believed that caritation does not play a decisive role in causing degradation and based his explanations of the break-down of longchain molecules on the assumption that frictional forces are developed when a solution is exposed to ultrasonic waves. He also believed that the mechanical properties (e. g., flexibility) of the polymer molecule are of importance in this connection. However, more recent work by Weissler [28] and Prudhomme [29] shows that cavitation plays a very important part in bringing about degradation. This means, of course, that Schmid's theoretical considerations are not immediately applicable to the experimental results. Thus, Jellinek and White [30] found that their results obtained from the degradation of fractionated polystyrene samples in benzene solution could not be satisfactorily accounted for by theories assuming frictional or impact forces. Melville and Murray [31] have shown recently that degradation by ultrasonic waves is a complex process brought about by cavitation and to a lesser extent also takes place in the absence of cavitation.

As in the case of thermal degradation, it will not be attempted here to describe all the experiments in detail; rather, it is intended to bring out the most important features of the ultrasonic degradation process. Apparatus and experimental technique will not be considered and reference should be made to the original papers.

\section{Experimental Results}

The main experimental facts concerning the ultrasonic degradation of vinyl polymers are as follows:

(1) In presence of cavitation:

(a) The rate of degradation is dependent on chain length; at a certain chain length the degradation either stops or becomes very slow.

(b) Degradation is not caused by chemical action such as oxidation, as degradation also proceeds when air has been replaced by other gases, such as nitrogen and helium.

(c) The final chain length is dependent on the intensity of the waves.

(d) Degradation is decreased when an outside pressure is applied to the solution (cavitation diminished).

(e) The rate of degradation seems to be independent of the frequency of the ultrasonic waves (experiments carried out so far go down to $10 \mathrm{kc} / \mathrm{sec})$.

(f) If the temperature of the solution is raised, the ultrasonic degradation decreases.

(g) The degradation is a function of concentration and riscosity of the solution. Starting with a homogeneous sample of a definite chain length, the degradation goes through a maximum when increasing the concentration. 
(h) Degradation is only slightly influenced by the density of the solvent.

(i) If a nonsolvent is added to the solution, degradation is markedly diminished, even before the solution shows any turbidity.

(j) In presence of $\mathrm{CO}_{2}$, Weissler [28] reports normal degradation of 0.20 percent of polystyrene in toluene $(400 \mathrm{kc} / \mathrm{sec}, 50-\mathrm{w}$ output), and Prudhomme [29] reports no degradation taking place of 1 percent polystyrene in toluene $\left(960 \mathrm{kc} / \mathrm{sec}, 76-\mathrm{w}\right.$ output, $\left.6.8 \mathrm{w} / \mathrm{cm}^{2}\right)$.

(2) Degradation in vacuum; in absence of cavitation:

(a) According to Weissler [28] and Prudhomme [29], degradation becomes negligible; however, according to Melville and Murray [31], degradation is appreciably reduced but still quite marked.

Weissler exposed a 1-percent toluene solution of polystyrene, polymerized at room temperature, in the presence of air for $3 \mathrm{hr}$ to ultrasonic waves $(400 \mathrm{kc} / \mathrm{sec}, 50-\mathrm{w}$ output). The flow time measured in a viscometer dropped from 929.3 to 270.9 sec. When the solution was degassed, the flow time only dropped to 925.7 sec. Prudhomme exposed a 1-percent toluene solution to ultrasonic waves for $120 \mathrm{~min}$. (960 kc/sec, $76-\mathrm{w}$ output, $6.8 \mathrm{w} / \mathrm{cm}^{2}$ ). With cavitation the flow time dropped from 23.6 to $18.3 \mathrm{sec}$. Without cavitation the flow time remained constant. Melville and Murray exposed a 0.1 -percent toluene solution of polystyrene $\left(M_{n}=700,000\right)$ to ultrasonic waves for $100 \mathrm{~min}\left(213 \mathrm{kc} / \mathrm{sec}, 30-\mathrm{w}\right.$ output, $\left.7.7 \mathrm{w} / \mathrm{cm}^{2}\right)$; the intrinsic viscosity, in absence of cavitation, dropped from 2.70 to 1.12.

(b) The rate of degradation is dependent on the intensity of the ultrasonic waves, slowing down with time and reaching a final value. It is not known yet with certainty whether the same final chain length is reached with different intensities.

(c) The dependence of the rate of degradation on chain length has not yet been studied.

(d) Copolymers that showed weak links on thermal degradation, do not show any special characteristics on degradation by ultrasonic waves in vacuum.

Some of the experimental results may be considered in more detail. Schmid and Rommel [27] degraded fractionated polystyrene samples in presence of cavitation and found that the rate of degradation increased with chain length, but that the final chain length was independent of the initial chain length. Schmid developed kinetic equations for the degradation process but disregarded size distributions and did not distinguish between number and weight average chain lengths.

Jellinek and White [30] degraded fractionated polystyrene samples, determining size distributions and intrinsic viscosities at definite stages of the degradation process. They also developed kinetic expressions for the chain length and distributions during the degradation process. The experimental results could be described satisfactorily by the kinetic equations. They also investigated the dependence of rate constants on the chain length (fig. 1.13), and the rate of degradation as a function of concentration (fig. 1.14). The kinetics and theories of ultrasonic degradation will be discussed subsequently. A typical example of the decrease of weight average chain length with time is given in figure $1.15\left(0.5 \mathrm{Mc} / \mathrm{sec}, 15.7 \mathrm{w} / \mathrm{cm}^{2}, 2 \% \mathrm{w} / \mathrm{v}\right.$ of polystyrene in benzene). It is seen that a very rapid decrease in chain length takes place initially, slowing down until a final chain length is reached. Figure 1.16 shows some of the experimental size distributions. 


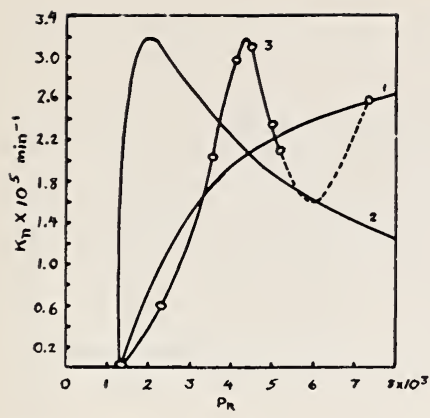

Figure 1.13. Ultrasonic rate constants for polystyrene as a function of initial chain length.

$P_{n}[30] 1$ and 2, theoretical curves; 3 , experimental curve.

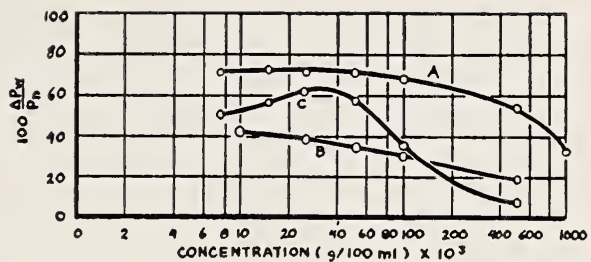

Figure 1.14. Percentage of decrease in weight-average chain length as a function of concentration during ultrasonic degradation (1/4-hr exposure of polystyrene [30]).

$\mathrm{A}$ is $\left(P_{n}=6850\right) ; \mathrm{B},\left(P_{n}=2019\right)$; and $\mathrm{C},\left(P_{n}=6785\right)$.

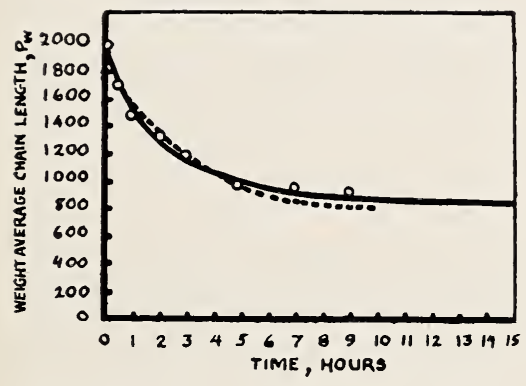

FiguRE 1.15. Decrease of weightaverage chain length of a fractionated polystyrene sample during ultrasonic degradation [30].

(......, theoretical curve). $P_{o w}=3 \xi P_{a}$ $=840 ; P_{e}=1260$.

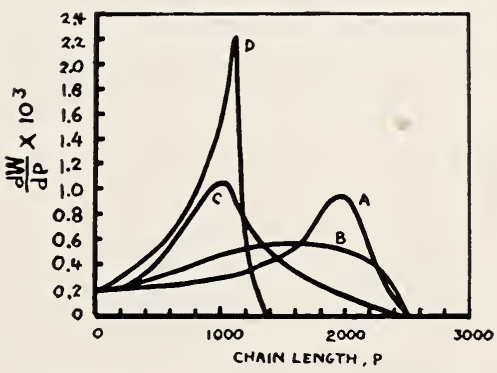

Figure 1.16. Weight distributions for a fractionated polystyrene sample at definite stages of ultrasonic degradation [30].

A, Untreated sample; B, 1/2-hr treatment; C, 3 hr.; D, 9 hr.

Schmid and Beutenmüller [27] studied the influence of the density of the solvent on the degradation. Some of their results are given in table 1.4 .

TABLE 1.4. Ultrasonic degradation of polystyrene at $55^{\circ} \mathrm{C}$ in a mixture of toluene and carbon tetrachloride [27] a

\begin{tabular}{|c|c|c|c|}
\hline \multirow{2}{*}{ Time } & \multicolumn{3}{|c|}{$\begin{array}{l}\text { Weight average molecular weight in } \\
\text { solution of initial density- }\end{array}$} \\
\hline & 0.867 & 1.058 & 1.234 \\
\hline $\min$ & & & \\
\hline 0 & 208,000 & 203,000 & $197,0 \mathrm{w} 0$ \\
\hline 10 & 140,000 & 158,000 & 157,000 \\
\hline 30 & 100,000 & 124,000 & 129,000 \\
\hline 60 & 80,000 & 100,000 & 108,000 \\
\hline 120 & 61,000 & 78,000 & 85,000 \\
\hline
\end{tabular}

a The concentration of the solutions was 0.038 base mole/liter. The density of polystyrene is $1.058 \mathrm{~g} / \mathrm{cm}^{3}$. 


\section{Theoretical}

As mentioned above, Jellinek and White [30] derived kinetic expressions for the degradation of homogeneous polymer samples. These equations, of course, do not give any direct information as to the actual mechanism of the ultrasonic degradation but take account of the fact that degradation rate constants decrease with chain length and become zero when the final chain length is reached. This final chain length is dependent on the intensity of the ultrasonic waves, the solvent (viscosity), the concentration of the polymer, and the intrinsic properties of the polymer molecules. They pertain to experiments carried out in the presence of cavitation but are most likely also applicable to ultrasonic degradation in vacuum.

Following Schmid [27, 32], the reasonable assumption was made that the rate constant $k_{x}$ is given by an expression:

$$
k_{x}=\alpha^{\prime}\left(P_{x}-P_{e}\right),
$$

where $P_{x}$ is any one chain length, $P_{e}$ the final chain length, and $\alpha^{\prime}$ a constant.

Even if no specific assumptions are made as to how the rate constants decrease with chain length, as long as $k=0$ at a definite chain length $P_{e}$, the final number and weight distribution functions are known, as the number of chains for 1 to $P_{e}$ must be equal for any one chain length [30]. The number and weight distributions for this case are given in figure 1.17.

However, the mathematical treatment for chains larger than $P_{4}$ becomes very complicated if eq (54) is taken as the basis for the calculations. The simplified assumption has to be made that the rate constants are constant down to chain length $P_{e+1}$ and zero for smaller chain lengths. 'The whole number distribution function for the number of moles of any one chain length in a system containing 1 base mole of polymer reads then as follows.

For chains from $P_{n-1}$ to $P_{c+1}$ :

$$
n_{x}=\frac{\alpha}{P_{n}}(1-\alpha)^{P_{x}^{-1}}\left\{2+\left(P_{n}-P_{x}-1\right) \alpha\right\} .
$$

For chains smaller than $P_{e}$ :

$$
n_{x}=\frac{2}{P_{n}}\left\{\frac{P_{n}-P_{e}-1}{P_{e}+1}\left[(1-\alpha)^{P_{e}+1}-1\right]-\frac{P_{n}-P_{e}}{P_{e}}\left[(1-\alpha)^{P_{e}}-1\right]\right\} .
$$

For chains of length $P_{n}$ :

$$
n_{n}=\frac{1}{P_{n}}(1-\alpha)^{P_{n}-1},
$$
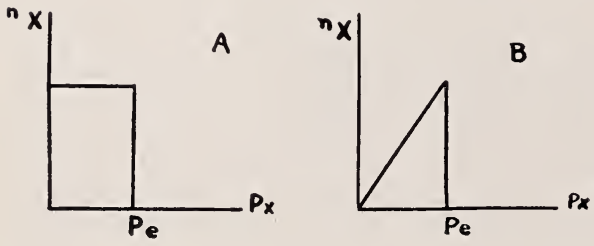

FIGURE 1.17. Final theoretical number and weight distributions at the end of ultrasonic degradation [30]. A, Final number distribution; B, final weight distribution. 
where $\alpha=1-e^{-k t}, k$ is a rate constant, and $t$ the time.

The weight distributions are obtained by multiplying these equations by $P_{x}$ and $P_{n}$, respectively. They give then the amount of polrmel in grams of a certain chain length for $1 \mathrm{~g}$ of initial material. A typical example of the decrease of $P_{w}$ with time is given in figure 1.15. From these experimental results an average rate constant was derived and a theoretical curve calculated, which is also shown in figure 1.15. As was to be expected, the theoretical curve lies somewhat above the experimental one for the initial part of the reaction and slightly below for the latter part of the reaction. This means that the actual degradation is initially faster than the theoretical one, but becomes eventually slower than the theoretical one due to the fact that an average $k$ value is only an approximation to the actual rate process. The theoretical distribution curves agreed satisfactorily with those found by experiment.

The question arises as to the elementary mechanism of the breakdown of a long-chain molecule by ultrasonic waves. Schmid [32], who believed that cavitation is not important for the degradation process, suggested that frictional forces are responsible for the breakdown. He considered first a long-chain molecule in solution rigidly fixed on one end and exposed to ultrasonic waves. Then the oscillating solvent will exert a frictional force; this force is given by Stokes' law:

$$
f_{o}=P_{n} \cdot 6 \pi \eta r v_{s}
$$

where $\eta$ is the viscosity of the solvent, $r$ is the radius of one monomer unit (the chain being considered as a string of pearls, the monomer units being spheres), and $v_{s}$ is the maximum relocity of the solvent relative to the chain. Equation (55) can be written in the form

$$
f_{o}=\alpha \cdot P_{n}
$$

For the experimental conditions chosen by Jellinek and White [30], $\alpha$ amounts to $1.79 \times 10^{-i}$ dyne, hence, for a chain length $P_{n}=3 \times 10^{3}$, $f_{0}=5.37 \times 10^{-4}$ dyne. This force is of the right order to break a $\mathrm{C}-\mathrm{C}$ bond (about $5.64 \times 10^{-4}$ dyne [32a]):

Howerer, in reality, the chain molecuie is not rigidly fixed. Therefore, it will move to a greater or less extent with the solvent, depending on its apparent mass. The frictional force in that case becomes, according to Schmid,

$$
f_{o}=P_{n} \frac{m_{o}}{N} \omega v_{s}
$$

where $m_{0}$ is the molecular weight of the monomer, $N$ is Aragadro's number, $\omega$ is $2 \pi / T$ equals angular frequency, $T$ is the periodic time of the ultrasonic waves. Equation (57) can again be written in the form $f_{o}=\alpha P_{n}$. In this case the forces under the conditions considered, are quite insufficient to break a $\mathrm{C}-\mathrm{C}$ bond $\left(\alpha=2.77 \times 10^{-14} \mathrm{dyne}\right)$.

Schmid made the reasonable suggestion that the chain molecules are entangled in the solution, thus increasing the mass of the particle; this mass will be further increased by solvent molecules immobilized by the entanglement. Then the inertia of such a system may become 
large enough to develop frictional forces sufficient to break a few $\mathrm{C}-\mathrm{C}$ bonds.

According to the theory outlined above, the ultrasonic degradation at very low concentrations should decrease and eventually cease when the apparent mass of the particles becomes too small. On the other hand, when the concentration becomes too high, the solvent is immobilized and again no frictional forces will be developed. Hence, the degradation should go through a maximum when increasing the concentration. This was found to be the case (see fig. 1.14). However, this cannot be taken as a confirmation of the theory, as it is known now that degradation is a complex process taking place with and without cavitation. These experiments were carried out in the presence of cavitation.

A further consequence of the theory is that degradation should cease completely when the density of the solute and solvent is equal, as then the apparent mass becomes zero. As stated above, it was found that the density of the solvent did not influence the degradation (see table 1.4). Here again this result does not prove that the theory is at fault, as the theory would only be expected to hold in the absence of cavitation. However, the experiments were carried out in the presence of cavitation.

It can also be assumed that the long-chain molecules may be broken down by the impact of the solvent molecules. This case was considered by Jellinek and White [30]. A chain molecule was imagined to be fixed at both ends and assumed to be rigid during the time of impact. If a layer of solvent molecules of thickness $\lambda / 4$ (where $\lambda$ is the wavelength of the ultrasonic waves) impinges on the cross section of the molecule, then the force acting on the molecule is

$$
f_{o}=\frac{m \cdot v}{t}
$$

where $m$ is the mass of solvent layer, $v$ is the average velocity of the solvent layer $(2 / \pi) v_{s}$, and $t$ is the time of impact $(\pi / 2 \omega)$. Equation (58) can be written in the form

$$
f_{o}=\frac{\rho v P_{n} d \frac{\lambda}{4} \frac{2}{\pi} v_{s}}{\frac{\pi}{2 \omega}},
$$

whare $\rho$ is the density of the solvent, $d$ is the width of the chain molecule, $v_{s}$ is the maximum solvent velocity, and $v$ is the spacing between monomer units. For constant conditions eq (59) can again be written

$$
f_{o}=\alpha P_{n}
$$

Calculation shows that under the experimental conditions chosen, the force is about 20 times too small to break a C-C link in a chain of length $P_{n}=3 \times 10^{3}$. Here again, the force becomes very much smaller when the chain is not fixed; entanglement has again to be assumed. It does not seem unlikely that break-down of chains in absence of cavi- 
tation might be brought about by a combination of frictional and impact forces. A consequence of the impact theory is that as the warelength of the ultrasonic wares gets longer, degradation should decrease because the time of impact becomes longer and the chain molecules cannot be considered rigid during this time interval. The effect of frequency was investigated by Schmid and Poppe [27], who could not find any decrease in rate of degradation in a range of frequencies from 300 to $10 \mathrm{kc} / \mathrm{sec}$. Here again, degradation was carried out in the presence of cavitation, so that a proper comparison between experiment and theory cannot be made.

The theoretical considerations were carried a stage further by Jeliinek and White [30] in order to find a relationship between the rate constants and chain length. It was assumed that the force acting on one chain molecule is given by

$$
f=f_{o} \sin (\omega t-\phi),
$$

where $\omega$ is the angular frequency, $t$ the time, and $\phi$ a phase angle. Two cases were considered:

Case 1:

The number of chains broken in a time interval $\delta t$ in the period when $f>\gamma$ (where $\gamma$ is the force required to break a $\mathrm{C}-\mathrm{C}$ link) is given by

$$
\delta_{n}=\beta(f-\gamma) n_{n} \delta t,
$$

where $\beta$ is a constant, and $n_{n}$ is the number of chains of length $P_{n}$. Case 2:

In this case the assumption is made that the number of chains broken in the time interval $\delta t$, when $f>\gamma$, is independent of $(f-\gamma)$ :

$$
\delta n_{n}=\beta n_{n} \delta t .
$$

Further, it is assumed that $f_{o}=\alpha P_{n}$, where $\alpha$ is a constant for a definite system, and $P_{n}$ is the initial chain length of the polymer.

This theory includes any ultrasonic degradation process as long as the force follows a sine function and $f_{o}$ is proportional to the chain length; hence it includes the frictional and impact theory and would include a degradation process in presence or absence of cavitation as long as these assumptions hold.

In cases 1 and $2, k_{n}=0$ when $f_{o}=\gamma$. Therefore,

$$
\frac{\gamma}{\alpha}=P_{e}
$$

where $P_{e}$ is the final chain length at the end of the degradation process, as $k_{n}$ must then be equal to zero.

The final equations obtained for the two cases are

$$
\text { (Case 1) } \quad k_{n}=\frac{2 \beta}{\gamma \pi P_{n}}\left\{\sqrt{\frac{P_{n}{ }^{2}}{P_{e}^{2}}-1}-\cos ^{-1} \frac{P_{e}}{P_{n}}\right\} \text {. }
$$




$$
\text { (Case 2) } \quad k_{n}=\frac{2 \beta}{\pi P_{n}} \cos ^{-1} \frac{P_{e}}{P_{n}} \text {. }
$$

By choosing suitable values of $\beta$, it was attempted to fit the experimental curve of the initial rate constants versus chain length (fig. 1.13). As can be seen, neither of the theoretical curves fits the experimental one. The assumptions made appear to be too simple, which is understandable, as it is now known that ultrasonic degradation is a complex process taking place with and without cavitation. Moreover, it must be kept in mind that the viscosity of the solution has a great effect on the process of cavitation and hence on the degradation [33]. Also the question of turbulence due to the fountain has to be considered [29]. Only an experimental separation of all these factors will lead to further progress in the elucidation of the ultrasonic degradation process.

I Ielville and Murray [31] tried to ascertain whether free radicals are produced when a long-chain molecule is broken down by ultrasonic wares. If that were the case, polymerization of monomer added during the degradation process should be accelerated by the free radicals. However, the effects observed were too small to draw any definite conclusions from these experiments.

A detailed theory of ultrasonic degradation of long-chain molecules by cavitation does not seem to have been advanced. It is fairly clear that this break-down must have some connection with the very high pressures developed when a cavity collapses [34,35]. Such a pressure may amount to several thousand atmospheres. As the collapse of a carity is rapid, a theory on the lin as considered above may be of value, that is, the chain molecules may be broken down by the impact generated during the collapse of a carity. Gases dissolved in th- liquid will behare differently during such collapse according to their intrinsic properties. Permanent gases will probably develop higher pressures than gases that condense during the collapse and that are quickly dissolved [36]. However, it must not be forgotten that high local temperatures may be produced during the collapse of a cavity, as the gas may be considered to be compressed adiabatically [37]. The action of caritation thus presents many interesting problems. However, it would lead too far to go into any more details at this stage. More experimental data are needed to clarify the position, and the numerous contradictory experimental results have to be investigated before any more progress can be expected.

\section{Conclusion}

The bulk degradation of vinyl polymers in vacuum shows that usually the degradation follows, in its main course, a mechanism that approaches what is termed "true" degradation or depolymerization. It remains now to fill in the details of the reaction mechanism, such as the determination of the rate constants for propagation, initiation, and termination. Especially polymerization degradation equilibria have not ret been explored fully.

The reaction in presence of oxidizing agents seems to follow a random degradation process. Also here, many problems remain to he solved as to the detailed mechanism of the reaction. 
The ultrasonic degradation process is still very obscure, in spite of the fact that a number of investigations have been carried out. Only a clear separation of all the experimental factors involved will bring any progress in this field.

\section{Summary}

Theories of degradation are discussed; a chain theory, involving the reversal of polymerization, is presented including degradation polymerization equilibria. The thermal degradation of vinyl polymers in vacuum is considered in the light of these theories.

Degradation of vinyl polymers in the presence of oxidizing agents is discussed.

The ultrasonic degradation of such polymers is described, and the experimental factors and theories of importance for this type of degradation are considered.

\section{References}

[1] R. Simha, L. A. Wall, and P. J. Blatz, J. Polymer Sci. 5, 615 (1950) ; R. Simha and L. A. Wall, J. Polymer Sci. 6, 39 (1951).

[2] W. Kuhn, Ber. deut. chem. Ges. 63, 1503 (1930); Z. physik. Chem. A159, 368 (1932); F. Klages, Z. physik. Chem. A159, 357 (1932); W. H. Durfee and I. Z. Kertesz, J. Am. Chem. Soc. 62, 1196 (1940); H. Mark and R. Simha, Trans. Faraday Soc. 36, 611 (1940); G. V. Schulz, Z. physik. Chem. B51, 127 (1942); R. Simha, J. Applied Phys. 12, 569 (1941); E. Montroll and R. Simha, J. Chem. Phys. 8, 721 (1940); I. Sakurada and S. Okamura, Z. physik. Chem. A187, 389 (1940); E. Montroll, J. Am. Chem. Soc. 63, 1215 (1941); J. Rehner, Ind. Eng. Chem. 36, 118 (1944). R. H. Boundy and R. F. Boyer, Styrene, polymers, copolymers, and derivatives (see chapter 13, "Styrene", by H. H. G. Jellinek. Reinhold Publ. Corp., New York, N. Y., 1952).

[3] G. V. Schulz, Z. physik. Chem. B52, 50 (1942); H. H. G. Jellinek, Trans. Faraday Soc. 40, 266, (1944); H. H. G. Jellinek, Trans. Faraday Soc. 44, 345 (1948).

[4] H. H. G. Jellinek, J. Polymer Sci. 4, 1 (1949).

[5] H. H. G. Jellinek, J. Polymer Sci. 4, 13 (1949).

[6] G. V. Schulz, Makromolekulare Chem. 3, 146 (1949).

[7] T. Alfrey, A. Bartovics, and H. Mark, J. Am. Chem. Soc. 65, 2319 (1943).

[8] C. E. H. Bawn, R. F. J. Freeman, and A. R. Kamaliddin, Trans. Faraday Soc. 46, 1107, (1950).

[9] H. H. G. Jellinek, J. Polymer Sci. 3, 850 (1948).

[10] W. Jackson and J. S. A. Forsyth, J. Inst. Elec. Engrs. (London) 92, Pt. III, $23(1945)$.

[11] S. L. Madorsky and S. Straus, J. Research NBS 40, 417 (1948) RP1886; Ind. Eng. Chem. 40, 848 (1948).

[12] W. G. Oakes and R. B. Richards, J. Chem. Soc. 2931 (1949).

[13] J. L. Bolland and G. Gee, Trans. Faraday Soc. 42, 244 (1946).

[14] L. H. Cross, R. B. Richards, and H. A. Willis, Faraday Soc. No. 9, Discussions $235,321(1950)$.

[15] N. Grassie and H. W. Melville, Proc. Roy. Soc. (London) [A] 199, 1, 14, 24,39 (1949).

[16] E. E. Lewis and M. A. Naylor, J. Am. Chem. Soc. 69, 1968 (1947).

[17] H. Staudinger and A. Steinhofer, Ann. Physik 517, 35 (1935); Th. Midgley, A. W. Henne, and H. M. Leicester, J. Am. Chem. Soc. 58, 1961 (1936); G. B. Bachman, etc., J. Org. Chem. 12, 108 (1947); R. B. Seymour, Ind. Eng. Chem. 40, 524 (1948); L. A. Wall, J. Research NBS 41, 315 (1948) RP1928.

[18] S. L. Madorsky, S. Straus, D. Thompson, and W. Williamson, J. Research NBS 42, 499 (1949) RP1989; S. L. Madorsky, Science 111, 360 (1950). 
[19] R. Mesrobian and A. V. Tobolsky, J. Am. Chem. Soc. 67, 785 (1945); J. Polymer Sci. 2, 463 (1947); P. J. Blatz and A. V. Tobolsky, J. Phys. Chem. 49, 77 (1945); H. S. Taylor and A. V. Tobolsky, J. Am. Chem. Soc. 67, 2063 (1945).

[20] J. O. Thompson, etc., J. Phys. \& Colloid Chem. 54, 338 (1950).

]21] D. S. Montgomery and C. A. Winkler, Can. J. Research [B] 28, 407, 416, $429(1950)$.

[22] A. Votinov, P. Kobeko, and F. Marey, J. Phys. Chem. (U. S. S. R.) 16, 106 (1942).

[23] J. Lacau and M. Magat, Faraday Soc. No. 2. Discussions, 388 (1947).

[24] Shih Wei-Chen, J. Phys. \& Colloid Chem. 53, 486 (1949).

[25] H. Staudinger, K. Frey, P. Grabsch, and S. Wehrli, Ber. deut. chem. Ges. 62, 2912 (1932).

[26] H. Staudinger and W. Heuser, Ber. deut. chem. Ges. 67, 1159 (1934); H. Staudinger and E. Dreher, 69, 1091 (1936). K. Hess, E. Steurer and H. Fromm, Kolloid-Z. 98, 149, 290 (1942).

[27] G. Schmid and O. Rommel, Z. physik. Chem. A185, 97 (1939); Z. Elektrochem. 45, 659 (1939); G. Schmid, Z. physik. Chem. A186, 113 (1940); G. Schmid, Kunststofftechnik 13, 65 (1943); G. Schmid and Beutenmüller, Z. Elektrochem. 49, 325 (1943); G. Schmid and W. Poppe, Z. Elektrochem. 53, 28 (1949).

[28] A. Weissler, J. Applied Phys. 21, 171 (1950).

[29] R. O. Prudhomme, J. Chim. Phys. 47, 795 (1950).

[30] H. H. G. Jellinek and G. White, Parts I-IV, J. Polymer Sci. 6, 745, 757; 7, 21, 33 (1951).

[31] H. W. Melville and A. J. R. Murray, Trans. Faraday Soc. 46, 996 (1950).

[32] G. Schmid, Physik Z. 41, 326 (1940).

[32a] H. J. de Boer, Trans. Faraday Soc. 32, 10 (1936).

[33] H. Okuyama, Chem. Soc. Bul. Japan 18, 397 (1943).

[34] Lord Rayleigh, Phil. Mag. [6] 34, 94 (1917).

[35] L. Bergmann, Der Ultraschall, p. 574, 605 (S. Hirzel, Zurich, 1949).

[36] A. Weissler, J. Chem. Phys. 18, 1513 (1950).

[37] V. Griffing, J. Chem. Phys. 18, 997 (1950). 


\title{
2. Mechanism and Kinetics of Thermal and Photodegradation Reactions
}

\author{
By Robert Simha ${ }^{1}$
}

\section{Introduction}

The complete kinetic analysis of a reaction involves four steps, namely, the postulation of a mechanism, its mathematical formulation in terms of rate equations and constants, the determination of these constants from or the rejection of the mechanism by experiment, and, finally, calculation of the absolute magnitudes of these rate parameters from first, that is, quantum mechanical, principles. Increasing attention has been devoted in recent years to an interpretation of the rate of initiation of radicals or ions, since this represents the rate-determining step in polymerization. In this paper, however, attention will be devoted exclusively to the first three points in the kinetic program.

The experimental quantities available are the over-all rate of reaction; for instance, the rate of appearance or disappearance of monomer, and where free radicals are involved, their mean life times. In polyreactions we have available, in addition, the rate of change of molecular weight with conversion and, in principle at least, the molecular weight distribution. Furthermore, in the case of thermal or photodepolymerization we have the monomer yield, that is, the relative amount of unchanged monomer appearing in the volatile products.

From the thoroughly studied situation in polymerization reactions, it is well known that two fundamentally different attitudes can be taken. The one treats the reaction as a step process, in which one and the same step is iterated. The example par excellence is polycondensation reaction, which involves the repeated formation of ester linkages in the build up of long chains. Vinyl polymerization on the other hand proceeds through a chain mechanism. It consists of an initiation of free radicals or ions, followed by a rapid addition of monomer, transfer of the active radical with solvent, monomer, or even polymer, and finally a termination by combination or disproportionation, at least where free radicals are involved.

Although more complicated, the situation is fundamentally the same in degradation. Also here we encounter reactions proceeding through a stepw ise splitting of bonds. The classical example is the hydrolysis of polysaccharides, which has played such an important role in the establishment of the structure of the monomer unit and the linkage in cellulose. The simplest case corresponds to a completely random breaking of bonds. This is essentially what one finds in cellulose, apart from end effects and deviations due to crystallinity. The situation is completely analogous to that in polyesterification reactions, where the rate of formation of an ester linkage is independent of the

1 New York University, New York, N. Y. 
size of the chain to which it is attached. The correspondence extends to the last mathematical details of the distribution curve, disregarding end effects. The theories of random degradation, due to Kuhn, Montroll, Simha, Schulz, and others, were directly inspired by the work on polysaccharides. More recently, cases where more than one type of linkage is involved have been considered [1]. ${ }^{2}$

Here, however, we shall be concerned with thermal or photoactivated depolymerization. Eleven years ago when the author outlined a more general kinetic theory of step degradation [2], it was apparent that pyrolysis could not be generally interpreted as a random splitting of bonds, because of the rapid evolution of monomer observed. As an extreme alternative, it was suggested that exclusive breaking of terminal bonds takes place. Figure 2.1 depicts the two assumptions. Here the probability $K_{i}$ of breaking link $i$ is plotted versus $i$, the ordinal number of the link. The two mechanisms yield profoundly different results in respect to rate and molecular weight. The number average molecular weight, excluding monomer, decreases sharply at once for random degradation. In the other case it decreases linearly with increasing conversion. However, this also represents only a special result of a more general situation. In order to fit experimental data, we could make various assumptions about the "spectrum" $K_{i}$, as indicated by the lines C and D in figure 2.1. This is reminiscent of the phenomenological theory of dispersion and absorption in dielectric or viscoelastic media. There one assumes a distribution of time constants and deduces the character of the distribution from experiment. A similar procedure could be adopted here. Partly with that in mind, the most general possible equations with no apriori assumptions about the spectrum had been formulated [2].

In the cases of interest here, at any rate, such an approach is fundamentally unsatisfactory. It still remains to account for the pronounced differences in the spectrum of, for example, polyethylene and polymethyl methacrylate. This can only be done by considering the process as the analog of a chain reaction, that is, of vinyl polymerization, as was very early suggested by Chalmers [3]. As will become apparent in the following, this leads naturally to a spectrum of rate constants.

It was thinking along these lines which induced Wall, Blatz, and the author to reconsider the theory of chain depolymerization in the

2 Figures in brackets indicate literature references on p. 54 .

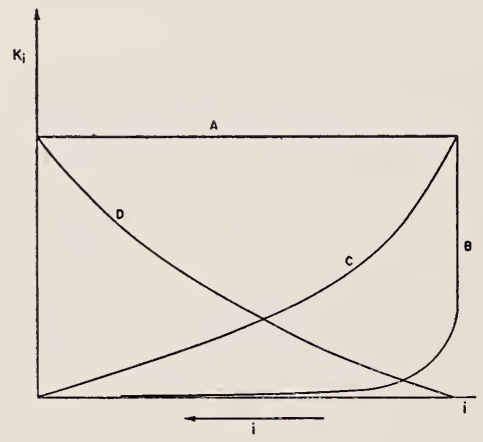

Figure 2.1. Spectrum of decomposition constants.

A, Random degradation; $B$, exclusive breaking of terminal bonds; $\mathrm{C}$ and $\mathrm{D}$, intermediate possibilities. The bonds are successively numbered from the end, starting at the right. 
light of recent experimental results. The basis for a quite general approach was developed. It rests on the assumption of a chain reaction consisting of the elementary acts familiar in polymerization. The theory and quantitative interpretation of experiments has been discussed in detail previously $[4,5,6,7]$. An account of the physical picture and a summary of the principal resulting relations are presented in this paper.

\section{Mechanism}

We postulate the following steps:

\section{Initiation}

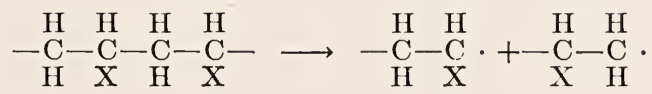

Propagation

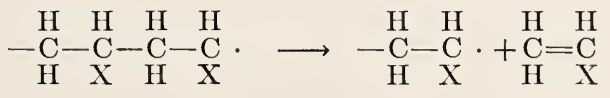

\section{Transfer}

(intermolecular)

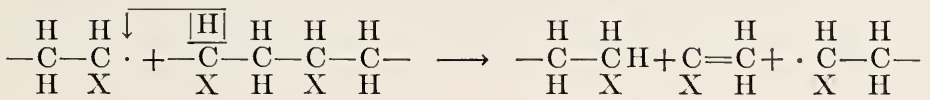

Transfer

(intramolecular)

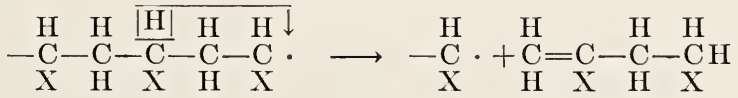

\section{Termination}

(disproportionation)

\section{2 radical $\longrightarrow 2$ polymer}

In order to fix our ideas, it has been assumed that initiation proceeds through breaking of a $\mathrm{C}-\mathrm{C}$ bond. Alternative possibilities, such as the attack of impurities in the chain, can be taken into account by suitably defining the rate constant $k_{1}$ (eq (1)) for initiation. The experimental results for thermal, and particularly for photoactivation, make it necessary to assume two modes of initiation, randomly along the chain or exclusively at a few points in the molecule, in particular the chain ends. The propagation is the reverse of radical growth in polymerization. The transfer, favored by the high temperatures involved, stabilizes a given radical and produces a new one, as in polymerization. However, this radical splits into a shorter radical and a stable chain. It is important to note that transfer acts, according to this picture, in two ways. It prevents, as in polymerization, further propagation. At the same time it breaks a stable molecule at random. Thus, whatever the spectrum of rate constants in figure 2.1 will turn out to be, transfer will contribute a random component. 
We indicate above also a mechanism for intramolecular transfer. This enhances effectively the propagation. Our mathematical analysis does not include this step, and this affects somewhat the subsequent conclusions about the monomer yield. For reasons of simplicity, disproportionation is exclusively admitted as the termination mechanism. It will be noted that cases such as the aging of polyvinyl chloride are not included, because only the break-down of the backbone is under consideration here. Recently a chain mechanism has been proposed by Jellinek [8] that represents a special case of the one described above. Nevertheless, the results derived are at variance with ours. The reason for this will become apparent as we proceed.

Equation (1) shows the four steps in symbolic form

\section{Initiation}

or

$$
P_{n} \stackrel{k_{1}}{\longrightarrow} R_{i}+R_{n-i}
$$

Propagation

$$
P_{n} \stackrel{k_{1}}{\longrightarrow} R_{n-2}+R_{2} ; \quad 2 \leqq i \leqq n-2 .
$$

Transfer

Termination

$$
R_{n} \stackrel{k_{3}}{\longrightarrow} R_{n-2}+P_{2}
$$

$$
\begin{aligned}
& R_{i}+P_{n} \stackrel{k_{3}}{\longrightarrow} P_{i}+R_{\mathrm{j}}+P_{n-j} \quad 2 \leqq j \leqq n-2 \\
& 4 \leqq n \leqq N
\end{aligned}
$$

$$
R_{i}+R_{n} \stackrel{k_{4}}{\longrightarrow} P_{i}+P_{n} .
$$

$P_{n}$ indicates the concentration of stable chains with $n$ C-atoms in the backbone; $R_{n}$, the corresponding radical. $N$ is related therefore to the initial degree of polymerization, $D P$. Any stable molecule with two $\mathrm{C}$-atoms will be called a monomer, as far as the over-all kinetics is concerned. In what follows, a quantity $Q_{n}=P_{n}+P_{n+1}$ is introduced. Assuming the existence of a steady state, as is customary, in respect to the radical concentration, $R$, two parameters, $\epsilon$ and $\sigma$, are characteristic for the theory:

$$
1 / \epsilon=\frac{\text { probability of propagation }+ \text { termination }+ \text { transfer }}{\text { probability of termination }+ \text { transfer }}
$$

or

$$
\left.\begin{array}{rl}
1 / \epsilon & =1+\frac{k_{2}}{k_{4} R+\frac{k_{3} R_{4}}{2 k_{1}} R^{2}}=1+\frac{k_{2}}{k_{4} R(1+\sigma / 2)} ; \text { random initiation, } \\
1 / \epsilon & =1+\frac{k_{2}}{k_{4} R\left(1+\frac{k_{3}}{k_{4} R} \Sigma i Q_{i}-3 \sigma / 2\right)} ; \text { initiation at ends, } \\
\sigma & =k_{3} R / k_{1} .
\end{array}\right\}
$$


It is seen that with increasing magnitude of transfer, $1 / \epsilon$ decreases. To clarify the physical significance of the first parameter, we consider that after initiation has taken place, the propagation will consume a number of monomer units by "zipping" down the line. All zip lengths are possible, although the average will be the larger, the larger the propagation at the expense of transfer and termination. The probability $p_{i}$ of consuming $i$ monomers in one zip equals

$$
p_{i}=\epsilon(1-\epsilon)^{i},
$$

and the number average $i_{n}$ is $(1 / \epsilon)-1$. We shall refer to $1 / \epsilon$ as the kinetic chain length for a given molecule. The usage of the term here differs from the customary one, since $1 / \epsilon$ depends on the transfer constant. It is the analog of the number average $D P$ in polymerization.

\section{Monomer Yield}

We can now establish an approximate relationship between the kinetic chain length and an observable quantity, namely, the monomer yield. Each termination or transfer act produces two altered units in the chain, although some of these disappear again in the course of events. Thus at complete conversion, the molar ratio $r$ between monomer and "impure" monomer should be a measure of the relative probabilities of propagation and termination plus transfer. Thus

$$
r=\frac{1}{2}\left(\frac{1}{\epsilon}-1\right)
$$

This disregards intramolecular transfer, which increases the probability of propagation and, at the same time, decreases the monomer yield. Thus the kinetic chain length so calculated represents probably a minimum value. Furthermore, the evaluation becomes inaccurate when $r$ becomes very large. Also in this case, or for small molecular weights, the irregularities in the terminal units introduced by the polymerization process should not be disregarded. With this in mind, we consider the values for three polymers for which the yields have been reported $[6,7]$.

For polymers of ethylene, styrene, isobutylene, and methyl methacrylate, respectively, one estimates $1 / \epsilon$ equal to $1.01,4.1,4.1$, and at least $2.10^{2}$.

In attempting an interpretation of these large differences, we note that a small kinetic chain length may result from a large transfer probability. This in turn implies a reactive radical and a labile $\mathrm{H}$-atom or other group for the transfer act. Thus the low monomer yield from polyethylene becomes understandable. Similarly, methyl acrylate has a lower yield than methyl methacrylate [9]. A comparison of styrene with $\alpha$-deuterostyrene and $\alpha$-methyl styrene is of interest, the monomer yields increasing in the order indicated [9]. No explanation for the position of polyisobutylene in the series can be offered. 


\section{Molecular Weight and Rate Behavior (Qualitative)}

Next we must examine how $\epsilon$ and $\sigma$ affect the other two important quantities, namely, the molecular weight and the rate. A qualitative picture is readily obtained. Let us neglect transfer, to begin with, and consider two limiting cases. When $1 / \epsilon$ approaches unity, the molecular weight of the degrading molecule will be determined by the initial point of attack, and so will the rate at which small molecules are produced. If initiation occurs exclusively at the ends, bonds in the body of the chain have hardly a chance to be attacked. We revert to the case of a step reaction with exclusive breaking of terminal bonds. The rate of conversion will be proportional to the number of chain ends, that is, inversely proportional to the initial molecular weight. The molecular weight change has been discussed before. In turn, if initiation proceeds at random, we approach a random step reaction with a slow evolution of monomer and a very rapid decrease of molecular weight.

If, on the other hand, the kinetic chain length is extremely large in comparison with the original DP, the locus of initiation is unimportant. A radical, once formed, will be completely annihilated. Monomer will come off rapidly, but the molecular weight of the residue will remain almost constant until the last stages of the reaction.

Our main concern here is the response of chain molecules to thermal and radiative attack. However, there are degradation processes that exhibit a striking similarity to the behavior outlined in the preceding two paragraphs, although the detailed picture is entirely different. In the deterioration of textile materials, some agents attack the fabric in such a way as to leave the tensile strength of the residue unimpaired, whereas others produce a gradual deterioration. The break-down of proteins by proteolytic enzymes seems to occur principally in two ways, one leaving intact material and small species, the other producing molecules of intermediate sizes. A kinetic description of these processes would be similar to ours, which involves in one extreme a slow process, followed by more rapid ones, and in the other a single, moderately rapid step [11].

It remains to consider intermediate cases of moderate kinetic chain length. If initiation is possible only near the chain ends, bonds in the inside are less likely to be reached by the "zip" down than those located near the periphery. If initiation is random, the situation is reversed. We have in effect a spectrum of disintegration probabilities of the type sketched in figure 2.1. This, it must be stressed, has nothing to do with actual weak links, which, one should assume, would be mainly the locus of attack for the chain initiation. All it implies is a statistical effect, produced by the number of ways of getting at a particular linkage.

What influence does transfer have on this picture? As mentioned previously, it introduces a random component, because it tends to strike anywhere with equal probability. Furthermore, it reduces the kinetic chain length. The total effect then is to produce a more rapid decrease of molecular weight and a slower rate of conversion. In the limit of very large $\epsilon N$, the process degenerates into a purely random degradation. 
Without any further calculation we are ready to establish the following parallelism between three important experimental quantities, namely, the monomer yield $Y$, the molecular weight change with conversion $-d M / d C$, and the rate of reaction $d C / d t$ :

$$
\begin{aligned}
& \text { Large } Y \rightarrow \text { small }-d M / d C \rightarrow \text { large } d C / d t \\
& \text { Small } Y \rightarrow \text { large }-d M / d C \rightarrow \text { small } d C / d t .
\end{aligned}
$$

Methyl methacrylate is an example for the first, and polyethylene for the second type of behavior.

\section{The General Rate Equations}

We must now consider the rate equations for the depolymerizing species $Q_{n}$, in order to obtain the molecular weight distribution and to place the above picture on a quantitative basis. We can intuitively anticipate the result in the simplest case, where transfer is negligible and initiation occurs at the ends. Stable chains $Q_{n}$ are destroyed by initiation. They are produced from all larger chains $Q_{n+2 i+2}$. However, these do not contribute equally. Very large molecules are less likely than smaller ones to be degraded down to the desired size before being terminated. This fact is expressed by a weight factor $K_{i}$. In our case $K_{i}$ is simply equal to the previously introduced quantity $p_{i}=\epsilon(1-\epsilon)^{i}$. Thus, we arrive at the following equation:

$$
\frac{d Q_{n}}{d \tau}=-Q_{n}+\sum_{i=0}^{\frac{N-n-3}{2}} K_{i} Q_{n+2 i+2} .
$$

$\tau=2 k_{1} t$ is a dimensionless constant that expresses time on a scale that makes $2 k_{1}$ equal to unity. It is the appearance of the sum that distinguishes the kinetics of depolymerization from the much simpler case of polymerization. There all chains grow from the same nucleus, a catalyst fragment or activated monomer, to an average size determined by the average kinetic chain length. A chain, once terminated, is not reactivated again. Here, however, there exists a whole distribution of nuclei for reactivation and this is reflected in the sum.

At this point we are ready to compare our conclusions with those of Jellinek [8], which he bases on a chain mechanism with no transfer and terminal initiation, as we have done for the moment. However, his treatment follows the one customary in polymerization kinetics, which states that:

Rate of consumption of monomer equals the number average kinetic chain length times the rate of production of radicals. Instead of the above equation, this leads to $d Q_{n} / d \tau=-Q_{n}+Q_{n+(1 / \epsilon)-1}[12]$. This is correct only in the two limiting cases of zero and infinitely large kinetic chain length, when Jellinek's and our expression become identical. In the former case, this is so because our sum reduces to the first term, as it is highly improbable to produce a given chain 
from any but the next largest. In the other case, there are no intermediate species $Q_{n}$ present at all.

The complete theory is epitomized in eq (5a) and (5b). Random initiation

$$
\begin{aligned}
& d Q_{n} / d \tau=-(1+\sigma)(n-3) Q_{n}+2\left(K_{0}-\sigma / 4\right) Q_{n+2}+ \\
& 2 \sum_{i=1}^{\frac{N-n-3}{2}} K_{i} Q_{n+2 i+2} ; \quad 4 \leqq n \leqq N-3, n \text { even } \\
& d Q_{N-1} / d \tau=-(1+\sigma)(N-4) Q_{N-1} ; \\
& K_{i}=(2+\sigma)\left[1-(1-3 / 4 \epsilon)(1-\epsilon)^{i}\right]+\sigma .
\end{aligned}
$$

with

Terminal initiation

$$
\begin{gathered}
d Q_{n} / d \tau=-[2+(n-3) \sigma] Q_{n}+2\left(K_{0}-\sigma / 4\right) Q_{n+2}+ \\
2 \sum_{i=1}^{\frac{N-n-3}{2}} K_{i} Q_{n+2 i+2} ; 4 \leqq n \leqq N-3 \\
d Q_{N-1} / d \tau=-[2+(N-4) \sigma] Q_{N-1} ;
\end{gathered}
$$

with

$$
K_{i}=\sigma\left[1-(1-3 / 4 \epsilon)(1-\epsilon)^{i}\right]+\sigma+\epsilon(1-\epsilon)^{i} .
$$

They are of the expected form in view of the foregoing discussion, eq (5b) for $\sigma=0$ reducing to our previous intuitive result. These rate equations represent formally a step reaction with a spectrum of rate constants $K_{i}$. Depending on the locus of initiation, peripheral bonds are more or less vulnerable to attack than those in the body of the molecule. In any case, however, there is a contribution $\sigma$ to $K_{i}$ independent of $i$, reflecting the randomizing eff ect of transfer.

The formal solution of the linear set $(5 \mathrm{a}, 5 \mathrm{~b})$ has been given. However, the numerical evaluation is another matter. It has been carried out so far for two cases, a finite but large kinetic chain length, by means of expansions in terms of the ratio between initial $D P$ and $1 / \epsilon$, and for very large values of this ratio, when the mechanism reduces to the kind of step reactions analyzed earlier. In addition, we have the solution of the set in the limit $t \rightarrow 0$. This is important because significant conclusions can be drawn from the steady state rate of reaction extrapolated to zero conversion, as shown later. Plans for a complete numerical evaluation were drawn up some time ago with the Computation Section of the Bureau, but no results have been forthcoming at the time of this writing. The limiting solution of eq $(5 \mathrm{a}, 5 \mathrm{~b})$ is

$$
\lim _{\tau \rightarrow 0} Q_{n}=2 Q_{N-1}(0) \frac{K_{N-n-3}}{2} \tau ; \quad n \neq N-1
$$




\section{Molecular Weight and Rate of Reaction}

In the following, $M_{n}(\tau)$ indicates the number arerage molecular weight of the mixture, excluding monomer or larger volatile species, and $C$ the relative extent of conversion, $0 \leqq C \leqq 1$. More detailed results are giren in the orignial papers. Here we summarize the main equations.

1. Random initiation; large kinetic chain length, $\epsilon N / 2 \ll 1, \sigma \rightarrow 0$ :

$$
\left.\begin{array}{c}
\left.M_{n}(\tau) / M_{n}(\tau=0)=\frac{1-\frac{2 \epsilon}{N}\left[e^{(N-1) \tau} \frac{d S}{d \tau}+\frac{N^{2}}{4}\right]+0\left(\epsilon^{2}\right)}{1+2 \epsilon\left[e^{(N-1) \tau} S-\frac{N}{2}\right]+0\left(\epsilon^{2}\right)}\right\} \\
S=\sum_{4}^{N-3} e^{-n \tau} . \\
1-C=e^{-(N-4) \tau}\left\{1-\frac{2 \epsilon}{N}\left[e^{(N-1)} \frac{d S}{d \tau}+\frac{N^{2}}{4}\right]\right\} \\
\frac{d C}{d \tau}=N(1-C)+2 \epsilon e^{3 \tau}\left(\frac{d S}{d \tau}+\frac{1}{N} \frac{d^{2} S}{d \tau^{2}}\right) \\
\lim _{\tau \rightarrow 0} \frac{d C}{d \tau}=N\left(1-\epsilon \frac{N}{6}+\cdots\right) .
\end{array}\right\}
$$

2. Random initiation; small kinetic chain length, $\epsilon \rightarrow 1, \sigma \neq 0$ :

$$
\left.\begin{array}{c}
\frac{M_{n}(t)}{M_{n}(t=0)}=\frac{1+(\alpha / N)(N-L)(L-1)}{1+\alpha(N-L)} \\
\alpha=1-e^{-k t} ; \quad k=k_{1}(1+\sigma)_{;}
\end{array}\right\}
$$

In eq (6.2) and (7.2) it has been assumed that molecules below a critical chain length $L$ evaporate under the conditions of the experiment before being degraded further.

The molecular weight as a function of $C$, eq (6.1) and (7.1), shows the expected slow decrease. It is similar to that in terminal initiation exhibited later. This may be contrasted with the result of eq (6.2) and (7.2) shown in figure 2.2. The initial drop is pronounced, but considerably mitigated by the evaporation of short chains. In polyethylene, for example, the low kinetic chain length and the pronounced possibility for transfer leads to the assumption of a process at least 


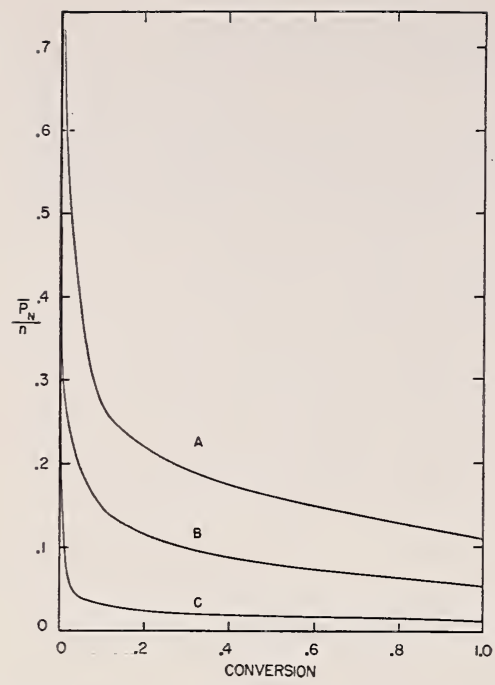

Figcre 2.2. Relative number-average molecular weight as a function of conversion for various values of $L$ and $N=1,000$, equations (6.2) and (7.2). $A: L=101 . B: L=51$. $C: L=$ 11 .

FIGURE 2.4. Rate as function of conversion for random depolymerization, equation (7.2) for various values of $L$ and $N=1,000$.

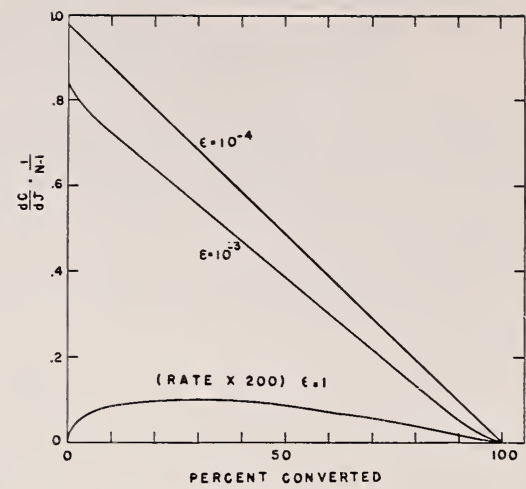

Figure 2.3. Rate as function of conversion and kinetic chain length, random initiation, equations (\%.1) and (7.2), with $L=2$ and $N=1,000$.

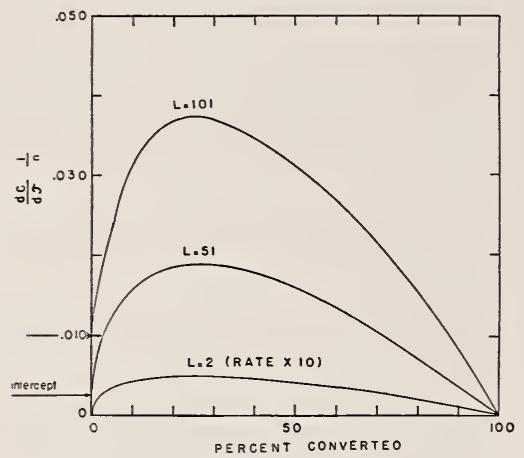

approaching random degradation. Furthermore, the cohesive energy is low enough so that $L$ should be measurably large. From the average molecular weight of the volatiles we have estimated $L$ to correspond to about 70 methylene units [5].

The rates of conversion, eq (7.1) and (7.2), also differ considerably in order of magnitude and in respect to variation with $C$. This is illustrated in figure 2.3. The initial rate is in one case proportional to the initial molecular weight, because each molecule can be attacked in $N$ places and produces in each case $N$ monomer units, considering only orders of magnitude. The number of molecules, however, is inversely proportional to $N$. In random degradation the desired products ( $L$-mers) can be obtained only from the chain ends, hence the initial rate is inversely proportional to $N$. For small $\epsilon N$, the rate is almost first order over the whole range. For $\epsilon \rightarrow 1$, on the other hand, there is a maximum rate at 25-percent conversion for $L=2$ or 3, the case corresponding to evaporation of monomer. With increasing $L$ the maximum remains practically stationary at 26 percent, as long, of course, as $L \ll N / 2$. However, its height and the intercept vary, as shown in figure 2.4. It can be shown that this maximum rate appears when the total number of nonvaporizable molecules and therefore 
molecule ends is at a maximum, which is understandable. The fraction of bonds broken, excluding volatile material, at this point is

$$
\alpha=\frac{N-2 L+1}{L(N-L)} \doteq 1 / L .
$$

If we start with a very low molecular weight material, the maximum shifts to lower conversions and in the limit $L \sim N / 2$ the rate decreases monotonously.

The change of intrinsic viscosity with time has been analyzed for polyethylene by Oakes and Richards [13]. However, conversion data were not published, although the relation between $[\eta]$ and $M$ has been determined [14]. The rate does not show the anticipated maximum [15]. It has been tentatively suggested that this is the consequence of the presence of two mechanisms, each of which would contribute a peak, if present alone [6]. Figure 2.5 shows the molecular weight change for a styrene sample [15]. The drop is sharp, but does not correspond to random depolymerization, unless unreasonably high values for $L$, of the order of 100 , are substituted. This is in qualitative agreement with the small but finite kinetic chain length estimated from the monomer yield. In conformity with this, the rates studied by Madorsky [15] show a maximum, as seen in figure 2.6. However, it is shifted to 40 percent instead of 26 percent, as predicted by random theory, which is again reasonable.

Experimental results such as these indicate again the necessity of evaluating our theory for intermediate cases. We can only present the initial rate, derived from eq $\left(5^{\prime}\right)$. For random initiation we obtain

$$
\begin{aligned}
\lim _{\tau \rightarrow 0} d C / d \tau=-(1+\sigma) & \left(1-\frac{8}{N-1}\right)+\frac{4}{(N-1) \epsilon}(2+\sigma)\left(1-\frac{3 \epsilon}{4}\right) \\
& {\left[\frac{N-1}{2}-1 / \epsilon+\frac{(1-2 \epsilon)}{\epsilon}(1-\epsilon)^{\frac{N-5}{2}}\right]+\frac{\sigma}{2} \frac{(N-3)}{(N-1)} . }
\end{aligned}
$$
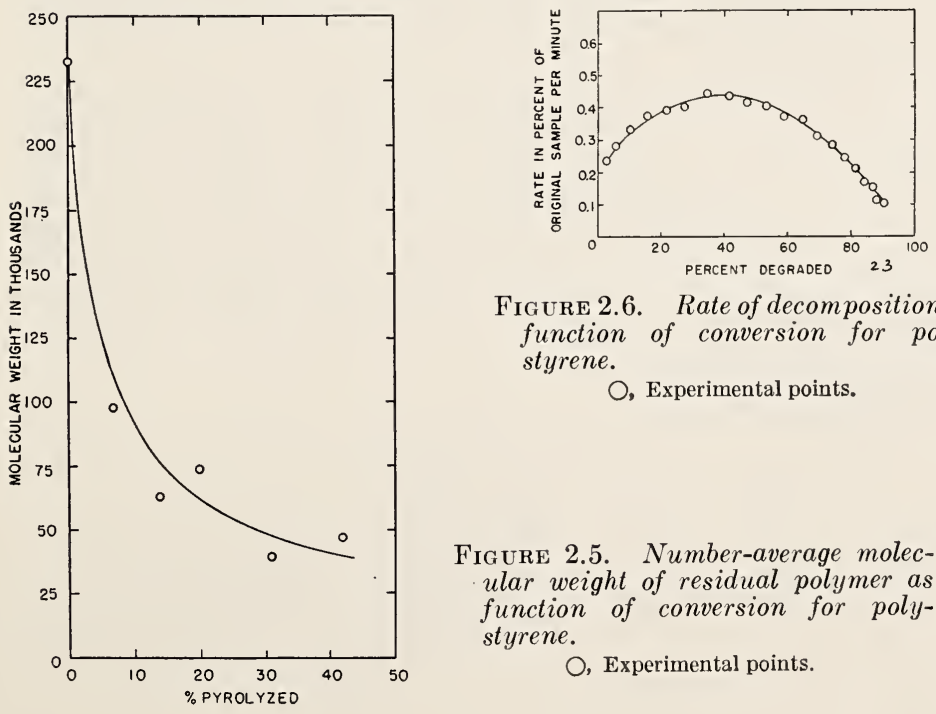

FIgURE 2.6. Rate of decomposition as function of conversion for polystyrene.

, Experimental points.

FIGURE 2.5. Number-average molec- ular weight of residual polymer as function of conversion for polystyrene.

O, Experimental points. 
Because $\epsilon$ is a function of $\sigma$, the rate is not linear in $\sigma$. Equations (7.1) and (7.2) are special cases of $\left(5 \mathrm{a}^{\prime}\right)$. Of further particular interest is the condition $N / 2 \gg 1 / \epsilon \gg 1$. The production of monomer by initiation alone is negligible, and at the same time a molecule is most likely to be only partially degraded. Thus we have the same situation as in polymerization, of course only at the beginning. The rate should be equal to the product of $1 / \epsilon$ and the rate of production of radicals. Indeed, eq $\left(5 \mathrm{a}^{\prime}\right)$ reduces to

$$
\lim _{t \rightarrow 0} d C / d t \doteq k_{1} 4 / \epsilon(1+\sigma / 2)=4 k_{2}\left(\frac{k_{1}}{k_{4}}\right)^{1 / 2} \frac{1}{\left[2(N-1) Q_{N-1}(0)\right]^{1 / 2}}
$$

It follows from (7.1) that for large $1 / \epsilon$ and small $N$ the energy of activation of the whole reaction is essentially that of the initiation step. As the molecular weight increases, we have $E_{\text {act }}=E_{1} / 2+E_{2}-$ $E_{4} / 2$, and the rate is independent of molecular weight. Actually, Madorsky [15] finds the same activation energy, about $60 \mathrm{kcal} \mathrm{mole}^{-1}$, for two polystyrene fractions of molecular weights $1.06 \times 10^{5}$ and $5.84 \times 10^{5}$, respectively. The initial rate for the former is about 10 percent higher than for the latter.

The previous estimate of $1 / \epsilon$, figure 2.6 extrapolated to zero conversion, and eq $\left(5 \mathrm{a}^{\prime}\right)$ yield $k_{1}(\sigma+2) \doteq k_{1} \sigma=k_{3} R=4.5 \times 10^{-6} \mathrm{sec}^{-1}$. The assumption $\sigma \gg 2$ is presumably justified by the fact that the true kinetic chain lengths $k_{2} / k_{4} R$ are of the same order in the thermal polymerization of styrene and methyl methacrylate, and that this should hold also for depolymerization. Consequently, the difference in the magnitude of $1 / \epsilon$ is principally due to the large value of $\sigma$ in styrene. As shown elsewhere [6], one arrives at a reasonable estimate of the propagation constant by assuming further that $k_{3}$ has a value determined in polymerization for transfer between polymer radical and styrene monomer, corrected only for temperature. This gives $k_{2} \approx$ $10^{12} \exp (-23100 / R T) \mathrm{sec}^{-1}$, where the energy of activation is the sum of the heat of polymerization and the energy $E_{2}$ observed in polymerization. Furthermore $R \approx 3 \times 10^{-8}$ mole $1^{-1}$. It is noteworthy, and follows also for methyl methacrylate, that the radical concentrations are of the order of one hundred times as large as in thermal polymerization at room temperature.

Without any assumptions, one deduces from the experimental value of $E_{\text {act }}=60$ and the theoretical expression valid for styrene, that $E_{1} \geqq 74 \mathrm{kcal}_{\text {mole }}^{-1}$. When $\epsilon$ approaches unity, the rate is determined by the sum of initiation and transfer rate, as evident in eq (7.2). Thus $E_{\text {act }}$ must vary with molecular weight, if the molecular weight range studied is wide enough. No specific over-all rate in terms of an invariant reaction order can be defined at finite conversions, except in special cases. Hence, a meaningful activation energy can, in general, be derived only for $C \rightarrow 0$ or 1 .

We now turn to

1. Terminal initiation; large kinetic chain length, $\epsilon N / 2 \ll 1, \sigma \rightarrow 0, z=2 \tau$ :

$$
\frac{M_{n}(z)}{M_{n}(z=0)}=\frac{1+\frac{\epsilon N}{4} z-\frac{\epsilon^{2} N^{2}}{24}\left(z-\frac{z^{2}}{2}\right)+\frac{\epsilon^{3} N^{3}}{192}\left(z-z^{2} / 2+z^{3} / 6\right)+\ldots}{1+\frac{\epsilon N}{2} z-\frac{\epsilon^{2} N^{2}}{8}\left(z-\frac{z^{2}}{2}\right)+\frac{\epsilon^{3} N^{3}}{48}\left(z-z^{2} / 2+z^{3} / 6\right)+\ldots} .
$$




$$
\left.\begin{array}{rl}
1-C & =e^{-2}\left[1+\frac{\epsilon N}{4} z-\frac{\epsilon^{2} N^{2}}{24}\left(z-\frac{z^{2}}{2}\right)+\frac{\epsilon^{3} N^{3}}{192}\left(z-\frac{z^{2}}{2}+\frac{z^{3}}{6}\right)+\cdots\right] \\
d C / d z & =(1-C)-e^{-z} \frac{\epsilon N}{4}\left[1-\frac{\epsilon N}{6}(1-z)+\frac{\epsilon^{2} N^{2}}{48}\left(1-2 z+\frac{z^{2}}{2}\right)+\cdots\right] \\
\lim _{z \rightarrow 0} \frac{d C}{d z} & =1-\frac{\epsilon N}{4}\left[1-\frac{\epsilon N}{6}+\frac{\epsilon^{2} N^{2}}{48}\right] .
\end{array}\right\}
$$

2. Terminal initiation; small kinetic chain length, $\epsilon \rightarrow 1, \sigma \rightarrow 0$ :

$$
\begin{gathered}
M_{n}(z) / M_{n}(z=0)=1-C \\
d C / d z=2 / N .
\end{gathered}
$$

In the limit $\epsilon N \rightarrow 0$, the initial rate is independent of $N$, whereas for $\epsilon \rightarrow 1$, the rate is inversely proportional to $N$. Grassie and Melville [16] examined the rates for a series of methyl methacrylate polymers of widely differing molecular weights, prepared with benzoyl peroxide as catalyst. The dependence on $N$ is slight, and therefore the initiation ought to occur only at a few loci per molecule. Their results are presented in figure 2.7. It is noted that above a certain range of values $N$ the initial rate starts to drop noticeably. This is just what eq (9.1) predicts. Discarding higher than linear terms in $\epsilon N$, we draw the best possible straight line through the experimental points. The rate is plotted as a function of the square root of molecular weight, since $\epsilon$ reduces to $k_{4} R / k_{2}$ when $\sigma=0$ and the radical concentra-
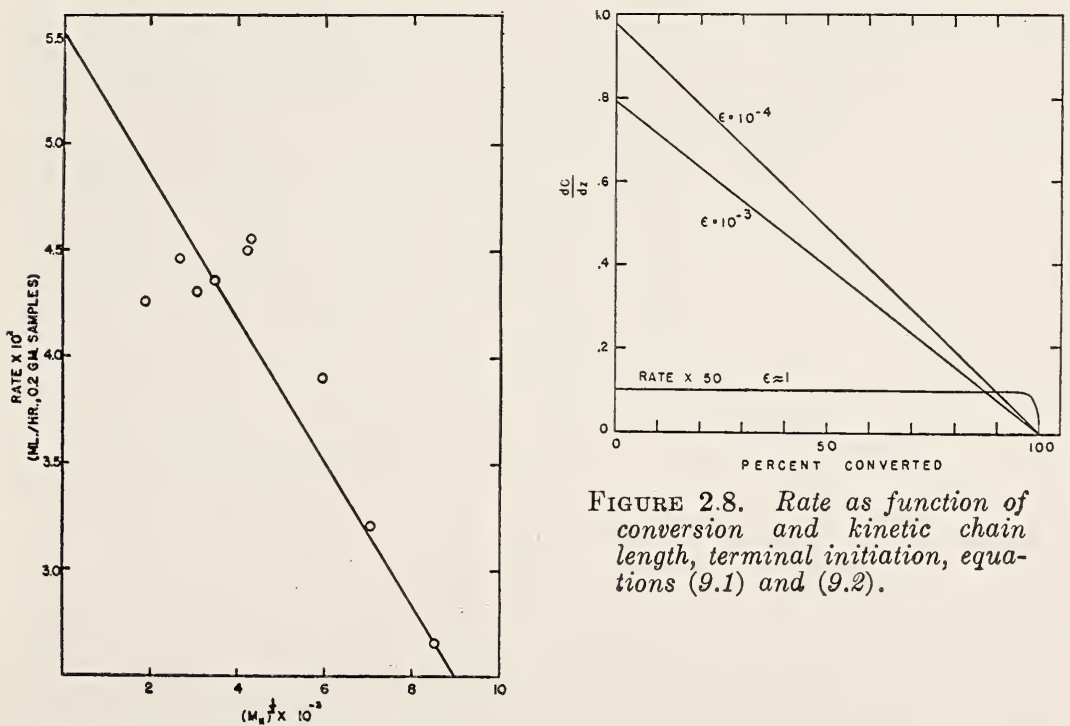

Figure 2.8. Rate as function of conversion and kinetic chain length, terminal initiation, equations (9.1) and (9.2).

FIGURE 2.7. Initial rates as function of initial DP for methyl methacrylate.

O, Experimental points. 
tion is proportional to the square root of the concentration of chain ends. Hence, one can write $\epsilon=\epsilon_{1} \cdot N^{-\frac{1}{2}}$. where $\epsilon_{1}$ is independent of $N$. From the intercept one finds $k_{1}=4 \times 10^{-5} \mathrm{sec}^{-1}$, and from the slope $\epsilon_{1}=1.73 \times 10^{-2}$. For $N=1880, \epsilon=4.1 \times 10^{-4}$, in agreement with the value previously estimated from molecular weight data [4]. For $N=3580, \epsilon=2.9 \times 10^{-4}$.

The change of the rate with conversion is illustrated in figure 2.8. As before, for $\epsilon N \rightarrow 0$, the rate is practically of first order in the conversion. This has been observed by Grassie and Melville [16] in a methyl methacrylate that had been polymerized in the presence of diphenyl cyanomethyl radicals. It is assumed that these both initiate and terminate the chain. With benzoyl peroxide a slight curvature in the graph of rate versus conversion appears, and this is ascribed by the authors to the existence of two types of chain ends with different initiation constants. For $\epsilon \rightarrow 1$, the rate is of zero order.

Before turning to the analysis of the molecular weight, we give the general expression for the initial rate:

$$
\begin{gathered}
\lim _{\tau \rightarrow 0} \frac{d C}{d \tau}=2-\sigma\left(1-\frac{8}{N-1}\right)-\frac{4}{N-1}\left[\frac{N-1}{2}-\frac{1}{\epsilon}+\frac{1-2 \epsilon}{\epsilon}(1-\epsilon)^{\frac{N-5}{2}}\right]+ \\
\frac{4 \sigma}{(N-1) \epsilon}\left(1-\frac{3}{4} \epsilon\right)\left[\frac{N-1}{2}-\frac{1}{\epsilon}+\frac{(1-2 \epsilon)}{\epsilon}(1-\epsilon)^{\frac{N-5}{2}}\right]+\frac{\sigma}{2} \frac{(N-3)}{(N-1)} .
\end{gathered}
$$

For $N / 2 \gg 1 / \epsilon \gg 1$, this reduces to

$$
\lim _{t \rightarrow 0} d C / d t=\frac{k_{1}}{N} 4 / \epsilon(1+\sigma N / 2) .
$$

Since the radical concentration is now proportional to $\left[Q_{N-1}(0)\right]^{1 / 2}$, the initial rate is inversely proportional to the square root of the molecular weight. As in the case of random initiation, the activation energy varies with the initial molecular weight [7].

Grassie and Melville [16] have measured the osmotic molecular weights of a number of polymethyl methacrylate fractions prepared with benzoyl peroxide, as a function of conversion. The initial molecular weights ranged from 44,000 to 775,000 . In the first instance, the molecular weight remained practically constant up to 60 -percent conversion. This is not surprising in view of the magnitude of the kinetic chain length estimated before for this polymer. The results for two samples of molecular weight 94,000 and 177,000 are shown in figures 2.9 and 2.10. For low conversion, the data can be fitted by assuming values of $\epsilon$ between $10^{-4}$ and $4 \times 10^{-4}$, in good agreement with the previous deduction from the initial rate. At higher conversions the decrease is more pronounced than our theory predicts. It is true that eq (8.1), which represents an expansion in $z$, gives too high values for large $C$, because it tends to a limiting value of $1 / 4$ instead of practically zero. However, it is doubtful that this can account fully for the discrepancy. For the highest molecular weight investigated [16], $\epsilon N / 2$ is of the order of unity. Nevertheless, the measured points lie closely to the diagonal, that is, the lower limit set by eq (8.2) in the absence of transfer. A similar behavior has been reported for a sample of about equal molecular weight, irradiated with ultraviolet light at about $100^{\circ}$ to $150^{\circ} \mathrm{C}$ less than the temperature of the thermal reaction 


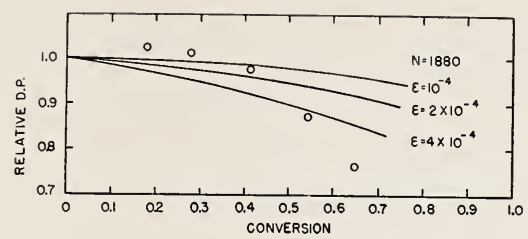

Figure 2.9. Molecular weight as function of conversion for methyl methacrylate, $N=1880$.

$\bigcirc$, Experimental points; full lines, theory.

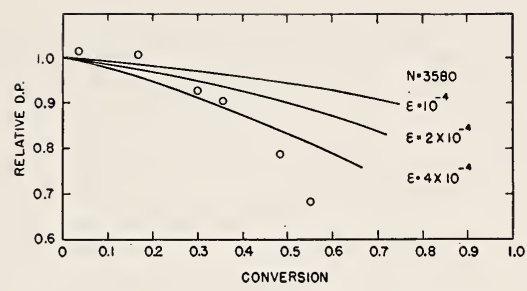

Figure 2.10. Molecular weight as function of conversion for methyl methacrylate, $N=3,580$.

$\bigcirc$, Experimental points; full lines, theory.

[17]. It would be of interest to see whether a further increase in $N$ pushes the curve decisively below the diagonal. This would indicate either that initiation does not take place at the chain ends only, or that some transfer process becomes relatively important as the reaction proceeds. This would affect particularly the larger molecular weights. The fact that the activation energy increases from 31 to $42 \mathrm{kcal} \mathrm{mole}^{-\mathrm{i}}$ with conversion, is not inconsistent with this suggestion, since it should be indicative of a decrease of the initiation rate. In the polymer containing diphenyl cyanomethyl radicals, the activation energy is 47 kcal mole ${ }^{-1}$ and constant. A determination of the molecular weight change for this polymer would be very pertinent.

Possibly these results indicate that the basic reaction scheme requires refinement in order to achieve closer agreement. At the same time it must be recalled, that so far, the numerical consequences of the theory have not been exploited fully.

Our theory is based exclusively on a chain process without explicit recourse to weak links. Such may well have been introduced into the polymerizing molecule through accelerator fragments, in the combination of two radicals or by means of other impurities. These would then act as initiating sites and determine the mechanism of initiation. We have made only two extreme assumptions regarding this step in order to allow for a molecular-weight drop more rapid than linear in $C$ and a maximum rate on the one hand, and the results for methyl methacrylate on the other. However, it must be remarked that transfer, if preponderant enough, will have the same qualitative effect as a random initiation. It would, therefore, be very desirable to investigate the effect of molecular weight on the initial rate of degradation, in cases other than methyl methacrylate, to have more information on this point. We have no explanation to suggest for the initiation taking place at the chain ends in methyl methacrylate and at random in styrene.

\section{Conclusions}

The achievements of the theory may be summarized as follows. First, it accounts for the parallelism existing between monomer yield, rate of decomposition, and rate of change of molecular weight. Secondly, it closes the gap between as diverse systems as polyethylene and methyl methacrylate. Finally, it is sufficiently general to provide a basis for further extension. We have already mentioned other 
processes that seem to follow the same general pattern, particularly the enzymatic degradation of proteins [11].

As far as experimental analysis is concerned, an analogy with the problem of sedimentation and diffusion rates and intrinsic viscosity comes to mind. Once a model has been adopted for the macromolecule, and the molecular mass is known, each of these quantities can be calculated from the other at infinite dilution. Yet we have no satisfactory picture of the macromolecule until more than one type of measurement has been carried out and the model chosen has been shown to be self-consistent, that is, to behave as one and the same thing regardless of the nature of the experiment. In the same manner, it is not sufficient for a satisfactory analysis to be content with a knowledge of the monomer yield, which is difficult to interpret. The other two quantities are necessary to decide whether the model of the reaction used is self-consistent.

The quantities that are most structure sensitive are the initiation and transfer rates and to a certain extent, the propagation. The investigations of Grassie and Melville, by concentrating on a polymer in which transfer should be more or less suppressed and by studying the effect of varying the molecule ends, have contributed in an important manner to the problem. Similarly, the experiments started by Wall, in which the possibility of transfer is systematically varied by the introduction of substituents, such as deuterium, should prove significant.

\section{References}

I[1] L. G. Sillén, Svensk Kem. Tid. 55, 221 (1943).

[2] R. Simha, J. Applied Phys. 12, 569 (1941).

'[3] W. Chalmers, J. Am. Chem. Soc. 56, 912 (1934).

[4] R. Simha, L. A. Wall, and P. J. Blatz, J. Polymer Sci. 5, 615 (1950).

[5] R. Simha and L. A. Wall, J. Polymer Sci. 6, 39 (1951).

[6] R. Simha and L. A. Wall, J. Phys. Chem. 56, 707 (1952).

[7] R. Simha, Trans. N. Y. Acad. Sci. 14, 151 (1952); J. Polymer Sci. 9, 465 (1952).

[8] H. H. G. Jellinek, paper 1 of this Symposium.

[9] L. A. Wall, private communication.

[10] K. V. Linderstrem-Lang, lecture presented at the twelfth International Congress of Pure and Applied Chemistry, New York, N. Y., Sept. 9-13, 1951 .

[11] R. Simha, Arch. Biochem. Biophys. (in press).

[12] R. Simha, J. Polymer Sci. 10, 499 (1953).

[13] W. G. Oakes and R. B. Richards, J. Chem. Soc. 2929 (1949).

[14] F. Bebbington, E. Hunter, and R. B. Richards, paper presented at the twelfth International Congress of Pure and Applied Chemistry, New York, N. Y., Sept. 9-13, 1951.

[15] S. L. Madorsky, paper 15 of this Symposium.

[16] N. Grassie and H. W. Melville, Proc. Roy. Soc. London [A] 199, 14, 24, 39 (1949).

[17] P. R. E. J. Cowley and H. W. Melville, paper 3 of this Symposium.

\section{Discussion}

Dr. G. F. Bloomfield, British Rubber Producers' Research Association, Welwyn Garden City, England: The experiments of Blow and Wood ${ }^{3}$ seemed to indicate that oxygen played an essential role in break-down of rubber during mastication because when an oxygenfree atmosphere was maintained in an internal mixer no break-down was observed during $40 \mathrm{~min}$ at each of the three temperatures $50^{\circ}$,

${ }_{3}$ Trans. Inst. Rubber Ind. 25, 309 (1950). 
TABLE 1. Mastication (30 minutes) of smoked sheet of initial Mooney 98

\begin{tabular}{|c|c|c|c|c|c|c|}
\hline \multirow{3}{*}{ Additive ( $1 \%$ on rubber) } & \multicolumn{6}{|c|}{ Final Mooney } \\
\hline & \multicolumn{3}{|c|}{ Under nitrogen at- } & \multicolumn{3}{|c|}{ In air at- } \\
\hline & $50^{\circ} \mathrm{C}$ & $100^{\circ} \mathrm{C}$ & $142^{\circ} \mathrm{C}$ & $50^{\circ} \mathrm{C}$ & $100^{\circ} \mathrm{C}$ & $142^{\circ} \mathrm{C}$ \\
\hline $\begin{array}{l}\text { None } \\
\text { MBT } \\
\text { Pepton } 22\end{array}$ & $\begin{array}{r}100 \\
66 \\
40\end{array}$ & $\begin{array}{l}95 \\
89 \\
83\end{array}$ & $\begin{array}{l}97 \\
86 \\
74\end{array}$ & $\begin{array}{l}24 \\
22 \\
28\end{array}$ & $\begin{array}{l}66 \\
11 \\
11\end{array}$ & $\begin{array}{l}34 \\
\text { Very low } \\
\text { Do. }\end{array}$ \\
\hline
\end{tabular}

TABLE 2. Mastication ( 30 minutes) of rubber (USF) of initial Mooney $90 \pm 3$

\begin{tabular}{|c|c|c|c|c|}
\hline \multirow{3}{*}{$\begin{array}{l}\text { Additive }(0.0925 \mathrm{~g} \text { moles } \\
\text { per } 1,000 \mathrm{~g} \text { of rubber })\end{array}$} & \multicolumn{4}{|c|}{ Final Mooney (all values \pm 3 ) } \\
\hline & \multicolumn{2}{|c|}{$\begin{array}{c}\text { Under nitrogen } \\
\text { at- }\end{array}$} & \multicolumn{2}{|c|}{ In air at- } \\
\hline & $55^{\circ} \mathrm{C}$ & $140^{\circ} \mathrm{C}$ & $55^{\circ} \mathrm{C}$ & $140^{\circ} \mathrm{C}$ \\
\hline None & 95 & 95 & 22 & 13.5 \\
\hline Benzoquinone.. & 36 & 91 & 23 & 34 \\
\hline Pyrogallol & 63 & 97 & 27 & \\
\hline Phenol & 76 & 88 & 25 & 19 \\
\hline Hyaroqu & 86 & & 20 & \\
\hline
\end{tabular}

$100^{\circ}$, and $140^{\circ}$ C. An unexplained feature of Blow and Wood's results (table 1) was the ready break-down under nitrogen at $50^{\circ} \mathrm{C}$, but not at $100^{\circ}$ or $140^{\circ} \mathrm{C}$, in the presence in the rubber of mercaptobenzthiazole (MBT) or $0, o^{\prime}$-dibenzamidodiphenyl disulfide (Pepton 22).

More recent work of Pike and Watson ${ }^{4}$ has resolved the anomaly in Blow and Wood's results. It now seems certain that primary bonds in rubber molecules are ruptured and thus produce chain fragments terminating in free radicals, if shearing forces of sufficient magnitude are developed during mastication. The radical ends would normally have a high probability of recombination unless there is also present an auxiliary substance capable of competing with the recombination reaction. In air-mastication oxygen normally fulfills this role, and its action is seen to be a secondary one of radical destruction rather than a primary one of causing scission by an oxidative chain reaction. Pike and Watson have shown that certain compounds (see table 2) that interact readily with radicals in polymerization systems may act similarly to oxygen in preventing recombination of mechanically ruptured polymer chains. Because their efficiencies are considerably lower than that of oxygen, these auxiliary substances are unable to compete effectively with oxygen, and they have practically no effect on break-down in air at low temperatures.

In mastication at temperatures in the region of $140^{\circ} \mathrm{C}$ the shearing forces are not sufficiently great to rupture molecules, and little or no break-down under nitrogen is observed, irrespective of whether

1 India Rubber J. 120, 1033, (1951). 
radical-combining additives are present or absent. The extensive break-down in air at this temperature is accordingly attributed to an oxidative chain mechanism. This view is supported by the observation that some of the additives now exhibit antioxidant activity.

The two unrelated mechanisms involved in hot and cold mastication explain the shape of the rate of break-down curve observed in mastication in air. The efficiencr of break-down by rupture (lowtemperature effect) decreases with increasing temperature, whereas the efficiency of oxidative scission (high-temperature effect) increases with increasing temperature. At some temperature between $50^{\circ}$ and $140^{\circ} \mathrm{C}$ the efficiency will be at a minimum. It has in fact long been known that the rate of break-down at temperatures around $100^{\circ} \mathrm{C}$ is considerably lower than that prevailing at either $50^{\circ}$ or $140^{\circ} \mathrm{C}$.

Dr. H. H. G. Jellinek, University of Adelaide, Adelaide, Australia: I wish to say a few words about the chain reactions. I am confining my remarks, here, to the experimental material available up to date. I do not think it is necessary to assume chain transfer. The experimental data arailable, relerant to this discussion, all refer to degradation in bulk; degradation in solution has, so far, been mainly carried out in the presence of catalysts such as benzoyl peroxide and presents a different problem. The bulk degradation takes place in a highly viscous medium. Now, the propagation reaction is a very fast one, and before chain transfer can become appreciable, this propagation will have been terminated. That is what I think happens in the case of polystyrene. In the case of polymethyl methacrylate, which has not an $\alpha$-hydrogen atom but an $\alpha$-methyl group, there is no experimental evidence for chain transfer at all.

It has been suggested that polyethylene degrades mainly by a random process, I take it, by a random degradation in the ordinary sense, not a chain reaction with random initiation. I am afraid I cannot share this view. It can very easily be calculated what the degree of degradation $s / P_{0}-1$ (where $s$ is the average number of breaks per original chain $P_{0}$ ) should be, if, for instance, 40 percent of the original polymer had volatilized, not even assuming that these 40 percent all consist of monomer units but of fragments comprising about 10 units or so. The degree of degradation would be so high for such a stage of degradation that the residual polymer in the reaction ressel would have such a small chain length that it should vaporize in a matter of seconds or minutes. As a matter of fact, a zero-order reaction was found for the loss of weight, which continued for more than an hour after a 40-percent loss of weight had been suffered by the polymer. For a random degradation, the loss-ofweight versus time curve would not be a straight line but would deviate very rapidly from the straight line, the loss of weight rapidly increasing.

I do not mean to say that chain transfer will not occur at all in degradation reactions. On the contrary, for degradation reactions in solution, chain transfer is likely to occur, especially by solvent molecules.

Dr. Simha mentioned, if I understood him correctly, that it would be more difficult to initiate a smaller than a longer chain. I have investigated in the case of polystyrene a number of fractions of different chain lengths. I did not find any indication that it is more difficult to initiate a smaller than a longer chain. It is the same for 
all lengths, and the rate of degradation depends only on the number of chain ends in the system. If you have smaller chains, you get a higher rate of monomer production per gram of polymer than if you would have a longer chain. The energy of activation for all fractions remains the same.

Dr. S. L. Madorsky, National Bureau of Standards: If I understand correctly, Dr. Jellinek stated that polyethylene breaks mostly from the end of the chain. How does he reconcile this with the fact that in the degradation of polyethylene a very small amount of monomer fragments come off, whereas the bulk of the rolatile product has an average molecular weight of about 700 ?

Dr. JellineK: It is an experimental fact that if you degrade polyethylene and you plot the loss of weight in percent against time, you get a straight line over a considerable range of the degradation. This means that you have a zero-order reaction or, in other words, that fragments are broken off mainly from the chain ends. This does not necessarily mean that you get only monomer units. It might be the case that larger fragments are broken off the chain ends. I can only see one other possibility as to how polyethylene might degrade at fairly elevated temperatures. In case the loss-of-weight curves are not quite straight but somewhat curved, then the degradation could be explained by assuming that as soon as one chain is initiated or actirated, it is completely shattered into a multitude of small fragments.

Dr. R. B. Richards, Imperial Chemical Industries Ltd., England: What I have to say is rather in support of what Dr. Madorsky has just said. Our own work has been done at rather lower temperatures than Dr. Jellinek's. We have worked in the range $290^{\circ}$ to $360^{\circ} \mathrm{C}$ for two reasons. First, we wanted to define the maximum temperature at which one could handle polyethylene without degradation. Second, we were interested in a process for the manufacture of a hydrocarbon wax with a molecular weight of about 3,000 . Up to about $360^{\circ} \mathrm{C}$, one gets scarcely any ethylene at all formed, despite the fact that you reduce the molecular weight of the polymer from about 25,000 to about 2,000 , or even lower. That, I think, supports Dr. Simha's idea of transfer as a dominant feature.

Dr. Jellinek: My remarks on polyethylene are only intended for the region of degradation where the loss of weight is appreciable, about $360^{\circ} \mathrm{C}$ and above. In this region the chain character of the reaction becomes predominant, as pointed out above, and the degradation cannot then be explained by a random process. The situation is probably similar to the case of polystyrene where at moderate temperatures, reactions are predominantly different from those at more elevated temperatures.

Dr. Simha: I should like to add first a few comments to what preceding speakers have said about transfer. 'The pronounced differences in monomer yield encountered are indicative of differences in the average zip length $(1 / \epsilon)-1$. The chemical evidence indicates that this means primarily differences in the termination and/or transfer rates, unless steric factors effect the propagation. From that point of view only an absolute determination of the elementary constants, that is, an additional measurement of the mean radical lifetime would settle this question. Howerer, the termination constants should not vary too much, judging from the experience in polymerization and the differences primarily reside in the transfer act. 'The chemical evidence 
in polyethylene, polymethyl acrylate, poly- $\alpha$-methyl and $\alpha$-deuterostyrene, and polyisoprene has already been discussed. Results obtained on copolymers of methyl methacrylate and acrylonitrile represent another case in point.

Now as to the molecular weight behavior: For polyethylene we have found no data of $D P$ versus conversion $C$ in the literature, but only versus time. Exclusive splitting of terminal fragments, advocated by Dr. Jellinek, requires a linear decrease of $D P$ with $C$. A theory of random degradation that allows for the evaporation of relatively large fragments gives a markedly reduced rate of decrease of $D P$ as compared with the situation where only monomer comes off. The monomer yields and cryoscopic determinations indicate that fragments containing $70 \mathrm{CH}_{2}$ units evaporate. The number varies during the reaction by something like 10 to 15 units due to the thermodynamic vapor pressure lowering. Considering the uncertainty in correcting heats of vaporization for the high temperatures in question, this represents a reasonable estimate. Moreover, one can calculate the resulting molecular weight distribution of the volatiles. The ratio between weight and number average is in agreement with experiment.

The question of the overall rate for polyethylene does not seem settled. For $1 / \epsilon \rightarrow 1$, one expects either a zero order or the maximum rate at a certain $C$. Madorsky obtains neither. The presence of branch points may "blur" the maximum curve, as has been suggested by Wall and experiments on a pure polymethylene system are pertinent.

One more word about weak points. The possibility of intrinsic differences in bond strength depending on position and chain length has been pointed out by several authors, in order to account for variations in the rate of prrolysis in the series ethane-butane, etc. Such a spectrum of weak links may be superimposed on the statistical spectrum $K_{i}$, discussed in my paper. However, it can hardly be of primary importance. The results of theories that operate on the assumption of equivalent bonds (apart from initiation, which would preferentially start at weak spots), ought to be fully exploited before introducing further refinements.

In polystyrene, a maximum rate is observed. Since $1 / \epsilon \doteq 4$ to 5 , we have so far no numerical solutions for the rate and molecularweight curves. Both random degradation and stepwise splitting of terminal bonds vield an initial over-all rate inversely proportional to the $D P$. Both mechanisms are irreconcilable with the relatively large monomer yield and the rather sharp molecular-weight decrease. Initiation may or may not occur at random. Kinetically, this is not too significant, in view of the predominance of randomizing transfer. I did not imply that the rate of initiation is dependent on chain size. In polystyrene, $N \gg 1 / \epsilon \gg 1$, approximately, the initial rate of depolymerization is independent of molecular weight and proportional to $k_{2}\left(k_{1} / k_{4}\right)^{1 / 2}$ in analogy to the well-known relation in polymerization. Results obtained at the NBS give an activation energy independent of molecular weight. The rate dependence is not conclusive. If at all, the results on two fractions point to a slight increase of initial rate with increasing $D P$. 


\title{
3. Photodegradation of Polymethyl Methacrylate
}

\author{
By P. R. E. J. Cowley ${ }^{1}$ and H. W. Melville ${ }^{1}$
}

\section{Introduction}

This paper is concerned with the mechanism of the degradation of vinyl polymers, particularly that of polymethyl methacrylate. In earlier studies of the thermal degradation of this polymer [1] ${ }^{2}$ the rate of evolution of monomer and the size and number of the residual polymer molecules were measured and the general features of the mechanism of degradation determined. However, no determination of the individual velocity constants was possible. The position was similar to that which prevailed a few years ago in the study of the mechanism of polymerization. Here the rate and chain length of the reaction had been measured, but the absolute rate constants of the elementary reactions could not be determined until accurate measurements were made of the lifetime of the polymer radicals.

In the present work it has been found that degradation to monomer is induced by radiation of wavelength $2537 \mathrm{~A}$ at much lower temperatures than those employed in the studies of thermal degradation. The kinetics of this reaction have been studied, and the lifetime of the degrading polymer radicals measured by a modification of the rotatingsector technique. From these measurements it has been possible to evaluate the concentration of radicals and the rate constants involved in the degradation reaction. By combining these data with published values for the corresponding polymerization reaction, it is possible to obtain a value for the entropy of polymerization in fair agreement with that obtained independently by direct measurement of the equilibrium pressure of monomer over a polymer-monomer mixture [2].

\section{Apparatus}

The apparatus used was similar to that used in the study of the thermal reaction, the essential features being that the reaction was carried out in vacuum in a molecular still, the rate of evolution of monomer being measured on a calibrated Pirani gage. Provision was made for more accurate temperature control $( \pm 1 \mathrm{deg} C$ ), and the polymer film (about $10^{-3} \mathrm{~cm}$ in thickness) was irradiated by a lowpressure mercury-vapor lamp emitting mainly 2537 A radiation. The galvanometer in the Pirani bridge network could be replaced by a d-c amplifier. The bridge was balanced at zero rate of monomer evolution. When monomer was evolved, the out-of-balance current was amplified and fed into a pen-recorder. The deflection of the pen was directly calibrated in terms of rate-of-monomer evolution, and

1 Chemistry Department, University of Birmingham, Birmingham, England.

2 Figure in brackets indicate the literature references on p. 67 . 
by using this arrangement it was possible to measure the rate of reaction during sector experiments with intermittent illumination by integrating under the pen-deflection versus time curves.

The polymers and copolymers used were made by conventional methods and were cast as very thin films on a heated copper tray inside the molecular still. The polymer residue after a degradation experiment could be dissolved from the tray and used for numberaverage molecular-weight determinations by the osmotic-pressure method [3], or by viscometric methods, using the relation between intrinsic viscosity and number-average molecular weight due to Baxendale, Bywater, and Evans [4].

\section{Preliminary Experiments}

It was found that residual volatiles (solvents and precipitants) in the polymer film could be removed in vacuum by heating to temperatures above the softening point of the polymer. After these had been removed, irradiation with ultraviolet light was started. The products were collected in a liquid-air trap and distilled into calibrated capillaries. Monomer was the only major product, and this evolved very rapidly in the temperature range $130^{\circ}$ to $200^{\circ} \mathrm{C}$ when ultraviolet light was incident on the polymer, initial rates of degradation being of the order 100 percent an hour. Similar high rates of degradation have been found for samples of polyisopropyl methacrylate, but several other common polymers investigated show very different behavior. Thus no evolution of volatiles occurred on irradiation in vacuum of polymethyl acrylate, polymethacrylic acid, polyacrylonitrile, polyisobutene, or polyvinyl acetate, though the latter two do degrade thermally to volatile products. Polystrene showed a relatively slow rate of photodegradation (less than 1 percent an hour at $280^{\circ} \mathrm{C}$ ), and a sample of poly- $\alpha$-methylstyrene photodegraded rapidly to volatile products in the range $240^{\circ}$ to $270^{\circ} \mathrm{C}$ when there was also some thermal degradation. These preliminary experiments were concerned only with finding out whether the materials degraded in such a way that the existing techniques could be used, and no attempt was made to measure any molecular-weight changes that may have occurred.

\section{Over-all Quantum Efficiency}

The lamp used in most of the photochemical experiments was a water-cooled low-pressure type emitting at least 90 percent of its radiation at $2537 \mathrm{~A}$. The total intensity incident on the polymer film was measured with a uranyl oxalate actinometer, and the absorption of a series of films at 2537 A measured on a Hilger Uvispek photoelectric spectrophotometer. A plot of $\log _{10}\left(I_{t} / I_{0}\right)$ against thickness of film (where $I_{0}$ is the incident intensity and $I_{t}$ the transmitted intensity) gave a good linear plot, and extrapolation to zero film thickness enabled a correction to be found for scattering and reflection. Thus, knowing the percentage absorption (about 6 percent) and the incident intensity, the number of quanta/sec absorbed by the film could be found, and dividing this in to the measured initial rate (num- 
ber of molecules monomer evolved/sec) gave a value for the over-all quantum efficiency in the initial stages of the reaction. For a sample of polymethyl methacrylate (the polymerization of which was initiated by benzoyl peroxide, $\left.\bar{M}_{n}=125,000\right)$, approximately 220 monomer molecules were evolved for each quantum of 2537 A radiation absorbed. This result shows clearly that the main feature of the reaction is a chain process involving elimination of monomer molecules.

\section{Intensity Exponents}

The intensity exponent could be measured by finding the rate-ofmonomer evolution at full light intensity, and then measuring the corresponding rate at a lower light intensity, the reduction in intensity being achieved by using wire-gauze screens of known transmission. Exponents could be measured to about \pm 0.05 in most cases. The results in table 3.1 show that for a variety of methyl methacrylate polymers of different molecular weights and for acrylonitrile-methyl methacrylate copolymers, the intensity exponents are very close to 0.50 , except for one or two polymers of relatively low molecular weight.

TABLE 3.1. Intensity exponents of polymers and copolymers of methyl methacrylate.

\begin{tabular}{|c|c|c|}
\hline Polymer & $\begin{array}{l}\text { Tempera- } \\
\text { ture of } \\
\text { measure- } \\
\text { ment }\end{array}$ & $\begin{array}{l}\text { Intensity } \\
\text { exponent }\end{array}$ \\
\hline & ${ }^{\circ} \mathrm{C}$ & \\
\hline $\begin{array}{l}0.5 \% \mathrm{BP} \text { a polymer, } \overline{\mathcal{M}}_{n}=125,000 \\
\text { Benzoin photosensitized polymer }\end{array}$ & $\begin{array}{l}150 \\
150\end{array}$ & $\begin{array}{r}0.51 \\
.48\end{array}$ \\
\hline $0.1 \%$ BP polymer, $\bar{M}_{n}=770,000$ & 180 & $\left\{\begin{array}{r}45 \\
50\end{array}\right.$ \\
\hline $\begin{array}{l}0.5 \% \text { BP polymer, at } 5 \% \text { degradation } \\
0.5 \% \text { B P polymer, at } 65 \% \text { degradation } \\
4 \% \text { BP polymer }\end{array}$ & $\begin{array}{l}171 \\
192.5 \\
163\end{array}$ & $\begin{array}{l}.6 \\
.57 \\
.49\end{array}$ \\
\hline BP polymer, $\bar{M}_{n}=70,000$ & 163 & .57 \\
\hline $0.1 \%$ BP polymer, $\bar{M}_{n} \bumpeq 900,000$ & 163 & .52 \\
\hline $\bar{M}_{n} \bumpeq 480,000 \ldots$ & 163 & .48 \\
\hline Ethyl benzene transfer polymer $\left\langle\overline{\underline{M}}_{n} \bumpeq 190,000 \ldots\right.$ & 163 & .59 \\
\hline $\mid \bar{\Lambda}_{n}<100,000 \ldots$ & 163 & .74 \\
\hline Acrylonitrile-methyl methacrylate copolymer $\left\{\begin{array}{lll}1 & A: 40 \\
1 & A: 410 & M M\end{array}\right.$ & $\begin{array}{l}192.5 \\
192.5\end{array}$ & .08 \\
\hline
\end{tabular}

a BP is benzoyl peroxide initiated.

Evidently in most cases the termination reaction must involve mutual interaction of two of the chain carriers. By analogy with similar reactions it is to be expected that the over-all process is a free radical reaction. The present results, particularly the pronounced effect of ultraviolet light and the intensity exponent of 0.5 , support this, and confirmation is provided by the effect of retarders to be discussed later. The essential features of the photoreaction thus appear to be the same as those deduced for the thermal reaction, namely, an initiation reaction providing free radicals, a propagation reaction that involves the successive removal of monomer units from a degrading polymer radical, and a termination reaction that in most cases involves mutual reaction of two radicals. 


\section{Molecular-Weight Changes}

Two polymers of initial number-average molecular weights 125,000 and 770,000 , respectively, were photodegraded to varying extents at $175^{\circ} \mathrm{C}$, the total percentage degradation in each experiment being found by weighing the reaction tray and polymer residue after the run. The polymer residues were dissolved from the tray and used for osmotic-pressure determinations.

The results are reasonably similar to those already published for the corresponding thermal reaction, and are shown with the earlier results in figure 3.1 .

The decrease in molecular weight of the residue of the highermolecular-weight polymer is approximately proportional to the amount of monomer lost. 'This agrees with the mechanism outlined above, in which termination of the chain reaction is by mutual interaction of two radicals. On the other hand, for low-molecular-weight polymers, the molecular weight of the residue stays nearly constant over considerable extents of reaction, gradually falling in the later stages of degradation. 'This behavior has been interpreted as being due to a depolymerization reaction involving rapid and complete elimination of whole polymer molecules, leaving a residue of unchanged polymer. However, this does not explain the gradual fall in molecular weight nor does it explain why the intensity exponent is 0.5 or nearly so for these polymers. These features can be explained if a chain transfer process occurs to a small extent. This type of reaction is favored at high temperatures, and if the reactivity of a degrading polymer radical is transferred to another polymer that then proceeds to degrade, we have the possibility of the kinetic chain length being greater than the degree of polymerization. In such a case the termination can still be a mutual reaction leading to an intensity exponent of 0.5 , and there will be a gradual increase in the number of stabilized polymer residues of lower molecular weight, which will cause the average molecular weight to fall in the later stages of reaction. A similar type of reaction scheme has recently been treated mathematically by Simha, Wall, and Blatz [5], whose curves of $\bar{M}_{n} / \bar{M}_{n o}$ versus percentage

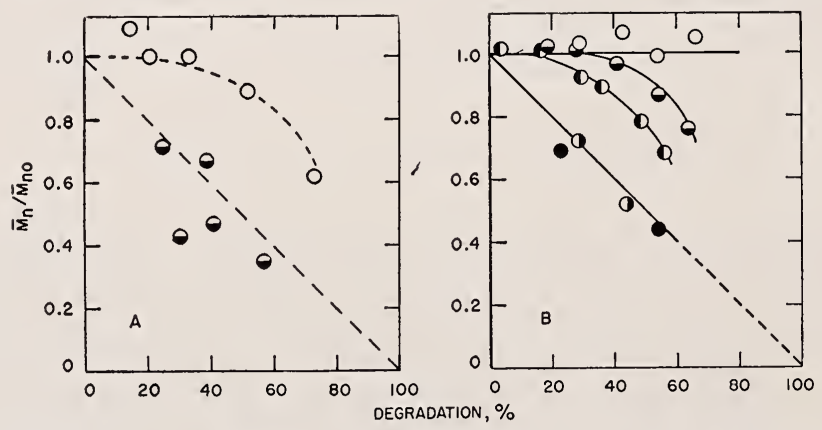

Figure 3.1 Molecular weight divided by initial molecular weight $\left(\bar{M}_{n o}\right)$ versus percent degradation to monomer.
A, Photodegradation, $\bigcirc, \bar{M}_{n o} 125,000 ; \ominus, \bar{M}_{n o} 770,000$
B, Thermal degradation (after Grassie and Melville).
○, $\bar{M}_{n o} 44,300 ; \Theta, \bar{M}_{n o} 94,000 ; \bigcirc, \bar{M}_{n o} 179,000$;
), $\bar{M}_{n o} 650,000 ; \bigcirc, \bar{M}_{n o} 725,000$. 
of degradation for cases where the kinetic chain length is greater than the degree of polymerization are similar to those obtained experimentally in the present work.

If this explanation is correct, the molecular-weight versus percentage-of-degradation curres indicate that the kinetic chain length is greater than the degree of polymerization of the lower-molecularweight polymer (1250) but less than that of the higher polymer (7700). Similarly, the treatment of Simha, Wall, and Blatz leads to a value of the kinetic chain length of approximately 3000. This may be compared with the minimum value of 200 deduced from the over-all quantum efficiency. The over-all rate decreases rapidly as the reaction proceeds, and it is to be expected that the kinetic chain length will increase as degradation proceeds. The value of 200 is derived as a minimum value at the high initial rate of degradation, and the mean value during the first 50 percent of degradation might well be of the order 1000. At all events, it is clear that the primary quantum efficiencr is of the order 0.1 or greater, and that the initial fission reaction is very efficient, considering the highly viscous nature of the polrmer. Knowing the rate and the kinetic chain length, the rate of initiation can be deduced as approximately $1.56 \times 10^{-6}$ (mole/liter of polymer)/sec. This value is supported by retardation experiments now to be described.

\section{Retardation by Tetraphenyl Succinodinitrile}

The photodegradation reaction is carried out in a very thin polymer film in vacuum at $150^{\circ}$ to $180^{\circ} \mathrm{C}$. Under these conditions all the usual inhibitors are useless, as the material has to be either in volatile at these temperatures or the molecules must be sufficiently large to have an excessively low rate of diffusion through polymethyl methacrylate. The only satisfactory material found so far as a retarder for the photoreaction is tetraphenyl succinodinitrile. In figure 3.2 the

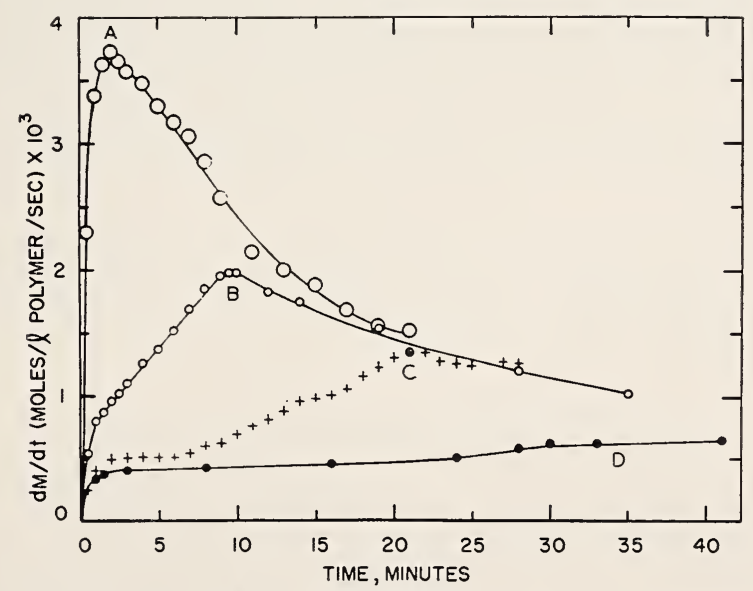

FIGURE 3.2. $d M / d$ t versus time curves showing the effect of addition of tetraphenyl succinodinitrile.

A, No added dinitrile; B, 0.04 percent of dinitrile; C, 0.10 percent of dinitrile; $D, 0.40$ percent of dinitrile. 
instantaneous rate of production of monomer as measured on the Pirani gage is plotted for a period after irradiation was started. For a pure polymer sample (curve $A$ ) the rate rises rapidly, reaching a maximum after about $2 \mathrm{~min}$, and thereafter decreases as the polymer is used up. Curves B, C, and D show the effect of adding 0.04, 0.10, and 0.40 percent by weight of tetraphenyl succinodinitrile, respectively. (An equimolecular mixture would contain 0.3 percent for a polymer of molecular weight 125,000). Consider, for example, curve C. After 2-min irradiation the monomer evolution reaches a rate that is much smaller than the corresponding rate for the pure sample at point $\mathrm{A}$. This reduced rate is maintained for a few minutes and then gradually rises until it reaches a value at point $C$ that is the same as a pure sample would have at the same time. Thereafter the polymer continues to degrade as though no retarder is present. The nearly steady rate attained after 2 min gives a measure of the reduction in rate due to the amount of retarder put in the polymer initially. Owing to the high rate of initiation, the retarder is used up relatively fast, and the over-all retarded rate gradually rises until at point $\dot{C}$ all the retarder is used up. The rate is then the same as that for a pure sample at the same time, and thereafter the degradation reaction is unaffected.

The pronounced effect of such small quantities of added material is fully consistent with the idea of a free radical chain reaction put forward earlier in this paper. Tetraphenyl succinodinitrile is known to dissociate into diphenyl cyanomethyl radicals at elevated temperatures $[6,7]$, and our results are consistent with the idea that these radicals react with the degrading polymer radicals. A complete mathematical treatment of the kinetics in the retardation stage is not possible, because the usual assumption of rate of initiation $(I)$ being constant is not correct in this case, and eren if $I$ is expressed as a function of time, the termination reaction during the period of retardation changes from predominantly first-order to second-order with respect to polymer radicals. In these circumstances three approximate treatments have been used to evaluate a rate of initiation from the retardation experiments. Making the assumption that each diphenyl cyanomethyl radical stops one degrading polymer chain, the values obtained for $I$ are all very close to $4 \times 10^{-6}$ (mole/liter)/sec. This agrees to within a factor of 3 with the value obtained from molecularweight measurements. The values obtained for $I$ are given in table 3.2 .

Considering the nature of the problem, the values obtained are in very fair agreement. The lowest value, $I=1.56 \times 10^{-6}$ (mole/ liter)/sec. will be used. If this is incorrect, it cannot be wrong by more than $10^{-1}$.

TABLE 3.2. Rate of initiation by different methods

\begin{tabular}{|c|c|c|}
\hline Method & $I$ & Remarks \\
\hline $\begin{array}{l}\text { Overall quantum efficiency } \\
\text { Retardation experiments } \\
\text { Molecular-weight measurements. }\end{array}$ & $\begin{array}{l}\text { (Mole/liter)/ } \\
\text { sec } \\
17.0 \times 10^{-6} \\
4.0 \\
1.56\end{array}$ & $\begin{array}{l}\text { Maximum possible value for initial rate. } \\
\text { May be low, as it may refer to an average } \\
\text { rate over } 0 \text { to } 50 \% \text { of degradation. }\end{array}$ \\
\hline
\end{tabular}




\section{Evaluation of Absolute Rate Constants, Using a Rotating Sector}

In the absence of retarders the degradation process may be represented by the scheme

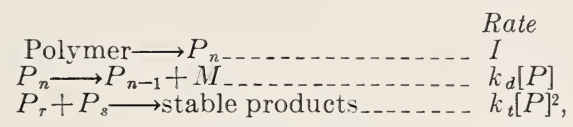

where $M$ is a monomer, $P_{n}, P_{r}, P_{s}$ are polymer radicals, $[P]$ is the total concentration of such radicals, $k_{t}$ is the bimolecular termination rate constant, and $k_{d}$ is the unimolecular rate constant for the splitting off of monomer from a polymer radical (the "depropagation" step). Assuming that $k_{d}$ and $k_{t}$ are independent of the radicals concerned and that the reaction chains are long, then under stationary state conditions, the rate of formation of monomer, $d M / d t$, is given by

$$
\frac{d M}{d t}=k_{d}[P]=k_{d} I^{\frac{1}{2}} k_{t}^{-\frac{1}{2}} .
$$

If $I$ and $d M / d t$ are known, it is necessary to measure one more relation between the various constants before the individual constants can be evaluated. The extra relation required can be obtained by measuring the lifetime $(\tau)$ of a kinetic chain by the rotating-sector technique. The lifetime in the stationary state being related to $I$ and $k_{t}$ by the equation $\tau=k_{t}^{-\frac{1}{2}} I^{-\frac{1}{2}}$, all the constants can then be evaluated.

Preliminary experiments with a rotating sector showed that the rate of evolution of monomer increased during light periods and decreased during dark periods, and a continuously recording Priani gage was introduced so that the mean degradation rates at different rates of section flash could be found. The precautions taken are described elsewhere. Measured lifetimes at $167^{\circ} \mathrm{C}$ gare a mean value of $4 \mathrm{sec}$ at a rate of degradation of $120 \% / \mathrm{hr}$, that is, $3.66 \times 10^{-3}$ (mole of monomer/liter of polymer)/sec.

$$
\frac{k_{d}}{k_{t}}=\frac{d M}{d t}=4 \times 3.66 \times 10^{-3}=1.46 \times 10^{-2} \text { mole liter }^{-1} .
$$

Taking

$$
I=1.56 \times 10^{-6} \text { mole liter }{ }^{-1} \sec ^{-1} \text { and using } \frac{k_{d}}{k_{t}^{\frac{1}{2}}}=\frac{d M}{d t} \times I^{-\frac{1}{2}},
$$

we have

$$
\begin{aligned}
& \frac{k_{d}}{k_{t}^{\frac{1}{2}}}=2.9(\text { mole liter } \\
& \left.-1 \mathrm{sec}^{-1}\right)^{\frac{1}{2}} . \\
& k_{t}^{\frac{1}{2}}=\frac{1}{\tau I^{\frac{1}{2}}}=2.0 \times 10^{2}\left(\text { liter mole }{ }^{-1} \sec ^{-1}\right)^{\frac{1}{2}} .
\end{aligned}
$$

Thus

$$
k_{t}=4.0 \times 10^{4} \text { liter mole } \mathrm{sec}^{-1} \text {, and } k_{d}=2.9 k_{t}^{\frac{1}{2}}=5.8 \times 10^{2} \mathrm{sec}^{-1} .
$$




\section{Discussion of Results}

The termination step involves the reaction of two radicals in the highly viscous polymer phase. It is of interest to compare the value obtained $\left(k_{t}=4.0 \times 10^{4}\right.$ liter mole $\left.\mathrm{sec}^{-1}\right)$ with data obtained for the corresponding step during the polymerization reaction. Mackay and Melville [8] measured $k_{t}$ for the reaction of two methyl methacrylate polymer radicals in dilute solution during polymerization, obtaining the value $k_{t}=6.6 \times 10^{7}$ liter mole $\mathrm{sec}^{-1}$ at $23.6^{\circ} \mathrm{C}$. Thus the value of $k_{t}$ in the viscous polymer is about 1,000 times smaller than that in dilute solution, even though the temperatures are widely different. This type of result might have been predicted because the physical conditions during depolymerization in a viscous polymer must be similar in character to those in the gel phase of polymerization, and Matheson, Auer, Bevilacqua, and Hart [9] have found that in the polymerization of methyl methacrylate $k_{t}$ at 33 percent conversion to polymer is less than its initial value in dilute solution by a factor of $1 / 200$.

The activation energy of the depropagation step, $E_{d}$, is to be expected to equal the sum of the actiration energy for the propagation reaction in polymerization, $E_{p}$, plus the heat of polymerization, $13.0 \mathrm{kcal} / \mathrm{mole}$ [10]. Taking $E_{p}$ as $5.5 \mathrm{kcal}$ (from the results of Mackay and Melville, and Matheson, Auer, Bevilacqua, and Hart) we expect $E_{d} \simeq$ $5.5+13.0 \simeq 18.5 \mathrm{kcal}$. The experimentally measured over-all activation energy is much smaller than this (about $8 \mathrm{kcal}$ ). From the kinetic scheme outlined above the over-all activation energy is $E_{d}-\frac{1}{2} E_{t}$. Thus $E_{t}$ is probably about $20 \mathrm{kcal}$. The low value of $k_{t}$ shows that radicals interact slowly in the molten polvmer, possibly because this rate of reaction is diffusion controlled. The activation energy for diffusion of methrl isobutrrate through methyl methacrylate polvmer is about $30 \mathrm{kcal}$ [11], and the activation energr of viscous flow in polymers is about $20 \mathrm{kcal}$ or more [12], so a high value for $E_{t}$ was not unexpected. Owing to the difficulties involved, it has not yet been possible to find $E_{t}$ directly by measurement of the lifetime of kinetic chains orer a range of temperatures. At high temperatures a thermal rate complicates matters, and at low temperatures the diffusion of monomer from the polymer film is relatively slow. In these circumstances, a high stationary concentration of monomer $[I /]$ is held in the film, and as this has the opportunity to recombine onto the degrading radical ends at a rate $k_{p}[P][M]$, where $k_{p}$ is the propagation rate constant for the polymerization reaction, the net rate of evolution of monomer from the polymer film may be less than the time rate of degradation $k_{d}[P]$.

Taking $E_{d} \simeq 18.5 \mathrm{kcal} / \mathrm{mole}$ and the measured value of $k_{d}$, we calculate the frequencr factor, $A_{d}$, in the expression $k_{d}=A_{d} e^{-E_{d}} /{ }^{R T}$ as $0.89 \times 10^{12} \mathrm{sec}^{-1}$. This order of magnitude is to be expected, for as Dainton and Ivin [13] point out, the depropagation reaction is a unimolecular decomposition, and as such would be expected to have a very small entropy of activation and a nearly normal frequency factor.

Talues are now arailable for the kinetic constants for both the addition of monomer to a polymer radical, and the splitting off of monomer from a radical. Unfortunately, it has not ret been possible to evaluate the two constants under similar physical conditions at the 
same temperature, but nevertheless the data can be used to obtain a value for the entropy of polymerization and, using the known heat of polymerization, to evaluate the corresponding free-energy change. At this stage it is necessary to assume the value for $E_{d}$ of 18.5 kcal given by the sum of the heat of polymerization and the activation energy for the propagation reaction. Small has recently obtained the relation

$$
\Delta S_{l c}=R \ln \left(A_{p} / V_{1} A_{d}\right)-R(1-\mu) V_{p} / V_{1},
$$

where $\Delta S_{l c}$ is the entropy change on converting 1 mole of liquid monomer to condensed polymer at $25^{\circ} \mathrm{C}, V_{p}$ is the volume fraction of polymer, $V_{1}$ is the volume fraction of monomer, and $\mu$ is Huggins' coefficient for polymethyl methacrylate in monomer solution. From the results of Mackay and Melville, and Matheson, Auer, Bevilacqua, and Hart, we use a value of $k_{p}$ at $25^{\circ} \mathrm{C}=2.8 \times 10^{2}$ liter mole $\mathrm{sec}^{-1}$, and $E_{p}=5.5 \mathrm{kcal}$, whence $A_{p}=3.03 \times 10^{6}$ liter mole $\mathrm{sec}^{-1}$. $A_{d}$ has been evaluated above as $0.89 \times 10^{12} \mathrm{sec}^{-1}$. Using these values, with $V_{1}=0.1086$ liter, $V_{p}=0.0843$ liter and $\mu=0.3$, we obtain $\Delta S_{l_{c}}=$ $-21.7\left(\mathrm{cal} /{ }^{\circ} \mathrm{C}\right) /$ mole. Small $[2]$ has recently carried out direct measurement of the equilibrium pressure of monomer in the methyl methacrylate monomer/polymer system, obtaining the value $\Delta S_{l c}=$ $-24.8\left(\mathrm{cal} /{ }^{\circ} \mathrm{C}\right) /$ mole. Considering the difficulty in obtaining and evaluating the kinetic data, the agreement between these values is satisfactory. The kinetic studies can at present yield a value that is correct to about $\pm 5 \mathrm{eu}$.

The author's are indebted to Dr. P. A. Small for permission to quote work in the course of publication, and to Imperial Chemical Industries, Ltd., Plastics Division, for allowing one of us (P.R.E.J.C.) to undertake this work.

\section{References}

[1] N. Grassie and H. W. Melville, Proc. Roy. Soc. [A] 199, 14 (1949).

[2] P. A. Small, Trans. Faraday Soc. (In press).

[3] Masson and H. W. Melville, J. Polymer Sci. 4, 323 (1949).

[4] Baxendale, Bywater, and Evans, J. Polymer Sci. 1, 237 (1946).

[5] R. Simha. L. A. Wall, and Blatz, J. Polymer Sci. 5, 615 (1950).

[6] Schulz, Z. Electrochem. 47, 265 (1941).

[7] MeLaren, Ph. D. thesis (Aberdeen 1948)

[8] Mackay and H. W. Melville, Trans. Faraday Soc. 45, 323 (1949).

[9] Matheson, Auer, Bevilacqua, and Hart, J. Am. Chem. Soc. \%1, 497 (1949).

[10] Tong and A. S. Kenyon, J. Am. Chem. Soc. 68, 1355 (1946).

111] P. A. Small, private communication (1950).

[12] Spencer and Dillon, J. Colloid Sci. 4, 241 (1949).

[13] Dainton and Ivin, Trans. Faraday Soc. 46, 331 (1950).

\section{Discussion}

Robert Simha, New York University, New York, N. Y.: I should like to take up three points regarding this very interesting work. The first has to do with the over-all rate as a function of conversion. As long as $\epsilon N / 2 \ll 1$, this should be a straight line. The curves obtained 
instead in the present work and in the pyrolysis of polymethyl methacrylate have been interpreted by Grassie and Melville as indicative of different chain ends. Has the polymer prepared in the presence of diphenyl cyanomethyl radicals been investigated?

Possibly connected with this is the change of molecular weight with conversion. For $M=(7$ to 8$) \times 10^{5}$, the decrease follows closely the diagonal both in the thermally and photoactivated process. This is somewhat surprising, because, judging from the results with lower molecular weights, the critical ratio $\epsilon N / 2$ should be only of the order of unity. Even for those smaller molecular weights the decrease is more pronounced at higher conversions than predicted by the theory. Formally, it is as if $\epsilon$ were increasing during the reaction. This might be indicative of an increasing relative importance of transfer, favored by the presence of several initiation mechanisms and/or of an inverse viscosity effect on the rate of termination. Larger molecular weights would be more sensitive to both effects. Investigations of still larger molecular weights are desirable to see whether a decisive drop below the diagonal occurs.

The third point has to do with the estimate of the over-all energy of activation from the initial rate. On going from small to large molecular weights one expects a change in the activation energy; in the case of photodegradation, an increase. The transition should occur in the region in which the factor $1-(1-\epsilon)^{N / 2}$ increases from $\epsilon N / 2$ to 1 , or $\epsilon N / 2 \approx 0.7$. Substitution of $\epsilon$ values deduced from pyrolysis data leads to $M \approx 3 \times 10^{5}$. On the other hand, the small rate of termination observed may result in much smaller values of $\epsilon$, unless transfer is not negligible. In this case the expression for the activation energy used would not be applicable. Has any such variation with $D P$ been observed? 


\title{
4. Effect of Heat and Light on Polyvinyl Chloride
}

\author{
By Donald Druesedow ${ }^{1}$ and C. F. Gibbs ${ }^{1}$
}

The thermal degradation of polyvinyl chloride is primarily a dehydrochlorination reaction. In an atmosphere of nitrogen the rite tends to approach a steady state and is not affected by a build-up of hydrogen chloride. The intrinsic viscosity of the polymer increases with the state of dehydrochlorination. If oxygen is present, the rate increases with time, and the reaction is autocatalytic with respect to hydrogen chloride. A drop in intrinsic viscosity in the early stages of degradation followed by an increase indicates that chain scission and cross-linking are competing reactions in the presence of oxygen. Heavy metal stabilizers absorb hydrogen chloride in a nitrogen atmosphere, but when oxygen is present they appear to function through some other mechanism.

Exposure to ultraviolet irradiation at room temperature results in the evolution of small amounts of hydrogen chloride. Measurable quantities of oxygen are taken up during this process. The irradiated polymer is more susceptible to thermal degradation than the original.

Possible mechanisms for these processes are discussed.

\section{Introduction}

The structure of polyvinyl chloride has been well established as essentially a linear polymer of "head to tail" configuration [1].2 Many details, such as branching and terminal groups, remain unexplained. If the structure was linear and all of the chlorine atoms typically secondary, the thermal stability should be quite high. The fact, however, that the best grades of polyvinyl chloride begin to lose hydrogen chloride at temperatures as low as $100^{\circ} \mathrm{C}$ indicates that activating influences are present. The character and location of these points of activation have not been demonstrated.

In a given polymer chain, once a molecule of hydrogen chloride is lost and a double bond produced, the neighboring chlorine atom is converted to an allyl chloride with a high degree of lability. From this point progressive dehydrochlorination takes place at a fairly high rate until a more stable configuration is reached.

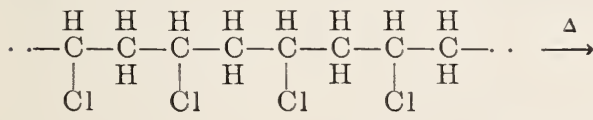

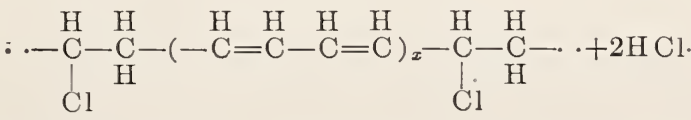

The resultant conjugated polyene is colored, the depth of which probably is a function of the total number of conjugated double bonds present [2]. Other physical properties, such as tensile strength, modulus, hardness, etc., are affected to a much less extent.

1 B. F. Goodrich Research Center, Brecksville, Ohio.

2 Figures in brackets indicate the literature references on p. 79 . 
Degradation by light is similar to thermal break-down in that hydrogen chloride is lost in the process, although at a very slow rate. risible light has little effect, the real damage being done by the ultraviolet. In plasticized stocks the predominant effect is stiffening, frequently accompanied by bleeding of the plasticizer. Prolonged exposure results in discoloration similar to that produced br heat.

The use of stabilizers in plasticized polyvinyl chloride stocks is common practice. These are commonly organic metal salts, although epoxides, monoglycerides, and some known ultraviolet absorbers are also used. In Germany, sodium carbonate, sodium phosphate, $\alpha$-phenyl indole, diphenylthiourea, and urea have been used with some success $[3,4]$. The primary function of these stabilizers is to inhibit the development of color in the stock. The general supposition has been that color stabilization results from inhibition of autocatalysis through remoral of liberated hydrogen chloride.

A number of criteria of break-down are available for study. These include hydrogen chloride evolution, color development of the polymer, infrared absorption, mass spectrographic analysis of break-down products, and molecular-weight measurements. This paper reports studies made on hydrogen chloride evolution at carefully controlled temperatures. Particular consideration was given to the effect of oxygen in the atmosphere surrounding the sample. Some attention was given to effect on molecular weight, and a brief study was made on plasticized stocks containing stabilizer. Geon 101 was used throughout.

\section{Experimental Methods}

\section{Thermal Decomposition}

The apparatus shown in figure 4.1 is closely similar to that used in ASTAI D 793-49. A 1-g sample was placed in the heating tube and immersed in an oil bath held within $\pm 0.5 \mathrm{deg} \mathrm{C}$ of the desired temperature. Air, oxygen, and nitrogen were used as sweeping gases at $150 \mathrm{~cm}^{3} / \mathrm{min}$. The hydrogen chloride was collected in water kept alkaline to phenolphthalein by intermittent titration with $0.01 \mathrm{~N}$ sodium hydroxide. Studies on unplasticized polymer were made on granular, commercial grade polyvinyl chloride. Plasticized samples were given identical heat histories during processing, then cut by hand to an approximete dimension of $1-\mathrm{mm}$ cubes. Samples containing $1 \mathrm{~g}$ of polymer were used.

\section{Ultraviolet Irradiation}

This was a qualitative study only with samples exposed for various lengths of time under a Hanovia $500-w$ mercury arc. A current of air was passed over the sample to aroid heating from the lamp. Although many plasticized stocks have been studied in this manner, only experiments with unplasticized, granular polyvinyl chloride will be reported here. Since the effect of oxygen was a suspected factor in ultraviolet action, the apparatus shown in in figure 4.2 was used to demonstrate oxygen absorption as a direct result of irradiation. The sample of polymer was placed in the quartz cell, which was exposed to the direct radiation of a mercury arc. A side tube contained 


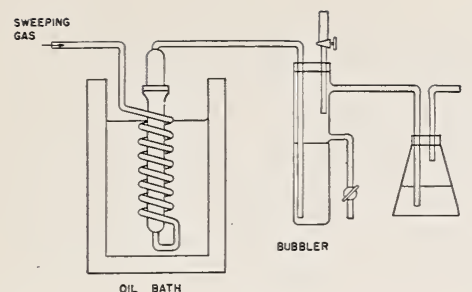

FigURE 4.1. Apparatus for thermal decomposition of polyvinyl chloride.

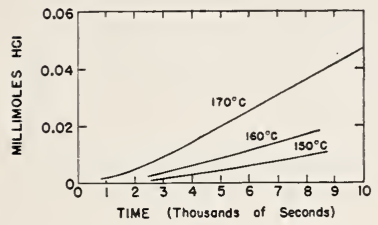

Figure 4.3. Thermal decomposition of polyvinyl chloride in $\mathrm{N}_{2}$.

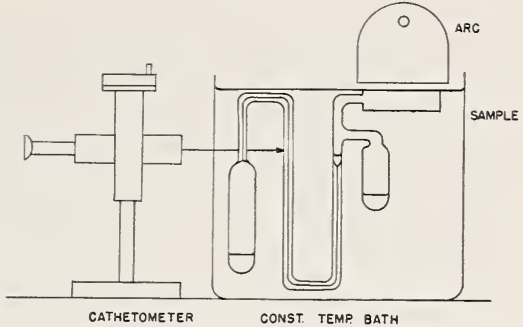

FigURE 4.2. Apparatus for ultraviolet irradiation.

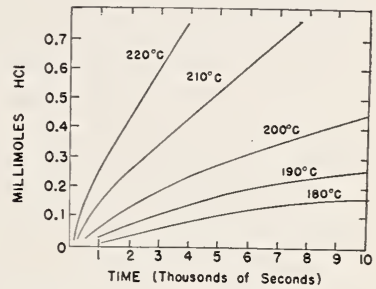

Figure 4.4. Thermal decomposition of polyvinyl chloride in $\mathrm{N}_{2}$.

standard sodium hydroxide solution, and the manometric system permitted small changes of pressure to be read by means of a cathetometer. The entire apparatus was immersed in a water bath, with the surface of the quartz cell barely on the surface of the water.

\section{Discussion of Results}

\section{Thermal Decomposition in Nitrogen}

An appraisal of the results of thermal decomposition reveals that choice of temperature and the concentration of oxygen in the system has much to do with the rate of dehydrochlorination. This may account for differences of opinion that have existed regarding the nature of the thermal reaction, particularly with respect to autocatalysis. With nitrogen (commercial crlinder grade) as the sweeping gas and evolved hrdrogen chloride plotted against time, a family of curves is obtained, the slopes of which vary with temperature (see figs. 4.3 and 4.4). From $150^{\circ}$ to $170^{\circ} \mathrm{C}$, after an appreciable induction period, the rate of hydrogen chloride evolution increases with time, giving a slight upward currature to the plot. At higher temperatures, the induction period is less evident, and a slight decline of rate prevails after an initial period of acceleration, and the curves appear to flatten out at the longer periods of time. Both the induction period and the change in rate are probably related to the state of decomposition, as the character of the curvature appears to be the same at all temperatures for the same loss of hydrogen chloride. 
The induction period may be likened to the induction periods common to polymerization reactions. These are dependent upon a buildup of active centers. In this case the active centers already exist as points of chlorine lability, but await a build-up of energy to the critical level before the first molecule of hydrogen chloride is lost. The progressive dehydrochlorination that follows is fairly rapid, but soon reaches a point of termination. The controlling factor is the activation energy of a small number of activated alkyl chlorides. The character of these points of activation is speculative, but the following estimates appear fairly logical:

1. A small amount of branching probably takes place during the polymerization process. These branches are likely on carbon atoms carrying chlorine, thus producing tertiary chloride, which could dehydrochlorinate or hydrolyze and dehydrate, giving rise to allyl chloride units. It is a known fact that small amounts of hydrogen chloride are split out during the polymerization process.

2. Direct oxidation reactions by peroxide catalysts during polymerization would result in a degree of activation of the chlorine atom nearest to the point of oxidation.

3. Catalyst residues may be present that are capable of attacking the polymer chain during the degradation process, thus producing points of increased chlorine activity.

As these active chlorine atoms diminish in number (in the order of their reactivity), the rate falls off and theoretically should reach a steady state. Although this steady state was never reached in our experiments, the rates in nitrogen were observed to fall with time throughout the longest periods of dehydrochlorination.

To determine the effect of hydrogen chloride on the thermal decomposition rate, a run at $190^{\circ} \mathrm{C}$ under a nitrogen stream was allowed to go $16,500 \mathrm{sec}$, then the nitrogen flow was discontinued (fig. 4.5). The stagnant system was continued at $190^{\circ} \mathrm{C}$ for an additional 5,000 sec, whereupon the flow of nitrogen was resumed. The total hydrogen chloride collected was the same as if the flow of nitrogen had not been interrupted. Apparently the thermal reaction in an inert atmosphere is not sensitive to hydrogen chloride concentration.

The effect on molecular weight may be inferred from figure 4.6. A

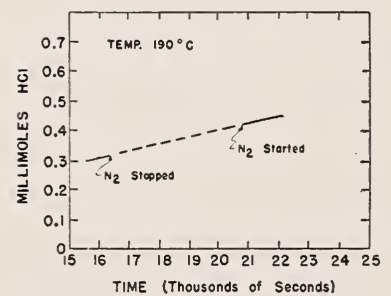

FigURE 4.5. Effect of accumulated $\mathrm{HCl}$ in the absence of $\mathrm{O}_{2}$ on the thermal decomposition of polyvinyl chloride.

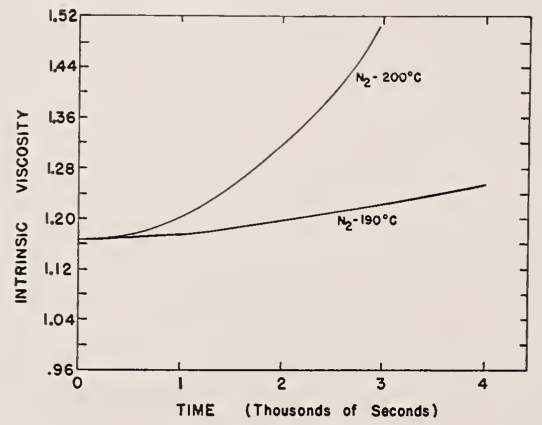

FigURE ${ }^{-4.6 . ~ E f f e c t ~ o f ~ t h e r m a l ~ d e c o m p o-~}$ sition of polyvinyl_chloride in $\mathrm{N}_{2}$ on intrinsic_viscosity. 
pretty good correlation exists between hydrogen chloride loss and intrinsic viscosity. Not indicated by the chart is the fact that at $190^{\circ} \mathrm{C}$ polymer insoluble in cyclohexanone appears at 5,000 sec, and at $7,000 \mathrm{sec}$ the sample is largely insoluble. At $900 \mathrm{sec}$ and $200^{\circ} \mathrm{C}$ the hydrogen chloride loss is 0.06 millimole/g and the molecular weight by intrinsic viscosity is approximately 66,000 as compared with 64,000 for the original polymer. Since the $1-\mathrm{g}$ sample contained a potential of 15.6 millimoles of hydrogen chloride, theloss is 0.385 percent, or at a DP of $1,024,3.94$ molecules per polymer chain. Computing the cross bonds developed in going from 64,000 to 66,000 molecular weight, one finds 3.12 cross bonds per 100 polymer chains. This gives $3.94 \times 100 / 3.12=126$ molecules or hydrogen chloride lost per cross bond. At $2,700 \mathrm{sec}$ and $200^{\circ} \mathrm{C}$, by the same calculation, one finds 38.8 molecules of hydrogen chloride evolved per cross bond. At $5,000 \mathrm{sec}$, the approximate point of insolubilization, 2.7 percent of the total hydrogen chloride has been lost, or 17.3 molecules per cross bond if all of the polymer chains are assumed to be linked together. The same calculation made for the point of insolubility $(\overline{7}, 000 \mathrm{sec})$ at $190^{\circ} \mathrm{C}$ gives 14.2 molecules of hydrogen chloride lost per cross bond.

Several possible mechanisms can be proposed for the cross-linking reaction. These include (1) copolymerization between polyene groups, (2) chain transfer between a polyene group and a segment of a normal polymer chain, (3) a Diels-Alder reaction, and (4) cross dehydrochlorination between a point of high chlorine activity on one chain and a hydrogen atom on another. Of these mechanisms, the last seems the most likely when the experimental facts are considered.

Copolymerization between polyene groups seems unlikely due to the small numbers of such groups present and their isolation from each other. The same may be said for a Diels-Alder reaction. A chaintransfer mechanism appears more plausible and mar take place to some extent, but initiators normally required for such reactions are absent. Cross dehydrochlorination seems to fill all the requirements of the situation. It is reasonable to assume that as progressive dehydrochlorination proceeds, a point will be reached sooner or later at which the most readily arailable hydrogen atom will be on another polymer chain.

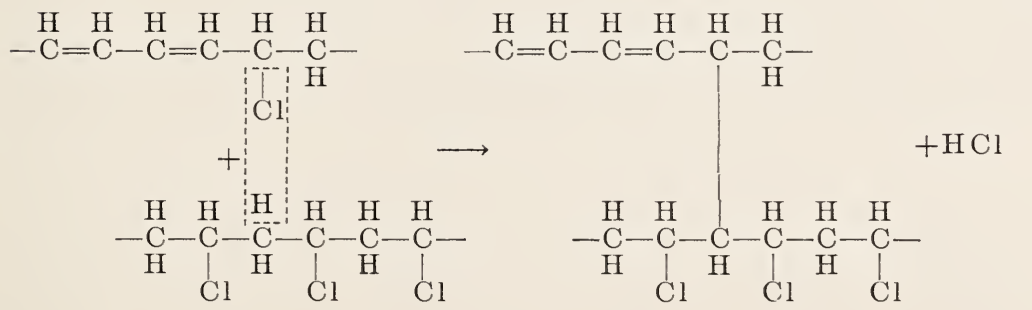

This would stabilize the system and account for the approach to a steady-state condition. Without a block in the dehydrochlorination process, a steadily increasing rate would be observed. This mechanism allows for a maximum number of polyene groups and their preservation as such, which would be in keeping with the progressive development of color in the polymer mass. 


\section{Thermal Decomposition in the Presence of Oxygen}

The curves in figures 4.7 and 4.8 reveal that a different situation exists when air is used as the sweeping gas. The rates of evolution of hydrogen chloride are faster at all temperatures and increase with time, regardless of the degree of decomposition. This indicates that atmospheric oxygen is effective in producing new points from which dehvdrochlorination is initiated.

The effect of oxygen concentration is shown in figure 4.9. The curve for lamp-grade nitrogen (4 parts per million of oxygen) represents the reaction for an essentially oxygen-free system. A direct oxygen attack upon the polymer, thereby increasing its susceptibility to dehydrochlorination, is indicated by the fact that the rate is dependent upon concentration.

The autocatalytic influence of hydrogen chloride in the presence of oxygen may be inferred from figure 4.10. A run was made at $190^{\circ} \mathrm{C}$, starting with the system filled with air. After 4,000 sec with a stagnant-air atmosphere, a flow of nitrogen was started and the total liberated hydrogen chloride titrated. The rate of hydrogen chloride erolution during the period of stagnation was approximately twice that for the air-sirept system, using the nitrogen curve as a base. This experiment demonstrates the effect of hydrogen chloride concentration on the dehydrochlorination reaction, as well as the fact that catalysis takes place.

Figure 4.11 presents curves relating intrinsic viscosity to thermal treatment in a current of air. It is evident that chain scission and cross-linking are taking place simultaneously. In this case insolubilization occurs at a somewhat earlier point than in the nitrogen system. The relatively large number of molecules of hydrogen chloride lost per cross-link in the early stages of the nitrogen reaction would provide ample double bonds for oxygen attack and chain scission. The more rapid hydrogen chloride loss as compared with the decomposition in nitrogen would provide for the faster rate of cross-linking that shortly overtakes the rate of chain scission and leads to early insolubilization. Probably some double-bond polymerization takes place also, but the most likely source of cross-linkage is cross-dehydrochlorination.

In considering possible mechanisms involved in the above experiments, it is evident that in the presence of oxygen, a number of reactions can take place, giving rise to a more rapid rate of production of activated chlorine atoms and to clearage at points of susceptibility in the polymer molecule. That the polyene groups are attacked as well as other points, is indicated by the fact that at the same hydrogen

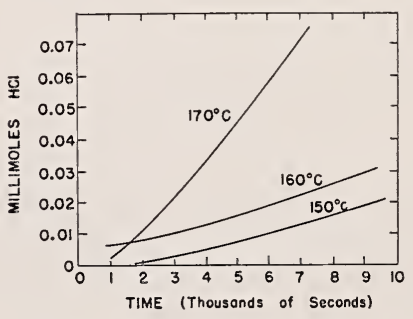

FIGLRE 4.7. Thermal decomposition of polyvinyl chloride in air.

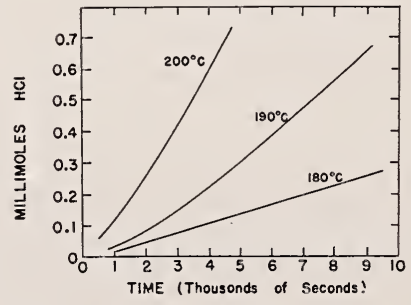

FIGLRE 4.8. Thermal decomposition of polyvinyl chloride in air. 


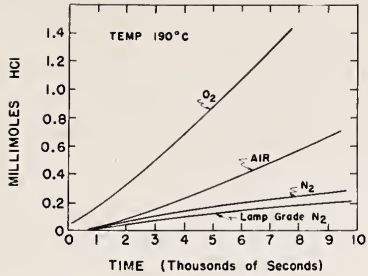

Figure 4.9. Thermal decomposition of polyvinyl chloride in $\mathrm{O}_{2}$, air, and $\mathrm{N}_{2}$.

FiguRE 4.11. Effect of thermal decomposition of polyvinyl chloride in air on intrinsic viscosity.

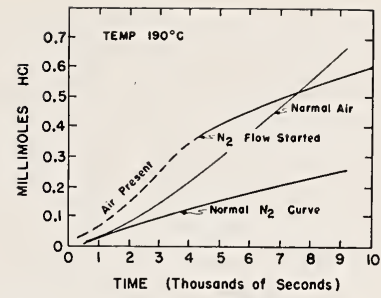

FIGURE 4.10. Effect of accumulated $\mathrm{HCl}$ on thermal decomposition of polyvinyl chloride in air.

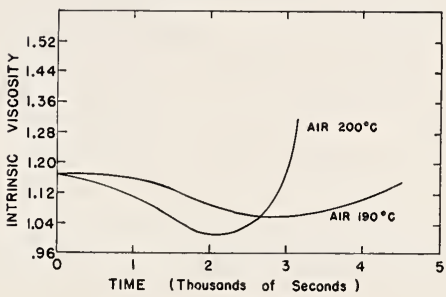

chloride loss, the polyvinyl chloride sample was less colored in the system swept by air than in the one swept by nitrogen. Also, it is a well-known fact that strong oxidizing agents will decolorize aged polyvinyl chloride either in the milling process or in solution. Reaction with halogens likewise will decolorize the polymer. The polyene attack may be responsible indirectly for the creation of labile chlorine atoms through the formation of peroxides, which in turn react with a normal chain segment by chain transfer. The active segment is then susceptible to reaction with oxygen, which in turn would produce a more labile chlorine atom.<smiles>CC(C)=CC(C)=CCCCC1(C)OOC(C)(C)C(C)=C1C</smiles><smiles>CC1=C(C)C(C)(C)OOC(C)(C)OO1</smiles><smiles>CC(C)(C)C(C)(O)C(C)(O)C(C)(O)C(C)(O)C(C)(O)C(C)(C)Cl</smiles><smiles>CC(Cl)(Cl)OOC(C)(Cl)OO</smiles> 
This is but one of many similar mechanisms that could be written to support the experimental evidence. This situation is closely related to the oxidation of unsaturated fats, particularly those with conjugated double bonds. Most of these mechanisms would account for the presence of carbonyl groups in degraded polyvinyl chloride, the presence of which has been reported previously [5].

The activating effect of hydrogen chloride upon the decomposition rate in the presence of oxygen finds a corollary in recent work of Raley, Rust, and Vaughan [6]. These authors report sensitization of the vapor-phase decomposition of di-t-butyl and di-t-amyl peroxides by hydrogen chloride. They propose the following mechanism:

$$
\begin{gathered}
\mathrm{R}_{3} \mathrm{COOCR} \mathrm{C}_{3} \longrightarrow 2 \mathrm{R}_{3} \mathrm{CO} . \\
\mathrm{R}_{3} \mathrm{CO}+\mathrm{HCl} \longrightarrow \mathrm{R}_{3} \mathrm{COH}+\mathrm{Cl} . \\
\mathrm{R}_{3} \mathrm{CO} \longrightarrow \mathrm{R}_{2} \mathrm{C}=\mathrm{O}+\mathrm{R} . \\
\mathrm{R}+\mathrm{HCl} \longrightarrow \mathrm{RH}+\mathrm{Cl} .
\end{gathered}
$$

It is reasonable to assume a similar mechanism taking place during the decomposition of polyvinyl chloride in the presence of air. The increase in the rate of production of free radicals by the action of hydrogen chloride would readily account for an increase in the development of points of lower activation energy for the dehydrochlorination reaction.

\section{Action of Stabilizers}

Stabilizers can be tested properly only in plasticized stocks. This produces a condition that is different from tests on granular polymer. In the first place, the polymer molecules are solvated by the plasticizer, and second, the polymer has undergone an exposure to heat and oxygen in the milling process. In any event, different results were obtained between stocks that only had been milled and those that had been molded for 3 min at $340^{\circ} \mathrm{F}$ after incorporation of plasticizer and stabilizer by milling. Reproducibility was not too good, but the differences that were observed were with respect to time rather than the slopes of the curves. Figures 4.12 and 4.13 present results obtained on molded plasticized stocks with and without stabilizer at $170^{\circ} \mathrm{C}$ in nitrogen and in air.

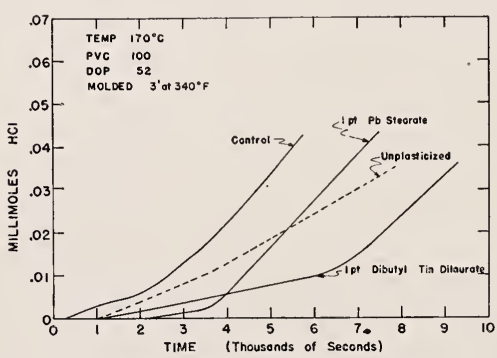

Figure 4.12. Thermal decomposition of plasticized polyvinyl chloride in $\mathrm{N}_{2}$.

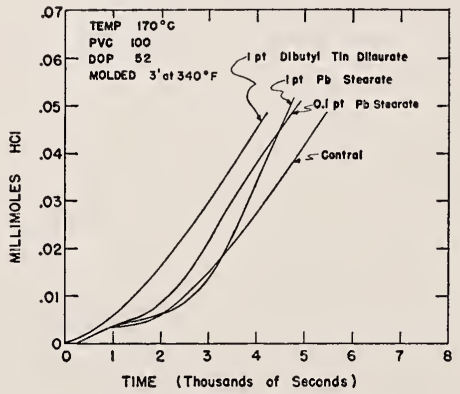

Figure 4.13. Thermal decomposition of plasticized polyvinyl chloride (molded stock) in air. 
In a current of nitrogen the basic decomposition rate of the plasticized stock is appreciably faster than for unplasticized polymer. After a prolonged induction period the rate approaches a steady state. The presence of lead stearate results in an inhibition of hrdrogen chloride erolution, presumably by reaction forming lead chloride, followed by a rate of evolution comparable with that of the unstabilized stock. This checks fairly well with the quantity of lead stearate present ( 0.026 milliequivalent) as compared with the hydrogen chloride absorbed ( 0.017 millimole). The dibutyl tin dilaurate stock, on the other hand, liberates hydrogen chloride slowly for an extended induction period, then assumes a rate comparable with that of the unstabilized control. The 0.05 millimole differential between the dibutyl tin dilaurate curve and the control suggests the absorption of 4 moles of hydrogen chloride per mole of stabilizer. The formation and retention of stannic chloride is difficult to accept unless one assumes the possibility of a complex with dioctyl phthalate, or possibly lauric acid. The stable pentahydrate of stannic chloride suggests such a possibility. It is noteworthy that only a slight difference in color existed between the control and the two stabilized stocks.

The results obtained when air was used as the sweeping gas (fig. 4.13) present an entirely different picture. In the presence of air, more hydrogen chloride is given up by the stabilized stocks than by the control, and for the lead stearate stock the rate following the induction period is definitely faster. Dibutyl tin dilaurate does not seem to be a hrdrogen chloride acceptor as a change in rate was not observed. The fact that the function of the stabilizer is to prevent color rather than to inhibit the evolution of hydrogen chloride is probably the key to the answer. Heary metal stabilizers may function as oxidation catalysts for the disruption of the chromophoric polrene groups. A parallel of this action is found in the use of hearymetal soaps (including lead soaps) as oxidation catalysts in drying oils.

In the case of milled, but not molded, stocks a considerable amount of hydrogen chloride inhibition is produced by both lead stearate and dibutyl tin dilaurate (fig. 4.14). In the light of the results with molded stocks, this is not easily explained. Complete solvation of the polymer molecules has not occurred at this stage nor has the stabilizer been completely dispersed throughout the system. These facts may interfere somewhat with oxidation catalysis. At the present time, a clear picture is lacking.

FigURE 4.14. Thermal decomposition of plasticized polyvinyl chloride (milled stock) in air.

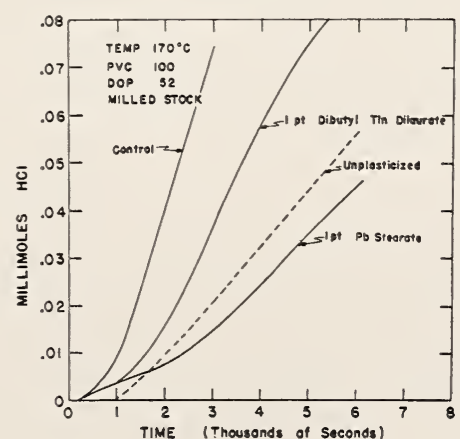




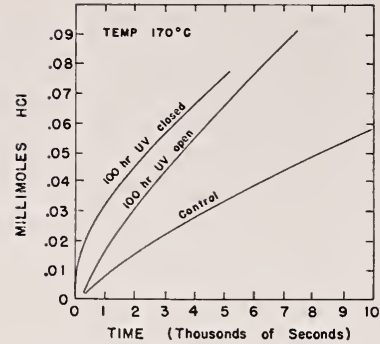

Figure 4.15. Thermal decomposition of irradiated polyvinyl chloride in $\mathrm{N}_{2}$.

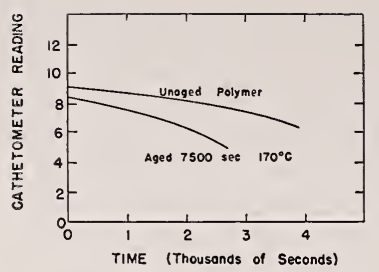

Figure 4.16. Absorption of $\mathrm{O}_{2}$ by irradiated polyvinyl chloride.

\section{Action of Light}

The quantitative aspects of light exposure were not made a part of this study. Rather, a qualitative examination was made and from which certain generalizations may be drawn. The amount of hydrogen chloride liberated upon prolonged exposure of polyvinyl chloride to ultraviolet light is small. The most important effect is the sensitization to thermal break-down, in which oxygen probably plays an important role. Figure 4.15 demonstrates the significant effect of ultraviolet exposure upon thermal stability. Here, the unplasticized polymer was placed in Corex tubes and rotated under a source of ultraviolet light. The increased sensitivity of the polymer in the closed tube over the one open to the atmosphere is further evidence of hydrogen chloride catalysis of the oxrgen mechanism. The fall in rate may be attributed to exhaustion of the points of activation initiated by the combined effect of ultraviolet light and oxygen.

To demonstrate that oxygen was taken up in the process of irradiation, the apparatus shown in figure 4.2 was used. Of the two polymers studied, one had been subjected to heating for $7,500 \mathrm{sec}$ at $170^{\circ} \mathrm{C}$, whereas the other was unaged. The pressure drop was measurable and decidedly greater for the aged sample (fig. 4.16). Oxygen attack on the polyene groups is indicated by the fact that ultraviolet exposure in the presence of air will bleach thermally discolored polymer.

It can be assumed with reasonable confidence that ultraviolet exposure results in the formation of free radicals on the polymer chain, which react with a vailable atmospheric oxygen. The resulting peroxide groups usually are in such position that they activate a chlorine atom, thus giving rise to a weak spot on the polymer. Oxygen attack on polyene portions of the polymer system may not affect a chlorine atom directly, but indirect at tack through peroxide free radical action may result.

\section{Conclusions}

The thermal degradation of polyrinyl chloride in an atmosphere of nitrogen proceeds at a diminishing rate as the points of higher chlorine-atom lability, which are normally present in the polymer molecule, disappear through dehydrochlorination. In the presence of oxrgen, a rising rate is noted due to the continuous generation of new labile chlorine atoms by an oxidative process. Liberated hydro- 
gen chloride acts as a catalyst for thermal degradation when oxygen is present.

The steady increase in molecular weight during the thermal degradation in nitrogen indicates a cross-dehydrochlorination mechanism. In the presence of oxygen, both chain scission and cross-linking take place, with the latter predominating after the early stages.

Stabilizers of the heavy-metal type probably function as oxidation catalysts in the destruction of polyene chromophores.

Ultraviolet light in the presence of oxygen produces points of higher chlorine lability, which are sensitive to thermal exposure. The points may be due to the proximity of oxygen-carbon linkages resulting from the attack of oxygen on free radicals produced by irradiation.

The authors thank V. L. Folt and M. R. Walters for providing portions of the data used herein and for their helpful consultation.

\section{References}

[1] C. S. Miarvel, J. H. Sample, and Max F. Roy, J. Am. Chem. Soc. 61, 3241 (1939).

[2] R. F. Bover, J. Phys. \& Colloid Chem. 51, 80 (1947).

[3] G. M. Kline, Modern Plastics 23, 1527 (Oct. 1945).

[4] J. M. DeBell, W. C. Goggin, and W. E. Gloor, German plastics practice, p. 63 (DePell \& Pichardson, Inc, Springfield, Mass., 1946).

[5] V. IV. Fox, J. G. Hendricks, and H. R. R atti, Ind. Eng. Chem. 41, 1774 (1949).

[6] J. H. Raley, F. F. Rust, and IV. E. Vaughan, J. Am. Chem. Soc. 70, 2767 (1948).

\section{Discussion}

Dr. E. H. Wood, Bakelite Co., Division of Union Carbide and Carbon Corp., Bound Brook, N. J.: It is my understanding that your molecular weights were intrinsic viscosities. In that case your ratio between molecular-weight change and $\mathrm{HCl}$ loss would be erroneous. Because intrinsic viscosities increase exponentially with increase in true molecular weights, if the $\mathrm{HCl}$ loss per unit increase in molecular weight were constant, the $\mathrm{HCl}$ loss per unit increase in viscosity would decrease. Thus the observation that the loss of $\mathrm{HCl}$ per unit increase in the viscosity-based "molecular weight" decreases as the degradation proceeds does not necessarily mean that less $\mathrm{HCl}$ is being evolved per cross-link formed.

Dr. GiBBs: That would be true after insolubility had set in.

Dr. Wood: No, before. As soon as you start branching something, you will get this result.

Dr. GibBs: That is very true. I do not claim to be too accurate. It was just a trend. It seemed to me that we were finding a range that we could interpret as meaning the approximate length of the runs. The cross-linking reaction seemed to me to be the controlling factor in the process.

Dr. C. Havens, Dow Chemical Co., Saran Development Laboratory, Midland, Mich.: In Dr. Gibbs' experiments he had the polyvinyl chloride exposed to a sweeping gas, nitrogen or air, over the specimen. He found an induction period and then the $\mathrm{HCl}$ evolution. I suggest that before he observes any $\mathrm{HCl}$ evolution the specimen must become saturated with $\mathrm{HCl}$ and then any excess over and above saturation point is what he measures. So that actually if this 
$\mathrm{HCl}$ saturation were included, there would be no induction period.

There is $\mathrm{HCl}$ evolution right from the beginning.

In the exposure under nitrogen, nitrogen was swept through up to a certain point. Then the sweeping of nitrogen was stopped and started at a later point. He found that the $\mathrm{HCl}$ evolution was exactly the same as though nitrogen had continuously swept the specimen. That was taken to indicate that $\mathrm{HCl}$ did not have a catalytic effect. However, since the specimen itself is already saturated with $\mathrm{HCl}$ and only the surface is swept, stopping the flow of nitrogen for a short time is not a measure of whether or not $\mathrm{HCl}$ is a catalyst.

Dr. A. R. Burgess, Imperial Chemical Industries Ltd., Welwyn Garden City, England: Such studies as those described by Dr. Gibbs are extremely valuable, and at the Plastics Division of Imperial Chemical Industries, Dr. Burnett has made preliminary experiments along the same lines. In our experiments, small weighed amounts of powdered vinyl chloride polymer were sealed up in a series of tubes and heated. The amount of chloride ion formed was followed by breaking open and titrating the contents of a tube at predetermined times. We agree that hydrogen chloride exerts no catalytic effect in the absence of oxygen, but we disagree with Dr. Gibbs in that we found no true catalytic effect even in the presence of oxygen. In oxygen the curve of chloride ion formed versus time was certainly convex to the time axis. If, however, after a certain time we pumped out the hydrogen chloride and oxygen and then replaced the oxygen and continued heating, the subsequent evolution of chloride ion was the same as if we had not removed the hydrogen chloride already formed. Te concluded then that the presence of oxygen rather than hydrogen chloride was responsible for the autocatalytic appearance of the decomposition curve.

In another experiment, we incorporated sodium carbonate into such a partly decomposed polymer, and here we did find a decreased rate of formation of chloride ion. This led us to believe that the carbonate itself had a stabilizing effect other than by absorbing hydrogen chloride. As we were measuring chloride ion and not acidity, the presence of the carbonate should have made no difference to the rate of chloride ion evolution.

Dr. Gibbs also stated that compared with simple secondary alkyl chlorides, polyvinyl chloride is much less stable. However, one would expect from the stoichiometry of polymerization that there should be a double bond at the end of the chain. This would make it much easier to dehydrochlorinate the next monomer unit.

Dr. GibBs: I find your observations on sodium carbonate very interesting. I have been puzzled as to why sodium carbonate should behave as a stabilizer. If hydrogen chloride is a catalyst in the presence of oxygen and if we can slow down that reaction or remove that possibility, then we will have stabilized the system to some extent at least. The fact that there may be double bonds at the end of the polymer chain, I think, is quite accepted. We have visualized this as a result of a chain-transfer mechanism. This would mean that if chlorine were lost, then you would have a double-bond structure. Now, if hydrogen was transferred, then the terminal group would have a stable configuration and would not be a point from which dehydrochlorination could proceed easily. However, if the double bond participates in a copolymerization, then you have a branch from this point giving a tertiary chlorine. 


\title{
5. Photodegradation of Polyvinyl Chloride
}

\author{
By A. S. Kenyon ${ }^{1}$ \\ Introduction
}

The photodegradation of polyvinyl chloride has been a problem of concern for several years. Many stabilizers of various types are on the market, but none has been completely satisfactory, especially for clear plastics. Many of the stabilizers were found by empirical methods.

It seemed from an analysis of previous work that a fundamental study of the photodegradation would aid in the problem of light stabilization. Some work has been done on the polymer itself in order to determine what effects various conditions have upon the degradation and to determine the products of the reaction. After it was found that considerable difficulty would be experienced with the polymer itself, it was considered that a simple compound such as sec-butyl chloride as a prototype might give more information concerning the mechanism of the degradation.

\section{Experimental Procedures}

Polyvinyl chloride powder was irradiater in a quartz tube by means of an S-4 lamp and in later experiments by means of an $\mathrm{AH}-6$ mercury lamp. Various filter systems were used to isolate the desired wavelength regions. Photodegradations were carried out either in an atmosphere of oxygen or in an atmosphere of nitrogen, using the S-4 lamp as the light source. The amount of hydrogen chloride evolved and the oxygen absorbed, when present, were measured for various periods of time (see table 5.1.).

TABLE 5.1. Hydrogen chloride liberated and oxygen absorbed by polyvinyl chloride irradiated at wavelengths of $2800 \mathrm{~A}$ and greater [1] ${ }^{2}$

\begin{tabular}{|c|c|c|c|}
\hline Exposure & \multicolumn{2}{|c|}{$\mathrm{HCl}$ evolved } & $\mathrm{O}_{2}$ absorbed \\
\hline $\begin{array}{r}24 \mathrm{hr} \text { in } \mathrm{O}_{2} \\
86 \mathrm{hr} \text { in } \mathrm{O}_{2} \\
24 \mathrm{hr} \text { in } \mathrm{N}_{2} \\
114 \mathrm{hr} \text { in } \mathrm{N}_{2}\end{array}$ & $\left\{\begin{array}{c}m e q / g \\
0.0024 \\
.0027 \\
.016 \\
.003 \\
.0078\end{array}\right.$ & $\begin{array}{c}(m e q / g) / h r \\
0.10 \times 10^{3} \\
.11 \\
.19 \\
.13 \\
.068\end{array}$ & \} $\begin{array}{c}m e q / g \\
0.016 \\
.0577 \\
-0\end{array}$ \\
\hline
\end{tabular}

It is seen that hydrogen chloride is evolved in both the presence and in the absence of oxygen, and therefore hydrogen chloride evolution, though catalyzed by the presence of oxygen, can occur independently.

1 Central Research Department, Monsanto Chemical Co., Dayton, Ohio.

2 Figures in brackets indicate the literature references on p. 93. 


\begin{tabular}{|c|c|}
\hline Wavelength & $\begin{array}{c}\mathrm{HCl} \\
\text { evolved }\end{array}$ \\
\hline $\begin{array}{l}A \\
3400 \text { to visible } \\
3000 \text { to visible } \\
2800 \text { to visible } \\
2350 \text { to visible }\end{array}$ & $\begin{array}{l}(\text { meq/g)/hr } \\
\text { None. } \\
0.00010 \\
.00016 \\
.00110\end{array}$ \\
\hline
\end{tabular}

The rate of hydrogen chloride liberated from polyvinyl chloride is also dependent upon the wavelength (see table 5.2). Light from the S-4 lamp was used with various glass filters, which isolated the desired wavelength regions. The lower wavelength of $2350 \mathrm{~A}$ was obtained from an S-4 lamp from which the glass envelope had been removed, leaving only the quartz mercury lamp. At low wavelengths, the amount of hydrogen chloride evolved is very large, since at these frequencies rupture of the $\mathrm{C}-\mathrm{Cl}$ bond would occur.

Porter and Iddings [2] have studied the absorption coefficient of a ketone material at various wavelengths in the ultraviolet region. They have shown in the case of acetone that light of wavelengths greater than $3300 \mathrm{~A}$ is not absorbed, and that a maximum absorption occurs at about 2700 to $2800 \mathrm{~A}$. From work to be discussed later, there seems to be a close resemblance of the effective absorption by a carbonyl group to the effective wavelengths for hydrogen chloride liberation from polyvinyl chloride. Alkyl chlorides are very stable compounds to light and show no absorption in the ultraviolet until the wavelength of 2300 to $2200 \mathrm{~A}$ is reached. Light of this frequency possesses sufficient energy to rupture the $\mathrm{C}-\mathrm{Cl}$ bond. Since photodegradation occurs at wavelengths much longer than $2300 \mathrm{~A}$, it is reasonable to assume that the degradation is caused by the presence of trace amounts of impurities that absorb the radiation and initiate degradation.

A polyvinyl chloride film was cast from a 5-percent solution of polymer in tetrahydrofuran and the infrared spectrum of the film was measured before and after irradiation by the $\mathrm{AH}-6$ lamp at wavelengths greater than $2700 \mathrm{~A}$. Figure 5.1 shows the infrared spectrum of the film in the wavelength region of 5.6 to $7.0 \mu$ before (curve A) and after irradiation in air (curve B). There is a very noticeable increase in the absorption in the region of $5.8 \mu$ indicating an increased concentration of the carbonyl group during the photo-

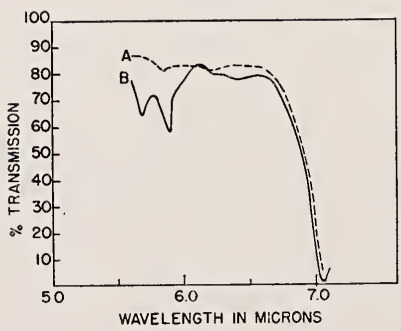

Figure 5.1. Infrared spectra of $P V C$ films.

$\mathrm{A}$ is PVC film before irradiation; $\mathrm{B}$, PVC film after 4-hour exposure; and thickness of films is 0.0024 inch. 
reaction. It is not possible to say from these data whether a carbonyl compound was initially present, but it is felt that such a group is very likely to be present, and that the degradation could be initiated by the photodecomposition of such a ketonic impurity.

If the concentration of carbonyl compounds in the polymer is increased br the addition of ketones, the quantity of hydrogen chloride produced is also increased. The presence of increased carbonyl impurity causes more light to be absorbed and therefore produces more radicals which are capable of attacking the polymer chain. The accelerated evolution of hrdrogen chloride has been observed in the presence of ketonic compounds, with both short and long aliphatic groups attached to the carbonyl group. Polrvinyl chloride in the presence of acetone produces 0.045 equivalent of hydrogen chloride per gram of polymer when irradiated $3 \mathrm{hr}$ with light of warelengths greater than $2700 \mathrm{~A}$, whereas octadecyl laurrl ketone produces 0.022 equiralent under the same conditions, and PVC alone produces only 0.01 equiralent of $\mathrm{HCl}$. The differences may be due to variation in the molar concentrations of the two ketones.

It has been shown by Norrish [3] that a ketone as simple as methyl $n$-butyl ketone upon photodecomposition gives appreciable amounts of acetone and proprlene. It is reasonable to expect that the highmolecular-weight ketones could split and eventually produce radicals that have sufficient activity to react with the polyvinyl chloride molecules.

Polrvinyl chloride also changes in molecular weight when irradiated either in the presence or absence of oxygen. When the degradation is carried out in an atmosphere of nitrogen or in a raccum, using Geon 102 powder, the molecular weight of the polrmer increases, indicating cross-linking. Irriadiation of polyrinyl chloride in the presence of oxrgen causes the molecular weight to decrease, which is indicative of chain scission (see table j.3).

The relative viscosities were measured, using 0.4 -percent solution of polymer in crclohexanone at $25^{\circ} \mathrm{C}$. The molecular weights were calculated bi a method of Mead and Fuoss [4], using the equivalent viscosity and assuming no change in slope due to molecular weight or distribution change. This is not true, and therefore the molecular weights are to be used only for comparison.

Color is also produced in the photodegradation of polyvinyl chloride. The color produced from the polymer irradiated in nitrogen is greater than that produced in an atmosphere of oxvgen. The amount of hrdrogen chloride liberated is greater in oxigen than the amount liberated in nitrogen. The hidrogen chloride is formed from attack

TABLE 5.3. Effect of irradiation of polyvinyl chloride at wavelengths from $2800 \mathrm{~A}$ to the visible and from $2350 \mathrm{~A}$ to the visible [1]

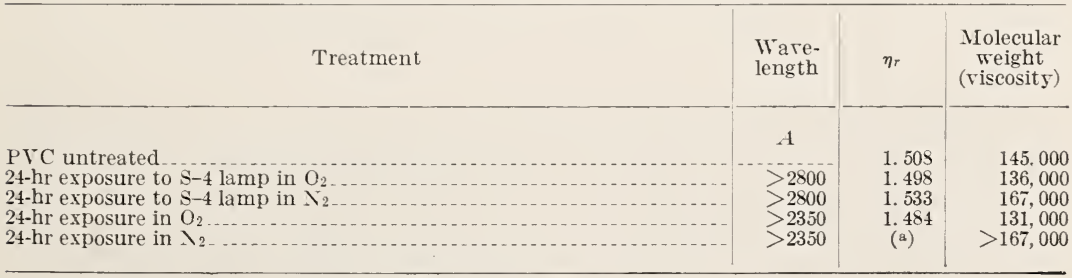

a Would not dissolve. 
on random sections of the polymer molecule. When the irradiation is carried out in the presence of oxygen, chain scission might occur at points that liberated hydrogen chloride, and thereby decrease the number of conjugate double bonds, thus reducing color groups.

Wilson [5] has studied the effects of irradiation upon colpoymers of polyvinylidine chloride and polyvinyl chloride. He observed that water was also produced. He followed the quantities of hydrogen chloride and water liberated by means of a vapor-pressure method.

As polyvinyl chloride has been shown to liberate hydrogen chloride and water upon irradiation with a change in molecular weight and production of color, it was considered that a thorough study on a prototype such as sec-butyl chloride would be a means of clarifying these many reactions.

The ultraviolet-absorption spectrum (fig. 5.2) of unpurified secbutyl chloride shows considerable absorption in the wavelength region 2700 to $3000 \mathrm{~A}$, which indicates the possibility of some carbonyl compound as an impurity. If the unpurified sec-butyl chloride is irradiated by light of wavelengths greater than $2700 \mathrm{~A}$, hydrogen chloride is a product. When sec-butyl chloride has been purified by washing with alkaline permanganate, the ultraviolet-absorption spectrum is shifted to the region of $2300 \mathrm{~A}$. No reaction has been observed with purified sec-butyl chloride either in the liquid or vapor phase when the wavelengths of the incident beam exceed $2700 \mathrm{~A}$, but if there is present an absorbing impurity such as a ketone, then photodecomposition will take place with evolution of hydrogen chloride.

In order to control the quantity of impurity present, pure acetone was added to the purified sec-butyl chloride. The acetone acts in this case as a photosensitizer as it is the only absorbing medium present. The formation of hydrogen chloride and other products are a result of secondary reactions.

The amounts of hydrogen chloride produced as a function of time were studied both in the presence and absence of oxygen. Irradiation was carried out in a system as shown in the schematic drawing (fig. 5.3) of the apparatus. Figure 5.4 shows a plot of equivalents of hydrogen
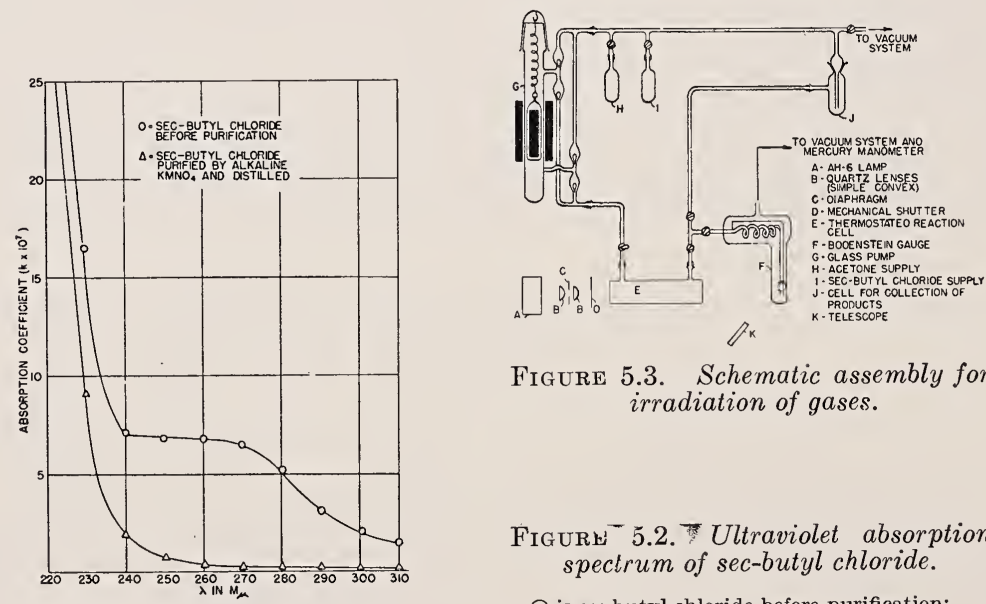

FigURE 5.3. Schematic assembly for irradiation of gases.

FIGURE 5.2. Ultraviolet absorption spectrum of sec-butyl chloride.

is sec-butyl chloride before purification; $\triangle$, sec-butyl chloride purified by alkaline $\mathrm{KMNO}_{4}$ and distilled. 


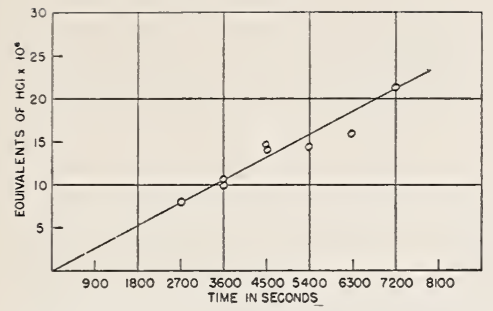

Figure 5.4. Photodecomposition of sec-butyl chloride in the presence of acetone and oxygen.

Temperature, $25^{\circ} \mathrm{C}$.

FIGURE 5.6. Effect of acetone concentration on the photo decomposition of $96 \mathrm{~mm}$ sec-butyl chloride.

Time of irradiation is $11 / 2$ hours; temperature $45^{\circ} \mathrm{C}$.

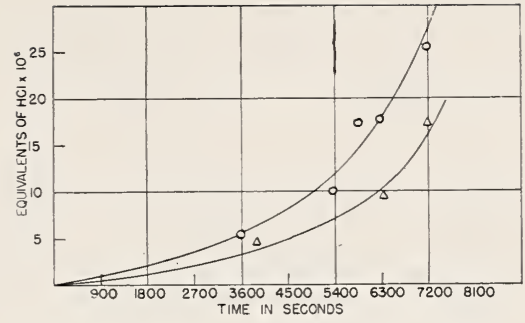

FiguRE 5.5. Photodecomposition of sec-butyl chloride in the presence of acetone.

$\mathrm{O}$ is $45^{\circ} \mathrm{C} ; \triangle, 25^{\circ} \mathrm{C}$.

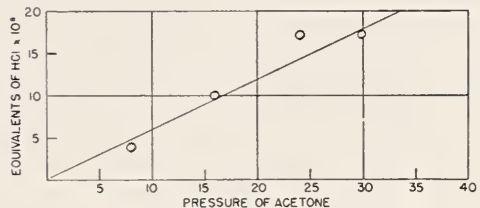

chloride produced as a function of time at $25^{\circ} \mathrm{C}$. when oxygen is present. In these experiments $98 \mathrm{ml}$ of sec-butyl chloride, $16 \mathrm{ml}$ of acetone, and $600 \mathrm{ml}$ of oxygen were used. The beam intensity was approximately $5 \times 10^{17}$ quanta per second. The quantity of hydrogen chloride produced is directly proportional to time, and the extrapolated curve passes through the origin, indicating little or no induction period.

Figure 5.5 shows the equivalents of hydrogen chloride produced in the absence of oxygen as a function of time at $25^{\circ}$ and $45^{\circ} \mathrm{C}$ when $98 \mathrm{ml}$ of sec-butyl chloride and $16 \mathrm{ml}$ of acetone are jrradiated in a cell having a capacity of $130 \mathrm{ml}$. There is an acceleration in the amount of hydrogen chloride liberated when sec-butyl chloride is irradiated in the absence of oxygen. Initially the rate of hydrogen chloride formation is slower in the absence of oxygen, but eventually it exceeds the rate in the presence of oxygen.

The amount of liberated hydrogen chloride in the photodegradation of sec-butyl chloride is proportional to the concentration of the absorbing substance, which in this case is acetone. The equiralents of hydrogen chloride produced by $96 \mathrm{ml}$ of sec-butyl chloride when irradiated for $1.5 \mathrm{hr}$ at a temperature of $45^{\circ} \mathrm{C}$ in the presence of varying amounts of acetone from 8 to $30 \mathrm{ml}$ are shown in figure 5.6 .

As the only absorbing substance in the system is acetone, the primary reaction is the decomposition of acetone into its radicals:

$$
\mathrm{CH}_{3} \mathrm{COCH}_{3}+h \nu \rightarrow \mathrm{CH}_{3} \cdot+\mathrm{CH}_{3} \mathrm{CO} \text {. }
$$

The acetyl and methyl free radicals then have the possibility of reacting with the sec-butyl chloride molecules. The effect of temperature on the quantity of hydrogen chloride liberated has been determined at $25^{\circ}, 45^{\circ}$, and $122^{\circ} \mathrm{C}$ for light of wavelengths greater than $2700 \mathrm{~A}$, using $98 \mathrm{ml}$ of sec-butyl chloride and $16 \mathrm{ml}$ of acetone in a volume of $130 \mathrm{ml}$. The amounts of hydrogen chloride produced in 
$1.5 \mathrm{hr}$ of irradiation are as follows:

$\begin{array}{cc}\text { Temperature } & \begin{array}{c}\text { Equivalents } \\ \circ \mathrm{C}\end{array} \\ 25 & \text { of } \mathrm{HCl} \times 10^{6} \\ 45 & 10 \\ 122 & 55\end{array}$

If these data are plotted in the form of an Arrhenius plot (fig. 5.7), a straight line is not obtained. The line is drawn through the points at $25^{\circ}$ and $45^{\circ} \mathrm{C}$ because at these temperatures the quantum vield for carbon monoxide production from the photolysis of acetone is of the order of $0.5^{3}$. If the increase in hydrogen chloride production is caused by a thermal increase alone, the vield at $122^{\circ} \mathrm{C}$ should have been approximately $27 \times 10^{-6}$ equivalent. The observed quantity of $55 \times 10^{-6}$ equivalent can be caused only by the increased rield of $\mathrm{CH}_{3}$. radicals at $122^{\circ} \mathrm{C}$. The production of hydrogen chloride from sec-butyl chloride is therefore initiated by the methyl radicals and not by the acetyl radical under the conditions of these experiments. Many investigators [6] have proved that the quantum yield for carbon monoxide from the photolysis of acetone at temperatures in excess of $100^{\circ} \mathrm{C}$ is unity, and that the primary reaction at these temperatures is

$$
\mathrm{CH}_{3} \mathrm{COCH}_{3}+h \nu \rightarrow 2 \mathrm{CH}_{3} \cdot \mathrm{CO} \text {. }
$$

When a carbonyl compound such as acetaldehyde or propionaldehrde was used as sensitizer, no hydrogen chloride was liberated. L sing a mixture of acetone and acetaldehyde as a sensitizer, the amount of hydrogen chloride was increased over that of acetone alone. Akerord and Norrish [7] and later Grahame and Rollefson [8] found that the presence of methyl radicals produced by the primary action of light with acetone catalyzed the decomposition of acetaldehyde, producing extremely long chains that form methyl radicals from the aldehrde. This is another indication that methyl radicals are responsible for the hydrogen chloride production from sec-butyl chloride. The reactions of the aldehyde in the presence of acetone have been given as follows:

$$
\begin{gathered}
\mathrm{CH}_{3} \mathrm{CHO}+h \nu \longrightarrow\left(\mathrm{CH}_{3} \mathrm{CO}\right)^{*} \nearrow_{\mathrm{CH}_{3}+\mathrm{H}+\mathrm{CO}}^{\mathrm{CH}_{4}+\mathrm{CO}} \\
\mathrm{H}+\mathrm{CH}_{3} \mathrm{CHO} \longrightarrow \mathrm{H}_{2}+\mathrm{CH}_{3} \mathrm{CO} \cdot \\
\mathrm{CH}_{3} \mathrm{CO} \cdot \longrightarrow \mathrm{CH}_{3} \cdot+\mathrm{CO} \\
\mathrm{CH}_{3}+\mathrm{CH}_{3} \mathrm{CO} \longrightarrow \mathrm{CH}_{4}+\mathrm{CH}_{3} \mathrm{CO} .
\end{gathered}
$$

The formation of $\mathrm{CH}_{3} \mathrm{CO}$. is the chain-propagating step.

In order to study more carefully the mechanism of the degradation reaction, a srstem for irradiating the sec-butyl chloride-acetone mixtures was used, as shown in figure 5.8. This system had no stopcocks in the reaction portion of the system, and the noncondensable gases, such as methane and carbon monoxide, could be isolated by freezing the condensable products with liquid nitrogen and pumping off the noncondensables by a Toepler pump. 


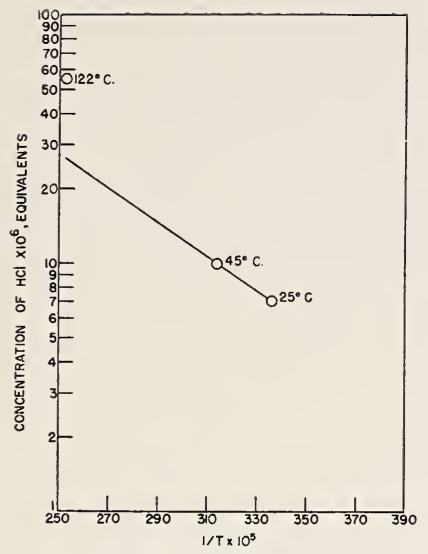

Figure 5.7. Amount of $\mathrm{HCl}$ produced in 1.50 hours of $\mathrm{ir}$ radiation.

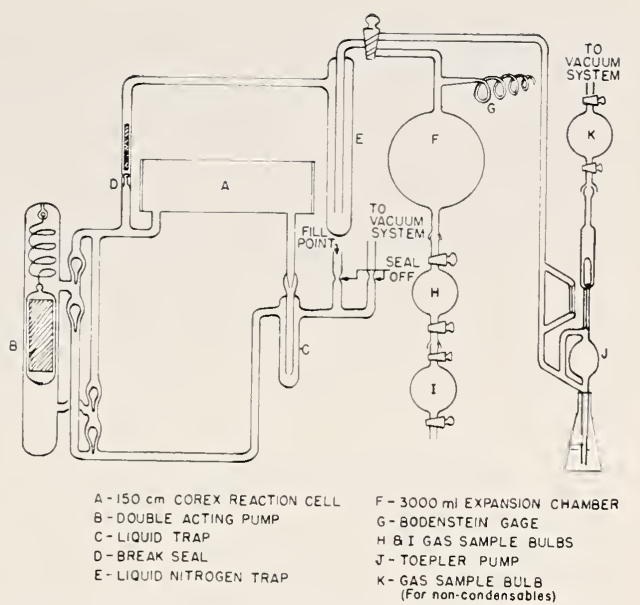

FIGURE 5.8. Schematic cissembly for irradiation of gases

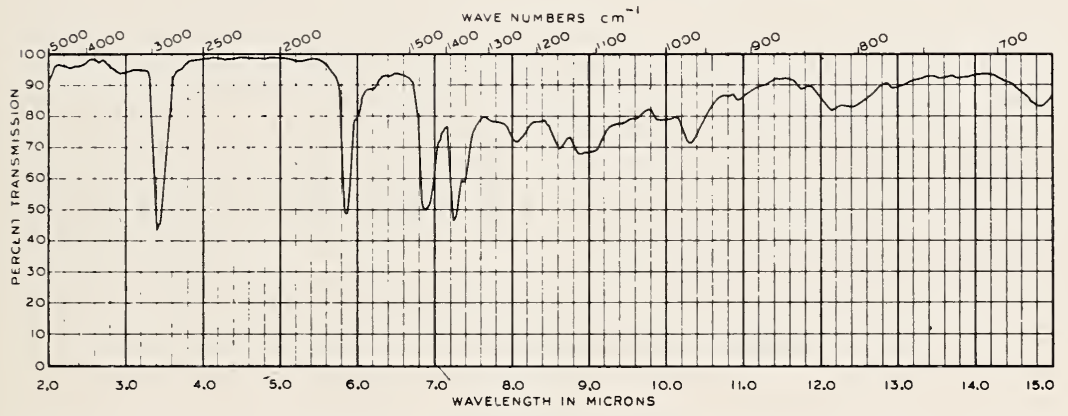

FigURE 5.9. Infrared spectrum of high-boiling fraction produced during irradiation of sec-butyl chloride and acetone.

The gaseous products were analyzed by a mass spectrometer. Analyses of the products show the presence of carbon monoxide, methane, hydrogen chloride, and masses of 95, 97, 110, 112 . If acetone is irradiated alone, there is present a large amount of ethane, but when sec-butyl chloride is added, there is little or no ethane formed. Steacie and coworkers [9] have found that the formation of ethane is suppressed in reactions of methyl radicals with hydrocarbons. This is expected, because reaction with hydrocarbons will prevent their combining to form ethane.

There existed the possibility of methyl chloride being formed as an intermediate step in the decomposition of sec-butyl chloride and acetone. No methyl chloride could be found from the mass spectra.

When a mixture of sec-butyl chloride and acetone is irradiated at $122^{\circ} \mathrm{C}$, there is formed a high-boiling liquid mixture having a carbonyl group, as shown by the infrared spectrum in figure 5.9. The mass analysis of this high-boiling liquid shows masses at practically all mass units up to mass 148 , which is the approximate limit of the mass spectrometer. No satisfactory chemical analysis could be ob- 
tained, because the high-boiling product lost hydrogen chloride and/or condensed, with resultant change in composition.

With these experimental facts, the following mechanism is proposed to account for the observed products:

$$
\begin{aligned}
& \mathrm{CH}_{3} \mathrm{COCH}_{3}+h \nu \stackrel{122^{\circ} \mathrm{C}}{\longrightarrow} 2 \mathrm{CH}_{3} \cdot+\mathrm{CO} \\
& \mathrm{CH}_{3} \cdot+\mathrm{CH}_{3} \mathrm{COCH}_{3} \longrightarrow \mathrm{CH}_{4}+\cdot \mathrm{CH}_{2} \mathrm{COCH}_{3}
\end{aligned}
$$
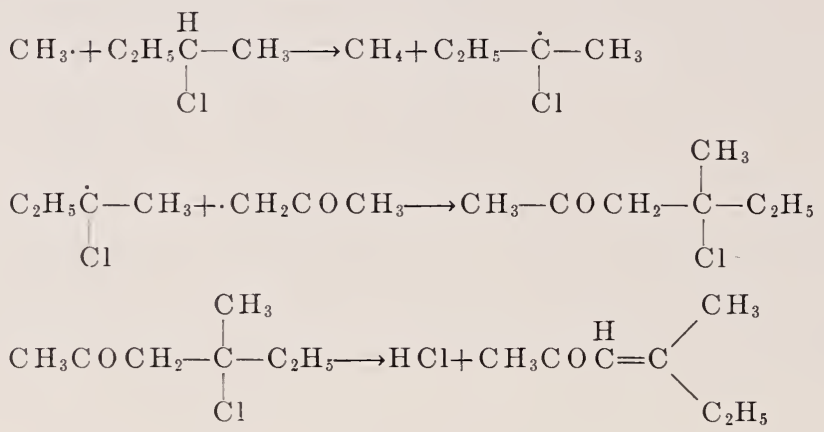<smiles>CCC(Cl)CCCC(C)(Cl)C(C)(Cl)CC</smiles>

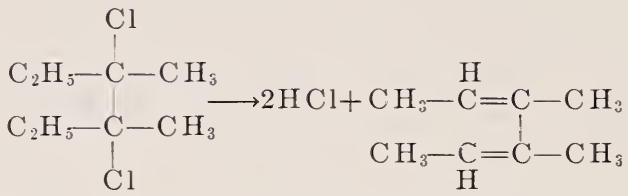

No evidence of the formation of acetonyl acetone has been observed in the mass spectra of the high-boiling fraction. This indicates that the acetonyl radical produced according to (2) reacts at a much faster rate with the sec-butyl chloride radical.

The carbonyl compound appearing in the high-boiling fraction can come only from the acetonyl group, because at $122^{\circ} \mathrm{C}$ the acetyl group is unstable and decomposes into carbon monoxide and a methyl free radical. The combination of a sec-butyl chloride radical and the acetonyl radical would produce a $\beta$-chloroketone (chlorine $\beta$ to the carbonyl). The chlorine in the $\beta$ position is unstable and would easily split out hydrogen chloride, leaving an unsaturated ketone (4-methylhexene-3-one-2).

There is also the possibility of the unsaturated ketone produced in the reaction condensing with acetone or itself in the presence of hydrogen chloride, thereby producing a conjugated double-bond system in the molecule, giving rise to color. One mole of water is also produced in the reaction for each condensation. If this explanation is true, then the quantity of hydrogen chloride liberated would not correlate with the color dereloped.

The mass analysis of the high-boiling liquid showed strong masses at $55,95,97,110$, and 112 . This indicates that more than one com- 
pound is in the high fraction. No possible combination of atoms containing oxygen could be written that would give mass indications of 55, 95, and 110. When mercury dimethyl, as a source of methyl radicals, and sec-butyl chloride were irradiated at $122^{\circ} \mathrm{C}$, only a trace amount of hydrogen chloride could be detected chemically. No other products could be identified by the mass spectrometer. If mercury dimethyl and sec-butyl chloride are heated to $300^{\circ} \mathrm{C}$, hydrogen chloride, methane, and a compound yielding masses of 55, 95, and 110 could be detected. It is concluded that the compound is<smiles>CC=C(C)C(C)=CC</smiles>

If sec-butyl chloride is heated alone at $300^{\circ} \mathrm{C}$, then the products of the reaction are only hydrogen chloride and butene-2. The action of methyl radicals therefore forms the sec-butyl chloride radicals, which then dimerize and split out hydrogen chloride, leaving the unsaturated hydrocarbon. The parent mass of this unsaturated hydrocarbon is 110. The mass of 112 arises from two sources: (a) the unsaturated ketone of reaction 5, and (b) the dimer (3,4-dichloro-3,4-dimethylhexane) less two chlorine atoms.

The quantum yields were determined for hydrogen chloride, methane, and the high-boiling fraction at a wavelength of $3130 \mathrm{~A}$. At temperatures above $100^{\circ} \mathrm{C}$, the quantum yield for carbon nonoxide from the photolysis of acetone has been determined by many workers [6] to be unity, at wavelengths of $3130 \mathrm{~A}$. The number of molecules of carbon monoxide produced in the reaction can thus be used as an internal actinometer. The following yields were obtained at $122^{\circ} \mathrm{C}$ :

$\begin{array}{llc}\phi \mathrm{HCl} & \phi \mathrm{CH}_{4} & \phi \mathrm{CO} \\ 1.04 & 2.12 & 1 \\ 1.29 & 2.20 & 1 \\ 1.00 & 2.00 & 1 \\ 1.02 & 2.00 & 1 \\ 1.09 \pm .11 & 2.08 \pm .09 & \end{array}$

After the reaction, the tube containing the condensed liquids was warnied to approximately $150^{\circ} \mathrm{C}$ to hasten the distillation into the expansion chamber. If this liquid trap is allowed to remain at room temperature, then the quant $x$ yield for $\mathrm{HCl}$ drops to 0.8 .

If the photodecomposition of sec-butyl chloride and acetone is carried o t at $25^{\circ} \mathrm{C}$, the quantum yieid for $\mathrm{HCl}$ is approximately 0.07 , and the quantum yield for methane is 0.08 . In all cases the total quanta absorbed have been nearly equal; therefore, the amount of photoreaction products formed was nearly equal, so that changes in the quantum yield of hydrogen chloride result from the thermal instability of the compounds produced in the photoreaction, such as the 4-chloro-4-methylhexanone-2, and the 3,4-dichloro-3,4dimethylhexane. The maximum possible quantum yield for the hydrogen chloride evolved would be 2 , but this value is not reached due to the incomplete thermal decomposition of the reaction products.

In order to follow the reaction more closely, deuterated acetone was used as the source of free methyl radicals, and the resulting products 
were analyzed by the mass spectrometer. If the deuterated acetone is 100 percent pure, then $\mathrm{CD}_{4}$ arises only from reaction of the $\mathrm{CD}_{3}$ radical with the deuterated acetone, and $\mathrm{CD}_{3} \mathrm{H}$ is produced by the action of the methyl $\left(\mathrm{CD}_{3}\right)$ radical with sec-butyl chloride. The $\mathrm{CD}_{4}$ and $\mathrm{CD}_{3} \mathrm{H}$ ratios give a measure of the distribution of methyl radicals between the two reactions when suitable corrections are applied for the amount of deuteration in the acetone. The following reactions are involved:

$$
\begin{gathered}
\mathrm{CD}_{3} \mathrm{C} \mathrm{OCD}_{3}+h \nu \longrightarrow 2 \mathrm{CD}_{3} \cdot+\mathrm{CO} \\
\mathrm{CD}_{3}+\mathrm{CD}_{3} \mathrm{C} \mathrm{OCD}_{3} \longrightarrow \mathrm{CD}_{4}+\mathrm{CD}_{3} \mathrm{C} \mathrm{O} \mathrm{CD}_{2} . \\
\mathrm{CD}_{3}+\mathrm{C}_{2} \mathrm{H}_{5}-\underset{\mathrm{C}}{\mathrm{C}}-\mathrm{CH}_{3} \longrightarrow \mathrm{CD}_{3} \mathrm{H}+\mathrm{C}_{2} \mathrm{H}_{5}-\underset{\mathrm{Cl}}{\dot{\mathrm{C}}-\mathrm{CH}_{3}} \\
\underset{\mathrm{Cl}}{l}
\end{gathered}
$$

When the rapor composition in the reaction cell consists of 36 $\mathrm{mm}$ of sec-butyl chloride and $157 \mathrm{~mm}$ of acetone containing deuterated acetone as a tracer at a temperature of $122^{\circ} \mathrm{C}, 11.1$ percent of the methyl radicals produced by the photolysis of acetone react with acetone to form methane and acetonyl radicals, and 88.9 percent of the methyl radicals attack the sec-butyl chloride to produce methane and sec-butyl chloride radicals. No acetonyl acetone could be detected in the mass spectrum of the high-boiling component, which indicates the acetonyl radicals must react with the sec-butyl chloride radicals to produce a $\beta$-chloroketone such as

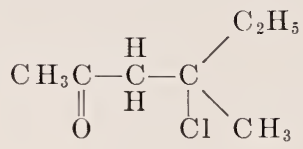

The excess sec-butyl chloride radicals probably dimerize to form 3,4-dichloro-3,4-dimethylhexane. Under these conditions, the highboiling liquid formed during the photodegradation would consist of 22.2 mole percent of $\beta$-chloroketone and 77.8 mole percent of dimer. A mixture of this composition would contain 36.0 percent by weight of chlorine, assuning no decomposition of the products. Chemical analyses for chlorine were made on the reaction products, and the total chlorine in the residue and evolved hydrogen chloride was 36.6 percent. The residue contained only 22.24 percent of chlorine, which indicates partial decomposition of the reaction products. Based upon this analysis, the dimer must be decomposed to the extent of 42.5 to 59 percent, depending upon the percentage decomposition of the $\beta$-chloroketone. After partial decomposition, the mixture would consist of a $\beta$-chloroketone, an alkyl dichloride, an unsaturated ketone, and an unsaturated hydrocarbon. The methyl radicals would be expected to react faster with the sec-butyl chloride than with the acetone because the energy of activation for the removal of a tertiary hydrogen is less than the removal of a primary hydrogen by methyl radicals. 
Hydrogen chloride, water, and color formation have been observed in the photodegradation of sec-butyl chloride and in polyvinyl chloride. It appears that some of the reactions must be similar.

If polyvinyl chloride is irradiated by ultraviolet light in the presence of oxygen and then thermally degraded, the rate of liberation of hydrogen chloride is very much greater than the rate for the thermal process alone. The following table shows the rate of hydrogen chloride evolved from polyvinyl chloride when irradiated by the S-4 lamp in oxygen for $24 \mathrm{hr}$ and then thermally degraded at $150^{\circ} \mathrm{C}$ compared with the thermal degradation alone:

\begin{tabular}{|c|c|c|}
\hline & $\begin{array}{l}\text { Nitrogen sweep- } \\
\text { ing } \mathrm{HCl}\end{array}$ & $\begin{array}{l}\text { Oxygen sweep- } \\
\text { ing } \mathrm{HCl}\end{array}$ \\
\hline $\begin{array}{l}\text { Irradiated }+1 \mathrm{hr} \text { at } 150^{\circ} \mathrm{C} \\
\text { Heat alone, } 1 \mathrm{hr} \text { at } 150^{\circ} \mathrm{C}\end{array}$ & $\begin{array}{c}\text { Millimoles } / g \\
0.009 \\
.0035\end{array}$ & $\begin{array}{c}\text { Millimoles/g } \\
0.021 \\
.0043\end{array}$ \\
\hline
\end{tabular}

Exposure to light causes the build-up of thermally unstable groups within the polymer, which liberate hydrogen chloride at a faster rate than when the polymer is only heated.

The reactions involved in the photochemical degradation of polyvinyl chloride must be different in the presence and absence of oxygen, as shown by molecular weight changes. In the absence of oxygen, considerable cross-linking has been detected, and presumably such compounds as the following are formed:

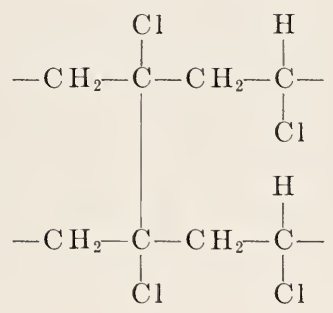

which could thermally split out hydrogen chloride, based on the evidence of instability or the 3,4-dichloro-3,4-dimethylhexane. In the case of photodegradation in the presence of oxygen, some oxidation must occur to give a ketonic group in the chain, which also causes hydrogen chloride to be produced through thermal decomposition.

C-14 labeled stabilizers have been used as means of detecting the course or the stabilization. When polyvinyl chloride is irradiated by light of wavelengths greater than $2700 \mathrm{~A}$ in the presence of $\mathrm{C}-14$ butyl-labeled dibutyl tin diacetate, there is observed an increase in the retained $\beta$ activity with time of irradiation. After irradiation, the stabilizer was extracted by dissolving and reprecipitating the polymer a number of times until constant retained activity was observed in a polymer film. Polyvinyl chloride treated with C-14 carboxyl-acetate-labeled dibutyl tin diacetate shows no change in the retained $\beta$ activity with time of irradiation, which shows that the 
acetate groups are not involved in the reaction. The following mechanism is proposed as a possible explanation for the $\beta$ activity due to reaction of the butyl groups with the polymer radical:

$$
\mathrm{R} \cdot+\left(\mathrm{C}_{4} \mathrm{H}_{9}\right)_{2} \mathrm{Sn}(\mathrm{AC})_{2} \longrightarrow \mathrm{RC}_{4} \mathrm{H}_{9}+\mathrm{C}_{4} \mathrm{H}_{9} \mathrm{~S} \mathrm{Sn}(\mathrm{AC})_{2},
$$

where $\mathrm{R}$. is a radical formed by a previous extraction of a hydrogen atom from the polyvinyl chloride. The tin compound acts also as a scavenger for hydrogen chloride and thus removes the condensation catalyst. The addition of the butyl group to the polymer radical has blocked further action to cross-linking and oxidation at this one particular point.

\section{Summary}

The amounts of hydrogen chloride evolved from the photodegradation of polyvinyl chloride have been determined in the presence and absence of oxygen. The degradation is accompanied by a decrease in molecular weight of the polymer in the presence of oxygen and an increase in molecular weight due to cross-linking in the absence of oxygen.

'The mechanism for the photodegradation of the prototype of polyvinyl chloride, sec-butyl chloride, has been determined in the presence of acetone. All hydrogen chloride is produced from the thermal decomposition of unstable compounds resulting from secondary reactions involving the radicals produced by the abstraction of hydrogen atoms from the sec-butyl chloride and acetone. The compounds producing the hydrogen chloride have been found to be a $\beta$-chloroketone (4-chloro-4-methylhexane-2) and 3,4-dichloro-3,4dimethylhexane. The extent of the methyl radical reactions have been determined by the use of deuterated acetone.

Quantum rields for the hydrogen chloride production from secbutyl chloride have been found to be thermally dependent. The quantum yield at $25^{\circ} \mathrm{C}$ is 0.08 , whereas at temperatures above $100^{\circ} \mathrm{C}$, the yield approaches unity. The quantum yield changes as a function of temperature eren at temperatures where the quantum yield for the production of carbon monoxide from the photolysis of acetone remains constant.

Polyvinyl chloride that has been exposed to ultraviolet irradiation degrades at a faster rate thermally than the polymer that has not been exposed to the light. This increased rate is caused by the formation of unstable compounds during the photolysis.

Butyl groups have been found to add onto the polymer when polyvinyl chloride is irradiated in the presence of dibutyl tin diacetate. A mechanism for this stabilization is proposed.

The work of Catherine Hogg on much of the polyvinyl chloride studies is gratefully acknowledged. The mass-spectrometer analyses performed by Richard Roberts have made parts of this study possible.

The author acknowledges the many helpful discussions and criticisms of Drs. Johns and Ruehrwein and Prof. George B. Kistiakowsky. 


\section{References}

[1] Unpublished work of Catherine Hogg, formerly Central Research Department Monsanto Chemical Co., Dayton, Ohio.

[2] C. W. Porter and C. Iddings, J. Am. Chem. Soc. 48, 40 (1926).

[3] R. G. W. Norrish, Trans. Faraday Soc. 33, 1521 (1937).

[4] J. D. Mead and R. M. Fuoss, J. Am. Chem. Soc. 64, 277 (1942).

[5] J. E. Wilson, J. Am. Chem. Soc. 72, 2905 (1950).

[6] W. A. Noyes, Jr., and L. M. Dorfman, J. Chem. Phys. 16, 788 (1948); W. Davis, Jr., Chem. Rev. 40, 201 (1947).

[7] E. I. Akeroyd and R. G. W. Norrish, J. Chem. Soc. 890 (1936).

[8] D. C. Grahame and G. K. Rollefson, J. Chem. Phys. 8, 98 (1940).

[9] A. F. Trotman-Dickenson and E. W. R. Steacie, J. Chem. Phys. 18, 1097 (1950).

A. F. Trotman-Dickenson, J. R. Birchard, E. W. R. Steacie, J. Chem. Phys. 19, 163 (1951).

A. F. Trotman-Dickenson and E. W. R. Steacie, J. Chem. Phys. 19, 169 (1951).

\section{Discussion}

Dr. J. E. Wilson, Bakelite Co., Division of Union Carbide and Carbon Corp., Bound Brook, N. J.: Dr. Kenyon's remarks on the formation of water as a product reminded me of a technique used by Deatherage, which may be of some interest. Methyl oleate was oxidized, and it was found that water was produced. In an attempt to find the source of the water, the analogous deuterium compound, methyl 9,10-dideuterooleate,

$$
\mathrm{CH}_{3}\left(\mathrm{CH}_{2}\right)_{7} \mathrm{CD}=\mathrm{CD}\left(\mathrm{CH}_{2}\right)_{7} \mathrm{COOCH}_{3} \text {, }
$$

was oxidized, the idea being that there should be a large proportion of deuterium in the water resulting. The result of the experiment was that there was no more deuterium in the product water than was in the original methyl 9,10-dideuterooleate, indicating that the olefinic hydrogen is probably not the source of the hydrogen in the water product. Deatherage and his associates ran another experiment, using 8,8,11,11-tetradeutero-cis-9-octadecene:

$$
\mathrm{CH}_{3}\left(\mathrm{CH}_{2}\right)_{6} \mathrm{CD}_{2} \mathrm{CH}=\mathrm{CHCD}_{2}\left(\mathrm{CH}_{2}\right)_{6} \mathrm{CH}_{3} \text {. }
$$

Again the deuterium did not appear in the water produced, indicating that the water hydrogen did not come from the alpha carbon atoms. Thus the results of these two experiments were negative, but they appear to eliminate two of the possible sources of the hydrogen of the water product. Deatherage also found that the rate of oxidation in the second experiment was reduced by 80 percent when hydrogen was replaced by deuterium, apparently because it is more difficult to snap the deuterium from the chain.

Dr. R. F. Boyer, Dow Chemical Co., Midland, Mich.: It is not necessary to postulate the presence of any extraneous grouping in the polyvinyl chloride, such as a $\mathrm{C}=\mathrm{O}$ group, in order to explain sensitivity to ultraviolet light. A simple calculation indicates that if polyvinylidene chloride loses just a few parts per million of $\mathrm{HCl}$ during heat fabrication, there will be sufficient absorption of ultraviolet energy to account for subsequent photodegradation. This 
situation then builds up very rapidly because once several double bonds are conjugated there is an even better site for energy absorption, and there is a better than average chance that this absorbed energy will be dissipated locally to cause further loss of $\mathrm{HCl}$.

I wonder how well model compounds can answer some of the problems that occur in the degradation of a long-chain molecule, particularly in the case of polyvinyl chloride, where one suspects a tendency to build up long runs of conjugated double bonds. You have to postulate polymerization reactions of the degraded model compounds in order to get a source for the light absorption at long wavelengths shown by degraded polyvinyl chloride.

Dr. Kenyos: The presence in the polyvinyl chloride of an impurity containing a carbonyl group is only a possibility. The point I would like to make is that pure polyvinyl chloride is theoretically a very stable compound at warelengths occurring in light, and therefore there has to be some impurity that will absorb the light and eventually cause degradation. Whether that be in the form of oxygen or double bonds, I am not in a position to say. Also as to the use of prototypes, it is rery difficult to take the degradation products of polyvinyl chloride and determine the exact composition of all the products. Prototypes might give us some simple picture whereby we can eventually work on the polymer. There is a long step between transferring the data from a prototype over to a polymer, and that is the step that is in the future. The use of a prototype is merely a means of getting some fundamental information as to what might go on and what can be expected in a polymer. It certainly does not give a complete mechanism for the degradation of the polymer itself. 


\title{
6. Role of Hydrogen Chloride in Polyvinyl Chloride Degradation
}

\author{
By Abb L. Scarbrough, ${ }^{1}$ W. L. Kellner, ${ }^{1}$ and P. W. Rizzo ${ }^{1}$
}

Polyvinyl chloride degradation is basically a manufacturing problem. It was the manufacturing plant in which the problem was first recognized, and in which early remedies were prescribed. The change of color and physical properties of vinyl resins under exposure to heat and light was the first major stumbling block to their ascendency as elastomeric and rigid plastic materials. Techniques have been developed to combat the problem, but in spite of the fact that polyvinyl chloride and its copolymers are the most used of contemporary plastic materials, their stabilization against heat and light break-down is still the most pressing single problem connected with them.

Hydrogen chloride was the first product of polyvinyl chloride degradation to be recognized. It was known to be troublesome not only due to its own odor and corrosive action, but because, in some way, the degradation became worse as more free hydrogen chloride formed. The value of substances forming insoluble or relatively un-ionized chlorides was recognized, their ability to remove free hydrogen chloride leading to improved thermal stability. These materials, basic lead carbonate and a few others, were the early stabilizeis.

Advances to this point, as has been stressed above, were largely the result of empirical axioms derired from plant experience. The first attempts to study systematically what happens when vinyl resins degrade also marked the end of the idea that hydrogen chloride catalysis was the sole significant factor in the process. In 1947, Boyer $[1]^{2}$ reported the presence of carbonyl $(\mathrm{C}=\mathrm{O})$ groups in degraded polymer, indicating that oxygen was involved in the reaction.

Fox, Hendricks, and Ratti [2] in 1949 established (1) a relationship of the structures formed in degradation of polyvinyl chloride under heat conditions to structures found in the oxidized unsaturates in drying oils, as reported by Holman, Lundberg, and Burr [3, 4]. 'These relationships were postulated as a result of similarities in absorption spectra in the range of 2500 to $3000 \mathrm{~A}$, and presumably may be attributed to polyene systems and carbony! groups, alone or in conjugation; (2) the presence of structures with many linearly conjugated double bonds in degraded polyvinyl chloride resins, through apparent relationships in the ultraviolet and visible light absorption spectrograms with those of carotene-type structures. These studies did much to establish the nature of the groups in degraded resin, but did little to show by what mechanism degradation occurred or in what way the reagents involved participate.

Kenyon [5] studied irradiation effects of ultraviolet light (3130 A) on sec-butyl chloride and on cast films of polyvinyl chloride, and

${ }^{1}$ National Lead Co., Research Laboratories, Brooklyn, N. Y.

2 Figures in brackets indicate the literature references on p. 105. 
showed the catalytic effects of reagents containing carbonyl groups on the decomposition of sec-butyl chloride. A mechanism was postulated involving free radical formation, an explanation which might account for carbonyl group formation in systems of this type. Credence was given to the procedure by previous work on polyvinylidene chloride by Matheson and Boyer [6], showing that during sunlight exposure virtually all of the degradation was accomplished by ultraviolet radiation of wavelength longer than $3000 \mathrm{~A}$.

The purposes of the present study are (1) to fix the role of hydrogen chloride in the polyvinyl chloride degradation process, (2) to clarify the relationship of oxygen to the course of the degradation, (3) to account for observed differences between the effects of heat and of light on the course of degradation through the above two hypotheses, and (4) to suggest approaches to combating degradation in view of any new concepts on the role of the participants in the reaction.

\section{Experimental Procedures}

Choice of a procedure for determining the effects of oxygen and hydrogen chloride on the course of polyvinyl chloride degradation is governed by three main considerations: (1) The procedure must permit a sufficient surface of the experimental samples to be exposed to insure that definite trends are produced by the variation in atmospheres. (2) The procedure must permit quantitative observation of the course of the degradation under various conditions, without the necessity for further treatment on the test samples in order to convert them into a suitable form for eraluation. Further exposure beyond that used in the actual tests might intensify or change the course of the induced degradation. (3) The procedure must permit exposure to both heat and light induced degradation under atmospheric and other conditions that are substantially identical.

\section{Preparation of Disks}

After a consideration of the alternatives, a procedure was adopted which is a modification of that used by Fox, et al. [2]. Transparent disks of high molecular weight polyvinyl chloride resin (Geon 101), 0.033 -in. thick, were molded at $110^{\circ} \mathrm{C}$ and $7.000 \mathrm{lb} / \mathrm{in}^{2}$ in a 20 -min cycle. Ultraviolet light absorption spectrograms in the range of 2000 to $4000 \mathrm{~A}$ were prepared on each of the transparent disks, using a Beckman DU spectrophotometer. Selected disks were then exposed to heat and light in each of the following atmospheres: (1) dry air; (2) nitrogen; (3) dry air (95\%)-hydrogen chloride (5\%); (4) nitrogen (95\%)-hydrogen chloride (5\%).

\section{Heat Tests}

Heat tests were done in a specially constructed glass cell, permitting constant circulation of the individual atmospheres. Circulation rates were such as to permit maintenance of $85^{\circ} \mathrm{C}$. The cells containing the vinyl disks were sealed with Pyrex plates, allowing removal of the samples after each $2 \mathrm{hr}$ of exposure for the purpose of preparing spectrograms of the ultraviolet light absorption. In this way, the course of the degradation was followed constantly during the exposure 
period. Original and final spectrograms were also taken in a Baird infrared spectrophotometer, model B, to determine any absorption changes in this region of the spectrum.

\section{Light Tests}

Light-exposure tests were made with an RS sunlamp. This source has strong radiation bands in the vicinity of 3024,3650 , and $4047 \mathrm{~A}$, and according to the work of Matheson and Borer [6] should be well suited to inducing vinyl degradation. Moreover, the practical value of such a light source is shown by the work of Kohler [7] and others $[8,9]$, who have found that radiations less than $3000 \mathrm{~A}$ amount to less than 5 percent of solar incident light on the earth's surface. To take advantage of this energy source, the Prrex window used to seal the samples into the cell during the heat-exposure tests was replaced br a plate of Vycor glass No. 791 , which admits the effective radiation of the RS sunlamp into the cell, as seen in figure 6.1 [10]. The atmospheres in the cells were renewed at 2 -hr intervals, and good air circulation around the cells permitted maintenance of $30^{\circ} \mathrm{C}$. The disks were $18 \mathrm{in}$. from the light source. Ultraviolet absorption spectrograms were taken after approximately every $25 \mathrm{hr}$ of light exposure to follow the course of the degradation. In addition to the four atmospheres used in the studies on heat degradation, light action on one sample was observed in an atmosphere of pure oxygen.

\section{Scope of Method}

Although this procedure was believed to be the best available for the purpose, certain limitations are inherent. The transparent molded disks are somewhat thicker than is desirable from two standpoints: (1) greater exposure to the various atmospheres per unit weight is possible when thinner disks are used, (2) greater sensitivity in the various spectographic recording machines is possible through use of thinner sections.

Actually, the lower limit on the thickness of the disk is established by the speed at which the mold used in its preparation may be heated and cooled. The required temperature for fusion of the disk can be attained only by such amounts of applied heat as would degrade

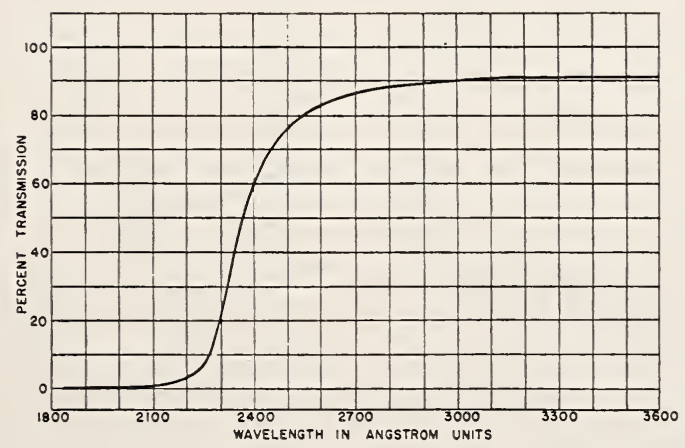

Figure 6.1 Ultraviolet transmission for Tycor glass No. 791. 
smaller amounts of resin to the point where further exposure studies would be impossible.

It would also be desirable to expose the samples to heat at temperatures more closely approximating those used in plant processing of vinyl resins. However, attempts to conduct heat tests at the $150^{\circ}$ $\mathrm{C}$ level caused warping of the plastic disks, making absorptionspectra determinations impossible. Repeated experiments showed $85^{\circ} \mathrm{C}$ to be the highest practical exposure temperature for this procedure.

A practical limitation of the selected test method is the elimination of plasticizers as a factor in the studies. Admittedly, most vinyl plastic articles contain plasticizers, and these plasticizers have a profound effect on the stability characteristics of the compound as a whole. Plasticizers, however, are complicating factors that direct attention away from the basic problem, the degradation behavior of the resin itself. They also obscure by their own effects the absorption spectra of the degraded resin, and so make attempts to determine anything by this method hopelessly complicated. The individual effects of plasticizers and other compounding materials on vinyl degradation are better accounted for at the practical level for particular cases.

\section{Results and Discussion}

Figure 6.2 shows the results obtained by exposing a molded disk to air at $85^{\circ} \mathrm{C}$ for $10 \mathrm{hr}$. The ultraviolet absorption curve taken on the original disk ( $0 \mathrm{hr})$ shows no peak at all. This was found to be a reliable index of the quality of the original disk, and considerable effort was necessary to develop a molding technique that affords satisfactory disks in a reproducible fashion.

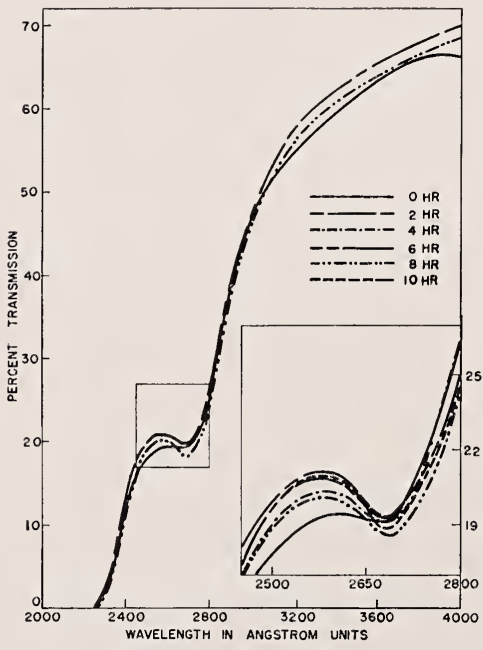

Figure 6.2 PVC degraded by heat under air.

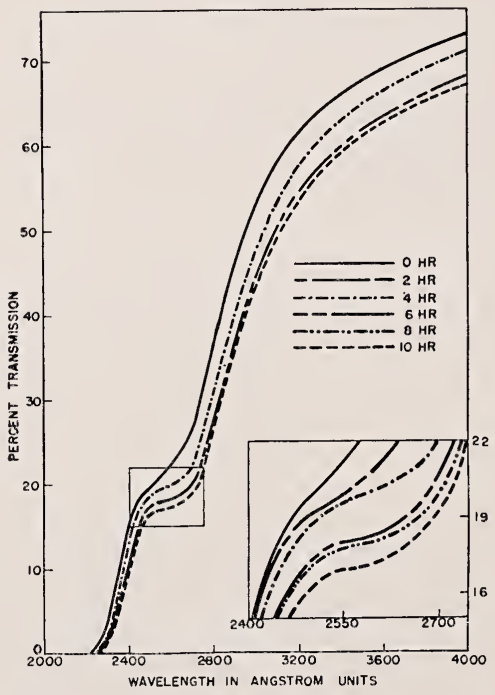

Figure 6.3 PVC degraded by heat under nitrogen. 
The successively greater breaks in the curves as the exposure to heat was continued are a definite measure of increased degradation. The inset showing the critical region on a larger scale shows a fairly close grouping of the curves, with small differences in the amount of break.

The extent of the break in each curve, obtained by subtracting the minimum values in the $2700-\mathrm{A}$ region from the maximum values in the 2550 - to 2600-A region, will be discussed later in comparing the effects of different atmospheres.

Figure 6.3 illustrates the effects of the same heat crcle in nitrogen. It is immediately apparent that there is no peak in the curves as was seen in figure $6 . \dot{2}$. The inert atmosphere prevents degradation to the extent that even after $10 \mathrm{hr}$ of heating, there is no appreciable break in the curve, though some displacement toward longer wavelengths is evident.

In figure 6.4, the addition of gaseous hydrogen chloride to the air system is seen to have caused a progressively greater break in the curves during heating. The curves are more clearly separated by a marked displacement toward longer wavelengths. Degradation is more severe than in air alone, as shown by the greater differences between maxima and minima.

An even more striking effect is obtained when hydrogen chloride is added to the nitrogen system, as shown in figure 6.5. Whereas there was some degradation in air which hydrogen chloride accelerated, the nitrogen system was changed from no apparent degradation to one in which the depth of the curve breaks and the displacement toward longer wavelengths are at least equal to those of the airhydrogen chloride system.

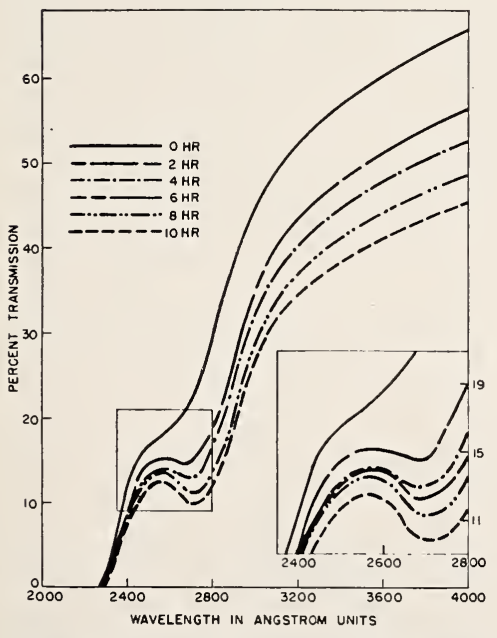

Figure 6.4 PVC degraded by heat under 95 percent of air and 5 percent $\mathrm{HCl}$.

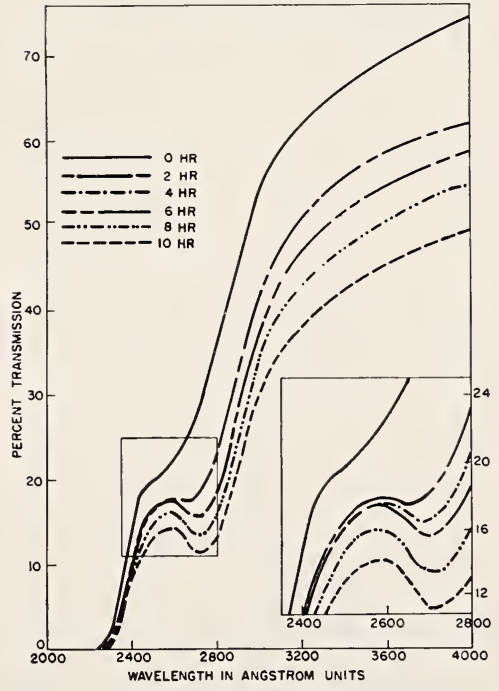

Figure 6.5 PIrC degraded by heat under 95 percent $\mathrm{N}_{2}$ and 5 percent $\mathrm{HCl}$. 
Figure 6.6 shows the result of light exposure in air. In the experimental conditions used, the degrading influence of light gave stronger breaks than those obtained in the heat tests. There is a fairly strong pattern of break-down in this system.

In figure 6.7 , the effect of light exposure in a nitrogen atmosphere is shown. The breaks in the curves are comparable to those obtained in air, though there is somewhat less displacement of the curves toward the right. The major point of interest is the change in behavior from the heat study, where there was no apparent degradation in nitrogen.

Addition of hydrogen chloride to the air system under light resulted in uniformly deeper breaks in the curves, as shown in figure 6.8. There is a smaller spread in the upper right-hand portions, and generally less shifting toward longer wavelengths.

Figure 6.9 shows the absorption curves after light exposure in a nitrogen-hydrogen chloride system. A pronounced increase in the amount of degradation is evident at each time interval. This parallels the results in heat studies, where hydrogen chloride promoted greater break-down in the inert atmosphere.

Figure 6.10 illustrates what happened when a disk was exposed to light in pure oxygen. The degradation pattern is generally similar to the one obtained in air, except that there is less displacement of the curves toward the right, and the breaks in the curves are considerably deeper. The differences appear to reflect a similar type of break-down with the higher proportion of oxygen being the only important variable.

Infrared and visible absorption spectra were made on the above disks and studied carefully. The absence of significant break differentials made it impossible to draw any conclusions whatsoever regarding the above experimental work. Either the extent of degradation was insufficient to produce significant changes or the thickness of the disks precluded adequately sensitive measurements.

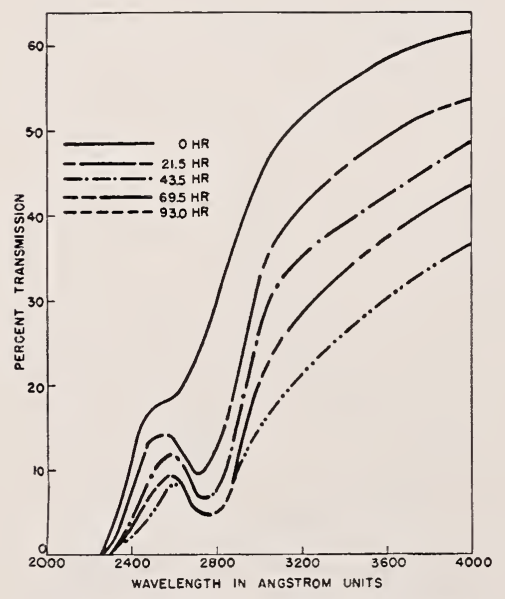

Figure 6.6. PIC degraded by light under air.

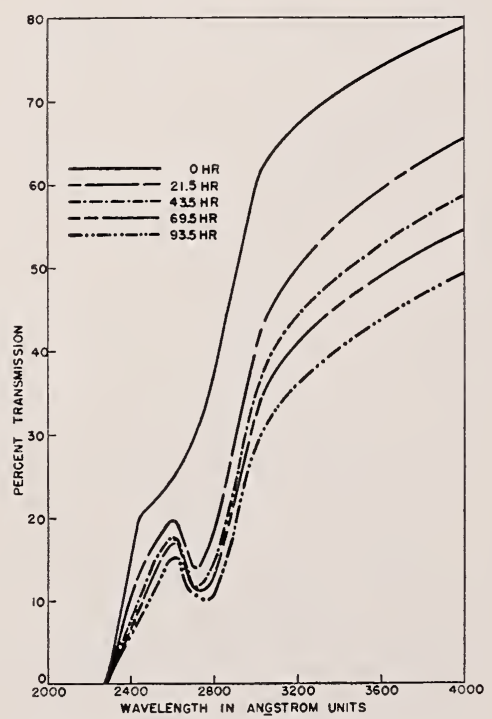

Figure 6.7. PVC degraded by light under nitrogen. 

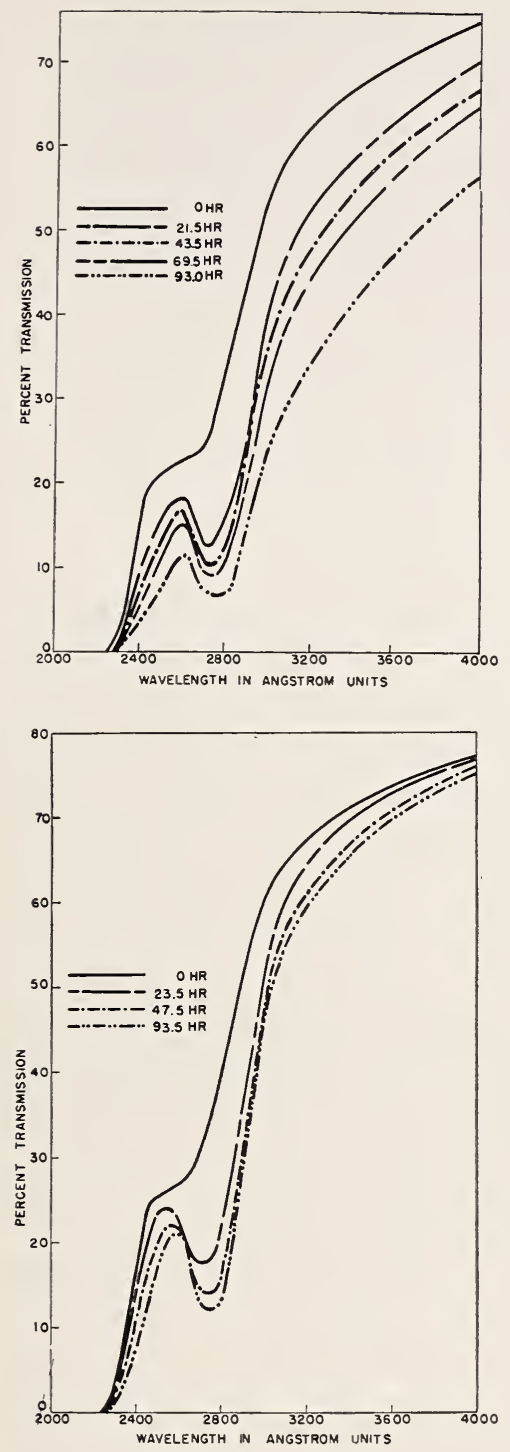

Figure 6.10. PVC degraded by light under oxygen.

\section{Interpretation of Data}

A substantial amount of data has been reported on ultraviolet light absorption of alkyl halides, ketones, and unsaturated compounds. We are not so fortunate when it comes to a study of poly vinyl chloride degradation, as very few data are available. We know that changes in the polymer molecules are taking place from the ultraviolet absorption curves reported above, and interpretation of these changes is best done in a rather general fashion. 
The characteristic absorption of light in the visible and ultraviolet portions of the spectrum is due to the displacement of electrons within the molecules. A substance that absorbs only in the ultraviolet is colorless, and the electrons exist at a relatively high degree of restraint. If the structure of such molecules is altered so as to decrease the electron restraint successively, the frequencies of light absorbed progressively decrease. Thus, ultimately the absorption bands enter the visible range of the spectrum. The first visible light to be absorbed will be in the violet, and therefore, to the ere, the substance in question will appear yellow.

The yellow to brown color dereloped by polyvinyl chloride upon exposure to light is due to this type of shift. Just as the characteristic absorption occurs at increasingly lower frequencies (longer wavelengths) as we progress from alkyl halides to unsaturated alkyl halides to conjugated olefinic structures, so does the absorption pattern of polyvinyl chloride correlate reasonably well with the accepted theory of degradation through the process of dehydrochlorination.

While the appearance of structures resulting from oxidative attack, such as the carbonyl group, plars some part in the absorption curves in the 2500- to 3000-A region, the amount of absorption these structures contribute is probably rather small. This was shown by the relatively small differences between nitrogen and air in the light exposures, and even oxygen. The situation is complicated by the fact that absorption due to a carbonyl group is probably obtained at the expense of absorption due to a double bond, so that the net change is one of difference in the two structures. Our interpretation, therefore, is not based on attaching significance to one structure or the other in these curves, but on the changes in this region as measures of total degradative change.

The loss of hydrogen chloride can be initiated by thermal energy and by energy resulting from the absorption of light. These two degradation influences operate along somewhat different paths, and a major purpose of the present work is to account for the role of hydrogen chloride in these two processes.

Figure 6.11 shows one means of analyzing the effect of hydrogen chloride on the degradation mechanism while operating under the influence of heat in two types of atmospheres. The data for the curves in this figure were obtained from the earlier figures showing the degradation effects in nitrogen, in air, and in mixtures of these gases with 5-percent hydrogen chloride. The maxima-minima differences (the height of the humps in the curves) were determined for each curve. Then, for each exposure interval, this value in the nonhydrogen chloride containing system was subtracted from the value in the same srstem containing hydrogen chloride. These values were plotted against time of exposure. It will be noted that these curves are formed by connecting the points directly without attempting to draw a smooth curve.

The similarity between the nitrogen systems and the air systems, as indicated by the two curves, is rather striking. Through the first $8 \mathrm{hr}$ of heating there is obviously a parallel course of reaction in the two systems. This represents only one basic degradation mechanism, since the effect of hydrogen chloride in both atmospheres as represented by these curves is parallel and almost linear. The almost identical courses of reaction shown br these curves leads us to the conclusion that molecular oxygen is taking no apparent part in the degradation 


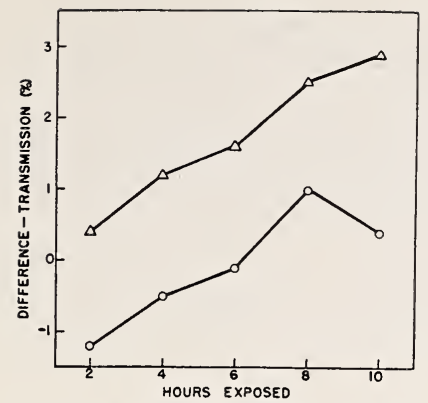

Figure 6.11. Effect of $\mathrm{HCl}$ in heat degradation of $P V C$.

$\triangle$, Nitrogen systems; $\bigcirc$, air systems.

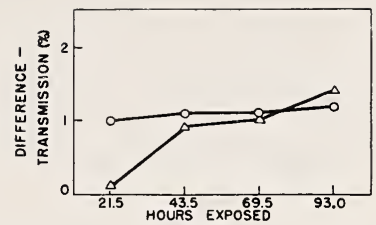

Figure 6.12. Effect of $\mathrm{HCl}$ in light degradation of $P Y^{Y} C$.

$\triangle$, Nitrogen systems; $\bigcirc$, air systems.

mechanism up to a point. The divergence of the air curve after $8 \mathrm{hr}$ of heating indicates that oxidation is beginning to participate in the break-down of the polvmer. Presumably, a certain amount of dehydrochlorination must occur before oxidative attack begins to take place. This practically amounts to an induction period under heat before oxidative degradation starts. The curves also afford an excellent indication that hydrogen chloride plays no important part in catalyzing oxidative attack in the absence of light. On the other hand, hydrogen chloride is a uniform catalyst for the dehydrochlorination reaction, at least up to the point where we lose track of it in the air system because of the advent of the oxidative process.

Figure 6.12 shows a similar plot of weighted curves illustrating the effect of hydrogen chloride in the two systems under light exposure. Here we have quite different results in the nitrogen and air systems. In nitrogen, hydrogen chloride has little effect on degradation characteristics during a short induction period. It then begins to act as a reasonably uniform catalyst. We conclude that under both ultraviolet light and heat, the presence of oxygen is not necessary to the dehydrochlorination process, and in fact has no effect until advanced stages are reached.

The air curve under ultraviolet light is significantly different from the one for the nitrogen systems. While there is a parallel behavior suggesting only dehydrochlorination as the major factor in the nitrogen systems under heat, there is clearly an entirely different type of degradation mechanism at work in air under ultraviolet light.

The "induction period" of about $8 \mathrm{hr}$ observed in the heat treatment of the air system is lacking under ultraviolet light. The latter curve shows the immediate onset of the oxidative mechanism through catalysis by the ultraviolet light. There is ample support for the catalytic effect of ultraviolet light in the oxidation of alkyl halides [11] and polyene systems [3, 12].

The oxidative attack on the vinyl polymer under light is not catalyzed by hydrogen chloride, as is shown by the level curve with no increase in the rate of reaction after it has started. The initial oxidative at tack does depend on preliminary dehydrochlorination to provide points on the chain that are particularly susceptible to oxidation. Thus, we may conclude that hydrogen chloride, which is a catalyst 
for the dehydrochlorination step, speeds up the initial attack of oxygen in the presence of ultraviolet light, even though hydrogen chloride is not an oxidation catalyst in this reaction. At the same time, the dehydrochlorination reaction does not appear to continue at the rates observed in an inert atmosphere. This is due to the effective blocking of conjugation in a chain through disruption of double bonds by formation of carbonyl groups or by actual scission.

A more detailed consideration of this action of oxygen in decreasing the rate of dehydrochlorination will also help to explain certain other experimental effects that were noted. The initial random split-out of hydrogen chloride from the polymer chain leaves a structure of the allyl type in the molecule.

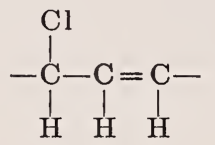

The chlorine atom remaining on the chain is exceptionally labile to further split-out as hydrogen chloride because of its activation by the adjacent double bond. The high reactivity of the "allyl" structure is well known. As pointed out by previous investigators [1, 2], progressive occurrence of this type of reaction accounts in large part for the color-bearing conjugated polyene systems present in degraded vinyl resin.

Oxygen counteracts operation of this mechanism in three ways: (1) Oxidation catalyzed by ultraviolet light reduces the number of labile chlorine atoms in the polymer due to reaction with the double bonds which create these "allylic excitation" centers. (2) Oxygen decreases the tendency of the polymer to absorb incident ultraviolet light energy. The points of greatest conjugated unsaturation are the most effective ultraviolet light absorbers, and oxygen, by reacting preferentially at these points, will break up conjugated unsaturation. (3) Oxygen under the action of ultraviolet light exerts a bleaching action on the degraded polymer. This is accomplished by reaction with the carotene-like structures consisting of many conjugated double bonds, known to be color-bearing structures. Operation of these three factors was indicated experimentally by the fact that the disks exposed in nitrogen atmospheres showed spot degradation and greater visible color formation.

It has been observed that polyvinyl chloride that has been previously exposed to light is far less stable to heat than the unexposed material [5]. This can be accounted for by the much shorter induction period for oxidative attack observed under ultraviolet light than is noted under heat treatment. The absorption of ultraviolet light catalyzes the initial take-up of oxygen. The induction period is thus disposed of rapidly, and the oxidative process proceeds readily under heat. A similar observation on the increased absorption of oxygen under ultraviolet light by polyvinyl chloride that has been previously exposed to heat has been made. This fits the above explanation on the basis that prior heat treatment has resulted in an increased number of double bonds, which will absorb more light energy and serve as points of oxidative attack. 
An effective antioxidant might be expected to prolong the induction period of the oxidative process because it does not permit the oxygen to obtain a foothold in the polymer chain. It must, however, also be an acid acceptor, or be used in conjunction with one, otherwise the degradation will proceed rapidly through the catalyzed dehydrochlorination mechanism. The transfer of the degradation mechanism from one process to the other can be visualized by consulting the curves in figure 6.12 .

\section{Conclusions}

(1) Under heat exposure, oxidation is not a factor until a certain minimum level of dehydrochlorination has been reached.

(2) Hydrogen chloride does not catalyze oxidation in the absence of light.

(3) Hydrogen chloride is a uniform catalyst for the dehydrochlorination process.

(4) Ultraviolet light catalyzes oxidation after a very short induction period.

(5) There are three distinct effects of oxidation. It disrupts dehydrochlorination, ultraviolet light absorption, and color formation.

(6) An antioxidant, to be a good light stabilizer for polyvinyl chloride, must be to some extent an acid acceptor, or be used with an acid acceptor.

(7) The thermal instability of irradiated polyvinyl chloride is attributed to the premature completion of the induction period preceding oxidative attack.

The authors express their appreciation to Walter C. Weithas and other members of the National Lead Company Research Laboratories' staff for their assistance in experimental work and the preparation of the illustrations.

\section{References}

[1] R. F. Boyer, J. Phys. \& Colloid Chem. 51, 80 (1947).

[2] V. W. Fox, J. G. Hendricks, and H. J. Ratti, Ind. Eng. Chem., 41, 1774 (1949).

[3] R. T. Holman, W. O. Lundberg, and G. O. Burr, J. Am. Chem. Soc. 67, 1386 (1945).

[4] R. T. Holman, W. O. Lundberg, and G. O. Burr, J. Am. Chem. Soc. 67, 1390 (1945).

[5] A. S. Kenyon, The photodegration of sec-butyl chloride and polyvinyl chloride, presented at 119th Meeting of American Chemical Society at Boston, Mass., April 3, 1951.

[6] L. A. Matheson and R. F. Boyer, Light stability of polystyrene and polyvinylidene chloride, presented at 118th Meeting of American Chemical Society at Chicago, Ill., September 1950.

[7] W. W. Coblentz and H. Kohler, BS Sci. Pap. 16, 233 (1920) S378.

[8] W. E. Forsythe and F. Christison, Gen. Elec. Rev. 32, 664 (1929).

[9] II. E. Forsythe and F. Christison, Gen. Elec. Rev. 32, 667 (1929).

[10] M. E. Nordberg, J. Am. Ceramic Soc. 27, 299 (1944).

[11] H. Stobbe and P. Schmitt, Z. wiss. Phot. 20, 51 (1920).

[12] E. H. Farmer, G. F. Bloomfield, A. Sundralingham, and D. A. Sutton, Rubber Chem. Tech. 15, 756 (1942). 


\section{Discussion}

Dr. A. R. Burgess, Imperial Chemical Industries Ltd., Welwyn Garden City, England: I should like to draw the attention of the meeting to a complicating effect we have noticed in the estimation of color in plasticized polyrinyl chloride. Such a composition goes brown in sunlight. If it is then put in the dark, the brown color develops still further, but this can be reversed to some extent by putting it into the sunlight again. A possible explanation for this is that the polyenes formed are isomerized to their predominantly cis forms in sunlight and revert to the more deeply colored trans forms in the dark.

Another point is that the colors generated in the presence of oxygen are much deeper and less like those of polyenes than the colors formed by irradiation in the absence of oxygen. If the oxygen were incorporated in the form of carbonyl groups and these became conjugated with the polyene chains, then one would not expect polyene-like light absorption, since the carbonyl gives rise to an extra long wave band. However, despite much infrared absorption work, we have not yet succeeded in proving the presence of conjugated carbonyl groups in the degraded polymer. Fox, Hendricks, and Ratti claimed to have done so, but we are of the opinion that the band they attributed to conjugated carbonyl is due to some other constituent like a catalyst or granulating agent. We do not find this band in all makes of polyvinyl chloride, and when it is present it is there in the undegraded material and does not increase in intensity on degradation.

Dr. R. E. Burк, E. I. du Pont de Nemour's \& Co., Inc., Wilmington, Del.: I have not heard any mention of the possible part played by metals in the stability of polyringl chloride. Could it not be that other things could be playing a part in these polymers?

Mr. Scarbrocgh: I was taking that influence for granted, and I did not go into it in the paper. It is a pretty well established fact that the metal chlorides lead to the break-down of the polymer, just as you would expect from the way ther behare as catalysts, and, as a matter of fact, the comparison is quite interesting. The dehydrochlorination step, which can be pictured largely as an ionic process, seems to be tied up rery definitely with the catalytic effect of the chlorides of a number of common metals. If we compare the activity of a number of common catalysts in the degradation of pure polyvinyl chloride with their activity in Friedel-Crafts alkylations, we find that there is almost an exact order of similarity. The general order is aluminum chloride, ferric chloride, zinc chloride, stannic chloride, and cupric chloride. The order of break-down with these salts in polyvinyl chloride has not been completely established, but is certainly very close.

The interesting point is that this order appears to be tied up with the ability of these salts to form $\mathrm{HCl}$ complexes, which in itself is a point that has always led me to think that hydrogen chloride is a catalyst. There is an important difference between putting the chlorides of these different metals into a vinyl polymer to determine how effective they are in degradation and putting in the metal itself because there is a rate control factor in the ability to form chlorides. If you put aluminum, zinc, copper, or iron powder in, you would not find the same order of reaction at all. That seems to be dependent on the ability of a giren metal to go from the metallic state to the metallic chloride. 


\title{
7. Aging of Vinyl Chloride and Vinylidene Chloride Polymers
}

\author{
By C. B. Havens ${ }^{1}$
}

\section{Introduction}

Polymers and copolymers of vinyl chloride and vinylidene chloride have shown a rapid industrial growth. The behavior of these polymers under the influence of heat and light is of interest to the manufacturer, the fabricator, and the consumer. Increased diversity of applications and higher standards of performance demand more and more stable polymers. Hence there is a need for a better understanding of polymer degradation.

\section{Materials}

The polyvinyl chloride used in these studies was prepared by emulsion polymerization. The polyvinylidene chloride and the vinylidene chloride-vinyl chloride copolymers were prepared by suspension polymerization. They were prepared from commercial monomers and hence presumably contain any trace impurities normally found present. For the sake of brevity, the term polyvinylidene chloride has been used for copolymers containing less than 10 percent of vinyl chloride, as well as for polyvinylidene chloride. All tests were run on the unstabilized resin, and in most cases no plasticizer was present.

\section{Apparatus and Procedure}

A sketch of the apparatus used in measuring hydrogen chloride evolution is shown in figure 7.1 . The bath was maintained at the desired temperature $\pm 0.2 \mathrm{deg} \mathrm{C}$. The sample, usually $5 \mathrm{~g}$ in powder form, was placed in tube A, and distilled water was placed in tube B. After any selected time interval, the pair of tubes were removed from the bath and allowed to cool. As the tube A cooled the resulting vacuum caused water from tube $B$ to be drawn in upon the polymer. The slurry of polymer and water was filtered and the polymer washed and dried.

The filtrate was titrated with a standard sodium hydroxide solution and the percent decomposition, based upon the hydrogen chloride evolved, was calculated. For polyvinylidene chloride, the percentage of decomposition was calculated according to the reaction

$$
-\mathrm{CH}_{2} \mathrm{C} \mathrm{Cl}_{2}-\longrightarrow-\mathrm{CH}=\mathrm{C} \mathrm{Cl}-+\mathrm{HCl} \text {. }
$$

As a measure of discoloration, a molding was prepared from the degraded polymer and its light transmission measured. In most cases, moldings 0.015-in. thick were used for polyvinyl chloride, whereas moldings 0.010 -in. thick were used for polyvinylidene chloride.

Light exposure tests were run in a Fade-Ometer, and the light transmission was measured with an Aminco photometer.

\footnotetext{
${ }^{1}$ Saran Development Laboratory, The Dow Chemical Co., Midland, Mich.
} 


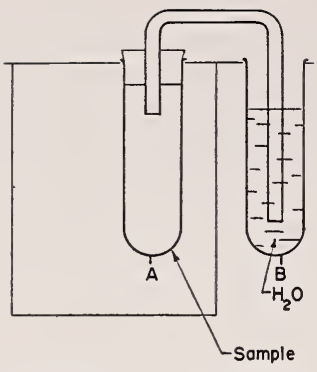

Figure 7.1. Decomposition test apparatus.

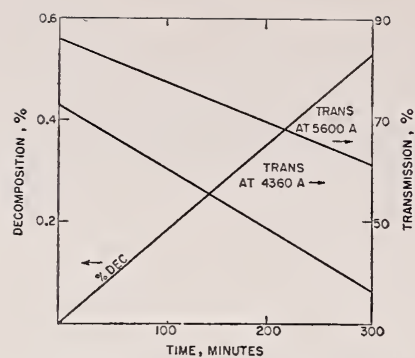

FIGURE 7.2. Aging of polyvinyl chloride at $130^{\circ} \mathrm{C}$.

\section{Pyrolysis}

\section{Aging Polyvinyl Chloride at $130^{\circ} \mathrm{C}$}

The results of aging an emulsion polymerized polyvinyl chloride at $130^{\circ} \mathrm{C}$ are shown in figure 7.2. It is seen that the percentage of decomposition is proportional to the exposure time at least during the early stages. At higher temperatures the decomposition rate is known to increase as decomposition proceeds. The light transmission is inversely proportional to the time of exposure. The light transmission then is inversely proportional to the percent decomposition and we may write

$$
D=K \frac{I_{o}-I_{f}}{I_{o}}
$$

where $D$ is percentage of decomposition, $K$ is a constant, $I_{o}$ is original transmission, and $I_{f}$ is final transmission. Using a 0.015 -in.-thick molding, $K=1.45$ for light transmissions at $5600 \mathrm{~A}$ and $K=1.04$ for light transmissions at $4360 \mathrm{~A}$. This technique enables one to get a fair quantitative measure of degradation even when the $\mathrm{HCl}$ evolved cannot be accurately determined.

It is interesting to note that only a trace of polymer insoluble in tetrahydrofuran was formed during 5-hr exposure at $130^{\circ} \mathrm{C}$. Furthermore, there was only a very slight change in the solution viscosity of the polymer (see fig. 7.2).

\section{Aging Polyvinylidene Chloride at $176.4^{\circ} \mathrm{C}$}

These studies were run above the fusion point of the polymer. The hydrogen chloride evolved was swept off by a slow stream of nitrogen. The results are shown in table 7.1, and in figure 7.3. It is seen that there was a period of slow decomposition followed by a period of rapid decomposition, the maximum rate being about 5.6 percent per $\mathrm{hr}$. It is notable that the rate of decomposition decreased markedly as the amount of hydrogen chloride evolved approached 50 percent of the theoretical. 
Figure 7.3. Decomposition of polyvinylidene chloride at $176.4^{\circ} \mathrm{C}$.

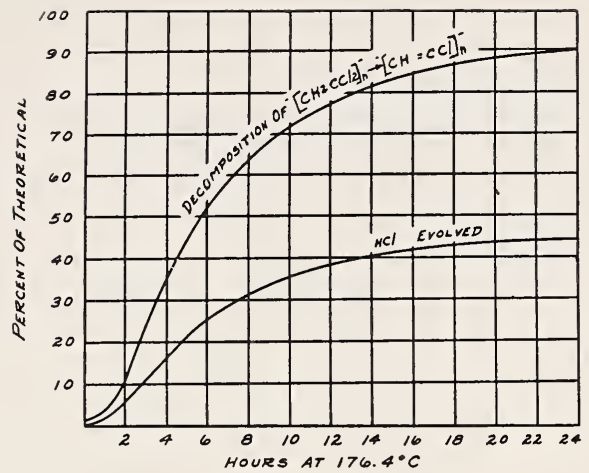

TABLE 7.1. Aging of polyvinylidene chloride at $176.4^{\circ} \mathrm{C}$

\begin{tabular}{|c|c|c|}
\hline $\begin{array}{c}\text { Time at } \\
176.4^{\circ} \mathrm{C}\end{array}$ & HCl evolved & $\begin{array}{c}\text { Calculated } \\
\text { decomposition } \\
\text { to }(\mathrm{CH}=\mathrm{CCl})_{n}\end{array}$ \\
\hline$h r$ & \% of total & $\%$ \\
0 & 0 & 0 \\
1 & 1.8 & 3.6 \\
2 & 5.8 & 11.6 \\
4 & 17.2 & 34.4 \\
6 & 27.2 & 54.4 \\
24 & 45.0 & 90.0 \\
\hline
\end{tabular}

\section{Comparison of Polyvinyl Chloride and Polyvinylidene Chloride}

A comparison of the decomposition of polyvinyl chloride and of polyvinylidene chloride is shown in table 7.2 . It is seen that, although the polyvinylidene chloride evolved hydrogen chloride four times as rapidly as did the polyvinyl chloride, it discolored less than one-half as rapidly.

The effect of partial degradation upon polymer stability is shown in table 7.3. The previously degraded polymers were washed free of hydrogen chloride and dried prior to the aging test. It is seen that for both polymers previous degradation of about 0.5 percent roughly doubled the rate of decomposition.

TABLE 7.2. Degradation at $130^{\circ} \mathrm{C}$

\begin{tabular}{|c|c|c|}
\hline Polymer & $\begin{array}{c}\text { Rate of } \\
\text { decomposition }\end{array}$ & $\begin{array}{c}\text { Loss in light } \\
\text { transmission }\end{array}$ \\
\hline & $\% / h r$ & $\% / h r$ \\
Polyvinyl chloride & 0.01 \\
Polyvinylidene chloride & 8.5 \\
& 3.5 \\
\hline
\end{tabular}

TABLE 7.3. Stability of virgin polymer versus degraded polymer

\begin{tabular}{|c|c|c|c|}
\hline Polymer & $\begin{array}{c}\text { Initial } \\
\text { decomposition }\end{array}$ & $\begin{array}{l}\text { Decomposition } \\
\text { during further } \\
\text { aging }\end{array}$ & $\begin{array}{l}\text { Increase in } \\
\text { decomposition } \\
\text { rate }\end{array}$ \\
\hline $\begin{array}{l}\text { Polyvinyl chloride } \\
\text { Do } \\
\text { Polyvinylidene chloride } \\
\text { Do }\end{array}$ & $\begin{array}{l}\% \\
0.00 \\
.55 \\
.00 \\
.45\end{array}$ & $\begin{array}{l}\% \\
\text { b } 0.53 \\
\text { b } 1.03 \\
\text { ० } 0.45 \\
\cdot 0.95\end{array}$ & 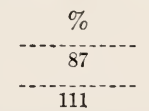 \\
\hline
\end{tabular}

a The polymer was degraded, washed free of $\mathrm{HCl}$, and dried. b Aged $6 \mathrm{hr}$ at $130^{\circ} \mathrm{C}$. ${ }^{\circ}$ Aged $1 \mathrm{hr}$ at $154^{\circ} \mathrm{C}$. 
The results of aging polyvinyl chloride in the presence of gases at atmospheric pressure and at $130^{\circ} \mathrm{C}$, are shown in table 7.4 . It is seen that for air, nitrogen, and oxygen decomposition was in the order of 0.04 percent, and the loss in light transmission was 32 percent of the original. Hydrogen chloride caused a 59-percent decrease in light transmission.

In table 7.5 the results of studies at $17 \mathrm{lb} / \mathrm{in} .^{2}$ and at $130^{\circ} \mathrm{C}$ are shown. About 0.04 percent of decomposition occurred in a nitrogen atmosphere as compared with about 0.07 percent of decomposition in an oxygen atmosphere. Oxygen also caused somewhat more discoloration. Fox, Hendricks, and Ratti have shown that polyvinyl chloride reacts with oxygen to form carbonyl groups, which can act as chromophores. ${ }^{2}$ Again, hydrogen chloride had the most marked effect, resulting in a light transmission of 28 percent as compared with 56 percent for oxygen and 60 percent for nitrogen.

The effects of gases at atmospheric pressure upon polyvinylidene chloride are shown in table 7.6. In the first column it is seen that after $1 \mathrm{hr}$ at $154^{\circ} \mathrm{C}$ there was no marked difference in the amount of darkening, regardless of whether air, nitrogen, or carbon dioxide were present. Hydrogen chloride caused slightly more darkening than was obtained in the presence of nitrogen or air. From the second and third columns of table 7.6 it is seen that oxygen had little effect upon $\mathrm{HCl}$ erolution, but it did result in slightly greater darkening. Hydrogen chloride caused the greatest darkening.

The effects of gases at high pressure are shown in table 7.7 . It is seen that at $10 \mathrm{~atm}$ under nitrogen more discoloration occurred than at $1 \mathrm{~atm}$ in air. Oxgyen caused slightly more darkening than occurred

TABLE 7.4. Effect of gases upon the thermal degradation of polyvinyl chloride a

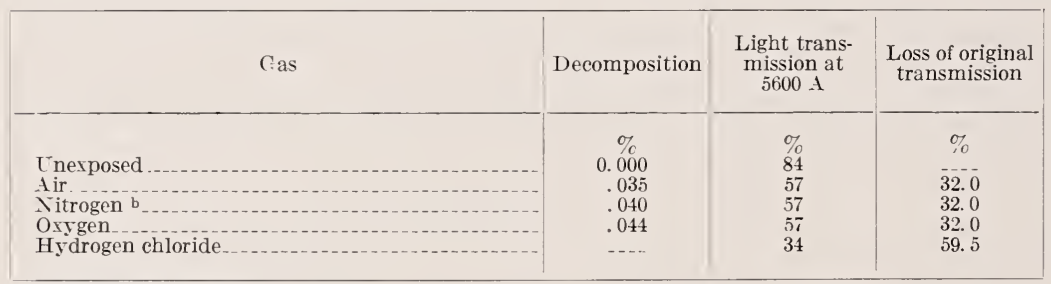

a Exposed $31 / 4 \mathrm{hr}$ at $130^{\circ} \mathrm{C}$.

b 99.5 percent pure.

TABLE 7.5. Effects of gases under pressure a

\begin{tabular}{|c|c|c|}
\hline Gas & Decomposition & ¿Transmission \\
\hline $\begin{array}{l}\text { Nitrogen } \\
\text { Do } \\
\text { Oxygen } \\
\text { Hydrogen chloride }\end{array}$ & $\begin{array}{r}07 \\
0.037 \\
.039 \\
.068 \\
-\end{array}$ & $\begin{array}{l}\% \\
60 \\
59 \\
56 \\
28\end{array}$ \\
\hline
\end{tabular}

a Polyvinyl chloride aged $31 / 4 \mathrm{hr}$ at $130^{\circ} \mathrm{C}$ at a pressure of $17 \mathrm{lb} / \mathrm{in} .{ }^{2}$.

${ }^{2}$ V. W. Fox, J. G. Hendricks, and H. J. Ratti, Ind. Eng. Chem. 41, 1774 (1949). 
TABLE 7.6. Effect of gases upon polyvinylidene chloride at $154^{\circ} \mathrm{C}$

\begin{tabular}{|c|c|c|c|}
\hline \multirow{2}{*}{ Gas } & \multicolumn{2}{|c|}{ Light transmission- } & \multirow{2}{*}{$\begin{array}{c}\text { Decomposition } \\
\text { after } 2 \mathrm{hr} \text { at } \\
154^{\circ} \mathrm{C}\end{array}$} \\
\hline & $\begin{array}{l}4360 \mathrm{~A} \text { after } 1 \\
\text { hr at } 154^{\circ} \mathrm{C}\end{array}$ & $\begin{array}{l}5600 \AA \text { after } 2 \\
\text { hr at } 154^{\circ} \mathrm{C}\end{array}$ & \\
\hline $\begin{array}{l}\text { Air } \\
\text { Carbon dioxide } \\
\text { Nitrogen } \\
\text { Oxygen } \\
\text { Hydrogen chloride } \\
\text { Hydrogen chloride at } 17-1 \mathrm{~b} / \mathrm{in}^{2}{ }^{2} \text { pressure }\end{array}$ & $\begin{array}{c}\% \\
38 \text { to } \\
44 \\
44 \\
38 \\
\overline{34} \\
--\end{array}$ & $\begin{array}{l}\% \\
54 \\
69 \\
50 \\
45 \\
28\end{array}$ & $\begin{array}{l}\% \\
1.44 \\
1.39 \\
1.46 \\
-. .-\end{array}$ \\
\hline
\end{tabular}

TABLE 7.7. Effect of gases at high pressure upon the thermal degradation of a vinylidene chloride polymer a

\begin{tabular}{|c|c|c|c|}
\hline Gas & Pressure b & $\begin{array}{c}\text { Light trans- } \\
\text { mission at } \\
5600 \mathrm{~A}^{\mathrm{c}}\end{array}$ & Color \\
\hline & atm & $\%$ & \\
Air & 1 & 54 & Light tan. \\
Nitrogen & 10 & 30 & Brown. \\
Oxygen & 10 & 24 & Do. \\
Hydrogen chloride & 10 & 5 & Very dark. \\
\hline
\end{tabular}

a A copolymer containing 85 percent of vinylidene chloride and 15 percent of vinyl chloride.

b All reactions held at $1 \mathrm{~atm}$ for first $1 / 2 \mathrm{hr}$ to eliminate possible differences due to thermal conductivity.

c After $2 \mathrm{hr}$ at $154^{\circ} \mathrm{C}$.

under nitrogen. Hydrogen chloride caused marked darkening.

Both polyvinyl chloride and polyvinylidene chloride were only slightly affected by oxygen at the temperatures and pressures investigated, whereas hydrogen chloride markedly increased discoloration.

\section{Effects of Reagents}

Aging studies at $130^{\circ} \mathrm{C}$ were run on samples of polyvinyl chloride containing 1 percent of various reagents. From table 7.8 it is seen that water and 2-ethylhexanol had little effect. Phenol, $\mathrm{HCl}$, and especially $\mathrm{H}_{2} \mathrm{SO}_{4}$ resulted in increased decomposition.

A vinylidene chloride copolymer was studied in a similar manner at $154^{\circ} \mathrm{C}$. From table 7.9 it is seen that the decomposition of polyvinylidene chloride was not affected by water, but it was increased by 2-ethylhexanol and $\mathrm{HCl}$. Phenol and $\mathrm{H}_{2} \mathrm{SO}_{4}$ markedly increased decomposition.

TABLE 7.8. Effect of reagents upon the thermal degradation of polyvinyl chloride a

\begin{tabular}{|c|c|c|}
\hline Reagent & Decomposition & $\begin{array}{l}\text { Light trans- } \\
\text { mission at } \\
4360 \mathrm{~A}\end{array}$ \\
\hline $\begin{array}{l}\text { None } \\
\text { Water } \\
\text { 2-Ethylhexanol } \\
\text { Phenol } \\
\text { Concentrated } \mathrm{HCl}_{2} \\
\text { Concentrated } \mathrm{H}_{2} \mathrm{SO}\end{array}$ & $\begin{array}{c}\% \\
0.033 \\
.028 \\
.034 \\
.080\end{array}$ & $\begin{array}{l}07 \\
68 \\
65 \\
63 \\
35 \\
32 \\
11\end{array}$ \\
\hline
\end{tabular}

a Aged $3 \frac{1}{4} \mathrm{hr}$ at $130^{\circ} \mathrm{C}$ in the presence of 1 percent of the reagent. 
TABLE 7.9. Effect of reagents upon the thermal degradation of a vinylidene chloride polymer a

\begin{tabular}{|c|c|c|}
\hline \multicolumn{2}{|l|}{ Reagent } & \multirow{2}{*}{$\begin{array}{c}\text { Decomposi } \\
\text { tion of } \\
\text { polymer }\end{array}$} \\
\hline Type & Amount & \\
\hline None.. & $\%$ & \multirow{4}{*}{$\begin{array}{c}\% \\
0.53 \\
.55 \\
1.09 \\
1.97 \\
0.82 \\
1.72\end{array}$} \\
\hline $\begin{array}{l}\text { Water } \\
\text { 2-Ethylhexanol }\end{array}$ & $\begin{array}{l}2 \\
1\end{array}$ & \\
\hline $\begin{array}{l}\text { Phenol } \\
\text { Concentrated } \mathrm{HCi} 1\end{array}$ & 1 & \\
\hline Concentrated $\mathrm{H}_{2} \mathrm{SO}_{4}$ & 0.4 & \\
\hline
\end{tabular}

a A copolymer containing 85 percent of vinylidene chloride and 15 percent of vinyl choride; aged $1 \mathrm{hr}$ at $154^{\circ} \mathrm{C}$.

\section{Light Exposure}

\section{Behavior of Polyvinyl Chloride}

In running light aging tests on polyvinyl chloride, unusual effects with regard to fading or darkening have been reported from time to time. In the present study, molded specimens 0.015-in. thick were exposed in a Fade-Ometer. The results are tabulated in table 7.10 and are shown graphically in figure 7.4. It is seen that storage in the Fade-Ometer while masked has very little effect upon the specimen. Exposure to the light results in darkening. Very little darkening occurs during the first $40 \mathrm{hr}$. However, the rate of discoloration increases as the darkening proceeds. Masking the specimen after $40 \mathrm{hr}$ exposure does not stop the darkening process. In fact, the specimen continues to darken at least as rapidly as an exposed specimen. However, at the end of $320 \mathrm{hr}$, the specimen that was masked after $40 \mathrm{hr}$ is completely soluble in tetrahydrofuran, whereas the specimen exposed to light for $320 \mathrm{hr}$ contains much insoluble polymer.

The question arises as to whether exposure to light will result in darkening during subsequent storage under normal conditions. To study this effect, specimens were exposed in the Fade-Ometer for various lengths of time and then stored in the dark at $25^{c} \mathrm{C}$. The data obtained are shown in figure 7.5. It is seen that no darkening

TABLE 7.10. Fade-Ometer exposure tests on polyvinyl chloride

\begin{tabular}{|c|c|c|c|}
\hline \multicolumn{2}{|c|}{ Time } & \multirow{2}{*}{$\begin{array}{l}\text { Light } \\
\text { transmis- } \\
\text { sion at } \\
5600 \mathrm{~A}\end{array}$} & \multirow{2}{*}{$\begin{array}{l}\text { Solubility of exposed polymer in } \\
\text { tetrahydrofuran }\end{array}$} \\
\hline Uncovered & Covered & & \\
\hline $\begin{array}{r}h r \\
0 \\
40 \\
80 \\
160 \\
320\end{array}$ & $\begin{array}{r}h r \\
0 \\
0 \\
0 \\
0 \\
0\end{array}$ & $\begin{array}{r}\% \\
84 \\
79 \\
72 \\
48 \\
5\end{array}$ & $\begin{array}{l}\text { Soluble. } \\
\text { Do. } \\
\text { Trace of insoluble polymer. } \\
\text { Some insoluble polymer. } \\
\text { Much insoluble polymer. }\end{array}$ \\
\hline $\begin{array}{r}0 \\
40 \\
80 \\
160\end{array}$ & $\begin{array}{l}320 \\
280 \\
240 \\
160\end{array}$ & $\begin{array}{r}77 \\
9 \\
0 \\
0\end{array}$ & $\begin{array}{l}\text { Soluble. } \\
\text { Do. }\end{array}$ \\
\hline $\begin{array}{l}40 \\
40 \\
40 \\
40\end{array}$ & $\begin{array}{r}40 \\
80 \\
120 \\
160\end{array}$ & $\begin{array}{l}47 \\
54 \\
46 \\
50\end{array}$ & \\
\hline
\end{tabular}




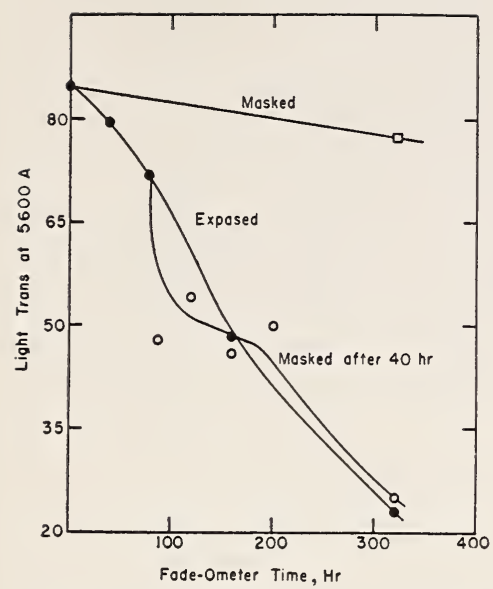

Figure 7.4. Fade-Ometer exposure test on polyvinyl chloride.

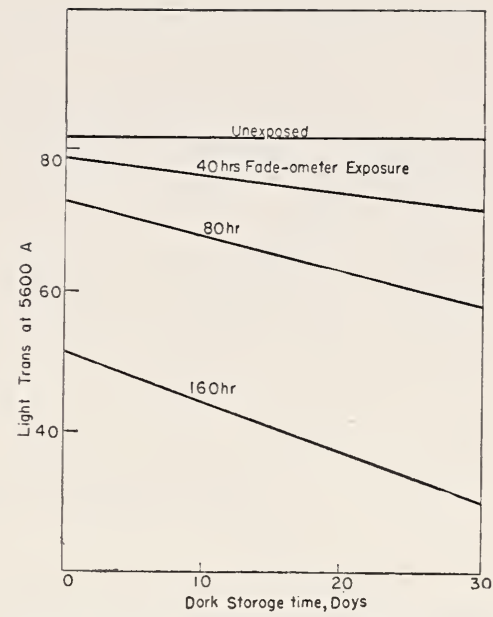

Figure 7.5. Effect of Fade-Ometer exposure upon the darkening of polyvinyl chloride during storage.

occurred for a specimen not exposed to light. The longer the exposure to light, the more rapid was the darkening during subsequent storage. Other data not presented here show that darkening still continues up to at least 2 months.

Exposure to light markedly activates degradation during subsequent exposure to heat. A Fade-Ometer exposure of $6 \frac{1}{2} \mathrm{hr}$ markedly increased the loss in light transmission during subsequent aging at $140^{\circ} \mathrm{C}$. The loss in light transmission was increased from 30 to 54 percent (note table 7.11).

Does previous exposure to heat result in decreased light stability? To answer this question, specimens which had been heat aged for various lengths of time were exposed in the Fade-Ometer. A remarkable thing occurred. Specimens which had lost as much as 50 percent of their original transmission due to heat exposure, rapidly lost their color upon exposure in the Fade-Ometer. After from 5 to $40 \mathrm{hr}$, all darkening had disappeared. After the disappearance of the original color, darkening proceeded in a more or less normal manner. The data are shown in figure 7.6.

Since heat aging does not affect light stability, what about $\mathrm{HCl}$ ? Is it a catalyst for photodecomposition? The answer is emphatically yes, as shown in figure 7.7 .

TABLE 7.11. Activation of heat degradation by light

\begin{tabular}{|c|c|c|c|}
\hline $\begin{array}{c}\text { Fade-Ometer } \\
\text { exposure } \\
\text { time }\end{array}$ & $\begin{array}{c}\text { Transmission } \\
\text { at 4360 A }\end{array}$ & $\begin{array}{c}\text { Transmission } \\
\text { after 1 hr at } \\
140^{\circ} \mathrm{C}\end{array}$ & $\begin{array}{c}\text { Loss of } \\
\text { original } \\
\text { transmission }\end{array}$ \\
\cline { 1 - 2 }$h r$ & $\%$ & $\%$ & $\%$ \\
0.0 & 46 & 32 & 30 \\
6.5 & 52 & 24 & 54 \\
\hline
\end{tabular}




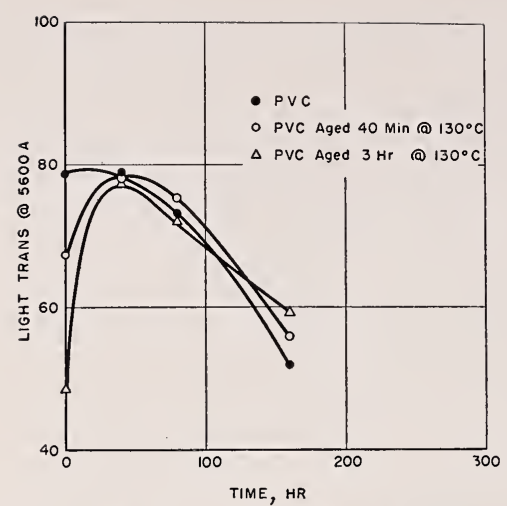

FiguRE 7.6. Fade-Ometer exposures on PVC.

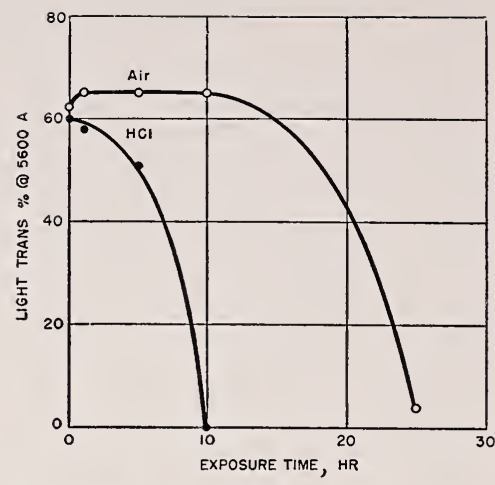

Figure 7.7. Effect of $\mathrm{HCl}$ upon light stability of PIC.

\section{Comparison of Vinyl Chloride and Vinylidene Chloride Polymers and Copolymers}

Exposures of 60-hr duration were made in the presence of various gases, as listed in table 7.12. Unfortunately, the polyvinyl chloride darkened so much that it could not be compared. For the 25:75 vinylidene chloride copolymer, it is seen that oxygen caused the least darkening, whereas $\mathrm{HCl}$ caused the most. With oxygen present the transmission was 43 percent as compared with 2 percent for a nitrogen atmosphere and 0 percent for $\mathrm{HCl}$. It is interesting to note that a 50:50 mixture of oxygen and $\mathrm{HCl}$ gave a transmission of 20 percent.

Polyvinylidene chloride appeared to be least affected by the type of gas present during exposure.

The activating effects of Fade-Ometer exposure are illustrated in table 7.13. A polyvinyl chloride specimen exposed for $40 \mathrm{hr}$ and then masked decreased in light transmission from 59 to 5 percent during $320 \mathrm{hr}$ of masked storage in the Fade-Ometer. Similarly, a specimen stored in the dark for 1 week decreased in light transmission from 59 to 50 percent.

Vinylidene chloride-vinyl chloride copolymers showed less change than polyvinyl chloride during storage, whereas polyvinylidene chloride showed the least change.

TABLE 7.12. Effects of gases during light exposure

\begin{tabular}{|c|c|c|c|c|c|c|}
\hline \multirow{2}{*}{ Polymer a } & \multicolumn{6}{|c|}{ Light transmission at $4360 \mathrm{~A}$ after Exposure for $60 \mathrm{hr}$} \\
\cline { 2 - 7 } & Original & In $\mathrm{N}_{2}$ & In air & In $\mathrm{O}_{2}$ & In $\mathrm{HCl}$ & In $\mathrm{HCl}+\mathrm{O}_{2}$ \\
\hline & $\%$ & $\%$ & $\%$ & $\%$ & $\%$ & $\%$ \\
Polyvinyl chloride & 65 & 0 & 0 & 0 & 0 & 0 \\
25:75 Vinylidene chloride-vinyl \\
chloride copolymer
\end{tabular}

a All polymers contained 5 percent of dioctyl phthalate. 
TABLE 7.13. Effect of light upon storage behavior of various polymers

\begin{tabular}{|c|c|c|c|}
\hline \multirow[b]{2}{*}{ Polymer a } & \multicolumn{3}{|c|}{ Light transmission after-b } \\
\hline & $\begin{array}{l}\text { Fade-Ometer } \\
\text { exposure } \\
40 \mathrm{hr}\end{array}$ & $\begin{array}{l}\text { Exposed } 40 \mathrm{hr} \\
\text { and masked } \\
320 \mathrm{hr}\end{array}$ & $\begin{array}{c}\text { Exposed } 40 \mathrm{hr} \\
\text { and stored } \\
168 \mathrm{hr}\end{array}$ \\
\hline $\begin{array}{l}\text { Polyvinyl chloride } \\
\text { 25:75 vinylidene chloride-vinyl chloride copolymer } \\
85: 15 \text { vinylidene chloride-vinyl chloride copolymer } \\
\text { Polyvinylidene chloride }\end{array}$ & $\begin{array}{l}\% \\
5 y \\
65 \\
4 \times \\
22\end{array}$ & $\begin{array}{r}\% \\
5 \\
56 \\
44 \\
20\end{array}$ & $\begin{array}{l}\% \\
50 \\
65 \\
48 \\
23\end{array}$ \\
\hline
\end{tabular}

a All specimens contained 5 percent of diostyl phthalate.

b Transmissions at $4360 \mathrm{~A}$ for moldings 0.015 -in. thick.

\section{Theoretical Considerations}

It is of interest to consider the behavior of these polymers in the light of classic organic chemistry. The relative stability of a series of alkyl halides are shown in table 7.14 . It is seen from this table that polyvinylidene chloride is analogous to No. 5 and would be expected to be thermally less stable than polyvinyl chloride, which is analogous to No. 4. Theory and fact are in agreement. It is further well known that strong acids catalyze the hydrolysis of alkyl halides. They also catalyze the degradation of the analogous polymers. Consider now the degraded polymer. Polyvinyl chloride evolves $\mathrm{HCl}$, leaving an unsaturated hydrocarbon residue, $(\mathrm{CH}=\mathrm{CH})_{n}$. From table 7.14, it is seen that a halogen on a double-bonded carbon atom is very stable. Hence from classic organic chemistry one would expect one-half the chlorine in polyvinylidene chloride to split off with the remaining half very tightly bound which indeed is the case as can be seen by referring to figure 7.6. The residue then has the structure $(\dot{\mathrm{CH}}=\mathrm{CCl})_{n}$. The presence of a nega tive group, such as chloride, retards the oxidation of a double bond, thus one would expect degraded polyvinylidene chloride to be less affected by oxygen than degraded polyvinyl chloride. Unsaturated polyene systems are capable of reacting by a Diels-Alder type of reaction, and this may be one of the ways in which cross-linking occurs. Behavior of the degraded polymer may be considered as analogous to the behavior of the drying oils and rubbers.

Due to the activating effect of the carbon to carbon double bond, it is reasonable to expect that the hydrogen chloride is split off zipperfashion along the chain. This would lead to color formation even at a low degree of decomposition (see No.6, table 7.14).

The bleaching in light and darkening in storage is reminiscent of the sun checking of rubber tires. ${ }^{3}$ This sun checking appears to be an oxidation process and actually proceeds more rapidly during the dark periods than during exposure to sunlight. This peculiarity may well explain some of the difficulty in attempting to correlate accelerated tests of continuous light exposure with the results obtained by outdoor exposure where there are alternating dark periods and light periods.

Studies regarding the effects of oxygen need to be extended to higher temperatures.

${ }^{3}$ ASTM Symposium on Aging of Rubbers, Special Tech. Bul. No. 89, American Society for Testing Materials, Philadelphia, $\mathrm{Pa}$. 
TABLE 7.14. Reactivity of alkyl chlorides

\begin{tabular}{|c|c|c|c|}
\hline Number & Type & Structure & Reactivity \\
\hline 1 & Primary (unsaturated) & $-\mathrm{CH}=\mathrm{CCl}$ & Inert except to alcoholic potash, \\
\hline 2 & Primary (saturated) .. & $-\mathrm{CH}_{2} \mathrm{Cl}$ & Decomposes only at high tempera- \\
\hline 3 & Secondary (unsaturated)... & $-\mathrm{CH}=\mathrm{CCl}-$ & $\begin{array}{l}\text { tures. } \\
\text { Inert except to alcoholic potash, }\end{array}$ \\
\hline 4 & Secondary (saturated) & $-\mathrm{CH}_{2} \mathrm{CHCl}-$ & Decomposes more readily than pri- \\
\hline 5 & $\begin{array}{l}\text { Secondary dichloride (sat- } \\
\text { urated). }\end{array}$ & $-\mathrm{CH}_{2} \mathrm{CCl}_{2}-$ & $\begin{array}{l}\text { mary alkyl chlorides. } \\
\text { Less stable than secondary alkyl } \\
\text { chlorides. }\end{array}$ \\
\hline 6 & $\begin{array}{l}\text { Primary (allylic arrange- } \\
\text { ment). }\end{array}$ & $-\mathrm{CH}=\mathrm{CH}-\mathrm{CH}_{2} \mathrm{Cl}$ & $\begin{array}{l}\text { In many reactions from four to sev- } \\
\text { eral hundred times as reactive } \\
\text { as a saturated primary alkyl } \\
\text { chloride. }\end{array}$ \\
\hline 7 & Tertiary.... & $\mathrm{C}-\stackrel{\stackrel{\mathrm{C}}{\mathrm{C}}-\mathrm{Cl}}{\mathrm{l}}$ & $\begin{array}{l}\text { Unstable. Tertiary halides con- } \\
\text { taining more than six carbon } \\
\text { atoms decompose upon distilla- } \\
\text { tion at atmoshperic pressure. }\end{array}$ \\
\hline
\end{tabular}

\section{Summary}

It has been shown that the general aspects of the degradation of both polyvinyl chloride and polyvinylidene chloride follow the concepts of organic chemistry. In common with simple alkyl halides, these halogenated polymers tend to be hydrolized by water, and their degradation is catalyzed by the presence of strong acids. The unsaturated residues appear to undergo the reactions normally expected of such unsaturated compounds.

\section{Discussion}

Mr. G. H. Taft, Deecy Products Co., Cambridge, Mass.: The most obvious characteristic of plasticized polyvinyl chloride is that it changes color on aging. The ICI has set up a system by which to describe a color by three functions-red, green, and blue colors-designated as $\mathrm{X}, \mathrm{Y}$, and $\mathrm{Z}$, respectively. We tried such measurements and found that the results did not always correlate with our own personal observation. Errors crept in, such as diffusion of light by the sample. So we said, "Let's take the red, or X, beam as a standard; then we can compensate for all errors except those inherent in the photo cell." When we did that, we immediately began to get results that checked amazingly well with what we see. By this method we obtain a value A, which equals green transmission divided by red transmission, and a value $B$, which equals blue transmission divided by red transmission. In 4 years we have found no case where this method has not correlated with the visual values. Inasmuch as one of the big difficulties in color work is recording what you observe, we think this method is more accurate in recording colors than our own visual observations.

Figure 1 is based on data by K. L. Kelly. ${ }^{4}$ Samples with $\log$ B near 1 and $\log A$ less than 1 are yellow. As you come down from the B axis, you find the sample getting darker and darker; in other words, there is more and more red in it. We find that our values check visually very well with this chart except that we are able now to read in the portion up towards the origin.

4 J. Opt. Soc. Am., 33, 627 to 632 (1943). 
In figure 2 we have plotted the data for a stabilized plasticized polvvinyl chloride compound milled in air and then heated for rarious times under various conditions. The data seem to fall in a line, starting at the origin, going out to about $\mathrm{B}=0.3$, and then falling off. When the eye looks at these samples, it rates them all as a yellow orange until about $\mathrm{B}=0.3$ and then a red color begins to appear. The lower points are definitely brown. You can interpret a chart like this visually and say that at the red point a break begins to appear. These samples were treated under rarious conditions, not all at the same time, spread over several months after the stock was milled. You will see that the data fit a straight line very well. If the transmission follows Beer's law, which is a controversial point, as we add color to a medium, these $A$ and $B$ values should go out on a straight line. The slope of the line is determined by the kind of color body. The distance from the origin is proportional to the amount of color body.

Now we are getting to a point where we can use this color as an indicator. In figure 3 we have data for a compound somewhat similar to the other one, treated in two different ways. Any point can be defined by the slope, which we will call $H$, and the distance from the origin, $K$. You will notice that the points generally fall very well on

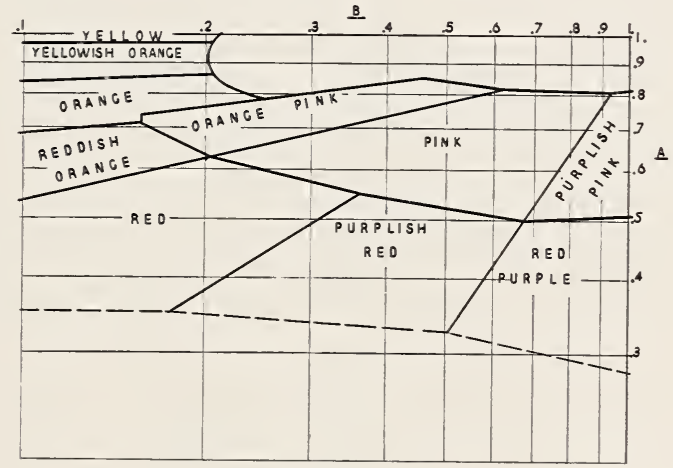

FIGURE 1.

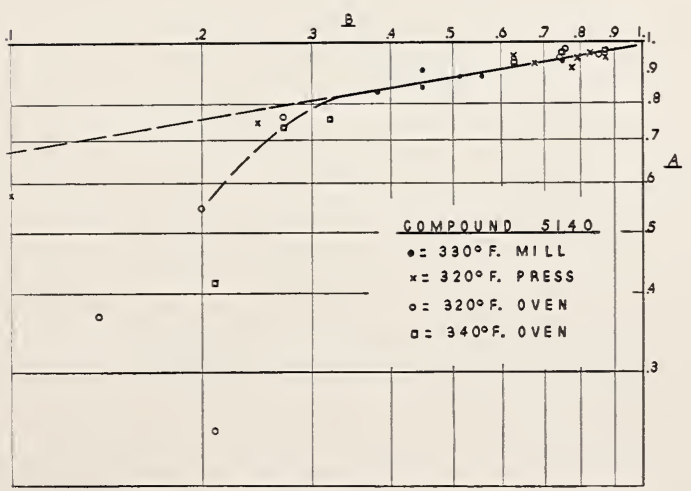

FIGUFE 2. 


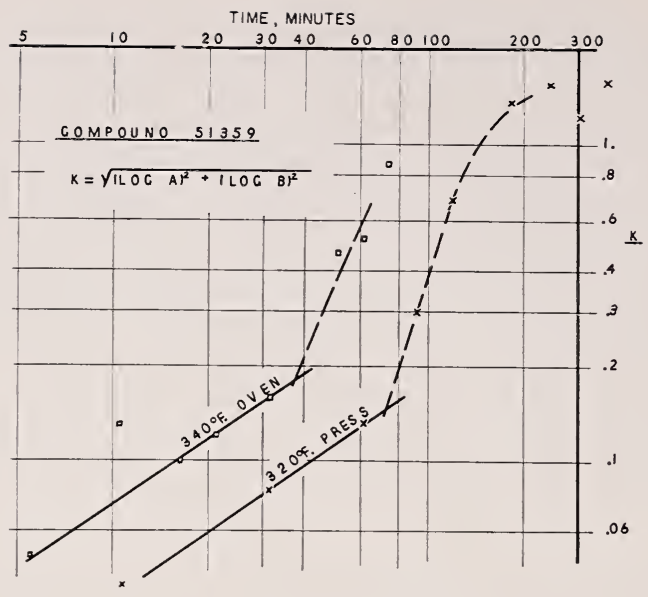

Figure 3.

a curve. The point where $K$ starts going up rapidly corresponds to the point in figure 2 where the sample fell off toward the red. We have other data showing that a sample below this break point shows no signs of what we call accelerated break-down. If we take such a sample and store it in a desk drawer, it bleaches. If we are beyond the break point, the sample in the desk drawer does not bleach. 'The farther out vou are, the more rou notice the difference. Break-down spots do not appear before the break point.

Apparently we have two cycles that have to be watched. Data obtained to the low side of that point mean something to the actual user of the product. 'The data to the right does not, except that it indicates you had better stay out of that region or you will be in trouble.

A good many of the data presented at this meeting, as well as others, fall on the right-hand or higher portion of that curve, which we have not studied. On the lower portions there is a very interesting situation. Br stabilizing we are able to change the slope, $H$; a good stabilizer can lower $H$ into the rellow region. We can vary it back and forth by changing the components, but we can not get very far off the average curve shown in figure 3 . We can change the break point and in some cases move it down appreciably.

You must be careful in using these data so that a side reaction does not cover up your color effects. A strong base added, in itself, produces a color.

We say that the resin can break down to give a colorless material plus HCl. This decomposition is subject to acid catalysis. This colorless material reacts with a developer to give a color body. We do not know if this reaction occurs, but by using this approach we have been able to explain most effectively variables in color. TTe can change the dereloper and change the value of $H$. We can change the break-down point by what we put in to absorb the HCl. Usually it has no effect on the color.

The metals like zinc, iron, and cadmium tend to lower $H$ values and give a low-colored stock. We say it is well stabilized. Such a stock has a lower color during its life, but a shorter life. 
Mr. B. G. Achhammer, National Bureau of Standards: This discussion concerns the gaseous products evolved when polyvinyl chloride powder is exposed to heat and to ultraviolet radiant energy. The procedure and equipment used to obtain these results are described in paper 14 of this Symposium.

The data in figure 1 were obtained by exposing polyvinyl chloride to fluorescent sunlamp radiant energy at $45^{\circ} \mathrm{C}$ in vacuum and detecting the gases evolved by mass spectrometry. Analyses of the gaseous components were made after $1,3,5,10,30,50$, and $100 \mathrm{hr}$ of exposure. The gases are plotted as progressive pressure, that is, the treatment was interrupted for mass spectrometric analysis after $1 \mathrm{hr}$, at which time the gases were removed from the tube; then the treatment was continued until the next analysis at $3 \mathrm{hr}$. In plotting, the pressure obtained after $3 \mathrm{hr}$ was added to that of the 1-hr analysis to give the point for $3 \mathrm{hr}$, and so forth.

Water is the major product detected, followed by carbon monoxide, carbon dioxide, and hydrogen. The dotted line represents the start of a 100-hr storage of the treated powder in darkness at room temperature. Water production continued during storage, and traces of carbon monoxide and carbon dioxide were detected. Hydrogen evolution stopped. No hydrogen chloride was detected either during the treatment or subsequent storage. This absence of hydrogen chloride is more interesting when it is noted that the originally, white polymer became pale brown, or perhaps sunburned is more apropos, as a result of the treatment.

The conditions of this treatment are perhaps less severe than those described in the papers of this symposium so far since the treatments here were designed primarily to study the initial step of the reaction. The initial step of the degradation mechanism in polyvinyl chloride is considered to be important because, theoretically, this polymer, if pure, should be reasonably stable to ultraviolet radiation and for that matter to reasonable degrees of heating.

Figure 2 shows that exposing the polymer to the same conditions for a longer time will result in the loss of hydrogen chloride. The sample used here was slightly larger than in the previous test. The dotted lines are extrapolated on the basis of previous work, that is, the mass spectrometric analysis made at $40 \mathrm{hr}$ showed no hydrogen chloride, whereas the 200-hr analysis did, but as no hydrogen chloride was found in the first $100 \mathrm{hr}$ in the previous test, it seemed logical to assume that the hydrogen chloride was evolved some time during the second $100 \mathrm{hr}$ of treatment. Actually, this merely serves to give a line rather than a point and has no particular significance. The hydrogen chloride might be produced as a secondary reaction. The powder in this case is dark brown after the 200-hr exposure. There was no observed change in color of the treated powder after 500 hr of postradiation storage, and no hydrogen chloride was detected during the storage period. Undoubtedly you are concerned with the presence or oxygenated components, such as carbon monoxide and dioxide in the gaseous products evolved, because the work was done in vacuum. Although some air leakage might be suspected, the oxygen content of the powder of 0.17 to 0.18 percent, as determined by the method of Walton, McCulloch, and Smith, ${ }^{5}$ provides

\footnotetext{
5 J. Resıarch NBS 40, 443 (1948) R P1889
} 
more than enough oxygen for the gases produced here.

Figure 3 shows that when the polymer is heated at $100^{\circ} \mathrm{C}$ in vacuum, water is the major product evolved and carbon dioxide, hydrogen, and carbon monoxide follow in that order. After $200 \mathrm{hr}$ the pressure of hydrogen chloride is well off the graph. Hydrogen chloride appears to come off at a rapid rate once it has started. The yield of $\mathrm{HCl}$ with heat is approximately 13 times that observed by exposure to ultraviolet radiant energy.

The white polymer powder discolors to a pale purple after the first hour of this heat treatment. No $\mathrm{HCl}$ was detected at this point. In fact, no $\mathrm{HCl}$ was found after $100 \mathrm{hr}$ of heat treatment, at which time the powder was a weak red. After $200 \mathrm{hr}$ of heating, $\mathrm{HCl}$ was detected in quantity and the polymer was a dusky red and showed no subsequent color change during at least 1,000 hr of storage at laboratory conditions in the dark.

In another experiment involving heat, the polymer was accidentally treated in oxygen due to a leak which, of course, is readily detectable by mass spectrometry. Although carbon monoxide assumed the role of major component involved, no $\mathrm{HCl}$ was detected up to $100 \mathrm{hr}$ of treatment.

Some preliminary work with the infrared spectrum of polyvinyl chloride has been done; however, there are some severe limitations. Film work was discarded because the solvent used must not be subject to oxidation or hydroperoxidation. Mulling of the powder for solid phase spectroscopy showed that the mulling produced oxidation of the polymer giving infrared absorption. However, a technique now being used experimentally gives an infrared spectrum for untreated polyvinyl chloride powder, showing no carbonyl or hydroxyl absorption, but there is absorption at approximately 12 microns. O. D. Shreve, et al. ${ }^{6}$ have shown this to be the region where hydroperoxide might be expected to show absorption.

The results to date seem to indicate that the initial step of the degradation of polyvinyl chloride is not associated with loss of hydrogen chloride, but can possibly be correlated with the break-down of residual thermolabile oxvgenated structures in the polymer in conjunction with a loss of thermolabile hydrogen atoms. The discolora-

${ }^{6}$ Anal. Chem. 23, 282 (1951).

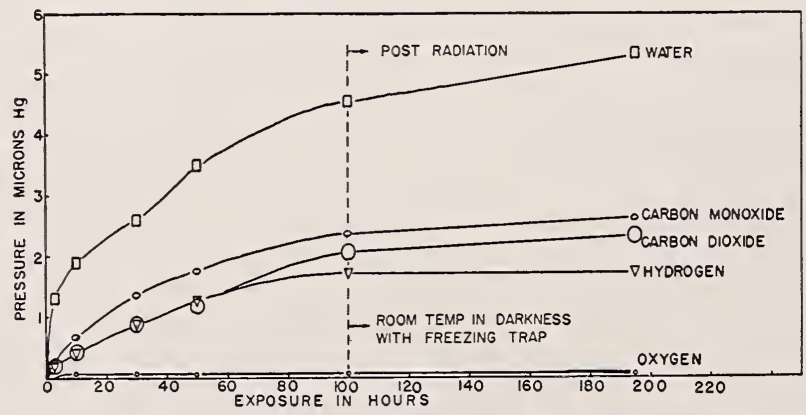

FiguRE 1. Gaseous degradation products from 0.1218 gram of polyvinyl chloride exposed under fluorescent ultraviolet lamp at $45^{\circ} \mathrm{C}$ in vacuum. 


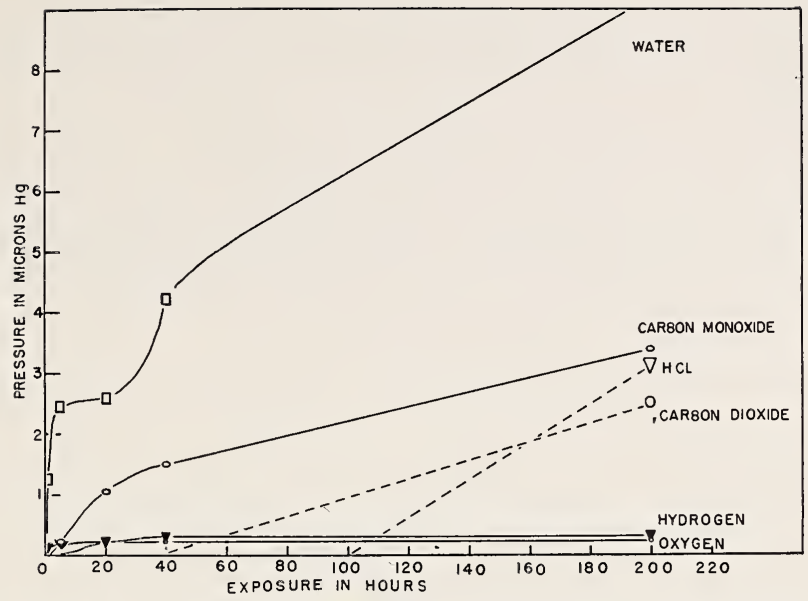

FigURE 2. Gaseous degradation products from 0.1474 gram of polyvinyl chloride exposed under fluorescent ultraviolet lamp at $45^{\circ} \mathrm{C}$ in vacuum.

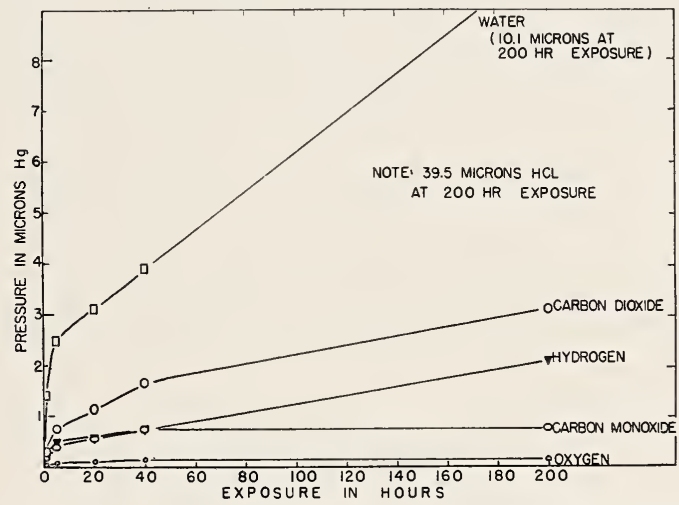

Figure 3. Gaseous degradation products from 0.1744 gram of polyvinyl chloride exposed at $100^{\circ} \mathrm{C}$ in vacuum.

tion of the polymer is apparently not connected with the loss of hydrogen chloride from the polymer. This is most marked on heat treatment, which causes a very early discoloration of the material. These conclusions, which are drawn from preliminary and incomplete work, may need modification as more data are obtained, and the statements made should be considered as essentially a hypothesis.

Dr. Havens: Some results that we have obtained witb polyvinyl chloride substantiate what Mr. Achhammer has had to say. Water is formed when polyvinyl chloride is exposed to oxygen. We suspect that it is the result of hydrogen peroxide decomposition:

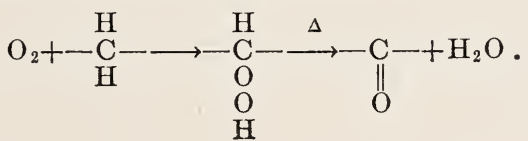


Dr. A. S. Kenyon, Monsanto Chemical Co., Dayton, Ohio: Is the failure to detect hydrogen chloride in some of the earlier stages of the reaction due to the fact that the gases are being analyzed by the mass spectrometer?

Dr. Leo Wall, National Bureau of Standards: Measurements of hydrogen chloride with the mass spectrometer are inaccurate, and small relative amounts may not be detected. However, in these experiments all components are produced in small amounts, so that failure to detect hydrogen chloride certainly means that very little is produced.

Dr. R. J. Wolf, B. F. Goodrich Chemical Co.: We have tried for a long time to get a simple test that would give us an indication of the color stability of vinyl chloride polymers of various types. It has been our experience that there is no direct relationship between $\mathrm{HCl}$ evolution and color development in a group of polymers that varied somewhat in chemical structure. We have found that in some cases a considerable amount of $\mathrm{HCl}$ is evolved with no change of color to speak of and in other cases a considerable color change appears with a relatively small amount of $\mathrm{HCl}$.

Dr. R. B. Mesrobian, Brooklyn Polytechnic Institute, Brooklyn, N. Y.: In connection with the aging of polyvinyl chloride I should like to suggest the following type of experiment. Prepare first a copolymer of vinyl chloride and ethylene that is sufficiently low in vinyl chloride content so that each vinyl chloride segment is isolated between ethylene segments. Such a copolymer would have essentially the structure

$$
-\left(\mathrm{CH}_{2} \mathrm{CH}_{2}\right)-{ }_{n} \mathrm{CH}_{2} \mathrm{CH}-\left(\mathrm{CH}_{\mathrm{Cl}} \mathrm{CH}_{2}\right)-{ }_{n} \mathrm{CH}_{2} \mathrm{CH}-\left(\mathrm{CH}_{2} \mathrm{CH}_{2}\right)-{ }_{n}
$$

Prepare next a polymer from 1-chlorobutadiene-1, 3. Disiegarding for the moment any 1,2 or 3,4 polymerization, the polymer should have the structure<smiles>CCC=CC(Cl)CC=CC(Cl)CC=CC(Cl)Cl</smiles>

Measurements of the rate of $\mathrm{HCl}$ loss of the above two types of polymers should provide information along the following lines. In the first case (polymer I) the rate of loss of $\mathrm{HCl}$ is observed when there is no activating influence of adjacent double bonds. This would be analogous to the situation with polyvinyl chloride in the earliest stages of aging when no double bonds are present along the polymer chain. In the second case (polymer II), the rate of loss of $\mathrm{HCl}$ is measured when each chlorine atom is situated on a carbon atom adjacent to a double bond and would be analogous to the situation with polyvinyl chloride in the later stages of aging.

I do not know whether these two polymers can be prepared and unfortunately we are not equipped at the Polytechnic Institute for high pressure ethylene polymerization. However, we should be very happy to do the aging studies if the samples could be prepared by industry. It seems to me that it would be of some interest to obtain as much data as possible on the kinetics of $\mathrm{HCl}$ evolution of the two polymers and the beneficial action that various stabilizers might exhibit in each case. 


\title{
8. Alkaline Degradation of Polyacrylonitrile
}

\author{
By J. R. McCartney ${ }^{1}$
}

Studies of the degradation of polymers by heat and chemical reagents, in addition to aiding in defining use properties, are valuable in elucidating chemical structure and other molecular features of polymers, such as molecular-weight distribution. In the course of research on polyacrylonitrile, it was found that concentrations of base of the order $10^{-3}$ normal in dilute polyacrylonitrile solutions produced rapid reductions in the intrinsic viscosity to 5 to 10 percent of the original value. The purpose of this paper is to present (1) the results of a study of this reaction, believed to be unique with polyacrylonitrile, and (2) a method that utilizes this reaction for qualitatively defining molecular weight distribution of polyacrylonitrile and which is applicable to any polymer that can be degraded in a controlled manner.

A specific example of the reaction will illustrate its principal features. A 1-percent solution of polyacrylonitrile in dimethyl formamide was made $0.002 N$ in $\mathrm{NaOH}$ by addition with rapid stirring of $0.2 \mathrm{~N}$ aqueous $\mathrm{NaOH}$. A bright-yellow color developed immediately, which darkened to orange and, on standing overnight, to deep red-orange. Upon addition of acid ( $\mathrm{HCl}, 0.004 \hat{N}$ in the final mixture) the deep color disappeared, leaving only a bright yellow. The polymer was precipitated by pouring the solution into hot aqueous $\mathrm{NaCl}$, and the intrinsic viscosity and number average molecular weight (by osmotic pressure) of the recorered polymer determined.

The results are summarized in table 8.1, and the osmotic pressure data are represented in figure 8.1. The difference in slope of the two

TABLE 8.1. Alkaline degradation of polyacrylonitrile

\begin{tabular}{|c|c|c|c|}
\hline Sample & $\begin{array}{c}\text { Intrinsic } \\
\text { viscosity }\end{array}$ & $\begin{array}{c}\text { Molecular } \\
\text { weight (number } \\
\text { average) }\end{array}$ & Osmotic slope \\
\hline Untreated & $\begin{array}{c}3.3 \\
1.5\end{array}$ & $\begin{array}{c}120,000 \\
19,000\end{array}$ & $\begin{array}{c}3.6 \times 10^{-3} \\
1.1\end{array}$ \\
\hline
\end{tabular}

1 Technical Dirision, Textile Fibers Department, E. I. du Pont de Nemours \& Co., Wilmington, Del. The work described in this paper has been briefly reported by R. C. Houtz, Orlon acrylic fiber: Chemistry and properties, Textile Research J. 20, 786 to 801 (1950). 
FIG URE 8.1. Osmotic pressure measurements of caustic degraded polyacrylonitrile.

$P / c R T$ is $(1 / M+B c) ; P$, osmotic pressure (atm); $c$, concentration $\left(\mathrm{g} / \mathrm{cm}^{3}\right) ; R$, gas constant $\left(\mathrm{cm}^{3} \mathrm{~atm} / \mathrm{deg}\right) ; T$, temperature $\left({ }^{\circ} \mathrm{K}\right) ; \mathcal{M}$, molecular weight (g/mole); $B$, osmotic slope $\left(\mathrm{cm}^{3} / \mathrm{g}^{2}\right)$.

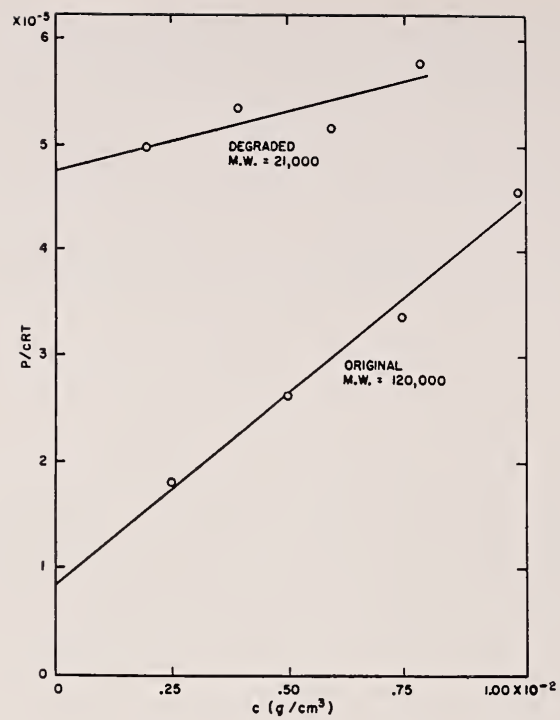

osmotic-pressure curves indicates that some chemical modification of the polymer took place during degradation. However, the difference in molecular weight shows clearly that a degradation in chain length and not chemical change is primarily responsible for the intrinsic viscosity change.

\section{Effect of Solvent on Rate and Extent of Reaction}

The degradation is extremely rapid in dimethyl formamide; both the rate and amount of degradation may be modified by the addition of a nonsolvent. In one set of experiments four 100-ml portions of 1 -percent polymer in dimethyl formamide were made up and treated as follows:

A. $2 \mathrm{ml}$ of $1 \mathrm{~N}$ aqueous $\mathrm{NaOH}$ was added with rapid stirring, followed immediately by $5 \mathrm{ml}$ of $1 N$ aqueous $\mathrm{HCl}$, so that active base was present for only 1 to $2 \mathrm{sec}$.

B. $2 \mathrm{ml}$ of $1 \mathrm{~N}$ aqueous $\mathrm{NaOH}$ was added with rapid stirring, and the solution allowed to stand $3 \mathrm{hr}$ before the addition of $5 \mathrm{ml}$ of $1 \mathrm{~N}$ aqueous $\mathrm{HCl}$.

C. $10 \mathrm{ml}$ of dioxane was added. $2 \mathrm{ml}$ of $1 \mathrm{~N}$ aqueous $\mathrm{NaOH}$ was then added with rapid stirring, followed immediately by $5 \mathrm{ml}$ of $1 \mathrm{~N}$ aqueous $\mathrm{HCl}$.

D. $10 \mathrm{ml}$ of dioxane was added. $2 \mathrm{ml}$ of $1 \mathrm{~N}$ aqueous $\mathrm{NaOH}$ was then added with rapid stirring and the solution allowed to stand $3 \mathrm{hr}$ before the addition of $5 \mathrm{ml}$ of $1 N$ aqueous $\mathrm{HCl}$.

Thus two pairs of solutions were reacted, one pair containing dioxane. After addition of base, the reaction was stopped in one solution of each pair as quickly as possible by addition of acid and allowed to proceed for $3 \mathrm{hr}$ in the other. 
All four solutions immediately developed a yellow color, although $\mathrm{C}$ and $\mathrm{D}$ were considerably lighter than $\mathrm{A}$ and $\mathrm{B}$. B and D darkened on standing. In $3 \mathrm{hr}, \mathrm{B}$ was deep orange-red and D was orange. Precipitates recovered by addition of the solutions to hot aqueous $\mathrm{NaCl}$ were colorless with the exception of that from B, which was bright yellow. Solutions of concentration $0.2 \mathrm{~g} / 100 \mathrm{ml}$ were made up, relative flow times measured, and intrinsic viscosities calculated (table 8.2 ).

TABLE 8.2. Effect of solvent on rate and extent of degradation

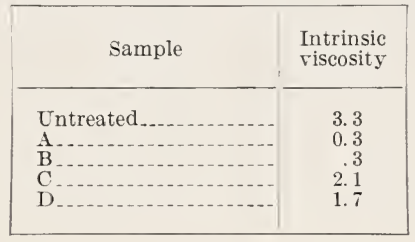

The reaction was thus essentially complete in 1 to 2 sec in dimethyl formamide, whereas the addition of dioxane to dimethyl formamide resulted in a decreased rate and orer-all effect. The reaction of alkali with dimethyl formamide to form dimethylamine competes with the degradation reaction, so the decrease in over-all effect is probably a consequence of the decreased rate which permits the base to be partially neutralized by the solvent. In another experiment, for example, in which tetramethylene sulfone (which does not react with dilute alkali) was used as solvent, the rate of degradation was considerably decreased, but the over-all effect was the same as in dimethyl formamide solution. The importance of the competing reaction between solvent and base was emphasized by an experiment in which 1-percent polymer was dissolved in dimethyl formamide that had been made $0.05 \mathrm{~N}$ in $\mathrm{NaOH}$ just before the polymer was added. The base had been completely neutralized by the solvent during the interval required for the polymer to dissolve appreciably, as shown by the fact that no detectable degradation was produced.

\section{Type of Degradation: "Unzipping" Versus Random Scission}

As a step in clarifying the nature of the reaction, it was considered essential to establish as closely as possible the stoichiometric relation between the amount of caustic added and the extent of degradation produced. Two distinct possibilities exist for the formation of a degraded polymer by depolymerization: (a) splitting off monomer units ("unzipping") or (b) random scission. Work of Jellinek on polystyrene degradation ${ }^{2}$ has shown that when mechanism (a) prevails, the yield of monomer is $10^{3}$ to $10^{4}$ times that calculated from mechanism (b), so it is possible to distinguish between (a) and (b) by determining the percentage of degraded polymer recovered by precipitation from solution, presuming the monomer formed is lost in this recovery process. The following set of experiments was accordingly carried out.

${ }^{2}$ H. H. G. Jellinek, Trans. Faraday Soc. 40, 266-279 (1944); 44, 345-349 (1948). 
To 500-ml portions of 2-percent polymer in dimethyl formamide were added $20-\mathrm{ml}$ portions of aqueous $\mathrm{NaOH}$ of various normalities selected to cover a wide range of degradation. After standing overnight each solution was divided into two equal parts; part B was acidified with $10 \mathrm{ml}$ of $2 \mathrm{~N} \mathrm{HCl}$, whereas part A was not acidified. The polymers were precipitated by pouring the solutions into hot aqueous $\mathrm{NaCl}$, filtered, washed thoroughly with water followed by acetone, dried in vacuum at $80^{\circ} \mathrm{C}$, ground to a fine powder, and redried to constant weight. The recovery data are given in table 8.3.

There is no significant difference between recoveries of the caustictreated samples and the control. Although there is some scatter in the recoveries, there is no evident trend with amount of caustic added or no difference between $\mathrm{A}$ and $\mathrm{B}$ samples. The average number of grams recovered in the 10 samples $2 \mathrm{~A}$ to $6 \mathrm{~B}$, inclusive, is $4.50 \pm 0.16$, not significantly different from the control $(4.47 \pm 0.06)$. Losses of monomer approaching 100 percent of the original weight would be expected for the most highly degraded samples if an unzipping mecha-

TABLE 8.3. Recovery and nitrogen content of degraded polymer

\begin{tabular}{|c|c|c|c|c|}
\hline Sample & $\begin{array}{l}\text { Normality } \\
\mathrm{NaOH} \text { added }\end{array}$ & $\begin{array}{l}\text { Intrinsic } \\
\text { viscosity }\end{array}$ & $\begin{array}{l}\text { Polymer re- } \\
\text { covered }\end{array}$ & $\begin{array}{c}\text { Nitrogen } \\
\text { content }\end{array}$ \\
\hline $\begin{array}{l}1 \mathrm{~A} \\
1 \mathrm{~B} \\
2 \mathrm{~A} \\
2 \mathrm{~B} \\
3 \mathrm{~A} \\
3 \mathrm{~B} \\
4 \mathrm{~A} \\
4 \mathrm{~B} \\
5 \mathrm{~A} \\
5 \mathrm{~B} \\
6 \mathrm{~A} \\
6 \mathrm{~B}\end{array}$ & $\begin{array}{l}0 \text { (control) } \\
0.05 \\
.075 \\
.10 \\
.20 \\
1.00 \\
0\end{array}$ & $\begin{array}{c}3.3 \\
0.8 \\
.6 \\
.4 \\
.2 \\
.1\end{array}$ & $\begin{array}{c}g \\
4.51 \\
4.42 \\
4.61 \\
4.61 \\
4.19 \\
4.55 \\
4.29 \\
4.58 \\
4.51 \\
4.34 \\
4.66 \\
4.70\end{array}$ & $\begin{array}{c}\% \\
25.6 \\
25.8 \\
25.6 \\
25.5 \\
25.6 \\
25.6 \\
25.5 \\
25.6 \\
24.9 \\
25.7 \\
21.7 \\
22.3\end{array}$ \\
\hline
\end{tabular}

nism prevailed, so it may be concluded that the degradation proceeds by random chain scission. Further support for this conclusion is given by the observation that the extent of unzipping would be affected by the catalyst used in the polymerization. The extent of degradation has been independent of catalyst in all the polymers tested.

Nitrogen analyses of the recovered samples are included in table 8.3 ; essentially 100 percent of the nitrogen was retained by the degraded samples, with the exception of $6 \mathrm{~A}$ and $6 \mathrm{~B}$, which were the most highly degraded.

\section{Stoichiometric Relation Between Moles of Base Added and Degradation}

It is characteristic of degradation reactions that proceed by random scission that for moderately high initial molecular weight and degree of degradation, the average molecular weight of the degraded polymer is not very dependent on either the original molecular weight or the original molecular weight distribution. This is evident from considerations developed in detail in the appendix, to which reference 
should be made for the derivation of equations used in this and the following sections.

In eq (1) to (9) of the appendix, it is shown that the degraded weight average molecular weight, $M_{w}$, resulting from random scission can be expressed as a simple function of the total weight of polymer, $W$, and the total number of chain cuts (that is, bonds broken), $R$; $M_{w}=2 W / R$, provided the original molecular weight and extent of degradation are moderately high.

Two experiments may be cited to show that the present system degrades according to the requirements of this representation of random scission.

\section{Influence of original molecular weight on degradation:}

One-hundred-milliliter samples of 1-percent dimethyl formamide solution of two polymers of different intrinsic viscosities (samples A and $\mathrm{B}$ ) were degraded by addition of $2 \mathrm{ml}$ of aqueous $\mathrm{NaOH}$ of different concentrations to $0.1 \mathrm{~N}$. Relative flow times of the reacted solutions were measured and intrinsic viscosities calculated. The results are given in figure 8.2 , in which intrinsic viscosity is plotted versus moles of base added per gram of polymer.

It will be noted that the decrease in intrinsic viscosity for the polymer of higher intrinsic viscosity is much more pronounced than that for the polymer of lower intrinsic viscosity in the range of 0 to $10 \times 10^{-5}$ mole base, but further addition of base affects the two partially degraded polymers in essentially the same way. The influence of the original intrinsic viscosity has already disappeared when only a few bonds per molecule have been broken. Consider, for example, sample $B$ degraded to an intrinsic viscosity of 1.3 (about 80 percent of the original value); $10^{-4}$ mole base/g of polymer are required for this amount of degradation. To degrade sample $A$ to the same intrinsic viscosity requires $1.33 \times 10^{-4} \mathrm{~mole} / \mathrm{g}$, an excess of $3.3 \times 10^{-5} \mathrm{~mole} / \mathrm{g}$. Shifting the upper curve to the left, a distance corresponding to $3.3 \times 10^{-5}$ mole/g makes the curves coincide for further increments of base.

Figure 8.2. Alkaline degradation of two polyacrylonitriles $(A$ and $B)$ of different intrinsic viscosity.

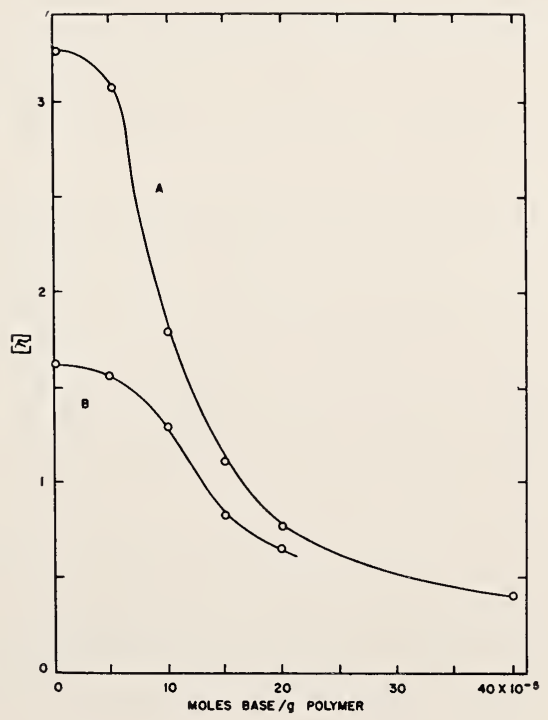




\section{Influence of original molecular-weight distribution:}

A blend of 57 percent by weight of polymer of intrinsic viscosity 1.0 and 43 percent of polymer of intrinsic viscosity 4.6 was made up in 1-percent concentration in dimethyl formamide. The intrinsic viscosity of the mixture was 2.5 . To $50 \mathrm{ml}$ of solution was added $1 \mathrm{ml}$ of $0.1 \mathrm{~N}$ aqueous $\mathrm{NaOH}$. The solution was diluted to $C=0.4$ $\mathrm{g} / 100 \mathrm{ml}$ and the relative flow time determined. A whole polymer of about the same intrinsic viscosity as the blend was treated in the same way. The data are summarized in table 8.4.

TABLE 8.t. Effect of molecular weight distribution on degradation

\begin{tabular}{|c|c|c|}
\hline \multirow{2}{*}{ Sample } & \multicolumn{2}{|c|}{ Intrinsic viscosity } \\
\cline { 2 - 3 } & Original & Degraded \\
\hline Blend & 2.5 & $\begin{array}{c}0.71 \\
.66\end{array}$ \\
\hline
\end{tabular}

The blend, which had a considerably broader distribution than the whole polymer, thus degraded to essentially the same intrinsic as the whole polymer. Under the conditions mentioned (moderately high initial molecular weight and degree of degradation), then, interpretation of results unambiguously in terms of a stoichiometric relation does not require either sharp fractions for starting material or a survey of molecular weight dependence.

The intrinsic viscosity of the degraded polymer as a function of the moles of $\mathrm{NaOH}$ added was calculated from flow time measurements on 1-percent solutions of a polymer that had been reacted with $2 \mathrm{ml}$ of aqueous $\mathrm{NaOH}$; the amounts of base varied from 0 to $2 \times 10^{-3}$ mole of base per gram of polymer. The derivation of an expression relating the degraded intrinsic viscosity and the moles of base added to the efficiency $k$ of the base in producing scission is given in the appendix, eq (10) to (14). The result is

$$
[\eta]^{-3 / 2}=\frac{k}{2} \frac{\left(M_{w}\right)_{o}}{[\eta]_{o}^{3 / 2}} \frac{B}{W}
$$

where $[\eta]$ is degraded intrinsic viscosity; $[\eta]_{o}$, original intrinsic viscosity; $\left(M_{w}\right)_{o}$, original weight average molecular weight; $B$, moles base added; $W$, weight of polymer; and $k$, efficiency of base in producing scission, defined by $R=k \dot{B}$, where $R$ is total number of chain cuts produced by $B$ moles of base. In figure 8.3 the data are plotted according to this result $\left([\eta]^{-3 / 2}\right.$ versus $\left.B / W^{\top}\right)$. Aside from a deviation near the origin (in which region the analysis leading to eq (14) does not apply) a linear relation was found with slope equal to $1.2 \times 10^{-4}$, from which

$$
k=\frac{1.4 \times 10^{5}}{\left(M_{w}\right)_{o}} .
$$

$M_{w}$ has not been determined for this polymer; estimating it to be twice $M_{n}$, that is, about 200,000 , leads to a value of $k$ of the order unity. This estimate cannot be refined without a determination of 


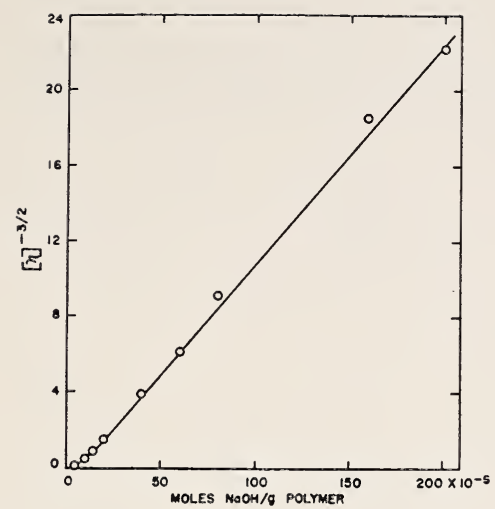

Figure 8.3. Stoichiometric relation in the alkaline degradation of polyacrylonitrile.

$M_{w}$, but to a good approximation it can be said that only one or two base molecules are required to break one bond.

It is evident from figure 8.3 that the degradation still follows the theoretical expectations for random scission at the highest degree of degradation. There is no indication of a decrease in slope of the curve, such as would occur, for example, if the base attacked flaws or "weak links" in the chain. Furthermore, to produce the observed intrinsic viscosity at the highest degree of degradation, about 300 bonds per molecule on the average (nearly 10 percent) have to be broken and the number of flaws per molecule according to infrared observations must be considerably less than this.

\section{Proposed Mechanism for the Reaction}

From the experiments just discussed the following facts may be noted about the reaction between polyacrylonitrile and dilute base:

1. The reaction proceeds by random scission.

2. Only one or two base molecules are required to break a bond.

3. The reaction rate is solvent dependent, and proceeds very rapidly in dimethyl formamide.

4. Reaction between solvent and base reduces the orer-all effect.

5. Depolymerization by "unzipping" the chain does not occur to any measurable extent.

6 . Reaction between base and flaws in the molecules is not important in the degradation.

7. The reaction is characterized by the rapid formation of a bright yellow color which deepens on standing to a deep red-orange. The latter stage of color formation is partially reversible in the presence of excess acid.

A theory of the reaction, then, should involve a reaction between base and "normal" (1,2 addition) polymer. Marvel ${ }^{3}$ advanced a theory that is in agreement with the observations, and which is supported by the results of further experiments suggested by the theory. It was suggested that polyacrylonitrile in dilute dimethyl formamide

\footnotetext{
${ }^{3}$ C. S. Marvel, pricate discussion.
} 
solution reacts with $\mathrm{NaOH}$ as follows:

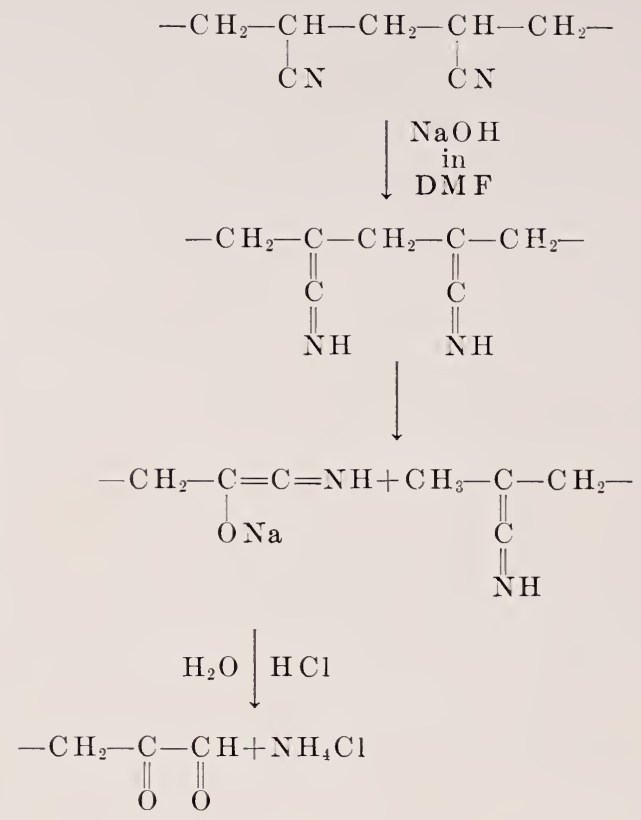

(a

The importance of the $\alpha$-hydrogen in this theory suggested as a first experiment an investigation of the degradation of polymethacrylonitrile. Because this latter polymer lacks the $\alpha$-hydrogen characterizing polyacrylonitrile, it would not react according to the above outline. To $40 \mathrm{ml}$ of 1-percent polymethacrylonitrile in dimethyl formamide was added $1 \mathrm{ml} 0.2 \mathrm{~N}$ aqueous $\mathrm{NaOH}$. A portion of this solution was reserved for flow time measurement and $30 \mathrm{ml}$ of the remainder reacted with $1 \mathrm{ml}$ of $0.2 \mathrm{~N}$ aqueous $\mathrm{NaOH}$. The flow times relative to $1-$ percent solution containing the same amount of water are recorded in table 8.5.

A slight drop in intrinsic viscosity with the initial addition of base is indicated, but this was probably due to a change of configuration of the molecules in solution due to basic solvent environment as shown by the fact that further addition of base produced only a very slight further change in flow time. Polyacrylonitrile degraded under the same conditions would have a solution flow time (relative to the unreacted solution) of only about 0.2 . Polymethacrylonitrile, therefore, does not degrade in dilute base.

It was observed that although polymethacrylonitrile did not degrade in dilute base, the dimethyl formamide solution went through essentially the same color cycle as do polyacrylonitrile solutions; that is, a bright yellow color formed which deepened to orange on standing. Thus the scission reaction is not involved in the color formation.

The role of the solvent in the color formation was checked by adding aqueous caustic to an acetone solution of polymethacrylonitrile; the color cycle was essentially the same as in dimethyl formamide solution, although the final orange color was lighter. The initial yellow and orange colors observed in the reaction of base with polyacrylo- 
nitrile accordingly do not involve either the degradation reaction or interaction with the solvent, but are characteristic of a reaction involving the cyano side groups. One possibility is the formation of naphthyridine-type structures induced by the presence of base:

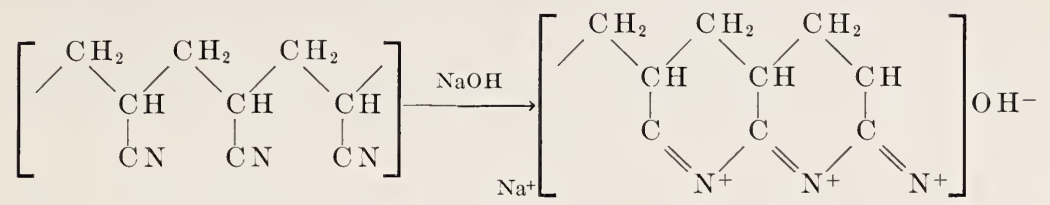

The deep red-orange color that develops in highly degraded solutions may be partially due to after-reactions of the split chains. For example, the $\alpha$-keto aldehyde product in step (d) of the proposed mechanism above may split out $\mathrm{HCN}$ to give conjugated colored structures. However, the most important reaction in this step

TABLE 8.5. Reaction of sodium hydroxide and polymethacrylonitrile

\begin{tabular}{|c|c|}
\hline $\begin{array}{c}\text { Moles of base } \\
\text { per gram of } \\
\text { polymer }\end{array}$ & Flow times \\
\hline $\begin{array}{c}5 \times 10^{-4} \\
12\end{array}$ & $\begin{array}{c}0.88 \\
.87\end{array}$ \\
\hline
\end{tabular}

s Relative to unreacteds olution containing the same weight of polymer and water as reacted solution.

of the color formation is probably the reaction of the cyano groups with dimethylamine, formed by reaction of the base with dimethyl formamide.

Due to the difficulty in preparing and maintaining strictly anhydrous dimethyl formamide solutions, the importance of water in the reaction was not established. Alcoholic $\mathrm{KOH}$, however, was observed to give the same effect as aqueous $\mathrm{NaOH}$.

Further experimental work will be necessary to elucidate the mechanism of the reaction. A study of the addition to the reaction system of such reagents as $o$-phenylene diamine, which add to dicarboxy groups, would check the proposed step (d).

\section{Determination of Molecular Weight Distribution From Degradation Data}

The initial stage of degradation by random scission is distribution dependent. Because the reaction between polymer and dilute base furnishes a means of degrading the polymer rapidly, the use of the reaction in determining molecular weight distribution in polyacrylonitrile polymer was indicated.

The specific decrease in weight-average molecular weight $1-M_{w}$ / $\left(\Lambda_{w}\right)_{o}$ produced by random scission degradation is proportional to the $Z$ average molecular weight $M_{z}$ (Appendix, eq (15) to (19)). Distribution may be characterized by comparing $M_{z}$ and $M_{w}$, analo- 
gously to a characterization by the ratio $M_{w} / M_{n}$ from a comparison of light scattering and osmotic pressure data. ${ }^{4}$ In order to use intrinsic viscosity measurement exclusively in the characterization, proportionality between $M_{w}$ and $[\eta]^{3 / 2}$ is assumed. A distribution characterizing parameter $D$, proportional to $M_{z} / M_{u}$, is then defined by

$$
D\left(\frac{B}{W^{\prime}}\right)-=[\eta]^{-3 / 2}\left[1-\left([\eta] /[\eta]_{0}\right)^{3 / 2}\right]
$$

and can be determined by measuring two instrinsic viscosities. The notation $D(B / W)$ indicates that $D$ is a function of the moles of base added per gram of polymer; therefore, to compare distribution in a set of polymers, $B / W$ must be kept constant. While this method necessarily assigns distribution to a more arbitrary scale than a comparison of two absolute measurements, it has the decided advantage of employing only the most conveniently measured index of molecular weight, intrinsic viscosity. Results on a series of partial conversion acrylonitrile polymers are given in table 8.6 to illustrate the

TABLE 8.6. Conversion and molecular weight distribution in polyacrylonitrile

\begin{tabular}{|c|l|}
\hline Conversion & \multicolumn{1}{c|}{$D$} \\
\cline { 1 - 2 } Percent & \\
6.5 & \\
12.1 & $2.4 \times 10^{2}$ \\
32.5 & 2.7 \\
46.7 & 1.1 \\
76.0 & 5.0 \\
\hline
\end{tabular}

method. Solutions of the polymers were reacted with $\mathrm{NaOH}$ $\left(B / \mathrm{W}=10^{-4}\right.$ mole base $/ \mathrm{g}$ of polvmer $)$, allowed to stand $1 \mathrm{hr}$, neutralized with $\mathrm{HCl}$, and intrinsic viscosities determined by relative flow time measurement. The difference between the first three samples is not significant. The distribution at intermediate conversion (46.7 percent) is sharper than in the lower conversion samples, and there is a significant broadening of distribution at the highest conversion. This technique is expected to be useful where a rapid indication of the effect of process variables on molecular weight distribution is desired.

\section{Appendix}

Nontroll and Simha ${ }^{5}$ have derived an expression for the weight average molecular weight $M_{w}$ of the distribution of molecules resulting from the random scission of a sharp fraction of initial molecular weight $M_{o}$ :

$$
M_{w}=M_{o} \frac{a^{2}(1+C)+2(1-a)\left[(1-a)^{C+1}+a(1+C)-1\right]}{a^{2}(1+C)^{2}},
$$

4 W. D. Lansing and E. O. Kraemer, J. Am. Chem. Soc. 5 ; , 1369-1377 (1935).

${ }^{5}$ E. W. Montroll and R. Simha, J. Chem. Phys. 6, 721-727 (1940). 
where $C$ is the initial number of bonds per molecule; $1+C$, initial number of monomer units per molecule; $a$, degree of degradation $=r_{o} / C$, where $r_{o}$ is the average number of cuts (that is, bonds broken) per molecule. This equation will serve as the basis of discussion of two subjects referred to in the body of the paper.

\section{$M_{w}$ When $a$ and $C$ Are Large}

When $a$ and $C$ are so large that $(1-a)^{C+1}$ is negligibly small, eq (1) simplifies to

$$
M_{w}=\frac{2 M_{o}}{r_{o}} .
$$

If there are $n$ molecules of polymer originally present,

$$
r_{o}=R_{o} / n,
$$

where $R_{o}$ is the total number of cuts. But,

$$
n=\frac{N W}{M_{o}}
$$

where $W$ is the total weight of polymer and $N$ is Avogadro's number. Combining eq (2), (3), and (4),

$$
M_{w}=2 \frac{W}{R}
$$

where $R$ is the total number of cuts expressed on a molar basis, that is,

$$
R=R_{o} / N \text {. }
$$

Thus $M_{w}$ does not depend upon the original molecular weight, but only upon the total number of cuts per gram of polymer.

Consider now the degradation of a distribution of molecules of different weights $\left(M_{o}\right)_{i}$, where the index $i=1,2,3$, etc., is used to distinguish between the different molecular weights present in the mixture. The weight-average molecular weight $\left(M_{w}\right)_{i}$ of the distribution resulting from making $r_{i}$ cuts per molecule in the $n_{i}$ molecules of molecular weight $\left(M_{o}\right)_{i}$ is, from eq $(2)$,

$$
\left(M_{w}\right)_{i}=\frac{2\left(M_{o}\right)_{i}}{r_{i}} .
$$

$M_{w}$ of the mixture obtained from degrading all the molecules of species $i$ in the original distribution is

$$
M_{w}=f_{i}\left(M_{w}\right)_{i}=2 \Sigma \frac{\left(f_{i} M_{o}\right)_{i}}{r_{i}} .
$$

But, from eq (3) and (4) (since the total number of cuts in the molecules of species $i$ is proportional to the weight fraction of that species)

$$
r_{i}=\frac{f_{\imath} R_{o}}{n}=\frac{f_{i} R_{o}\left(M_{o}\right)_{i}}{N W_{i}}=\frac{R_{o}\left(M_{o}\right)_{i}}{N W}
$$

where $f_{i}$ is the weight fraction of species $i$. Therefore, combining eq (6), (8), and (9),

$$
M_{w}=2 \frac{W}{R}
$$

which is identical with eq (5), derived with the assumption that all the initial molecular weights were equal. Thus distribution does not affect the final average molecular weight. 
The stoichiometric relation between moles of base added, $B$, and the degradation produced in the reaction of base with polyacrylonitrile can be obtained from the above relations. Denoting by $k$ the efficiency of the base in producing scission, that is,

$$
R=k B \text {, }
$$

equation (5) becomes (dividing both sides of the equation by $\left(M_{w}\right)_{o}$, the original weight average molecular weight)

$$
\frac{M_{w}}{\left(M_{w}\right)_{o}}=\frac{2}{\left(M_{w}\right)_{o}} \frac{W}{k B} .
$$

It follows from the intrinsic viscosity- molecular weight relation

$$
[\eta]=K M^{s}
$$

that for broad distributions of polymers, such as polyacrylonitrile, which have $s=\frac{2}{3}$, that

$$
\frac{M_{w}}{\left(M_{w}\right)_{o}}=\left[\frac{[\eta]}{[\eta]_{0}}\right]^{3 / 2} \text {. }
$$

Combining eq (11) and (13) and rearranging, proportionality between $[\eta]^{-3 / 2}$ and $B / W$ is obtained:

$$
[\eta]^{-3 / 2}=\frac{k\left(M_{w}\right)_{o}}{2[\eta]^{3 / 2}} \frac{B}{W}
$$

This equation is the theoretical basis of figure 8.3 , from which $k$ was evaluated.

\section{$M_{w}$ When $r_{o}$ is Small}

For the early stages of the degradation, that is, when $r_{o}$ is small, $(1-a)^{C+1}$ may be represented by the first few terms of a power series, and eq (1) becomes (using eq (3) and (4))

$$
M_{w}=M_{o}-\frac{r_{o}}{3} M_{o}=M_{o}-\frac{R}{3 W} M_{o}^{2}
$$

Thus the initial molecular weight change in a degradation reaction depends upon the square of the initial molecular weight.

Considering again the degradation of a distribution of different molecular weights, from eq (15),

Since

$$
M_{w}=\Sigma f_{i}\left(M_{w}\right)_{i}=\Sigma f_{i}\left(M_{o}\right)_{i}-\frac{R}{3 W} \Sigma f_{i}\left(M_{o}\right)_{i}^{2} .
$$

$$
\begin{gathered}
\Sigma f_{i}\left(M_{o}\right)_{i}=\left(M_{w}\right)_{o}, \\
\frac{M_{w}}{\left(M_{w}\right)_{o}}=1-\frac{R}{3 W} \frac{\Sigma f_{\imath}\left(M_{o}\right)_{i}^{2}}{\left(M_{w}\right)_{o}} .
\end{gathered}
$$

The quantity $f_{i}\left(M_{o}\right)_{i}^{2} /\left(M_{w}\right)_{o}$, which has the dimensions of a molecular weight, occurs in ultracentrifuge theory and has been defined as the $Z$-average molecular weight $M_{Z}$ (see footnote 4):

$$
M_{Z}=\frac{\Sigma f_{i} M_{i}^{2}}{\Sigma f_{i} M_{i}}
$$

Since $M_{Z}$ depends upon distribution, the early stages of the degradation must depend upon distribution. Conversely, a study of the initial degradation furnishes an insight into the original distribution. 
The degradation data may be analyzed in terms of a distribution characterizing parameter $D$, defined by

$$
D=\frac{R}{3 W} \frac{M_{Z}}{[\eta]^{3 / 2}}
$$

which is proportional to $M_{Z} / M_{w} . \quad M_{Z} / M_{w}$ is unity for a sharp fraction and increases with the broadness of the distribution; for example, for high conversion condensation polymerization it is 1.5 . Thus the parameter $D$ increases with the broadness of the distribution. Combining eq (13), (18), and (19) an expression for $D$ involving only the initial and final intrinsic viscosities is obtained:

$$
D=[\eta]^{-3 / 2}\left[1-\left([\eta] /[\eta]_{0}\right)^{3 / 2}\right] \text {. }
$$

\section{Discussion}

Dr. C. F. Gibis, B. F. Goodrich Co., Brecksrille, Ohio: What is the effect of dilute caustic solution upon polyacrylonitrile powder and oriented fibers?

Dr. McCartney: Studies on the effect of caustic on the solid polymer have been conducted and polyacrylonitrile is considered among the more inert polymers in that respect. The rapid degradation discussed here is peculiar to dilute solutions where, of course, the molecule is in a considerably more vulnerable condition than it is in the solid form.

Dr. J. D. Cotmax, Plastics Division, Monsanto Chemical Co., Springfield, Mass.: Carbanion elimination can occur as a very rapid, almost immediate reaction. In a highly polar medium, the hydrogen transfer equilibration<smiles>[R]CC([Al])C[R]OC#CCC([R])([CH])CO</smiles>

is probably extremely rapid. The ion may then react irrerersibly to cleave the chain:

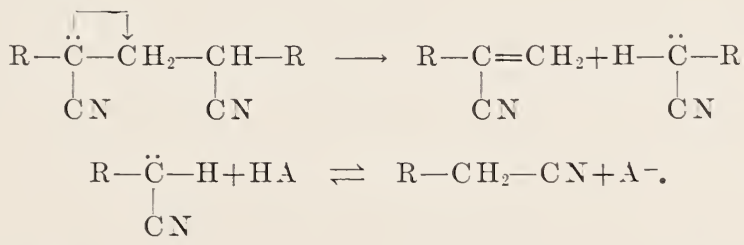

Dr. E. H. Wood, Bakelite Co., Division of Union Carbide and Carbon Corp., Bound Brook, N. J.: Hare you checked your distribution function by actual fractionation?

Dr. McCARtNey: We used this method so that we would not have to fractionate. If you have different $M_{v} / M_{z}$ ratios, you will get different $D$ values by this method; the distributions are different, but you can not say more about the details of the difference without applying a more detailed procedure, such as fractionation. 
Dr. H. H. G. Jellinek, University of Adelaide, Adelaide, Australia: I take it that all these calculations imply that Staudinger's rule holds.

Dr. McCartney: The intrinsic viscosities that we have measured fall within the range where we have empirically established the intrinsic-molecular weight relation.

Dr. Robert Simha, New York University, New York, N. Y.: A quantitative kinetic study of this reaction would be of interest. The sharp initial drop in viscosity seems to indicate that one is not far from a random case. There is one way of establishing whether the process is random, but it requires a direct determination of the fraction of bonds broken or an analysis for the monomer produced. The results so obtained, for instance, the maximum rate at 25 -percent conversion to monomer, are independent of the initial molecular weight distribution. 


\title{
9. Aging of Polyethylene
}

\author{
By B. S. Biggs ${ }^{1}$ \\ Introduction
}

Polyethylene became available as a commercial material in England in the late 1930's and production was started in this country in 1943. Many people, being familiar with the reputed chemical inertness of paraffin wax, expected this new hydrocarbon polymer to have great inherent stability. It became apparent very soon, however, that pure unprotected polyethylene was oxidized rapidly on outdoor exposure and would not retain its original properties more than a few months. During this development period some serious misapplications of polyethylene were made. As experience grew, these were largely eliminated, and today it is being used to advantage in many ways. As with many polymers, it has been necessary to suit the material to the application by appropriate compounding modifications. The purpose of this paper is to discuss the nature of the deterioration reactions of polyethylene and to describe some means of retarding them.

There are many papers in the literature testifying to the ease of thermal oxidation of paraffinic hydrocarbons. Francis and his associates $[1,2,3,4]^{2}$ oxidized paraffin wax at temperatures in the neighborhood of $100^{\circ} \mathrm{C}$ and obtained as products water, formic acid, ketones, hydroxy compounds, and long fatty acids. Haslam and Frolich [5] oxidized highly refined mineral oil at $130^{\circ} \mathrm{C}$ and found acids and other oxygenated compounds in the product. Dornte [6], Balsbaugh and Oncley [7], Von Fuchs and Diamond [8], and Larsen, Thorpe, and Armfield [9] carried out extensive experiments with saturated aliphatic hydrocarbons and found that they are easily oxidized and follow an autocatalytic oxidation curve. It was also well established that their oxidation is subject to inhibition and is therefore presumably a chain reaction. In the light of these facts it should not have been surprising to find that polyethylene, which is a larger homologue of these compounds, is also easily attacked by oxygen at elevated temperature. Its susceptibility to photooxidation is surprising, and this will be discussed at greater length later.

\section{Mechanism of Oxidation}

The oxidation of polyethylene is most easily observed by milling the plastic on a hot mill. The viscosity decreases and the power factor, initially very low, increases rapidly. Antioxidants are effective in preserving the high molecular weight and the low power factor (fig. 9.1). The oxidation and its inhibition are also observed in oxygen absorption studies carried out at $150^{\circ} \mathrm{C}$. Figure 9.2 shows curves of such oxygen absorption for polyethylene with and without antioxidant.

1 Bell Telephone Laboratories, Murray Hill, N. J.

2 Figures in brackets indicate the literature references on p. 145 

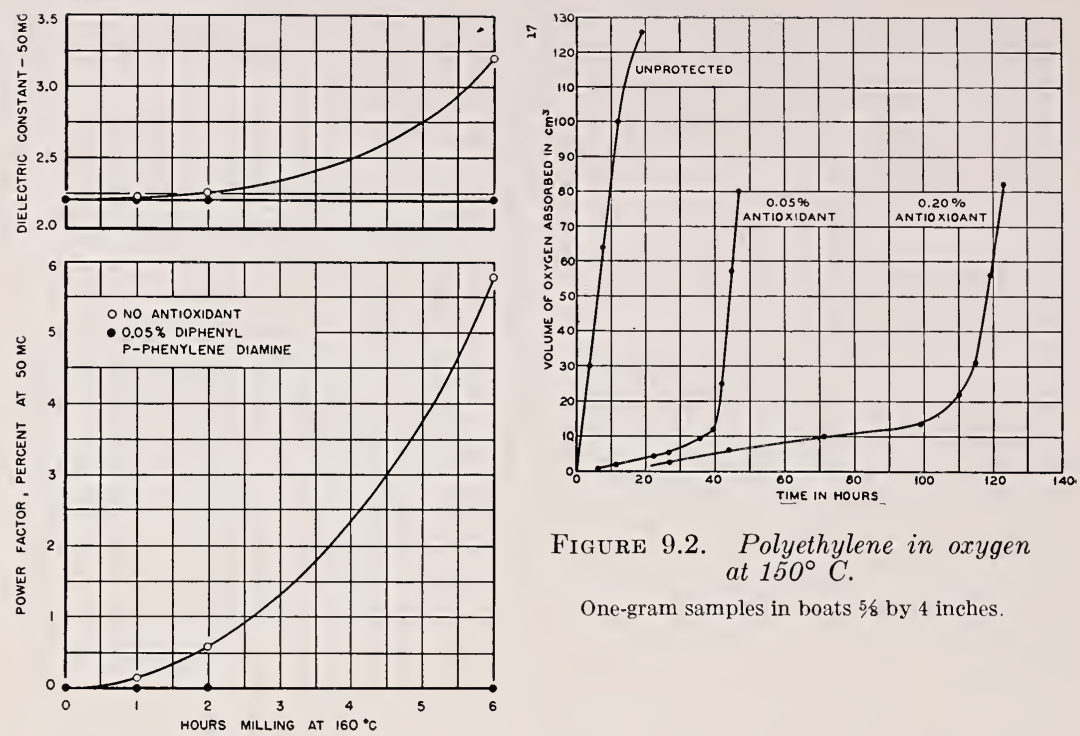

\section{Figure 9.2. Polyethylene in oxygen at $150^{\circ} \mathrm{C}$.}

One-gram samples in boats $5 / 8$ by 4 inches

Figure 9.1 .

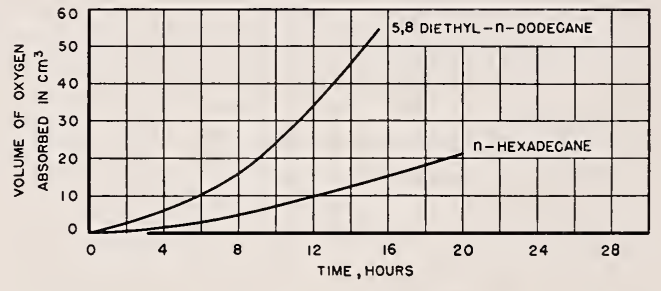

Figure 9.3. Isomeric hexadecanes in oxygen at $105^{\circ} \mathrm{C}$.

Three-cubic centimeter samples.

The question of where and how the polyethylene chain is attacked is an interesting one and something can be learned about it by turning attention again to small molecules. The order of decreasing stability of hydrogen atoms in saturated hydrocarbons is known to be primary, secondary, tertiary $[10,11,12,13,14,15]$. Thus, highly branched hydrocarbons are resistant to oxidation if all the methyl groups are paired (neopentane) but are reactive if single methyl or other alkyl groups (and therefore a tertiary hydrogen) occur along a chain. The stability of a methyl silicone polymer, which is about 40 percent hydrocarbon, undoubtedly resides in the fact that all the hydrocarbon portion is in the form of methyl groups. It is of interest to compare the rates of oxidation of two isomeric compounds of formula $\mathrm{C}_{16} \mathrm{H}_{34}$, one being normal hexadecane and the other being diethyl dodecane, which contains two tertiary hydrogens (fig. 9.3). Polyethylene has 
been reported on the basis of infrared absorption evidence [16, 17, 18] to contain branches, and the concomitant tertiary hydrogen atoms would no doubt increase its susceptibility to oxidation. However, the secondary groups of normal hexadecane are seen to be quite reactive enough, and we can assume that polyethylene would be subject to thermal oxidation even if it were a perfect straight chain paraffin.

The mechanism of the oxidation is assumed to be that generally accepted for aliphatic hydrocarbons uncomplicated by unsaturation. The presence of occasional double bonds, like occasional tertiary hydrogens, would undoubtedly facilitate the initiation step by formation of the first peroxide group, but if we can judge from the easy oxidation of simple paraffins, such points of increased vulnerability are not necessary. The source of the first radicals is a matter of speculation. It may be that radicals are formed by dissociation of $-\mathrm{C}-\mathrm{H}$ or $-\mathrm{C}-\mathrm{C}-$ bonds in the segments of highest energy, or perhaps hydroperoxide groups can be formed by direct union with oxygen, and their decomposition can produce the first radicals. Once hydrocarbon radicals are produced it can be assumed that they react with oxygen to form peroxidic radicals, and these in turn abstract hydrogen from other molecules of substrate and repeat the process. Due principally to the work of the British Rubber Producers Research Association $[19,20,21]$ these steps are now well known and widely accepted and need not be repeated here. Antioxidants act as chain stoppers by interposing themselves in the chain reaction at an early stage and terminating it by leaving a radical too inert to react with oxygen [22]. That they are quite effective in molten polyethylene is shown by figure 9.2 cited above.

The free radical chain reaction described above results in the introduction of hydroperoxide groups into the polyethylene chain. Its autocatalytic nature derives from the decomposition of these peroxides into new free radicals. These reactions would be adequate to account for the increase in power factor of polyethylene. The fact that there is an attendant decrease in viscosity means that somewhere in the series of reactions scissions of the carbon chains occur. There is abundant evidence that decomposition of tertiary hydroperoxides can result in chain scission. Stephens [23] showed about $20 \mathrm{yr}$ ago that dialkyl phenyl methanes on oxidation give phenyl alkyl ketones, the longer alkyl group being split off:

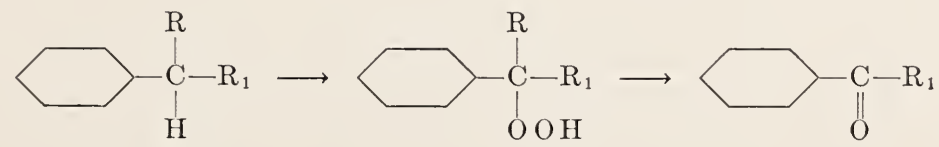

Cumene hydroperoxide similarly gives acetophenone. George and Walsh [24] have described similar scissions of carbon bonds in the decomposition of tertiary hydroperoxides of methyl cyclohexane and methyl cyclopentane.

There is less direct evidence for chain scission in secondary peroxides, although it is apparent that scission occurs at some point in the sequence of reactions because short-chain fatty acids are produced in the oxidation of paraffin wax and octadecane. 
Bateman [25] has recently suggested on kinetic grounds that the chain scission in rubber occurs in the decomposition of the peroxidic radical ROO. rather than in that of the hydroperoxide. Regardless of which mechanism is correct, the fact is that unprotected polyethylene does undergo chain scission and is ultimately degraded to a material with little more structural value than paraffin wax.

\section{Thermal Oxidation}

Although the most rapid degradation of polyethylene in service has been in outdoor exposure, it is of interest in the laboratory to study the purely thermal oxidation. The work reported here was carried out in a manometric system, which permits a sample contained in suitable glass tubes to be heated in an oven and the absorption of oxygen to be measured in burettes outside. The parts of the apparatus are connected by capillary glass tubing, flexible joints being provided outside the oven by use of very short sections of polyvinyl chloride tubing.

The measurement of the true rate of oxygen absorption of a given mass of polyethylene is difficult because the slow rate of diffusion of oxygen limits the reaction. Even at $150^{\circ} \mathrm{C}$ melted polyethylene is still too viscous to permit any effective agitation except on a hot mill. It has been found that the limiting thickness of polyethylene film that will permit reaction at a rate proportional to mass rather than surface area is between 1 and 5 mils. At $90^{\circ} \mathrm{C}$, samples of equal weight prepared by slicing a block of material with a microtome oxidize at equal rates at thicknesses of $\frac{1}{2}$ and 1 mil but not at 5 mils. Such thin chips cannot be used above the melting point, of course, because they fuse together. Attempts have been made to support thin films of melted polyethylene on glass cloth, but it is difficult in such experiments to be certain that the maximum thickness is kept below the critical level. The oxygen absorption curves shown in figure 9.2 were obtained on samples contained in aluminum boats of fixed size and in which only the upper surface is exposed to oxygen. This method is satisfactory for obtaining comparative rates, particularly when comparing the effectiveness of different antioxidants.

One way in which this method has been useful is in determining whether there is any marked difference in rate of oxygen absorption on passing from the liquid state into the solid state. In figure 9.4

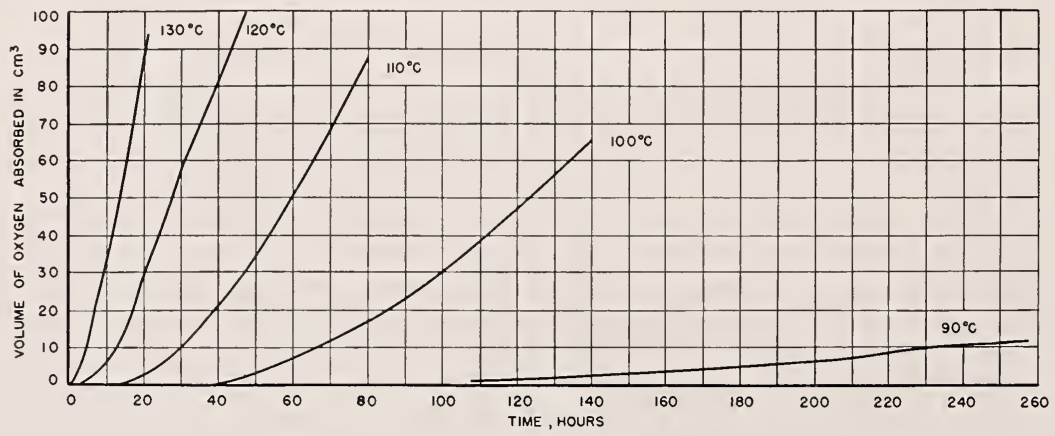

Figure 9.4. Polyethylene in oxygen.

75 mils deep in boats $5 / 8$ by $4 / 3 / 4$ inches. 
are plotted data on oxygen absorption at $130^{\circ}, 120^{\circ}, 110^{\circ}, 100^{\circ}$, and $90^{\circ} \mathrm{C}$ by samples of pure polyethylene contained in aluminum boats $\frac{5}{8}$ by $4 \frac{3}{4}$ in. The thickness of the polyethylene was about 75 mils, the samples having been prepared by cutting pieces the exact size of the boat from a 75 -mil sheet and melting them in place in the boats before the expcriment was started. These curves are not exact indices of the differences in rate of reaction at the various temperatures because the depth of penetration of adequate supplics of oxygen is not constant, but they are interesting nevertheless. There is not a major change in rate between the liquid state and the solid state at the melting point, which is about $110^{\circ} \mathrm{C}$, but there appears to be a falling off between $100^{\circ}$ and $90^{\circ}$. It is known that polyethylene is largely amorphous just below its melting point and that the degree of crystallinity increases with decreasing temperature, and this may account for the observed result.

It was observed by $\mathrm{V}$. T. Wallder and his associates in this Laboratory that carbon black retarded the lowering of viscosity of polyethylene during hot milling just as do chemical antioxidants. Oxygen absorption experiments, using the boat method, confirm the effect (fig. 9.5) and show that, in general, the channel blacks or high-acid blacks are more effective than the furnace blacks. Calcining the channel blacks lowers their effectiveness. It is interesting to note that the presence of carbon black produces an induction period which is finally overcome just as is the case with chemical antioxidants. It is known that carbon black is effective in deactivating free radicals, and presumably the antioxidant action is related to this property.

Study of the oxidation of polyethylene at high temperatures is important because the polymer is normally compounded and processed at temperatures above its melting point, but in service it is always

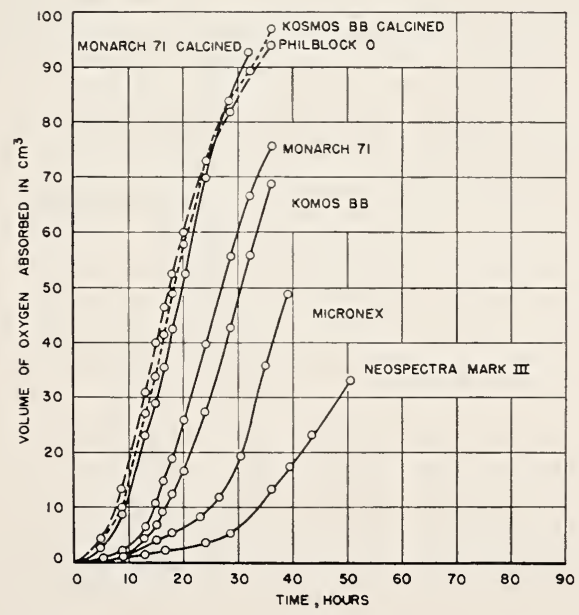

Figure 9.5. Polyethylene plus 1 percent of carbon black in oxygen.

One-gram samples in boats $5 / 5$ by $4 \frac{34}{4}$ inches. 
near the temperature of the atmosphere, and hence it is of great interest to see how it ages in the solid state.

As has been pointed out by Wallder [26], measurement of the lowtemperature brittle point is a convenient method of following the deterioration of polyethylene. By use of this method it has been found that a sample of unprotected polyethylene that has been stored in the dark at room temperature for $10 \mathrm{yr}$ still has a brittle point below $-76^{\circ} \mathrm{C}$, so its rate of oxidation under those conditions can be considered negligibly slow. At $90^{\circ} \mathrm{C}$ the rate is fast enough to be measured, and it is found that the brittle point of strips $\frac{1}{4}$ in. wide and $\frac{1}{16}$ in. thick was raised to above $-40^{\circ} \mathrm{C}$ by 500 -hr exposure to oxygen at that temperature. At this point the weight of oxygen absorbed is $0.0085 \mathrm{~g} / \mathrm{g}$. Samples containing antioxidant reached this brittle point after $5,500 \mathrm{hr}$. Brittle-point measurement by the impact method is quite sensitive to changes in the brittleness of the surface layer, and undoubtedly the deterioration of these samples was limited largely to the surface.

Antioxidants are extremely effective in lowering the rate of oxygen absorption of polyethylene even in the solid state. This is shown in figure 9.6, which presents oxygen absorption curves at $90^{\circ} \mathrm{C}$ for two samples of "pure" polyethylene representing different batches of material and for a third sample, which was supposedly pure polyethylene but which had been stored for about $2 \mathrm{yr}$ in a drawer that also contained inhibited material. The trace of antioxidant that migrated to the pure material was enough to produce a very long induction period. All these samples were in the form of shavings 1 mil thick. Even fresh samples of pure polyethylene vary considerably in the length of the induction period probably because of traces of impurities picked up during processing operations. Thus, of the two samples studied in this experiment, one had an induction period of about 7 days and the other of about 12 days. Such behavior is the common experience with small molecules that have been given only superficial purification, so it is not surprising to find it with a polymer which cannot normally be given any effective purification.

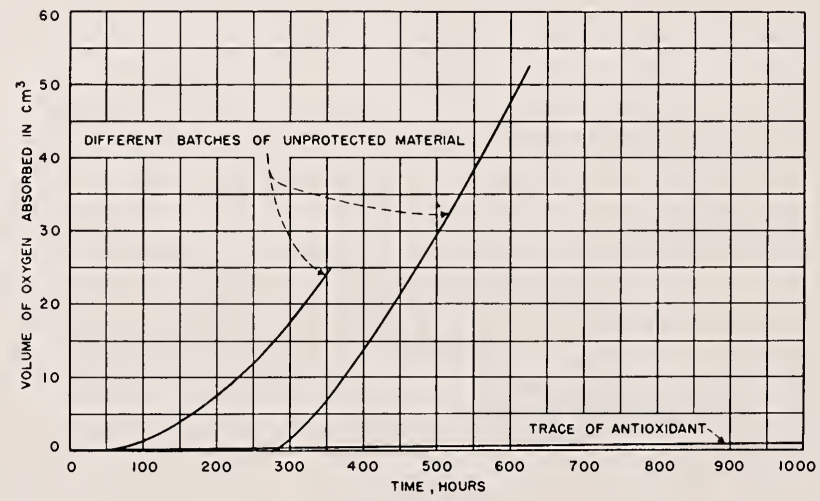

Figure 9.6. Polyethylene in oxygen at $90^{\circ} \mathrm{C}$ at $135^{\circ} \mathrm{C}$. One gram of shavings 1 mil thick. 


\section{Photo-Oxidation}

The evidence cited above indicates that for applications at normal temperatures and not involving exposure to light, properly inhibited polyethylene can be expected to last indefinitely. Such is not the case when the material is to be used outdoors. Polyethylene free of protective agents is deteriorated beyond use by a few months' exposure to summer sunshine in New Jersey. A sample of unprotected polyethylene, which had been exposed outdoors for about $3 \mathrm{gr}$ and had become quite brittle (fig. 9.7) was analyzed for oxygen by difference and was found to contain 3 percent of oxygen. Even heary dosages of antioxidant do not prolong the life of polyethylene to more than 1 or 2 yr. Apparently, the rate of initiation of chains by sunlight is high enough to orerwhelm any practical amount of antioxidant, particularly because the antioxidant is also being destroyed at the same time by its own independent oxidation.

While the most reliable information on outdoor exposure of various polyethylene compounds obviously is obtained br actual outdoor test (fig. 9.8) and this constitutes a necessary adjunct to any program in the study of deterioration, this method is too slow to use in guiding development work. Wallder and his associates have found that a bare carbon arc can be used to great advantage as an accelerated test for polyethylene, and they justify this use of light waves shorter than are found in sunlight by an empirical calibration against $10 \mathrm{yr}$ of outdoor exposure. They arrived at a rough correlation that states that 100 hr of exposure to the bare carbon arc under prescribed conditions is equal to $1 \mathrm{gr}$ of exposure outdoors. The test is probably less fair to transparent samples than to opaque ones but that is unimportant since only the opaque ones have any practical ralue for outdoor use.

The rapid photo-oxidation of polvethylene outdoors is surprising when one considers that it is a saturated aliphatic hydrocarbon and therefore should not absorb light of the solar spectrum. Octadecane, for example, is not measurably oxidized at room temperature by exposure to ultraviolet light. The light absorption curve of polyethylene has been reported by Maibauer and Myers [27] and by Pross and Black [28], and shows a low but definite absorption throughout the lower end of the solar spectrum, although there are no apparent resonant peaks above $2500 \mathrm{~A}$. Whether this absorption is due to very low concentrations of known groups is not established. Pross and Black attribute it to small traces of carbonyl. In any case, it can be demonstrated in the laboratory that polyethylene, in contrast with octadecane, oxidizes at room temperature under exposure to ultra riolet light.

It has already been stated that there is little hope of giving polyethylene a usefully long outdoor life by the use of chain-stopping additives alone. It is necessary to shut off the light. This has been done with some success in other polymers by use of organic absorbers of ultra violet light such as phenyl salicylate [29] and dihydroxybenzophenone [30], but these substances have been found of little practical benefit in polyethylene. Studies have been made of the protective effect of pigments and a few have been found helpful, notably lead chromate, iron oxide, and carbon black. The last is by far the best. 


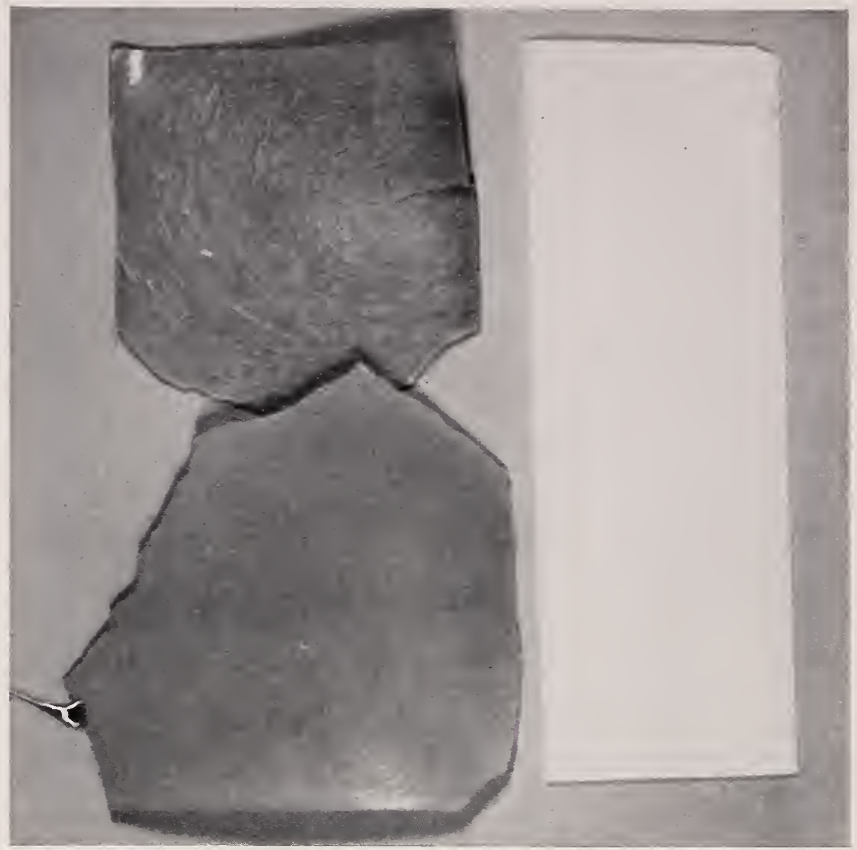

FIGURE 9.7. Samples of poyethylene aged 3 years outdoors compared with the original.

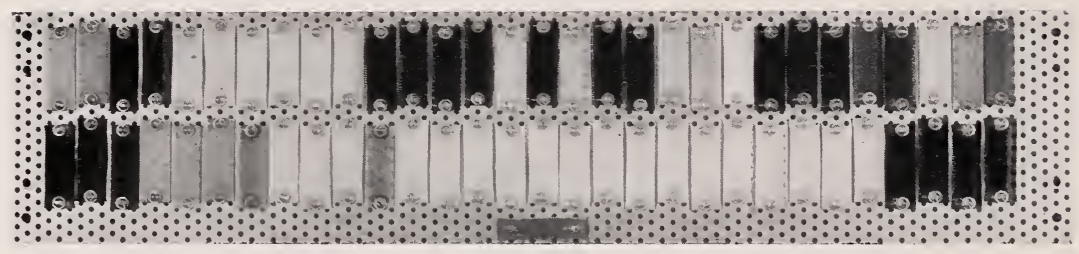

Figure 9.8. A typical test panel containing miscellaneous polyethylene compounds after 1 year of exposure at Yuma, Ariz.

Wallder, Clarke, DeCoste, and Howard [26] have published the results of an extensive study of the protection of polvethylene by carbon black, and their paper should be consulted for details. They show that 2 percent of a finely dirided black well dispersed in the polymer increases the life in accelerated tests at least thirty fold. 'They also report actual outdoor aging of $9 \mathrm{yr}$, and show that samples containing 1 percent of black are still in good condition after that period of exposure in Florida. On the basis of this work, a polyethylene compound containing 0.2 percent of antioxidant and 2 percent of carbon black has been used in large amount as a sheath for outdoor cable. Because the oldest samples of this cable have been out only $4 \mathrm{yr}$, it is too early to know what its useful life will be. 


\section{Summary}

The thermal oxidation of polyethylene is found to follow the pattern set by lower homologues, such as paraffinic waxes and oils. It is an autocatalytic free radical chain reaction and is subject to inhibition by typical antioxidants. Oxygen absorption curves for inhibited and uninhibited samples are shown for temperatures both above and below the melting point. The rate of degradation in the dark at room temperature is found to be extremely low.

Photo-oxidation of polyethylene is rapid in contrast with that of saturated low molecular weight aliphatic hydrocarbons. Furthermore, antioxidants are of little benefit in exposure to light. Opaque pigments are of great value in reducing the effects of light, finely divided carbon black being particularly effective. By proper compounding polyethylene can be made to last many years outdoors.

This outline of the oxidation of polyethylene cites several experiments that have been carried out by my associates, and I acknowledge my indebtedness to them. The published work of Wallder, DeCoste, Howard, and Clarke is referred to in the text. Experiments by J. Crabtree, W. L. Hawkins, and R. H. Erickson will be published in greater detail at an appropriate time.

\section{References}

[1] F. Francis, J. Chem. Soc. 121, 496 (1922).

[2] F. Francis, W. F. Millard, C. H. Rutt, C. M. Watkins, R. W. Wallington, and C. P. Garner, J. Chem. Soc. 125, 381 (1924).

[3] F. Francis and H. F. Gauntlett, J. Chem. Soc. 2377 (1926).

[4] F. Francis and N. E. Wood, J. Chem. Soc. 1897 (1927).

[5] R. T. Haslam and P. K. Frolich, Ind. Eng. Chem. 19, 292 (1927).

[6] R. W. Dornte, Ind. Eng. Chem. 28, 26 (1936).

[7] J. C. Balsbaugh and J. L. Oncley, Ind. Eng. Chem. 31, 318 (1939).

[8] G. H. Von Fuchs and H. Diamond, Ind. Eng. Chem. 34, 927 (1942).

[9] R. G. Larsen, R. E. Thorpe, and F. A. Armfield, Ind. Eng. Chem. 34, 183 (1942).

[10] H. S. Taylor and J. O. Smith, J. Chem. Phys. 8, 543 (1940).

[11] M. S. Karasch and M. Z. Fineman, J. Am. Chem. Soc. 63, 2776 (1941).

[12] H. N. Stephens, J. Am. Chem. Soc. 48, 2920 (1926).

[13] N. G. Lovell, J. M. Campbell, and T. A. Boyd, Ind. Eng. Chem. 23, 26 (1931).

[14] J. C. Pope, F. J. Dykstra, and G. Edgar, J. Am. Chem. Soc. 51, 2209 (1929).

[15] A. D. Walsh, Trans. Faraday Soc. 42, 269 (1946).

[16] W. M. D. Bryant, J. Polymer Sci. 2, 550 (1947).

[17] J. J. Fox and A. E. Martin, Proc. Roy, Soc. (London) [A] 175, 226 (1940).

[18] H. W. Thompson and P. Torkington, Trans. Faraday Soc. 41, 248 (1945).

[19] E. H. Farmer, G. F. Bloomfield, A. Sundralingham, and D. A. Sutton, Trans. Faraday Soc. 38, 348 (1942).

[20] J. L. Bolland, Proc. Roy. Soc. (London) [A] 186, 218 (1946).

[21] L. Bateman and G. Gee, Proc. Roy. Soc. (London) [A] 195, 376 (1949).

[22] J. L. Bolland and P. TenHave, Trans. Faraday Soc. 45, 93 (1949).

[23] H. N. Stephens, J. Am. Chem. Soc. 5\%, 2380 (1935).

[24] P. George and A. D. Walsh, Trans. Faraday Soc. 42, 94 (1946).

[25] L. Bateman, Trans. Inst. Rubber Ind. 26, 246 (1950).

[26] V. T. Wallder, W. J. Clarke, J. B. DeCoste, and J. B. Howard, Ind. Eng. Chem. 42, 2320 (1950).

[27] A. E. Maibauer and C. S. Myers, Trans. Electrochem. Soc. 90, 449 (1946).

[28] A. W. Pross and R. M. Black, J. Soc. Chem. Ind. 69, 113 (1950).

[29] L. W. A. Meyer and W. M. Gearhart, Ind. Eng. Chem,. 3\%, 232 (1945).

[30] R. F. Boyer, U. S. Paterit 2,429,155. 


\section{Discussion}

Dr. J. E. Wilson, Bakelite Co.: Dr. Biggs has mentioned that there is kinetic evidence that chain scission occurs in the $\mathrm{RO}_{2}$. radical. It might be of interest to summarize some of this evidence very briefly, most of which was obtained by Bolland, Bateman, and Gee in England. The essential oxidation mechanism is

\section{Initiation:}

Propagation:

Production of $\mathrm{R} \cdot$ or $\mathrm{RO}_{2}$. radicals

$$
\begin{gathered}
\mathrm{R} \cdot+\mathrm{O}_{2} \longrightarrow \mathrm{R} \mathrm{O}_{2} . \\
\mathrm{R} \mathrm{O}_{2} \cdot+\mathrm{RH} \longrightarrow \mathrm{R} \mathrm{O}_{2} \mathrm{H}+\mathrm{R} .
\end{gathered}
$$

Termination:

$$
\left.\begin{array}{r}
\mathrm{R} \cdot+\mathrm{R} \cdot \longrightarrow \\
\mathrm{R} \cdot+\mathrm{R} \mathrm{O}_{2} \cdot \longrightarrow \\
\mathrm{R}_{2} \cdot+\mathrm{R} \mathrm{O}_{2} \cdot \longrightarrow
\end{array}\right\} \text { xonradical products. }
$$

In the mechanism the $\mathrm{RO}_{2}$. radical reacts with the hydrocarbon to remove a hydrogen atom, form a hydroperoxide, and regenerate an $R$. radical. The $R$. radical mar combine with oxrgen, attack the $\mathrm{RH}$, and go through the crcle again.

The chain scission efficiency is the ratio of the number of chain scissions to the number of molecules of oxygen taken up. In order to determine where chain scission is occurring in the reaction mechanism it is necessary to consider the effect of specific changes in the nature of the initiation, termination, or propagation step on the chain scission efficiency.

The effect of a change in the initiating step can be determined by making a change in the peroxide used as initiator. But in the case of polyisoprene, for example, an oxidation initiated by either benzoyl peroxide or polvisoprene peroxide has the same scission efficiency. This would indicate that a profound change in the initiation step does not affect the scission efficiency, and hence that scission does not occur in the initiation step.

Turning next to the termination step, it is possible to alter this step by the introduction of an antioxidant. It is generally accepted that an antioxidant such as $\alpha$-naphthol inhibits oxidation by introducing an additional termination step. But it has been found that the scission efficiency remains unchanged when the mode of termination is altered by $\alpha$-naphthol, implying that chain scission does not occur in the termination step.

Finally, considering the propagation step, the propagation is carried out by $\mathrm{R}$ - and $\mathrm{RO}_{2}$. radicals. The $\mathrm{R}$-radical, in carrying on the chain, combines with oxygen in a reaction so rapid that the average lifetime of the $\mathrm{R}$. radical is only $10^{-8} \mathrm{sec}$. The $\mathrm{RO}_{2}$. radical, on the other hand, snaps the $\mathrm{H}$ atom from the hydrocarbon, which is believed to be the slow and rate-determining step of the oxidation. Thus the $\mathrm{RO}_{2}$. radical has a much longer lifetime, of approximately $10^{-2} \mathrm{sec}$, and a much greater chance to decompose. Other things being equal, the probability of spontaneous scission will thus be one 
million times greater for the $\mathrm{RO}_{2}$. radical. Further evidence on the decomposition of the $\mathrm{RO}_{2}$. radical was obtained by introducing 1 percent of mercaptobenzthiazole or 2,2'-dibenzamidodiphenyl disulfide in polyisoprene. The former reduces oxygen absorption while the latter has no effect on oxidation rate, but both compounds produce chain scission or softening of the polyisoprene. The reaction of such materials with $\mathrm{RO}_{2}$. radicals is highly probable, especially in view of the well-known oxidation of sulfides by peroxides or hydroperoxides.

Thus, the facts on hand indicate that chain scission does not occur in the initiation or termination step, but that it may well occur by some sort of decomposition of the $\mathrm{RO}_{2}$. radical.

Dr. H. J. Ratri, National Lead Co., Brooklyn, N. Y.: We have found that the acetylene black has an antioxidant or inhibiting effect, indicating that it is an exception to the idea that furnace blacks are not as effective.

Dr. R. B. Richards, Imperial Chemical Industries, Ltd.: I would like to mention one or two points arising from our own work on thermal oxidation of polyethylene which is related to Dr. Biggs' paper. First of all, about the change at the melting point. We also have studied the rate of oxidation of polyethylene in the melted and in the solid state almost exactly the same as Dr. Biggs - on a glass sheet in the molten state up to about $200^{\circ} \mathrm{C}$ and in thin films down to temperatures of about $75^{\circ} \mathrm{C}$. We found no apparent discontinuity in the oxidation rate at the melting point. We also carried out some experiments in the range $35^{\circ}$ to $95^{\circ} \mathrm{C}$ in which the film was immersed in water. This had no effect on the oxidation rate. The water still permitted oxygen to get at the polyethylene at the rate at which the oxidation rate required it to be there.

Second, about the point of attack. We have measured the rate of oxidation in the melted and the solid states of a series of polymers of different degrees of chain branching. The general effect observed is, as Dr. Biggs suggested, that the more branched polymers oxidize more readily.

We have observed both in the melted and in the solid state that oxidation is autocatalytic. If you plot the log of the oxygen content against time, you get a straight line up to an oxygen content of 3 or 4 percent of oxygen. It then tails off. If you oxidize to a certain extent, stop the experiment, put the sample away in a drawer for a month, start oxidizing again later on, it will start again at exactly the same rate as when you left off. It is tempting to suggest that the formation of a peroxide is indicated here, but I feel that we need rather more definite evidence. One suggestion I could make is that the carbonyl group, which is the main group found in thermally oxidized polyethylene, is the center for further oxidation. To test that, we made a copolymer of ethylene containing a very small portion of carbon monoxide and found that it oxidized at an initial rate similar to that of a partially oxidized polyethylene of the same oxygen content. That led us to believe that when you put in a carbonyl group, presumably by oxidation, there is further rapid oxidation occurring at that point, leading to a rate of oxidation proportional to the oxygen content and hence to a linear log [O]-time plot.

We have tried to follow the build-up of peroxide in thermal oxidation of low molecular weight polyethylene. We can certainly detect 
it, but the peroxide content seems to remain approximately constant at a very low value.

Dr. Biggs: I cannot comment very extensively on the point Dr. Richards makes. I think his suggestion about the carbonyl effect is a good one. We have measured peroxides in an oxidized polyethylene as I have mentioned and have found quite substantial amounts but we have not followed it from sample to sample with time to see whether it is building up. I would like to ask, though, if this is necessary. Might you not expect it to be decomposing and showing up finally as carbonyl? And might you not expect that the concentration of peroxide would never get very high? 


\title{
10. Photo-oxidation and Stabilization of Polythene
}

\author{
By A. R. Burgess ${ }^{1}$
}

The break-down of polythene in sunlight is due to an oxidative chain reaction. The chain is initiated by the photolysis of carbonyl groups to form free radicals, and one of the main products is more carbonyl groups. The chain length is probably very short ( $<10$ in strong sunlight) and this provides one explanation for the failure of the thermal antioxidants to prevent the photo-oxidation. Also many of the thermal antioxidants absorb ultraviolet radiation powerfully, and it is believed that the energy so absorbed can be used to sensitize the oxidation.

The conditions under which a light absorber will act as a sensitizer or a stabilizer and possible mechanisms for sensitization are considered. Besides having high absorptive power for the damaging radiation, the stabilizer must be able to dispose of the energy harmlessly. Visible color may be necessary for it to do this as efficiently as carbon black.

\section{Introduction}

The phenomenon of the photochemical break-down of polythene when exposed outdoors has been fully described by Dr. Biggs in the preceding paper. The most surprising aspect of the photo-oxidation is that the phenolic and amino antioxidants that suppress thermal oxidation during processing $\left(140^{\circ}\right.$ to $\left.200^{\circ} \mathrm{C}\right)$ have very little effect. Some of the antioxidants even accelerate the photodegradation. So far the only satisfactory solution to the problem of protecting polythene from sunlight is the incorporation of 1 to 2 percent finely divided carbon black. Black is not a desirable color in some applications and the object of the work described in this paper was a sufficient understanding of the mechanism to explain the failure of the thermal antioxidants and to explore other possible methods of stabilization to sunlight. Our general conclusions are probably applicable to other light-sensitive polymers as well.

\section{Mechanism of Photo-oxidation}

It is not generally realized what a powerful potential initiator of chemical change intense tropical sunshine is. In figure 10.1, we see that the ultraviolet tail of the sun's spectrum extends down to 2950 $A$, where one quantum is $95 \mathrm{kcal} /$ mole. This is sufficient to break most single bonds and is some 3 to 4 times the activation energy of many thermochemical reactions. Even yellow light quanta are 50 $\mathrm{kcal} / \mathrm{mole}$. It is also clear from figure 10.1 that a mercury arc lamp (Hanovia $500 \mathrm{w}$ screened with $0.5-\mathrm{cm}$ Prrex to eut off at $2900 \mathrm{~A}$ ) is weak compared with intense sunshine, except in the 3000-A region.

Saturated hydrocarbons do not absorb radiation of wavelengths longer than $2000 \mathrm{~A}$, so we must attribute the light absorption to groups foreign to the basic $\left(\mathrm{CH}_{2}-\mathrm{CH}_{2}\right)_{n}$ structure. Both the thermal and photo-oxidation lead to aldehydic or ketonic earbonyl groups that are detectable in the infrared spectra of most virgin polythenes and all processed polythenes. Because aldehydes and ketones absorb and

\footnotetext{
${ }^{1}$ Imperial Chemical Industries, Ltd., Welwyn Garden City, England.
} 
TABLE 10.1. Quantum efficiency of carbonyl formation of light radiation

\begin{tabular}{|c|c|c|}
\hline Wavelengths & Intensity & $\begin{array}{c}\text { Quantum } \\
\text { efficiency of } \\
\text { carbonyl } \\
\text { formation }\end{array}$ \\
\hline$A$ & & \\
2537 & $\begin{array}{c}\left(\text { Quanta/cm } / \mathrm{cm}^{2}\right) / \mathrm{sec} \\
3.0 \times 10^{15}\end{array}$ & 0.04 \\
2500 to 3500 & 0.9 & .11 \\
3500 to 4500 & 8.0 & .00 \\
\hline
\end{tabular}

are broken down to free radicals by wavelengths shorter than about $3300 \mathrm{~A}$, we attribute the sensitization of polythene to them. We suppose that the free radicals so formed combine with oxvgen giving the normal chain reaction leading to peroxides. As peroxides are not found in the oxidized polythene to any great extent, they presumably break down to carbonyl groups. These conclusions are supported by a number of experiments. Thus, only light within the carbonyl absorption region brings about photo-oxidation (table 10.1).

The products of photo-oxidation also support the idea that carbonyl photolysis is important. Besides the normal oxidation products, such as oxides of carbon and water, acetaldehyde and acetone are found in the gas phase and vinyl groups in the polymer. These probably arise from the Norrish type II decomposition of carbonyl groups, which is known to take place parallel to the type I free radical decomposition. Thus, for an aldehyde group:

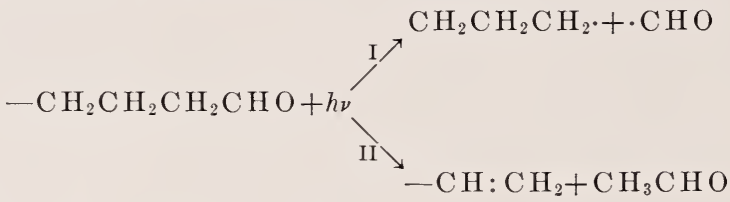

Figure 10.2 shows the growth of carbonyl, hydroxyl, and vinyl groups in a sample of polythene using monochromatic $3130 \mathrm{~A}$ radiation, and the carbonyl increase appears to be linear with time in the early stages. Oxygen uptake can also be followed and is likewise linear.

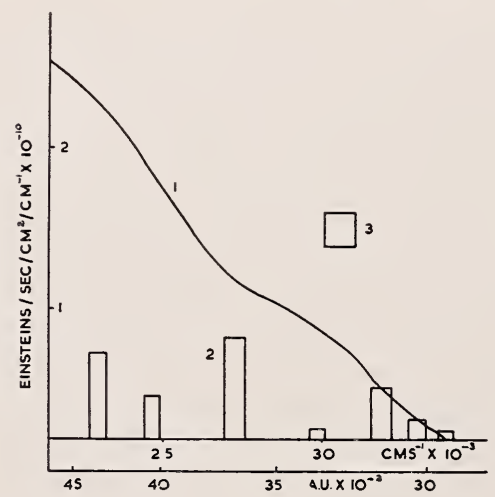

Figure 10.1. Energy distribution of sunlight and mercury-vapor arc.

1, Noon summer sun at Cragmor, Colo. (Greider and Downes, Trans. Illum. Eng. Soc. 25, 561 (1931)); 2, intensity $10 \mathrm{~cm}$ from $500-w$ Hanovia arc behind $0.5 \mathrm{~mm}$ of Pyrex; 3 , square represents $10^{16}\left(\right.$ quanta $\left./ \mathrm{cm}^{2}\right) / \mathrm{sec}$. 
The rate of oxygen uptake is roughly proportional to the square root of the incident light intensity, which again is support for a free radical initiation. Figure 10.3 shows the calculated quantum efficiencies of carbonyl formation at various stages in the same experiment as figure 10.2. It is noteworthy that it drops very rapidly to a value of about 0.1 .

Bamford and Norrish ${ }^{2}$ found a primary quantum efficiency of 0.01 for the photolysis to free radicals of di-n-propyl ketone in the liquid at $20^{\circ} \mathrm{C}$. If we adopt this figure for $\phi$, the quantum efficiency of initiation, and our figure of 0.1 for the over-all quantum efficiency, $\Phi$, then substituting in the equation $\Phi=\lambda \phi$, we obtain for $\lambda$, the kinetic chain length, the value 10 . Hence, the oxidation chains are short, even at the low intensity used in these experiments. Since $\lambda$ should be an inverse function of light intensity, it is clear that the chain length will probably be very much smaller in intense sunlight. Here we have the fundamental explanation for the spectacular failure of the chain-stopping antioxidants, that the rate of initiation is so high and the chains so short that effective inhibition is impossible. The relative immobility of the antioxidants in the solid phase may also contribute.

This cannot be the whole story, as it does not explain the fact that some thermal antioxidants actually promote photo-oxidation. Many thermal antioxidants of the amine class are in fact powerful absorber's of ultraviolet radiation. Although pure phenol itself does not absorb in the sunlight region, the maximum in the absorption spectrum is pushed to longer wavelengths by nuclear substitution and many typical phenolic antioxidants do absorb sunlight. Incorporation of 1 percent of an amine antioxidant can put the absorption of the damaging radiation by a $1 \mathrm{~mm}$ sheet of compositon from a fraction of 1 percent to nearly 100 percent. If only 1 percent of this extra energy is used to promote break-down, the oxidation will be increased manyfold.

2 J. Chem. Soc. 1544 (1938).

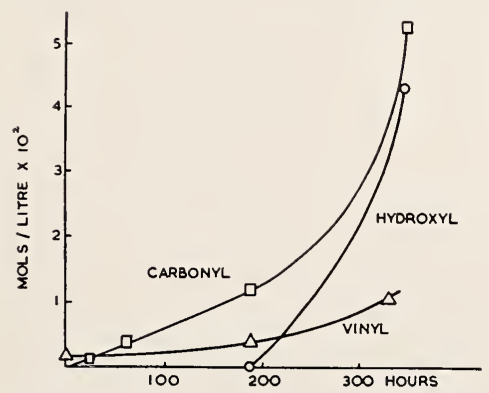

Figure 10.2. Photo-oxidation of polythene.

Increase of concentration of products with time. Monochromatic $3130-\mathrm{A}$ radiation of intensity $2 \times 10^{14}\left(\right.$ quanta $\left./ \mathrm{cm}^{2}\right) / \mathrm{sec}$.

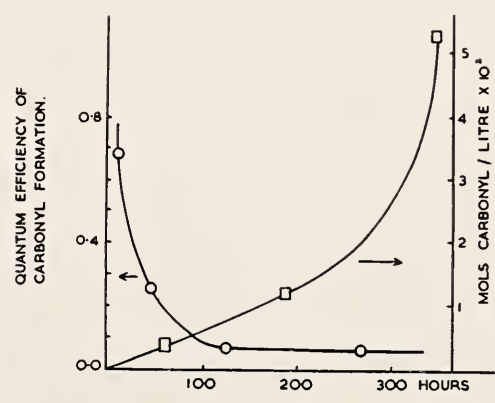

Figure 10.3. Photo-oxidation of polythene.

Same conditions as in figure 10.2. 


\section{Light Absorption and Stabilization}

We concluded at this stage that because of the difficulty of stopping the short oxidation chains once they had started, it was better to try and prevent them from starting at all. This could only be done by absorbing the damaging radiation with a more powerful light absorber than carbonyl groups. It was clear howerer that the absorber would have to have special properties otherwise it might, like some of the antioxidants, be a sensitizer rather than a stabilizer.

It should be pointed out that the classification of a light absorber as a sensitizer or a stabilizer depends upon the method of test employed. Figure 10.4 shows how the distribution of oxidation through the thickness of the sample is changed by the incorporation of a sensitizing material, and figure 10.5 shows the same effect integrated up to various thicknesses. It is clear that the surface tends to be sacrificed to protect the bulk of the material. Hence the over-all effect will depend on the thickness of the sample and on the method of test. A bend test will tend to show up surface deterioration, whereas power factor or light radiation transmission will measure only the arerage orer the thickness of the sample. So long as the rate is proportional to the square root of the light intensity, even a light absorber which is 100 percent efficient at sensitizing would be rated a stabilizer at sufficient thickness using, say, a power factor test. Another result of these considerations is that even a powerful nonsensitizing light absorber cannot protect the very surface layer of the material.

An experiment illustrating the thickness effect is shown in figure 10.6,

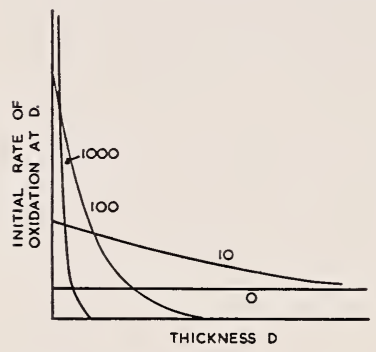

Figure 10.4. Effect of 100 percent-efficient light sensitizer on distribution of initial rate of oxidation through a slab of polymer.

The absorption of the polymer is assumed to be small. Absorption by sensitizer/absorption by polymer ratio is indicated by figures a)tached to curves.

FigLre 10,6. Effect of various light absorbers on distribution of photooxidation in a slab of polythene.

1, No additive; 2, 1 percent of phenyl betanaphthylamine; 3,1 percent of triphen rlamine 4, 1 percent of diphenyl alpha-naphthylamine.

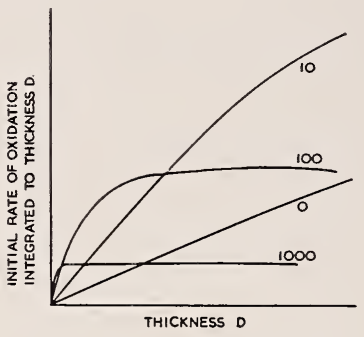

Figure 10,5. Curves of figure 10.4 integrated with respect to thickness.

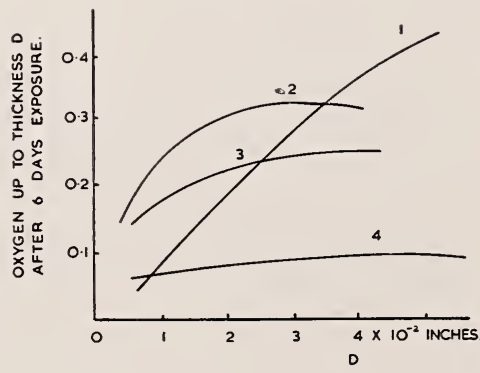


and the curves do resemble in form those predicted theoretically. They also illustrate the sensitizing action of a typical amine antioxidant, phenyl $\beta$-naphthylamine. This sensitizing effect is considerably reduced when the reactive hydrogen is substituted, so we are led to the conclusion that labile hydrogen of the secondary amine can undergo reaction (2), the $\mathrm{HO}_{2}$. radical starting a chain:

$$
\begin{aligned}
\mathrm{C}_{10} \mathrm{H}_{7} \mathrm{NHC}_{6} \mathrm{H}_{5}+h \nu & \longrightarrow\left(\mathrm{C}_{10} \mathrm{H}_{7} \mathrm{NHC}_{6} \mathrm{H}_{5}\right)^{*} \\
\left(\mathrm{C}_{10} \mathrm{H}_{7} \mathrm{NHC}_{6} \mathrm{H}_{5}\right)^{*}+\mathrm{O}_{2} & \longrightarrow \mathrm{C}_{10} \mathrm{H}_{7} \mathrm{~N} \cdot \mathrm{C}_{6} \mathrm{H}_{5}+\mathrm{H} \mathrm{O}_{2} .
\end{aligned}
$$

We have examined a large number of ultraviolet absorbing substances as possible light stabilizer's for polythene ${ }^{3}$ and have concluded that even compounds that do not contain labile hydrogen atoms can sensitize polythene photo-oxidation. To account for this, we have to postulate additional chain starting reactions such as (3), (4), and (5).

$$
\begin{gathered}
\mathrm{AH}^{*}+\mathrm{O}_{2} \longrightarrow \mathrm{A}+\mathrm{HO}_{2} . \\
\mathrm{AH}^{*} \longrightarrow \text { free radicals } \\
\mathrm{AH}+\mathrm{RH} \longrightarrow \mathrm{A} \mathrm{H}_{2} \cdot+\mathrm{R} .
\end{gathered}
$$

A H* + carbonyl $\longrightarrow$ A H + carbonyl* $\longrightarrow$ free radicals.

There is evidence for all these reactions with particular compounds, both in the photo-oxidation of polythene and in other photochemical reactions.

To prevent the excited light absorber $\mathrm{AH}^{*}$ from entering into chemical reactions it is necessary for it to lose its electronic energy rapidly. Our experience is that materials which fluoresce in the visible or ultraviolet are in general sensitizers for polythene, so clearly fluorescence is too slow to compete with reactions (2) to (5). Again this is in line with evidence from other photoreactions. We need a rapid loss of electronic energy as thermal energy, ${ }^{4}$ either right down to the ground state or to a level that is relatively inactive, say $\mathrm{AH} \ddagger$. 'The electronic energy of this lower level could then be lost by fluorescence. This is illustrated by the energy diagram in figure 10.7; either

or

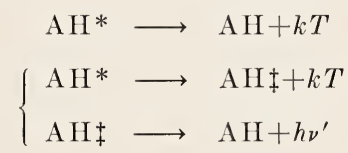

From the large number of compounds tested, no simple general rules have emerged except that on the whole soluble compounds are better than nonsoluble (table 10.2) and fluorescent compounds are sensitizers (table 10.3).

Table 10.2 shows that all the "good" stabilizers $(S>10)$ were soluble in cold paraffin, although clearly this is an insufficient condition.

\footnotetext{
3 The compositions were tested by exposing them to the light of a 500-w Hanovia lamp screened with 0.5-mm Pyrex to eut out radiation below $2900 \mathrm{~A}$ (fig. 10.1). Pressed sheet san ples of 20 -mil thickness were rotated round the lamp at a distance of $10 \mathrm{~cm}$ and kept conl $\left(25^{\circ} \mathrm{C}\right)$ by air suction which also prevented ozone diffusing to the samples. The increase in carbonyl was followed by infrared. The test does not claim to be an "accelerated weathering test", but merely to reproduce the relevant part of the sun's spectrum in the laboratory.

4 The internal conversion of electronic to thermal energy in complex molecules is a fascinating field of study and highly relevant to our considerations. Reference should be made to the recent work of Kasha, Discussions of the Faraday Society 9, 14 (1950).
} 


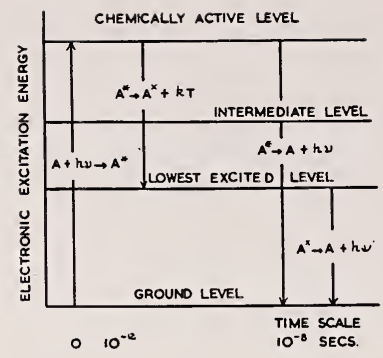

Figure 10.7. Energy diagram for light absorber with internal conversion of electronic to thermal energy.

TABLE 10.2. Light stabilization versus solubility of light absorber

Number of compounds falling in various classes

\begin{tabular}{|c|c|c|c|}
\hline \multirow{2}{*}{$\begin{array}{c}\text { Stabilization } \\
\text { factor }(S)^{\mathrm{a}}\end{array}$} & \multicolumn{3}{|c|}{ Solubility in paraffin oil } \\
\cline { 2 - 4 } & Soluble cold & Partly soluble & Insoluble \\
\hline$>10$ & 6 & 0 & 0 \\
\hline 1 to 10 & 1 & 9 & 6 \\
\hline 10 & 2 & 1 & 8 \\
\hline
\end{tabular}

a $S=$ (increase in carbonyl in blank)/(increase in earbonyl in composition with 1 percent of additive).

TABLE 10.3. Light stabilization versus fluorescence of absorber

Number of compounds falling in various classes

\begin{tabular}{|c|c|c|}
\hline \multicolumn{1}{|c|}{$S$} & $\begin{array}{c}\text { Fluores- } \\
\text { cent }\end{array}$ & $\begin{array}{c}\text { Nonfluo- } \\
\text { rescent }\end{array}$ \\
\hline$>10$ & 0 & 8 \\
\hline 1 to 10 & 0 & 20 \\
& 5 & 14 \\
\hline
\end{tabular}

It is remarkable that all the fluorescent materials were sensitizers $(S<1)$, although nonfluorescence does not guarantee stabilizing power.

We were not interested in materials of only moderate stabilizing power, say $S$ ranging from 1 to 10 , but only in those having a large effect. The highest $S$ values observed with 1 percent of carbon black were in the range 30 to 60 , and the only compounds which equalled it were certain metal chelate complexes of copper and nickel. The two most successful are

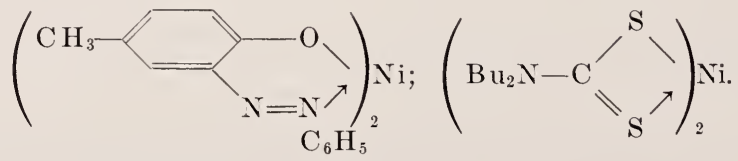

Unfortunately both of these compounds are highly colored and do not offer much advantage over carbon black. 
The fact that no really efficient colorless stabilizer has been found mav have fundamental significance. If we suppose that polythene will not tolerate an excited molecule having more than $70 \mathrm{kcal}$ electronic energy without chemical reaction eq (2) to (5), then clearly we must design our stabilizer to have its lowest excited level lower than $70 \mathrm{kcal}$ above the ground state. Transitions to this lowest excited state from the ground state will then lie in the visible, and the compound will be colored. The risible absorption merely indicates the existence of low energy states which will assist in the safe disposal of the chemical reactivity acquired by absorption of the dangerous radiation in the ultraviolet.

The problem of finding an ultraviolet absorbing stabilizer for a plastic is similar to that of finding nontendering dyes for fibers. Although the mechanism of degradation may well be different, it is interesting that yellow dyes are the most active sensitizers. These dyes absorb in the blue, say $60 \mathrm{kcal}$ above ground level. Other colors all have an excited level lower than this and are less efficient. Light stability can also be improved by chelation with metals, for example, "chroming" and "coppering".

\section{Conclusion}

Although likely stabilizers must still be tested by actual exposure and accelerated testing in polythene itself, we feel that further fundamental advances in our understanding of light stabilizers can only come about by a breakaway from polymers. By using a simpler liquid substrate like cyclohexene we can avoid the initial high temperature compo unding with the additive to be tested and get over the difficulties of nonsolubility, bad dispersion, and sweating-out. Further the reaction can be halted at the peroxicie stage and kinetic analysis becomes possible with the simpler reaction. For this reason we have taken up the study of the influence of molecular structure on photosensitizing action, using the system

Cyclohexene + light $+\mathrm{O}_{2}+$ light absorber $\longrightarrow$ cyclohexene hydroperoxide.

\section{Discussion}

Mr. H. A. Perry, Jr., Naval Ordnance Laboratory, White Ork, Md.: Are reflective rather than absorptive materials a possible solution?

Dr. Burgess: We have tried milling in powdered aluminum, but although it showed some effect, it was by no means so effective as carbon black.

Dr. B. S. Biggs, Bell Telephone Laboratories, Murray Hill, N. J.: We have wiped flake aluminum on the surface and obtained an improvement beyond that contributed by the carbon black alone.

Dr. W. L. Hawkiss, Bell Telephone Laboratories, Murray Hill, N. J.: Tetralin alpha-hydroperoxide can undergo a scission or can form tetralone. $\mathrm{By}$ the addition of mercaptobenzimidazole we changed the course of the reaction from 100-percent decomposition to tetralone to one in which 66-percent scission occurs with only 33-percent tetralone formed. We did not exclude light. It may be a factor in the work. 
Dr. R. F. Boyer, Dow Chemical Co., Midland, Mich.: If you expose a filament of polyvinylidene chloride to sunlight, in the beginning degradation occurs throughout but very quickly a dark material builds up on the surface which screens the inside of the sample. This surface layer can get very black and be just as effective as carbon black in screening the interior. The result is that the interior seals itself and such samples, depending on the thickness, may well retain 95 percent of their strength for years. I recently examined some filaments that had been outdoors for $8 \mathrm{yr}$ and they seemed just as strong and pliable as when they were first put out but they were jet black. Your curves give a perfect explanation of that.

One other interesting analog of what you found occurs in the case of stabilizer's for vinylidene chloride, for which very good ultraviolet stabilizers are 2,2-dihydroxybenzophenone and some of the orthonitrophenols. These molecules presumably gain stability to ultraviolet radiation because of internal hydrogen bonding. Since they are good ultraviolet absorbers, they protect the base polymer in which they are placed.

Dr. Burgess: We have investigated some nitrocompounds as stabilizers for polyethylene, but they were not very successful. We feel that this was to be expected, since they tend to act as oxidizing agents when illuminated and lose an oxygen atom, becoming reduced to nitroso compounds.

We have tried to follow up the sensitizing and stabilizing action of various light absorbers by examining their effect in a model system. Cyclohexene is a good choice because the kinetics of its oxidation have been fully worked out by Bateman of the British Rubber Producers Research Association. Using monochromatic 3600-A ultraviolet radiation, we have shown that benzophenone is a very efficient photosensitizer for cyclohexene oxidation. If one plots the rate of oxygen uptake against the square root of the absorbed light intensity, a good straight line is obtained, showing that the benzophenone is a pure initiator and does not interfere with the chain termination steps. This is in line with our observation that benzophenone, although a good ultraviolet absorber, accelerates the photodegradation of polyethylene. We imagine that oxidative chain initiation takes place in the following: way:

$$
\begin{aligned}
\phi_{2} \mathrm{CO}+h \nu & \longrightarrow \phi_{2} \mathrm{CO}^{*} \\
\phi_{2} \mathrm{CO}^{*}+\mathrm{HR} & \longrightarrow \phi_{2} \dot{\mathrm{CO}} \mathrm{H}+\mathrm{R} .
\end{aligned}
$$

The radical $R$. then starts the oxidation chain. If one irradiates benzophenone in alcohol solution, the dimer of the radical $\phi_{2} \dot{\mathrm{COH}}$ crystallizes out. The radical probably also dimerizes in our experiments, but the concentration we employed was very low, and no dimer' was isolated.

Now we had found that 2-hydroxybenzophenone, although not an outstanding stabilizer for polyethylene, was a far less efficient sensitizer. In the thicknesses we were using the over-all sensitization/ stabilization balance was about even. We assume that as the carbonyl in this molecule was already hydrogen-bonded to the hydroxyl, it 
would, when excited by ultraviolet, attack this hydrogen instead of the hydrogen of the hydrocarbon.
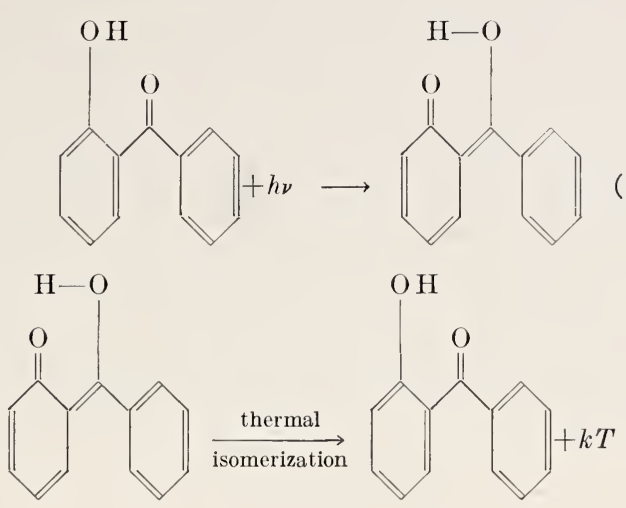

Net result:

$$
h \nu \rightarrow k T
$$

We need not assume that the quinonoid form (A) is completely formed; it is probably some resonance hybrid with the hydrogen more equally shared between the oxygens. However, we clearly have a mechanism here for disposing of the potentially harmful ultraviolet energy as less harmful thermal energy.

When we examined 2-hydroxybenzophenone in cyclohexene, we found that the behavior was by no means simple. A plot of rate ver'sus the square root of the absorbed light intensity fell rapidly off from the initial slope, which was less than that for benzophenone, and as the concentration was increased the rate eventually fell towards zero. This could not be accounted for by screening action in such low concentrations. We concluded that the 2-hydroxybenzophenone was acting in two ways: (1) As an inefficient sensitizer; its efficiency in starting chains is reduced by the mechanism outlined above. (2) As an oxidation inhibitor; as the concentration is increased the phenolic nature of the 2-hydroxybenzophenone may introduce an extra chaintermination step in addition to the normal mutual destruction of radicals. It is possible to account for the observed rate versus concentration curve by such a mechanism.

Mr. J. B. DeCoste, Bell Telephone Laboratories, Murray Hill, N. J.: Before we leave the subject of the oxidation of polyethylene, would Dr. Burgess want to say something regarding the cross linking which occurs quite quickly in the oxidation process and is evidenced by infusibility and insolubility in the polymer.

Dr. Burgess: Our appioach was that if we could stop the oxygen accumulating, then the improvement in the other properties would follow. We have not investigated cross linking as such.

Mr. DeCoste: Don't you have to account for cross linkages in your infrared analysis of the reaction products that form in polyethylene on photo-oxidation?

Dr. Burgess: I don't think they would show up. We have followed the cross linking at the same time as carbonyl and other things. You get cross linking occurring very quickly in the early 
stages with very little oxygen uptake. It is probably a carboncarbon link which does not show up in the infrared sp zctrum.

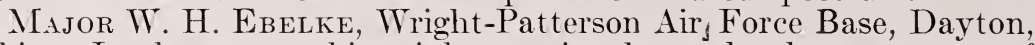
Ohio: Is there something inherent in the molecular structure of polyethylene which precludes the possibility of optical clarity?

Dr. E. H. Wood, Bakelite Co. Division of Union Carbide and Carbon Corp., Bound Brook, N. J.: In a microcrystalline material the crystals will scatter light and give a poor transparency.

Major Ebelke: Is there any possibility of preventing the formation of crystallites?

Dr. R. B. Richards, Imperial Chemical Industries Ltd.: Then you would not have the mechanical properties required. The only hope is to alter the way in which the crystalites arrange themselves. There are crystalline transparent polymers, but for some reason the crystals in polyethylene seem to arrange themselves in large aggregates rather too well oriented and too big for transparency. 


\title{
11. Oxygen-absorption Studies on Olefins with Structures Related to GR-S
}

\author{
By J. Reid Shelton ${ }^{1}$
}

\begin{abstract}
Phenyl-substituted olefins representing some structural units of GR-S have been oxidized with molecular oxygen at temperatures in the range of $70^{\circ}$ to $100^{\circ} \mathrm{C}$. The rate of oxidation appears to be a function of peroxide concentration and the concentration of unreacted olefin. Qualitative and quantitative analyses for the products of oxidation indicate the formation of such products as carbonyl, carboxyl, hydroxyl, ester, carbon dioxide, and water. Phenyl 1-propenyl ketone and benzoic acid have been isolated from the oxidation of 1-phenyl-2-butene. Higher temperatures produce lower concentrations of peroxide. Oxidation at the alpha methylene group is indicated, although some initial attack at the point of unsaturation is also probable. The oxidation products detected are probably the result of peroxide decomposition and secondary oxidation reactions. The data suggest that ketones may be oxidized to esters in the presence of peroxide.
\end{abstract}

\section{Introduction}

Oxidation studies carried out with polymeric materials are always complicated by the difficulty of isolation and identification of the oxidation products. Oxygen-absorption studies on natural and synthetic rubber, for example, are difficult to interpret for this reason. Consequently, it is often preferable to study simpler compounds with comparable structural units, and apply the information by analogy to the more complex polymer systems.

The oxidation of hydrocarbons, both saturated and unsaturated, has been studied by numerous investigators. Farmer and his coworkers [1], ${ }^{2}$ for example, have studied a number of olefins, including some related to natural rubber, and Bolland [2] has recently summarized the kinetic interpretations of their data. Chavanne and coworkers [3] identified some products of the oxidation of certain derivatives of cyclopentane and cyclohexane, and thus provided the evidence on which George and Walsh [4] based their interpretation of the possible mechanism of the oxidation reactions. Rust, Vaughan, and coworkers [5] have identified a number of products formed by the hydrogen bromide catalyzed oxidation of various types of hydrocarbons. Tobolsky, Metz, and Mesrobian [6] have related oxygenabsorption data with the extent of chain scission in certain polymeric hydrocarbons.

In spite of the extensive investigations which have been made, and the considerable body of information which has been built up regarding the oxidation of hydrocarbons, there is still much that is not yet established with respect to the sequence of reactions by which the various types of oxidation products are formed. Even in the case of relatively simple olefins, for example, the reactions and reaction products appear to be quite complex.

We have been studying the oxidation and aging of natural and synthetic rubber in this laboratory $[7,8]$, and as an aid to the interpretation of the data, we have simultaneously investigated the oxidation of certain olefins of comparable structure. In particular, we have studied some phenyl-substituted olefins with structures related to GR-S as illustrated in figure 11.1. (The deriation from the preferred

1 Case Institute of Technology, Cleveland, Ohio.

2 Figures in brackets indicate the literature references on p. 169. 
SOME POSSIBLE STRUCTURES IN A GR-S POLYMER CHAIN<smiles>CC=CCCC(CC)CC(O)CC=CCCC(C)O</smiles>

COMPOUNDS WITH SIMILAR STRUCTURAL RELATIONSHIPS<smiles>CC=CCCCc1ccccc1</smiles>

1-PHENYL - 4- HEXENE<smiles>CC=CCCCc1ccccc1</smiles>

1- PHENYL - 3 - PENTENE

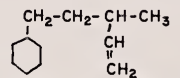

I- PHENYL - 3 - VINYLBUTANE

$\mathrm{CH}_{3}-\mathrm{CH}_{2}-\mathrm{CH}_{2}-\mathrm{CH}_{2}-\mathrm{CH}_{2}-\mathrm{CH}=\mathrm{CH}-\mathrm{CH}_{3}$

2- OCTENE

Figure 11.1. Comparison of a series of unsaturated compounds with some structural units in $G R-S$.

Figure 11.2. Oxygenabsorption apparatus.
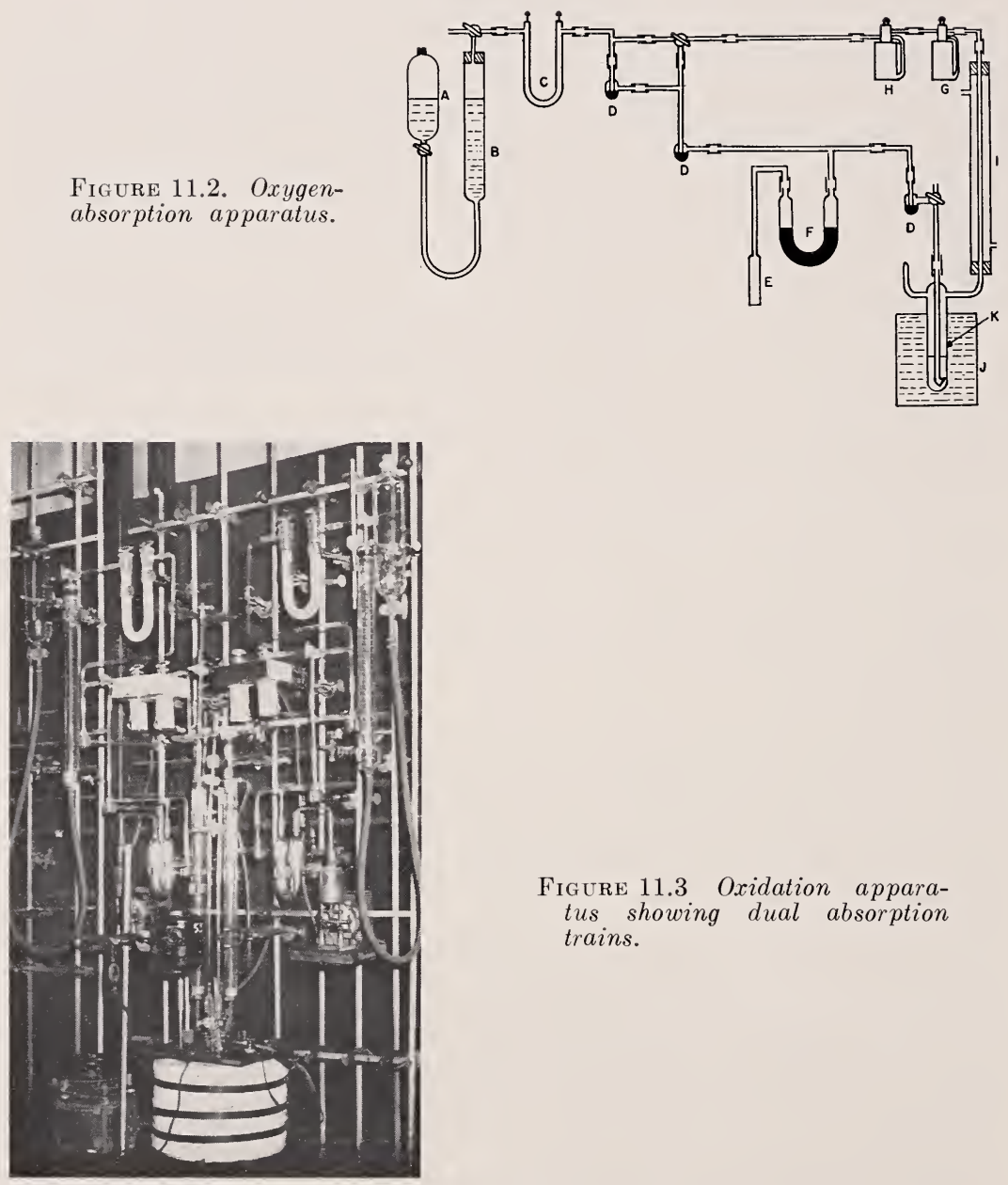

Figure 11.3 Oxidation apparatus showing dual absorption trains. 
IUC system of numbering in the names of these compounds is intended to emphasize the 1-2,1-3, and 1-4 relationship of the phenyl group and the double bonds.) It is the object of this paper to briefly review and summarize both the published $[9,10]$ and unpublished [11] data obtained in this study and to indicate the direction of our continuing investigation.

The equipment and procedure are described in the references cited above. A diagram of the apparatus is presented in figure 11.2, and a photograph of the actual setup is shown in figure 11.3. Oxygen is circulated by means of the pumping arrangement, $\mathrm{E}$ and $\mathrm{F}$, and the check valves, $D$, through the sample tube, $K$, which is maintained at constant temperature. The condenser, I, returns any condensible vapors, and tubes, $\mathrm{G}$ and $\mathrm{H}$, contain absorbents for water and carbon dioxide. Oxygen absorbed at constant temperature and pressure is measured by means of the gas burette, $B$.

\section{Oxidation Data}

Typical oxygen-absorption curves are shown in figure 11.4. The short induction period is barely discernible here as the uninhibited compounds rapidly enter the autocatalytic stage of rapid oxidation. The leveling off to a constant rate should not be confused with the constant-rate period observed with an inhibited rubber vulcanizate. These curves are rather comparable to those obtained with an uninhibited polymer. The leveling off results, at least in part, from a decrease in the concentration of unreacted material, and frequently there may also be a limitation of rate by a lower rate of diffusion of oxygen since the oxidized product becomes more viscous in the later stages of oxidation. Due to the uncertainty as to the magnitude of the contribution of the limitation by diffusion, the observed rates at the higher stages of oxidation may not be completely valid although they seem to be fairly reproducible.

All four olefins oxidized at about the same rate initially. The 1-phenyl-2-butene, in which the methylene group is alpha to both the double bond and the ring, appears to oxidize somewhat more extensively in the autocatalytic stage. The two phenylhexene isomers behave differently in that the 1-phenyl-3-vinylbutane levels off at a much lower rate. This may be due to the formation of a tertiary hydroperoxide, in the case of the isomer with the vinyl side chain, which would be expected to be more stable than the corresponding secondary hydroperoxide from the straight-chain isomer.

An aliphatic olefin, 2-octene, is shown in figure 11.5 to oxidize as rapidly as the phenylhexene in the autocatalytic stage, but the octene subsequently levels off at a much lower rate.

The lower rate of oxidation of the 1-phenyl-3-pentene as compared to 1-phenyl-4-hexene may be due to the fact that in the first case the two methylene groups, activited by phenyl and double bond, respectively, are adjacent, while in the phenylhexene, they are separated by a third methylene group. The over-all rate constants (see, for example, table 11.1) at $90^{\circ} \mathrm{C}$, were calculated to be 0.63 for 1 -phenyl3 -pentene, and 1.33 for 1-phenyl-4-hexene.

The effect of olefin concentration is demonstrated by the curves for phenylpentene at 80- and 98-percent purity. At comparable purity, however, the curves for the three olefins in figure 11.5 are 


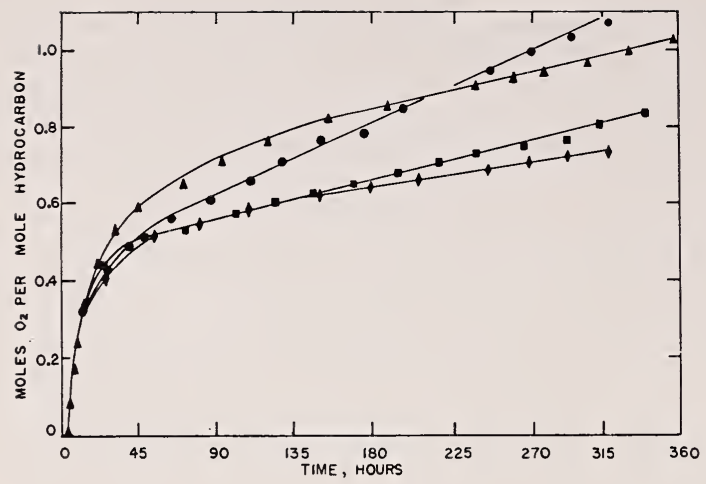

Figure 11.4. Tariation of oxygen absorption with time for a series of unsaturated hydrocarbons.

Temperature $100^{\circ} \pm 0.1^{\circ} \mathrm{C}$, pressure approximately $760 \mathrm{~mm}$. $\mathbf{\Delta}$, 1-Phenyl-2-butene; O, 1-phenyl4-hexene; 1-phenyl-3-vinyl butane, $\$$ - -phenyl-3-pentene.

Figure 11.5. Effect of structure and purity on oxygen absorption at $100^{\circ} \mathrm{C}$

O, 1-Phenyl-4-hexene, $100 \%$; $\times$, 2-octene, $95 \%$; $\triangle$, 1-phenyl-3pentene, $98 \%$ pentene, $80 \%$.

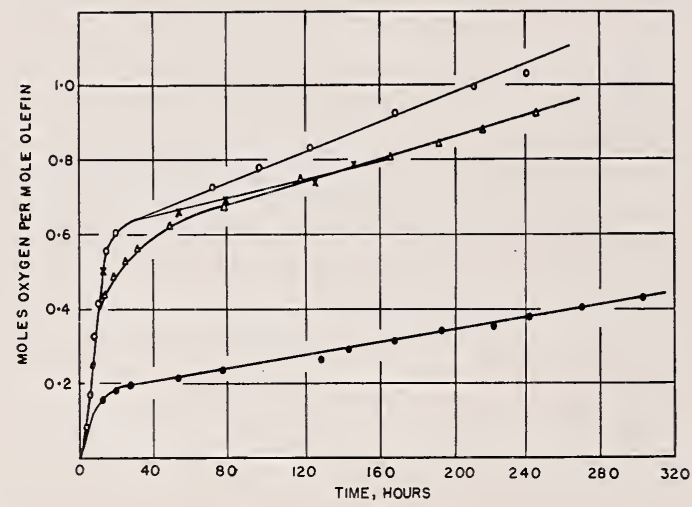

TABLE 11.1. Over-all rate constant for oxidation of 1-phenyl-3-pentene at $90^{\circ} \mathrm{C}$

\begin{tabular}{|c|c|c|c|}
\hline \multicolumn{4}{|c|}{ Rate, $d \cdot M / d t=k$ (unreacted olefin) (peroxide) } \\
\hline Time & $k \times 100$ & Time & $k \times 100$ \\
\hline $\begin{array}{c}h r \\
6.0 \\
12.0 \\
18.0 \\
25.0 \\
31.0 \\
44.0\end{array}$ & $\begin{array}{r}1.06 \\
0.84 \\
.79 \\
.69 \\
.49 \\
.44\end{array}$ & $\begin{array}{c}h r \\
68.0 \\
98.0 \\
163.0 \\
189.5 \\
216.7 \\
237.0\end{array}$ & $\begin{array}{r}0.28 \\
.27 \\
.56 \\
.57 \\
.59 \\
60\end{array}$ \\
\hline \multicolumn{4}{|c|}{ A verage $k=0.63$} \\
\hline
\end{tabular}

quite similar, and it seems probable that the same fundamental mechanism is involved in both aliphatic and phenyl-substituted olefins.

The effect of temperature on the autocatalytic rate is shown in figure 11.6 for 1-phenyl-4-hexene at three temperatures. It will be noted that the rate increases with temperature. The leveling off of these curves appears to be influenced by other factors (for example, the tendency to polymerize to form a more viscous liquid), and consequently the subsequent behavior may not be significant.

Samples of the oxidized olefins were removed at various stages of 
Figure 11.6. Effect of temperature on oxygen absorption of 1-phenyl4-hexene.

$\mathrm{O}, 99.9^{\circ} \mathrm{C} ; \times, 89.8^{\circ} \mathrm{C} ; \triangle, 80.1^{\circ} \mathrm{C}$.
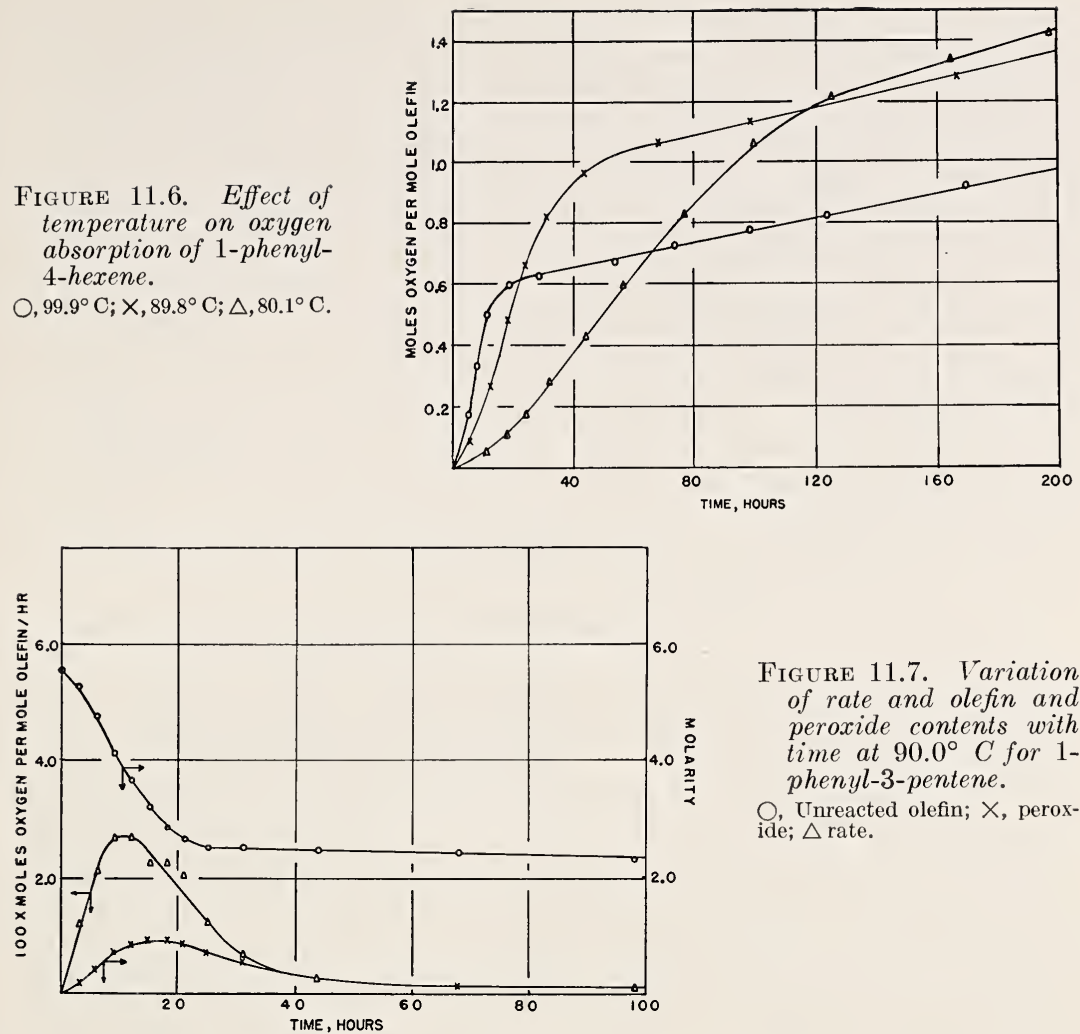

FIgure 11.7. Variation of rate and olefin and peroxide contents with time at $90.0^{\circ} C$ for 1 phenyl-3-pentene.

0 . Unreacted olefin; $x$, peroxide; $\triangle$ rate.

oxidation and analyzed for peroxide, unsaturation, and other functional groups as will be described later. The instantaneous rates obtained graphically from enlarged plots of the oxygen-absorption curve for 1-phenyl-3-pentene at $90^{\circ} \mathrm{C}$ are compared in figure 11.7 with the peroxide concentration and the unreacted olefin remaining at any given time. The rate increases as peroxide concentration increases, and is accompanied by a corresponding drop in unreacted olefin concentration. (The peroxide was assumed to be hydroperoxide which would still be unsaturated, and consequently the olefin analyses were corrected by subtracting the molar equivalent of the peroxide.) Both rate and peroxide concentration go through a maximum and then decrease. This is no doubt due in part to depletion of the unreacted starting material, although another factor may be the formation of products such as acids which catalyze peroxide decomposition. Comparable data at other temperatures show that the peroxide maximum is lower at higher temperatures, indicating that temperature accelerates peroxide decomposition even more than peroxide formation.

The rate should be proportional to both olefin and peroxide concentration, and a simple rate equation such as the following is consistent with the kinetic mechanism proposed by Bolland [2]:

$$
d M / d t=k \text { (unreacted olefin) (peroxide). }
$$


The rate is expressed as moles of oxygen absorbed per mole of original olefin per hour, and the parentheses represent molar concentrations. Using the data from the curves of figure 11.7 for 1-phenyl-3-pentene oxidized at $90^{\circ} \mathrm{C}$, we may evaluate $k$ to see if a constant value is obtained. Some calculated values for this over-all rate constant are given in table 11.1. There is considerable variation, but when it is recalled that the times listed include not only the autocatalytic stage, but also the later stage where the rate leveled off, it is surprising that the values do approximate a constant. Part of the error is inherent in the assumption that all the peroxide is hydroperoxide and thus would also analyze as olefin. Calculated energies of activation based on the over-all rate constants obtained at different temperatures for 1-phenyl-3-pentene and 1-phenyl-4-hexene [10] give values within the range of 20 to $25 \mathrm{kcal}$, which is of the right order of magnitude for a chemical reaction. In any event, the data support the theoretical expectation that the rate should be a function of both peroxide concentration and the concentration of unreacted hydrocarbon.

\section{Products of Oxidation}

The products formed by the oxidation of hydrocarbons are quite varied. Peroxides appear to be the principal product of the initial oxygen attack, but secondary oxidation products include acids, esters, aldehydes, and ketones, as well as carbon dioxide and water. The results of some qualitative tests on oxidized samples of three phenylsubstituted olefins are given in table 11.2. Aldehyde was found only in the short-term oxidation which was terminated in the autocatalytic stage where the rate and peroxide concentration would both be near the maximum 'The aldehydes formed thus appear to be oxidized readily to acids. (The presence of aldehyde in the early stages, but not in the later stages has also been observed in subsequent studies on 1-phenyl-3-pentene.) No free alcohol was found, although a considerable amount of ester was present. No procedure for testing for epoxides was available. Ketones were detected in each sample tested, and in the case of the oxidation of 1-phenyl-2-butene, the ketone, phenyl 1-propenyl ketone, was isolated and identified. Benzoic acid was also isolated in pure form from the oxidized samples. Other acids are also formed, as will be shown later.

Quantitative analyses for certain functional groups were run on samples removed at various stages of oxidation. The change in concentration with time is plotted in figure 11.8 for peroxide, carbonyl, and ester. The oxygen-absorption curve is also shown for compari-

TABLE 11.2. Oualitative tests for oxidation products

\begin{tabular}{|c|c|c|c|c|c|}
\hline Product & $\begin{array}{l}\text { Oxidation } \\
\text { time }\end{array}$ & Alcohola & Ketoneb & Aldehydec & Compounds isolated \\
\hline $\begin{array}{l}\text { 1-Phenyl-2-butene } \\
\text { Do } \\
\text { 1-Phenyl-3-pentene } \\
\text { 1-Phen yl-4-hexene... } \\
\text { 1-Phen yl-3-vinylbu- } \\
\text { tane. }\end{array}$ & $\begin{array}{r}h r \\
359 \\
\\
6 \\
\\
316 \\
369 \\
370\end{array}$ & 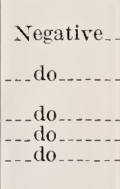 & 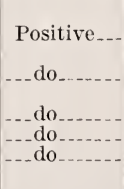 & $\begin{array}{l}\text { Negative_- } \\
\text { Positive_-- } \\
\text { Negative_- } \\
\text {-_do_._._...- }\end{array}$ & $\begin{array}{l}\text { Benzoic acid, phenyl } \\
\text { 1-propenyl ketone. } \\
\text { Phenyl 1-propenyl ke- } \\
\text { tone. } \\
\text { Benzoic acid. } \\
\text { Do. } \\
\text { Do. }\end{array}$ \\
\hline
\end{tabular}

a Ceric ammonium nitrate test.

b 2,4-Dinitrophenylhydrazine reagent.

c Fuchsin aldehyde reagent. 


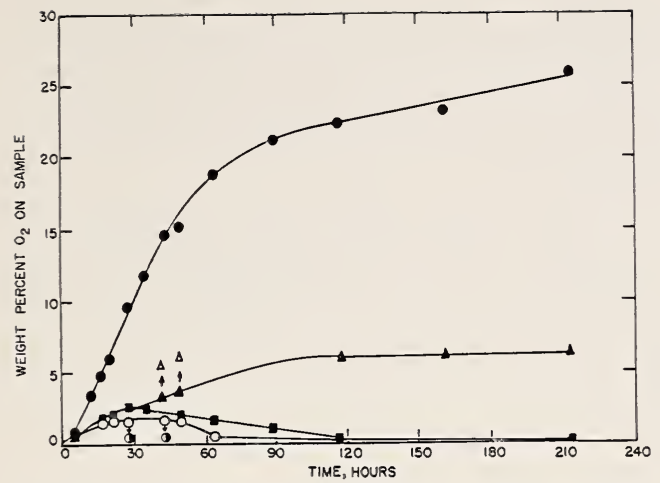

Figtre 11.8. Oxygen absorption and peroxide, carbonyl, and ester analyses in the $80^{\circ} \mathrm{C}$ oxidation of 1-phenyl-3-pentene.

-. Oxygen absorption; 0 . peroxide; $\bigcirc$, carbonyl, $\mathbf{0}$, after 7 days at $0^{\circ} \mathrm{C} ; \boldsymbol{\Delta}$, ester, $\triangle$, after 11 days at $0^{\circ} \mathrm{C}$.

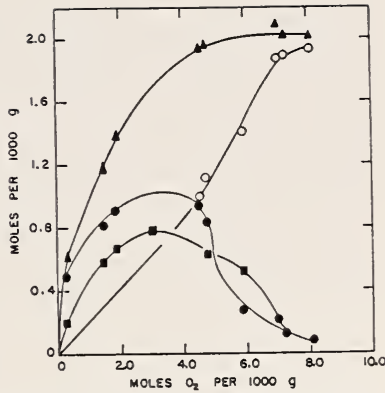

Figure 11.9. Concentrations of some oxidation products of 1-phenyl-3-pentene as a function of oxygen absorption at $80^{\circ} \mathrm{C}$.

- Peroxide; , carbonyl; 0 . ester; $\triangle$, carbonyl plus ester.

son. Peroxide and carbonyl follow a similar trend with a maximum at about the same time, suggesting the probable formation of ketone by dehydration of peroxide. However, the ketone must also be converted to other products since the concentration decreases as the rate of oxidation diminishes. The carbonyl content also decreased during storage of the oxidized sample, while ester content increased.

The change in concentration of peroxide, carbonyl, and ester with oxygen absorption is plotted on a mole basis in figure 11.9. The peroxide and carbonyl both diminish at higher stages of oxidation, but the ester content continues to increase. However, the sum of ester plus carbonyl is essentially constant in the region where carbonyl is decreasing and ester is increasing. These data indicate that carbonyl is being converted to ester, and that this reaction, rather than esterification of alcohol and acid, is the chief source of ester formed. Further eridence for this conversion of ketone to ester is the fact that analyses on the same samples after storage for a week at $0^{\circ} \mathrm{C}$ gave much lower ralues for carbonyl, while the ester concentration increased. Examples of these analyses at a later date are indicated in figure 11.8 .

The conversion of ketone to ester in the presence of peroxide is not without precedent, since comparable reactions have been reported with ketones and peracids $[12,13,14]$. In the case of alpha,betaunsaturated ketones with peracetic acid, enol esters were obtained [13]. A comparable reaction with peroxide in place of peracid might proceed as follows in the oxidation of 1-phenyl-3-pentene:

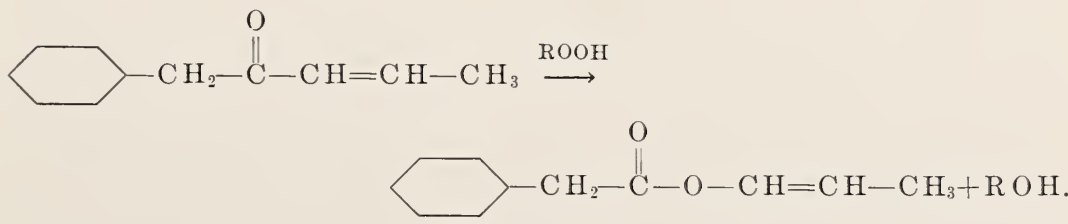

The change in unsaturation with oxygen absorption is plotted in figure 11.10 . The data indicate a nearly constant relationship al- 
though the change was somewhat greater in the early stages. Most of the decrease in unsaturation must be attributed to oxygen-induced polymerization, however, rather than to direct saturation with oxygen, since the sum of the oxygen found by analysis as peroxide, carbonyl, ester, acid, carbon dioxide, and water was sufficient to account for most of the oxygen absorbed.

The formation of water, carbon dioxide, and acids as a function of oxygen absorbed is shown in figure 11.11. A regular increase with oxygen absorbed is shown in each case up to about 6.0 moles of oxygen per $1,000 \mathrm{~g}$ of sample. At higher stages of oxidation, increasing amounts of water and carbon dioxide are liberated. This break occurred at about $65 \mathrm{hr}$ at $80^{\circ} \mathrm{C}$ and coincides with the time at which the oxygen-absorption curve leveled off. Beyond this point most of the oxygen consumed can be accounted for by the liberation of an equivalent amount of water and carbon dioxide.

Chromatographic analyses were used to determine the nature of the acids formed. The method of Marvel and coworkers [15] was employed. A typical chromatogram is shown in figure 11.12 by way of example. A sharp peak is shown in the 50- to $60-\mathrm{ml}$ region, with broader peaks at 170 to $200 \mathrm{ml}$ and at $290 \mathrm{ml}$. Known samples were used to calibrate the procedure, and the acids which appeared to be formed in the oxidation of 1-phenyl-3-pentene at $80^{\circ} \mathrm{C}$ are listed in table 11.3. Formic and acetic acid were only detected after hydrolysis, and thus were apparently present mainly as esters.

It should be pointed out that while the original sample of 1-phenyl3-pentene analyzed 97.3 percent pure as olefin, infrared data indicated that as much as 20 percent of the isomeric compound, 1-phenyl-4pentene, was probably present. This complicated the picture somewhat, but at the same time, the mixture corresponds more closely to GR-S by analogy, since the second isomer contains a terminal double bond like that of the side-chain vinyl groups formed to the extent of about 20 percent by 1,2-polymerization of the butadiene.

The acid formed in greatest amount in the oxidation of phenylpentene appeared to be phenylpropionic acid. This could arise in part from the 1,4-isomer by way of ketone and ester, and also by direct oxidative scission of the 1,3-isomer at the double bond.

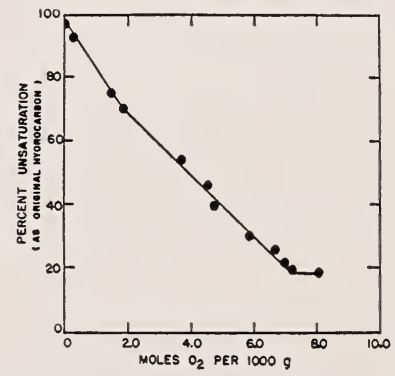

Figure 11.10. Unsaturation of 1-phenyl-3-pentene as a function of oxygen absorption at $80^{\circ} \mathrm{C}$.

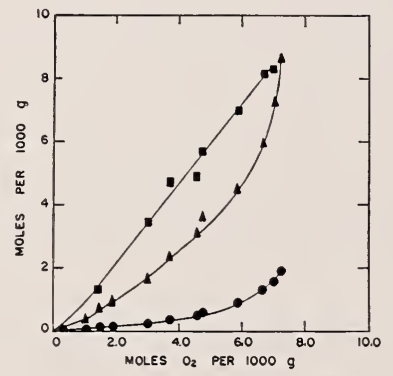

Figure 11.11. Formation of water, carbon:dioxide, and acids as a function of the oxygen absorption of 1phenyl-3-pentene at $80^{\circ} \mathrm{C}$.

A, Water;, carbon dioxide; $\mathbf{\square}$, acid 
Figure 11.12. Total acids chromatogram for oxidized 1-phenyl-3-pentene.

$80^{\circ} \mathrm{C} ; 160.5$ hours; 1.08 moles of $\mathrm{O}_{2}$ per mole of original hydrocarbon

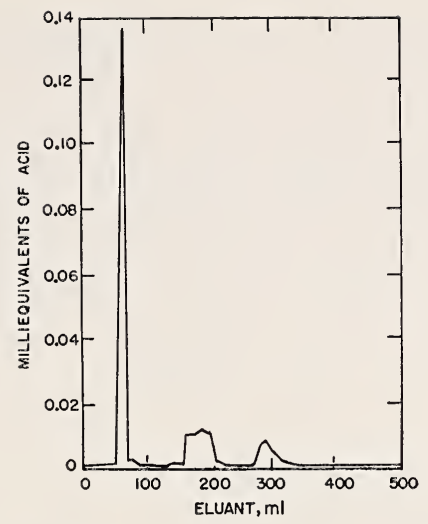

TABLE 11.3. Chromatographic analysis for acids formed by oxidation of 1-phenyl-3-pentene at $80^{\circ} \mathrm{C}$.

\begin{tabular}{|c|c|c|c|}
\hline \multicolumn{2}{|l|}{ Calibration } & \multicolumn{2}{|c|}{ Analysis a } \\
\hline Acid & $\begin{array}{l}\text { Absorption } \\
\text { band }\end{array}$ & Free acids & $\begin{array}{l}\text { Total acids } \\
\text { (after hydrol- } \\
\text { ysis) }\end{array}$ \\
\hline $\begin{array}{l}\text { Crotonic } \\
\text { Phenylacetic } \\
\text { Phenylpropionic } \\
\text { Benzoic } \\
\text { Acrylic } \\
\text { Acetic } \\
\text { Formic }\end{array}$ & $\begin{array}{c}m l \text { of eluant } \\
50 \\
60 \\
60 \\
160 \\
170 \\
200 \\
290\end{array}$ & $\begin{array}{l}\stackrel{\times}{\times} \\
\stackrel{x}{\times} \\
\stackrel{x}{\times} \\
-1\end{array}$ & $\begin{array}{l}x \\
x \\
x \\
x \\
x \\
x \\
x\end{array}$ \\
\hline
\end{tabular}

a $X$ indicates detection of acid.

\section{Indicated Reactions}

The main reactions which appear to be involved in the initiation and propagation stages of the oxidation of an olefin are illustrated in figure 11.13. Initiation may be brought about by attack at either the alpha-methylene group to give a radical by removal of a proton, or by direct addition of oxygen to the double bond to form a radical. In the autocatalytic stage, the predominant initiation is probably peroxide decomposition with the formation of radicals. Regardless of the method of initiation, the propagation chain would be the same, with hydroperoxide as the main primary oxidation product.

There appear to be many secondary reactions which take place simultaneously, and lead to a variety of products. This complicates both kinetic and chemical investigation of the oxidation reaction, and makes it difficult to interpret the data. For example, both R. and $\mathrm{RO}_{2}$. radicals may add to double bonds and initiate polymerization reactions as indicated in figure 11.14. There may also be some direct oxygen attack at the double bond with resultant cleavage at that point.

Peroxide decomposition thus gives radicals which may initiate oxidation or polymerization, or they may undergo reactions leading to such products as aldehydes, ketones, esters, acids, etc. Some of the probable reactions, based on the oxidation products which have been characterized, are summarized in figure 11.15. The dehydration of 

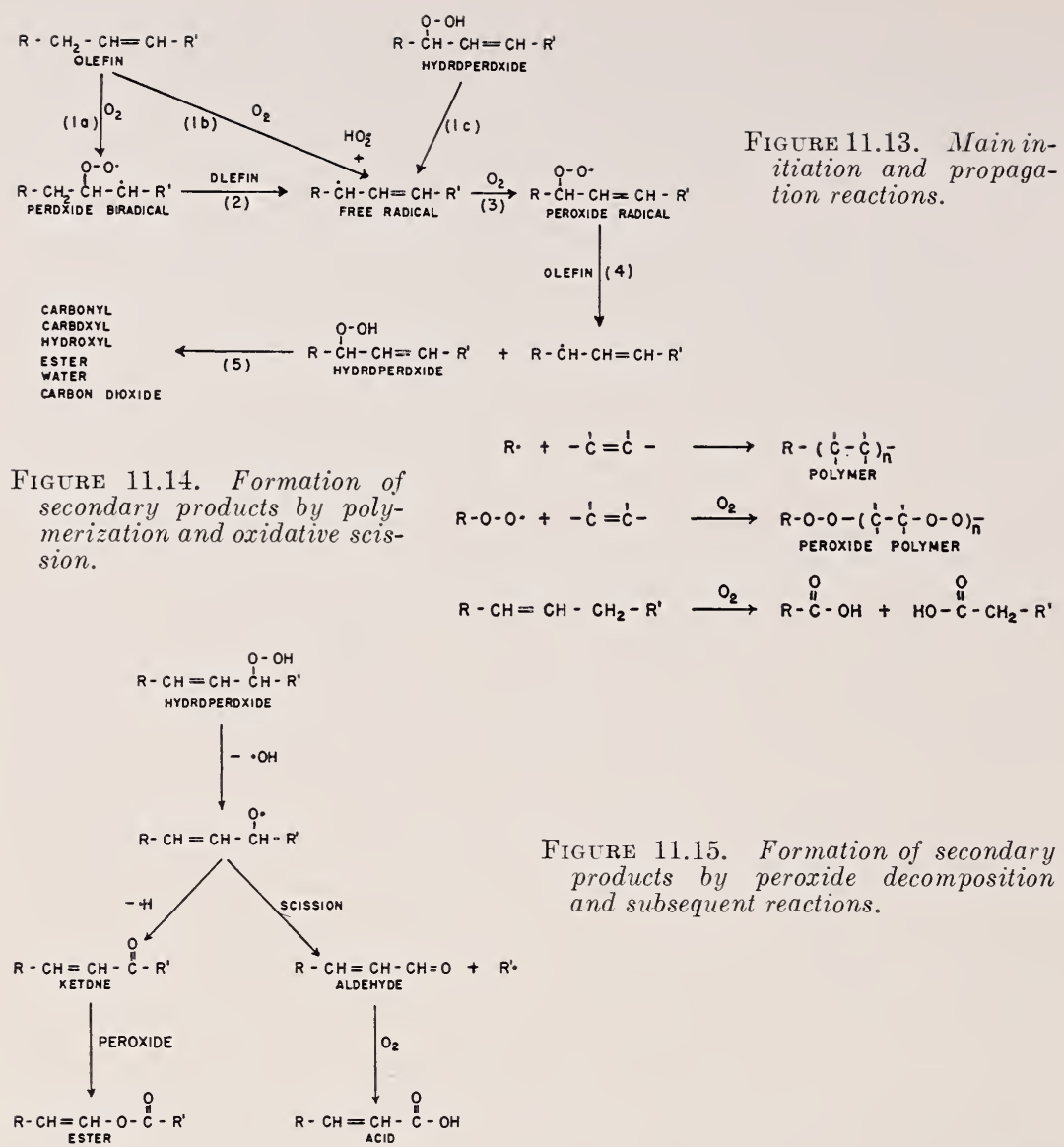

FIGURE 11.15. Formation of secondary products by peroxide decomposition and subsequent reactions.

hydroperoxide to carbonyl and the conversion of ketone to ester in the presence of peroxide are important examples. Chain scission may well result from cleavage of an RO. radical to form an alkyl radical and an aldehyde, which would ultimately oxidize to an acid. Any alcohols formed as intermediates are apparently either dehydrated, or esterified by the excess of acids, under the conditions of constant remoral of water. Carbon dioxide may be formed by decarboxylation of ketoacids, and by complete oxidation of such products as formic and oxalic acids.

These studies on the oxidation of olefins with structures related to natural and synthetic rubber have been made possible by a sequence of fellowship grants from the Firestone Tire and Rubber Co. J. W. Lawrence, ${ }^{3}$ W. C. Warner, ${ }^{4}$ and G. R. Nitchell ${ }^{5}$ have contributed data included in this paper. W. L. Cox ${ }^{6}$ has assisted in the preparation of the manuscript.

3 Present address, Atlas Powder Co., Tamaqua, Pa.

4 Present address, General Tire \& Rubber Co., Akron, Ohio.

3 Present address, Olin Industries, New Haren, Conn.

6 Research Assistant, Case Institute of Technology, Cleveland, Ohio. 


\section{References}

[1] E. H. Farmer, et al., J. Chem. Soc. 139 (1942).

[2] J. L. Bolland, Quart. Rev. Chem. Soc. 3, 1 (1949); Publication No. 113, british Rubber Producers Research Association.

[3] O. Chavanne, et al., J. Am. Chem. Soc. 52, 1609 (1930); Bul. soc. chim. belg. 40, 611 (1931); 41, 630 (1932); 42, 527 (1933).

[4] P. George and A. D. Walsh, Trans. Faraday Soc. 42, 94 (1946).

[5] F. F. Rust, W. F. Vaughan, et al., Ind. Eng. Chem. 41, 2595, 2597, 2604, $2609,2612(1949)$

[6] A. V. Tobolsky, D. J. Metz, and R. B. Mesrobian, J. Am. Chem. Soc. 72 , $1942(1950)$.

[7] J. R. Shelton and H. Winn, Ind. Eng. Chem. 38, 71 (1946).

[8] J. R. Shelton and W. L. Cox, Ind. Eng. Chem. 43, 456 (1951).

[9] J. W. Lawrence and J. R. Shelton, Ind. Eng. Chem. 42, 136 (1950).

[10] W. C. Warner and J. R. Shelton, Ind. Eng. Chem. 43, 1160 (1951).

[11] G. R. Mitchell, Ph. D. thesis, Case Institute of Technology (1951).

[12] Baeyer and Villiger, Ber. Deut. chem. Ges. 32, 3625 (1899); 33, 858 (1900).

[13] J. Boeseken, et al., Rec. trav. chim. 50, 827 (1931); 52, 874 (1933); 55, 786 (1936).

[14] S. L. Fries, et al., J. Am. Chem. Soc. 71, 14, 2571 (1949); 72, 2611, 5518 (1950).

[15] C. S. Marvel, et al., J. Am. Chem. Soc. 7\%, 2642, 3887 (1950).

\section{Discussion}

Dr. D. Swern, Oil and Fat Division, Eastern Regional Research Laboratory, Bureau of Agricultural and Industrial Chemistry, U. S. Department of Agriculture, Philadelphia, Pa.: We have been studying the photochemical autoxidation of methyl oleate and have been following the buildup of oxygen contained in functional groups. In one study we checked the oxygen introduced by combustion analysis as well as by chemical analysis, and we found that the total oxygen introduced, determined chemically, was possibly 130 to 200 percent of the oxygen introduced, as shown by a combustion analysis. This worried us, of course, and we decided to go back and do a study with a series of model compounds that contained these oxygen-carrying: groups, including a series of hydroperoxides, carbonyl, hydroxy compounds, and other's. It was found that peroxides made the analyses invalid. On mixtures containing 20 percent of carbonyl, 20 percent of peroxide, and 60 percent of inert material, one could obtain by chemical analysis values for carbonyl of 40 or 60 percent. When the sample was reduced by sulfide, then the functional group analysis was correct.

Dr. Shelton: With respect to the oxygen balance obtained in these studies, it was not 100 percent, but it did run close to it in some cases. In general, we were able to account for from 90 to 100 percent of the oxygen absorbed. It must be recognized that certain functional groups may interfere in some measure in an analytical procedure for determination of another group, and that some oxygen was probably present in forms that we did not determine. Perhaps some of these errors were compensating. At any rate, we did not encounter discrepancies of the magnitude cited by Dr. Swern.

Dr. J. E. Wilson, Bakelite Co., Division of Union Carbide and Carbon Corp., Bound Brook, N. J.: In Dr. Shelton's very interesting discussion he did not stress epoxide formation during oxidation. 
Bricker studied the oxidation of 10,11-undecylenic acid and concluded that one of the steps of the mechanism was as follows:

$$
\mathrm{H}_{2} \mathrm{C}=\mathrm{CH}\left(\mathrm{CH}_{2}\right)_{8} \mathrm{COOH} \stackrel{\mathrm{O}_{2}}{\longrightarrow} \mathrm{H}_{2} \mathrm{C} \underset{\mathrm{O}}{\longrightarrow} \mathrm{CH}\left(\mathrm{CH}_{2}\right)_{8} \mathrm{COOH}
$$

I

In order to account for the very small amount of II in the product, it was postulated that II reacted with I:

$$
\mathrm{I}+\mathrm{II} \longrightarrow \mathrm{H}_{2} \mathrm{C}=\mathrm{CH}\left(\mathrm{CH}_{2}\right)_{8} \underset{\|}{\mathrm{C}}-\mathrm{O}_{\mathrm{O}}-\mathrm{O}-\mathrm{CH}_{2} \mathrm{CH}\left(\mathrm{CH}_{2}\right)_{8} \mathrm{C} \mathrm{O} \mathrm{OH}
$$

III

Hydrolysis of III at the dotted line would produce 10,11-dihydroxyhendecanoic acid, which was actually isolated as a component of the products. Several literature references describe reactions of the type I+II. Furthermore, experiments on the oxidation of methyl 10, 11-dihydroxyhendecanoate resulted in an increased yield of epoxide product, which is reasonable, because the epoxide could not react in this way with the methyl ester. This work may tie in with Dr. Shelton's finding of a considerable amount of esters in his oxidation products.

Dr. Shelton: I think we would be inclined to agree that epoxides probably do play a part. We are still in the process of analyzing some of the samples. Because we did not have reliable data on epoxides to present, we more or less ignored them. Analyses for epoxide have been run since the paper was written, but unfortunately it appears that $\alpha,-\beta$-unsaturated ketones and esters interfere in the procedure, because they, too, tend to add anhydrous HCl. After correcting for the probable interference, it still appeared that about 1 percent of epoxide oxygen was present in the oxidized samples. Thus, to the extent that epoxide is formed, this would be a very plausible means of accounting for some of the ester. We certainly have acids present, and if epoxides are also present, we could obtain ester by this mechanism, as well as by the direct ketone to ester reaction for which there are also definite precedents. We intend to study this latter reaction in the near future.

It must be recognized that double bonds do shift under favorable conditions, and some of the acids may have been formed by a mechanism involving such a rearrangement prior to or during oxidation. Certainly a free electron on the alpha carbon with respect to a double bond, would be expected to undergo a resonance interaction with the electrons of the double bond as illustrated by these resonance structures:

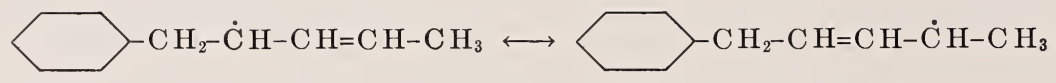

Products resulting from the oxidation of both forms of the radical would thus be expected. 


\title{
12. Stabilization of Cellulose Esters
}

\author{
By G. C. DeCroes ${ }^{1}$ and J. W. Tamblyn ${ }^{1}$
}

Some of the causes of the degradation occurring when cellulose ester plastic srstems are exposed to excessive heat were investigated. It was found that the plasticizer is often the chief contributor toward break-down. Most of the common plasticizers were found to be more easily oxidized than the cellulose ester itself, and the oxidation process induced break-down of the chains. Antioxidants inhibited this type of break-down, whereas various metal soaps and titanium dioxide catalyzed it. In systems containing an oxidation-resistant plasticizer, a similar break-down could be produced by addition of a peroxide. A severe but nonoxidative type of break-down was found to occur when the plasticizer contained ferric salts of strong acids. This thermal break-down could be greatly reduced by the addition of a suitable chelating agent.

In the process of break-down by ultraviolet light, oxvgen also was observed to play an important part. The oxidizable plasticizer dibutyl sebacate was found to contribute to this type of break-down but to a far lesser extent than to breakdown by heat. Ultraviolet-break-down inhibitors are discussed, and it is shown that antioxidants are useful only if combined with an additive that filters out the ultraviolet efficiently. In studies of weathering inhibitors, the thickness of the test piece is an important variable. Mechanisms by which ultravioiet breakdown inhibitors may be effective are briefly speculated upon.

\section{Introduction}

This paper presents the results of an investigation of the breakdown of cellulose ester plastic systems under conditions apt to be encountered in processing and use. Attention was also given to means of minimizing the changes that occur in these systems during exposure to heat, air, and ultra violet light.

Then cellulose esters are orerheated, many changes occur, all of them unfortunately detrimental from the viewpoint of the user. Evans and McBurney [1] ${ }^{2}$ bave recently reviewed the earlier work on the stability of cellulose acetate toward heat and extended this with their own study of the oxvgen absorption by the solid ester in granular form at $160^{\circ} \dot{\mathrm{C}}$. They found that oxidation proceeded at a conveniently slow rate at this temperature. Evidence was obtained of the release of the volatile products, acetic acid, carbon dioxide, carbon monoxide, and water. These decompositions were accompanied by progressive orange-yellow discoloration, increase in apparent acetyl content, and chain-length break-down in the residue. Measurements by Erans and McBurney on the production of rolatile matter, color, and chain-breaking showed linear relationships between these quantities and the amount of oxygen absorbed, up to about one-fourth of an oxygen atom per anhydroglucose unit.

The break-down of cellulose esters by natural or artificial weathering is, in many ways, a more complicated process than the thermal degradation. It is to be expected, howerer, that oxidation and other reactions occurring at elevated temperatures will still go on, even if more slowly, under outdoor conditions or in a weathering machine. The effects of oxygen and water on the photodegradation of cellulose acetates have been investigated by Montonna and Winding [2], Lawton and Nason [3], and Heuser and Chamberlin [4]. Some of the puzzling inconsistencies have been resolved by the interesting studies on cellulose by Launer and Wilson [5], who showed that these effects

1 Tennessee Eastman Co., Dirision of Eastman Kodak Co., Kingsport, Tenn.

2 Figures in brackets indicate the literature references on p. 189. 
were markedly dependent on the wavelength of the ultraviolet light being used. It seems to be now generally agreed that, in the sunlight range, oxygen and water both assist in the break-down processes.

Weathering produces marked changes in the appearance and physical properties of cellulose ester plastics. These effects have been well described by Meyer and Gearhart [6]. The photochemical aspects of degradation of cellulose acetate butyrate have been discussed by Tichenor [7].

In our work, the break-down of cellulose esters themselves at elevated temperatures was studied briefly, but most of our experiments were made on plasticized systems. Inhibitors and catalysts for degradation in these systems were extensively investigated. Some of the variables involved in the weathering process were also studied. Possible mechanisms for the protection of cellulose ester plastics from ultraviolet break-down by inhibitors are suggested.

It was found that oxidizable plasticizers play a very important role in the break-down by heat, but a less important role in weathering.

\section{Materials}

Because the investigation was primarily exploratory in nature, no purification treatments of the materials used were undertaken except in a few cases. In most of the experiments to be described, a regular production grade of powder-precipitated cellulose acetate butyrate containing 13 percent of acetyl and 37 percent of butyryl was used. Additives, such as plasticizers, antioxidants, inhibitors, break-down catalysts, and so on, were mostly the best grades of commercially available materials.

The nitrogen used in experiments run under an "inert" atmosphere was further purified before use. Gas from a tank containing 0.3 percent of oxygen was led through a drying train and passed at $350^{\circ} \mathrm{C}$ over fine copper oxide wire that had been reduced under hydrogen at $300^{\circ} \mathrm{C}$ to bright copper. By this means the oxygen content of the nitrogen was reduced to 0.001 percent before the latter entered the reaction vessel.

\section{Apparatus and Procedures}

\section{Thermal Break-down}

Degradation by heat was carried out in 125-ml Erlenmeyer flasks shaken back and forth at $50 \mathrm{c} / \mathrm{min}$ through an arc of $60^{\circ}$ in an oil bath. Except for some of the experiments with pure cellulose esters that were run at $180^{\circ} \mathrm{C}$, the temperature of the bath was held at $150^{\circ} \pm 1^{\circ}$ C. The atmosphere above the cellulose ester system was confined by a gas burette and mercury reservoir kept manually adjusted to atmospheric pressure at frequent intervals throughout the run.

When a plasticizer was used, $1 \mathrm{~g}$ of the cellulose ester was mixed with $25 \mathrm{ml}$ of the plasticizer (or $25 \mathrm{~g}$, if the latter was a solid). Other additives, such as antioxidants, if used, were added in amounts of $0.02 \mathrm{~g}$, except where otherwise noted.

\section{Weathering Break-down}

\section{Natural Weathering}

Outdoor exposures were made on the roof of the Research Laboratory of the Tennessee Eastman Co. at Kingsport, Tenn. Test strips of plastic, usually 2.5 by 0.5 by 0.050 in., cut from rolled and com- 
pression-molded sheets, were mounted facing south without backing at an angle with the horizontal of $36.5^{\circ}$, the latitude at Kingsport. All samples were washed with mild detergent and rinsed with distilled water once each month. The basic formulation used was 12 parts of dibutyl sebacate to 100 parts of cellulose acetate butyrate, which gives a plastic of medium-soft flow. If used, soluble additives, such as ultraviolet inhibitors or antioxidants, were usually held to 1 part, and pigments to 2 parts, unless specified otherwise. Some measurements were also made on sheets of thickness varying from 0.025 to 0.187 in. which had been exposed at Phoenix, Ariz., on conventional racks [6]. These samples were obtained through the courtesy of the Cellulose Esters and Plastics Development Laboratory of the Tennessee Eastman Co.

\section{Accelerated Weathering}

The Twin-Arc Weather-ometer, with carbon ares enclosed by Pylex globes, was used at $60^{\circ} \mathrm{C}$ on a cycle consisting of $51 \mathrm{~min}$ without water spray followed by 9 min with water spray. An exposure of 50 to $150 \mathrm{hr}$, or even longer for some materials, was required in this machine to equal the break-down obtained during one midsummer month outdools at Kingsport. The plastic test pieces were again usually 2.5 by 0.5 by 0.050 in. In some experiments, the Weatherometer was modified by the addition of eight $20-w$ fluorescent sun lamps to raise the shorter ultraviolet output. The test samples thus received radiation more nearly like sunlight. These lamps were mounted rertically at a distance of about $2 \mathrm{in}$. from the sample drum. Factors correlating this test with outdoor exposures have not yet been determined.

\section{Analyses}

\section{Determination of Acid}

The acid formed during degradation was titrated to a phenolphthalein end point with $0.1 \quad \mathrm{~N}$ sodium bydroxide solution in $1: 1$ ethanol: acetone medium.

\section{Determination of Peroxide}

Peroxides were measured by an iodimetric procedure in 1:1 ethanol: acetone medium at a cellulose ester concentration of $0.23 \mathrm{~g}$ per $100 \mathrm{ml}$ of solvent. The method of Kokatnur and Jelling [8] was modified to eliminate the 5-min heating period. When the solution containing free iodine was heated, as required by the unmodified procedure, low peroxide values were obtained, probably because of reaction of the iodine with some of the degradation products. Our peroxide values may thus still be lower than the true ralues if certain relatively stable peroxides were formed during degradation. The nature of the peroxidic matter present was such that the peroxide ralues obtained did not change on standing at room temperature for $15 \mathrm{hr}$.

\section{Determination of Percentage of Apparent Acetyl}

The procedure recommended by Malm, Genung, Williams, and Pile [9] was followed. These measurements were made in the Analytical Department of the Research Laborators.

\section{Determination of Viscosity}

The procedure of Wagner and Russell [10] was used. Inherent viscosities [11] were calculated from measurements on solutions containing $0.23 \mathrm{~g}$ of cellulose ester per $100 \mathrm{ml}$ of acetone. In the thermal experiments, in which excess plasticizer was used, the solution con- 
tained as much as 6 percent of plasticizer. Appropriate blanks on the solvent were run in all such cases. When desired, number-average molecular weights were estimated from published viscosity-molecular weight relationships for cellulose acetate butyrate [12] and cellulose acetate [13].

\section{Determination of Flexural Strength}

This property was calculated for unweathered and weathered plastic samples from data obtained by the Tour-Marshall test for stiffness in flexure (ASTM D 47-43T).

\section{Determination of Brittleness}

Brittleness was defined to have developed in the plastic test strip when a break occurred at a bend angle of less than $90^{\circ}$ in the TourMarshall test.

\section{Experimental Results and Discussion}

\section{Break-down of Unplasticized Cellulose Esters by Heat}

Our experiments on the break-down of the solid unplasticized esters in powder or sheet form at $150^{\circ}$ and $180^{\circ} \mathrm{C}$ were in agreement with those of Evans and McBurney [1]. However, we interpret our results to indicate that the observed break-down was not entirely oxidative. Table 12.1 gives illustrative results for an unplasticized cellulose acetate butyrate powder heated under various conditions for $24 \mathrm{hr}$. The molecular-weight break-down observed in a cellulose acetate containing 38 percent of acetyl was somewhat less than that in the acetate butyrate. Although the degradation at both temperatures was considerably less under nitrogen than under oxygen, the former was always a considerable fraction of the latter.

The break-down rates exhibited by the esters themselves are rather too slow to have an important effect in the processing of cellulose ester products. Since, in actual practice, we are more concerned with plasticized cellulose esters, we have concentrated our studies on these systems. Here it was soon found that the type and purity of plasticizer used could have a very important influence on the oxidation and break-down occurring at processing temperatures.

TABLE 12.1. Oxidative and thermal degradation of cellulose acetate butyrate a

\begin{tabular}{|c|c|c|c|c|}
\hline Temperature & Atmosphere & $\begin{array}{l}\text { Apparent } \\
\text { acetyl }\end{array}$ & Color & Degradation \\
\hline $\begin{array}{l}{ }^{\circ} C \\
\text { Unexposed } \\
150 \\
150 \\
180 \\
180\end{array}$ & $\begin{array}{l}\text { Unexposed } \\
\mathrm{N}_{2}-1- \\
\mathrm{O}_{2} \\
\mathrm{~N}_{2} \\
\mathrm{O}_{2}\end{array}$ & $\begin{array}{l}\text { Percent } \\
34.7 \\
36.6 \\
37.2 \\
37.2 \\
37.2\end{array}$ & $\begin{array}{l}\text { White-- } \\
\text { Brown } \\
\text { Black }\end{array}$ & $\begin{array}{c}\% \text { mol wt } \\
0 \\
64 \\
81 \\
48 \\
90\end{array}$ \\
\hline
\end{tabular}

a 24-hr exposure.

\section{Break-down of Plasticized Cellulose Esters by Heat}

\section{Oxidation of the Plasticizer}

Oxidizability of the plasticizer alone was first investigated. The amount of oxygen absorbed by $25 \mathrm{ml}$ of the plasticizer, if liquid, or $25 \mathrm{~g}$, if solid, during $3 \mathrm{hr}$ of shaking at $150^{\circ} \mathrm{C}$ under $1 \mathrm{~atm}$ of oxygen 
was measured. The amounts of acid and peroxide present at the end of each run were also determined. 'Table 12.2 indicates the large differences observed in oxidizability of several commercial plasticizers. No absolute significance is to be attached to the values of absorbed oxygen or to the quantities of oxidation products formed, since the volatile oxidation products were not absorbed, nor were the diffusion rates of oxygen through the different liquids determined. However, it is quite apparent that dimethyl phthalate and triphenyl phosphate are highly resistant to oxidation at $150^{\circ} \mathrm{C}$, whereas the rest are rather easily oxidized. 'The distinguishing feature of the oxidation-resistant pair of plasticizers is the absence in their molecules of methylene or methylidyne groups, which are well known to be susceptible to oxidative attack [14].

The rather high susceptibility of such useful plasticizer's as dibutyl sebacate and dioctyl phthalate to oxidation at $150^{\circ} \mathrm{C}$ seemed surprising to us. We had obtained no oxidation of dibutyl sebacate at $100^{\circ} \mathrm{C}$ during $60 \mathrm{hr}$ of exposure under oxygen at $100 \mathrm{lb} / \mathrm{in}^{2}$ in the standard bomb test for oxidizability used by Universal Oil Products for gasolines. Atkins, Baker, Murphy, and Zisman [15], however, have observed appreciable oxidation of a number of high-boiling diesters under oxygen at $125 \mathrm{lb} / \mathrm{in} .^{2}$ over much longer periods of time at $100^{\circ} \mathrm{C}$.

\section{Oxidative Break-down Induced by the Plasticizer}

When cellulose ester's were heated under oxygen in the presence of these oxidizable plasticizers, a marked increase in the rate of chainlength break-down was observed. Several plasticizers were evaluated in this way, as described previously. It should be pointed out that the concentration of plasticizer used in this test is many times higher than that used in practical compounding. It would, therefore, be expected that the oxidative effects would be exaggerated. Although the plasticizer itself was not in every case tested for autoxidizability, it was assumed that if the whole mixture oxidized the plasticizer alone would likewise oxidize. Table 12.3 summarizes results obtained for cellulose acetate/butyrate (13 percent of acetyl; 37 percent of butyryl) with a 3-hr heat test. Very little molecular-weight break-down occurred under these conditions, either in the absence of plasticizer or in the presence of the oxidation-resistant plasticizers. Table 12.4 gives further results with other plasticizer's, this time for a 2 -hr heat test. All of these compounds behaved according to expectations, the three not containing methylene or methylidyne groups being the only oxidation-resistant members of the group.

TABLe 12.2. Oxidizability of plasticizers a

\begin{tabular}{|c|c|c|c|}
\hline Plasticizer & $\begin{array}{l}\text { Apparent oxy- } \\
\text { gen absorbed }\end{array}$ & Acid produced & $\begin{array}{l}\text { Peroxide pro- } \\
\text { duced }\end{array}$ \\
\hline $\begin{array}{l}\text { Dimethyl phthalate } \\
\text { Diethyl phthalate. } \\
\text { Dibutyl phthalate } \\
\text { Bis(2-ethylhexyl) phthalate } \\
\text { Dibutyl sebacate. } \\
\text { Tripropionin } \\
\text { Triphenyl phosphate. }\end{array}$ & $\begin{array}{r}m l \\
0 \\
26 \\
52 \\
42 \\
67 \\
20 \\
0\end{array}$ & $\begin{array}{c}\text { mil eq } \\
0 \\
2.0 \\
2.3 \\
1.5 \\
1.7 \\
1.8 \\
0\end{array}$ & $\begin{array}{c}\text { mil } e q \\
0 \\
1.3 \\
0.5 \\
.7 \\
1.6 \\
1.0 \\
0\end{array}$ \\
\hline
\end{tabular}

- Under $\mathrm{O}_{2}$ for $3 \mathrm{hr}$ at $150^{\circ} \mathrm{C}$. 
TABLE 12.3. Induced oxidative degradation of cellulose acetate butyrate a

\begin{tabular}{|c|c|c|}
\hline • & $\begin{array}{l}\text { Apparent } \\
\text { oxygen } \\
\text { absorbed }\end{array}$ & Degradation \\
\hline $\begin{array}{l}\text { None- } \\
\text { Dimethyl phthalate } \\
\text { Diethyl phthalate } \\
\text { Dibutyl phthalate } \\
\text { Bis(2-ethylhexyl) phthalate } \\
\text { Dibutyl sebacate } \\
\text { Tripropionin } \\
\text { Bis(2-ethylhexyl) adipate } \\
\text { 2-Methoxyethyl palmitate } \\
\text { Triphenyl phosphate }\end{array}$ & $\begin{array}{r}m l \\
0 \\
0 \\
29 \\
32 \\
84 \\
66 \\
39 \\
80 \\
63 \\
0\end{array}$ & $\begin{array}{c}\% \text { mol wt } \\
0 \\
11 \\
86 \\
69 \\
76 \\
71 \\
80 \\
63 \\
71 \\
1.5\end{array}$ \\
\hline
\end{tabular}

a $1 \mathrm{~g}$ of ester in $25 \mathrm{ml}$ (or g) of plasticizer heated $3 \mathrm{hr}$ at $150^{\circ} \mathrm{C}$ under $\mathrm{O}_{2}$.

TABLE 12.4. Induced oxidative degradation of cellulose acetate butyrate a

\begin{tabular}{|c|c|c|}
\hline Plasticizer & $\begin{array}{l}\text { A pparent } \\
\text { oxygen } \\
\text { absorbed }\end{array}$ & Degradation \\
\hline $\begin{array}{l}\text { Diisopropyl phthalate } \\
\text { Bis(2-methoxyethyl) phthalate } \\
\text { Tris(2-ethylhexyl) phosphate } \\
\text { Tetrahydrofurfuryl oleate } \\
\text { Bis(2-ethylhexyl) diglycolate } \\
\text { 2,2-Dimethyl-1,3-propanediol bis (2-ethylbutyrate) } \\
\text { Methyl benzoate } \\
\text { Propyl benzoate } \\
\text { Diphenyl 2,2-dimethylmalonate } \\
\text { Bis ( } p \text {-tert-butylphenyl) 2,2-dimethylmalonate }\end{array}$ & $\begin{array}{r}m ! \\
8 \\
8 \\
65 \\
150 \\
27 \\
56 \\
3 \\
34 \\
7 \\
0\end{array}$ & $\begin{array}{c}\% \text { mol wt } \\
57 \\
57 \\
60 \\
40 \\
60 \\
46 \\
10 \\
79 \\
0 \\
19\end{array}$ \\
\hline
\end{tabular}

a $1 \mathrm{~g}$ of ester in $25 \mathrm{ml}$ (or g) of plasticizer heated $2 \mathrm{hr}$ at $150^{\circ} \mathrm{C}$ under $\mathrm{O}_{2}$.

TABLE 12.5. Induced oxidative degradation of cellulose acetate butyrate a

\begin{tabular}{|c|c|c|}
\hline Plasticizer & $\begin{array}{l}\text { Apparent } \\
\text { oxygen } \\
\text { absorbed }\end{array}$ & Degradation \\
\hline $\begin{array}{l}\text { Triethyl phosphate } \\
\text { Ethylenc glycol diacetate } \\
\text { 2,2-Dimethyl-1,3-propanediol diacetate } \\
\text { 2,2-Dimethyl-1,3-propanediol dibenzoate } \\
\text { 2,2-Dimethyl-1,3-propanediol bis (p-tert-butylbenzoate) } \\
\text { 2,2-Dimenthyl-1,3-propanediol } \\
\text { tert-Butyl benzoate }\end{array}$ & $\begin{array}{r}m l \\
0 \\
6 \\
2 \\
0 \\
0 \\
0 \\
170\end{array}$ & $\begin{array}{c}\% \text { mol wt } \\
12 \\
10 \\
20 \\
12 \\
26 \\
0 \\
51\end{array}$ \\
\hline
\end{tabular}

a $1 \mathrm{~g}$ of ester in $25 \mathrm{ml}$ (or g) of plasticizer heated $2 \mathrm{hr}$ at $150^{\circ} \mathrm{C}$ under $\mathrm{O}_{2}$.

In table 12.5 are listed several plasticizers that did not react in the manner that might have been expected from their molecular structure. As before, a 2-hr heating period under oxygen at $150^{\circ} \mathrm{C}$ was used. Except for the last one in the list, all of these compounds contain methylene groups, yet their oxidizability and break-down-inducing capacity are reduced far below normal. It is possible that the methylene groups in these materials are sterically hindered to some degree, especially in the case of 2,2-dimethyl-1,3-propanediol and its esters. The tert-butyl benzoate may have contained some impurity which caused the unexpected oxidation and break-down. A result equally difficult to understand was obtained with dimethyl adipate, which failed to oxidize or cause break-down of cellulose acetate. Here again, some impurity, this time an inhibitor, may have been present. 


\section{Inhibition of Induced Oxidative Break-down}

The oxidative break-down of cellulose esters at $150^{\circ} \mathrm{C}$, promoted by autoxidizable plasticizers, may be very effectively prevented by the addition of a small amount of inhibitor, or antioxidant. Table 12.6 shows the protection given by a concentration of 0.08 percent of several types of inhibitor in a 2 -hr heat test under oxygen with dibutyl sebacate as the plasticizer. Similar results were obtained with other autoxidizable plasticizers. We found that a large number of aromatic amines and phenols were effective, although there were a few puzzling exceptions. Some of these may be explainad on the basis of interference with the functional group, such as by a hydrogen-bonding. For example, table 12.7 gives antioxidant ratings, based on a 2 -hr heat test with dibutyl sabacate as plasticizer, for phenyl salicylate and a number of its isomers. These compounds were obtained from L. W. A. Meyer of the Cellulose Esters and Plastics Development Laboratory. The isomers containing the hydroxyl group in a position ortho to the ester linkage showed little, if any, antioxidant activity. On the other hand, little impairment of activity resulted from the presence of fairly bulky hydrocarbon substituents in the ortho position (for example, see the first phenol listed in table 12.6). Many so-called hindered phenols are finding varied application as antioxidants in the industry today.

\section{Catalysis of Induced Oxidative Break-down}

Oxidations are generally recognized to be chain reactions [16], and, as such, it is to be expected that they will exhibit the phenomena of inhibition, as illustrated above, and of catalysis as well. The latter has been observed, for example, when peroxides are added to a system

TABLE 12.6. Inhibition of oxidative degradation of cellulose acetate butyrate plasticized with dibutyl sebacate ${ }^{\text {a }}$

\begin{tabular}{|c|c|c|}
\hline Antioxidant & $\begin{array}{l}\text { Apparent } \\
\text { oxygen } \\
\text { absorbed }\end{array}$ & Degradation \\
\hline $\begin{array}{l}\text { None } \\
\text { 2, 6-Di-tert-butyl-p-cresol } \\
\text { N-tert-Butylaniline. } \\
\text { N-Phenylglycine } \\
\text { 1, 3-Diphenyl-2-thiourea } \\
\mathrm{N}, \mathrm{N}^{\prime} \text {-Diphenylacetamidine } \\
\alpha \text {-A cetyl-4-(bis 2-hydroxyethyl amino) 2-methylcinnamonitrile } \\
\text { 2-[N-Ethyl-N-( } p \text {-nitro- } p \text {-phenylazoanilino)] ethanol }\end{array}$ & $\begin{array}{l}m l \\
35 \\
0.5 \\
2 \\
0 \\
2 \\
2 \\
0.5 \\
1\end{array}$ & $\begin{array}{l}\% \text { mol wt } \\
49 \\
3 \\
1.5 \\
4 \\
14 \\
13 \\
16 \\
0\end{array}$ \\
\hline
\end{tabular}

a $1 \mathrm{~g}$ of ester in $25 \mathrm{ml}$ of plasticizer heated $2 \mathrm{hr}$ at $150^{\circ} \mathrm{C}$ with $0.02 \mathrm{~g}$ of antioxidant under $\mathrm{O}_{2}$.

TABLE 12.7. Inhibition of oxidative degradation of cellulose acetate butyrate a

\begin{tabular}{|c|c|c|}
\hline Additive & $\begin{array}{l}\text { A pparent } \\
\text { oxygen } \\
\text { absorbed }\end{array}$ & $\begin{array}{c}\text { Degrada- } \\
\text { tion }\end{array}$ \\
\hline $\begin{array}{l}\text { None } \\
\text { Phenyl salicylate } \\
\text { Phenyl } m \text {-hydroxybenzoate } \\
\text { Catechol myonobenzoate } \\
\text { Resorcinol monobenzoate. } \\
\text { Hydroquinone monobenzoate }\end{array}$ & $\begin{array}{l}m l \\
35 \\
44 \\
25 \\
24 \\
16 \\
8 \\
0\end{array}$ & $\begin{array}{c}\% \text { mol wt } \\
49 \\
40 \\
23 \\
27 \\
42 \\
8 \\
0\end{array}$ \\
\hline
\end{tabular}

a $1 \mathrm{~g}$ of ester in $25 \mathrm{ml}$ dibutyl sebacate heated $2 \mathrm{hr}$ at $150^{\circ} \mathrm{C}$ with $0.02 \mathrm{~g}$ of additive under $\mathrm{O}_{2}$. 
consisting of a cellulose ester and an oxidation-resistant plasticizer. In table 12.8 it is shown that the effect of addition of 0.05 percent of acetyl peroxide to a triphenyl phosphate system is to induce a breakdown similar to that occurring in the presence of an autoxidizable plasticizer without addition of peroxide. It seems likely that the reaction proceeds by way of relatively unstable peroxidic intermediates [17], either radicals or molecules or both. The peroxidic material formed during the autoxidation is, in some cases, probably more reactive than the relatively stable peroxides, such as acetyl peroxide, which are handled commercially. Peroxidic break-down is utilized in the burn-out process for remoring cellulose acetate from mixed fabrics [18].

Oxidative break-down may also be catalyzed by a number of heavymetal soaps. The results of some of these experiments have been included in table 12.8. A 2-hr heating period was used in these experiments. According to Robertson and Waters [17], copper and cobalt compounds accelerate oxidation reactions by catalyzing the decomposition of the peroxidic intermediates. Soaps of metals like aluminum, zinc, and cadmium did not act catalytically in our experiments. Titanium dioxide, however, did exert a pronounced catalytic effect on the oxidative break-down under the conditions of the experiments summarized in table 12.9. Addition of an antioxidant prevented this degradation. In these experiments, cellulose acetate butyrate plasticized with 12 parts of dibutyl sebacate was heated for $48 \mathrm{hr}$ in an oren at $130^{\circ} \mathrm{C}$. Pigment and antioxidant, when used, were present in a concentration of 1 percent.

A very interesting phenomenon was noted in the case of cupric stearate. This compound catalyzed oxidation and break-down in the dibutyl sebacate system, as shown in table 12.8 , but retarded these reactions in the diethyl phthalate system. Figure 12.1 illustrates

TABLE 12.8. Catalysis of oxidative degradation of cellulose acetate butyrate a

\begin{tabular}{|c|c|c|c|}
\hline Plasticizer & Catalyst & Atmosphere & Degradation \\
\hline $\begin{array}{r}\text { Triphenyl phosphate. } \\
\text { Do } \\
\text { Dibutyl sebacate } \\
\text { Do } \\
\text { Do } \\
\text { Do } \\
\text { Do } \\
\text { Do } \\
\text { Do } \\
\text { Do }\end{array}$ & $\begin{array}{l}\text { None } \\
\text { Acetyl peroxide } \\
\text { None } \text { Cupric stearate } \\
\text { Cobaltous oleate } \\
\text { Manganous naphthenate } \\
\text { Ferrie stearate } \\
\text { Nickelous stearate. } \\
\text { Chromic stearate } \\
\text { Silrer stearate }\end{array}$ & $\begin{array}{l}\mathrm{T}_{2} \\
\mathrm{~N}_{2} \\
\mathrm{O}_{2} \\
\mathrm{O}_{2} \\
\mathrm{O}_{2} \\
\mathrm{O}_{2} \\
\mathrm{O}_{2} \\
\mathrm{O}_{2} \\
\mathrm{O}_{2} \\
\mathrm{O}_{2}\end{array}$ & $\begin{array}{l}\% \text { mol wt } \\
1.5 \\
36 \\
49 \\
82 \\
60 \\
74 \\
67 \\
73 \\
89 \\
67\end{array}$ \\
\hline
\end{tabular}

a $1 \mathrm{~g}$ of ester in $25 \mathrm{ml}$ (or g) of plasticizer at $150^{\circ} \mathrm{C}$.

TABLE 12.9. Catalysis of oxidative degradation of cellulose acetate butyrate a by titanium dioxide and its inhibition by antioxidant

\begin{tabular}{|c|c|c|}
\hline Pigment & Antioxidant & Degradation \\
\hline $\begin{array}{l}\text { Tone } \\
\text { Titanium dioxide } \\
\text { Do } \\
\text { Do }\end{array}$ & $\begin{array}{l}\text { None-1o- } \\
\text { p-tert-Butylphenol } \\
\text { 2,6-Di-tert-butyl-p-cresol- }\end{array}$ & $\begin{array}{c}\% \text { mol wt } \\
3 \\
87 \\
2 \\
3\end{array}$ \\
\hline
\end{tabular}

a $1 \mathrm{~g}$ of ester with $0.12 \mathrm{~g}$ of dibutyl sebacate and $0.01 \mathrm{~g}$ of pigment (if any) and $0.01 \mathrm{~g}$ of antioxidant (if any) heated $48 \mathrm{hr}$ in $\mathrm{O}_{2}$ at $130^{\circ} \mathrm{C}$. 
Figure 12.1. Positive and negative catalysis of oxidation by cupric stearate.

A, Dibutyl sebacate plus 0.08 percent of cupric stearate; $\mathrm{B}$, dibutyl sebacate; C, diethyl phthalate; D, diethyl phthalate plus 0.08 percent of cupric stearate.

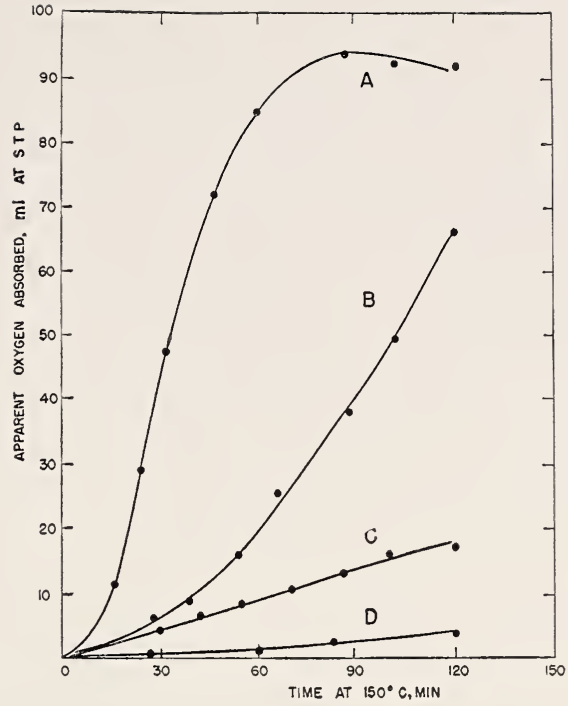

the effect of addition of 0.08 percent of cupric stearate on the rate of oxygen takeup by these two plasticizers at $150^{\circ} \mathrm{C}$. George and Robertson have discussed similar opposing effects [19], or positive and negative catalysis, exhibited by hear $y$-metal compounds in oxidation. reactions. These phenomena emphasize the importance of proper choice of stabilizer to be used with any given plastic system. In the stabilization of polyvinyl chloride plastics, Lally and Hansen [20] have pointed out the necessity of matching the stabilizer to the plasticizer, colorant, and other components of the plastic system.

\section{Thermal, Nonoxidative Break-down}

Cellulose ester plastics can be thermally unstable for reasons other than oxidizability. The pronounced degrading action of traces of strong mineral acids in cellulose ester systems is well known [1]. Cellulose esters must be carefully purified and stabilized against release of acidic matter on heating [21] before they can pass the specifications for heat stability. Plasticizers must also be acid-free. The degrading effect of 0.08 percent of concentrated sulfuric acid is illustrated by table 12.10 . In these experiments, $1 \mathrm{~g}$ of cellulose acetate butyrate was heated for $3 \mathrm{hr}$ at $150^{\circ} \mathrm{C}$. in $25 \mathrm{ml}$ of diethyl phthalate. To guarantee a minimum of oxidative break-down, $0.02 \mathrm{~g}$ of an effective antioxidant was added, and the heating was carried out under an atmosphere of nitrogen. Addition of $0.2 \mathrm{~g}$ of the acid acceptor, 4phenylthiosemicarbazide, counteracted the degrading effect of the sulfuric acid.

The catalytic action of certain metallic impurities on the thermal break-down is perhaps not so well known. It was found that some ferric salts of strong acids were remarkably active in this respect, but that other iron compounds, including iron powder itself, were quite inert. Many other metallic compounds (for example, cupric sulfate, chromic sulfate, nickelous chloride, cobaltous acetate, manganous acetate, and vanadium stearate) were also without effect on this 
TABLE 12.10. Thermal degradation of cellulose acetate butyrate a

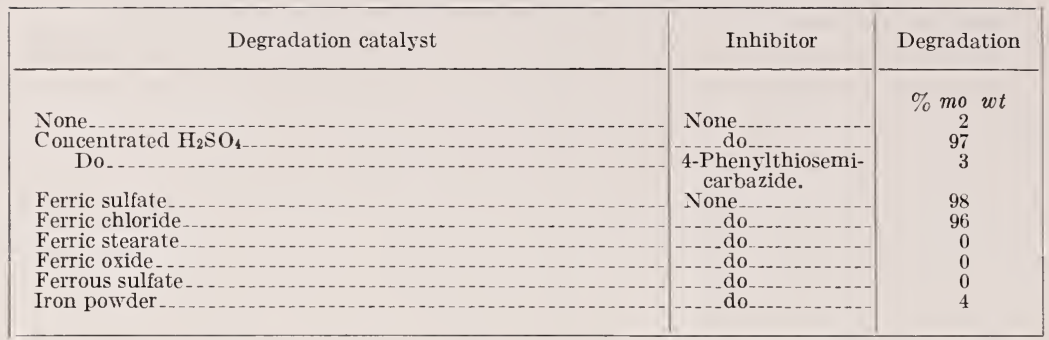

a $1 \mathrm{~g}$ of ester in $25 \mathrm{ml}$ diethyl phthalate heated at $150^{\circ} \mathrm{C}$ under $\mathrm{N}_{2}$ with $0.02 \mathrm{~g}$ of antioxidant added.

TABLE 12.11. Inhibition of thermal degradation of cellulose acetate butyrate a

\begin{tabular}{|c|c|c|c|c|}
\hline $\begin{array}{l}\text { Quality of diethyl } \\
\text { phthalate }\end{array}$ & $\begin{array}{l}\text { Added degradation } \\
\text { catalyst }\end{array}$ & Inhibitor & $\begin{array}{c}\text { Heating } \\
\text { time }\end{array}$ & $\begin{array}{l}\text { Degra- } \\
\text { dation }\end{array}$ \\
\hline 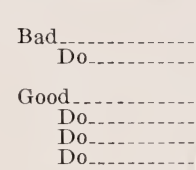 & $\begin{array}{l}\text { None } \\
\text { Ferric chloride } \\
\text { Ferric sulfate }\end{array}$ & $\begin{array}{l}\text { None } \\
\text { amine. } \\
\text { ame } \\
\text { 1,3-Diphenyl-2-thiourea } \\
\text { None } \\
\beta \text {-Isothioureidopropionic acid }\end{array}$ & $\begin{array}{l}h r \\
3 \\
3 \\
\\
2 \\
2 \\
2 \\
2\end{array}$ & $\begin{array}{c}\% \text { mol wt } \\
99 \\
6 \\
91 \\
17 \\
92 \\
3\end{array}$ \\
\hline
\end{tabular}

a $1 \mathrm{~g}$ of ester in $25 \mathrm{ml}$ of plasticizer heated at $150^{\circ} \mathrm{C}$ under $\mathrm{N}_{2}$ with $0.02 \mathrm{~g}$ of antioxidant added.

type of break-down. These tests were carried out in the same way as those for acid break-down and are included in table 12.10. Each metallic compound was added to the extent of 0.08 percent.

Occasionally, batches of plasticizer (for example, diethyl phthalate) which cause objectionable thermal break-down when incorporated into cellulose ester plastics are encountered. Such batches are, of course, screened out by the heat stability tests which are carried out as a part of the regular quality control procedures. These batches may contain neither free acid nor peroxide detectable by the ordinary chemical analyses. Iron contamination was found, howerer, by spectrographic analysis. The stability of the plastic prepared from such a plasticizer could be greatly improved by the addition of a suitable metal deactivator, or chelating agent. 'Table 12.11 summarizes these experiments, which were run under the same conditions as those of table 12.10 .

Batches of diethyl phthalate, made unstable by the addition of ferric salts, could likewise be corrected br the addition of certain chelating compounds. The examples included in table 12.11 were 2-hr tests with $0.1 \mathrm{~g}$ of chelating inhibitor and $0.002 \mathrm{~g}$ of ferric chloride instead of the usual $0.02 \mathrm{~g}$.

\section{Break-down by Ultraviolet Light}

\section{Effect of Plasticizer}

Since the plasticizer was found to play such an important part in the break-down by heat, this variable was investigated in the weathering process. In addition to variations in photo-oxidizability from one plasticizer to another, there are, of course, the variations in ultraviolet absorption to be considered. Meyer and Gearhart [6] have 
reported, for example, that aromatic plasticizers contribute some protection against uitraviolet break-down by virtue of their increased absorption over that of aliphatic plasticizers.

Table 12.12 shows the extent to which the concentration of dibutyl sebacate affects the degradation taking place during 4 mo of exposure to the weather at Kingsport, Tenn. That the effect is not greater is probably due to the fact that in this system the light is primarily absorbed by the cellulose ester. Thus it might be expected that the photo-oxidation of the plasticizer and the break-down induced thereby in the cellulose ester would play a secondary role.

Another experiment apparently illustrating the deleterious effect of an oxidizable plasticizer on the weathering resistance of the plastic was one in which the break-down, occurring in a thin sample, was compared with that occurring in an equally thin outside layer of a thick sample. In table 12.13 are listed the decreases in molecular weight during both natural and accelerated weathering of clear and pigmented thin plastic sheets as compared with the corresponding decreases in equally thin outside layers of thick samples, milled off after exposure. Twelve parts of dibutyl sebacate were used in both clear and pigmented formulations, the latter containing in addition 2 parts of pigment. The plasticizer content of the thin pieces, as the result of evaporation and leaching during exposure, would remain well below that of the outside layers of the thick pieces, which would be kept supplied with fresh plasticizer by diffusion from the internal reservoir. Break-down in the outside layers of the thick pieces was

TABLE 12.12. Effect of plasticizer concentration on outdoor weathering break-down in cellulose acetate butyrate plastic ${ }^{\text {s }}$

\begin{tabular}{|c|c|}
\hline $\begin{array}{c}\text { Dibutyl sebacate } \\
\text { concentration }\end{array}$ & Degradation \\
\cline { 1 - 2 } $\begin{array}{c}\text { Parts/100 parts of } \\
\text { cellulose ester } \\
0\end{array}$ & \\
1 & \\
2 & 9.5 \\
4 & 11.8 \\
8 & 13.4 \\
12 & 16.3 \\
16 & 17.7 \\
20 & 16.5 \\
25 & 14.7 \\
& 16.5 \\
& \\
\hline
\end{tabular}

a Plasticizer: dibutyl sebacate; sample thickness, 0.050 in.

$\mathrm{T}_{\mathrm{ABLE}}$ 12.13. Effect of sample thickness on weathering break-down in cellulose acetate butyrate plastic a

\begin{tabular}{|c|c|c|c|}
\hline \multirow{2}{*}{ Pigmentation } & \multirow{2}{*}{$\begin{array}{l}\text { Sample } \\
\text { thickness }\end{array}$} & \multicolumn{2}{|c|}{ Outer-layer degradation } \\
\hline & & Outdoor b & Accelerated \\
\hline $\begin{array}{l}\text { None } \\
\text { Do.... } \\
\text { Titanium dioxide. } \\
\text { Do }\end{array}$ & $\begin{aligned} & \text { in. } \\
& 0.010 \\
& \text { d. } .187 \\
& .010 \\
& \text { d. } 187\end{aligned}$ & $\begin{array}{c}\% \text { mol wt } \\
15.1 \\
49.0 \\
3.3 \\
14.3\end{array}$ & $\begin{array}{l}\% \text { mol wt } \\
21.2 \\
85.0 \\
18.6 \\
65.0\end{array}$ \\
\hline
\end{tabular}

a Plasticizer content: 12 parts of dibutyl sebacate per 100 parts of cellulose ester.

b 6 months in Kingsport, Tenn.

c $1,000 \mathrm{hr}$ in Twin-Are Weather-ometer.

d Layer thickness, 0.010 in. 


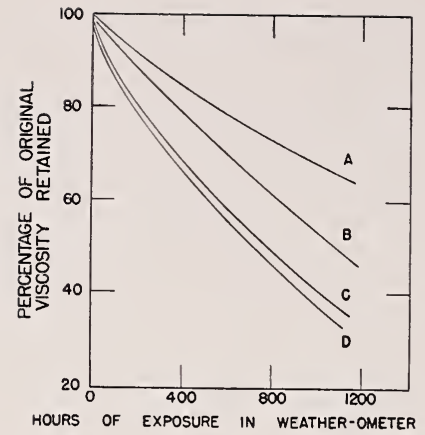

Figure 12.2. Post-irradiation break-down in cellulose acetate butyrate plastic.

$A$, In acetone, no heating; $B$, in pyridine, no heating; $C$, in pyridine, heated 15 hours at $80^{\circ} \mathrm{C} ; \mathrm{D}$, in pyridine, heated 35 hours at $80^{\circ} \mathrm{C}$.

considerably greater than in the corresponding thin samples from which the plasticizer was allowed to escape during exposure.

The apparent effect of plasticizer concentration in this indirect experiment is so much greater than would have been expected from the previous direct experiment that we must suspect an additional effect acting in the same direction to cause the milled-off layer to show more break-down. This is perhaps to be found in the well-known thermal instability characteristic of cellulosic compounds which have been irradiated by ultraviolet light $[4,5,22]$. The heat generated by the milling operation may have been sufficient to cause considerable additional break-down. "The extent to which such a dark reaction proceeds in cellulose acetate butyrate after exposure may be seen in figure 12.2. Viscosities of dilute pyridine solutions of exposed samples were markedly lowered by heat treatments too mild to affect the viscosity of unexposed material. These viscosity decreases were accompanied by the development of a yellowish-brown color, the intensity of which was proportional to the previous irradiation received.

\section{Effect of Oxygen}

The effects of oxygen in the photodegradation process itself may be demonstrated easily by accelerated exposures in sealed quartz tubes. Table 12.14 shows a comparison of the break-downs observed in unplasticized cellulose acetate butyrate during exposure in the Weatherometer, both in the usual unprotected manner and sealed inside quartz tubes, either under air or nitrogen atmosphere. Elimination of oxygen reduced break-down considerably.

$\mathrm{T}_{\mathrm{ABLE}}$ 12.14. Break-down of cellulose acetate butyrate in sealed quart $z$ tubes ${ }^{\mathrm{a}}$

\begin{tabular}{|c|c|}
\hline Exposure conditions & Degradation \\
\hline & $\%$ mol wt \\
& 44 \\
Unprotected .............. & 12 \\
Sealed in air & 12 \\
\hline
\end{tabular}

a $800 \mathrm{hr}$ of exposure in Weather-ometer.

\section{Haze Formation in Unplasticized Ester}

A curious effect was noted in the weathering of the unplasticized cellulose acetate. During unprotected exposure, either outdoor's or in the Weather-ometer, an opaque, white layer developed on both front and back surfaces. Since this did not occur in either sealed-tube 


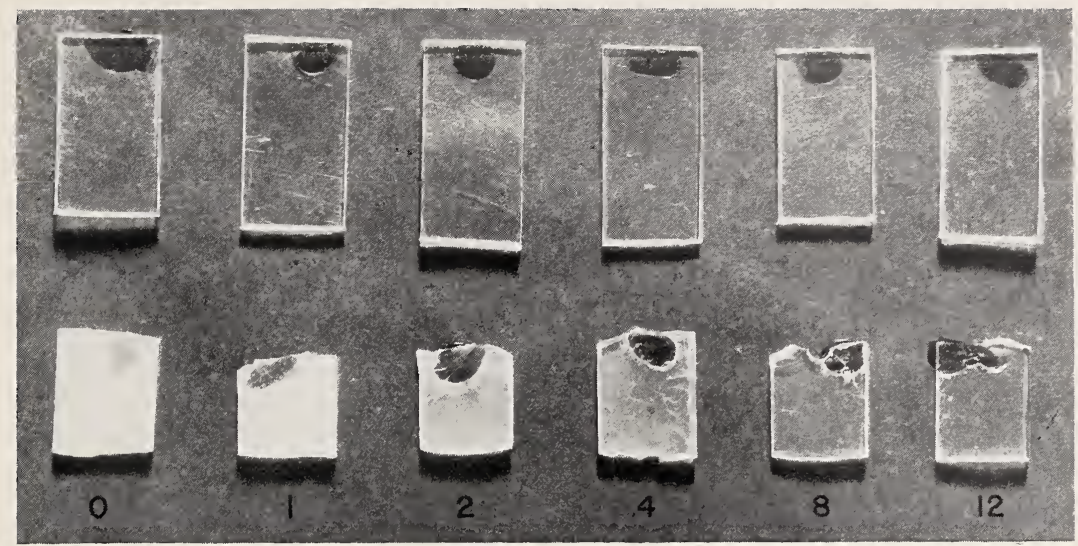

Figure 12.3. Effect of plasticizer concentration on development of haze during weathering of cellulose acetate butyrate plastic.

Numerals under each pair of pieces denote parts of dibutyl sebacate.

exposure, it is assumed to be the result of a photochemical reaction involving moisture. The presence of only small concentrations of plasticizer is required to prevent the formation of this white haze during weathering. This effect is illustrated in figure 12.3, which shows the appearance, before and after $350 \mathrm{hr}$ of exposure in the modified Weather-ometer, of cellulose acetate butyrate samples containing 0 to 12 parts of dibutyl sebacate.

\section{Phenyl Salicylate as an Ultraviolet-Break-down Inhibitor}

In actual practice, a break-down inhibitor, such as phenyl salicylate, is added to cellulose ester plastics for outdoor use. Figure 12.4 illustrates the remarkable effectiveness of 1 percent of phenyl salicylate in preventing break-down in 3/16-in.-thick samples of cellulose acetate butyrate, plasticized with 12 parts of dibutyl sebacate, during exposure in Arizona [6]. It has been found difficult to improve on this inhibitor for a colorless and noncoloring outdoor plastic formulation. The great weakness of phenyl salicylate is its escaping tendency, which limits its use to relatively thick articles. As shown by table 12.15 , the protection afforded by 1 percent of phenyl salicylate, in a plastic the same as that of figure 12.4, decreases quite rapidly with decreasing thickness. Layers 0.025-in. thick were milled off the thicker pieces for comparison with the thinnest piece which had a total thickness of 0.025 in. These pieces had each received 20 mo of exposure in Arizona. The situation with the protected plastic is thus seen, by comparison with table 12.13 , to be just the reverse of that in the unprotected plastic. In the latter case, the thin surface layers on thick pieces were more degraded than corresponding individual thin pieces. The excessive break-down in the layer milled from the 0.050 -in.-thick piece may be the result of additional thermal breakdown by milling a material already considerably photodegraded (table 12.13).

When a similar plastic composition containing 1 percent of phenyl salicylate was sealed in air in a quartz tube and exposed to Weatherometer radiation, the break-down rate was sharply reduced from that observed in the same sample exposed outside the tube. The former 


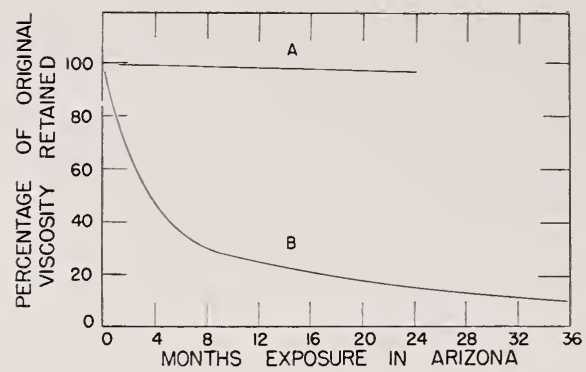

F I G URE 12.4. T'iscosity break-down in sunlight of cellulose acetate butyrate plastic.

A, With 1 percent of phenyl salicylate; $\mathrm{B}$, uninhibited.

TABLE 12.15. Effect of sample thickness on outdoor-weathering break-down in cellulose acetate butyrate plastic protected by phenyl salicylate a

\begin{tabular}{|c|c|}
$\begin{array}{c}\text { Sample thick- } \\
\text { ness }\end{array}$ & $\begin{array}{c}\text { Outer layer } \\
\text { degradation } \mathrm{b}\end{array}$ \\
\cline { 1 - 1 } In. & $\%$ mol wt \\
0.125 & 20 \\
.100 & 30 \\
.075 & 46 \\
.050 & 85 \\
.025 & 70 \\
\hline
\end{tabular}

a Exposed 20 months in Arizona.

b Layer thickness, 0.025 in.

TABLE 12.16. Ultraviolet-filter action of phenyl salicylate in cellulose acetate butyrate plastic

\begin{tabular}{|c|c|c|}
\hline \multirow{2}{*}{$\begin{array}{l}\text { Phenyl sali- } \\
\text { cylate concen- } \\
\text { tration }\end{array}$} & \multicolumn{2}{|c|}{$\begin{array}{l}\text { Relative degradation } \\
\text { rate }\end{array}$} \\
\hline & Calculated & Observed \\
\hline $\begin{array}{c}\text { Percent } \\
0.00 \\
.01 \\
.03 \\
.1 \\
.3 \\
1.0\end{array}$ & $\begin{array}{r}100 \\
92 \\
78 \\
49 \\
25 \\
11\end{array}$ & $\begin{array}{r}100 \\
84 \\
78 \\
55 \\
27 \\
11\end{array}$ \\
\hline
\end{tabular}

sample showed only a 14-percent decrease in molecular weight after $800 \mathrm{hr}$ of exposure, whereas the latter lost 46 percent, probably because of the greater loss of phenyl salicylate.

It is perhaps not generally realized that the best inhibitor for a plastic article of substantial thickness may not be the best for a thin sheet. In the latter case, it may be better to sacrifice some initial efficiency in the inhibitor for the sake of a gain in its permanence.

Phenyl salicylate appears to function primarily as an inert inner filter for the ultraviolet light, according to studies made by Morey and Tichenor [23]. In table 12.16 is shown the agreement they obtained between observed and calculated rates of break-down, under an S-4 lamp, of plastic samples containing various concentrations of phenyl salicylate. These experiments were made on 0.01-in.-thick films of cellulose acetate butyrate plasticized with 10 percent of triphenyl phosphate. In the calculations, a uniform quantum yield of breakdown was assumed for wavelengths below $3500 \mathrm{~A}$.

As table 12.7 indicated, phenyl salicylate shows much less anti- 
oxidant activity than its isomer, resorcinol monobenzoate. This may be an important factor in determining the superiority of the latter as a weathering inhibitor [24]. The primary requirement for a good inhibitor, however, is that it have the proper molecular make-up for absorbing ultraviolet energy and disposing of this absorbed energy in a harmless manner. Many efficient antioxidants do not fulfill this condition and are of little use by themselves as weathering inhibitors. Resorcinol monobenzoate combines both absorbing and antioxidant functions to a marked degree.

\section{Value of Antioxidant in Pigmented Plastic}

We found that the addition of an antioxidant can be particularly valuable for increasing the resistance of certain pigmented plastic formulations toward accelerated weathering. For example, when titanium dioxide was the pigment used, almost any antioxidant was highly effective in preventing the break-down that otherwise occurred at depths within the plastic not directly reached by the actinic light. The photosensitization of oxidation reactions by titanium dioxide has been discussed by Weyl and Forland [25]. Table 12.17 gives some illustrative examples of this protection in cellulose acetate butyrate containing 12 parts of dibutyl sebacate and 2 parts of pigment. In each case, 1 percent of antioxidant was used. In this formulation, phenyl salicylate gave no added protection. By contrast, in the unpigmented plastic, phenyl salicylate gave better protection than $p$-tert-butylphenol and very much better protection than the "hindered" phenol.

TABLE 12.17. Protective action of antioxidants in cellulose acetate butyrate plastic pigmented with titanium dioxide a

\begin{tabular}{|c|c|c|}
\hline \multirow{2}{*}{ Antioxidant } & \multicolumn{2}{|c|}{$\begin{array}{l}\text { Time in weather-ometer re- } \\
\text { quired for 25-percent loss of- }\end{array}$} \\
\hline & $\begin{array}{l}\text { Inherent } \\
\text { viscosity }\end{array}$ & $\begin{array}{l}\text { Flexural } \\
\text { strength }\end{array}$ \\
\hline & $h r$ & $h r$ \\
\hline Phenyl salicylate & $\begin{array}{l}1,900 \\
1,900\end{array}$ & 700 \\
\hline 2,6-Di-tert-butyl-p-cresol & 3,500 & 800 \\
\hline $\begin{array}{l}p \text {-tert-Butylphenol } \\
\mathrm{N}^{\prime}, \mathrm{N}^{\prime} \text {-Diphen ylacetamidine }\end{array}$ & $\begin{array}{l}>3,200 \\
>4,400\end{array}$ & $\begin{array}{r}3,500 \\
>4,400\end{array}$ \\
\hline Propyl gallate & $>4,600$ & $>4,600$ \\
\hline$p$-Butylaminophenol.. & $>4,700$ & $>4,700$ \\
\hline
\end{tabular}

a Sample thickness, $0.050 \mathrm{in}$.

\section{Mechanisms of Inhibition}

The first necessary property of a weathering inhibitor is that it be the primary absorber of the ultraviolet light entering the plastic system. Unfortunately, this is not a sufficient requirement, for then it would be easily possible to combine several compounds whose absorptions blanketed the range of harmful radiation. The absorbed ultraviolet energy must be disposed of in a manner harmless to the polymer chains, and this turns out to be a much more exacting requirement. No major fraction of the absorbed quantum must be transferred to the polymer chain in a single packet of energy. The inhibitor should have the ability to fritter the ultraviolet quantum rapidly away into small, harmless increments of vibrational magnitude. The remarkable photochemical stability of crotonaldehyde has been ascribed to this sort of property [26]. 
Molecular rearrangements offer a mechanism for achieving safe disposal of energy. Since it is equally essential to preserve the inhibitor in its active form, the rearrangement should reverse itself in the dark. A number of azo dyes are known to be phototropic on cellulose acetate yarn, changing to a different color when exposed to ultraviolet or visible light and returning to the original shade in the dark. These compounds are effective inhibitors of Weather-ometer break-down when incorporated into cellulose ester plastic, as table 12.18 shows. The plastic formulation used in these experiments was cellulose acetate butyrate with 12 parts of dibutyl sebacate and 1 part of inhibitor. The mechanism of rearrangement occurring here may be the cis-trans isomerism discussed by Hartley [27], or in some cases, an azo-hydrazone shift [28].

An oxidation-reduction mechanism for regeneration of the inhibitor may play a part in the functioning of certain types of inhibitors. Such a mode of action has been discussed by Murphy, Ravner, and Smith in connection with the use of phenothiazine as an antioxidant for ester-type lubricants [29].

Aryl amidines and aryl imino ethers undergo easy thermal rearrangements by an $\alpha-\gamma$ shift of a phenyl group or other substituent [30]. Possibly this type of rearrangement also occurs photochemically. At any rate, these compounds are effective weathering inhibitors for cellulose ester plastic, as illustrated by table 12.19. The plastic formulation in this series of experiments was the same as that in table 12.18,

TABLE 12.18 Effectiveness of phototropic azo dyes as weathering inhibitors for cellulose acetate butyrate plastic a b

\begin{tabular}{|c|c|c|}
\hline \multirow{2}{*}{ Inhibitor } & \multicolumn{2}{|c|}{$\begin{array}{l}\text { Time in Weather-ometer re- } \\
\text { quired for- }\end{array}$} \\
\hline & $\begin{array}{l}\text { 25-percent loss } \\
\text { in inherent } \\
\text { viscosity }\end{array}$ & $\begin{array}{l}\text { Development } \\
\text { of brittleness }\end{array}$ \\
\hline $\begin{array}{l}\text { None } \\
\text { Phenyl salicylate- } \\
\text { p-Phenylazodiphenylamine-- } \\
\text { 2,5-1imethoxy-4-phenyl-azoaniline } \\
\text { 4-(o-Chlorophenylazo)-N-methyl-m-toluidine } \\
p \text {-[( } p \text {-Dimethylamino)-phenylazo] acetanilide] }\end{array}$ & $\begin{array}{r}h r \\
400 \\
1,000 \\
>1,700 \\
1,500 \\
>1,700 \\
>2,600\end{array}$ & $\begin{array}{r}h r \\
400 \\
800 \\
>1,700 \\
>1,700 \\
>3,100\end{array}$ \\
\hline
\end{tabular}

a $1 \mathrm{~g}$ of ester with $0.12 \mathrm{~g}$ of dibutyl sebacate and $0.01 \mathrm{~g}$ of inhibitor exposed in Weather-ometer.

b Sample thickness, 0.050 in.

TABLE 12.19. Effectiveness of rearrangeable compounds as weathering inhibitors for cellulose acetate butyrate plastic a b

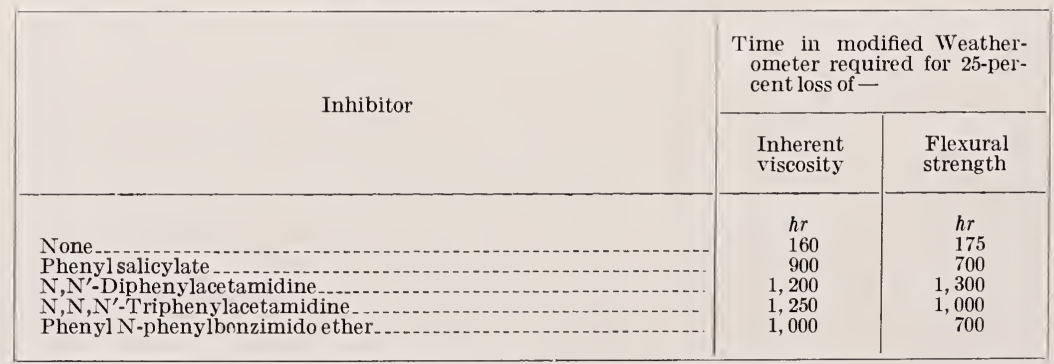

a $1 \mathrm{~g}$ of ester with $0.12 \mathrm{~g}$ of dibutyl sebacate and $0.01 \mathrm{~g}$ of inhibitor exposed in Weather-om?ter modified by addition of 8 fluorescent sun lamps.

b Sample thickness, 0.050 in. 
but the exposures were made in the modified Weather-ometer. Morey and Tichenor [31] recommended the use of $\mathrm{N}, \mathrm{N}^{\prime}$-diphenylacetamidine as a weathering inhibitor for cellulose ester plastics.

Many derivatives of the molecular configuration,

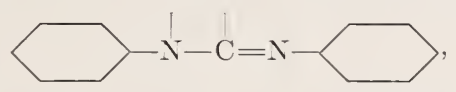

were found to confer a high degree of weathering protection on cellulose ester systems. Many effective inhibitors, such as phenyliminobenzothiazolines [32], phenylaminobenzothiazoles, aryl amidines [31], aryl ureas, and aryl thioureas, fall into this classification. In all of these compounds, similar $\alpha-\gamma$ shifts of substituent groups are possible.

\section{Aggregative Changes Caused by Light}

In addition to chain-breaking processes, chain-building processes also were observed during weathering. Tavlor and Tobolsky [33] have discussed the simultaneous occurrence of scission, cross-linking, and branching in vinyl polymers. Figure 12.5 illustrates the very delicate balance that can exist between these disaggregative and aggregative processes during Weather-ometer exposures of cellulose acetate butrrate plastic treated with tetraethyl silicate. The formulation used in these experiments contained 12 parts of dibutyl sebacate and 2 parts of titanium dioxide.

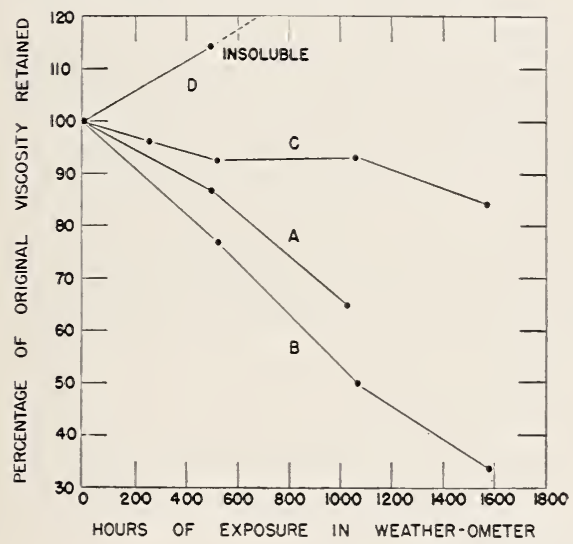

Figure 12.5. Weathering of cellulose acetate butyrate plastic treated with tetraethyl silicate.

Minutes soaking in 1:1 ethanol-tetraethyl silicate: A, 0; B, 15; C, 85; D, 290 (dotted line indicates insolubility).

\section{Kinetics of Break-down}

\section{Thermal Break-down}

Suppose we heat $m$ grams of cellulose ester whose number-average degree of polymerization is $\bar{P}_{o}$ for a time $t$ at some fixed elerated temperature so that the degree of polymerization drops to $\bar{P}_{t}$. The number of chain links in the system, originally $N_{o}$, will correspondingly drop to $N_{t}$, where

and

$$
N_{o}=m\left(\bar{P}_{o}-1\right) / u \cdot \bar{P}_{o},
$$

$$
N_{t}=m\left(\bar{P}_{t}-1\right) / u \cdot \bar{P}_{t}
$$

Here, $u$ represents the mass of the anhydroglucose unit of the cellulose ester chain. If the number of links breaking per unit of time is pro- 


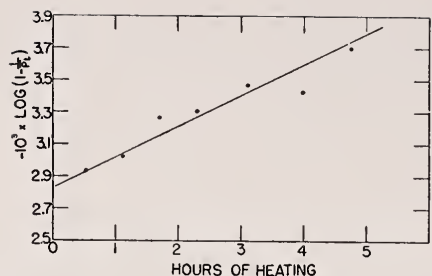

FIgURE 12.6. Molecular-weight break-down of cellulose acetate butyrate at $190^{\circ} \mathrm{C}$.

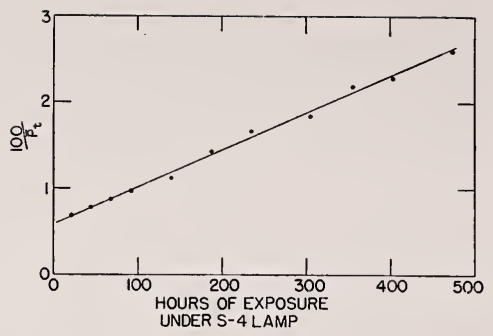

FiguRE 12.7. Molecular-weight break down of cellulose acetate butyrate by ultraviolet light.

portional to the total number of links present, that is, if breakage is random, then

or

Thus

$$
-d N / d t=k N
$$

$$
N_{t}=N_{o} e^{-k t}
$$

or

$$
\left(\bar{P}_{t}-1\right) / \bar{P}_{t}=\left[\left(\bar{P}_{o}-1\right) / \bar{P}_{o}\right] e^{-k t}
$$

$$
\ln \left(1-1 / \bar{P}_{t}\right)=\ln \left(1-1 / \bar{P}_{o}\right)-k t \text {. }
$$

This is the equation given by Wolfrom, Sowden, and Lassettre [34] for the acid-catalyzed hydrolysis of methylcellulose. Data obtained for degradation of cellulose acetate butyrate at $190^{\circ} \mathrm{C}$. also obeyed eq (1) reasonably well, as shown in figure 12.6. Heating was carried out in a glass tube containing $7.5 \mathrm{~g}$ of the cellulose ester and $10 \mathrm{ml}$ of dimethyl phthalate. This was held in an oil bath with the tube open to the atmosphere and was kept stirred by a Brookfield viscometer bob. Dilute solution viscosities, run on samples withdrawn from the melt from time to time, were used [12] to estimate the number-average molecular weights shown in figure 12.6.

\section{Ultraviolet Break-down}

If, as in the case of thermal break-down, we assume random breakage of chain links, the kinetics are quite simple. The number of links breaking per unit of time is constant, at least in the initial stages of degradation. Calling this constant $k^{\prime}$, we may write

$$
N_{t}=N_{o}-k^{\prime} t
$$

or

$$
\frac{1}{\bar{P}_{t}}=\frac{1}{\bar{P}_{o}}+k^{\prime \prime} t
$$

where

$$
k^{\prime \prime}=\frac{u}{m} k^{\prime} .
$$

Figure 12.7 shows how well eq (2) fits data calculated from viscosity measurements [35] of Walker, Tichenor, and Morey on 0.012-in-thick films of cellulose acetate butyrate plasticized with 10 percent of triphenyl phosphate. Exposures were made with an S-4 lamp at a temperature of $55^{\circ} \mathrm{C}$. The linear relationship of reciprocal molecular 
weight with exposure time was preserved to a remarkably high degree of degradation.

The agreement of the thermal and ultraviolet break-down data with eq (1) and (2) is taken to indicate that chain-link breakage was random in both types of break-down. This inplies that the original cellulose ester contained no weak links.

\section{Summary and Conclusions}

Man plasticizers containing methrlene or methrlidrne groups were found to be susceptible to oxidation at $150^{\circ} \mathrm{C}$ and therebr induced chain-length break-down in cellulose esters. Antioxidants prevented this type of degradation; hearr-metal soaps and titanium dioxide catalyzed it.

Severe thermal break-down was caused br traces of strong mineral acid or by certain ferric salts. The former type of break-down could be largely prevented by the addition of acid acceptors, the latter by metal deactivators.

Oxidative degradation, induced by the presence of autoxidizable plasticizers, still played a part, though a less important one, in the break-down produced by irradiation with ultraviolet light.

Agreement of calculated with observed rates of ultraviolet breakdown indicates that the inhibiting action of phenyl salicylate is due to its ability to function as an inert inner filter.

It is postulated that compounds such as arrl amidines and azo dres are effective inhibitors, because they can safely dispose of absorbed energy by processes of molecular rearrangement.

The kinetics of break-down br heat and light were shown to be consistent with the theory that breakage of chain links is a random process in both thermal and ultrariolet degradation.

We are greatly indebted to many of our associates for advice and assistance, especiall $Y$ to Harmon Long for conducting experiments on heat break-down; to G. M. Armstrong for supervising the compounding of plastic samples and the phrsical testing of weathered specimens; to G. R. Greear and G. N. Nerland for help with the weathering experiments and viscosity measurements; and to L. IV. A. Meyer for furnishing us with many of the plastic test materials and for continued helpful adrice.

\section{References}

[1] Eran F. Erans and Lane F. McBurner, Ind. Eng. Chem. 41, 1260 (1949).

[2] Ralph E. Montonna and C. C. Winding. Ind. Eng. Chem. 35, 782 (1943).

[3] T. S. Lawton, Jr, and H. K. Nason, Ind. Eng. Chem. 36, 1128 (1944).

[4] E. Heuser and George $\mathrm{N}$. Chamberlin, J. Am. Chem. Soc. 68, 79 (1946).

[5] Herbert F. Launer and William K. W'ilson, J. Research NBS 30, 55 (1943); RP1517: J. Am. Chem. Soc. i1, 958 (1949).

[6] L. W. A. Meyer and W. M. Gearhart, Ind. Eng. Chem. 3i, 232 (1945).

[7] R. L. Tichenor, J. Polymer Sci. 1, 217 (1946).

[8] V. R. Kokatnur and IIurray Jelling. J. Am. Chem. Soc. 63, 1432 (1941).

[9] C. J. Malm, L. B. Genung. R. F. Williams, Jr., and M. A. Pile, Ind. Eng. Chem., Anal. Ed. 16, 501 (194t).

[10] R. H. Wagner and John Russell, Anal. Chem. 20, 151 (1948).

[11] L. H. Cragg, J. Colloid Sci. 1, 261 (1946).

[12] J. IT. Tamblyn, D. R. Morey, and R. H. Wagner, Ind. Eng. Chem. 3i, $573(1945)$.

[13] W. J. Badgley, Polymer Bul. 1, 17 (1945).

[14] R. Criegee, H. Pilz, and H. Flygare, Ber. deut. chem. Ges. 22, 1799 (1939). P. George and A. D. Walsh, Trans. Faradar Soc. 42, 94 (1946). 
[15] D. C. Atkins, Jr., H. R. Baker, C. M. Murphy, and W. A. Zisman, Ind. Eng. Chem. 39, 481 (1947).

[16] General Discussion on Oxidation, Trans. Faraday Soc. 42, 99-398 (1946).

[17] Alan Robertson and W. A. Waters, Trans. Faraday Soc. 42, 201 (1946).

[18] Louis Diserens, Chemical technology of dyeing and printing, II, p. 245 (Reinhold Publishing Co., New York, N. Y., 1951).

[19] Philip George and Alan Robertson, Trans. Faraday Soc. 42, 217 (1946).

[20] R. E. Lally and F. R. Hansen, Modern Plastics 2\%, No. 4, 111 (1949).

[21] Carl J. Malm and Charles R. Fordyce, Cellulose and cellulose derivatives, p. 675 (Interscience Publishers, Inc., New York, N. Y. 1943).

[22] Robert A. Stillings and Robert J. Van Nostrand, J. Am. Chem. Soc. 66, 753 (1944).

[23] D. R. Morey and R. L. Tichenor, unpublished work.

[24] L. W. A. Meyer and W. M. Gearhart, Ind. Eng. Chem. 43, 1585 (1951).

[25] W. A. Weyl and Tormod Forland, Ind. Eng. Chem. 42, 257 (1950).

[26] F. E. Blacet and J. G. Roof, J. Am. Chem. Soc. 58, 73 (1936).

[27] G. Spencer Hartley, J. Chem. Soc. 633 (1938).

[28] J. Nils Ospenson, Acta Chem. Scand. 4, 1351 (1950).

[29] C. M. Murphy, Harold Ravner, and Nathan L. Smith, Ind. Eng. Chem. 42, 2479 (1950).

[30] Arthur Wm. Chapman et al., J. Chem. Soc. 2138 (1929) ; 2458, 2462 (1930); $1770(1932)$.

[31] D. R. Morey and R. L. Tichenor, U. S. Patent 2393802 (Jan. 29, 1946).

[32] D. R. Morey and R. L. Tichenor, U. S. Patent 2393801 (Jan. 29, 1946).

[33] H. S. Taylor and A. V. Tobolsky, J. Am. Chem. Soc. 67, 2063 (1945).

[34] M. I. Wolfrom, John C. Sowden, and E. N. Lassettre, J. Am. Chem. Soc. 61, 1072 (1939).

[35] W. F. Walker, R. L. Tichenor, and D. R. Morey, unpublished work.

\section{Discussion}

Dr. A. J. Warner, Federal Telecommunication Laboratories, Inc. Nutlıy, N. J.: We have found that in the case of the vinyl resins it is most important that the purification of the plasticizer be taken into account. It is known that plasticizers exist in several commercial grades and the life of vinyl compounds is very dependent on the quality of the plasticizer used. We have found that a very useful tool for examining plasticizers to be used for compounds having the greatest probability of long life is the measurement of the electrical resistivity particularly at elevated temperatures. These measurements detarmine and detect quite small amounts of ionizable materials. It is of course known that you can purify plasticizers from adventitious ionic materials by subjecting the material to an electrical field and, in effect, "plating out" the impurities.

Dr. R. J. Wolf, B. F. Goodrich Chemical Co., Avon Lake Experimental Station, Avon Lake, Ohio: Have lead compounds been tested as to their effect on the oxidative break-down?

Dr. Tamblyx: The only one we tested was lead stearate. Compared with many other heavy-metal salts of fatty acids it acted only as a very mild catalyst of oxidative break-down. It did not, however, contribute to the thermal break-down in the presence of an antioxidant.

Major W. H. Ebelke, Wright Air Development Center, Dayton, Ohio: Have you studied the attack of fungi on cellulose esters?

Dr. TamblyN: We find that dibutyl sebacate is hospitable to fungi, whereas phthalate plasticizers are more repellent. Some of these materials have been exposed in Florida and come back covered with a black deposit of fungus, which interferes very much with the weathering study. Some copper organic compounds would act as fungicides and would also have some value as weathering inhibitors. 


\title{
13. Oxidative Degradation of Ethyl Cellulose
}

\author{
By L. F. McBurney ${ }^{1}$
}

In recent years major advances have been made in the chemical theory of polymer behavior. These contributions have been based upon a more precise knowledge of free-radical reactions as well as of electron-transfer mechanisms in general. One of these developments has been the realization of the importance of oxidation processes, and especially those that are the result of molecular oxygen attack by means of free-radical propagation.

The cellulose chemist, on the other hand, has long been aware of the importance of oxygen relative to final properties of the polymer. For example, in viscose manufacture, commercial use is made of the oxidation of alkali cellulose by atmospheric oxygen to achieve the desired product viscosity. This has been a general practice for many years, although only very recently have Entwistle and coworkers [1] ${ }^{2}$ published an excellent series of papers that shed considerable light upon the mechanism of this autoxidation reaction.

In the field of cellulose ethers it has been recognized industrially that raw films would lose tensile strength and become brittle when exposed to heat and light. Furthermore, the loss of strength by the polymers was found to progress at a fairly rapid rate. This knowledge has led to the practice of incorporating antioxidants into the plastic formulations in order to reduce the loss of strength. The mechanism involved in the reaction was not known, however, and the literature contained many inconsistencies regarding the types and effectiveness of antioxidants. The present investigation was undertaken to establish the nature of the degradation reactions so that a basic approach to stabilization would be possible. The commercial importance of ethyl cellulose made it the obvious choice for this study.

An examination of the structure of this polymer, with its high concentration of ethyl ether linkages, would lead to the immediate assumption that degradation under weathering conditions of oxygen, heat, and light is an oxidation reaction similar to those of the simple ethers $[2,3,4,5]$.

To test this hypothesis, the rate of oxygen absorption by ethyl cellulose was measured under controlled conditions of temperature, pressure, and oxygen concentration. The oxygen measurements were made in a system described in a recent publication [6]. The ethyl cellulose was in all cases, unless otherwise noted, cast as a film from a $70: 30$ benzene:alcohol solution. After drying the film, it was ground in a Wiley mill to pass a 20-mesh screen. The ground film was oxidized as such, in the absence of any solvent. Solvent was excluded for two major reasons. First, because of the viscosity characteristics of ethyl cellulose, it was necessary to use dilute solutions which involved large quantities of solvent and complicated attempts to isolate and identify any oxidation products. Second, over long periods of time all of the solvents investigated were affected by the

\footnotetext{
1 Hercules Experiment Station, Hercules Powder Co., Wilmington, Del.

${ }^{2}$ Figures in brackets indicate the literature references on p. 203.
} 
peroxides formed during oxidation, and it was not possible to ascertain how much of the oxygen absorption was due to ethyl cellulose and how much was due to peroxide-catalyzed oxidation of the solvent. All of the oxidations were conducted in an atmosphere of pure oxygen, and the volume of oxygen absorbed was calculated to standard conditions.

It became evident in the preliminary investigation that the rate of oxygen absorption by ethyl cellulose was a function of time as well as of temperature during the early stages of the reaction. To obtain a clear picture of this effect, a series of ethyl cellulose samples was oxidized under identical conditions; the samples, however, differed in both chain length and ethoxyl content. The results of this study are illustrated in figure 13.1. It can be readily seen from this figure that the samples showed varying induction times and initial rates of oxidation. However, after oxidation had been initiated, the rate of oxygen absorption was quite similar in all cases. Thus, it is possible to conclude that the steady-state oxidation is not a function either of the degree of substitution of the polymer or of its chain length. On the other hand, an explanation is required for the variance observed in the induction periods of the different samples.

An induction period may be an indication either of the presence of inhibitors such as transition state metals which are destroyed as the reaction proceeds, or of a truly autocatalytic process. In the case of ethyl cellulose, it might be assumed that reaction would be initiated on an aldehyde group at chain ends. Such a process might be illustrated as follo:vs:

$$
\begin{aligned}
\mathrm{R}_{\mathrm{ec}} \mathrm{CHO}+\mathrm{O}_{2} & \longrightarrow\left(\mathrm{R}_{\mathrm{ec}} \mathrm{CO} \cdot\right)+\left(\mathrm{HO}_{2} \cdot\right) \\
\left(\mathrm{R}_{\mathrm{ec}} \mathrm{CO} \cdot\right)+\mathrm{O}_{2} & \longrightarrow \mathrm{R}_{\mathrm{ec}} \mathrm{CO}(\mathrm{OO} \cdot) \\
\mathrm{R}_{\mathrm{ec}} \mathrm{CO}(\mathrm{OO} \cdot)+\mathrm{R}_{\mathrm{ec}} \mathrm{H} & \longrightarrow \mathrm{R}_{\mathrm{ec}} \mathrm{CO}(\mathrm{OOH})+\left(\mathrm{R}_{\mathrm{ec}} \cdot\right)
\end{aligned}
$$

If this interpretation is correct, then the length of the induction period would be a function of the concentration of aldehyde groups as well as of the degree to which the oxidation may have progressed during storage or prior handling of the ethyl cellulose sample. Because of this it is reasonable to assume that different samples will show varying induction periods which will depend upon the past history of the sample.

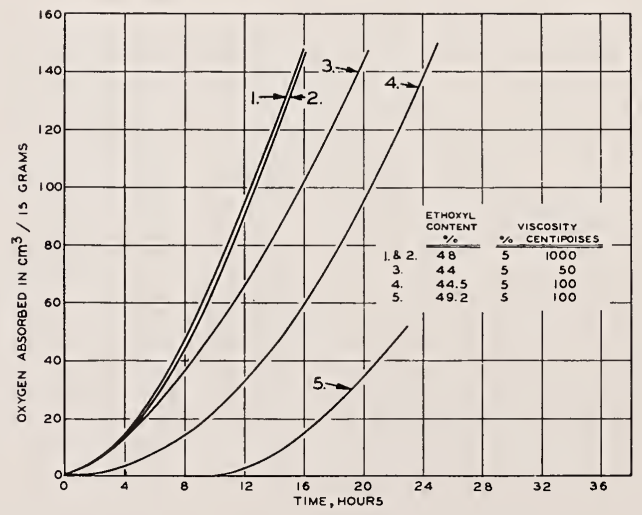

Figure 13.1. Rate of oxygen absorption by various types of ethyl cellulose at $90^{\circ} \mathrm{C}$. 
To establish this possibility, a sample of ethyl cellulose was oxidized in the usual manner and compared with another portion of the same sample that was given a series of purifications designed to remove low-molecular-weight material, peroxides, and metallic impurities. The results of this experiment are shown in figure 13.2. Curve 1 of figure 13.2 is the oxygen-absorption curve of the untreated sample, and curve 2 is that of the highly purified sample. It is apparent from these results that the induction period is greatly extended by removal of possible centers of initiation: furthermore, since the method employed also would remove any metallic impurities, the results seem to indicate that the length of the induction period is not a function of the presence of retarders but rather is due to the process being autocatalytic. Further justification can be found by a consideration of curves 3 and 4 in figure 13.2. Curve 3 , for example, shows the oxygen-absorption rate of another portion of the same sample (as represented by curve 2) after the addition of 0.1 percent of an oxidized ethyl cellulose containing peroxides. Curve 4 represents the oxygen-absorption rate of another portion of the sample (again represented by curve 2) to which a trace of paraldehyde has been added. The use of both the oxidized ethrl cellulose and paraldehyde resulted in a major reduction in the length of the induction period; the rate of oxidation at the steady state, however, was unchanged orer the sample (curve 1) that had had no special purification treatment. A similar reduction in induction period was observed when catalytic quantities of typical free-radical sources, such as benzoyl peroxide, were added. This evidence tends to indicate that the oxidation of ethyl cellulose by molecular oxygen is an autocatalytic reaction that proceeds by means of a peroxide mechanism.

If this conclusion is correct, it should be possible to measure the peroxide formation during degradation and also to determine whether or not other functional groups are developed as a result of this reaction.

In figure 13.3 is sbown the effect of oxidation on peroxide development, on carboxyl derelopment, and on the ethoxyl content of the ethyl cellulose sample. The peroxide content shows a rapid initial

Figure 13.2. Initiation of oxidation of ethyl cellulose

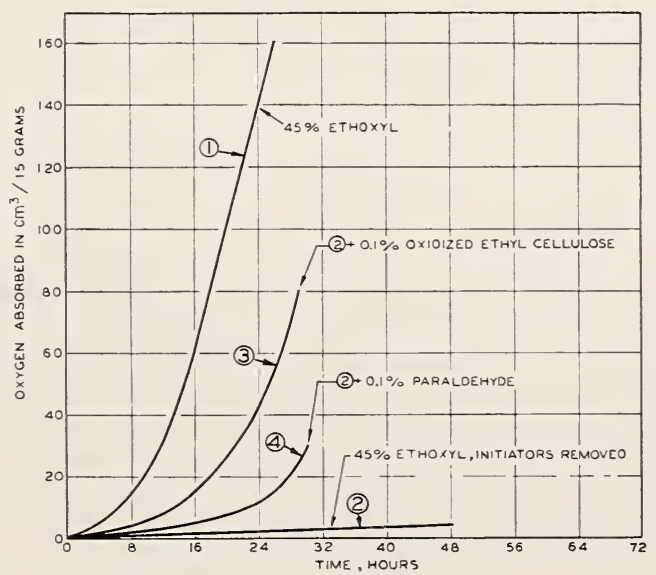




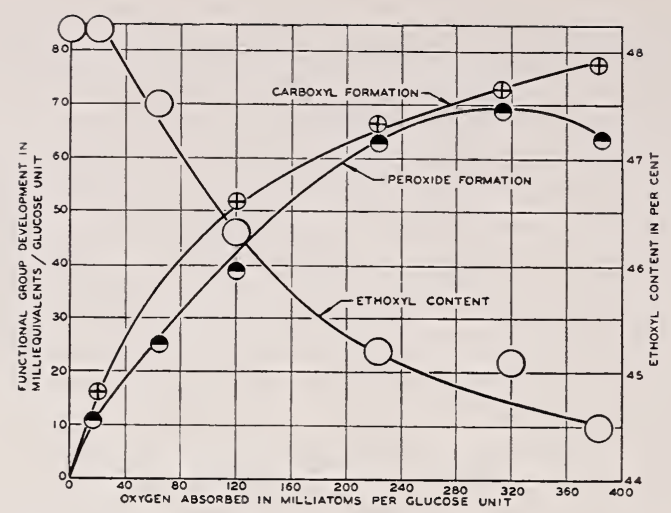

FIGURE 13.3 Chemical changes accompanying oxygen oxidation of ethyl cellulose at $90^{\circ} \mathrm{C}$.

development, and in this case it reached a maximum of approximately 70 milliequivalents per glucose unit at an oxygen absorption of 320 milliatoms per glucose unit. At higher degrees of absorption the peroxide content then showed a decrease in magnitude. At the same time, the carboxyl content increased rapidly initially and then appeared to show a decreasing rate of formation, although it gave no indication of reaching a maximum value. The ethoxyl content, on the other hand, showed a short interval of no change followed by a rapid and then a diminishing rate of decrease. The ethoxyl determination is not as accurate as the carboxyl and peroxide methods, and for that reason the experimental points are represented by circles of much greater diameter to indicate the range of accuracy.

These findings are of major importance in assigning an over-all mechanism to ethyl cellulose peroxidation. First, the nature of the peroxide-formation curve is indicative of the controlling step in the oxidation, that of peroxide formation by oxygen attack, followed by a peroxide decomposition. In all probability the peroxide decomposition is initially thermal in nature in this case, since no salts, acids, or bases were present. However, as reaction progresses it is conceivable that further break-down is the resultant of thermal and acid catalysis, the latter being due to the carboxyls generated during the process. A point is reached at which the rate of decomposition is so great that the measured peroxide content shows a decrease, although the rate of oxygen absorption by the sample has shown no change.

The initial rapid carboxyl development may be a reflection of the induction mechanism previously postulated, in which a peracid is formed from the aldehyde end group present in the sample. The propagation of the reaction, however, does not require end groups alone, and for that reason the rate of carboxyl development shows a decrease and approaches a steady state with oxygen absorption.

This conclusion is further substantiated by the behavior of the ethoxyl curve. It is apparent that extensive oxidation is accompanied by a considerable loss in ethoxyl content. Therefore, it is justifiable to assume that a major portion of the attack must be on 
the ether linkage present in the molecule. The hydroperoxide formation may be depicted as occurring on the carbon atom alpha to the ether linkage, followed by a thermal or acid-catalyzed decomposition to form a celluloseoxy free radical and acetaldehyde. Thus

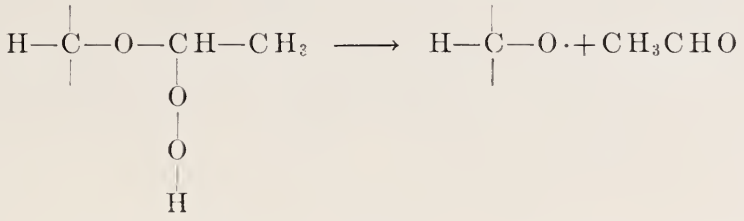

That such a reaction does occur has been demonstrated by isolating acetaldehyde from oxidized ethyl cellulose in appreciable quantities and identifying it by means of the 2,4-dinitrophenylhydrazone. The nature of the ethoxyl loss curve also lends further support to the proposed mechanism in that during the initiation stage of the reaction the ethoxyl content does not change. Such a behavior would be required because the oxygen attack has been postulated to be on an aldehyde group and not on ethoxyls at this point.

Another important consideration in dealing with reactions of this type lies in the establishment of the uniformity of attack of the oxygen along the polymer chain. In the case of cellulose, modern developments have served to show beyond any reasonable doubt that the molecular chains are arranged so that there is a gradation from three-dimensional order to complete disorder; in other words, cellulose is a composite of crystalline and amorphous material. It is not possible to point to any one area of a fibril and say that this is crystalline or amorphous, but rather there are regions in which there is more regularity than in others, and any individual molecular chain may pass through several of these regions of greater or lesser order. The question then may be asked, what is the significance of this with respect to the reaction mechanism? This question can be best answered by saring that in the ordered regions, the chains are closely packed, which results in a region of high density, whereas in the completely disordered area there exists a loose, open, less dense structure, with a continuous gradation of density between these two extremes. Thus, in the amorphous regions, penetration of reagents is not a problem in a heterogeneous reaction because of the open structure; in the crystalline regions, however, penetration is difficult, and for that reason reaction may be diffusion-controlled rather than controlled by the normal reaction kinetics. Because of these structural factors, it is of primary importance from mechanistic considerations to establish that the oxygen absorption rates are a true measure of the reaction kinetics and not simply a measure of rate of oxygen diffusion into the polymer.

This can be accomplished in two ways for such a material. First, a determination of the activation energy of the reaction gives an indication of the type of reaction in that most diffusionary processes have energies from 5 to $8 \mathrm{kcal} / \mathrm{mole}$. In the case of the ethyl cellulose oxidation, it was found that an Arrhenius plot between the temperatures of $69^{\circ}$ and $90^{\circ} \mathrm{C}$ gave a good straight line, from which a value of $25 \mathrm{kcal}$ could be calculated for the activation energy. A value of such a magnitude was considered to be sufficiently high to indicate that diffusion was not the controlling factor over this temperature range. 
The second type of evidence can be deduced from the viscosity and fluidity behavior of the polymer during degradation. Under certain conditions, that is, the assumption of a random process that has occurred so as to give a statistical distribution of chain lengths and which has been shown to be true for ethyl cellulose in this substitution range by Timell [7], the intrinsic fluidity change, $\Delta[\phi]$, is a measure of the number of chain breaks that have occurred in the degradation of a polymer. Thus, for a given weight, $W$, of a polymer that suffers degradation, the number of chain breaks, $Z$, is equal to the increase in the number of molecules, $\Delta N$ :

$$
Z=N_{s}-N_{o}=\Delta N=\Delta\left(\frac{W}{M_{n}}\right)
$$

where $M_{n}$ is the number-average molecular weight, and $N_{o}$ and $N$ are the number of molecules present originally and after degradation respectively. For polymers that have been randomly degraded, the number-average molecular weight is approximately one-half the weight-average molecular weight [8]. Also, when the constant $a$ in Mark's equation

$$
[n]=K M M^{a}
$$

is unity, weight-a verage molecular weights are directly proportional to the intrinsic viscosities:

$$
M_{w}=K[n]^{a},
$$

where $M_{w}$ equals weight-average molecular weight. Accordingly,

$$
Z=\Delta\left(\frac{W}{M_{n}}\right)=\Delta\left(\frac{2 W}{M_{w}}\right)=\Delta\left(\frac{2 W}{K[n]}\right)=K \Delta[\phi] .
$$

The condition of random degradation is undoubtedly met br the original ethyl cellulose, especially since it goes through a solution phase during its manufacture.

In figure 13.4 is shown the effect of oxidation on the intrinsic viscosity and fluidity of ethyl cellulose. The excellent linearity existing between the intrinsic fluidity change, and the oxygen absorption permits the conclusion that the number of chain breaks are a linear function of oxygen absorption and that the reaction is occurring uniformly along the polymer chain.

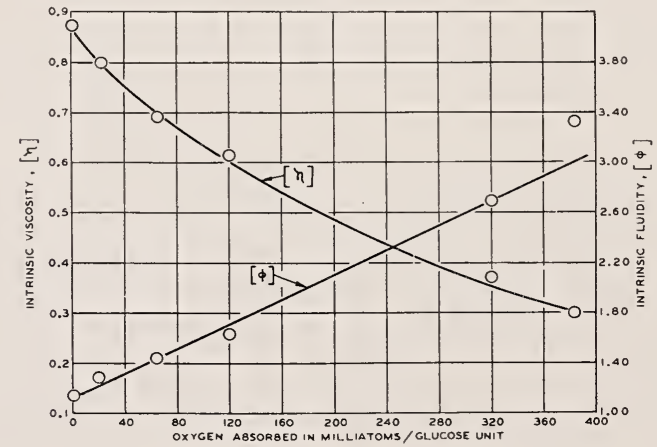

Figure 13.4. Heat degradation of ethyl cellulose at $90^{\circ} \mathrm{C}$. 
The evidence that has been presented thus far seems to fit into a free-radical chain-propagated mechanism. However, chain reactions of this type are known to be subject to catalysis, both positive and negative. It was demonstrated previously that the oxidation could be initiated by the free radicals in trace amounts, which would be expected of such a reaction. Frequently, such chain reactions are also subject to catalysis by photolysis, and for that reason the ethyl cellulose oxygen system was also investigated for its behavior under the influence of ultraviolet light.

The apparatus used in this study has been described previously [9] and differed from that used in the heat and oxygen experiments only in the reaction cell, which was designed to utilize the full intensity of four General Electric Co. Mazda H-4 mercury-vapor lamps. 'The use of Pyrex glass in the cell eliminated ultraviolet light of wavelength below $2900 \mathrm{~A}$, which is quite similar to that of sunlight at the earth's surface and in accordance with the suggestion of Staud [10]. The efficiency of illumination was determined by measuring the decomposition of uranyl oxylate in the apparatus, and the results indicated a minimum of $7 \times 10^{21}$ quanta per hour entered the reaction flask.

These reactions were conducted at $50^{\circ} \mathrm{C}$ in an atmosphere of pure oxygen, and absorption values were calculated to standard conditions. In all cases the experiments conducted under the influence of light were compared with identical runs in complete darkness so that any gross difference in the nature of the reaction could be observed:

Figure 13.5 illustrates the type of oxidation results obtained in this study. With illumination, the induction period is greatly reduced over the blank, and the rate of reaction at the steady state is also markedly increased. This figure also serves to illustrate another' important consideration, namely, the possibility of a post-irradiation effect. The ultraviolet lamps were turned off after oxidation had progressed for 3 days, and the reaction was allowed to continue in the dark. The result was an immediate dimunition in the rate of oxidation to the point that it then became equivalent to the rate of the blank sample at the same degree of oxidation. In fact, the two curves are superimposable. Thus it can be concluded that in this system there is no post-irradiation effect.

The development of peroxide and carboxyl groups showed similar trends, although their magnitudes were somewhat different. This is illustrated in figure 13.6. A maximum peroxide content observed at

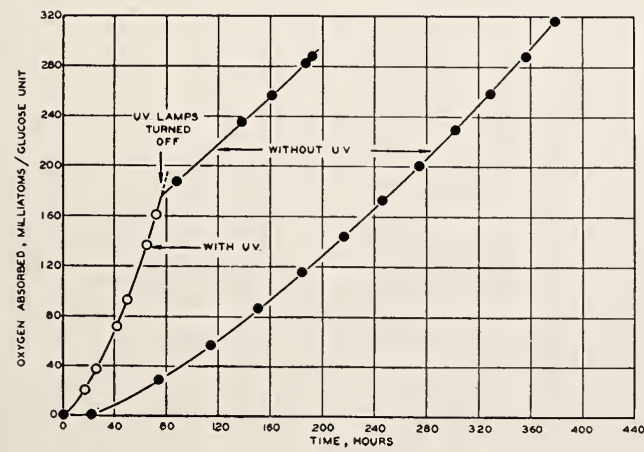

Figure 13.5. Oxidation of ethyl cellulose at $50^{\circ} \mathrm{C}$. 
$90^{\circ} \mathrm{C}$ was not reached in these reactions at $50^{\circ} \mathrm{C}$. Apparently the ratio of formation to decomposition of the peroxides is so greatly altered at the lower temperature that the maximum in the curve does not occur until much higher levels of oxidation have been achieved. An examination of these curves reveals the possibility that ultraviolet light catalyzes the decomposition of peroxides in this reaction.

The ethoxyl loss that occurs upon oxidation of ethyl cellulose is shown in figure 13.7. Oxidation under ultraviolet light caused a loss of ethoxyl that was linear with respect to the oxvgen absorbed. In the absence of irradiation, the ethoxyl loss paralleled the other curve, except for a slightly faster initial rate. The reason for this initial drop is not known.

The general similarity of data for the samples oxidized with and without ultraviolet light indicates that there was no essential difference in the mechanism of oxidation. The only striking difference between the two types of oxidation is the rate at which they take place. This conclusion is similar to that expressed by Milas [3] for the autoxidations of simple ethers under ultraviolet irradiation. The acceleration of the ethyl cellulose oxidation under ultraviolet light cannot be due to ozone or to the activation of the oxygen because oxygen does not absorb the longer wavelengths of the ultraviolet light that is transmitted by Pyrex glass. The ultraviolet therefore behaves entirely as a catalyst, as was postulated should be the case if the reaction was chain propagative in character.

Figure 13.6. Chemical changes accompanying ethyl cellulose oxidution at $50^{\circ} \mathrm{C}$.
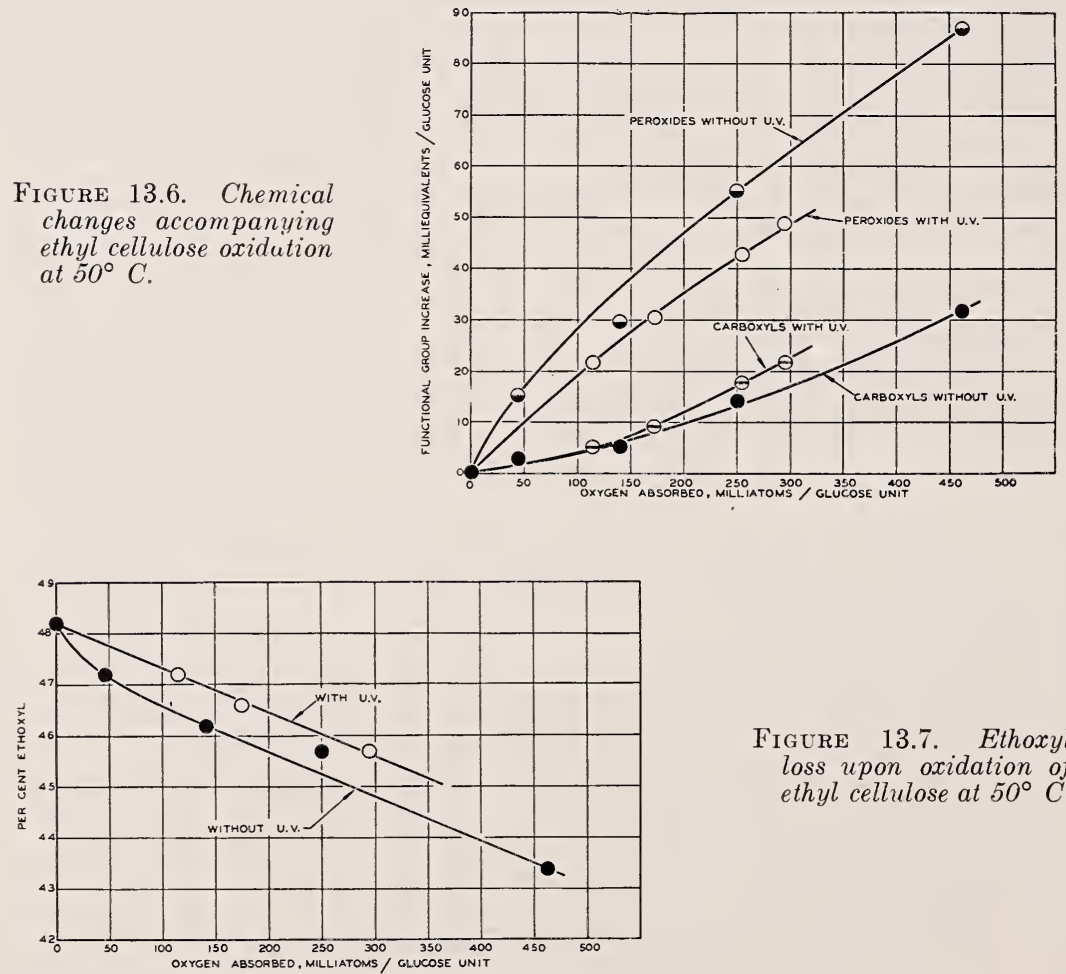

Figure 13.7. Ethoxyl loss upon oxidation of ethyl cellulose at $50^{\circ} \mathrm{C}$. 
It has been demonstrated that ethyl cellulose oxidation is subject to positive catalysis by sources of free radicals and by ultraviolet light. It is of equal importance to examine the effect of inhibitors or negative catalysts upon the same reaction.

This phase has been examined first by observing the effect of the monobenzyl ether of hydroquinone on the rate of oxygen absorption by ethyl cellulose at $90^{\circ} \mathrm{C}$. The results of this experiment are illustrated in figure 13.8. The basic curve of oxygen absorption versus time was obtained for a standard ethyl cellulose sample and found to show a typical behavior. Various concentrations of the inhibitors were then added to the sample, and the resulting oxidation rate was determined. As can be seen from the graph, the inhibitory effect is a function of concentration for the extremely low concentrations, that is, 0.026 and 0.33 percent. Increasing the concentration beyond this point, however, did not increase the effectiveness in proportion; for example, 5-percent monobenzyl ether of hydroquinone increased the retardation only slightly over the 0.33 -percent concentration. These results are quite typical of chain propagative mechanisms and again act as further support for the mechanism as postulated.

Figure 13.9 illustrates the effectiveness of several different com-

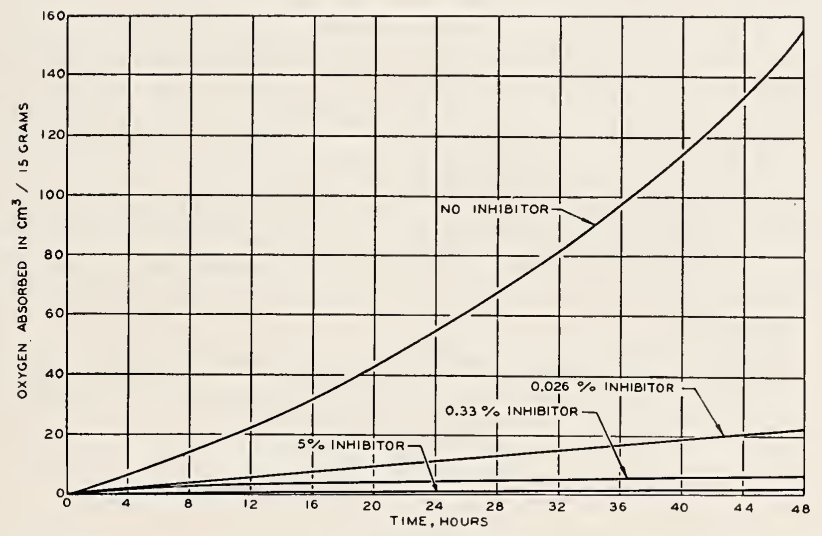

Figure 13.8. Inhibitory effect of hydroquinone monobenzyl ether on ethyl cellulose oxidation.

Figure 13.9. Effect of inhibitors on oxidation of ethyl cellulose at $90^{\circ} \mathrm{C}$

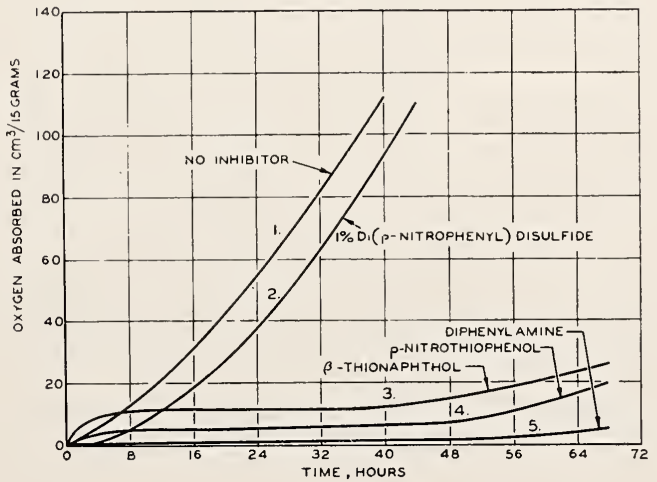


pounds in preventing oxidation of ethyl cellulose. As can be seen, secondary aromatic amines such as diphenylamine are most effective as illustrated by curve 5. Substituted phenols, such as $p$-menthylphenol, Belro phenol lactone, and similar compounds, were equally effective. In all these cases, oxidation was completely suppressed for almost $36 \mathrm{hr}$ and then progressed only very slowly over the entire range of measurement.

Mercaptans (thiols), on the other hand, showed somewhat peculiar results. For example, the sample containing $p$-nitrothiophenol as an inhibitor (curve 4) showed an initial rapid uptake of oxygen, followed first by a period of no absorption and then by a slow resumption of oxidation. A similar behavior was observed with 2-thionaphthol.

It was initially postulated that this rapid reaction might represent oxidation of the mercaptan to disulfide, and that the latter was acting as a chain breaker. The tendencr for aromatic disulfides to exist as free radicals lends further support to such a hypothesis. However, upon addition of di-( $p$-nitrophenyl)-disulfide to ethyl cellulose, very little effect was noted, as can be seen from curve 2, figure 13.9. Although contrary to expectations, this result might be explained on the basis of the very limited solubility of the disulfide in the benzenealcohol solvent used to incorporate it into the ethyl cellulose, which prevented the distribution of an adequate quantity in the films. Primary mercaptans were found to be far more effective than secondary; in fact the latter showed practically no inhibitory action.

The effect of several antioxidants on oxidation of ethyl cellulose under untraviolet irradiation is shown in figure 13.10. The rapid oxidation exhibited by the normal sample was reduced by the addition of antioxidants, although in this case a retardation was observed rather than a true inhibition. Under photolytic conditions, menthylphenol and the monobenzyl ether of hydroquinone were found to be effective oxidation retarders.

From the facts that have been discussed and also by analogy between this and similar reactions [11], it is possible to deduce a reason-

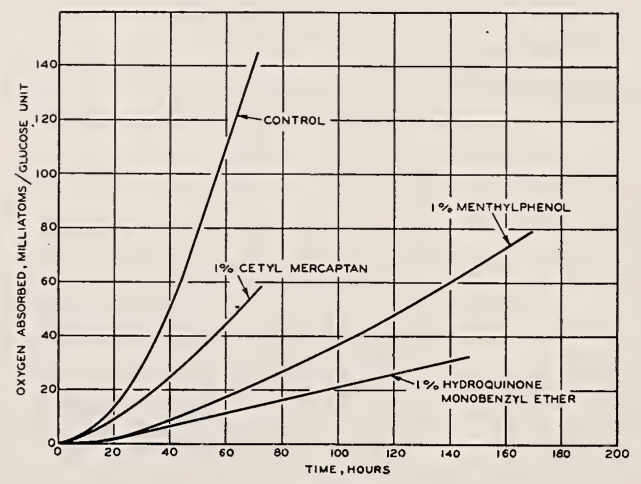

Figure 13.10. Effect of inhibitors on ethyl cellulose oxidation at $50^{\circ} \mathrm{C}$ under ultraviolet 
able mechanism for the oxidation of ethyl cellulose as follows:

(a) Initiation:

$$
\begin{gathered}
\mathrm{R}_{\mathrm{eo}} \mathrm{CHO}+\mathrm{O}_{2} \longrightarrow\left(\mathrm{R}_{\mathrm{ec}} \mathrm{CO} \cdot\right)+\left(\cdot \mathrm{O}_{2} \mathrm{H}\right) \\
\left(\mathrm{R}_{\mathrm{ec}} \mathrm{CO} \cdot\right)+\mathrm{O}_{2} \\
\longrightarrow \mathrm{R}_{\mathrm{ec}} \mathrm{CO}(\mathrm{OO} \cdot)
\end{gathered}
$$

$\mathrm{R}_{\mathrm{ec}} \mathrm{CO}(\mathrm{OO} \cdot)+\mathrm{H}-\stackrel{+}{\mathrm{C}}-\mathrm{OCH}_{2} \mathrm{CH}_{3} \longrightarrow \mathrm{R}_{\mathrm{ec}} \mathrm{CO}(\mathrm{OOH})+\mathrm{HCO}-\mathrm{CHCH}_{3}$

(b) Propagation:

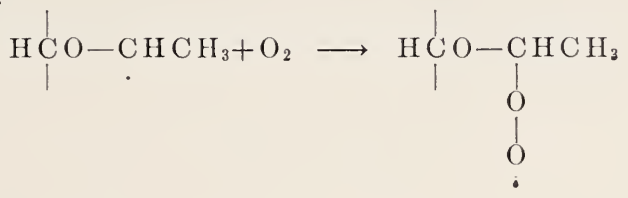<smiles>CCOC(C)CC(C)OO</smiles>

(c) Decomposition:

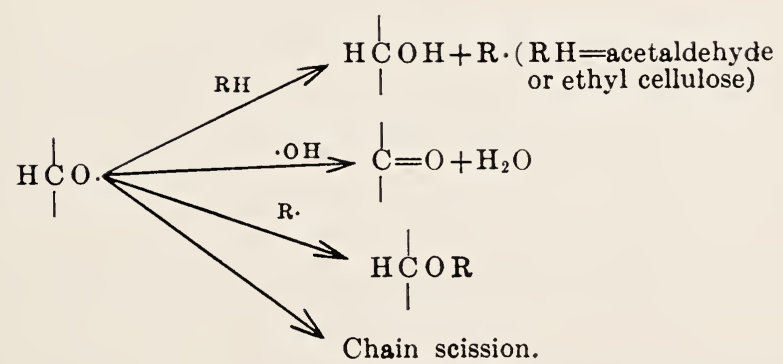

On the basis of this mechanism, the initiation of the reaction occurs on so-called chain ends or aldehyde groups. Propagation of the reaction, however, does not require those end groups but rather occurs on the ethoxyls along the chain. Chain termination is the resultant of the complex decomposition reaction of the celluloseoxy free radical. Reactions 16,17 , and 18 are hypothetical, insomuch as it is not possible to isolate material corresponding to those indicated in the reaction 
scheme. Qualitatively, it is possible to demonstrate a production of carbonyl groups during the reaction, as required by eq (17). However, the nature of the carbonyl group is such that a quantitative determination of sufficient accuracy cannot be made. Reaction (19), the chain scission reaction, is also complex, although well established experimentally. For example, if the celluloseoxy radical is assigned to either the 2 or 3 position in the glucose ring (formulas I and II), the following is obtained:
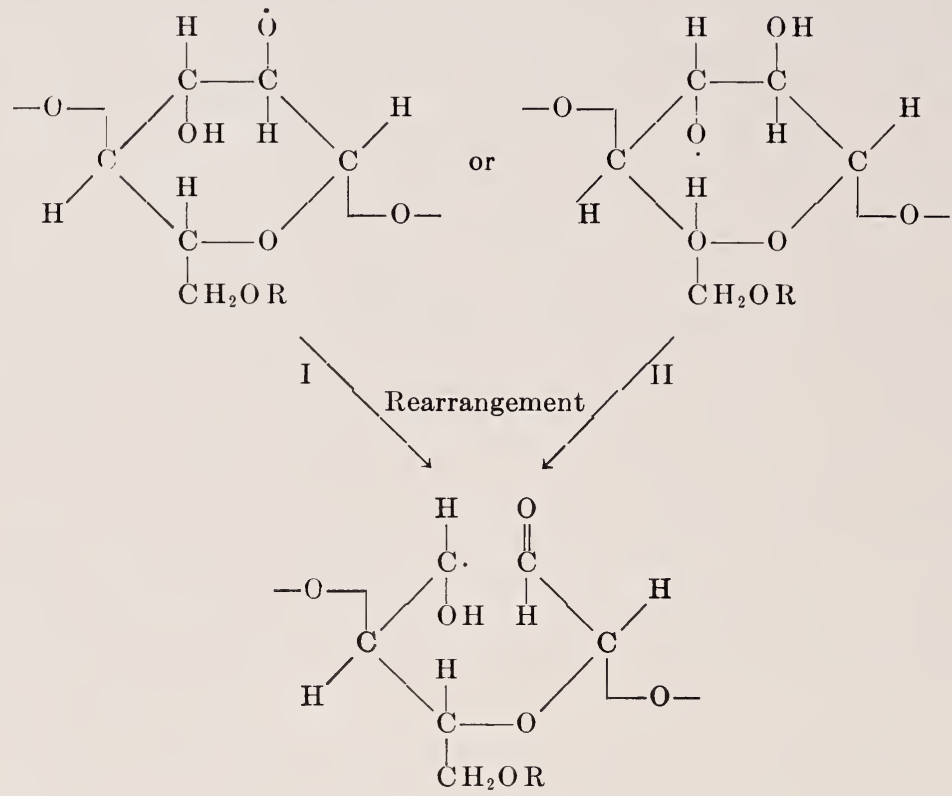

III

Compound III would be extremely unstable and tend to undergo a chain cleavage. If the radical is at ring position 1 or 2 , a similar unstable structure will be produced, which would lead to chain rupture.

In summary, then, it is possible to draw the following conclusions relative to the mechanism of ethyl cellulose oxidation:

1. Oxidation proceeds by means of a peroxide-catalyzed chainpropagated mechanism.

2 . The reaction appears to be initiated on the end groups or aldehydes formed during polymer chain cleavage either during the formation of the ethyl cellulose or on those originally present in the chemical cellulose.

3. Chain propagation is accomplished by reaction on the ethoxyl groups, substituted along the polymer chain, to form hydroperoxides.

4. Formation of carboxyl groups, loss of ethoxyl, and polymer chain scission are a result of the decomposition of ether hydroperoxides by thermal-catalyzed and/or an acid-catalyzed mechanism.

5. Oxidation is subject to positive catalysis by free radical sources or ultraviolet light, and can be inhibited or retarded by typical organic chain terminators. 


\section{References}

[1] D. Entwistle, E. H. Cole, and V. S. Wooding, Textile Research J. 19, 527, 609 (1949).

[2] Clover, J. Am. Chem. Soc. 44, 1107 (1922).

[3] N. A. Milas, J. Am. Chem. Soc. 52, 739-53 (1930); 53, 221-33 (1931).

[4] C. Moureu and C. Dufraisse, Chem. Rev. 3, 113 (1927); J. Soc. Chem. Ind. 47, 819, 848 (1928).

[5] Willard and Wingler, Ann. Physik 431, 317 (1923).

[6] L. F. McBurney, Ind. Eng. Chem. 41, 1251-56 (1949).

[7] T. Timell, Doctoral thesis, Stockholm (1950).

[8] P. J. Flory, J. Am. Chem. Soc. 58, 1877 (1936).

[9] E. Evans and L. F. McBurney, Ind. Eng. Chem. 41, 1256 (1949).

[10] C. J. Staud, Paint Oil, Chem. Rev. 89, No. 18, 8 (1930).

[11] Waters, Ann. Repts. on Progress Chem. (Chem. Soc. London) 42, 130 (1945).

\section{Discussion}

Dr. B. S. Biggs, Bell Telephone Laboratories, Murray Hill, N. J.: You said that you would not postulate what the chain scission step was. Were you referring to the cellulose chain? We know that the primary chain is broken.

Dr. McBurney: What I had referred to was rupture of the primary cellulose chain, which resulted from the oxidation of the ethyl cellulose molecule. Suppose, for illustration, we postulate what could occur in the case of one isolated glucose unit. We will assume that in this instance oxidation has occurred on the ethoxyl group in the 2 position. Cleavage of the hydroperoxide thus formed will result in the formation of a free radical as follows:

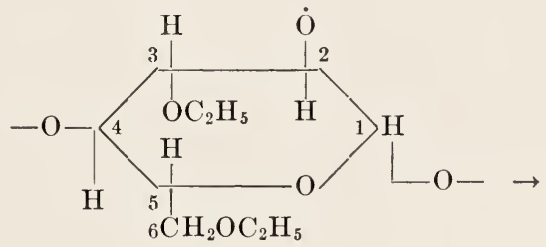

Migration of this free radical could occur in such a way that chain cleavage would occur in the glucosidic linkage in the 1 position. This migration and cleavage could occur thermally.

Dr. J. R. Shelton, Case Institute of Technology, Cleveland, Ohio: Then you are not excluding, in the propagation stage, the possibility of removal of hydrogen from the carbons of the primary cellulose chain, as well as from the ethoxyl groups. Reaction with these hydrogens would seem to be equally plausible.

Dr. McBurney: Oh, very definitely. In fact, in the case of cellulose acetate, it seems quite likely that the initial attack of oxidation will be on the tertiary hydrogen atoms in the 1 or 4 position. The decomposition of hydroperoxide formed at these points would result in a chain break.

Dr. J. W. Tamblyn, Tennessee Eastman Co.: The ether linkage in the cellulose chain must be considerably more stable than that in the ethoxyl group because cellulose acetate has greater stability than ethyl cellulose. 
Dr. McBurney: Yes; for example, ethyl cellulose oxidation occurs at a measurable rate at $50^{\circ} \mathrm{C}$, whereas with cellulose acetate a temperature of $150^{\circ}$ to $160^{\circ} \mathrm{C}$ is required for an equivalent speed of oxidation. The difference must involve the molecular structure of cellulose.

Dr. Biggs: I gather from what Dr. Tamblyn said earlier about removing cellulose acetate from cellulose that this linkage is even more stable in cellulose than in the acetate.

Dr. TAMBLyn: The effect in that burn-out process is probably one of greater absorption by the cellulose ester yarn because it is in an organic medium. The cellulose would not soak up much.

Dr. McBurnex: Alkali cellulose, for example, is extremely unstable to oxidation, even at room temperature, according to work published recently in England. The kinetics of the oxidation of cellulose have been studied very thoroughly.

DR. BigGs: Do they indicate that it is an ionic oxidation?

Dr. McBurner: No, they prove that it is free radical.

Dr. G. G. Stoner, General Aniline and Film Corp., Central Research Laboratories, Easton, Pa.: Does your method of analysis for peroxides distinguish between hydroperoxides and other kinds of peroxides?

Dr. McBurner: Hydroperoxides are much more sensitive than peroxides to the conventional iodometric method used in this analysis. 


\title{
14. Study of Degradation of Polystyrene, Using Mass Spectrometry
}

\author{
By B. G. Achhammer, ${ }^{1}$ M. J. Reiney, ${ }^{1}$ L. A. Wall, ${ }^{1}$ \\ and F. W. Reinhart ${ }^{1}$
}

The degradation of polystyrene by heat and ultraviolet radiant energy was followed by mass spectrometric identification of the gaseous products evolved as a function of time of exposure. The studies were made on films cast from methyl ethy, ketone solvent. The degradation involves two different processes: (1) the break-down of thermolabile groups, formed in the polvmer prior to degradative treatment, concomitant with the loss of residual materials, such as solvent, and (2) subsequent oxidation of the polymer. The first stage of the deterioration is caused by heat at $120^{\circ} \mathrm{C}$ in vacuum and $115^{\circ} \mathrm{C}$ in oxygen, and by ultraviolet radiant energy at $120^{\circ} \mathrm{C}$ in vacuum and $118^{\circ} \mathrm{C}$ in oxygen. It results in the evolution of gases, such as benzene, methyl ethyl ketone, dimethylbenzenes, and alcohols, as well as a decrease in oxygen content of the polymer; the polvmer was not discolored, but insolubility increased with progressive exposure. The oxidation of the polymer, which occurred on exposure to ultraviolet radiant energy at $118^{\circ} \mathrm{C}$ in oxygen, produced gaseous products, such as formaldehyde, formic acid, and acetic acid, and resulted in an increase in oxygen content along with discoloration and insolubility.

\section{Introduction}

It has been shown by infrared spectrophotometric measurements that carbonyl and hydroxyl groups are formed in polystyrene on exposure to heat and ultraviolet radiant energy in the presence of oxygen $[1,2,3] .^{2} \quad$ Ultraviolet spectrophotometric measurements have shown that monomeric styrene when present is removed from polystyrene on exposure to heat [4]. Polystyrene becomes discolored when exposed to ultraviolet radiant energy in the presence of oxygen $[3,4]$. This report presents the results of an investigation made to identify the volatile products given off and to determine the changes in solubility and oxygen content of polystyrene when exposed to heat and/or ultraviolet radiant energy in vacuum and in oxygen. This information offers further evidence for the mechanism of the oxidation reaction previously postulated [3].

The major problem in the past, in tracing the mechanism of the degradation of polymer's from the gaseous products given off when they are exposed to ultraviolet radiant energy and to heat except at pyrolytic temperatures, has been to collect sufficient material to enable these products to be identified. The development of the mass spectrometer as an analytical tool $[5,6,7$, and 8] makes it possible to identify individual compounds or groups of compounds in very small samples. Consequently, degradation reactions at conditions more comparable to service conditions can be investigated.

The work described herein is part of an investigation to determine the chemical reactions involved in the degradation of butadienestyrene synthetic rubber $(\mathrm{GR}-\mathrm{S})$. A knowledge of the manner in which polystyrene and polybutadiene individually degrade is helpful in understanding the degradation of GR-S. Information on the chemical changes involved when plastics and rubbers are exposed to

1 National Bureau of Standards, Washington, D. C.

2 Figures in brackets indicate the literature references on p. 217. 
ultraviolet radiant energy and to heat is needed to interpret the results of accelerated laboratory tests, to formulate more satisfactory accelerated methods of test, to learn how to increase the stability of the polymers, and to predict more accurately their life in service.

\section{Material and Procedures}

Films cast from a specially prepared and purified sample of polystyrene were used in this investigation. The films were inserted in quartz exposure tubes, which were then evacuated. The films in the evacuated tubes were exposed to various conditions of heat and/or ultraviolet radiant energy in vacuum and in the presence of oxygen. The experiments are described in table 14.1. After various periods of exposure, the treatments were interrupted, and mass spectrometric analyses of the gaseous degradation products were made, using a Consolidated mass spectrometer. When the exposures were complete, the films were removed from the tubes, and the infraredabsorption characteristics, solubility, color changes, and oxygen content of each film were determined.

TABLE 14.1. Description of exposure conditions used in experiments

\begin{tabular}{|c|c|c|c|c|}
\hline Experiment a & Exposure conditions $b \mathrm{c}$ & $\begin{array}{c}\text { Total } \\
\text { exposure }\end{array}$ & $\begin{array}{l}\text { Periods of exposure } \\
\text { after which gaseous } \\
\text { products were } \\
\text { analyzed }\end{array}$ & $\begin{array}{l}\text { A verage oxygen } \\
\text { content of film } \\
\text { at end of } \\
\text { experiment } d\end{array}$ \\
\hline $\begin{array}{l}\mathrm{A} \\
\mathrm{B} \\
\mathrm{C} \\
\mathrm{D}\end{array}$ & $\begin{array}{l}20^{\circ} \text { to } 30^{\circ} \mathrm{C} \text { in vacuum } \\
120^{\circ} \mathrm{C} \text { in racuum } \\
115^{\circ} \mathrm{C} \text { in } 20 \mathrm{~mm} \text { of oxygen } \\
\mathrm{UV} \text { at } 120^{\circ} \mathrm{C} \text { in vacuum } \\
30 \mathrm{hr} \text { of } \mathrm{U} \mathrm{V} \text { at } 120^{\circ} \mathrm{C} \text { in vac- } \\
\text { uum. } \\
40 \mathrm{hr} \text { of } \mathrm{UV} \text { at } 118^{\circ} \mathrm{C} \text { in } 20 \mathrm{~mm} \\
\text { of oxygen. } \\
180 \mathrm{hr} \text { of } \mathrm{UV} \text { at } 120^{\circ} \mathrm{C} \text { in vac- } \\
\text { uum. } \\
\mathrm{UV} \text { at } 118^{\circ} \mathrm{C} \text { in } 20 \mathrm{~mm} \text { of oxy- } \\
\text { gen. } \\
\mathrm{UV} \text { at } 115^{\circ} \mathrm{C} \text { in } 20 \mathrm{~mm} \text { of oxy- } \\
\text { gen e. }\end{array}$ & $\begin{array}{r}h r \\
2,000 \\
250 \\
250 \\
250 \\
30 \\
\\
70 \\
250 \\
\\
250 \\
\\
100\end{array}$ & $\begin{array}{l}\quad h r \\
2,000 . \\
5,10,30,70,150,250 . \\
5,10,30,70,150,250 . \\
70,150,250 . \\
5,10,30 . \\
70 . \\
150,250 . \\
5,10,30,70,150,250 . \\
100 .\end{array}$ & $\begin{array}{l}\% \\
0.33 \\
.11 \\
.10 \\
.13 \\
0 . \\
1.1 \\
1.4\end{array}$ \\
\hline
\end{tabular}

a The pieces of film used in these experiments weighed approximately $0.3 \mathrm{~g}$. The loss in weight as a result of the exposures is estimated to be 1 to 3 percent.

b All the films were subjected to a preliminary degassing period of $7 \mathrm{hr}$ at $105^{\circ}$ to $110^{\circ} \mathrm{C}$ in vacuum.

- UV, ultraviolet radiant energy from an RS sunlamp.

d Determined by the direct method of Walton, McCulloch, and Smith [11]. The films were degassed in a stream of purified helium at room temperature for $1 \mathrm{hr}$ prior to making the analysis.

e No polystyrene specimen in exposure tube.

\section{Material}

The polystyrene sample used in this investigation was made by polymerizing purified monomer without catalyst or solvents in a nitrogen atmosphere at $120^{\circ} \mathrm{C}$ for $48 \mathrm{hr}$ [9]. The sample was purified by dissolving in benzene, precipitating with methanol, and decanting the supernatant liquid. This treatment was then repeated. The twice-precipitated material was dissolved in benzene, and the solution was frozen. The benzene was removed with a vacuum pump while the frozen mixture was slowly brought to $24^{\circ} \mathrm{C}$. The resulting light, fluffy sample was kept under vacuum while being raised to $60^{\circ} \mathrm{C}$ and finally held at this temperature for $24 \mathrm{hr}$ to remove essentially all of the volatile materials. The sample was then stored in a dark- 
glass bottle until used. This procedure should remove monomer, low-molecular-weight polymer, and other impurities. The dried product contained less than 1 percent of benzene, based on mass spectrometric analysis of the gaseous products evolved from the polymer when heated to $250^{\circ} \mathrm{C}$ for $1 \mathrm{hr}$. The number average molecular weight determined from osmotic-pressure measurements was 230,000 [10]. The average oxygen content determined by the direct method of Walton, McCulloch, and Smith [11] was 0.33 percent.

\section{Preparation of Films}

The polystyrene was dissolved in methyl ethyl ketone. Frames 1 by 4 in. made from 0.13 -in.-diameter glass rod were placed on a flat, level regenerated cellulose surface in a desiccator. Sufficient solution to leave a film weighing approximately $0.3 \mathrm{~g}$ upon evaporation of the solvent was poured into the frame. The drying rate was controlled by partially opening the desiccator. The films obtained were slightly wedge-shaped, that is, thinner in the center of the film and thicker near the holder. The films attached to the holders were removed from the regenerated cellulose surface by wetting the opposite side of the cellulose with water, which swelled the cellulose and broke the bond between it and the polystyrene film. The films were then airdried to remove further solvent.

\section{Exposure Tubes}

The exposure tube used in this work is shown in figure 14.1. It is essentially a single-stage molecular still designed to facilitate the removal of primary degradation products before secondary degradation becomes appreciable. The frame holding the polystyrene film is placed in part A. For exposure to ultraviolet radiant energy, part $A$ of the tube is made of clear quartz and is attached to the Pyrex section, part B, by means of a graded seal. For exposure to heat alone, the entire tube is made of Pyrex glass. Part $\mathrm{C}$ is the cooling arm, which is immersed in dry ice-acetone mixture $\left(-78^{\circ} \mathrm{C}\right)$ in order to condense most of the volatile products given off as a result of the various treatments. Part $\mathrm{D}$, a stopcock with standard-taper adapters, simplifies the transfer of the gaseous degradation products into the mass-spectrometer inlet system.

Figure 14.1. Exposure tube.

Part A, (exposure arm) quartz or Pyrex for ultraviolet and heat exposures, respectively; part B, (adapter arm) Pyrex (graded seal necessary when part A is quartz; part C, (cooling arm) Pyrex; part D (mass spectrometer adapter) 3446 standard taper, 4-mm-bore vacuum stopcock, and 1, 20 standard taper. The over-all length and height of the tubes, that is, exterior measurements, varied somewhat. The meassurements shown are average.

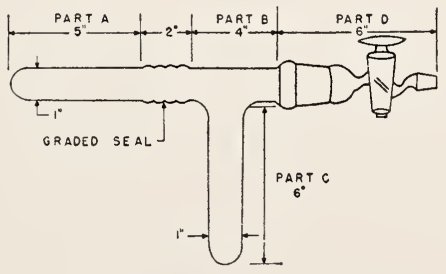

\section{Evacuation and Pretreatment of the Films}

The exposure tubes containing the polystyrene films are evacuated to less than $10^{-4} \mathrm{~mm}$ of $\mathrm{Hg}$ while heated for $7 \mathrm{hr}$ at $105^{\circ}$ to $110^{\circ} \mathrm{C}$ by cylindrical furnaces placed over the sections containing the films. After the evacuation is completed, the stopcocks to the tubes with 
the films intended for exposure in vacuum are closed. The system is flushed with tank oxygen that has been passed through a liquidnitrogen trap. When a final pressure of $20 \mathrm{~mm}$ is obtained, the stopcocks to the other tubes with the films intended for exposure in oxygen are closed.

\section{Exposure of the Films}

The exposure chamber, shown in figure 14.2 , consists of a rectangular transite box insulated by 3 in. of glass fiber from an inner duraluminum chamber, $30 \mathrm{in}$ long, $10 \mathrm{in}$. wide, and $34 \mathrm{in}$. high. Heating of the chamber is accomplished with 600 -w cone heaters (B). RS sunlamps (C) are used as a source of ultraviolet radiant energy. During ultraviolet exposure the temperature of the chamber is controlled by a blower operated by a thermoregulator, which in troduces air from the room. For exposure to ultraviolet radiant energy at $120^{\circ} \mathrm{C}$ sufficient heat is supplied by the 275-w RS sunlamps. For exposure to $120^{\circ} \mathrm{C}$ in the absence of ultraviolet radiant energy the cone heaters are controlled by a mercury relay operated by the thermoregulator. The temperature of the exposure chamber is controlled at $100^{\circ} \pm 2^{\circ} \mathrm{C}$. The temperatures reported in table 14.1 for the various experimental conditions include the effect of radiant heating on the films and were determined by imbedding a thermocouple in a film. subjected to the conditions of study. The air in the chamber is circulated continuously by the fan. $(\mathrm{F})$, and control of the conditions is adjusted manually by means of the damper (A). The control panel $(\boldsymbol{J})$ contains the necessary meters and switches for operation of the chamber. The dotted lines indicate the probable beam of the radiant energy, but do not take into consideration. the multiple reflections from the chamber walls.

The position of the exposure tubes containing the polystyrene films for treatment is shown in figure $14.2 \mathrm{~b}$. This particular illustration shows the setup for exposure to ultraviolet radiant energy. Part A of the exposure tube $(\mathrm{G})$, containing the film for exposure is inserted into the exposure chamber through a 1.25-in. aperture (D). The film is entirely within the exposure chamber. During the exposure, part $\mathrm{A}$ of the exposure tube is at the conditions of the chamber, part $\mathrm{B}$ is at laboratory conditions, and part $\mathrm{C}$ is cooled to $-78^{\circ} \mathrm{C}$ by insertion into a Dewar flask $(\mathrm{H})$ containing a mixture of dry ice and acetone.
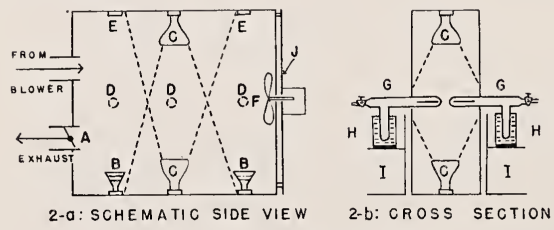

Figure 14.2. Diagram of exposure chamber.

A, Damper; B, 600-w cone heater; C, 275-w RS sunlamp; D, 11/4-in. aperture for inserting tubes (there are 6 apertures, 3 on each side of chamber); E, extra socket for cone heater or sunlamp; $F$, circulating tan; $\mathrm{G}$, exposure tube; $\mathrm{H}$, cooling Dewar; $\mathrm{I}$, ringstand; $\mathrm{J}$, control panel.

\section{Mass-Spectrometer Analysis}

After the treatment is completed, the exposure tube is removed from the exposure chamber without removing the cooling flask on part C. When part A attains room temperature the cooling Dewar is removed and part $\mathrm{C}$ is allowed to come to laboratory conditions. The exposure tube containing the gaseous degradation products is then transferred to the mass spectrometer. 


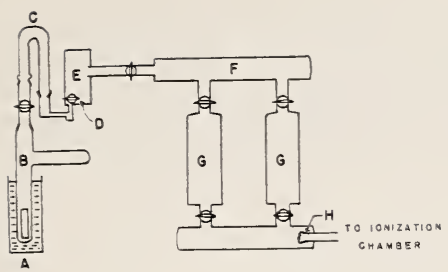

Figure 14.3. Simplified diagram of mass spectrometer inlet system.

$A$, Cooling Dewar (liquid nitrogen or dry ice and acetone mixture; removed for room-temperature fraction); $B$, exposure tube containing film and gaseous degradation products; C, adapter; D, control valre; E, inlet for sample; F, chamber; G, expansion bottle; H, leak derice.

Figure 14.3 shows the procedure used in attaching the exposure tube to the Consolidated mass-spectrometer inlet srstem. When the stopcocks are opened, the rolatile products are expanded into the inlet system. The total rolumes of the inlet system and the exposure tube are known. The partial pressure of each component of the gaseous degradation products is determined by means of the known pattern sensitivities. The sensitivity for a compound is the height of a characteristic peak produced when $1-u$ pressure of the pure substance is in the inlet srstem.

To simplify interpretation of the mass spectrometric data, the gaseous products of each treatment were fractionated. With the exposure tube attached to the inlet sistem as shown in figure 14.3, liquid nitrogen was applied to the exposure tube as indicated in this sketch. When the exposure tube contained $20 \mathrm{~mm}$. of oxygen, the oxrgen ras pumped off at this temperature $\left(-196^{\circ} \mathrm{C}\right)$; in the case of samples exposed in racuum, the gaseous degradation products, rolatile at this temperature, were expanded directly into the instrument. The stopcocks were then closed, the liquid nitrogen was replaced by the mixture of dry ice and acetone, and the gaseous products rolatile at this temperature $\left(-78^{\circ} \mathrm{C}\right)$ were analyzed. Finally, the exposure tube was allowed to come to laborator conditions $\left(25^{\circ} \mathrm{C}\right)$, and the remainder of the rolatile gaseous products were expanded into the mass spectrometer.

After analysis of the gaseous products obtained from a certain treatment for a known exposure period, the exposure tubes were again attached to the high-racuum manifold; and, with the exception of the preheating. were prepared as described above. The films were then exposed further to the same conditions to which ther had previously been exposed. This procedure was repeated for additional exposure periods until the desired data were obtained.

\section{Results of Tests}

The pressures of each component in the gaseous degradation product mixtures obtained from the mass spectrometric records are plotted against the hours of exposure to heat and to ultraviolet radiant energy in figure 14.4. These pressures are directly proportional to the amounts of the component given off. Wall [ $\bar{\tau}$ ] has shown that the results of prrolysis of polrmers by a sinilar technique vary considerably with the size of the sample that is prrolyzed. The weights of the polystrrene samples used in the exposure tests were maintained at approximately $0.3 \mathrm{~g}$.

The points on the curves in figure 14.4 represent cumulative totals of the pressures observed up to the times shown. A particular component is no longer coming off when the curve becomes horizontal. 
A continuous production of a component is shown by a continuously rising curve. It is probable that materials that come off in the initial part of the exposure are associated components, such as residual solvents and water or degradation products of weak structures, such as peroxides or hydroperoxides. The curves for the various compounds
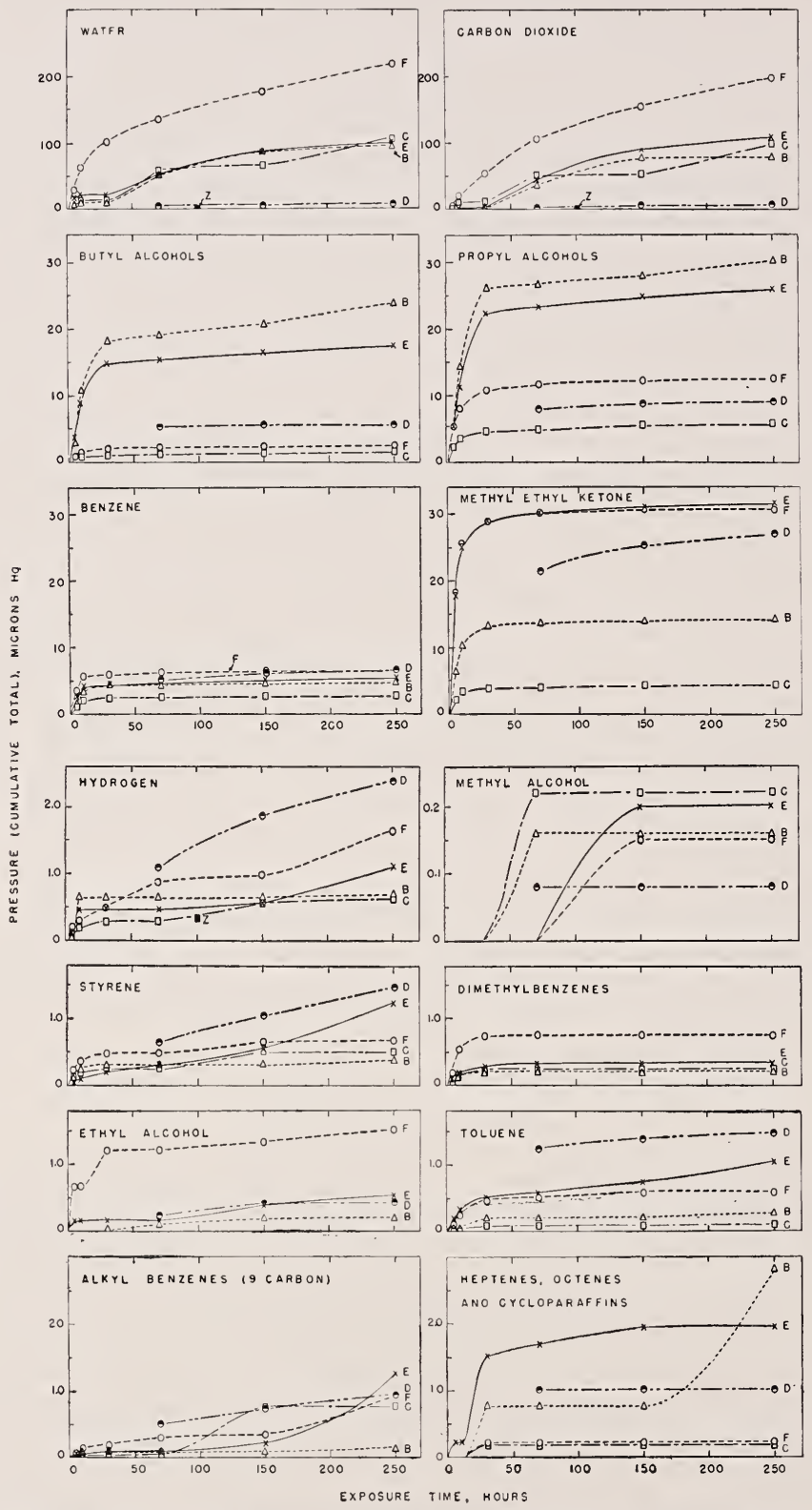

FIGURE 14.4 Gaseous degradation product: from polystyrene film. 

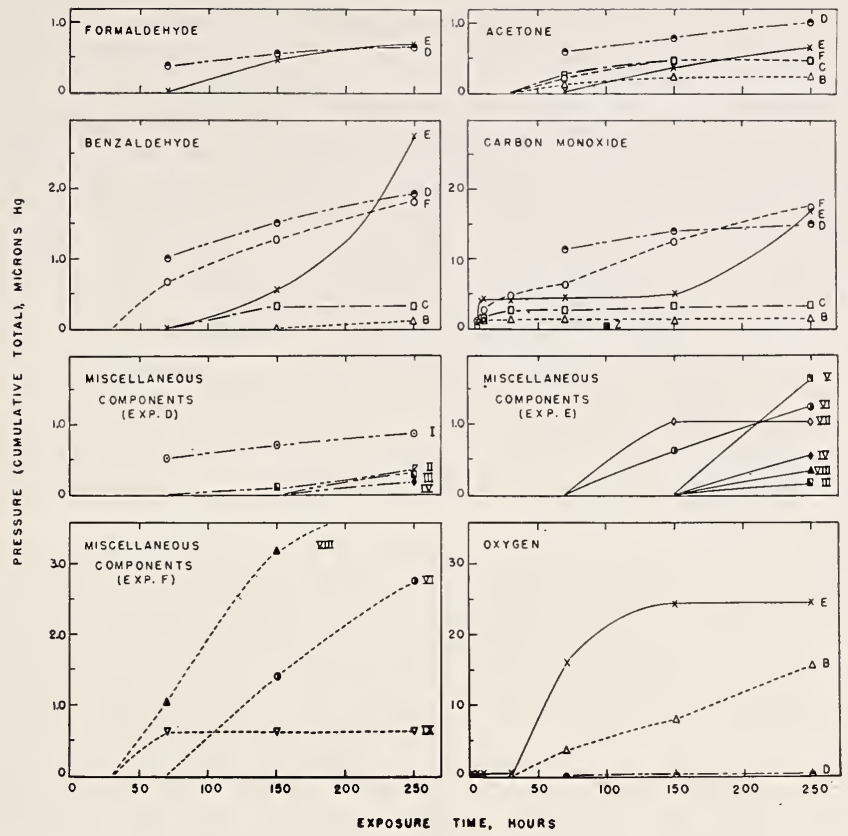

FIGURE 14.4 Gaseous degradation producto from polystyrene film-Continued.

$\triangle$, Experiment $\mathrm{B}, 120^{\circ} \mathrm{C}$ in vacuum; $\square$, experiment $\mathrm{C}, 115^{\circ} \mathrm{C}$ in $20 \mathrm{~mm} \mathrm{O}$; $\theta$, experiment $\mathrm{D}$, ultra violet at $120^{\circ} \mathrm{C}$ in vacuum; $\times$, experiment $\mathrm{E}$, ultraviolet at $120^{\circ} \mathrm{C}$ in vacuum ( 30 hours), ultraviolet at $118^{\circ} \mathrm{C}$ in $20 \mathrm{~mm}$ of $\mathrm{O}_{2}$ (40 hours), ultraviolet at $120^{\circ} \mathrm{C}$ in vacuum (180 hours); $O$, experiment $\mathrm{F}$, ultraviolet at $118^{\circ} \mathrm{C}$ in $20 \mathrm{~mm}$ of $\mathrm{O}_{2} ; \mathbf{D}$, blank experiment $\mathrm{Z}$, ultraviolet at $115^{\circ} \mathrm{C}$ in $20 \mathrm{~mm}$ of $\mathrm{O}_{2}$ with no film in tube. Miscellaneous components: methane, ethylene, methylstyrene, acetaldehyde, butene, acetic acid, dimethyl ether, formic acid, and methyl formate (I through IX, respectively).

are extrapolated to zero at the start of the exposure period in which the particular component was first detected except in experiment D, where the first mass spectrometric analysis was made after $70 \mathrm{hr}$ of exposure.

Methyl alcohol was produced in very small amounts in the intermediate periods of all the experiments. The amounts produced do not correlate with the type of exposure or the presence of oxygen.

Although styrene was given off in all the experiments, it was not a major product as is the case under pyrolytic conditions $[6,7]$. More styrene was produced on exposure to heat and to ultraviolet radiant energy than to heat alone.

Dimethylbenzenes were given off during the initial part of experiments B, C, E, and F only. Exposure to ultraviolet radiant energy in vacuum did not produce these compounds, whereas exposure to ultraviolet radiant energy in oxygen produced the greatest amount.

Ethyl alcohol was mainly produced on exposure to ultraviolet radiant energy. A very small amount was produced in one heat exposure test and none in the other.

Toluene was produced in all the experiments. Exposure to ultraviolet radiant energy produced more than exposure to heat. The presence of oxygen caused a reduction in the amount of toluene produced by exposure to ultraviolet radiant energy. 
Alkylbenzenes (nine carbon), and heptenes, octenes, and cycloparaffins were produced in all the exposures. The presence of oxygen reduced markedly the amount of olefins and crcloparaffins.

Water and carbon dioxide were given off under all exposure conditions. Larger amounts were obtained when the exposures were made in oxygen than in vacuum, and the greatest amounts were given off under ultraviolet radiation in oxrgen. Because oxrgen was found in experiment $B$, it is evident that there was a leak in the tube; it was intended that this experiment be made in vacuum. The experiment was not repeated because the results showed that extensive oxidation did not occur under these conditions.

Butyl and propyl alcohols were given off during all the exposure treatments. The major part of these two compounds was produced during the first $30 \mathrm{hr}$ of the exposures. The production of these alcohols by heat and ultraviolet radiant energy was much less when oxygen was present than in vacuum.

Benzene and methyl ethyl ketone were produced as a result of all the treatments. Since the greater part was given off during the first $30 \mathrm{hr}$ of exposure, it is probable that these compounds were present as residual solvent and do not result from a molecular break-down of the polystrrene. In view of the extensive drying and purification treatment to which the sample was subjected, it is obvious that small amounts of these compounds are held very tenaciously by the polymer. The residual benzene and methyl ethyl ketone are removed more effectively by exposure to heat and ultraviolet radiant energy than to heat alone; this effect is particularly pronounced for the methyl ethyl ketone. Methyl ethyl ketone may be broken down to some extent by exposure to the ultra violet energy, giving rise to other products.

Hydrogen was given off during all the exposures. More was produced on exposure to heat and ultra violet radiant energy than to heat alone. Most of the hydrogen resulting from heat alone was produced during the initial part of the exposure, whereas exposure to ultrariolet radiant energy resulted in a continuous evolution of hydrogen.

Formaldehyde was given off in experiments D and E, both of which involved exposures to ultra violet radiant energy in vacuum. Acetone, benzaldehyde, and carbon monoxide were given off in all the experiments, with the greatest amounts being produced in those exposures involving ultraviolet radiant energy.

Those experiments involving exposures to ultraviolet radiant energy produced a variety of compounds that were associated only with ultraviolet treatment. These compounds are indicated in figure 14.4 by roman numerals. Methane (I), ethylene (II), methylstyrene (III), and acetaldehyde (IV) were found in experiment D. Butene (V), acetic acid (VI), dimethyl ether (VII), acetaldehyde (IV), formic acid (VIII), and methylstyrene (III) were found in experiment E. Formic acid (VIII), acetic acid (VI), and methyl formate (IX) were produced in experiment $\mathrm{F}$.

The curves for gaseous oxygen show that a small amount of oxygen was present in experiment $\mathrm{B}$, probably because of a leak. However, the amount was smaller than that found in experiment $\mathrm{E}$, where a small amount was added at one point during the exposure. Practically no gaseous oxygen was present in experiment D. No curves for oxygen are given for experiments $\mathrm{C}$ and $\mathrm{F}$, in which large amounts of oxygen were added to the exposure tubes. 
No gaseous products were given off in experiment $A$, in which a film of polystyrene was stored in vacuum at room temperature for 83 days.

The oxygen. contents of the film specimens were determined at the conclusion of the exposures by the direct method of Walton, MeCulloch, and Smith [11]. The specimens were washed in a stream of helium at room temperature for $1 \mathrm{hr}$ prior to the determinations. The oxygen content of the unexposed polystyrene was 0.33 percent. Heating in vacuum and in oxygen and exposure to ultraviolet radiant energy in vacuum reduced the oxygen content to one-third of the original amount. Exposure to ultraviolet radiant energy in oxygen caused approximately a fourfold increase in the oxygen content.

The physical appearance of the polystyrene specimens after the exposures is shown in figure 14.5. The letters under the films in this figure correspond to the experiment numbers in table 14.1. Film A is still held in the glass frame, although a longitudinal shrinkage of about 7 percent and a horizontal shrinkage of 3 to 5 percent occurred. The bubbles in the lower right-hand corner of the specimen appeared during the degassing period. The shrinkage and bubble formation are attributed to loss of solvent. The shrinkage of film B is slightly more than that of film $A$, and the film is no longer attached to the glass frame. Film B is very brittle and curled; the highlights in the photograph are caused by the curling. Film $\mathrm{C}$ has about the same appearance and shrinkage as film A. Films D, E, and F, which were exposed to ultraviolet radiant energy, show a greater change in appearance than those exposed to heat alone. Film D curled, split, shrank about the same amount as film B, and became slightly discolored. Film E deteriorated badly; it curled, split, shrank greatly, became very brittle and badly discolored. Film F also deteriorated badly; it curled, split slightly, shrank about 20 percent longitudinally and 50 percent horizontally, and became very brittle and badly discolored.

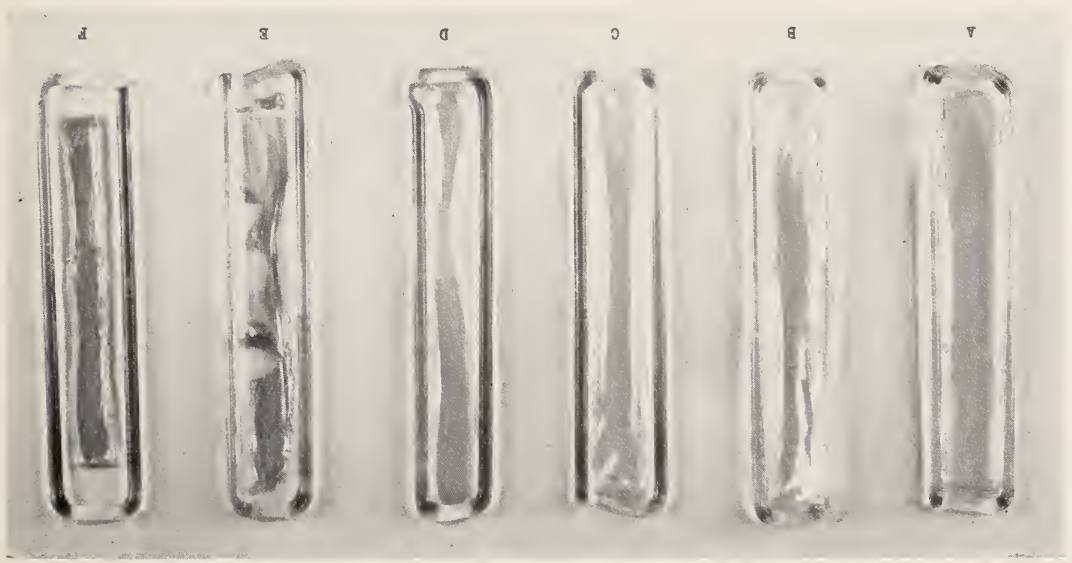

Figure 14.5. Physical appearance of polystyrene specimen..

A, Untreated polystyrene film; B, film after 250 hours at $120^{\circ} \mathrm{C}$ in vacuum; C, film after 250 hours at $115^{\circ}$ $\mathrm{C}$ in $20 \mathrm{~mm}$ of oxygen; $\mathrm{D}$, film after 250 hours of ultraviolet radiant energy at $120^{\circ} \mathrm{C}$ in vacuum; $\mathrm{E}$, film after 30 hours of ultraviolet radiant energy at $120^{\circ} \mathrm{C}$ in vacuum plus 40 hours of ultraviolet radiant energy at $118^{\circ} \mathrm{C}$ in $20 \mathrm{~mm}$ oxygen followed by 180 hours of ultraviolet radiant energy at $120^{\circ} \mathrm{C}$ in vacuum; $\mathrm{F}$, film after 250 hours of ultraviolet radiant energy at $118^{\circ} \mathrm{C}$ in $20 \mathrm{~mm}$ of oxygen. 
During the last two periods of exposure in experiments $\mathrm{E}$ and $\mathrm{F}$, the glass frames became coated with a white powdery deposit. This material tended to become clear in contact with air. The deposit was removed from the glass frames and the following properties observed under the microscope:

(a) Low birefringence, with refractive indices of 1.63 to 1.64 ; (b) insoluble, but swelled slightly in xylene; (c) insoluble, but swelled greatly in ethanol; (d) insoluble, but swelled greatly in acetone; (e) insoluble, but swelled greatly in chloroform; (f) birefringence persisted during the early stages of swelling, indicating that the birefringence was anomalous and not evidence of true crystallinity. The results indicate that the substance is probably a dense aromatic compound. Sufficient material was not available to make further tests.

The colors of the specimens were determined by the Munsell system with a white background in north light and are reported in table 14.2. Appreciable changes in color occurred on exposure to ultraviolet radiant energy in the presence of oxygen.

Solubility of the exposed specimens in benzene was determined by placing a small portion of film in a test tube with $10 \mathrm{ml}$ of distilled benzene and shaking the tube occasionally. The solubility observed with and without a microscope is reported in table 14.2. No change was found after the first $15 \mathrm{~min}$. Insoluble particles were observed in all specimens that were exposed to heat and to ultraviolet radiant energy. Exposure to ultraviolet radiant energy produced more insoluble material than exposure to heat.

TABLE 14.2. Color and solubility of polystyrene film specimens after exposure

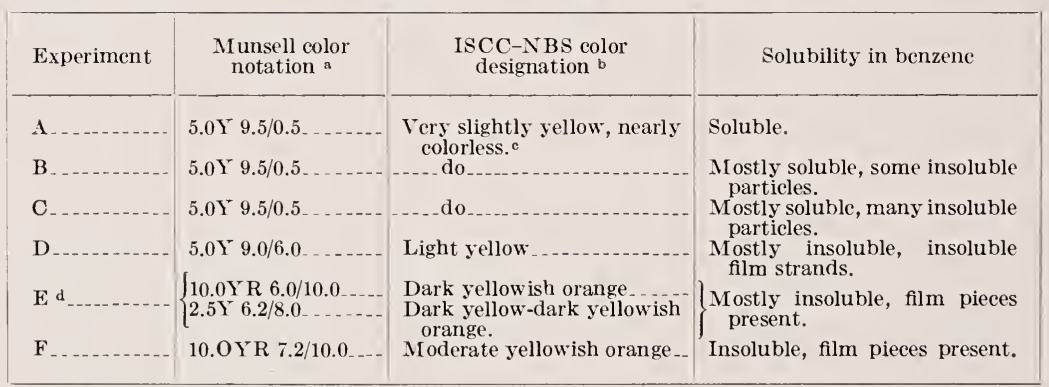

a All specimens are transparent.

b ISCC-NBS is Inter-Society Color Council-National Bureau of Standards [12].

c Not an ISCC-NBS color designation.

d The color in this specimen was in two shades; the darker shade was in the contracted areas.

\section{Discussion of Results}

The most striking aspects of the mass spectrometric data are the numerous products obtained and the lack of similarity between their structures and that of polystyrene. The relatively small amount of styrene monomer produced and the insolubility of the degraded polymer contrast with the results of pyrolysis studies $[6,7]$ and of solution degradation between $100^{\circ}$ and $200^{\circ} \mathrm{C}$ [13]. One important difference between conditions in the latter studies and this work is that the polymer remains solid here, whereas in the other investigations the material is in a fluid condition while decomposing. It is 
possible that in the essentially solid polymer cross-linking effects may be farored, while chain reaction may be suppressed. Many aspects of the thermal depolymerization of the simple addition polymers have been shown to be compatible with a mechanism composed of four elementary reactions: initiation, depropagation, transfer, and termination [14].

The general results reported here indicate a quite different process. The degradation of polystyrene under the conditions used in this study apparently involves: (1) loss of rolatile materials and decomposition of the more thermolabile structures, and (2) oxidation of the polymer.

The mass spectrometric data show that both heat and ultraviolet radiant energy, in vacuum and in the presence of oxygen, cause an initial evolution of gaseous products from polystrrene. These are attributable to loss of residual solvent and the decomposition of the more thermolabile structures, such as peroxides, present in the polymer. The thermolabile groups presumably result from the incorporation of oxygen in the polymer during polymerization or by addition of oxygen after the polymer is made. Relatively more hydrocarbons are evolved from the polymer by ultraviolet than by heat treatment. This might be indicative of direct attack on the polymer by the ultraviolet radiant energy.

The removal of trace impurities, such as methyl ethyl ketone and benzene, changes the properties to a minor extent because they have some plasticizing action, and their removal increases the brittleness of the films. The removal of such materials from various synthetic rubbers is complete on evacuation at $25^{\circ} \mathrm{C}$ for 3 dars, whereas for polystyrene it is not complete on evacuation at $60^{\circ} \mathrm{C}$ for a comparable period of time. Others have observed more rapid evolution of residual solvent when a polymer is held above its softening point [15].

In contrast to the slight effects of oxrgen and heat or heat and ultraviolet in racuum, oxygen in combination with ultraviolet at $118^{\circ} \mathrm{C}$ results in extensive deterioration of the polymer characterized by the evolution of oxidation products, such as formic acid, acetic acid, and acetaldehyde in the latter stages of treatment. The products collected are in general agreement with those to be expected from the oxidation reactions postulated in a previous paper [3], involving hydroperoxide formation in the polystyrene at the tertiary carbon adjacent to the benzene ring.

If a hydroperoxide intermediate accumulated in the polymer to a considerable extent on exposure to ultraviolet radiant energy at $118^{\circ} \mathrm{C}$ in oxygen, then subsequent exposure to ultraviolet radiant energy in racuum at $120^{\circ} \mathrm{C}$ might be expected to produce a definite amount of water comparable to the total concentration of ketones and aldehydes formed. Actually, in such an experiment water was the chief rolatile product, and only small amounts of acetone, acetaldehyde, and benzaldehyde were obtained. It seems probable that analogous nonvolatile products of the trpe $\mathrm{RCO} \phi$ are produced. Previous work $[2,3]$ shows that the oxidized polymer contains carbonyl groups possibly of this type. A similar experiment made in the absence of ultraviolet radiant energy in both phases produced a meager amount of rolatile products and even relatively little water.

Oxidation of the polymer is the most deleterious phase of the degradation and is concomitant with discoloration of the material. 
This discoloration of the films on exposure to ultraviolet radiant energy at $118^{\circ} \mathrm{C}$ in oxygen is not easily explained, although it is known [16] that diphenyl polyenes with as little as four conjugated double bonds in the chain absorb in the visible region of the spectrum. The compounds responsible for the yellow discoloration of polystyrene can be washed out in certain cases [2].

Insolubility was noted for all films treated, although there was only a slight effect due to heat alone. The degree of insolubility was roughly proportional to the extent of degradation. The experiments in vacuum indicate that this insolubility occurs simultaneously with the decomposition of oxygenated groups in the polymer, hence is not a consequence of the primary reaction with oxygen.

The production of benzaldehyde and presumably analogous solids is compatible with the formation of a hydroperoxide group on the tertiary carbon. Howerer, the simple decomposition of such an intermediate would seem to demand a break in the chain and hence an increase in solubility. Therefore, either a competitive reaction or subsequent reactions involving the products of the hydroperoxide decomposition is responsible for the insolubility. An alternate, but less likely, possibility is that the oxidation occurs preferentially at the chain ends and the resulting radical intermediates couple to give very large molecules.

Studies of the oxrgen content of a series of polystyrene samples [17] have shown that various "degassing" procedures employing temperatures of $70^{\circ}$ and $100^{\circ} \mathrm{C}$ for up to $100 \mathrm{hr}$ reduce the oxygen content of commercial polymers from approximately 0.3 to 0.1 percent. Changes of oxygen content of this magnitude were observed on heating the polystyrene films at $120^{\circ} \mathrm{C}$ for $250 \mathrm{hr}$ in vacuum and at $115^{\circ} \mathrm{C}$ in $20 \mathrm{~mm}$ of oxygen, and on exposure of a film to ultraviolet radiant energy at $120^{\circ} \mathrm{C}$ in vacuum for $250 \mathrm{hr}$. The difference in oxygen content as a result of these various treatments is undoubtedly a result of the decomposition of the thermolabile oxidation structures. The residual oxygen content may be due to oxygenated structures that are not readily decomposed by heat or ultraviolet radiant energy of the intensities and wavelengths employed in this study.

'The final oxygen content of a polymer exposed to ultraviolet radiant energy at $118^{\circ} \mathrm{C}$ in $20 \mathrm{~mm}$ of oxygen for $250 \mathrm{hr}$ was quadruple that of the untreated polymer. Thus the amount of stable oxygenated structures was augmented by the treatment. The presence of these stable structures is further substantiated by the fact that polystyrene exposed to oxygen and ultraviolet radiant energy at $118^{\circ} \mathrm{C}$ for $40 \mathrm{hr}$, and subsequently exposed to ultraviolet at $120^{\circ} \mathrm{C}$ in vacuum for 180 $\mathrm{hr}$, still contained three times the original amount of oxygen. The oxygen-content data indicate that oxidation of polystyrene does not occur on heating the polymer at $115^{\circ} \mathrm{C}$ in oxygen for $250 \mathrm{hr}$.

In summation, the general results discussed indicate that the mechanism of the degradation of polystyrene under the conditions used in this study consists of two processes: (1) the loss of volatile materials and decomposition of the more thermolabile structures; this results from exposure to ultraviolet radiant energy and/or heat in the range $115^{\circ}$ to $120^{\circ} \mathrm{C}$, both in vacuum and in oxygen; (2) oxidation of the polymer caused by exposure to ultraviolet radiant energy at $120^{\circ} \mathrm{C}$ in the presence of oxygen. Accordingly, the combination of ultraviolet, heat, and oxygen was the most degradative set of conditions 
employed in this study; oxygenated structures in the polymer increased, insolubility and discoloration were produced, and oxygenated gaseous products were evolved.

This investigation was sponsored by and conducted with financial assistance from the Office of the Quartermaster General, Department of the Army.

The assistance of other personnel at the Bureau is acknowledged, in particular the efforts of Robert M. Reese and Laura M. Williamson in operating the mass spectrometer and in calculating the mass spectrograms, respectively.

\section{References}

[1] A. J. Warner, A survey of recent research and development on high performance insulators as used or suggested for use in ion chamber instruments. Ionization chamber insulating material, Signal Corps Project No. 32-152-B-O (C540. 1), Report July 15-Oct. 15, 1949. Federal Telecommunication Laboratories, Inc.

[2] L. A. Matheson and R. F. Boyer, Light stability of polystyrene and polyrinylidene chloride, Paper presented at American Chemical Society Meeting September 1950, Chicago, Ill.

[3] B. G. Achhammer, M. J. Reiney, and F. W. Reinhart, Study of degradation of polystyrene, using infrared spectrophotometry, J. Research NBS 47, 116 (1951) RP2235.

[4] Unpublished work.

[5] R. C. Taylor, R. A. Brown, IV. S. Young, and C. E. Headington, The mass spectrometer in organic chemical analysis, Anal. Chem. 20, 396 (1948).

[6] S. L. Madorsky and S. Straus, High vacuum pyrolytic fractionation of polystyrene. Mass spectrometer analysis of some of the fractions, Ind. Eng. Chem. 40, 848 (1948).

[7] L. A. Wall, Mass spectrometric investigation of the thermal decomposition of polymers, J. Research NBS 41, 315 (1948) RP1928.

[8] S. L. Madorsky, S. Straus, D. Thompson, and L. Williamson, Pyrolysis of polyisobutene (Vistanex), polyisoprene, polybutadiene, GR-S, and polyethylene in a high vacuum, J. Research NBS 42, 499 (1949) RP1989.

[9] This polymer was prepared by the Dow Chemical Laboratories, Nidland, Mich.

[10] Unpublished work of Guy A. Hanks, Plastics Section, NBS.

[11] W. W. Walton, F. W. MeCulloch, and W. H. Smith, Determination of small amounts of oxygen in organic compounds, J. Research NBS $\mathbf{4 0}, 443$ (1948) RP1889.

[12] D. B. Judd and K. L. Kelly, Method of designating colors, J. Research NBS 23, 355 (1939) RP1239.

[13] R. B. Mesrobian and A. V. Tobolsky. Some structural and chemical aspects of aging and degradation of vinyl and diene polymers, J. Polymer Sci. 2, 463 (1947).

[14] R. Simha, L. A. Wall, and P. J. Blatz, Depolymerization as a chain reaction, J. Polymer Sci. 5, 615 (1950).

[15] N. Grassie, Determination of the Concentration of High Polymer Solutions, J. Polymer Sci. 6, 643 (1951).

[16] G. E. Branch and M. Calvin, The theory of organic chemistry-An advanced course, p. 161. (Prentice Hall, Inc., New York, N. Y., 1941).

[17] Unpublished work of F. IV. Reinhart, et al., NBS.

\section{Discussion}

Dr. H. H. G. Jelliner, University of Adelaide, Adelaide, Australia: Some years ago I studied the oxidative break-down of polystyrene, and I found, by plotting intrinsic viscosity against time, a fairly rapid decrease, which eventually tails off. If you try to work 
out the mechanism according to the random theory, then for the initial period of degradation you should get a straight line by plotting the average number of breaks per original chain against time. As a matter of fact, straight lines are not obtained; a rapid tailing off is found. The mechanisn, which fitted best the experimental results at the time is something like this:

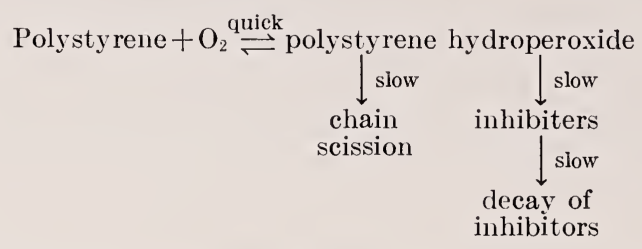

You get an equilibrium established first, which is maintained throughout the degradation reaction. The hydroperoxide can lead to chain scission, but this scission reaction is retarded by reaction products. I think this mechanism, on the whole, fits quite nicely your experimental results. Your experiments are somewhat more complex than mine; I did not use any ultraviolet radiation. You also mentioned that you have some oxygen in your polymer from the very beginning. That means, probably, that this oxygen must have been incorporated during the polymerization process. I just want to make a tentative suggestion as to the nature of these groups. During the polymerization of styrene, the following reactions might take place:<smiles>[R]/C=C\OC([R])C([2H])([2H])OC</smiles><smiles>[R]C(O)(CC1OOC1([R])NCC)C(C)O</smiles>

This radical then grows into a polymer chain. On degradation chain scission might take place as follows:<smiles>[R]C(=O)[C@H](C)O</smiles>

Mr. B. G. Achhammer: 'The infrared spectra of the various polystyrenes we studied showed no hydroxyl absorption before treatment. The hydroxyl absorption is quite easily detected by infrared, and I feel that we should have found hydroxyl groups if they were present to any extent in the untreated polymers.

DR. LeO A. WALL: In the experiments reported by $\mathrm{Mr}$. Achhammer, the temperature was approximately $100^{\circ} \mathrm{C}$. It is obvious, of course, that in the oxidation of polystyrene high conversions will result in reduced molecular weight. However, the conditions of the experi- 
ments just reported were very mild, the conversion being only a few percent. In this case, cross-linking, which may be favored by the bulk state of the polymer and the low temperature, apparently occurs. Solution oxidation and higher temperatures will favor scission reactions leading to a molecular weight decrease.

The reported copolymers of oxygen and styrene, would indicate that you can get peroxide links in the chain during polymerization. However, one might expect at the temperature of polymerization most of such peroxidic links will eventually decompose to form hydroxyl or ether groups.

We have obtained some preliminary results on the ultraviolet induced oxidation of poly-alpha-deuterostyrene. If the alpha bydrogen is replaced by deuterium and if the rate determining process is the removal of such atoms by $\mathrm{O}_{2}$, then the rate of oxidation may be slowed down by a factor of 6 . Actually, we find a factor of about 2 in the initial rate. This indicates that considerable oxidation occurs at the alpha position, although the low result may imply that an important amount of oxidation occurs at other positions, such as the beta position. Further work with poly-beta-deuterostyrene is in progress in order to evaluate the amount of oxidation occurring at the different positions in the molecule.

Dr. E. H. Wood, Bakelite Co., Division of Union Carbide and Carbon Corp., Bound Brook, N. J.: From the intrinsic viscosity curve against time, Dr. Jellinek figured that he was getting inhibition. Isn't that what one would expect from random breaking? In a material that is not homogeneous, the big molecules degrade faster than the others. In the big molecule you may break one bond and have a marked lowering of the molecular weight. Wouldn't you get some such curve even if you had no inhibition?

Dr. Jellinek: Yes, you would. If you work your curve out theoretically choosing a rate constant, which fits the initial part of the degradation, then you will find that the actual experimental curve tails off much more quickly than your theoretical curve. Hence, you have to assume that some slowing down of the reaction takes place. Something must happen to inhibit the reaction.

Dr. T. G. Fox, Rohm \& Haas Co., Philadelphia, Pa.: I would like to mention some observations on the thermal degradation of polystyrene that Dr. Flory and I made in the course of our study of the bulk viscosities of fractionated polystyrenes. 'They furnish additional information on the initiation of the thermal degradation in polystyrene and on the relative stability of various end groups.

In our nitial work the polymers were prepared by bulk polymerization of styrene at $60^{\circ} \mathrm{C}$ with 1 percent or less of benzoyl peroxide. On heating a relatively high molecular-weight fraction $(70,000)$ in an inert atmosphere (nitrogen) at $217^{\circ} \mathrm{C}$, both the bulk and the intrinsic viscosities were observed to fall. In several instances an insoluble portion appeared and an increase in the intrinsic viscosity of the soluble portion was observed. It appears that degradation and cross-linking reactions occur simultaneously under these conditions. Addition of 0.5 percent of phenyl $\beta$-naphthylamine eliminated all evidenes of gelation and reduced degradation to a negligible amount when polystyrene was heated at $217^{\circ} \mathrm{C}$ in nitrogen for $2 \mathrm{hr}$.

It was assumed that the lower molecular-weight polymer fraction in the presence of this inhibitor would be at least as stable as the higher molecular weight ones. Experience showed, however, that 0.5 -percent 
phenyl $\beta$-naph thylamine fails to inhibit degradation reactions in polystyrenes of molecular weight of 10,000 or lower. These low polymers were prepared in the presence of 4 percent of benzoyl peroxide. A connection between the observed instability and the presence of benzoate end groups on the polystyrene chains was indicated by the sublimation of a quantity of pure benzoic acid when these polymer fractions were heated at $217^{\circ} \mathrm{C}$ under reduced pressure. Since the polystyrenes had been subjected to fractional precipitation they would not have any peroxide molecules or fragments present except those chemically tied to the polymer chain.

Following this sad experience, low mo ecular weight polymers of styrene were prepared by thermal polymerization in diethylbenzene in the absence of added catalyst. Fractions prepared from these polymers were much more stable. When heated for several hours at $217^{\circ} \mathrm{C}$ in pure nitrogen, they discolored only slightly and exhibited no change in the bulk or intrinsic viscosities even in the absence of inhibitor.

The data indicate that the thermal stability of polystyrene is dependent on the nature of the chain ends, that is, that the degradation at $217^{\circ} \mathrm{C}$ is initiated at the chain ends. This appears to be at variance with the conclusion that below $340^{\circ} \mathrm{C}$ the degradation of polystyrene is initiated by the rupture of a limited number of we? links in the polymer chain. Furthermore, the ends consisting of fragments from benzoyl peroxide (benzoate ends) are less stable than the chain ends evolved when styrene is polymerized thermally in diethylbenzene. This is in contrast to Grassie and Melville's conclusion that polymethyl methacrvlate molecules with benzoyl peroxide fragment ends were more stable than similar chains with double or single bonded ends.

Finally, it would appear that any complete interpretation of the thermal degradation of polystyrene must take into consideration the possibility of cross-linking and branching reactions.

Dr. Jellinek: At about $250^{\circ} \mathrm{C}$ or so, you get a type of degradation of polystyrene that is characterized by the fact that the degradation comes to a standstill when a certain chain length is reached. It can be shown that this type of degradation $i$; due to weak links, which are distributed at random along the chains and when these have been broken down, nothing much more happens. But if you go to higher temperatures, say, about $400^{\circ} \mathrm{C}$, then you get a different type of degradation altogether. If you plot the percentage loss of weight against time, you get first a rapid loss of weight. This is due to the breaking of weak links; for each weak link ruptured, a number of monomer units are broken off. After this you get an induction period, probably indicating that inhibitors have been produced. After this you get a straight line, which indicates that the polystyrene breaks down from the chain ends. That means that the number of chain ends remains constant for a long period during the degradation. So you have two processes. First of all, the weak links break, then monomer units are broken off from the chain ends. Also the energies of activation for these two processes are different. The energy of activation for breaking of weak links is 24,500 cal, and that for splitting off monomer units is $45,000 \mathrm{cal}$. Polystyrene polymerized in the presence of benzoyl peroxide seems to have many more weak links than other polystyrenes. Also cross-linked polystyrene behaves differently, whether it was polymerized in the presence of benzoyl peroxide or not. In the first instance it degrades more readily than in the second. 


\title{
15. Rates of Thermal Degradation of Polystyrene and Polyethylene in a Vacuum ${ }^{1}$
}

\author{
By Samuel L. Madorsky ${ }^{2}$
}

When heated in a vacuum at $325^{\circ}$ to $375^{\circ} \mathrm{C}$, polystyrene vields mainly the monomer and some dimer, trimer, tetramer, etc., indicating that the breaks in the molecular chains occur largely at the ends. Polyethylene, under similar conditions, but at a somewhat higher temperature, yields fragments of an average molecular weight of about 700, indicating that the molecular chains break mostly at random positions.

A study was made of rates of thermal degradation of these two polymers by measuring rates of loss of weight of samples by means of a very sensitive tungstenspring balance inclosed in a vacuum. The samples were limited to 5 to 6 milligrams in order to avoid spattering during degradation.

Rate curves plotted against percentage loss of weight by volatilization indicate that in the case of polystyrene the process is intermediate between a zero and first-order reaction, while in the case of polyethylene, the process approximates a first-order reaction. Activation energies were calculated on the basis of rates of degradation at various temperatures. The values thus obtained for polystyrene and polyethylene are 58 and 68 kilocalories, respectively.

\section{In troduction}

It is only within the last few years that some important experimental work has been reported in the literature on rates and activation energies of thermal degradation of polvmers. Grassie and Melville $[1]^{3}$ measured the vapor pressure of volatile products formed in the pyrolysis of polymethyl methacrylate in a vacuum, using a Pirani gage. Jellinek [2] measured the rate of loss of weight of samples of polystyrene, poly-alpha-methylstyrene and polyethylene heated in. a vacuum. He used for this purpose a quartz-spring balance inclosed in a vacuum system. Atherton [3] repeated Jellinek's work on polystyrene, heating the same amount of material, that is, $20 \mathrm{mg}$, in a crucible of the same size, and found that a good deal of spattering occurred during the heating of the samples. Oakes and Richards [4] studied rate of degradation of polyethylene by measuring rate of change of intrinsic viscosity and of other properties of thermally degraded samples.

It was shown experimentally $[5,6]$ in some of the previous work at the National Bureau of Standards, in the case of some hydrocarbon polymers, such as polystyrene, polyisoprene, polyethylene, etc., that the volatile products obtained during thermal degradation in a vacuum, in the temperature range $300^{\circ}$ to $400^{\circ} \mathrm{C}$, consist of fragments varying in size from a monomer to a molecular weight of abut 1,000 . It was also shown that the chemical nature and relative amounts of these fragments are independent of the temperature at which degradation takes place or of the extent of volatilization. In the case of another

\footnotetext{
1 This work was supported by funds transferred from the Reconstruction Finance Corporation, Office of Rubber Reserve, Washington, D.C.

2 National Bureau of Standards, Washington, D. C.

3 Figures in brackets indicate the literature references on p. 235.
} 
group of polymers, such as polymethyl methacrylate, and poly-alphamethylstrrene, it was shown [7] that when these are heated in the temperature range $250^{\circ}$ to $350^{\circ} \mathrm{C}$, the volatile products consist almost exclusively of the monomer, regardless of temperature or extent of volatilization.

In the prrolysis of polystyrene it was found [5] that in the temperature range $360^{\circ}$ to $420^{\circ} \mathrm{C}$, where volatilization varied from 4 to 100 percent, the light fraction consisted, on the average, of 40 percent strrene and 2.4 percent toluene in terms of total volatilized part. The remaining 57.6 percent consisted of heavier molecules of an arerage molecular weight of 264. In the prrolysis of polyethylene [6], in the temperature range $400^{\circ}$ to $475^{\circ} \mathrm{C}$, where volatilization varied from 12 to 99 percent, the light fraction consisted of monomer and monomer-like molecules to the extent, on the average, of 3.4 percent of the total volatilized part. These small molecules were found to be in constant ratios to each other. The heavy, or less volatile fraction, had in each case an average molecular weight of 692 . Since the experiments were carried out under almost ideal conditions of molecular distillation, the rate of evaporation of any fragment, up to a molecular weight of about 1,000, would be extremely fast as compared with the rate of formation of these fragments by prrolysis.

While it is true that the smaller molecules would diffuse through the liquid polymer faster than the larger ones, the thickness and viscosity of the liquid did not affect the composition of the volatile product through the temperature ranges indicated above and through a change of thickness of the liquid from 40 to $0 \mu$. In view of the constancy of the degradation patterns, in the case of the abovementioned and similar polymers, the rate of loss of weight during their thermal degradation can serve as a basis for the calculation of the activation energies of the orer-all reaction.

The relative amounts of monomer or monomer-like molecules in the volatile thermal degradation products of a number of polymers are shown in table 15.1, which is based on data from investigations at the National Bureau of Standards [7,8]. It can be seen from this table that in the case of polymethyl methacrylate and polyalpha-methylstyrene the breaks occur exclusively at the ends of the chains. At the other extreme is polyethylene, which yields a small amount of monomer-like fragments, while the bulk of the product consists of fragments of average molecular weight of about 700 [6]. It can, therefore, be assumed that here the random breaking of the

TABLE 15.1. Amount of monomer or monomer-type molecules in the volatile products of thermal degradation of polymers

\begin{tabular}{|c|c|c|}
\hline \multirow{2}{*}{ Polymer } & \multicolumn{2}{|c|}{$\begin{array}{c}\text { Monomer or monomer-type } \\
\text { molecules in- }\end{array}$} \\
\hline & $\begin{array}{l}\text { Weight per- } \\
\text { cent of total } \\
\text { pyrolyzed part }\end{array}$ & $\begin{array}{l}\text { Mole percent } \\
\text { of total py- } \\
\text { rolyzed part }\end{array}$ \\
\hline Polymethylmethacrylate & 100 & 100 \\
\hline Poly- $\alpha$-methylstyrene & 100 & 100 \\
\hline Polyisobutene & 32 & 78 \\
\hline Polystyrene & 42 & 65 \\
\hline Poly butariene. & 14 & 57 \\
\hline $\mathrm{GR}-\mathrm{S}$ & 12 & 52 \\
\hline Polyisoprene & 11 & 44 \\
\hline Polyethylene & 3 & 21 \\
\hline
\end{tabular}


chains predominates. Polystyrene, polyisobutane, etc., occupy an intermediate position between the two extremes.

Polystyrene and polyethylane having 42- and 3 -weight percent or 65- and 21-mole percent, respectively, of the monomer-type fragments in their volatile products, were selected for the present investigation of rates and activation energies of thermal degradation. It is planned to extend this investigation in the future to the other polymers shown in table 15.1 and to additional polymers and copolymers.

\section{Apparatus and Experimental Procedure}

'The apparatus is shown diagrammatically in figure 15.1. 'The main part of the apparatus consists of a tungsten helical spring balance. A similar spring balance has been described briefly in a previous publication [9]. 'The spring was made from a 3-mil tungsten wire. It consists of 23 turns, each turn having a diameter of about $1.1 \mathrm{~cm}$; total weight of spring and extension down to the crucible suspended from it is about $100 \mathrm{mg}$; the sensitivity is about $525 \mu / \mathrm{mg}$. The crucible is made of platinum, has a diameter of $8.5 \mathrm{~mm}$, a height of $5 \mathrm{~mm}$, and weighs about $196 \mathrm{mg}$. The spring and crucible are inclosed in a Pyrex glass housing that is evacuated by means of an oil pump and a mercury diffusion pump to a sticking vacuum (about $\left.10^{-6} \mathrm{~mm} \mathrm{Hg}\right)$. A liquid-nitrogen condenser is located between the

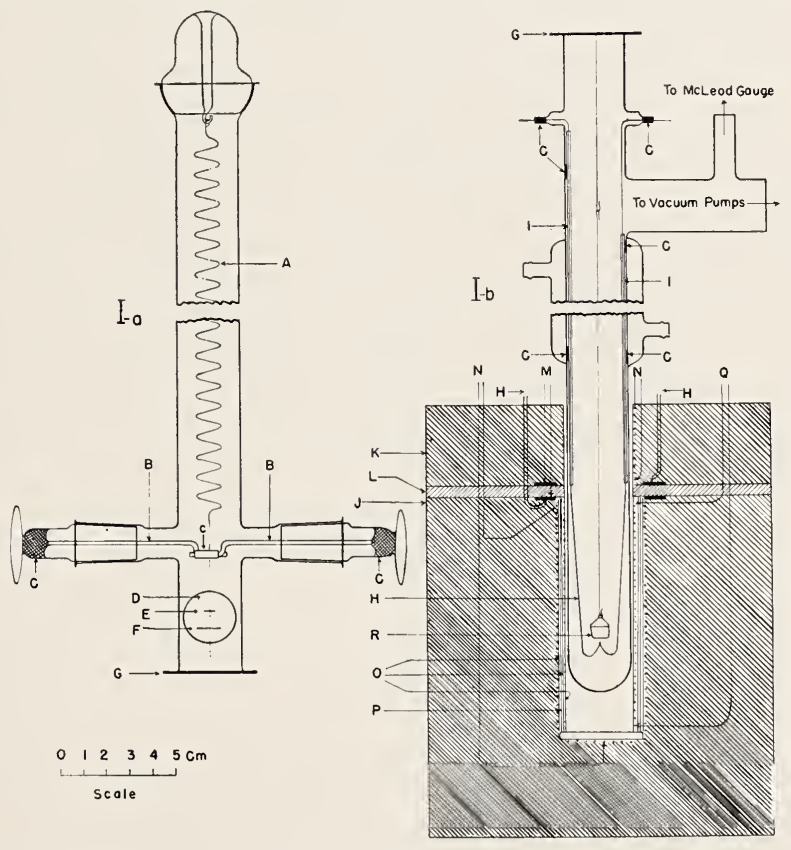

Figure 15.1. Apparatus for the study of rates of thermal degradation of polymers.

Part I-a fits on top of part I-b; A, tungsten spring; B, brass friction clasps padded with rubber sleeves $c$; $C$, hard apiezon wax; $\mid D$, flat Pyrex window; $E$, crossline on spring extension; $F$, crossline on window; $G$, ground flanges; H, chromel-constantan thermocouples; $\mathbf{I}$, glass capillaries for holding the thermocouple wires inside the apparatus in rigid position; $\mathrm{J}$, main heater; $K$, auxiliary heater; $L$, transite plate; $M$, brass disks; $N$, nichrome heating elements; $O$, thin mica sheets; $P$, thin brass sheet, between two mica shcets; Q, No. 40 pt-wire resistance thermometer; R, platinum crucible. 
mercury diffusion pump and the housing of the spring. The pressure is measured by means of a McLeod gage. The spring is suspended from a ball-joint at the top of the housing, so that the position of the crucible may be adjusted with respect to the center of the housing.

Fused-quartz spring balances have been used extensively for weighing in a vacuum. However, due to the low internal friction of quartz, the damping force is very small and when once disturbed, while in a high vacuum, it may take several hours for a quartz spring of the same sensitivity to come to rest. The tungsten spring used in this investigation came to rest in about 10 to $15 \mathrm{~min}$ after a disturbance.

A newly constructed tungsten spring continues to stretch under a constant load, due to elastic creep, for many days at a diminishing rate. The stretching becomes imperceptible after about 30 days. In order to minimize the effect of elastic creep when the spring is not in use and to avoid disturbance of the spring when the crucible is removed for charging and weighing, the balance is provided with a pair of friction clasps, as shown in figure 15.1. The clasps are operated by rotation in opposite directions until they grip the extension of the spring. A sensitive cathetometer, reading to $1 \mu$, was used in connection with the balance. The cathetometer includes a microscope capable of moving in a vertical or horizontal direction.

In order to avoid errors due to differences in the thermal expansion of the apparatus and its support, on the one hand, and the cathetomoter and its support, on the other, also due to physical disturbance of the cathetometer during operation, the position of the crossline on the spring extension was checked against a zero-point on the balance housing before or after each reading. This zero-point is in the form of a thin horizontal wire attached to a flat glass window in front of the cathetometer. Fortunately, the thermal coefficients of expansion of tungsten and Pyrex are about the same. Another source of error is the thermal coefficient of the modulus of rigidity of tungsten [10] which will affect the sensitivity of the balance. This error was minimized by operating the balance in a constant-temperature room.

A nichrome-wire wound electric heater fits around the lower part of the balance housing. This heater can be moved vertically by means of an elevator. A platinum resistance thermometer is incorporated in the heater and the temperature of the heater controlled by means of a "time-modulation" electronic thermostat [11]. The end effect at the top of this main heater is eliminated by means of an auxiliary heater. The temperature of the auxiliary heater is adjusted to the temperature of the main heater by means of a pair of chromel-constantan thermocouples at the interface of the two heaters in conjunction with another time-modulation electronic thermostat. Temperature of the platinum crucible was measured by means of a chromel-constantan thermocouple placed within 2 to $3 \mathrm{~mm}$ below the crucible. Although there may be some slight difference between the temperature of the thermocouple and that of the crucible, this difference would be constant through the temperature range employed and would, therefore, not affect the results appreciably. A condenser, through which water from a thermostated reservoir was circulated, kept the part of the glass housing just above the auxiliary heater at room temperature. The temperature, as indicated by the thermocouple below the platinum crucible, could be maintained by all these means constant to within $\pm 0.2^{\circ} \mathrm{C}$. 
The following procedure was used. The sample was weighed in the platinum crucible on a semimicro balance. It was found that if the sample weighed more than 5 to $6 \mathrm{mg}$, spattering occurred later during the heating. This spattering could be observed very clearly by the recoil produced in the spring. The crucible was placed in the apparatus and the system evacuated. The heater was kept in the lower position, that is, not embracing the crucible in the apparatus, and its temperature was brought to the approximate temperature required for a given run. After ascertaining that the system was evacuated to a sticking vacuum, the heater was raised gently by means of an elevator, not shown in figure 15.1. This operation did not disturb the spring balance. It usually lasted from 10 to $20 \mathrm{~min}$ before the thermocouple under the crucible registered the required temperature. During this time, and when the temperature reached about $175^{\circ} \mathrm{C}$, a zero reading of the spring cross line was taken. Ordinarily, by the time the required temperature was reached, a small amount of the material in the crucible was evaporated. The rates of evaporation were measured from the time the operating temperature was reached.

The crucible and residue were weighed at the end of the experiment. The sensitivity, $\lambda$, of the spring balance was determined by dividing the total change in length of the spring by the total loss in weight of the sample. The values of $\lambda$ for 22 experiments are shown in the last column of table 15.2 , the average value being $525.3 \pm 6.3 \mu / \mathrm{mg}$. The

$\mathrm{T}_{\mathrm{ABLE}}$ 15.2. Experimental data on thermal degradation of polystyrene and polyethylene

\begin{tabular}{|c|c|c|c|c|}
\hline $\begin{array}{c}\text { Tempera- } \\
\text { ture }\end{array}$ & Duration & $\begin{array}{l}\text { Weight of } \\
\text { sample }\end{array}$ & $\begin{array}{l}\text { Total loss } \\
\text { in weight }\end{array}$ & $\begin{array}{l}\text { Sensitivity } \\
\text { of springs a }\end{array}$ \\
\hline \multicolumn{5}{|c|}{ Polystyrene 230,000} \\
\hline $\begin{array}{l}{ }^{\circ} C \\
335 \\
340 \\
345 \\
350 \\
355 \\
360 \\
365\end{array}$ & $\begin{array}{r}\min \\
450 \\
450 \\
390 \\
300 \\
210 \\
155 \\
130\end{array}$ & $\begin{array}{c}m g \\
5.24 \\
5.49 \\
4.84 \\
5.42 \\
5.38 \\
5.29 \\
5.26\end{array}$ & $\begin{array}{c}m g \\
2.97 \\
3.95 \\
4.10 \\
4.96 \\
4.97 \\
4.75 \\
4.99\end{array}$ & $\begin{array}{c}\mu \\
511.4 \\
513.2 \\
517.8 \\
513.4 \\
514.3 \\
524.9 \\
515.4\end{array}$ \\
\hline \multicolumn{5}{|c|}{ Polystyrene 106,000} \\
\hline $\begin{array}{l}335 \\
340 \\
345 \\
350 \\
355\end{array}$ & $\begin{array}{l}450 \\
425 \\
375 \\
360 \\
310\end{array}$ & $\begin{array}{l}5.81 \\
5.54 \\
5.47 \\
5.45 \\
5.13\end{array}$ & $\begin{array}{l}3.55 \\
2.94 \\
3.68 \\
4.40 \\
4.56\end{array}$ & $\begin{array}{l}526.4 \\
526.9 \\
534.8 \\
533.4 \\
530.0\end{array}$ \\
\hline \multicolumn{5}{|c|}{ Polystyrene 584,000} \\
\hline $\begin{array}{l}335 \\
340 \\
345 \\
350 \\
355\end{array}$ & $\begin{array}{l}465 \\
420 \\
420 \\
360 \\
272\end{array}$ & $\begin{array}{l}4.62 \\
5.29 \\
5.25 \\
5.45 \\
5.54\end{array}$ & $\begin{array}{l}2.31 \\
3.25 \\
3.78 \\
4.40 \\
4.58\end{array}$ & $\begin{array}{l}532.4 \\
532.6 \\
532.5 \\
533.4 \\
538.2\end{array}$ \\
\hline \multicolumn{5}{|c|}{ Polyethylene 20,000} \\
\hline $\begin{array}{l}385 \\
390 \\
395 \\
400 \\
405\end{array}$ & $\begin{array}{l}510 \\
450 \\
430 \\
270 \\
270\end{array}$ & $\begin{array}{l}6.55 \\
6.47 \\
6.46 \\
6.07 \\
6.03\end{array}$ & $\begin{array}{l}3.24 \\
3.80 \\
4.54 \\
4.69 \\
5.16\end{array}$ & $\begin{array}{l}524.4 \\
521.8 \\
526.6 \\
525.2 \\
528.1\end{array}$ \\
\hline
\end{tabular}

a Average sensitivity of spring $525.3 \pm 6.3 \mu / \mathrm{mg}$. 
value of $\lambda$ for each experiment was used in the calculation of rates and residues for that experiment. Individual readings could be made with an accuracy of about $5 \mu$, which corresponds to an accuracy of about $0.01 \mathrm{mg}$.

\section{Thermal Degradation of Polystyrene}

An unfractionated sample of polystyrene of molecular weight 230,000 , similar to the one used previously in pyrolysis work [5], was also used in this investigation. In addition to this, two fractions of molecular weights 106,000 and 584,000, obtained from the above unfractionated material were also used. These materials will be designated, in the order given above, as samples I, II, and III.

First, a study was made with material I to determine change of molecular weight of residue with percentage of degradation. Small samples were pyrolyzed in a vacuum, by a method described previously [5], to various stages of degradation by heating at various temperatures for half-hour periods. The residues were then analyzed for average molecular weight by the osmotic-pressure method. ${ }^{4}$ The results of these experiments are shown in figure 15.2. There seems to be a rapid drop in molecular weight during the first 10-percent loss of weight, after which the drop is more gradual. This is in agreement with experiments carried out by Jellinek [2] on a polystyrene fraction of molecular weight of about 120,000 .

Rates of degradation of polystyrene I were studied at 5-deg intervals, from $335^{\circ}$ to $365^{\circ} \mathrm{C}$, and those of II and III were studied at similar intervals, from $335^{\circ}$ to $355^{\circ} \mathrm{C}$. Some of the more pertinent experimental data are shown in table 15.2. In figures $15.3,15.4$, and 15.5 cumulative losses of weight at various temperatures, expressed in percentage of original weight of the sample, are shown as a function of time of

4 The pyrolysis experiments on the polystyrene samples were carried out by S. Straus of the Mass Spec trometry Section, and the average molecular weights of the residues were determined by G. A. Hanks of the Organic Plasties Section of the National Burcau of Standards.

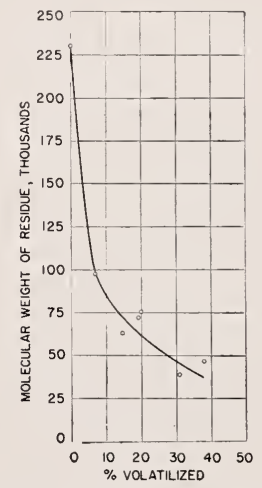

Figure 15.2. Numberaverage molecular weight of residual thermally degraded polystyrene as a function of percentage of volatilization.

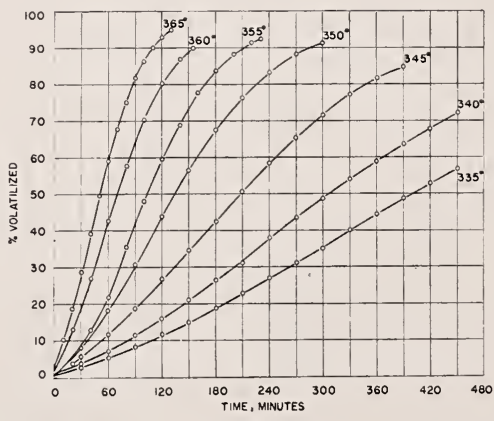

Figure 15.3. Cumulative degradation of unfractionated polystyrene, molecular weight 230,000, as a function of time. 
degradation for the three grades of polystyrene. At first glance it would seem from these graphs that the rates for each temperature are more or less constant through most of the volatilization range. However, a careful observation will reveal the fact that the rates are slower at both ends of the curves than in the middle. This becomes more obvious from tables $15.3,15.4$, and 15.5 , where average rates for short-time intervals are shown. In each of these tables there are three columns under each temperature heading. In the first column, under $D$, mean percentages of loss of weight by volatilization for short-time intervals are given. In the second column, under $K_{1}$, the mean rates of loss of weight for the same intervals are given in percentage of the original weights of the samples used. In the third column, under $K_{2}$, are given the mean rates of loss of weight in percentage of the mean weights of residues for the same time intervals.

Values of $K_{2}$ are plotted as a function of $D$ (percentage loss of weight) in figure 15.6, and those of $K_{1}$ are plotted in figure 15.7,

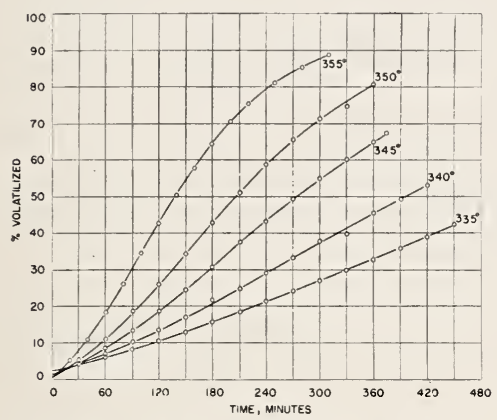

Figure 15.4. Cumulative degradation of polystyrene fraction, molecular weight 106,000, as a function of time.

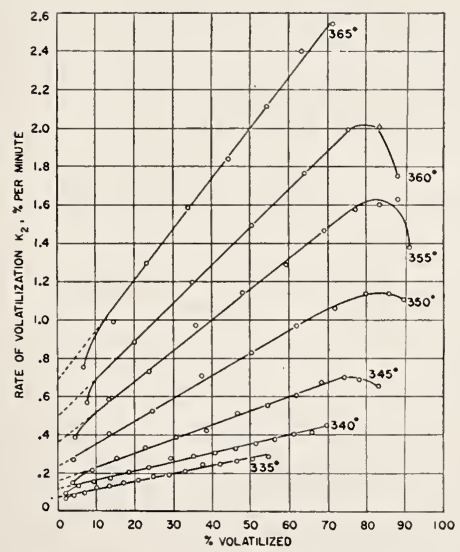

Figure 15.6. Rates of thermal degradation of polystyrene, molecular weight 230,000 in percentage of corresponding residues, as a function of volatilization.

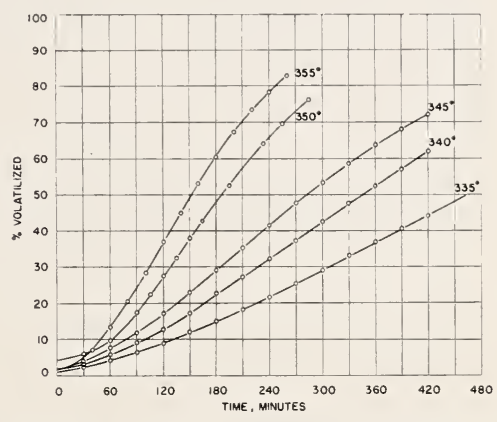

Figure 15.5. Cumulative degradation of polystyrene fraction, molecular weight 584,000, as a function of time.

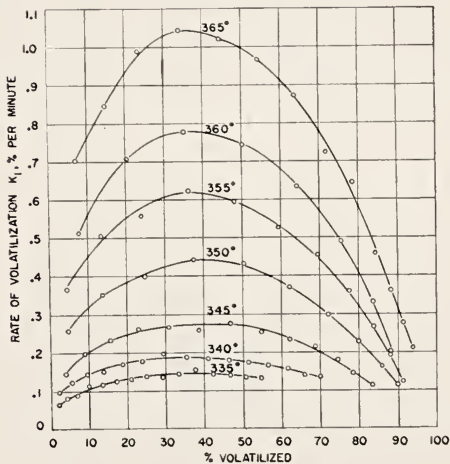

Figure 15.7. Rates of thermal degradation of polystyrene, molecular weight 230,000, in percentage of original weight of sample, as a function of volatilization. 
for polystyrene I. Polystyrene grades II and III give the same type of curves when plotted similarly. As shown in figure 15.6, the values of $K_{2}$ rise with percentage loss of weight in straight lines; and the higher the temperature, the steeper the slope of the line. However, the initial values, up to 2- to 10-percent degradation, and the final values, above 75 - percent degradation, fall below the straight lines. In figure 15.7 the $K_{1}$ curves at first rise with percentage loss of weight, reach a maximum between 30 and 40 percent, and then fall again. Comparing the data in tables 15.3, 15.4, and 15.5, we see that the unfractionated polystyrene I has higher rates of degradation than its fractions II and III; also, that III, with a molecular weight of 584,000 has higher rates than II, which has a molecular weight of 106,000. In view of these facts, and also in view of the fact that the molecular weight of the residue decreases unevenly with percentage loss of weight, as shown above (fig. 15.2), one is justified in using rates of loss of weight by volatilization at different temperatures for the purpose of calculating activation energies only when the residues corresponding to the rates used have the same average molecular weights and the same distributions. These conditions are met at zero degradation when the same material is used for all temperature experiments. By

TABLE 15.3. Rates of thermal degradation of polystyrene, molecular weight 230,000 at various temperatures

$D$ is volatilization in percentage of original sample. $K_{1}$ and $K_{2}$ are rates of degradation or of loss in weight per minute in percentage of original sample and of residue, respectively, at any given percent age of volatilization. Initial rates, $K_{2}$, were obtained by linear extrapolation.

\begin{tabular}{|c|c|c|c|c|c|c|c|c|c|c|c|}
\hline \multicolumn{3}{|c|}{$335^{\circ} \mathrm{C}$} & \multicolumn{3}{|c|}{$340^{\circ} \mathrm{C}$} & \multicolumn{3}{|c|}{$345^{\circ} \mathrm{C}$} & \multicolumn{3}{|c|}{$350^{\circ} \mathrm{C}$} \\
\hline$D$ & $K_{1}$ & $K_{2}$ & $D$ & $K_{1}$ & $K_{2}$ & $D$ & $K_{1}$ & $K_{2}$ & $D$ & $K_{1}$ & $K_{2}$ \\
\hline$\%$ & $\%$ & $\%$ & $\%$ & $\%$ & $\%$ & $\%$ & $\%$ & $\%$ & $\%$ & $\%$ & $\%$ \\
\hline 0 & 0 & 0.069 & 0 & 00 & 0.115 & 0 & 70 & 0.158 & 0 & - - & 0.235 \\
\hline 2.1 & 0.067 & .069 & 2.1 & 0.098 & .100 & 3.8 & 0.147 & .152 & 4. 2 & 0.259 & .271 \\
\hline 4. 3 & .082 & .086 & 5. 4 & .123 & .130 & 9. 0 & . 199 & .219 & 13.4 & .352 & .407 \\
\hline $\begin{array}{l}6.9 \\
99\end{array}$ & .090 & .096 & 9.4 & .143 & .158 & 15.4 & .233 & .280 & 24. 7 & .399 & .530 \\
\hline 9.9 & .113 & .126 & 13.8 & .151 & .175 & 22.9 & .262 & .339 & 37.3 & .442 & .701 \\
\hline 13.4 & .116 & .135 & 18. 6 & .169 & .208 & 30.7 & .266 & .396 & 50.2 & . 432 & .827 \\
\hline 17.1 & .125 & .154 & 23.8 & .177 & .232 & 38.6 & 259 & .421 & 61.9 & .370 & .973 \\
\hline 20.9 & .132 & .166 & 29.4 & .197 & .279 & 46. 6 & .276 & .517 & 71.9 & .297 & 1. 057 \\
\hline 24.9 & .138 & .181 & 35. 0 & .187 & .289 & 54. 5 & 251 & .552 & 79.8 & .229 & 1. 137 \\
\hline 29.0 & .136 & .191 & 40.8 & .183 & .307 & 61.8 & 233 & .609 & 85. 7 & .163 & 1. 140 \\
\hline 33.3 & .144 & .214 & 46. 1 & .178 & .330 & 68.5 & 214 & .680 & 89.8 & .113 & 1. 107 \\
\hline 37.7 & .155 & .249 & 51.4 & .173 & .356 & 74.4 & .179 & .699 & -..- & ---- & --.-- \\
\hline 42.2 & .144 & .250 & 56.5 & .165 & .378 & 78.2 & 143 & 687 & -- & -..... & -... \\
\hline 46.5 & .141 & .263 & 61.3 & .157 & .406 & 83.1 & .112 & .658 & -... & - & - - - \\
\hline 50.6 & .136 & .274 & 65. 8 & .141 & .411 & -.-- & --.-- & -...- & --- & $-\ldots$ & --.. \\
\hline 54.7 & .134 & .289 & 69. 9 & .136 & .452 & -... & $\cdots$ & 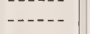 & $\ldots$ & $-1-1$ & $\ldots$ \\
\hline \multicolumn{4}{|c|}{$355^{\circ} \mathrm{C}$} & \multicolumn{4}{|c|}{$360^{\circ} \mathrm{C}$} & \multicolumn{4}{|c|}{$365^{\circ} \mathrm{C}$} \\
\hline$D$ & \multicolumn{2}{|c|}{$K_{1}$} & $K_{2}$ & $D$ & \multicolumn{2}{|c|}{$K_{1}$} & $K_{2}$ & $D$ & \multicolumn{2}{|c|}{$K_{1}$} & $K_{2}$ \\
\hline$\%$ & \multicolumn{2}{|c|}{$\%$} & $\%$ & $\%$ & \multirow{2}{*}{\multicolumn{2}{|c|}{$\%$}} & $\%$ & $\%$ & \multicolumn{2}{|c|}{$\%$} & $\%$ \\
\hline 0 & \multirow{2}{*}{\multicolumn{2}{|c|}{0.366}} & 0.358 & 0 & & & 0.496 & 0 & \multirow{2}{*}{\multicolumn{2}{|c|}{0.705}} & 0.680 \\
\hline 4. 5 & & & .384 & 7.8 & \multicolumn{2}{|c|}{5.515} & .570 & 7.0 & & & .758 \\
\hline 13.3 & \multicolumn{2}{|c|}{$\begin{array}{r}0.500 \\
.512\end{array}$} & .590 & 20.1 & \multicolumn{2}{|c|}{.706} & .884 & 14.7 & \multicolumn{2}{|c|}{.844} & .990 \\
\hline 23.9 & & 56 & .730 & 35.0 & \multicolumn{2}{|c|}{.779} & 1. 200 & 23.2 & \multicolumn{2}{|c|}{.988} & 1. 298 \\
\hline 35.8 & & 24 & 971 & 50.2 & \multirow{2}{*}{\multicolumn{2}{|c|}{$\begin{array}{r}743 \\
635\end{array}$}} & $\begin{array}{l}1.490 \\
\text { 1. } 492\end{array}$ & 34.0 & \multicolumn{2}{|c|}{ 1. 044} & 1. 582 \\
\hline 47.9 & & 997 & 1.146 & 64.0 & & & 1. 765 & 44.4 & \multicolumn{2}{|c|}{ 1. 020} & 1. 832 \\
\hline 59.2 & & 26 & 1. 288 & $\begin{array}{l}0.0 \\
75.3\end{array}$ & \multicolumn{2}{|c|}{$\begin{array}{r}.030 \\
.490\end{array}$} & 1. 978 & 54.3 & & 966 & 2.11 \\
\hline 69.0 & & 55 & 1.467 & 83.5 & & 33 & 2. 005 & 63.5 & & 374 & 2.40 \\
\hline 77.1 & & 359 & 1. 572 & 88.3 & & 00 & 1.702 & 71.5 & & 724 & 2. 54 \\
\hline 83.4 & & 266 & 1. 600 & ...... & & ... & - & 78.6 & & 645 & 2. 97 \\
\hline 88.0 & & 95 & 1. 625 & - & & & 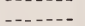 & 84.0 & & 483 & 3. 01 \\
\hline 91.2 & & 22 & 1. 380 & - & & & & 88.2 & & 361 & 3. 06 \\
\hline & & & & - & & & & 91.4 & & 276 & 3. 20 \\
\hline$\ldots$ & & & & - . & & & & 93.8 & & 211 & 3.42 \\
\hline
\end{tabular}


TABLE 15.4. Rates of thermal degradation of polystyrene, molecular weight 106,000, at various temperatures

See table 15.3 for meaning of symbols

(Values of $K_{1}$ and $K_{2}$ are in percent per minute)

\begin{tabular}{|c|c|c|c|c|c|c|c|c|c|c|c|c|c|c|}
\hline \multicolumn{3}{|c|}{$335^{\circ} \mathrm{C}$} & \multicolumn{3}{|c|}{$340^{\circ} \mathrm{C}$} & \multicolumn{3}{|c|}{$345^{\circ} \mathrm{C}$} & \multicolumn{3}{|c|}{$350^{\circ} \mathrm{C}$} & \multicolumn{3}{|c|}{$355^{\circ} \mathrm{C}$} \\
\hline$D$ & $K_{1}$ & $K_{2}$ & $D$ & $K_{1}$ & $K_{2}$ & $D$ & $K_{1}$ & $K_{2}$ & $D$ & $K_{1}$ & $K_{2}$ & $D$ & $K_{1}$ & $K_{2}$ \\
\hline$\%$ & $\%$ & $\begin{array}{c}\% \\
0.060\end{array}$ & $\begin{array}{l}c \% \\
0\end{array}$ & $\%$ & $\begin{array}{c}\% \\
0.093\end{array}$ & $\begin{array}{l}\% \\
0\end{array}$ & $\%$ & $\begin{array}{c}\% \\
0.128\end{array}$ & $\%$ & $\%$ & $\begin{array}{c}\% \\
0.193\end{array}$ & $\%$ & $\%$ & $\%$ \\
\hline 2. 7 & 0.071 & .072 & 2.5 & 0.105 & .107 & 2. 3 & 0.138 & .139 & 3.0 & 0.160 & .165 & 3.8 & 0.217 & $\begin{array}{r}0.200 \\
.225\end{array}$ \\
\hline 4. 9 & .074 & .078 & 5.6 & .102 & .108 & 6.5 & .150 & .160 & 10.2 & .193 & .210 & 8.3 & .286 & .312 \\
\hline 7.1 & .072 & .077 & 8.7 & .108 & .119 & 11.2 & .158 & .178 & 15.0 & .251 & .296 & 14.8 & 365 & .428 \\
\hline 9.4 & .080 & .089 & 12.0 & .106 & .120 & 16.2 & .177 & .212 & 22.5 & 252 & .323 & 22.3 & 394 & .515 \\
\hline 11.8 & .081 & .092 & 15.3 & 117 & .138 & 21.8 & .195 & .249 & 30.4 & .271 & .390 & 30.5 & 425 & 612 \\
\hline 14. 4 & .092 & .107 & 18.9 & .129 & .155 & 27.8 & 209 & .290 & 38.7 & .285 & .466 & 38.8 & 402 & .657 \\
\hline 17.1 & .092 & .112 & 22.9 & .135 & .175 & 34.2 & 212 & .322 & 47.1 & .275 & .520 & 46.8 & .397 & .746 \\
\hline 20.0 & .097 & .121 & 27.0 & .141 & .197 & $40 . \overline{5}$ & 207 & .347 & 55.0 & .252 & .559 & 54.4 & .363 & .795 \\
\hline 22.8 & .094 & .121 & 31.3 & .141 & .206 & 46.5 & .195 & .365 & 62.2 & .229 & .607 & 61.3 & .325 & .841 \\
\hline 25.7 & .094 & .126 & 35.5 & .141 & .219 & 52.2 & .188 & .393 & 68.6 & .191 & .609 & 67.6 & .303 & .934 \\
\hline 28.5 & .094 & .131 & 39.6 & .130 & .215 & 57.7 & .175 & .414 & 74.0 & .171 & .661 & 73. 2 & .257 & .959 \\
\hline 31.3 & .097 & .148 & 43.5 & .138 & .241 & 62.7 & .159 & .427 & 78.7 & .139 & .650 & 78.5 & .185 & .853 \\
\hline 34. 4 & .106 & .161 & 47.5 & .129 & .239 & 66.2 & .146 & .433 & - & - - & $\ldots$ & 83. 4 & .142 & .858 \\
\hline 37.6 & .107 & .171 & 51.2 & .123 & .253 & - . & & - . & & & & 87.2 & .111 & .870 \\
\hline 40.8 & .106 & .178 & & & & & & & & & & $-\ldots$ & $\ldots$ & -.... \\
\hline
\end{tabular}

TABLE 15.5. Rates of thermal degradation of polystyrene, molecular weight 584,000, at various temperatures

See table 15.3 for meaning of symbols

(Values of $K_{1}$ and $K_{2}$ are in percent per minute)

\begin{tabular}{|c|c|c|c|c|c|c|c|c|c|c|c|c|c|c|}
\hline \multicolumn{3}{|c|}{$335^{\circ} \mathrm{C}$} & \multicolumn{3}{|c|}{$340^{\circ} \mathrm{C}$} & \multicolumn{3}{|c|}{$345^{\circ} \mathrm{C}$} & \multicolumn{3}{|c|}{$350^{\circ} \mathrm{C}$} & \multicolumn{3}{|c|}{$355^{\circ} \mathrm{C}$} \\
\hline$D$ & $K_{1}$ & $K_{2}$ & $D$ & $K_{1}$ & $K_{2}$ & $D$ & $K_{1}$ & $K_{2}$ & $D$ & $K_{1}$ & $K_{2}$ & $D$ & $K_{1}$ & $K_{2}$ \\
\hline$\%$ & $\%$ & $\%$ & $\%$ & $\%$ & $\%$ & $\%$ & $\%$ & $\begin{array}{l}\% \\
0\end{array}$ & $\%$ & $\%$ & $\%$ & $\%$ & $\%$ & $\%$ \\
\hline 1. 0 & 0.025 & .025 & 0.6 & 0.035 & .035 & 0.7 & 0.044 & .044 & 1.8 & 0.125 & .127 & 2.0 & 0.148 & .152 \\
\hline 1. 7 & .065 & .066 & 2. 2 & .070 & .072 & 2.8 & .095 & .098 & 6.8 & .204 & .219 & 5.4 & .194 & .205 \\
\hline 3. 6 & .065 & .067 & 4. 6 & .094 & .099 & 6.0 & .123 & .132 & 13.8 & 262 & .304 & 11.0 & .313 & .357 \\
\hline 5.5 & .072 & .077 & 7.6 & .104 & .113 & 10.0 & .137 & .152 & 20.1 & .317 & .396 & 17. 1 & .347 & .419 \\
\hline 7. 9 & .090 & .097 & 12. 0 & .124 & .141 & 14.7 & .177 & .208 & 25.0 & .336 & .447 & 24.4 & .391 & .516 \\
\hline 10.8 & .096 & .108 & 15.1 & .147 & .173 & 20.2 & .190 & .239 & 30.0 & .333 & .476 & 32.6 & .428 & .636 \\
\hline 13.7 & .101 & .117 & 19.6 & .153 & .190 & 26.1 & .199 & .271 & 35.2 & .359 & .552 & 40.9 & .401 & .678 \\
\hline 16.8 & .103 & .124 & 24.1 & .160 & .211 & 32.1 & .203 & .314 & 40.3 & .330 & .554 & 49.0 & .409 & .801 \\
\hline 20.1 & .116 & .146 & 29.1 & $.1 € 2$ & .228 & 38.4 & .214 & .348 & 48.6 & .322 & .626 & 56.8 & .372 & .861 \\
\hline 23.6 & .118 & .155 & 34.1 & .173 & .262 & 44.6 & .195 & .354 & 58.3 & .303 & .727 & 63.9 & .340 & .943 \\
\hline 27.2 & .122 & .168 & 39.4 & .177 & .291 & 50.3 & .187 & .377 & 66.8 & .257 & .773 & 70.4 & .301 & 1. 015 \\
\hline 31.0 & .134 & .195 & 44.6 & .174 & .314 & 55.9 & .182 & .413 & 72.9 & .215 & .793 & 75.8 & .246 & 1. 020 \\
\hline 35. 0 & .128 & .197 & 50.4 & .161 & .324 & 61.1 & .169 & .436 & -.... & ---- & $\ldots$ & 80.5 & .219 & 1. 125 \\
\hline 38.7 & .121 & .197 & 54.4 & .160 & .351 & 65.8 & .145 & .428 & & & $\ldots$ & -..... & -.... & $\ldots$ \\
\hline 42. 3 & .121 & .209 & 59.2 & .154 & .376 & 70.0 & .133 & .444 & & & & & & ...... \\
\hline 47.1 & .128 & 243 & & & & & & & & & & & & \\
\hline
\end{tabular}

extrapolating the straight lines in figure 15.6 to zero degradation, we obtain intercepts that represent the initial rates. Jellinek [2] and Atherton [3] used average rates from the seemingly straight parts of the curves in figures $15.3,15.4$ and 15.5 as a basis for calculating the activation energy of polystyrene. However, judging from figure 15.7, where values of $K_{1}$ are plotted against percentage loss of weight, the rates are very far from constant throughout the degradation range.

The experimental initial low rates obtained in this work, may be explained on the ground that some different reaction, or combination of reactions, prevails in the initial stages of thermal degradation. The rate curves begin at some short distance from the ordinate, due to the fact that some material volatilizes before the crucible attains 

peratures.

See table 15.3 for meaning of symbols

(In percent per minute)

\begin{tabular}{|c|c|c|c|c|c|}
\hline \multirow{2}{*}{$\begin{array}{l}\text { Tempera- } \\
\text { ture }\end{array}$} & \multicolumn{3}{|c|}{$K_{2}$ for $\mathrm{I}$} & \multirow{2}{*}{$\begin{array}{c}K_{2} \text { for } 1 \mathrm{II} \\
\text { initial }\end{array}$} & \multirow{2}{*}{$\begin{array}{c}K_{2} \text { for III } \\
\text { initial }\end{array}$} \\
\hline & Initial & $\begin{array}{l}\text { At } 25 \text {-per- } \\
\text { cent volatil- } \\
\text { ization }\end{array}$ & $\begin{array}{l}\text { At } 50 \text {-per- } \\
\text { cent volatil- } \\
\text { ization }\end{array}$ & & \\
\hline${ }^{\circ} \mathrm{C}$ & & & & & \\
\hline 33.5 & 0.069 & 0.175 & 0.282 & 0.060 & 0.065 \\
\hline 340 & .115 & .232 & .350 & .093 & .100 \\
\hline 345 & .155 & .342 & .528 & .128 & .138 \\
\hline 350 & .235 & .530 & .827 & .193 & .215 \\
\hline 355 & .358 & .755 & 1. 156 & .265 & .275 \\
\hline 360 & .496 & .991 & 1. 485 & --.- & ........ \\
\hline 365 & .680 & 1. 340 & 2. 016 & .......... & - \\
\hline
\end{tabular}

the operating temperature. Degradation of polystyrene is largely due to break-offs at the ends of the chains, as indicated by the fact that the volatile product contains a large amount of monomer. Degradation may be initially retarded by groups such as $\mathrm{H}_{2} \mathrm{O}, \mathrm{O}_{2}, \mathrm{CO}_{2}$, etc., clinging to the free-radical ends of the chains. The drop in the rates above 75-percent degradation may be explained on the ground that cross-linking develops in the residue during the heating.

The extrapolated initial rates or initial values of $K_{2}$ at various temperatures for all three polystyrenes are giren in table 15.6. For the sake of comparison the values at 25 - and 50 -percent loss of weight, in the case of polystyrene I, are also given. Using Arrhenius' equation

in the form

$$
K=A e^{-(E / R T)}
$$

$$
-E=2.3 R \frac{d(\lg K)}{d(1 / T)},
$$

the logarithms of $K_{2}$ are plotted against the inverse of absolute temperature to obtain the activation energy of the three polystyrenes. These plots are shown in figure 15.8. The slopes of the solid lines represent activation energies based on initial rates, $K_{2}$, for the three polystyrenes used. They all have the same activation energy of 58 kcal. The lower dotted line shows an activation energy of 56 , and the upper dotted line an activation energy of $54 \mathrm{kcal}$, corresponding

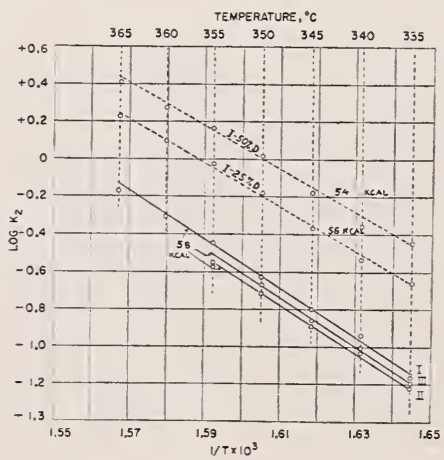

Figure 15.8. Activation energy of polystyrene based on extrapolated initial rates and on rates at 25- and 50-percent volatilization. 
to a 25-and 50-percent loss of weight, respectively, of polystyrene I. Thus, we see that the three polystyrenes, the mother substance (I) and its two fractions, one of molecular weight 106,000 (II) and the other of molecular weight 584,000 (III), have the same activation energy, although they have different initial rates of degradation. This is in agreement with the findings of Jellinek [2]. However, he found an activation energy of 44.7 kcal. Jellinek heated $20-\mathrm{mg}$ samples in a crucible of about the same size as was used in this work and most likely lost some material by spattering. Atherton [3] repeated Jellinek's work and found an activation energy of 50 kcal. Both Jellinek and Atherton assumed that the rates were the same throughout a considerable part of the degladation range and used the slopes of degradation curves, similar to those given in figures 15.3, 15.4 , and 15.5 of this work, to obtain average values of degradation rates. This method of calculating rates was shown above to be unreliable.

The degradation rates, $K_{2}$, and the activation energy based on these values should not be considered as referring to any one particular reaction taking place in the process of thermal degradation, but should be looked upon, as pointed out by Simha [12], Simha, Wall, and Blatz [13], and Simha and Wall [14], as composite values referring to several reaction mechanisms, such as initiation, propagation, etc.

The question of order of reaction involved in thermal degradation of polymers is of great interest because it may throw light on the mechanism of degradation. Actually, one cannot speak of any given order of reaction when several reactions are involved. However, one can speak here of a composite reaction that approximates a given order. Figure 15.9 shows a comparison between the experimental rate-curves, $K_{2}$ and $K_{1}$, for the unfractionated polystyrene at $350^{\circ} \mathrm{C}$, as given in figures 15.6 and 15.7, respectively, on the one hand, and theoretical curves of $K_{2}$ and $K_{1}$ based on the same initial value of $K_{2}$ and calculated on the assumption that the reaction is of zero order. The solid lines in figure 15.9 are the experimental curves, and the dotted lines are the theoretical ones. There seems to be some resemblance between the two pairs of curves, but all one can say is that the thermal degradation of polystyrene approaches a zero order. If polystyrene were to yield the monomer only by a simple mechanism of end breaks, the process could be expected to be of the zero order, since the number

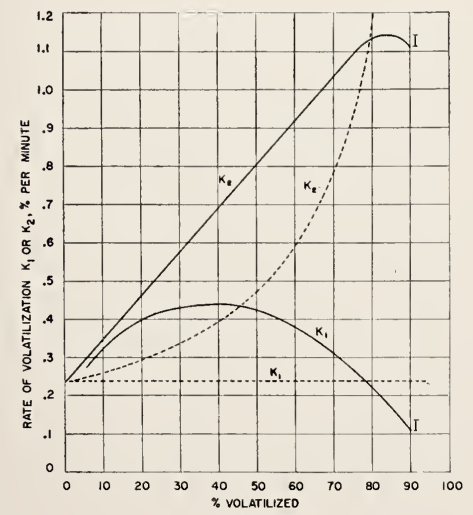

Figure 15.9. Comparison between experimental values of rates of degradation of polystyrene at $350^{\circ} \mathrm{C}$ and theoretical values calculated on the basis of a zero-order reaction. 
of ends in the sample would remain the same throughout the degradation. However, it is known that other fragments, such as dimers, trimers, and higher fragments $[5,15]$ are also formed. This seems to indicate that, in addition to end breaks, random breaks also take place to some extent. In view of this, it is not surprising that the reaction is of a type intermediate between a zero order and a first order.

\section{Thermal Degradation of Polyethylene}

The polyethylene used in these experiments was similar to that used previously in the pyrolysis work [6] and had an average molecular weight of 20,000. Like polystyrene, polyethylene has a tendency to spatter if too thick a layer is heated and the operating samples were, therefore, limited to about $6 \mathrm{mg}$. Some of the more pertinent experimental data at $385^{\circ}, 390^{\circ}, 395^{\circ}, 400^{\circ}$, and $405^{\circ} \mathrm{C}$, are given in table 15.2 .

Figure 15.10 shows cumulative percentage volatilization as a function of time. It is seen that degradation begins at an initial high rate and then slows down gradually as the reaction proceeds. Table 15.7 shows the mean values of $K_{1}$ and $K_{2}$ and the corresponding mean percentages of volatilization for successive time intervals. The values of $K_{2}$ and $K_{1}$ are plotted as functions of percentage volatilization in figures 15.11 and 15.12, respectively. At each temperature the rates start initially very high and drop with degradation in a straight line, $B$. Then a break occurs, and the rates follow again a straight line, $A$, but drop more slowly with degradation than the initial rates. The break itself occurs at 10 percent for the $385^{\circ} \mathrm{C}$ graph and moves toward higher percentage of volatilization with temperature until it is at about 40 percent for the $405^{\circ} \mathrm{C}$ graph. The reason for high initial rates and the breaks in the rate curves is not apparent from this work. A partial explanation may be found in the fact that the polyethylene used in this work is unfractionated and has a low average molecular

TABLE 15.7. Rates of thermal degradation of polyethylene, molecular weight 20,000, at various temperatures

See table 15.3 for meaning of symbols

(Values of $K_{1}$ and $K_{2}$ are in percent per minute)

\begin{tabular}{|c|c|c|c|c|c|c|c|c|c|c|c|c|c|c|}
\hline \multicolumn{3}{|c|}{$385^{\circ} \mathrm{C}$} & \multicolumn{3}{|c|}{$390^{\circ} \mathrm{C}$} & \multicolumn{3}{|c|}{$395^{\circ} \mathrm{C}$} & \multicolumn{3}{|c|}{$400^{\circ} \mathrm{C}$} & \multicolumn{3}{|c|}{$405^{\circ} \mathrm{C}$} \\
\hline$D$ & $K_{1}$ & $\boldsymbol{K}_{2}$ & $D$ & $K_{1}$ & $K_{2}$ & $D$ & $K_{1}$ & $K_{2}$ & $D$ & $K_{1}$ & $K_{2}$ & $D$ & $K_{1}$ & $K_{2}$ \\
\hline $\begin{array}{c}\% \\
0\end{array}$ & $\%$ & $\begin{array}{c}\% \\
0.160\end{array}$ & $\begin{array}{l}\% \\
0\end{array}$ & $\%$ & $\begin{array}{c}\% \\
0.225\end{array}$ & $\%$ & $\%$ & $\begin{array}{c}\% \\
0.340\end{array}$ & $\begin{array}{l}\% \\
0\end{array}$ & $\%$ & $\begin{array}{c}\% \\
0.480\end{array}$ & $\%$ & $\%$ & $\%^{\%}$ \\
\hline 4. 0 & 0.276 & 0.288 & 4.1 & 0.400 & .417 & 6.3 & 0.514 & $\begin{array}{r}.545 \\
.545\end{array}$ & 8.7 & 0.601 & $\begin{array}{r}.659 \\
\end{array}$ & 14.6 & 1.245 & 1.457 \\
\hline 7. 4 & .174 & .188 & 7.5 & .288 & .311 & 9.3 & .397 & .445 & 14.3 & .522 & .609 & 24.5 & 0.738 & 0.978 \\
\hline 9.9 & .157 & .174 & 11.3 & .228 & .257 & $14.4^{\circ}$ & .324 & .378 & 20.7 & .382 & .482 & 31.0 & .568 & .824 \\
\hline 12.1 & .134 & .153 & 15. 7 & .210 & .249 & 18.7 & .266 & .327 & 27.8 & .328 & .455 & 38.6 & .469 & .764 \\
\hline 14.0 & .132 & .154 & 19.4 & .163 & .202 & 23.8 & .240 & .315 & 33.9 & .276 & . 419 & 46.8 & .356 & .670 \\
\hline 16.0 & .128 & .153 & 22.6 & .160 & .206 & 30.0 & .189 & .271 & 41.2 & .230 & .382 & 53.6 & .320 & .690 \\
\hline 18. 6 & .113 & .139 & 25.6 & .139 & .187 & 37.0 & .163 & .259 & 50.0 & .211 & .422 & 59.5 & .269 & .665 \\
\hline 22.0 & .108 & .138 & 29.5 & .125 & .176 & 43.4 & .153 & .271 & 57.7 & .171 & .404 & 64.4 & .223 & .627 \\
\hline 25.1 & .099 & .133 & 34.6 & .130 & .198 & 49.0 & .128 & .251 & 63.8 & .137 & .378 & 68.5 & 186 & .592 \\
\hline 28.0 & .093 & .129 & 40.0 & .111 & .184 & 53.8 & .112 & .243 & 69.0 & .120 & .386 & 72.1 & .169 & .605 \\
\hline 30.6 & .082 & .119 & 44.4 & .085 & .152 & 58.0 & .103 & .246 & $\ldots-$ & - . - & $-\ldots$ & 75.5 & .172 & .700 \\
\hline 33.0 & .078 & .116 & 47.9 & .089 & .171 & 62.2 & .101 & .266 & & & & 78.5 & .127 & .593 \\
\hline 35.4 & .076 & .117 & 51.2 & .081 & .166 & 65.8 & .081 & .237 & & -. & $\ldots$ & 80.9 & .118 & .616 \\
\hline 37.6 & .070 & .112 & 54.4 & .077 & .169 & 68.9 & .071 & .228 & & & & 83.8 & .087 & .531 \\
\hline 39.6 & .067 & .111 & 57.4 & .069 & .162 & - & $\ldots$ & $\ldots$. & & $\ldots$ & & -.... & -...- & $-\infty-\cdots$ \\
\hline 41. 6 & .065 & .111 &.--- & $\ldots .$. & $\ldots \ldots$ & & & & $\ldots$ & $\ldots$ & & -.... & -....- & $-\ldots-n$ \\
\hline 43.5 & .060 & .106 & & & & $-\ldots$ & & & 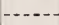 & 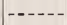 & $\ldots$ & -..... & & $\ldots \ldots$ \\
\hline 45. 2 & .058 & .106 & & $\ldots$ & & - & - & - & & $-\ldots$ & & - . & & $--\ldots$ \\
\hline 47.0 & .058 & .109 & & $-\ldots$ & & -.... & & & $\ldots$ & $-\cdots$ & & $-\cdots$ & -.... & \\
\hline 49.0 & .054 & .104 & & & & & & & & & & & & \\
\hline
\end{tabular}




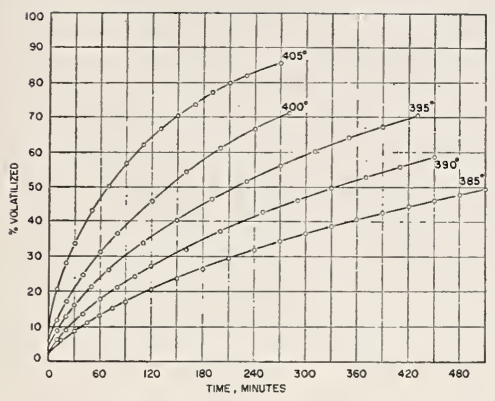

Figure 15.10. Cumulative degradation of polyethylene, molecular weight 20,000 , as a function of time.

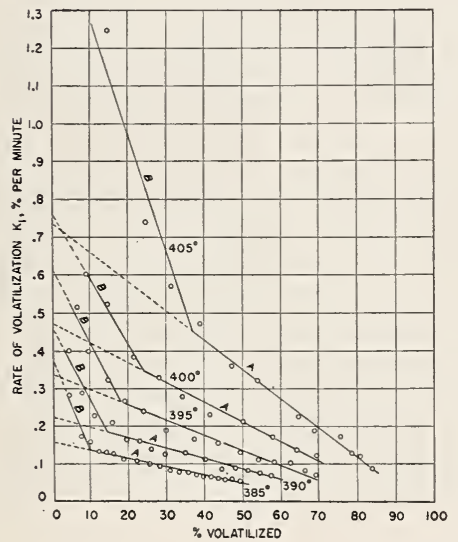

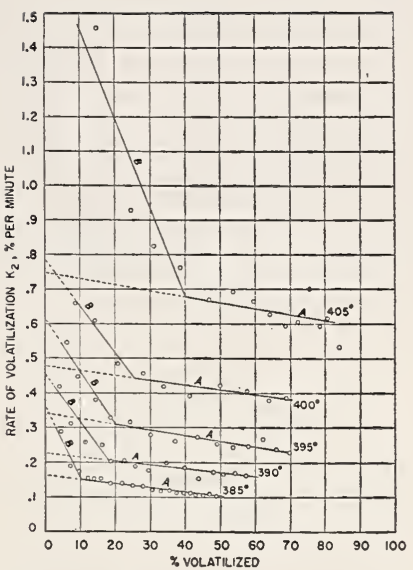

Figure 15.11. Rates of thermal degradation of polyethylene in percentage of corresponding residues, as a function of volatilization.
Figure 15.12. Rates of thermal degradation of polyethylene in percentage of original weight of sample, as a function of volatilization.

weight. At high temperatures the shorter chains escape in the initial stage of degradation either unbroken or after a few random breaks.

By extrapolating sections $A$ of the graphs in figures 15.11 and 15.12 , that is, those to the right of the breaks, we obtain intercepts that correspond theoretically to initial rates for these sections. The values of these initial rates, $K_{1}$ or $K_{2}$, also, those corresponding to 25- and 50 -percent volatilization, as given in figure 15.11 for $A$ sections of $K_{2}$ curves are given in table 15.8. By a similar extrapolation of sections $B$ in figures 15.11 and 15.12 , corresponding intercepts are obtained. These intercepts are also shown in table 15.8. In figure 15.13, logarithms of the rates shown in table 15.8 are plotted against $1 / \mathrm{T}$. The activation energies, $E$, as obtained from the slopes of the solid lines, are 68 and $48 \mathrm{kcal}$ for the $A$ and $B$ sections, respectively, of the $K_{1}$ or $K_{2}$ curves in figures 15.11 and 15.12. For comparison, the slopes of the dotted lines represent values of $E$ at 25 - and 50 -percent volatilization on the $A$ sections of the $K_{2}$ curves (fig. 15.11). These values are 71 and $80 \mathrm{kcal}$, respectively. 
TABLE 15.8. Rates of thermal degradation of polyethylene, molecular weight 20,000, at various temperatures

Sec table 15.3 for meaning of symbols

(In percent per minute)

\begin{tabular}{|c|c|c|c|c|}
\hline \multirow{2}{*}{ Temperature } & \multicolumn{3}{|c|}{ For $A$ sections of eurves: } & \multirow{2}{*}{$\begin{array}{c}\text { For } B \text { sections } \\
\text { of curves } K_{1} \text { or } \\
K_{2} \text {, intercepts }\end{array}$} \\
\hline & $\begin{array}{c}K_{1} \text { or } K_{2} \\
\text { intercepts }\end{array}$ & $\begin{array}{c}K_{2}, 25^{c_{0}} \\
\text { volatilization }\end{array}$ & $\begin{array}{c}\boldsymbol{K}_{2}, 50^{c} \\
\text { volatilization }\end{array}$ & \\
\hline${ }^{\circ} \mathrm{C}$ & & & & \\
\hline 385 & 0.160 & 0.130 & 0.103 & 0.360 \\
\hline 390 & .225 & .198 & .170 & .450 \\
\hline 395 & .340 & .300 & .260 & .610 \\
\hline 400 & .480 & .443 & .408 & .780 \\
\hline 405 & .745 & (1) & .665 & $(2)$ \\
\hline
\end{tabular}

${ }^{1} \mathrm{~A}$ section of $405^{\circ} \mathrm{C}$ curve begins at about 40-percent volatilization.

2 There is too much scattering on $B$ sections of curves $K_{1}$ and $K_{2}$ at $405^{\circ} \mathrm{C}$, due to rapid rate of volatilization.

Jellinek [2] found that the activation energy of polyethylene varies from 46 to $66.1 \mathrm{kcal}$, as the initial molecular weight of the polymer varies from 11,000 to 23,000. On the other hand, Oakes and Richards [4] report that, working with polyethylene of molecular weights from 9,000 to 23,000 , they found no significant variation of the activation energy with molecular weight; also, that the activation energy is around 60 to $70 \mathrm{kcal}$.

An indication of the order of reaction involved in the thermal degradation of polyethylene can be obtained from figure 15.14. Here the solid lines are the $K_{1}$ and $K_{2}$ curves for $400^{\circ} \mathrm{C}$, with 0.480 percent per min as the extrapolated initial rate (see figs. 15.11 and 15.12) and are plotted against percentage of volatilization. The dotted lines represent theoretical values of $K_{1}$ and $K_{2}$, with 0.480 as the initial rate and plotted on the assumption that the reaction follows the first order. There seems to be a resemblance between the $A$ sections of the experimental curves, on the one hand, and the theoretical curves, on the other. The deviation of the experimental

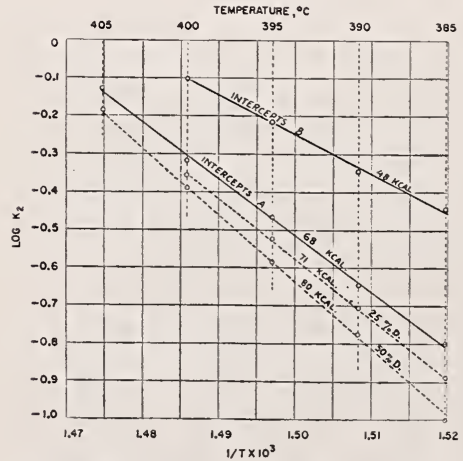

Figure 15.13. Activation energy of polyethylene based on extrapolated initial rates and on rates at 25- and 50-percent volatilization.

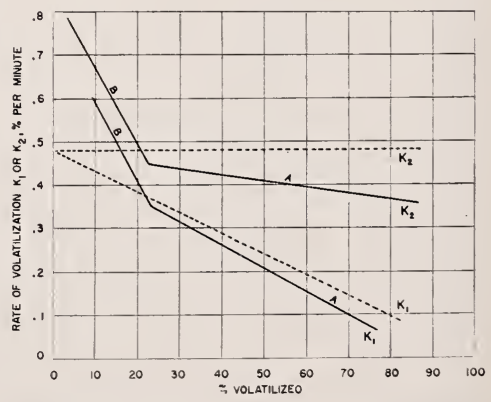

Figure 15.14. Comparison between experimental rates of volatilization or polyethylene at $400^{\circ} \mathrm{C}$ and theoretical values calculated on the basis of a first-order reaction. 
curves from a first-order reaction may be due to the fact that while random breaking is the principal mechanism of the process, other mechanisms, such as preferred breaking off at the ends of monomer or monomer-like fragments, either followed by propagation or not, may take place concurrently to some extent.

The constant $\mathrm{A}$ in the equation $K=A e^{-(E / R T)}$ can be calculated by substituting the ralue for $E$ in this equation. This constant is found to be, in the case of polystyrene, $6 \times 10^{17}$ and in the case of polvethylene $8 \times 10^{19}$ and $4 \times 10^{13}$, corresponding to the two values of $E, 68$ and $48 \mathrm{kcal}$, respectively. Talues of $A$ are per mole per second and are based on the assumption that the initial degradation is a first-order reaction.

\section{Summary}

Summarizing the results of this investigation, the average molecular weight of the polystyrene residues decreases with increasing extent of thermal degradation, at first rapidly to about 10-percent rolatilization and then more slowly. Rates of degradation of unfractionated polystyrene are higher than those of two fractions obtained from it. As to the fractions themselves, for some as yet unexplained reason, the lower molecular weight fraction has lower rates of degradation than the higher one. Howerer, the mother substance and its fractions exhibit the same activation energy of 58 kcal. The over-all reaction involved appears to be intermediate between a zero order and a first order, but is closer to the zero order. The inference is drawn that polystyrene degrades at temperatures between $300^{\circ}$ to $400^{\circ} \mathrm{C}$, preferably at the ends, but that some random degradation takes place simultaneously.

In the thermal degradation of polyethylene the activation energy was found to be $48 \mathrm{kcal}$ for the beginning of degradation and $68 \mathrm{kcal}$ for the subsequent degradation. The over-all process approximates a first-order reaction. It is concluded that the mechanism involved here is mainly that of a random breaking of the molecular chains.

\section{References}

[1] N. Grassie and H. W. Melville, Proc. Royal Soc. [A] 199, 1 (1949).

[2] H. H. G. Jellinek, J. Polymer Sci. 4, 13 (1949).

[3] A. Atherton, J. Polymer Sci. 5, 378 (1950).

[4] W. G. Oakes and R. B. Richards, J. Chem. Soc. 2929 (1949).

[5] S. L. Madorsky and S. Straus, J. Research NBS 40, 417 (1948) RP1886; Ind. Eng. Chem. 40, 848 (1948).

[6] S. L. Madorsky, S. Straus, D. Thompson, and L. Williamson, J. Research NBS 42, 499 (1949) R P1989; J. Polymer Sci. 4, 639 (1949).

[7] S. Straus and S. L. Madorsky, J. Research NBS 50, 165 (1953) RP2405.

[8] S. L. Madorsky, Science 111, $360(1950)$.

[9] S. L. Madorsky, Rev. Sci. Instr. 21, 393 (1950).

[10] IV. G. Brombacher, Rev. Sci. Instr. 4, 688 (1933).

[11] F. A. Ransom, unpublished work.

[12] R. Simha, Mechanism and kinetics of thermal and photodegradation reactions, see paper 2 of this Symposium.

[13] R. Simha, L. A. Wall, and P. J. Blatz, J. Polrmer Sci. 5, 615 (1950).

[14] R. Simha and L. A. Wall, J. Phys. Chem. 56, 707 (1952).

[15] H. Staudinger and A. Steinhofer, Ann. Physik 517, 35 (1935). 


\section{Discussion}

Dr. Leo Wall, National Bureau of Standards: We now have molecular-weight variation of the residue, over-all-rate data, and product analysis for the thermal depolymerization of both polystyrene (Dr. Madorsky's work) and polymethyl methacrylate (Dr. Melville's work). In addition, in the case of polymethyl methacrylate we have initial rate versus initial molecular-weight data for benzoyl peroxide catalyzed polymers. Referring to the theoretical treatment in Dr. Simha's paper, we can tabulate our conclusions as to the mechanism of decomposition for these two important polymers:

\begin{tabular}{|c|c|c|}
\hline Elementary reaction & Polystyrene & Polymethyl methacrylate \\
\hline Initiation.... & Probably random & Proportional to number of mole- \\
\hline Propagation. & Approximately five units per pure & Approximately $2.5 \times 10^{3}$ to $8.3 \times 10^{3}$ \\
\hline Transfer... & $\begin{array}{l}\text { 1nitiation or initiation by transfer. } \\
\text { Probably about } 10^{2} \text { times each pure }\end{array}$ & $\begin{array}{l}\text { units per initiation act. } \\
\text { Very small. }\end{array}$ \\
\hline Termination. & $\begin{array}{l}\text { Mnituation act. } \\
\text { Mainly disproportionation in the } \\
\text { case of large radical chains. }\end{array}$ & $\begin{array}{l}\text { Mainly disproportionation in the } \\
\text { case of large radical chains. }\end{array}$ \\
\hline
\end{tabular}

a Since the kinetic chain length is large and the initial rate is insensitive to initial molecular weight. However, the initiation could also occur at a point of termination formed during polymerization, since such points would also be proportional to the number of molecules. Eridence for the above is only adequate for benzoyl peroxide and photopolymers of methyl methacrylate. Differently prepared polymers, such as with azonitrile catalysts, may decompose by random initiation.

Thus, the polymethyl methacrylate case where the polymer unravels into monomer is contrasted with polystyrene where a transfer process cuts polymer chains and only about five monomer units unravel before the radical terminates either by transfer or pure termination. Transfer also occurs to a great extent in polyethylene. Transfer gives a random component to the decomposition mechanism which is compatible with the molecular weight variations in the residues and the type of products obtained. The over-all rates may be sensitive to impurities, and hence conclusions based on rates may in some cases be difficult to interpret.

Dr. H. H. G. Jellinek, University of Adelaide, Australia: I want to say a few words about the discrepancy in the energy of activation between Dr. Madorsky's and my work. Dr. Madorsky found for the energy of activation for polystyrene about $60 \mathrm{kcal}$, and I found $45 \mathrm{kcal}$. I think that is due to the manner in which you evaluate your experimental results. If you plot the loss of weight in percent against time, you get your curve, which is initially a straight line (compare fig. 1.2 , curve 1 , of $\mathrm{my}$ paper). I have taken the initial part of the curve, and Dr. Madorsky has taken, I believe, a latter part of the curve. If you assume the mechanism that monomer is coming off the chain ends by initiation, propagation, and termination, you can only expect a zero-order reaction for the initial part of the degradation. It follows from theory that you get only initially a straight line, which tails off later. That explains the fact that the rate of degradation falls off with the extent of the degradation.

The equation obtained for this type of degradation, where the kinetic chain length, $\gamma$, is smaller than the original chain, is as follows:

$$
\frac{m_{1}}{m_{0}}=1-\frac{1}{P_{n}} e^{-k_{4} t} \sum_{a=1=0}^{a-1} \frac{\left(k_{4} t\right)^{a-1}}{(a-1) !}\left[P_{n}-(a-1) \gamma\right]
$$


(see eq 2 of my paper). You get initially a straight line plotting $m_{1}$ against $t$; this straight line will be the longer the smaller $\gamma$. So you see that you cannot expect a constant rate throughout the degradation, and the energy of activation will be different whether derived from the straight-line part of the curve or whether it is derived during the latter stages of the degradation.

In the case of polyethylene, one can distinguish the following three cases of degradation: (1) Reverse polymerization, where $\gamma$ is smaller than the original chain length; (2) reverse polymerization, where $\gamma \geq P_{n}$; that means that once a chain is activated, it degrades completely; and (3) the ordinary random degradation. See figure 1.2 of my paper. Of course, you will never get the curve for monomer formation according to the random case as given in the graph in an open reaction system. Because if you degrade to such an extent, for instance, that 40 percent in weight is lost, then the residual polymer in your reaction vessel should have, according to the random theory, such a small chain length that it would evaporate in a matter of seconds. You will never get that monomer formation curve according to the random theory, but you will get a curve that very rapidly increases in the rate of losing weight. As a matter of fact, however, in the case of polyethylene, the reaction goes on in a fairly straight line fashion for at least another hour; this means, of course, that polyethylene does not degrade by a random process.

Dr. WALL: In the thermal decomposition of polyethylene, the largest fragment that can distill over has a molecular weight of 1,000 or a DP of 36 ethylene units. These fragments are about $1 / 20$ the size of the polyethylene used in the pyrolysis experiments. The relatively large size of these fragments alters the picture considerably. This type of process has been treated by Simha and Wall. ${ }^{4}$ The results are that for an initial molecular weight of 20,000 the residue will have a molecular weight of 3,400 when the degradation takes place at random. The residual molecular weight may actually be higher, since the calculation does not consider the effect of initial molecular-weight distribution. Thus, the fact that at 40-percent conversion the remaining material has a considerably higher molecular weight does not preclude the operation of a random process in the decomposition.

With reference to Dr. Madorsky's rate data for polyethylene, I want it to be clear that the data do not indicate a simple unique random process for decomposition. However, Dr. Richards ${ }^{5}$ has shown that there are at least two types of transfer reactions possible, a fast reaction proceeding via the tertiary hydrogen atoms present as a result of the branching in the polymer molecule and a slow reaction operating through the secondary hydrogen atoms. Thus, there are at least two superimposed random reactions, and in the cases studied the maximum in the rate versus conversion curve that is characteristic of a random process would be in the region of 5-percent conversion. Since no points were taken below this conversion due to experimental difficulties, the results cannot be interpreted unambiguously. A more decisive experiment would be to study the rate of decomposition of a linear polyethylene such as can be prepared from the decomposition of diazomethane.

4 J. Polymer Sci. 6, 39-44 (1951).

5 Oakes and Richards, J. Chem. Soc. 2929-2939 (1949). 
Dr. R. B. Richards, Imperial Chemical Industries Itd.: We were able to degrade polyethylene of 20,000 molecular weight down to 2,000 without losing any volatile material in a process for making a wax, and we were, of course, interested in not losing any material. Above $360^{\circ} \mathrm{C}$ you get volatile material off very rapidly, but below that temperature, the loss is very slight indeed. We could detect no ethylene among the volatiles at all.

DR. WALL: The observations of Dr. Richards clearly indicate random type of processes to be operating, which may of course be initiated by impurities. It is highly probable that the introduction of some catalyst will facilitate these changes at lower temperatures by initiating transfer reactions.

Dr. R. B. Mesrobiax, Brooklyn Polytechnic Institute: You can have weak linkages spaced at random throughout the polymer chain. They need not be just at the end, where the catalyst fragment is associated. This could be the case in polymethyl methacrylate, for example, where you get a weak inkage at head-to-head structures.

The question of whether or not the initial scission of polyethylene occurs at the end of the polymer chain or at random along the chain could be resolved in the following manner. If an inhibitor such as 1,4-diaminoanthraquinone (successfully employed by Melville and Grassie for polymethyl methacrylate) were incorporated into the polymer, and the change in molecular weight of the polymer under degradative conditions followed with time, two possibilities might be expected. In the case of initiation by preferred fission at chain ends there will be no measurable decrease in molecular weight with time. Alternatively, for random scission there will be a progressive decrease in molecular weight with time. It should be kept in mind that the role of the inhibitor is not to alter the rate of the initiation step but to prevent the zipper reaction from proceeding at the site of the initial fission. 


\title{
16. Pyrolysis of Copolymers
}

\author{
By Leo A. Wall ${ }^{1}$
}

The yields of the individual monomers obtained on pyrolysis of a series of copolymers have been determined by means of the mass spectrometer and the variation in vields with composition of copolymer studied. An understanding of the results is enhanced by the employment of copolymerization theory to derive mathematical expressions relating yields and composition. These equations are based on the assumption that at the boundaries between sequences of the two monomers forming the copolymer most of the processes occur that determine the yield of the various products. A parameter $\theta$ is introduced, which measures the probablility of losing original monomer units on decomposition, and in speciar cases is defined as twice the fraction of internal monomer bonds broken. A random decomposition leads to $\theta=1$. The values of $\theta$ obtained vary considerably and depend characteristically on the structure of the polymers.

\section{Introduction}

Although the study of pyrolysis products from polymers has contributed to our basic knowledge of their structure $[1,2]^{2}$ and considerable work $[3,4,5,6]$, both experimental and theoretical, on simple polymers has recently appeared, relatively little has been done on copolymers. It has been reported, however, that the fraction of the styrene recovered on pyrolysis of butadiene-styrene polymers is considerably lower than that from polystrrene $[\overline{7}, 8]$. Morthland [9] has shown recently that on the pyrolysis of $\beta$ - $\mathrm{C}^{1+1}$ styrene- $m$ - or $p$ methylstyrene copolymers, active methylstyrene is recovered. Also, Bachman, et al. [10] in a study on styrene-methyl methacrylate and on styrene-maleic anhydride copolymers, found that the relative yield with reference to pure polystyrene was not affected in the former case, whereas in the latter it was only one sixth. These results are presumably connected with the arrangement of monomeric units within the copolymer which can be calculated from copolymerization theory [11, 12, and 13]. It is possible to distinguish two ways in which the amount of original monomer recovered may be decreased; either preferential codimer or cotrimer formation at the boundaries between sequences of $A$ and $B$ units or by the formation of "hrbrid monomers" made up of fragments of both $A$ and $B$ units. If codimers are less favored, an increased original monomer yield can also occur.

The chief quantity studied in this work is the relative monomer yield, which is the yield of a given monomer from the copolymer divided by the yield from the simple polymer of the given monomer. Theoretical expressions are given for this quantity as a function of copolymer compositions and are used to evaluate the experimental data. 


\section{Theoretical}

In a simple polymer such as polystyrene, a sequence of $i$ units has $2 i$ ways of producing a monomer unit by a random splitting of bonds in the chain. In a copolymer let $i$ be the number of $A$ units in a sequence of $A$ units.

Since the arrangement in a copolymer facilitates the production of other species, we will define $\theta_{a}$ as the probability of losing $A$ units. It can be seen by inspection that in a sequence of $A$ monomers $\left(\mathrm{CH}_{2}=\mathrm{CHX}\right)$ bounded by $B$ monomers $(\mathrm{CHY}=\mathrm{CHY})$, splitting of the internal bonds of the original monomers $A$ and $B$ will lead to the production of hybrid monomers, $\mathrm{CH}_{2}=\mathrm{CHY}$ and $\mathrm{CHX}=\mathrm{CHY}$.

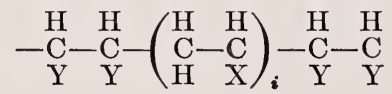

With $i=1$, random splitting, that is $\theta=1$, will produce equal quantities of all four species. In a situation where four such species are the principal products it is clear that

$$
\theta=2 \cdot\left(\frac{\text { Internal monomer bonds broken }}{\text { Internal monomer bonds in polymer }}\right) .
$$

The maximum possible value for $\theta$ in this case is 2 . The substitution of isotopes, $\mathrm{C}^{13}, \mathrm{C}^{14}$, or $\mathrm{H}^{2}$ in the beta position of monomers like styrene, would allow the preparation of copolymers capable of being decomposed into four distinguishable units, the study of which will give an insight into the mechanism of break-down. Where hybridmonomer production is the more important process, $\theta$ should have the same value when determined from any of the four species. However, if codimer or cotrimer production is important, different $\theta$ values may occur. In such cases the value of $\theta$ can exceed 2 .

From copolymerization theory there is obtained for small increments of conversion the relationship

$$
\frac{d B}{d A+d B}=\frac{z+r_{b}}{r_{a} z^{2}+2 z+r_{b}}
$$

which gives the composition, $d B /(d A+d B)$, of the copolymer formed from a mixture of monomers having the ratio $z=A / B$. The constants $r_{a}$ and $r_{b}$ are the reactivity ratios $[11,12,13]$. The probability $P_{i}(A)$ of forming in a given copolymer sequences of $i A$ units bounded by $B$ units can be calculated from the relationship

$$
P_{i}(A)=\left(\frac{z}{z+r_{b}}\right)\left(\frac{r_{a} z}{r_{a} z+1}\right)^{i-1}\left(\frac{1}{r_{a} z+1}\right)
$$

and is normalized over the total number of $A$ sequences. The product $[d B /(d A+d B)] P_{i}(A)$ is the fraction of $A$ sequences, based on the 
a verage concentration of $A$. The probability of obtaining the monomer $A$ from the copolymer is then equal to the sum of the products of the fraction of $A$ sequences times the probability of recovering $A$ units $\left[i\left(2 i-\theta_{a}\right) / 2 i\right]$ from such a sequence. The yield of monomer $A$ is then given by ${ }^{3}$

$$
Y(A)=Y_{o}(A) \frac{d B}{d A+d B} \sum_{i=1}^{i=\infty}\left[\frac{i\left(2 i-\theta_{a}\right)}{2 i}\right] P_{i}(A)
$$

when $Y_{o}(A)$ is the yield from the simple polymer of $A$. The relative yield, $Y(X) / Y_{o}(X)$, will in most cases be plotted, where $X$ is the $A$ or $B$ component. The final form of the equation is

$$
\frac{Y(A)}{Y_{o}(A)}=\frac{d A}{d A+d B}\left[1-\frac{\theta_{a}}{2} \cdot \frac{1}{\left(r_{a} z+1\right)}\right]=1-\frac{\left(1+0.5 \theta_{a}\right) z+r_{b}}{r_{a} z^{2}+2 z+r_{b}} .
$$

For the $B$ component there is obtained

$$
\frac{Y(B)}{Y_{o}(B)}=\frac{d B}{d A+d B}\left[1-\frac{\theta_{b}}{2} \cdot \frac{z}{z+r_{b}}\right]=1-\frac{\left(1+0.5 \theta_{b}\right) z+r_{a} z^{2}}{r_{a} z^{2}+2 z+r_{b}} .
$$

In the case of vinyl copolymers prepared from monomers of the type $\mathrm{CH}_{2}=\mathrm{CHX}$ and $\mathrm{CHY}=\mathrm{CHY}$, the equations for the yields, $Y^{\prime}(A)$ and $Y^{\prime}(B)$ of the two possible hybrid monomers, would be written

$$
\begin{aligned}
& \frac{Y^{\prime}(A)}{Y_{o}^{\prime}(A)}=\frac{d A}{d A+d B}\left(\frac{0.5 \theta_{a}^{\prime}}{r_{a} z+1}\right) \\
& \frac{Y^{\prime}(B)}{Y_{o}^{\prime}(B)}=\frac{d B}{d A+d B}\left(\frac{0.5 \theta_{b}^{\prime} z}{z+r_{b}}\right) .
\end{aligned}
$$

It can be shown that the right-hand quantities are identical when $\theta_{a}^{\prime}=\theta_{b}^{\prime}$. The eq (6) and (7) have a maximum at the point $z=$ $\left(r_{b} / r_{a}\right)^{\frac{1}{2}}$, which is also the monomer ratio that yields polymers of the composition $d A /(d A+d B)=0.5$. Hence, the value of the maximum (when $\theta_{a}^{\prime}=\theta_{b}^{\prime}=1$ ) is $0.25\left[1+\left(r_{a} r_{b}\right)^{\frac{1}{2}}\right]$ and is greatest for $r_{a} r_{b}=0$. If only monomeric products are obtained, then for the case of random splitting all the $\theta$ 's are unity, and where there is no effect due to the sequence boundaries the $\theta$ 's are zero. If splitting is nonrandom, but only monomeric materials are produced, a single value, differing from unity, would be expected for all the $\theta$ 's. When appreciable dimer is formed or when the dimer-monomer ratio on pyrolysis of the copolymer is different from that obtained from the simple polymers, the $\theta$ 's for products may differ. Equations (6) and (7) also apply to products that are produced by reactions other than pyrolysis, occurring at the boundaries between $A$ and $B$ sequences and may be useful in the study of low-temperature degradation and other reactions.

The chief experimental difficulty in exploring the above relationships lies in the fact that relatively few polymers on pyrolysis yield large amounts of monomers. In the experimental work on the dienecontaining copolymers, the variation in the yield of monomer $A$

${ }^{3}$ A previous deriviation, [7], omitted the first $i$ factor and led to relationships that behave similarly but are incorrect. 


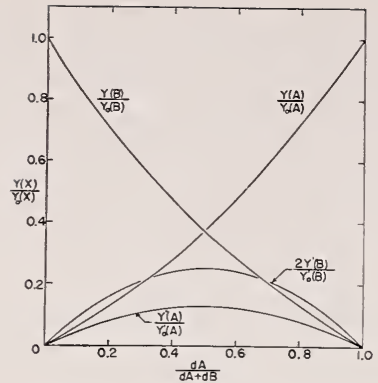

Figure 16.1. Plots of $Y(X) / Y_{o}(X)$ versus $d A$ $(d A+d B)$ according to equations (2), (3), (4), and (5), $\theta_{a}=\theta_{b}=\theta_{a}^{\prime}=\theta_{b}^{\prime}=$ $1, r_{a}=r_{b}=1$.

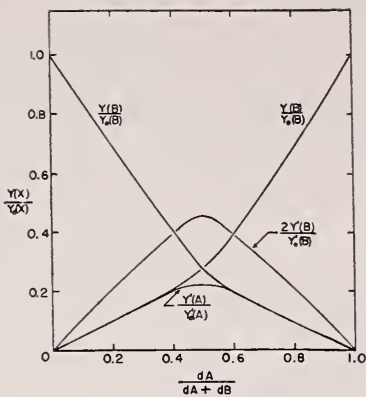

Figure 16.2. Plots of $Y(X) / Y_{o}(X)$ versus $d A$ ! $(d A+d B)$ according to equations (2), (3), (4), and $(5), \theta_{a}=\theta_{b}=\theta_{a}^{\prime}=\theta_{b}^{\prime}=$ $1, r_{a}=r_{b}=0.1$.

(styrene or methyl methacrylate) alone was studied. The $B$ monomer was not produced in yields above a few percent.

Figures 16.1 and 16.2 show plots of relative vields against polymer composition according to eq (4), (5), (6), and (7), all $\theta$ 's set equal to 1 .

In figures 16.1, $r_{a}=r_{b}=1$, that is, the rates of addition of $A$ type monomer or of $B$ type monomer to the growing radicals in polymerization were identical. Hence a broad distribution of sequences is found in the polymer. With $r_{a}=r_{b}=0.1$, figure 16.2, the growing radicals add to form $A B$ or $B A$ links 10 times more rapidly than $A A$ or $B B$ links. This produces a greater effect because the number of $A B$ or $B A$ links determines the difference between the depolymerization results from the simple polymers and the copolymers.

As $r_{a} r_{b}$ becomes very large (no alternation of monomers), the $Y^{\prime}$ curves will disappear and the $Y$ curves will become two diagonal lines. With a high degree of alternation, $r_{a} r_{b}=0$, the $Y^{\prime}$ curves reach a maximum and the $Y$ curves have a maximum curvature.

\section{Experimental Procedure}

The pyrolyses of the polymers were carried out in sealed tubes, each haring a side arm and 3 to $4 \mathrm{in}$. of $3-\mathrm{mm}$-tubing attached [7]. The volume of the tubes was approximately $50 \mathrm{ml}$. Benzene solutions of $1 \mathrm{mg} / \mathrm{ml}$ concentration were prepared from each polymer sample. One or in some cases $2 \mathrm{ml}$ of solution was placed in the side arm of a given tube and the benzene eraporated. After this, the tubes were sealed to a racuum apparatus and evacuated for 6 to $8 \mathrm{hr}$. After a vacuum of better than $10^{-4} \mathrm{~mm} \mathrm{Hg}$ was obtained the tubes were sealed off. The body of each tube was placed in liquid nitrogen, and the side arm was slipped into a small furnace maintained at $425^{\circ} \mathrm{C}$. After 25 to $30 \mathrm{~min}$ the tube was removed and another treated. Ten minutes of treatment was found adequate for complete pyrolysis. Following prrolysis the tubes were connected to the mass spectrometer via the attached $3-\mathrm{mm}$ tubing and the products expanded completely 
TABLE 16.1. Copolymers used in depolymerization studies

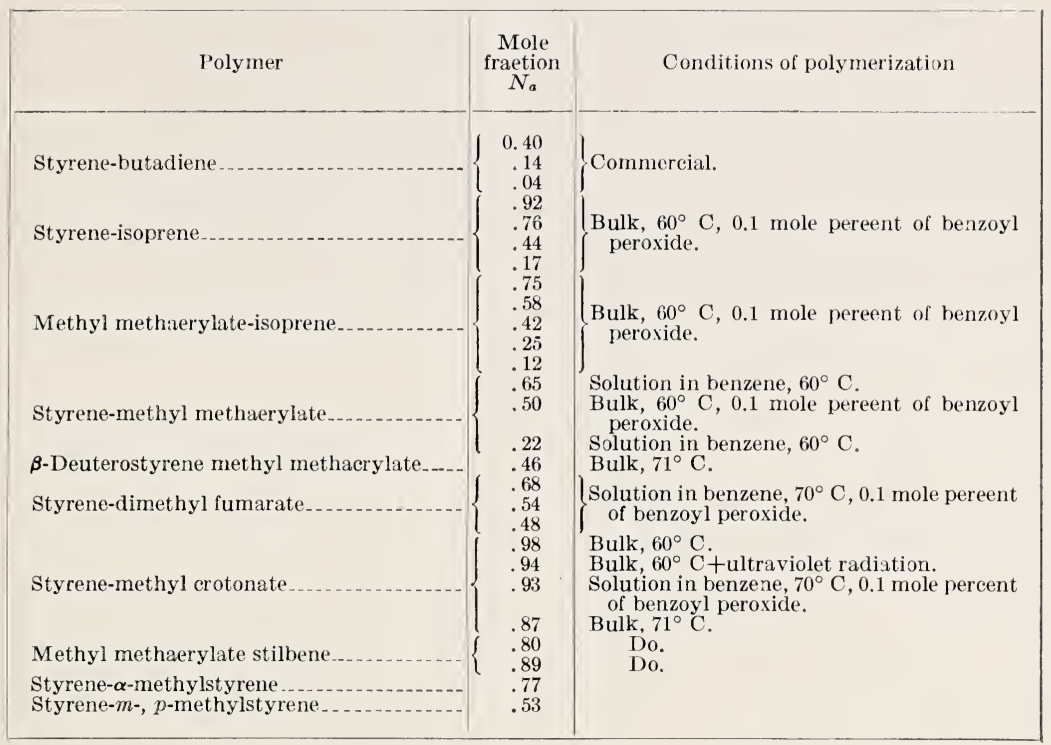

in to the inlet system (volume $=3.75$ liters) by breaking the tip of the 3 -mm tubing. Because all tubes were approximately the same volume, the heights of the mass spectral peaks are proportional to the yield of products. Usually, only the monomer peaks were recorded and corrected from yields based on weight of polymer to yields based on a given number of moles of monomer units contained in the polymer. As a rule three determinations were carried out on each sample of polymer and two polystyrenes were included in each group of runs as a check on technique and sensitivity of the mass spectrometer.

Table 16.1 contains composition data and conditions of polymerization of the copolymers studied. Purified styrene-butadiene copolymers, polybutadiene and polyisoprene polymers were obtained from Max Tryon of the National Bureau of Standards Rubber Section. The styrene content of the copolymers was determined by both the refractive-index method and by carbon-hydrogen analysis.

Polymers of $\alpha$-methylstyrene and $m, p$-methylstyrene, and also their copolymers with styrene were obtained from Dow Chemical Co. The composition was estimated from the polymerization charge, assuming complete conversion, and is probably somewhat inaccurate in the case of the $\alpha$-methylstyrene copolymer.

The composition of the styrene-isoprene and the methyl methacrylate-isoprene copolymers was calculated from the known reactivity ratios $[15,16]$ and the charge ratio used for the polymerization.

The composition of all other copolymers was determined by carbonhydrogen analysis. 


\section{Results}

\section{Diene Copolymers}

In order to ascertain the effect of catalysts or inhibitors on the depolymerization products, the .4 mole percent of styrene copolymer of styrene-butadiene was mixed in separate solutions with 2-percent amounts, based on the polymer weight, of phenyl beta-naphthylamine, cumate, potassium acetate, diphenylmercury, and benzoyl peroxide. Pyrolyses of each mixture were made in triplicate, and the yield of styrene was unchanged by the presence of the additives under the thermal decomposition conditions used.

It was also of interest to pyrolyze mixtures of polystyrene and polybutadiene to determine whether the low yields from the copolymers were the result of other factors than the structure of the copolymer. These runs also constitute a test of the analytical method. The yialds of styrene from the mixtures and from the styrene-butadiene copolymers are plotted against the composition of the polymer in figure 16.3. It is seen that the yields from the mixtures exhibit a linear dependence on composition. This dependence demonstrates the applicability of the technique as well as the expected behavior in the absence of any copolymer effect, that is, $\theta=0$.

In the experiments, most errors in technique, such as incomplete pyrolysis or incomplete expansion into the mass spectrometer, favor low results; hence, the average of the higher values is reported in this work after corrections are made for variation in the sensitivity of the mass spectrometer.

Using the values for the reactivity ratios for the styrene-butadiene system $r_{a}=0.78, r_{b}=1.40$ [14], the theoretical curves for $\theta=1, \theta=2$, $\theta=2.5$ were calculated, knowing the composition of the copolymers.

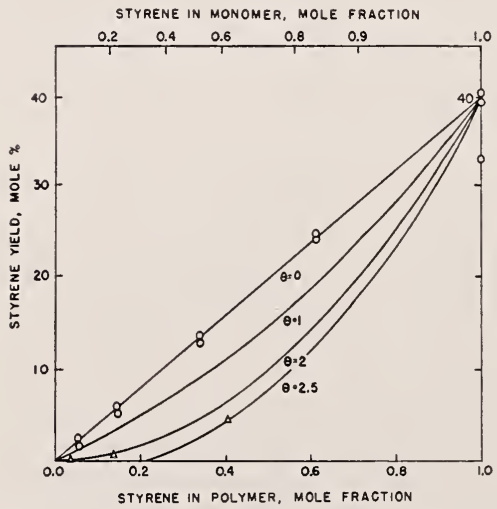

Figure 16.3. Yields of styrene from polystyrene-polybutadiene mixtures and styrene-butadienecopolymers,versus mole fraction styrene in polymer.

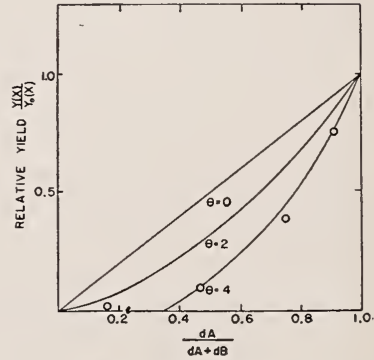

FIgure 16.4. Relative yields of styrene from styreneisoprene copolymers versus mole fraction styrene in copolymer.

O, experimental points, copolymer.

O, Experimental points, mixtures; $\triangle$. experimental points, copoly. mers. 
The values of $z$ necessary for the application of the eq (4), (5), (6), (7) were calculated, using eq (1). The experimental yields, figure 16.3 , are seen to fall on the lines for $\theta=2, \theta=2.5$. The copolymers, then, produce a much lower amount of monomer than would have been anticipated on the basis of their composition.

Figure 16.4 demonstrates a similar result for styrene-isoprene copolymers. Here the yields at higher compositions fall on or near the theoretical curve for $\theta=4$. The low composition point tends to $\theta=2$. It should be mentioned that for $\theta>2$ the equations give negative values in the low-composition region, since a value of $\theta=2$ means that each $A$ type sequence gives rise to the loss of one $A$ unit. At low mole fractions of $A$ in the polymer most sequences contain only one $A$ unit. 'Two units are lost per sequence if $\theta=4$, three for $\theta=6$, and so on. The number of units lost per sequence then can be larger for larger sequences; that is at high mole fractions of the given species. The values used for the reactivity ratios were $r_{a}=1.4$, $r_{b}=2.0[15]$.

Figure 16.5 presents the behavior of methyl methacrylate-isoprene copolymers. Here, again, the low-composition polymers in methyl methacrylate tend to fall near the theoretical curve $\theta=2$, whereas high compositions of methyl methacrylate approach the curve $\theta=4$. The values used for the reactivity ratios were $r_{a}=0.25, r_{b}=0.75$ [14], which are for the methyl methacrylate-butadiene system. There is not likely to be any large discrepancy due to the assumption that isoprene will behave similarly to butadiene (see tables of reactivity ratios in references [15] and [16]). The greater difference between $\theta=0$ and $\theta=2$ or 4 in the theoretical curves as compared with the previous two sets of copolymers, arises from greater alternation of units in the methyl methacrylate-isoprene copolymer and are reflected by the reactivity ratios in the depolymerization equations.

From these three diene-containing coplymers the yield of the diene unit is extremely low as it is from simple polymers of these two materials [7]. Mass spectrometer analysis, however, did show an increase in the yield of $B$ units up to an $A$ mole fraction of 0.7 . This would be expected, since the major products from the simple diene polymers are dimers, trimers, etc., and in the copolymers many of the $B$ units are isolated between $A$ units. The dimer production from the copolymers is thus diminished compared to the simple diene polymer.

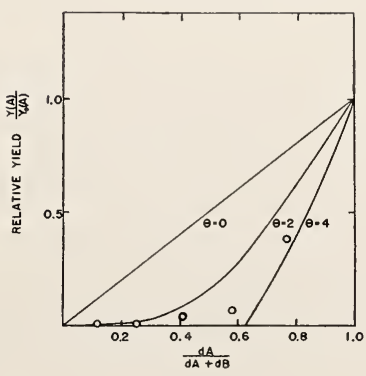

FJgure 16.5. Relative yields of methyl methacrylate from methyl methacrylateisoprene copolymers.

O, Experimental points copolymer. 
This leaves the question of how to account for the diminished production of monomer $A$. The copolymers yielded no new products of monomer size to any extent. One concludes, then, that compared to the simple polymers of $A$ the depolymerized products of the diene copolymer contain relatively greater amounts of dimer, etc. These larger fragments are presumably mixed dimers or trimers. In these copolymers, then, it appears that the increased relative production of $B$ monomers and the decrease of $A$ monomers is a consequence of the monomer to the dimer-trimer ratio, which in the copolymer is intermediate between the two simple polymers.

In figure 16.6, the parameter $\theta$ is plotted against the copolymer composition for all three systems. A linear dependence of $\theta$ on composition is the most reasonable interpretation of the data. In the light of the reasons just advanced for the behavior of these copolymers, and the variation in $\theta$, which indicates that parameter to be dependent on the length of the $A$ and $B$ sequences, it appears that arrangements occur that favor the production of specific codimers, cotrimers, etc. Our experimental values then represent an average $\theta$.

The use of the empirical equation for $\theta$ in terms of copolymer composition deduced from these data, permits the derivation of a semiempirical relationship for styrene yield and composition of copolymer for given conditions of pyrolysis. A useful analytical method is then possible.

\section{Vinyl Copolymers}

Several copolymers, where both units were ethylenic derivatives, were prrolyzed. The first of these was styrene-methyl methacrylate copolymers and the results are shown in figure 16.7. The yields of

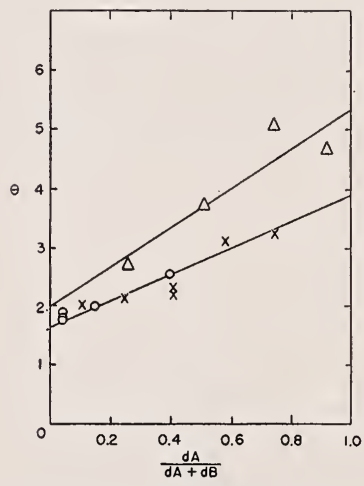

Figure 16.6. Plot of experimental $\theta_{s}$ versus $d A$ / $(d A+d B)$.

Experimental points: $\triangle$. Styreneisoprene copolymers; $O$, styrenebutadiene copolymers; $X$, methyl methacrylate-isoprene copolymers.

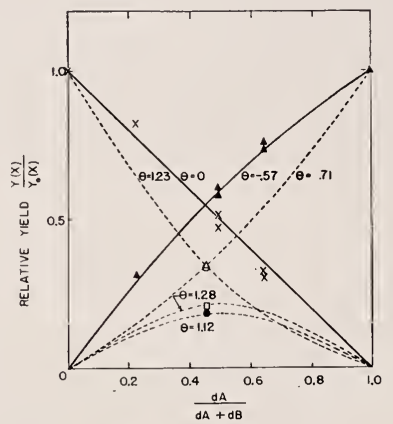

FIGURE 16.7. Relative yields of monomers from styrene-methyl methacrylate copolymers (solid lines) and $a \quad \beta$-deuterostyrenemethyl methacrylate copolymer (dashed lines).

Experimental points-Solid lines: . Styrene; $X$, methyl methacry late. Dashed lines: $\triangle, \beta$-Deuterostyrene;: $O$, methyl methacrylate; $\square$, styrene; $\boldsymbol{O}, \boldsymbol{\beta}$-deuteromethyl methacrylate. 
methyl methacrylate fall along the full straight line $\theta=0$, while the rields of strrene fall on the full line having a slight upward curvature $\theta=-0.57$. A negative $\theta$ indicates an increase in the relative yield of the given species from the copolymer.

The results for methyl methacrylate are as anticipated: independent of any copolymer effect, since it is structurally obvious that no hybrid monomers can deplete the rield, and dimer formation is not favored. Strrene shows an increased relative rield, which illustrates the decreased tendency of methyl methacrylate to form dimers compared to styrene. A certain number of styrene species being isolated between the other monomer units are thus restrained from dimerization with their own kind.

A copolymer of $\beta$-deuterostyrene and methyl methacrylate produced on prrolrsis four species. The four vields are plotted also in figure 16. $\overline{7}$. The dashed lines are calculated from $\theta$ values determined from the experimental points. As pointed out earlier, the four $\theta$ values should be identical when four species comprise almost the total product. When the low $\theta$ ralue 0.71 for $\beta$-deuterostrrene and the high value of styrene are corrected for the increase in relative vield found in the nondeuterated polrmers, the agreement is good. "The correction is made by subtracting $0.57 / 2$ from the high value for strrene and adding $0.57 / 2$ to the low value for $\beta$-deuterostyrene. Table 16.2 lists these corrected values. From our definition of $\theta$, it follows that the splitting process is completely random. Morthland's results on copolymers containing $\beta-\mathrm{C}^{14}$ styrene with $\alpha$-methylstyrene and $m$-, $p$-methylstyrene are in qualitative agreement with these results.

It would be of interast to make these and similar studies precisely enough to study differences in isotope effects.

Another series of polymers sturlied were the copolymers of styrenedimethyl fumarate. In this case the hybrid monomers, methyl acrylate and $\beta$-phenyl methyl acrylate, could be possible products. However, the volatility of $\beta$-phenylmethyl acrvlate and the dimethrl fumarate is such that they will not appear in the mass spectra. Methyl acrrlate was produced and the relative rields are shown in figure 16.8. The dotted line refers to methyl acrolate and was calculated by using the reactivity ratios $r_{a}=0.21, r_{b}=0.025$ [15], with $\theta=1.2 \tilde{5}$. The full line was calculated, using the same data, and refers to the strrene rields. The experimental results fall reasonably well on the two theoretical curres, both calculated by using the same value (1.25) for $\theta$. Again the indication is that from the point of riew of the products, an approximately random process is occurring, with some preferential splitting of the internal monomer bonds.

TABLE 16.2. Corrected $\theta$ values for $\beta$-deuterostyrene-methyl methacrylate copolymer

\begin{tabular}{|c|c|c|}
\hline$\theta_{a}$ & $\beta$-Deuterostyrene & 1. 00 \\
\hline$\theta_{b}$ & Methyl methacrylate_... & 1.23 \\
\hline$\theta_{a}^{\prime}$ & Styrene....... & 0.99 \\
\hline$\theta_{b}^{\prime}$ & $\beta$-Deuteromethyl methacrslate & 1.12 \\
\hline
\end{tabular}




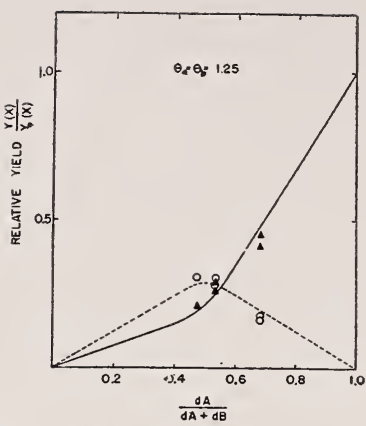

FIGURE 16.8. Relative yields of monomers from styrene-dimethyl fumarate copolymers.

Experimental points: $\boldsymbol{\Delta}$, Styrene; O, methyl acrylate.

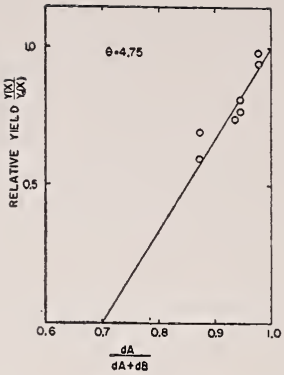

FIgURE 16.9 Relative yields of styrene. from styrene-methyl crotonate copolymer.

O,:Experimental points

The depolymerization results on a group of styrene-methyl crotonate copolymers are shown in figure 16.9. Here the only product detected was one of the original species, styrene. 'The production of methyl crotonate was neglible and may be due in part to the relatively small amount of the crotonate in the copolymers. The polymers have a high content of styrene due to the poor copolymerization behavior of the methyl crotonate. The reactivity ratios used in the pyrolysis calculations were $r_{a}=16, r_{b}=0$.

The $r_{b}$ was assumed to be zero, since polymerization of the methyl crotonate alone does not occur. The $r_{a}$ was then calculated by using the differential copolymer composition equation, and values ranging from 13 to 18 were obtained. The average value was then applied to the depolymerization results. The relative yields of styrene are quite low and fall along the line $\theta=4.75$. The data indicate a loss of all the styrenes attached at the sequence boundaries to crotonate units and the probable production of codimer or cotrimer.

The results for the copolymers of methyl methacrylate-stilbene, styrene- $\alpha$-methylstyrene and styrene- $m-, p$-methylstyrene are presented in table 16.3. The relative percentage recovery is the percentage recovery of the designated monomer from the copolymer, divided by the percentage recovery from the simple polymer, times

TABLE 16.3. Monomer production from copolymers

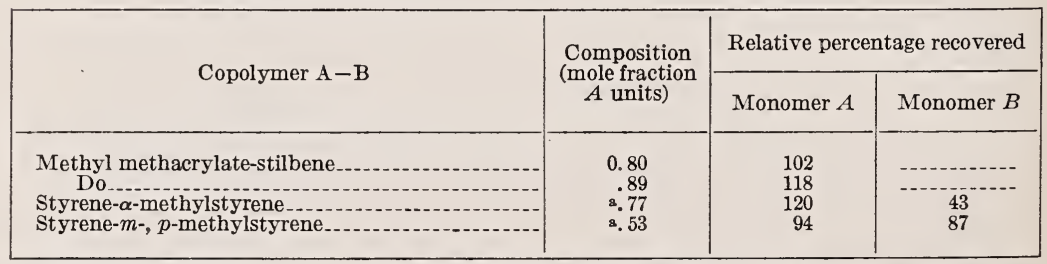

- Calculated from charge data and is probably considerably higher in the styrene- $\alpha$-methylstyrene case. 
100. If the tabulated yield were 100 percent, then $\theta=0$, that is, a linear relation between yield and composition is obtained. It is seen that all the results are in the vicinity of 100 when the probable error in the styrene- $\alpha$-methylstyrene composition is taken into account. This means that all $\theta$ 's are certainly less than one. In the styrene copolymers this is the expected result. The styrene- $\alpha$-methylstyrene polymer should behave similar to the styrene-methyl methacrvlate polymer. In the case of the methyl methacrylate-containing polymer the results contrast with those for the styrene-dimethyl fumarate copolymer in that none of the hybrid-monomer styrene was detected. The almost complete recovery of the methyl methacrylate and the absence of any hybrid monomer indicates that decomposition proceeded by the breaking of the bonds that formed during polymerization. The method of analysis would not detect stilbene due to its low volatility, although it is presumably produced. 'The results, in particular the absence of styrene, implies no splitting of the $\mathrm{CH} \phi-\mathrm{CH} \phi$ bond.

\section{Discussion}

Recent kinetic studies on depolymerization of polymers have shown a considerable diversity in the over-all rate and molecular-weight changes in the residual polymer [3, 17]. Polymethyl methacrylate apparently depolymerizes in large part by a mechanism that is essentially the reverse of polymerization. Polystyrene, on the other hand, decomposes by a mechanism having an important random component with some reverse polymerization. An alternate point of view [18] postulates definite weak points in polystyrene. Simha, Blatz, and the present author have developed $[6,19]$ a theoretical kinetic treatment of depolymerization based on the four processes, initiation, propagation, transfer, and termination, which are deemed sufficient to account for the behavior of a wide variety of polymers. This scheme is presented below:

\section{Initiation}

Propagation

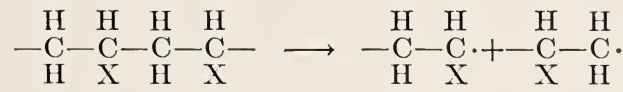

Transfer
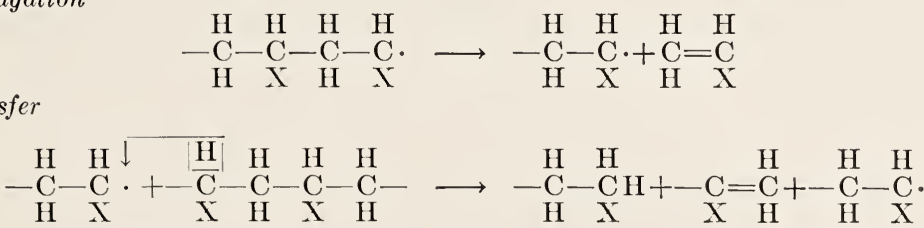

Termination

2 radical $\longrightarrow 2$ polymer

The important step which gives rise to differences between polymers is transfer. This depends on the activity of the radicals and the ease 
of removal of the hydrogen atoms concerned. Table 16.4 lists the yield of monomer from various polymers, and it is seen that the possibility for transfer increases as the yield decreases.

TABLE 16.4. Monomer yields from vinyl polymers

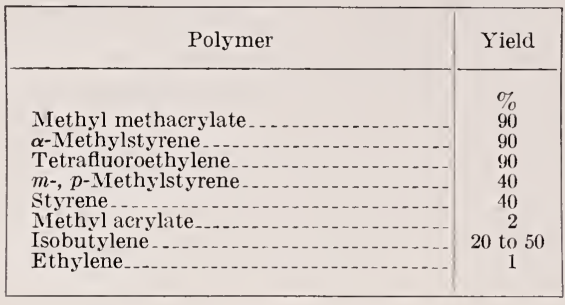

'There is a second type of transfer which is possible, termed intramolecular transfer:

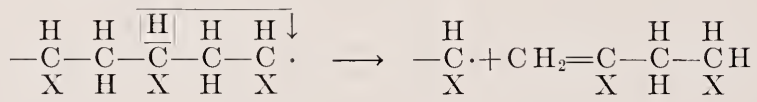

and which also decreases the vield of monomer. It influences the kinetics, however, in a manner similar to the propagation. The importance of this process could be estimated from the difference of transfer as determined from data on rate or molecular weight change and the yield of monomer. The coiling required of a radical in order for this process to occur, which may involve the removal of other hydrogens, would depend in part on steric factors. It may be pointed out that diene polymers produce small amounts of monomer presumably because dimer and trimer formation occurs readily. Little steric hindrance and ease of addition of the radical to nearby double bonds, in the same chain, instead of hydrogen abstraction, favors the dimer and trimer production. Table 16.5 illustrates this effect. Steric factors apparently account for the variation in monomer yield. The difference between the dimethylbutadiene and methylpentadiene polymers is particularly significant. These factors influencing the depolymerization of diene polymers have been previously discussed by Rice [20].

TABLE 16.5. Monomer yields from diene polymers

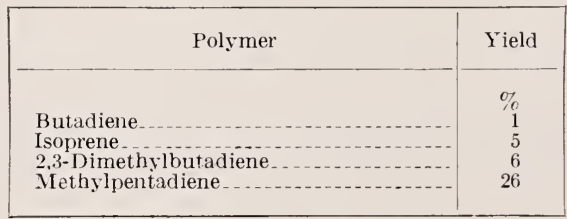


It seems clear, then, that production of dimer and larger fragments due to the probability of coiling, where diene units are concerned, is the most feasible explanation of the results in the depolymerization of the diene-containing copolymers.

The enhancement of the styrene yield from the styrene-methyl methacrylate copolymer would result through a decrease in the amount of either type of transfer. The results with the deuterated copolymer would be expected with simple initiation at random and no intermolecular transfer, or with selective initiation and intermolecular transfer, Such transfer will always introduce a random component in depolymerization reactions.

It may be possible to obtain a variation in $\theta$ depending on method of initiation used in polymerization for a copolymer of methyl methacrylate and $\beta$-deutero- $\alpha$-methylstyrene. Both materials have little possibility for transfer, hence the depropagation reaction predominates. If the termination in polymerization occurred by coupling, to give a specific weak point for the initiation of depolymerization, the polymer might be expected to unravel by splitting only bonds formed in the polymerization process, $\theta=0$.

If disproportionation occurred in polymerization producing a weak point, such a point may or may not induce specific type of unraveling. A catalytically prepared polymer may lack susceptible bonds, resulting in random initiation in the pyrolysis, which would give a $\theta=1$.

The high relative yield of methyl methacrylate from its copolymer with stilbene is a case where $\theta=0$ and where it appears that the bonds formed in polymerization were those broken in pyrolysis. The low activity of the methyl methacrylate radical and steric factors probably account for the results.

The absence of styrene formation in the stilbene polymer and the presence of methyl acrylate in the products from the dimethyl fumarate copolymer may have a bearing on the thermal stability of links formed by combination of radicals in the polymerization process. However, the difference of behavior here may be in part due to the difference in the comonomers.

The wide variety of values obtained for the parameter $\theta$ and their variation with composition in the cases of the diene copolymers indicate large differences in the mechanism of decomposition between the various polymers. The results, in general, fall within the scope of the scheme set forth, and indicate a random effect $\theta=1$, that is, random initiation or large degree of intermolecular transfer in many polymer systems. At the same time it appears that certain cases of nonrandom breakdown exist; as in the methyl methacrylate-stilbene copolymer. It also seems evident that the activity of the radicals involved, the possibility of abstracting hydrogen atoms or of adding to double bonds in the same or adjacent chains and steric factors, account for the behavior observed.

The author thanks R. Simha and F. O. Rice for their interest in this work and valuable suggestions. The cooperation of Robert Reese, in obtaining the mass spectra, and Daniel Brown, in calculating and plotting the data, is gratefully acknowledged. 


\section{References}

[1] T. Midgley and A. L. Henne, J. Am. Chem. Soc. 51, 1215 (1929).

[2] H. Staudinger and A. Steinhofer, Ann. Physik, 517, 35 (1935).

[3] N. Grassie and H. W. Melville, Proc. Roy. Soc. [A] 199, 15 (1949).

[4] H. H. G. Jellinek, J. Polymer Sci. 3, 850 (1948) ; 4, 1 (1949).

[5] S. L. Madorsky and S. Straus, J. Research NBS 40, 417 (1948) RP1886; S. L. Madorsky, S. Straus, D. Thompson, and L. Williamson, J. Research NBS 42, 499 (1949) RP1949.

[6] R. Simha, L. A. Wall, and P. J. Blatz, J. Polymer Sci. 5, 615 (1950).

[7] L. A. Wall, J. Research NBS 41, 315 (1948) RP1928.

[8] R. C. Crippen and C. F. Bonilla, Anal. Chem. 21, 927 (1949).

[9] F. W. Morthland, High Polymer Division, 119th Meeting American Chemical Society, Cleveland, Ohio, April 8 to 12, 1951.

[10] G. B. Bachman, H. Hellman, K. R. Robinson, R. W. Finhold, E. J. Kahler, L. J. Filar, L. V. Heisey, L. L. Lewis, and D. D. Micueci, J. Org. Chem. 12, 108 (1947).

[11] F. R. Mayo and F. M. Lewis, J. Am. Chem. Soc. 66, 1594 (1944).

[12] R. Simha and H. Branson, J. Chem. Phys. 12, 253 (1944).

[13] T. Alfrey and G. Goldfinger, J. Chem. Phys. 12, 205 (1944).

[14] F. M. Lewis, C. Walling, W. Cummings, E. R. Briggs, and W. J. Wenisch, J. Am. Chem. Soc. 70, 1527 (1948).

[15] F. R. Mayo and C. Walling, Chem. Rev. 46, 191 (1950).

[16] R. Simha and L. A. Wall, J. Research NBS 41, 521 (1948) RP1937.

[17] S. L. Madorsky, Paper 15 of this Symposium.

[18] H. H. G. Jellinek. Paper 1 of this Symposium.

[19] R. Simha and L. A. Wall, J. Phys. Chem. 56, 707 (1952).

[20] F. O. Rice and K. K. Rice, The aliphatic free radicals (Johns Hopkins Press, Baltimore, Md., 1935). 


\title{
17. Mechanism of the Degradation of Polyamides
}

\author{
By Bernard G. Achhammer, ${ }^{1}$ Frank W. Reinhart, ${ }^{1}$ \\ and Gordon M. Kline ${ }^{1}$
}

Films of the polyamides were exposed to heat, ultraviolet radiant energy, and different atmospheric conditions. The degradation products were collected in some cases and analyzed by mass spectrometric techniques. The unexposed and exposed specimens were examined by the following techniques to obtain information concerning the changes in chemical and physical structure of the polymer: Infrared absorption, ultraviolet absorption, viscosity of solutions, measurement of dielectric constant and dissipation factor, photomicrography, X-ray diffraction, electron microscopy, and effect of organic liquids. In addition, pyrolysis studies were made and some physical properties were determined. The results of the investigation show clearly that no single method gives a complete picture but that the results from several of the methods give an insight into the degradation mechanism. The general course of the degradation of polyamides may be described as follows: (1) Splitting of the polymer chain at the $\mathrm{C}-\mathrm{N}$ linkages, (2) changes in degree of crystallinity or local order, and (3) loss of dipole associated plasticizers.

\section{Introduction}

One of the major problems facing the plastics industry is the degradation of some plastics when exposed to certain service conditions. This problem has been investigated extensively by accelerated tests involving one or more physical properties. While empirical investigations of this type give information of value, they yield little or no information on the basic changes in the material. As a result, the value of the information now available is not only limited, but in too many instances the information cannot be used to predict behavior in actual service [1]. ${ }^{2}$ The physical changes observed during degradation may result from (1) changes in the chemical structure of the plastic material and (2) loss or changes in the compounding ingredients. The logical method of attack is to determine the specific chemical reactions involved in the degradation of the plastic and how these reactions are affected by the intensity of the conditions encountered.

The degradation of the plastic type of polyamides was investigated as part of a general research program to determine the mechanism or fundamental chemical reactions that occur when organic high polymeric materials are exposed to degrading forces such as heat, ultraviolet radiant energy, and constituents of the ambient atmosphere. The term "degradation" is used in this paper to describe irreversible changes in the chemical structure of the polymer as a result of exposure to external forces.

Boulton and Jackson $[2,3]$ found that treatment with sulfuric acid, exposure to ultraviolet radiant energy from a carbon arc, and exposure

1 National Bureau of Standards, Washington, D. C.

2 Figures in parentheses indicate the literature references on p. 279 . 
to sunlight degrade nylon. The degradation was followed by measurement of tensile strength and the viscosity of solutions in $m$-cresol and formic acid. They found that a loss of over 50 percent in tensile strength is accompanied by a relatively small change in relative viscosity. A smaller decrease in viscosity for a given loss in tensile strength was observed for the materials degraded photochemically than for those degraded by the acid.

Hosino [4] reported that heating nylon with concentrated hydrochloric acid for $12 \mathrm{hr}$ causes almost complete hydrolysis of the polymer into adipic acid and hexamethylenediamine.

Taylor [5] reported that ammonia is produced during the degradation of nylon. Peterson [6] depolymerized polyamides by heating them with an equal amount of water in an autoclave at $200^{\circ}$ to $210^{\circ} \mathrm{C}$ and 300 psi.

Fuller [7] found that heating linear polyamides for a short period of time to a suitable temperature in the presence of air or oxygen increases their toughness, flexibility, and resistance to embrittlement on aging. The temperature used, which must be above the melting point of the polyamide but below the decomposition temperature, may vary from $125^{\circ}$ to $450^{\circ} \mathrm{C}$, depending on the chemical structure of the polyamide. To obtain this effect the polyamide must be in the form of thin cross sections; otherwise the oxygen reacts with the material on the surface, leaving the interior unaffected.

\section{Materials}

The polyamide materials used in this investigation were copolymers of nylon salts with $\epsilon$-caprolactam and are described in table 17.1. The samples were obtained in the form of commercial films, 0.002 to 0.004 in. thick, which were presumably cast from hot ethanol solutions. There were some differences among the samples in transparency, attributable to differences in rates of cooling. It was necessary for some experiments to have thinner films, which were prepared by recasting from ethanol solution and washing out the ethanol with water. The discoloration and melting points reported in table 17.1 were determined in air by the usual capillary-tube method.

TABLE 17.1. Description of polyamides

\begin{tabular}{|c|c|c|c|c|c|}
\hline Sample & Chemical composition & $\begin{array}{l}\text { Code desig- } \\
\text { nation for } \\
\text { chemical } \\
\text { composition }\end{array}$ & $\begin{array}{l}\text { Nom- } \\
\text { inal } \\
\text { thick- } \\
\text { ness }\end{array}$ & $\begin{array}{l}\text { Approxi- } \\
\text { mate melt- } \\
\text { ing point } \\
\text { in air }\end{array}$ & $\begin{array}{l}\text { Approxi- } \\
\text { mate dis- } \\
\text { coloration } \\
\text { point in air }\end{array}$ \\
\hline \multirow{8}{*}{$\begin{array}{l}4405 \ldots \\
4406 \ldots \\
4407 \\
4408 \\
4409 \\
4410 \ldots\end{array}$} & \multirow{8}{*}{$\begin{array}{l}\text { Hexamethylenediamine/adipic acid salt, } \\
50 \% \text {; } \epsilon \text {-caprolactam, } 50 \% \text {. } \\
\text { Hexamethylenediamine/adipic acid salt, } \\
50 \% \text {; } \epsilon \text {-caprolactam, } 50 \% \text {. } \\
\text { Hexamethylenediamine/adipic acid salt, } \\
60 \% \text {; } \epsilon \text {-caprolactam, } 40 \% \text { - } \\
\text { Hexamethylenediamine/adipic acid salt, } \\
60 \% \text {; } \epsilon \text {-caprolactam, } 40 \% \text { - } \\
\text { Hexamethylenediamine/adipic acid salt; } \\
\text { hexamethylenediamine/sebacic acid salt; } \\
\epsilon \text {-caprolactam }{ }^{2} \\
\text { Hexamethylenediamine/adipic acid salt; } \\
\text { hexamethylenediamine/sebacic acid salt; } \\
\epsilon \text {-caprolactam (more than in } 4409 \text { ) }\end{array}$} & \multirow{6}{*}{$\begin{array}{l}66 / 6-50: 50 \\
66 / 6-50: 50 \\
66 / 6-60: 40 \\
66 / 6-60: 40\end{array}$} & \multirow{2}{*}{$\begin{array}{c}\text { in. } \\
0.002\end{array}$} & \multirow{2}{*}{$\begin{array}{c}{ }^{\circ} C \\
179 \text { to } 180\end{array}$} & \multirow{2}{*}{${ }^{\circ} \underset{172}{C}$} \\
\hline & & & & & \\
\hline & & & .004 & 179 to 180 & 174 \\
\hline & & & .002 & 188 to 190 & 177 \\
\hline & & & .003 & 186 to 190 & 177 \\
\hline & & & \multirow[b]{2}{*}{.002} & \multirow[b]{2}{*}{165 to 167} & \multirow[b]{2}{*}{160} \\
\hline & & $66 / 610 / 6 \mathrm{~A}$ & & & \\
\hline & & & .002 & 161 to 163 & 158 \\
\hline
\end{tabular}

a The percentage composition of sample 4409 is estimated to be 60 percent of 66 salt, 20 percent of 610 salt, and 20 percent of caprolactam. 
Figure 17.1. Apparatus to produce controlled degradation.

1, Gas cylinder; 2 , flowmeter; 3 , needle valve; 4 , drying tube; 5 , rheostat; 6 , copper gauze furnace; 7 , cold trap $\left(-78^{\circ} \mathrm{C}\right) ; 8$, carbon dioxide absorption tube; 9 , three-way stopcock; 10 , automatic temperature recorder with thermocouple cold junction; 11 , manometer; 12 , manometer; 13 , constant temperature bath; 14, source of ultraviolet radiant energy; 15 , heating platen; 16. Pyrex ring (not shown in figure) used to hold film flat on heating platen, $15 ; 17$, desiccator lid plaeed on 18 during evacuation to protect thin glass, 21 , by evacuating both sides simultaneously; 18, Pyrex plate; 19, gas collection trap; 20 , immersion pump; 21 , ultraviolet transmitting glass; 22 , cooling Dewar; 23 , carbon dioxide absorbent; 24 , drying tube; 25 , reverse bubbler for pressure regulation; 26 , water trap; 27 , aspirator; $\mathrm{A}-\mathrm{B}$, by pass valve; $\mathrm{J}$, end of purification train and start of exposure chamber unit; $M$, beginning of water aspirator and flow control apparatus.

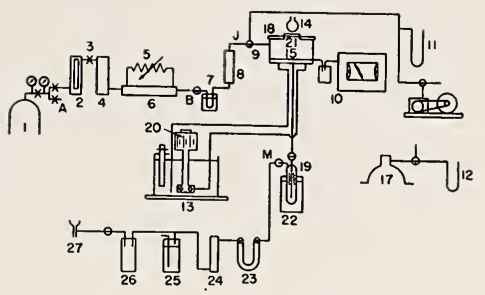

\section{Test Procedures}

\section{Apparatus to Produce Controlled Degradation}

The apparatus designed to degrade polymeric materials by exposure to controlled conditions of heat, ultraviolet radiant energy, and ambient atmosphere is shown in figure 17.1. It consists of three integrated units: (1) a gas purification train; (2) an exposure chamber suitable for exposing polymer films to ultraviolet radiant energy at controlled temperatures; and (3) a gas-collection trap suitable for collecting samples for mass spectrometric analysis of the gaseous degradation products. The apparatus and experimental procedure used are described in detail in another paper [8]. Equipment of a somewhat similar nature was used successfully by Stillings and Van Nostrand [9] in a study of the action of ultraviolet radiant energy on cellulose.

\section{Accelerated Weathering and Outdoor Exposure}

Accelerated weathering was conducted in accordance with Method 6021 of Federal Sepcification L-P-406a [10], involving cyclic exposure to the radiant energy from an $\mathrm{S}-1$ sunlamp and to fog. The total period of exposure to these two conditions is divided into multiples of $20 \mathrm{hr}$ of sunlamp radiation to $4 \mathrm{hr}$ of fog.

Outdoor exposure was accomplished by placing specimens fastened to glass frames on outdoor racks at $45^{\circ}$ facing south from February 17 to April 18, 1947. During this period of 61 days there were 12 clear, 23 partly cloudy, and 26 cloudy days. The precipitation was $4.36 \mathrm{in}$. The temperature varied from $15^{\circ}$ to $86^{\circ} \mathrm{F}$ and the relative humidity from 22 to 100 percent. The total sunshine was $330 \mathrm{hr}$.

\section{Mass Spectrometry}

Polyamide samples 4406, 4407, and 4409 were exposed to controlled conditions of heat, ultraviolet radiant energy, and ambient atmosphere in the apparatus described previously. The gaseous products were analyzed with a Model 21-102 Consolidated Engineering mass spectrometer. Some of the experiments were made with the nylon samples in the film form in which they were received and others with 
recast films. Most of these films were dried in a vacuum at room temperature for $16 \mathrm{hr}$ prior to use in the experiments.

\section{Pyrolysis}

Polyamide samples 4407 and 4409 were pyrolyzed at $400^{\circ} \mathrm{C}$, the products separated into fractions, and the gaseous products analyzed with the mass spectrometer. These experiments were made (a) to obtain reference patterns to assist in the interpretation of the mass spectrometric analysis patterns of the products obtained on exposure to heat and to ultraviolet radiant energy, and (b) to determine what bonds are broken when nylon molecules are ruptured by heat. The apparatus (fig. 17.2) and procedure are described in reference 11.

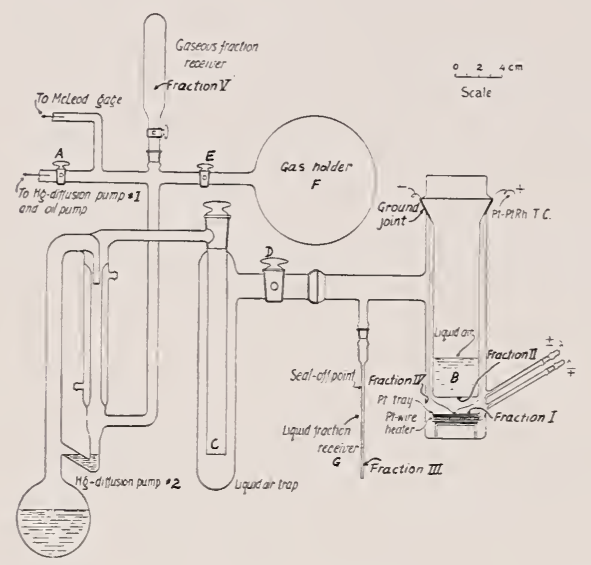

Figure 17.2. Molecular still for pyrolysis of high polymers.

\section{Ultraviolet Spectrometry}

The ultraviolet transmission characteristics of polyamide samples 4407 and 4409 , subjected to various controlled conditions of temperature, ultraviolet radiant energy, and ambient atmosphere, were determined with a model DU Beckman photoelectric quartz spectrophotometer, range 200 to 2,000 millimicrons. A critical factor in comparative ultraviolet absorption measurements is the thickness of the specimens being measured. The thicknesses of specimens of sample 4407 were measured, and the films were then heated in air at $100^{\circ} \mathrm{C}$ for $20 \mathrm{hr}$ and the thickness remeasured. The changes in thickness were less than 0.00004 in. The maximum change in ultraviolet transmission that could be attributed to this change in thickness was calculated to be 2 percent at $280 \mathrm{~m} \mu$. No differences were observed in the results of the experiments conducted in oxygen and nitrogen, respectively.

\section{Infrared Spectrometry}

Recast polyamide films were subjected to $20 \mathrm{hr}$ at $100^{\circ} \mathrm{C}$ in air and nitrogen, $217 \mathrm{hr}$ of accelerated weathering, and 2 months of outdoor exposure. The infrared transmission characteristics of the films were measured before and after the various exposures with a Baird 
recording spectrophotometer in order to determine whether changes in chemical structure had occurred. A change of at least 2 percent in the amount of a chemical group is required for detection by the infrared spectrometers used in this investigation. Recast films were used for this phase of the investigation because the original films were too thick to distinguish individual characteristic transmission bands.

\section{Viscosity}

Polyamide sample 4410 was subjected to controlled conditions of heat, ultraviolet radiant energy, and relative humidity and to outdoor exposure; the degradation of the polymer chains was determined from the measurement of the viscosities of dilute solutions of the exposed nylon specimens in $m$-cresol. This procedure can be used to determine whether or not the polymer chains are broken or cross-linked but will usually not reveal any other changes in chemical structure $[2,5]$. The viscosities of the solutions were measured at $30^{\circ} \mathrm{C}$, using calibrated Ostwald-Fenske viscometers $[12,13]$. Duplicate parallel runs were made, using the average of five efflux time measurements determined to the nearest $0.1 \mathrm{sec}$.

\section{Light Microscopy}

A petrographic microscope was used to examine films of polyamides for changes resulting from exposure to natural and accelerated weathering. Photomicrographs of untreated and treated samples 4406, $4407,4408,4409$, and 4410 were studied.

\section{Electron Microscopy}

An RCA type EMU transmission electron microscope with an accelerating voltage of $50 \mathrm{kv}$ and an operating pressure of less than $10^{-4} \mathrm{~mm}$ of mercury was used to examine films prepared by a dipping technique described in another paper [9]. Representative areas were selected and micrographs were taken at an exposure of about 10 sec. All the electron micrographs were taken at about 6,000 diam under constant conditions, except that the beam current intensity had to be altered slightly because of variation in film thickness. Shadow casting was done in an RCA type EMV vacuum unit at a pressure not higher than $10^{-4} \mathrm{~mm}$ of mercury.

\section{Electron Diffraction}

The diffraction stage of an RCA type EMU electron microscope was used to make transmission electron diffraction patterns of unexposed and exposed specimens of the samples. The thickness of films that can be used with this technique is limited because electrons are scattered a million times more effectively than X-rays [14]. The average thickness of the nylon films used was $0.4 \pm 0.1 \mu$ as determined by an interferometer.

\section{X-ray Diffraction}

The X-ray patterns were obtained with a Philips X-ray spectrometer with various modifications. $\mathrm{CuK} \alpha_{1}$ radiation at $40 \mathrm{kv}$ and 
$15 \mathrm{ma}$ was used. The spacings were obtained by diffraction of the $\mathrm{X}$-ray beam from the surface of the film and by measuring the intensities of the reflected X-ray beam from $20^{\circ}$ to $0^{\circ}$ with a Geiger counter spectrometer.

\section{Electrical Properties}

The dielectric constant and dissipation factor measurements were made with a conjugate Schering bridge in accordance with ASTM Method of Test D 150-47T [15]. Tin foil electrodes were applied to the film using petroleum jelly as an adhesive. To determine the electrical uniformity of the nylon samples, electrodes were placed on the specimens at three different positions, namely, in the center and at two positions off center. The latter two are designated as the second and third positions. The measurements were made at $25^{\circ} \mathrm{C}$ and 50-percent relative humidity after the test specimens had been at these conditions for $24 \mathrm{hr}$. The ultra riolet treatment was made by exposing specimens of the films to radiant energy from an RS sunlamp for $20 \mathrm{hr}$ in nitrogen at $100^{\circ} \mathrm{C}$.

\section{Effects of Water and Alcohols}

The effects of water, methanol, and ethanol on the polyamide samples were investigated by immersing the films in these liquids before and after exposure to heat, ultraviolet radiant energy, and outdoor weathering. The effects of these particular liquids were investigated because in any actual use the polyamide films come into contact with water and in casting the films alcohols are used as the solvents.

\section{Mechanical Properties and Weathering Resistance}

The tensile strength, elongation at failure in the tensile tests, tearing strength, resistance to accelerated weathering, and resistance to exposure outdoors of the six polyamide samples were determined. The results are reported in tables 17.2 and 17.3 .

No significant differences were observed in tensile strength and elongation between the two orientations of the specimens of each sample or between the rarious samples at $25^{\circ} \mathrm{C}$ and 50 -percent relative humidity. At $-29^{\circ} \mathrm{C}$ and 100-percent relative humidity, the differences between the samples are significant; the tensile strengths of samples 4405 and 4406 are slightly lower than those of samples 4407 and 4408 and higher than those of samples 4409 and 4410 ; the scatter in the elongation values was so great that the differences are not significant. The tensile strengths at $-29^{\circ} \mathrm{C}$ are higher than those observed at $25^{\circ} \mathrm{C}$; the increase on going to the low temperature is greater for samples $4405,4406,4407$, and 4408 than for samples 4409 and 4410. The elongation values are much lower at $-29^{\circ} \mathrm{C}$ than they are at $25^{\circ} \mathrm{C}$. The tensile strengths of the samples were reduced by exposure outdoors for 2 mo. Except for sample 4410, the elongations at break were also reduced by the exposure outdoors.

The tensile strength of sample 4408 increases with a decrease in water content. Although a similar trend is apparent for sample 4409 , it is not statistically significant. The elongations at break for 


\begin{tabular}{|c|c|c|c|c|}
\hline \multirow{2}{*}{ 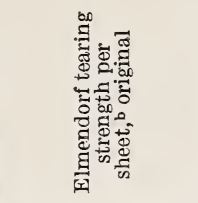 } & \multirow{2}{*}{\multicolumn{2}{|c|}{ 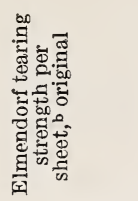 }} & 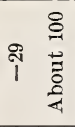 & 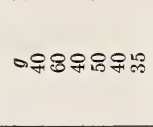 \\
\hline & & & 疋 & 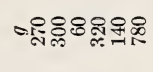 \\
\hline \multirow{6}{*}{ 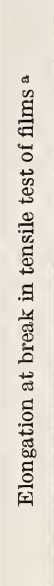 } & \multirow{3}{*}{\multicolumn{2}{|c|}{ 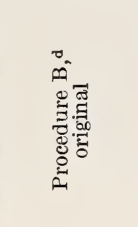 }} & ล & 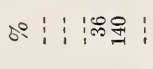 \\
\hline & & & $2{ }^{\infty}$ & 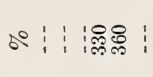 \\
\hline & & & 농 & o: $:$ : \\
\hline & \multirow{3}{*}{ 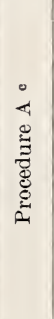 } & 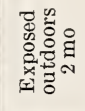 & 누 & 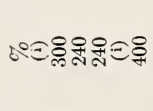 \\
\hline & & \multirow{2}{*}{ 㺃 } & 趈 & $\varrho \infty \pm$ \\
\hline & & & เి : & 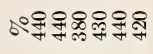 \\
\hline \multirow{7}{*}{ 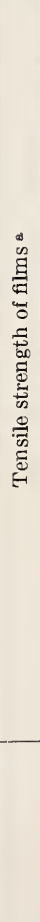 } & \multirow{3}{*}{\multicolumn{2}{|c|}{ 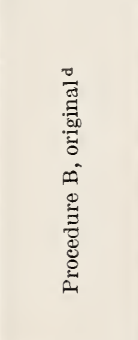 }} & ล & 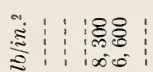 \\
\hline & & & 世 & 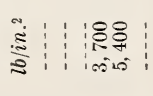 \\
\hline & & & is & こ્: \\
\hline & \multirow{3}{*}{ 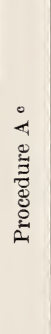 } & 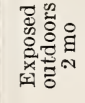 & 疋 & 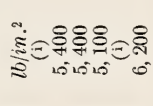 \\
\hline & & \multirow{2}{*}{ 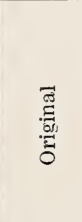 } & 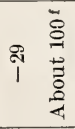 & 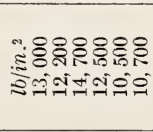 \\
\hline & & & 'i : & 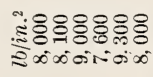 \\
\hline & & & 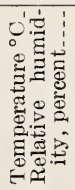 & 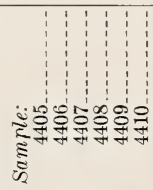 \\
\hline
\end{tabular}

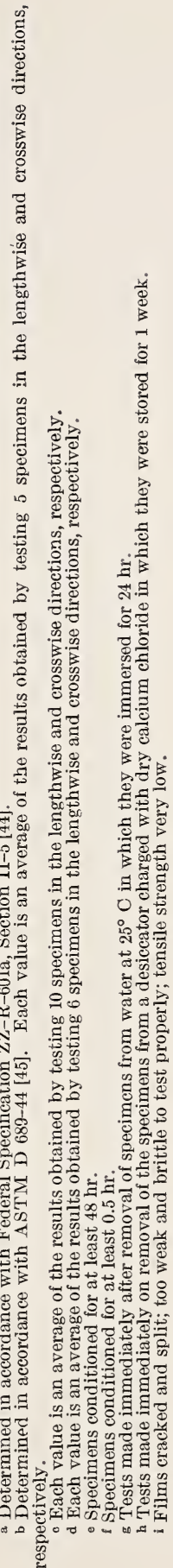


TABLE 17.3. Effects of sunlamp-fog accelerated weathering test on the properties of polyamides

\begin{tabular}{|c|c|c|c|}
\hline \multirow{3}{*}{ Sample } & \multicolumn{3}{|r|}{ Effects of accelerated weathering test a } \\
\hline & \multicolumn{2}{|c|}{ Change in } & \multirow{2}{*}{ Condition (after $240 \mathrm{hr}$ unless otherwise noted) } \\
\hline & Width & Length & \\
\hline $\begin{array}{l}4405 \\
4406 \\
4407 \\
4408 \\
4409 \\
4410\end{array}$ & $\begin{array}{c}\% \\
-3.4 \\
-1.3 \\
-1.3 \\
-10-\end{array}$ & $\begin{array}{c}\% \\
-2.6 \\
-1.7 \\
-1.7 \\
-10\end{array}$ & $\begin{array}{l}\text { Very brittle and weak; fell apart at } 180 \mathrm{hr} \text {. } \\
\text { Slightly brittle; edges curled. } \\
\text { Very brittle and weak; fell apart. } \\
\text { Slightly brittle along edges nearest to sunlamp. } \\
\text { Very brittle and weak; fell apart. } \\
\text { Do. }\end{array}$ \\
\hline
\end{tabular}

a Three specimens of each sample were tested.

samples 4408 and 4409 wet and at 50-percent relative humidity are not statistically different. The elongations of the dry specimens are much lower than those of the wet specimens of both samples. Thus it appears that water acts as a plasticizer. The stressed specimens of sample 4408 became cloudy, but those of 4409 remained clear.

The tensile stress-strain curves showed marked differences in characteristics between the wet and dry specimens. As the water content is reduced, the elongation at break is decreased. The failures were quick brittle breaks, particularly for the dry specimens. The shape of the stress-strain curves is typical of materials that undergo an instantaneous elastic response when stressed and that also workharden when stressed beyond the yield point $[16,17]$. The character of the rield point is also different for the wet and dry specimens. A comparison of the curves for the wet and dry specimens suggested that the crystalline order is different for the different conditions near the breaking point, but the character of this difference is not known. The curves for samples 4408 and 4409 indicated that these materials probably have different degrees of crystallinity.

The tearing strengths at $-29^{\circ} \mathrm{C}$ are markedly less than those at $25^{\circ} \mathrm{C}$. Since the thicknesses of samples $4405,4407,4409$, and 4410 are approximately the same, the results of the tear-strength tests show that samples 4405 and 4410 are inherently stronger in tearing strength at ordinary temperatures than samples 4406 and 4409 .

The effects of outdoor exposure and accelerated weathering on some properties of polyamide films are shown in tables 17.2 and 17.3. The accelerated weathering test conditions caused all the polyamide samples to become more brittle. The outdoor exposure tests show that the resistance to weathering of samples 4406 and 4408 is superior to that of the other samples. The greater resistance of samples 4406 and 4408 to the action of the ultraviolet radiant energy is attributed to their greater thickness, and implies a surface degradation that may retard further degradation in the interior of the films.

Marked differences in the strength properties of the various polyamides are indicated in the results reported in this section. Various techniques were utilized to obtain data relating the observed changes in physical properties with changes in chemical structure of the polyamides. The results of these studies are described in the subsequent sections of this report. 


\section{Evidence Regarding Degradative Changes in Molecular Constitution}

\section{Mass Spectrometry}

The presence of the products detected by mass spectrometry, namely, water, carbon dioxide, ethanol, carbon monoxide, and hydrocarbons (tables 17.4 and 17.5), may be explained as follows:

Most of the water was sorbed on the polymer molecules. A small part of the water may have resulted from a reaction of the following type:

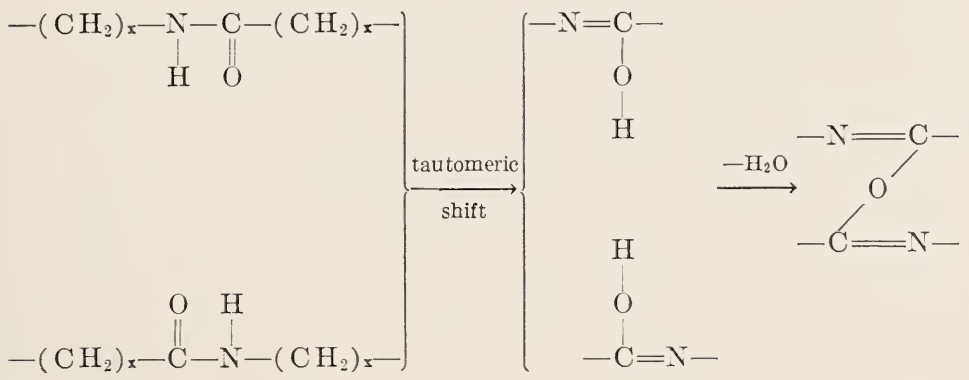

Such a reaction would result in some degree of cross-linking of the polyamide chains. However, if any cross-linking did occur as a result of the treatments, it was not sufficient to affect the solubility of the polymers in ethanol.

Carbon monoxide was evolved in all the experiments in which the specimens were exposed at temperatures of $90^{\circ}$ to $100^{\circ} \mathrm{C}$. Hydrocarbons were found in all experiments in which carbon monoxide was detected. A possible source of these two compounds is as follows:

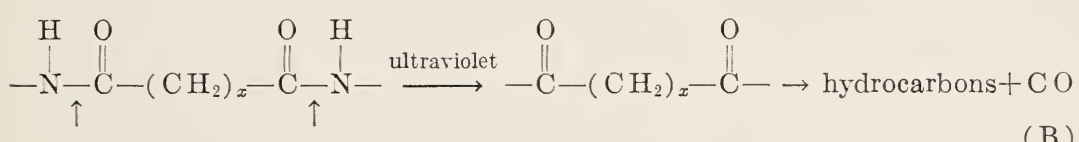

The arrows indicate the points of cleavage in the polymer chain. The carbonyl group of the peptide linkage absorbs energy at $280 \mathrm{~m} \mu$ and this energy apparently results in the scission of the bond between the imine group and the carbonyl group.

Presumably most of the carbon dioxide shown by the analysis was originally absorbed by the polymer. In the experiments made at the higher temperatures and/or with ultraviolet radiant energy, a small amount may be formed by the decarboxylation of the chain ends. Decarboxylation at temperatures of $100^{\circ} \mathrm{C}$ in the presence of ultraviolet radiant energy appears to be reasonable.

The ethanol evolved from these films is probably residual solvent. The specimens used in most of the experiments were studied in the condition in which they were receired. Residual ethanol could be attached at the peptide linkage during manufacture and act as a dipole associated plasticizer. 


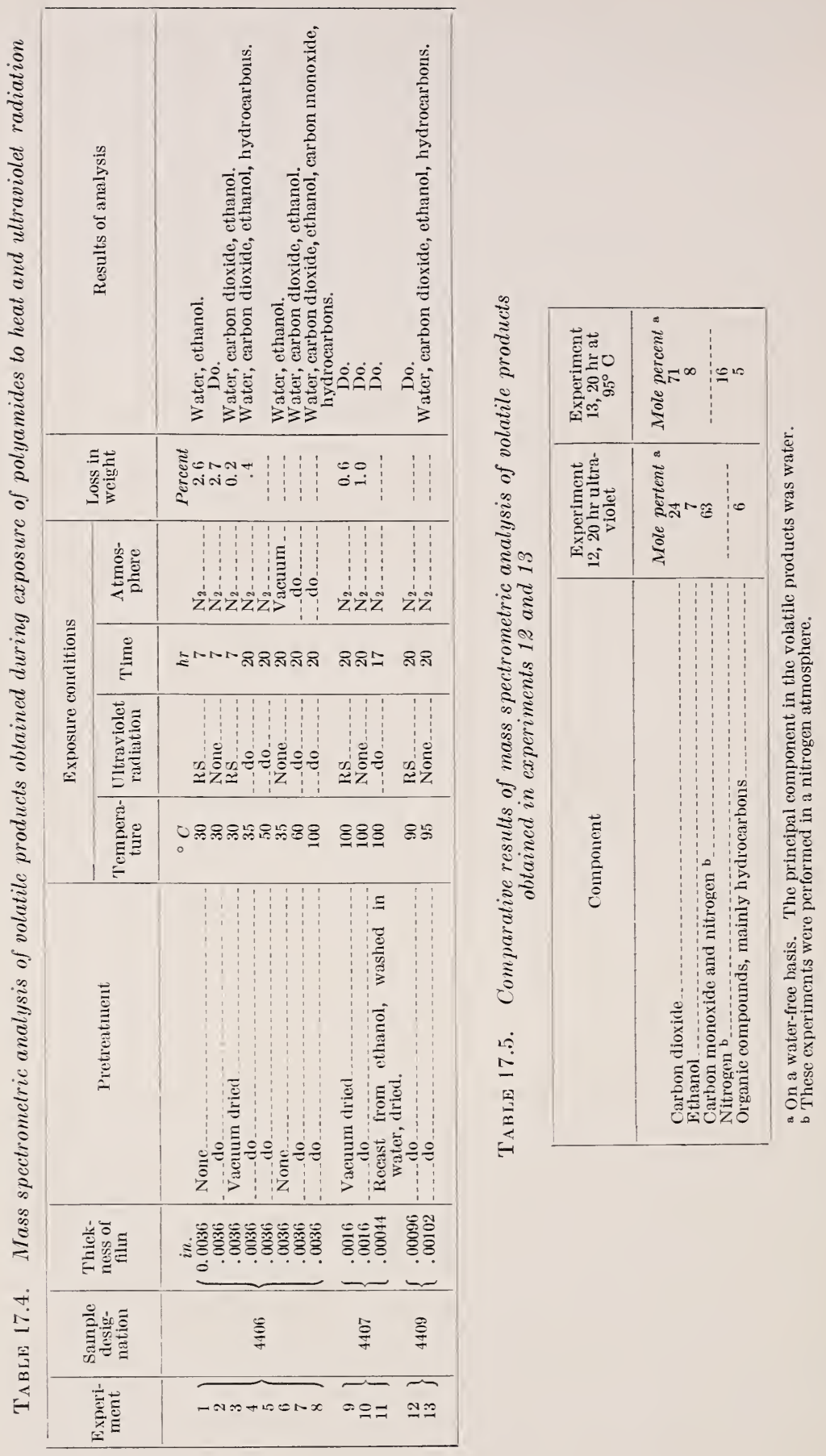




\section{Pyrolysis}

Water was one of the gaseous degradation products resulting from pyrolysis of these polyamides (table 17.6). The conditioning period in which the films were heated for $2 \mathrm{hr}$ at $150^{\circ} \mathrm{C}$ in vacuum should have removed sorbed water and gases. Water may be formed by reaction (A). The probability of reaction (A) occurring in nylon sample 4407, where there is the possibility of more peptide linkages in proximity to each other, is greater than in nylon sample 4409 where the formulation suggests a more dispersed dipole population. It is interesting to note that the water produced on pyrolysis of 4409 is almost insignificant. This water represents about 0.6 percent of the total oxygen of the polymer. With nylon 4407, 9.7 percent of the total oxygen of the polymer was evolved as water. The mechanism proposed above would lead to cross-linking of the polymer. A relatively small percentage of cross-linking leads to insolubility. The residues of these pyrolyses showed only partial solubility in ethanol, but no quantitative study of the relative solubility of samples 4407 and 4409 was made.

The cyclopentanone formed in the pyrolysis experiments may be accounted for in two ways. The polymer molecule may break as follows:

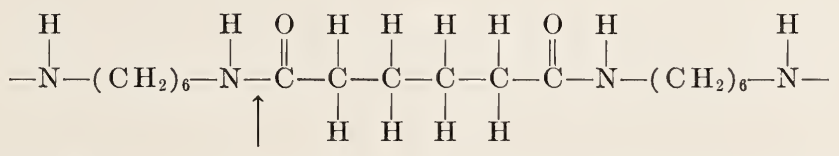

Scission at these places is logical since the $\mathrm{C}-\mathrm{N}$ bond is weaker than the $\mathrm{C}-\mathrm{C}$ bond, $66 \mathrm{kcal}$ compared to $82.5 \mathrm{kcal}$ [18]. The radical produced may split off carbon monoxide and form cyclopentanone from the fragment shown below:<smiles>CC(=O)CCCC(=O)CC1CCCC1C=O</smiles>

If this mechanism is correct and if it represents the only source of cyclopentanone, cyclopentanone and carbon monoxide should be produced in the molar ratio of $1: 1$. The molar ratio of cyclopentanone to carbon monoxide observed was $4: 1$.

Another possible mechanism to explain formation of the cyclopentanone is as follows. If adipic acid terminated the polymer chain, a reaction of the following type may occur:<smiles>[R]O[R]O[R]</smiles> 
TABLE 17.6 Results of pyrolyses of polyamides and mass spectrometric analyses of volatile fractions

\begin{tabular}{|c|c|c|c|c|c|}
\hline \multirow{2}{*}{ Fraction } & \multirow{2}{*}{ Product } & \multicolumn{4}{|c|}{$\begin{array}{c}\text { Portion of total weight, a experiment and } \\
\text { sample }\end{array}$} \\
\hline & & $1-4407$ & $2-4407$ & 3-4409 & 4- 4409 \\
\hline I. ........ & $\begin{array}{l}\text { Residue; dirty-brown flakes, slightly soluble } \\
\text { in warm ethanol. }\end{array}$ & $\begin{array}{l}\text { Percent } \\
\quad 7.87\end{array}$ & $\begin{array}{l}\text { Percent } \\
5.89\end{array}$ & $\begin{array}{l}\text { Percent } \\
2.09\end{array}$ & $\begin{array}{l}\text { Percent } \\
2.37\end{array}$ \\
\hline II, IV .... & $\begin{array}{l}\text { Semiliquid resinous mass, fairly soluble in } \\
\text { warm ethanol. }\end{array}$ & 86.71 & 88. 77 & 92.87 & 93.12 \\
\hline IIIA & $\begin{array}{l}\text { Volatile at }-75^{\circ} \mathrm{C} \\
\text { Carbon dioxide } \\
\text { Hydrocarbons } \\
\text { Methane } \\
\text { Ethane } \\
\text { Propane } \\
\text { Butanes } \\
\text { Ethylene } \\
\text { Butenes } \\
\text { Cyclopentanone }\end{array}$ & $\begin{array}{l}1.72 \\
1.67 \\
0.05 \\
.00 \\
.00 \\
.00 \\
.00 \\
.00 \\
.00 \\
.00\end{array}$ & $\begin{array}{l}1.80 \\
1.75 \\
0.05 \\
.01 \\
.01 \\
.01 \\
.00 \\
.01 \\
.01 \\
.00\end{array}$ & $\begin{array}{r}0.19 \\
.18 \\
.00 \\
.00 \\
.00 \\
.00 \\
.00 \\
.00 \\
.00 \\
.01\end{array}$ & $\begin{array}{l}0.59 \\
.56 \\
.02 \\
.00 \\
.01 \\
.00 \\
.01 \\
.00 \\
.00 \\
.01\end{array}$ \\
\hline IIIB & $\begin{array}{l}\text { Volatile at room temperature } \\
\text { Carbon dioxide } \\
\text { Hydrocarbons } \\
\text { Methane } \\
\text { Ethane } \\
\text { Butanes } \\
\text { Pentanes } \\
\text { n-Hexane } \\
\text { Ethylene } \\
\text { Propene } \\
\text { Butenes } \\
\text { Pentenes } \\
\text { Cyclohexene } \\
\text { Pentadiene } \\
\text { Cyclopentadiene } \\
\text { Benzene } \\
\text { Cyclopentanone }\end{array}$ & $\begin{array}{l}3.48 \\
1.48 \\
0.39 \\
.00 \\
.01 \\
.03 \\
.00 \\
.00 \\
.00 \\
.07 \\
.12 \\
.03 \\
.02 \\
.00 \\
.00 \\
.11 \\
1.61\end{array}$ & $\begin{array}{l}3.34 \\
1.89 \\
0.30 \\
.00 \\
.00 \\
.03 \\
.00 \\
.00 \\
.00 \\
.05 \\
.13 \\
.03 \\
.00 \\
.03 \\
.02 \\
.01 \\
1.15\end{array}$ & $\begin{array}{l}4.56 \\
4.38 \\
0.18 \\
.02 \\
.01 \\
.00 \\
.02 \\
.02 \\
.03 \\
.00 \\
.03 \\
.01 \\
.00 \\
.00 \\
.00 \\
.00 \\
.04\end{array}$ & 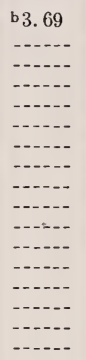 \\
\hline$V_{--.}$ & $\begin{array}{l}\text { Gaseous fraction } \\
\text { Carbon monoxide } \\
\text { Carbon dioxide } \\
\text { Methane }\end{array}$ & $\begin{array}{r}0.22 \\
.09 \\
.13 \\
.00\end{array}$ & $\begin{array}{r}0.20 \\
.12 \\
.07 \\
.01\end{array}$ & $\begin{array}{c}\text { c. } 29 \\
--1-- \\
-\cdots--\end{array}$ & $\begin{array}{r}0.23 \\
.00 \\
.20 \\
.03\end{array}$ \\
\hline & $\begin{array}{l}\text { Summary on dry basis: } \\
\text { Carbon monoxide. } \\
\text { Carbon dioxide } \\
\text { Hydrocarbons } \\
\text { Cyclopentanone }\end{array}$ & $\begin{array}{l}0.09 \\
3.28 \\
0.44 \\
1.61\end{array}$ & $\begin{array}{l}0.12 \\
3.71 \\
0.36 \\
1.15\end{array}$ & $\begin{array}{r}.00 \\
4.56 \\
0.18 \\
.05\end{array}$ & $\begin{array}{c}.00 \\
-2-1 \\
-2--\end{array}$ \\
\hline & Water & 1.67 & 1.66 & $<.1$ & $<.1$ \\
\hline
\end{tabular}

- On a dry basis except for percentage of water.

b Peaks on mass spectrometer off scale; detailed analysis not possible.

c Sample tube broken.

If a slight excess of adipic acid were used in the manufacture of the polymer, a large portion of the chain ends would be carboxyl groups. The possibility of hydrolysis of these terminal groups with subsequent decomposition of free adipic acid seems unlikely, owing to the pretreatment of films and the ease with which adipic acid could be distilled off under the conditions of pyrolysis.

It is possible that cyclopentanone is produced by both mechanisms described. There is sufficient carbon dioxide and carbon monoxide to account for these mechanisms. The moles of cyclopentanone, carbon dioxide, and carbon monoxide produced are about 0.017 , 0.08 , and 0.004 , respectively, for sample 4407 .

A very small amount of cyclopentanone was found in the products from sample 4409 . This polymer contains some adipic acid salt. The ratio of cyclopentanone to adipic acid component for sample 4407 
is about 40 times the same ratio for sample 4409. Evidently the sebacic acid component markedly affects the course of the reaction. If the 10 carbon unit breaks out in a manner similar to the reaction in eq (C), the production of cyclopentanone would not be expected. If a 10 carbon cyclic ketone formed, its existence would be transitory at the temperatures of the experiment, considering the well-known weakness of ten carbon rings. It should be noted that no carbon monoxide was produced by sample 4409 .

The carbon dioxide found is in excess of the maximum possible calculated on the basis of two carboxyl groups per molecular chain. If a molecular weight of 16,000 is assumed (table 17.7), the total possible mole percent of carbon dioxide that may be generated is about 0.3 percent; the mole percent of carbon dioxide found is about 3.5 and 4.6 percent for samples 4407 and 4409 , respectively. This shows that a large part of the carbon dioxide was sorbed on the original material or comes from parts of the chains other than the ends.

TABLE 17.7. Triscosity data for solutions of polyamide sample $4410 \mathrm{in} \mathrm{m-cresol}$

\begin{tabular}{|c|c|c|c|c|c|}
\hline \multirow{2}{*}{$\begin{array}{l}\text { Specimen desig- } \\
\text { nation }\end{array}$} & \multirow{2}{*}{ Treatment } & \multirow{2}{*}{$\begin{array}{l}\text { Concen- } \\
\text { tration of } \\
\text { solution }\end{array}$} & \multicolumn{2}{|c|}{ Intrinsic riscosity a } & \multirow{2}{*}{$\begin{array}{l}\text { Calculated mol- } \\
\text { ecular weight, } \\
\bar{M}_{0} \mathrm{~b}\end{array}$} \\
\hline & & & {$[\eta]$} & $\begin{array}{c}\text { Arerage } \\
{[\eta]}\end{array}$ & \\
\hline A.... & None & $\left\{\begin{array}{l}g / d l \\
1.032 \\
3.009 \\
0.998 \\
3.036 \\
4.988\end{array}\right.$ & $\begin{array}{l}1.33 \\
1.28 \\
1.32 \\
1.30 \\
1.30\end{array}$ & 1.31 & 16,300 \\
\hline B... & Ultraviolet, $22 \mathrm{hr}$..- & $\left\{\begin{array}{l}1.006 \\
3.001\end{array}\right.$ & $\begin{array}{r}0.98 \\
.97\end{array}$ & 0.97 & 10.800 \\
\hline C & Ultraviolet, $168 \mathrm{hr} . .$. & 0.984 & .43 & .43 & 3,470 \\
\hline D ........ & $105^{\circ} \mathrm{C}, 25 \mathrm{hr} \ldots$ & $\left\{\begin{array}{l}1.014 \\
3.005\end{array}\right.$ & $\begin{array}{l}1.03 \\
0.98\end{array}$ & \} 1.00 & 11,200 \\
\hline E.... & $105^{\circ} \mathrm{C}, 169 \mathrm{hr} \ldots$ & $\left\{\begin{array}{l}1.020 \\
2.997\end{array}\right.$ & $\begin{array}{l}.58 \\
.54\end{array}$ & \} 0.56 & 5,020 \\
\hline F.... & Outdoors, 2 mo... & $\left\{\begin{array}{l}1.011 \\
2.987\end{array}\right.$ & $\begin{array}{l}1.03 \\
0.99\end{array}$ & \} 1.01 & 11,400 \\
\hline
\end{tabular}

a The intrinsic riscosities were calculated as $3\left(\eta_{r}^{\frac{1}{3}}-1\right) / c$, where $\eta_{r}$ is the relative riscosity, and $c$ is the concentration in $g / d l$.

b Calculated riscosity arerage molecular weight [46] based on the applicability of Taylor's equation and on the use of a different solrent, since the value for intrinsic riscosity depends on the solvent [4i]. $\vec{M}_{0}=$ $13,000[\eta]^{1.30} ; 0.9[\eta] m$-cresol $=[\eta]$ formic acid.

The amounts of oxygen in nylon samples 4407 and 4409 were 15.2 and 14.2 percent, respectively. The amounts of oxygen found in the gaseous fractions were about 2.9 and 3.3 percent, respectively. Although the gaseous fractions constituted only 5 percent of the weight of the polymer, these fractions contained 19 percent of the oxygen in sample 4407 and 23 percent of the oxygen in sample 4409 . This can be explained on the basis that the $\mathrm{C}-\mathrm{N}$ bonds break as indicated in eq (C) and that the fragment broken out decomposes as indicated in eq (B) or (D).

An obvious postulate to account for the large amount of carbon dioxide and hydrocarbons formed on the pyrolysis of these nylon polymers is that the $\mathrm{C}-\mathrm{N}$ bonds break as indicated in eq $(\mathrm{C})$ and that the fragment, of either 6 or 10 carbon units, decomposes by some unknown mechanism to give carbon dioxide and hydrocarbons. In the case of the 6 carbon unit, part but not all of the fragment gives cyclopentanone and carbon monoxide as shown in eq (D). 


\section{Ultraviolet Transmission}

Nylon is a polypeptide and absorption characteristics similar to polypeptides and proteins would be expected. A region of selective absorption at approximately $280 \mathrm{~m} \mu$ has been attributed to the peptide group [19] and more particularly to the carbonyl unit of this group [20]. Thus it seems probable that the absorption in the region of $280 \mathrm{~m} \mu$ found in the polyamides (figs. 17.3 and 17.4) is caused by the peptide group and more specifically by the carbonyl unit in this group.

The absorption band of the polyamides between 250 and $310 \mathrm{~m} \mu$ may also be attributed in part to an associated structure between the peptide group and water or ethanol. The loss of the water and/or ethanol by the polyamides may cause an increase in the transmission in this region. The results obtained in this investigation show that exposure to ultraviolet radiant energy causes the disappearance of this absorption band; however, soaking the exposed specimens in water or ethanol does not cause it to return.

It is known that peptide groups form hydrogen bridges, possibly of the following nature:

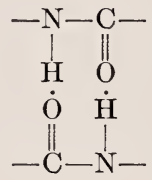

It is probable that denaturation by agents such as ethanol causes these hydrogen bridges to be broken. The ethanol used to dissolve the nylon in making the film may break the hydrogen bridge and give

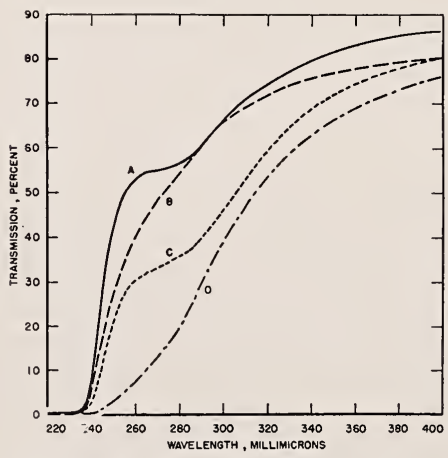

FigURE 17.3. Ultraviolet transmission of polyamide samples 4407 and 4409 before and after 120 hours of accelerated weathering.

A, Untreated sample $4407 ; \mathrm{B}$, sample 4407 after accelerated weathering; C, untreated sample 4409; D, sample 4409 after weathering.

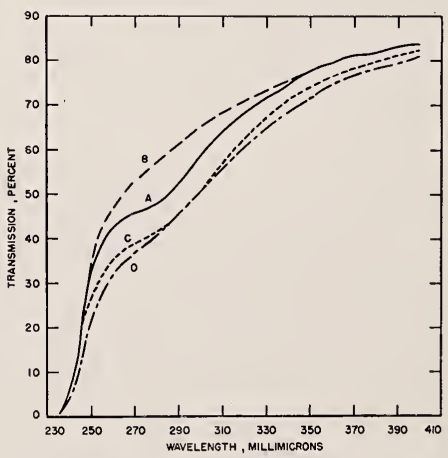

Figure 17.4. Ultraviolet transmission of polyamide sample 4407 before and after ultraviolet irradiation and/or heating.

A, Untreated sample 4407; B, after 20 hours of exposure to ultraviolet at $50^{\circ} \mathrm{C}$ in nitrogen; C, after 20 hours of exposure at $95^{\circ} \mathrm{C}$ in air oven; $\mathrm{D}$, after 20 hours of exposure to ultraviolet at $50^{\circ} \mathrm{C}$ in nitrogen and 20 hours at $95^{\circ} \mathrm{C}$ in air oven. 
a structure such as

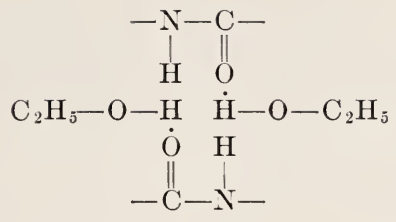

or some similar type of structure.

Exposure to heat and to ultraviolet radiant energy could eliminate the ethanol, since the energy involved in a hydrogen bridge is only about $5 \mathrm{kcal}$ [21]. However, if both these treatments remove ethanol, the structures left in the polyamide must be somewhat different; heating does not eliminate the absorption but does cause an over-all decrease in transmission, whereas ultraviolet radiant energy causes an increase in transmission so that the absorption band is removed. Water may be bound in a manner similar to that holding the ethanol.

The following hypothesis is suggested to explain the difference in the behavior of these polyamides on exposure to heat and to ultraviolet radiant energy. The heat may cause break-down of the associated complex between the carbonyl group and the ethanol and/or water, resulting in an over-all decrease in transmission. If the absorption in the region of $280 \mathrm{~m} \mu$ is attributed to the carbonyl group, it would not be appreciably affected by elimination of the complex. The ultraviolet radiant energy causes some reaction to take place, which eliminates in part the absorption supposedly characteristic of the carbonyl and peptide groups. The greatest change in transmission in the region of $280 \mathrm{~m} \mu$, observed with sample 4407 , was about 9 percent.

It may be that the characteristic dip can be attributed to structures such as that shown in $(G)$ and that the structure shown in (F) has a somewhat greater transmission.

Another type of cross-linking reaction has been suggested for proteins that eliminates the carbonyl group [20a] as follows:
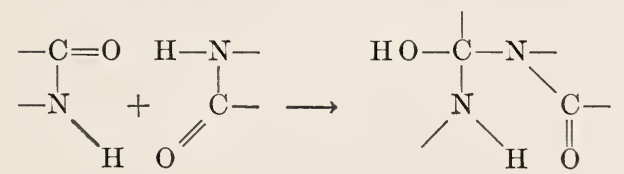

The splitting of the $\mathrm{C}-\mathrm{N}$ bond and removal of $\mathrm{C}=\mathrm{O}$ as a result of ultraviolet exposure are possible and could be responsible for the changes in the ultraviolet spectra.

\section{Infrared Transmission}

The infrared transmission curves of the polyamides (typical curves are shown in fig. 17.5) are interpreted as follows: The absorption of the imine group ( $\mathrm{NH}$ ), which is ordinarily at 2.83 to $2.93 \mu$, is relatively weak. However, a shift to a longer wavelength with increased intensity of absorption occurs with bridging, indicating that the $3 \mu$ band is probably caused by linear NH.O bridging [22-24]. Absorptions at 


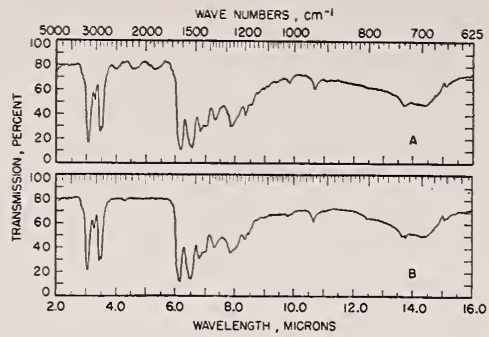

FIGURE 17.5. Infrared transmission of untreated polyamide samples 4407 and 4409.

A, Sample 4407, 0.0008 inch thick; B, sample 4409, 0.0009-inch thick.

3.36 and $3.42 \mu$ are usually a result of carbon-hydrogen bond stretching. The intense absorption at $6.1 \mu$ is attributed to the carbonyl group, which usually absorbs at 5.76 to $5.84 \mu$ but is displaced to longer wavelength in nylon 66 and amides in general probably as a result of interaction between the carbonyl and imine groups [22]. The intense absorption at $6.46 \mu$ may result from $\mathrm{N}-\mathrm{H}$ bending and could cause a harmonic effect at $3.2 \mu$.

The doublet band at 6.8 and $7.05 \mu$ has been observed in diallyl adipate [25]. This pair of bands may be connected with $\mathrm{CH}_{2}$ deformations, such as $-\mathrm{CH}_{2}-$ and $=\mathrm{CH}_{2}$ [22]. A band at $7.3 \mu$ is attributed, in some cases, to $-\mathrm{CH}_{3}$ deformation. Single-bond stretching might absorb in this region, and the band has been observed in diallyl adipate [25]. Thompson and Torkington [22] claim that "much of the absorption between 8.34 and $5.88 \mu$ must be due to vibrations of the $-\mathrm{C}-\mathrm{CO}-\mathrm{NH}-\mathrm{C}-$ part of the structure."

In the present study no extensive changes were revealed by the infrared-transmission measurements. In some cases, structural differences of significance for physical properties cannot be detected by infrared methods because the relative number of chemical linkages affected is too small [26]. Apparently the degradation of nylon under the conditions used in this study results in either a change in a small proportion of the chemical linkages or in alterations in chemical structure that are not accompanied by a change in infrared absorption, or both.

\section{Viscosity Measurements}

It seems probable that some chain scission occurred during the treatments, as shown by the lowering of $[\eta]$ (table 17.7 and fig. 17.6). It is of interest to estimate the average number of chain scissions. For this a rough estimate of molecular weight is provided by Taylor's equation [46]. Admittedly, this equation, having been derived for a $6 / 6$ polymer in formic acid, is not likely to yield accurate molecular weights for the systems used here. It can be assumed that the orders of magnitude are reasonable. The data obtained on solutions of specimens $\mathrm{B}$ and $\mathrm{C}$ and of specimens $\mathrm{D}$ and $\mathrm{E}$ show that the difference in degree of degradation is quite pronounced.

Taylor [5] has expressed the relation between the intrinsic viscosity of fractions of nylon 66 in formic acid and the molecular weight as $\bar{M}_{N}=13,000[\eta]^{1.39}$, where $\bar{M}_{N}$ is the number-average molecular weight, and $[\eta]$ is the intrinsic viscosity in formic acid. The measurements of intrinsic viscosity obtained in this laboratory on nylon 4410 were made in $m$-cresol. In 90-percent formic acid the intrinsic viscosity values are approximately 0.9 of those determined in $m$-cresol [27]. Applica- 
Figure 17.6. Effect of degradation conditions on intrinsic viscosity of polyamide sample 4410 .

$\mathrm{A}$, Heated in air oven at $105^{\circ} \mathrm{C}$ : B, irradiated with ultrariolet from an $\mathrm{S}-1$ in air at $60^{\circ} \mathrm{C}$. Value of intrinsic viscosity of sample weathered outdoors for 2 months was $1.01 \mathrm{dl} / \mathrm{g}$.

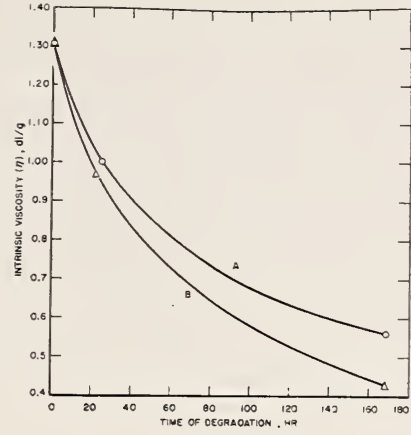

tion of Taylor's relation to the intrinsic riscosities of unfractionated polymers leads to a viscosity-arerage molecular weight $\left(\vec{M}_{v}\right)$.

Calculations from the results presented in table 17.7 indicate that 0.4 percent of all chain bonds were broken in the specimen degraded by exposure to ultraviolet radiation for $168 \mathrm{hr}$, the treatment that resulted in the greatest decrease in intrinsic viscosity. Since the strength of the $\mathrm{C}-\mathrm{N}$ bond is rather weak compared to the $\mathrm{C}-\mathrm{C}$ bond, the chain break probably occurs at the peptide linkage. If this is the case, about 3.5 percent of the peptide linkages were broken in this specimen.

\section{Evidence Regarding Degradative Changes in Molecular Orientation}

Investigations were made to detect any changes in molecular orientation or crystallinity of the polyamides under study. The terms "molecular orientation" and "crystallinity" are used in this discussion to describe a condition or state in which sections of the macromolecules are more or less situated spatially in some definite pattern or alinement to one another. The development of crystallinity in films of polvamides would be expected to lead to increased brittleness and splitting, with a corresponding limitation of plastic flow. A decrease in the water or alcohol content below a specific minimum ralue would also produce the same changes in phrsical properties.

There appear to be two trpes of molecular orientation possible in polyamides. One is hydrogen bridging through adjacent peptide groups. This is also referred to as intermolecular bridging and dipole association. The higher the degree of dipole associaton, the greater the degree of crystallinity attributable to hrdrogen bridging. The other type is hydrocarbon packing or alinement of the polymethylenic sections between the peptide groups. It has been shown that smaller polymer molecules increase the possibility of more crystallinity or local order [28] and that molecular size has a determining effect on the uniformity of properties [29].

The degree of crystallinity may be affected by the association of molecules of water and alcohol by hydrogen bridges at the peptide groups and by the dispersion of liquids or gases in the form of minute spherical inclusions in the body of the polymer. The presence of 
molecules, such as water and alcohol, in any way in the matrix will reduce the potential for molecular orientation because of spatial factors.

On the basis of chemical constitution, the polyamides used in this investigation can be divided roughly into two clases as regards possibility of molecular orientation. Samples 4405, 4406, 4407, and 4408 would be expected to have or develop a higher degree of dipole association than samples 4409 and 4410 because of the regularity in the spacing of the peptides. The 10-carbon acid along with the 6 -carbon acid creates an irregularity and causes the displacement of successive peptide groups along the chain. The caprolactam that is present in both classes also tends to create irregularity. This situation is analogous to the one described by Baker and Fuller [30] for a 50:50 composition of a $66 / 610$ polymer, which shows the effect of copolymerization in decreasing the concentration of polar groups in the average planes. The unequal polymethylene group spacings cause some of the polar peptide groups to fall along the polymer chain where they cannot contribute to dipole interaction.

\section{Microscopic Examination}

The results of the microscopic examination indicated that the exposures to heat and to ultraviolet radiant energy appeared to be attended by small changes in optic axial angle or orientation of the principal plane. The changes were little greater than the variations in each film, however, and it is concluded that the exposures have caused little alteration of the general molecular orientations within the films this technique is capable of revealing.

\section{Electron Microscopic Examination}

The electron micrographs of the unshadowed surfaces of the untreated specimens and of those exposed to ultraviolet radiant energy and to heat showed no appreciable differences as a result of the exposures. The electron micrographs of the shadowed surfaces of the untreated samples and of those exposed to ultraviolet radiant energy showed that the crystallinity of sample 4407 appeared to be increased and that of sample 4409 not affected as a result of the treatment. Exposure to heat appeared to have no effect on the crystallinity of samples 4407 and 4409. Exposure to ultraviolet radiant energy may permit rotation of a segment of the polymer chain into a more highly ordered state. If this is the situation, then sample 4407 would be affected more than sample 4409 .

\section{Electron Diffraction}

Assuming that the spacing in the region of $4.0 \mathrm{~A}$ (see table 17.8) represents hydrogen-bridged peptide linkage, the intensity of this ring should be proportional to the degree of order in the molecular chains. Positive determination of the crystallinity from such criteria as the intensity and width of the lines could not be made even with such measuring devices as the recording densitometer and the comparator because of the diffuse background and halolike character of the patterns. Visual estimation of the intensity and width of the rings was made by several experienced observers. There was no 
correlation between their observations and the treatments to which the films were subjected. These electron-diffraction studies revealed no changes in the degree of molecular orientation of the polyamides as a result of exposure to heat and to ultraviolet radiant energy.

TABLE 17.8. Electron-diffraction sparings of polyamides a

\begin{tabular}{|c|c|c|c|c|}
\hline $\begin{array}{l}\text { Diffraction } \\
\text { ring }\end{array}$ & \multicolumn{4}{|c|}{$\begin{array}{l}\text { Interplanar spacings of polya- } \\
\text { mide films }\end{array}$} \\
\hline \multirow{5}{*}{$\begin{array}{l}1 \\
2 \\
2 \\
3\end{array}$} & \multicolumn{4}{|c|}{4407 Before treatment } \\
\hline & A & B & $\mathrm{C}$ & $\mathrm{D}$ \\
\hline & $\begin{array}{c}A \\
25.15 \\
3.98 \\
2.09 \\
---\end{array}$ & $\begin{array}{c}A \\
23.89 \\
3.95 \\
2.13 \\
-.--\end{array}$ & $\begin{aligned} A \\
11.95 \\
3.59 \\
2.06 \\
1.18\end{aligned}$ & $\begin{aligned} \text { A } \\
11.95 \\
4.16 \\
1.97 \\
1.11\end{aligned}$ \\
\hline & \multicolumn{4}{|c|}{4407 After treatment $\mathrm{b}$} \\
\hline & $A^{\prime}$ & $B^{\prime}$ & $\mathrm{C}^{\prime}$ & $\mathrm{D}^{\prime}$ \\
\hline $\begin{array}{l}1 \\
2 \\
3 \\
4\end{array}$ & $\begin{array}{l}9.75 \\
4.08 \\
1.95 \\
1.07\end{array}$ & $\begin{array}{r}12.91 \\
3.89 \\
1.99 \\
1.16\end{array}$ & $\begin{array}{l}9.19 \\
\text { 3. } 85 \\
2.03 \\
1.09\end{array}$ & $\begin{array}{r}11.38 \\
3.92 \\
2.06 \\
-.--\end{array}$ \\
\hline \multirow{2}{*}{$4 \ldots$} & \multicolumn{4}{|c|}{4409 Before treatment } \\
\hline & A & $\mathrm{B}$ & $\mathrm{C}$ & $\mathrm{D}$ \\
\hline 1 & $\begin{array}{r}13.65 \\
4.02 \\
2.07 \\
1.08\end{array}$ & $\begin{array}{l}11.95 \\
3.92 \\
1.97 \\
1.096\end{array}$ & $\begin{array}{r}10.86 \\
4.27 \\
1.88 \\
1.11\end{array}$ & $\begin{array}{l}9.18 \\
4.12 \\
2.16 \\
1.99\end{array}$ \\
\hline \multirow{2}{*}{$4+\ldots$} & \multicolumn{4}{|c|}{4409 After treatment b } \\
\hline & $A^{\prime}$ & $\mathrm{B}^{\prime}$ & $\mathrm{C}^{\prime}$ & $\mathrm{D}^{\prime}$ \\
\hline $\begin{array}{l}1 \ldots \\
2 \ldots \\
3 \\
3 \\
\ldots\end{array}$ & $\begin{array}{l}9.75 \\
3.95 \\
1.89 \\
1.12\end{array}$ & $\begin{array}{r}10.39 \\
3.82 \\
1.96 \\
1.10\end{array}$ & $\begin{array}{l}9.75 \\
\text { 3. } 79 \\
1.99 \\
1.12\end{array}$ & $\begin{array}{r}10.17 \\
3.73 \\
1.87 \\
1.13\end{array}$ \\
\hline
\end{tabular}

a The patterns were made with an exposure of 15 sac.

b Code for treatment: $\mathbf{A}^{\prime}$, Sample of $\mathrm{A}$ after $168 \mathrm{hr}$ of ultraviolet (S-1) radiation; $\mathrm{B}^{\prime}$, sample of $\mathrm{B}$ after 336 $\mathrm{hr}$ of ultraviolet (S-1) radiation; $\mathrm{C}^{\prime}$, sample of $\mathrm{C}$ after $168 \mathrm{hr}$ at $100^{\circ} \mathrm{C}$ in air; $\mathrm{D}^{\prime}$, sample of D after $336 \mathrm{hr}$ at $100^{\circ} \mathrm{C}$ in air.

\section{X-ray Diffraction}

The results obtained with the X-ray-penetration photographs show some correlation with those expected on the basis of chemical constitution. Sample 4407 appeared to be more crystalline than sample 4409 ; the differences were sufficient to identify the samples.

It is possible that the $7-\mathrm{A}$ spacing observed (table 17.9) may be an identity period along the polymer chain. The increase in this spacing on exposure of sample 4407 to ultraviolet radiant energy may be attributed to the unkinking of the chain when dipole associated compounds, such as water, ethanol, or caprolactam, leave the polymer. This would permit bridging at the peptide groups and tend to 
extend the chain. In the case of sample 4409, the chain may tend to kink on exposure to ultraviolet radiant energy. This may be explained by interference between the polar groups and by a tendency of the longer methylene chain to twist. As the polar linkages are broken, the longer hydrocarbon chain section may coil, thus causing a decrease in the spacing. These phenomena would be related to the action of any dipole associated plasticizer.

TABLE 17.9. Spacings from X-ray-reflection measurements with polyamides

\begin{tabular}{|c|c|c|c|c|c|c|}
\hline $\begin{array}{l}\text { Nylon } \\
\text { sample }\end{array}$ & Treatment & $\underset{\text { thickness }}{\text { Film }}$ & \multicolumn{4}{|c|}{$\begin{array}{c}\text { Observed spacings in } \mathrm{X} \text {-ray } \\
\text { measurements }\end{array}$} \\
\hline 4409 & 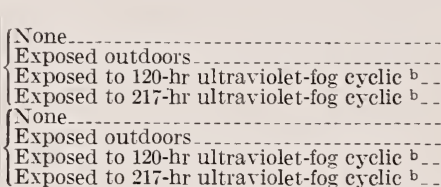 & $\begin{array}{l}\text { in. } \\
0.0016 \\
.0016 \\
.0016 \\
.00081 \\
.0023 \\
.0023 \\
.0023 \\
.00092\end{array}$ & $\begin{array}{r}\text { A } \\
3.8 \\
3.8 \\
3.9 \\
\text { c } 3.8 \\
3.9 \\
3.9 \\
3.9 \\
3.8\end{array}$ & $\begin{array}{r}\text { A } \\
4.3 \\
4.4 \\
4.4 \\
-4.3 \\
4.4 \\
4.4 \\
\text { d. } \\
4.4\end{array}$ & $\begin{array}{l}\text { A } \\
6.8 \\
6.9 \\
7.2 \\
7.5 \\
7.0 \\
7.1\end{array}$ & $\begin{array}{l}\text { A } \\
\text { a } 11.0 \\
\text { a } 11.0 \\
\text { ?11.0 } \\
\text { a } 11.0\end{array}$ \\
\hline
\end{tabular}

a Very diffuse, low peak.

b Method 6021 of Federal specification L-P-406a.

c Very weak, poor pattern.

d Poor pattern.

The splitting observed on exposure to heat and to ultraviolet radiant energy may be attributed to a rearrangement to a more crystalline state. In this case, evidence of increased crystallinity would be expected in the X-ray patterns. No increases in crystallinity were observed.

X-ray studies by Baker [31] and Brill [32] show that hydrogenbond rupture occurs on heating polyamides. A study of the rupture of intermolecular forces in molten nylon suggests that some of the intermolecular bonds rupture before melting, and thus permit "rotational movements and realinements inferred from X-ray-diffraction data" [33].

\section{Electrical Measurements}

The polyamides used in this investigation are electrically nonuniform to a high degree (table 17.10). This variation in electrical properties tends to obscure changes that might arise from alterations in molecular orientation due to exposure to heat, ultraviolet radiant energy, and other degrading forces. However, the results obtained indicate tentatively that exposure to ultraviolet radiant energy reduces the dielectric constant of sample 4406 slightly and that of sample 4407 markedly.

It seems highly probable that the nonuniform results reported here mar be connected with local variations in the degree of dipole association. The change in dielectric constant with respect to time when the electrodes are not moved may be attributed to a change in the degree of dipole association under the influence of the electrical field. Changes in the degree of association of water at the free dipoles may also be a factor.

Baker and Yager [34] have discussed the effect of hydrogen bridging in nylon on dielectric measurements. 
TABLE 17.10. Electrical uniformity of polyamides

\begin{tabular}{|c|c|c|c|c|c|c|c|c|}
\hline \multirow{2}{*}{ Sample } & \multirow{2}{*}{$\begin{array}{l}\text { Speci- } \\
\text { men }\end{array}$} & \multirow{2}{*}{$\begin{array}{l}\text { Exposed to } \\
\text { ultraviolet } \\
\text { radiation }\end{array}$} & \multirow{2}{*}{$\begin{array}{c}\text { Time } \\
\text { after } \\
\text { electrodes } \\
\text { were } \\
\text { applied }\end{array}$} & \multirow{2}{*}{$\begin{array}{l}\text { Position of } \\
\text { electrodes }\end{array}$} & \multicolumn{2}{|c|}{$\begin{array}{l}\text { Dielectric con- } \\
\text { stant at - }\end{array}$} & \multicolumn{2}{|c|}{$\begin{array}{l}\text { Dissipation } \\
\text { factor at-- }\end{array}$} \\
\hline & & & & & $10^{3} \mathrm{c} / \mathrm{s}$ & $10^{5} \mathrm{c} / \mathrm{s}$ & $10^{3} \mathrm{c} / \mathrm{s}$ & $10^{5} \mathrm{c} / \mathrm{s}$ \\
\hline $4406 \ldots$ & 12 & No & $\begin{array}{r}h r \\
0 \\
1 \\
96 \\
144 \\
312 \\
0 \\
0 \\
0 \\
72 \\
264 \\
0 \\
24 \\
0 \\
0 \\
0 \\
1 \\
2 \\
120 \\
0 \\
0 \\
24 \\
0 \\
0 \\
336 \\
0 \\
2 \\
24 \\
0\end{array}$ & 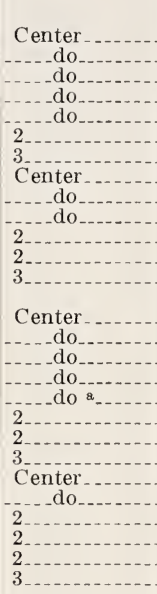 & $\begin{array}{r}6.5 \\
6.7 \\
6.8 \\
7.1 \\
7.1 \\
12.3 \\
11.7 \\
6.4 \\
6.2 \\
6.2 \\
10.8 \\
11.7 \\
11.0 \\
7.9 \\
7.9 \\
7.9 \\
8.3 \\
8.4 \\
9.4 \\
10.1 \\
9.5 \\
4.9 \\
4.8 \\
5.8 \\
-1 . \\
6.0 \\
5.4\end{array}$ & 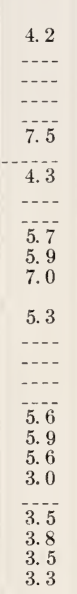 & $\begin{array}{l}0.17 \\
.17 \\
.17 \\
.17 \\
.18 \\
.18 \\
.17 \\
.16 \\
.15 \\
.15 \\
.23 \\
.23 \\
.25 \\
.15 \\
.15 \\
.15 \\
.14 \\
.13 \\
.17 \\
.18 \\
.17 \\
.13 \\
.15 \\
.16 \\
-.17 \\
.17\end{array}$ & 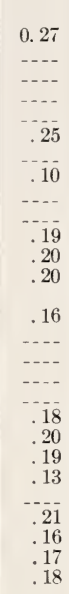 \\
\hline
\end{tabular}

a Reapplied.

\section{Effect of Water and Alcohols}

The apparent relation between the degree of molecular orientation and degree of attack by liquid (table 17.11) may be explained by the following hypothesis: Since the degree of intermolecular bridging in samples 4409 and 4410 is less than in the other samples, more unbridged carbonyl groups are available in samples 4409 and 4410 to associate with the liquid. The decreased degree of attack by the water in samples 4409 and 4410 is explained by the water molecules being sufficiently small to remain between the polymer chains without causing linear and lateral disorder sufficient to weaken the intermolecular forces holding them together. The alcohol molecules, on the other hand, are sufficiently large to cause enough disorder to weaken the intermolecular forces holding the polymer molecules together and permit further attack by the solvent.

The attack by water on nylon samples 4406, 4407, and 4408 may be directly at peptides that are hydrogen bridged. Water being a very strong polar solvent may cause dissociation of the existing bridge and be held between the peptides, causing a resultant strain on the molecular relations. In the case of nylon samples 4409 and 4410 , with more free dipoles and longer methylene chains, it may be that considerable water can be absorbed without creating extensive strains on the polymer chains.

Ethanol, a larger molecule, apparently cannot interleave between chains of samples 4406, 4407, and 4408. Absolute ethanol does not show effects that indicate that it is breaking intermolecular bridges between adjacent peptides. In the case of nylon samples 4409 and 
TABLE 17.11. Effects of water and alcohol immersion on untreated polyamides a

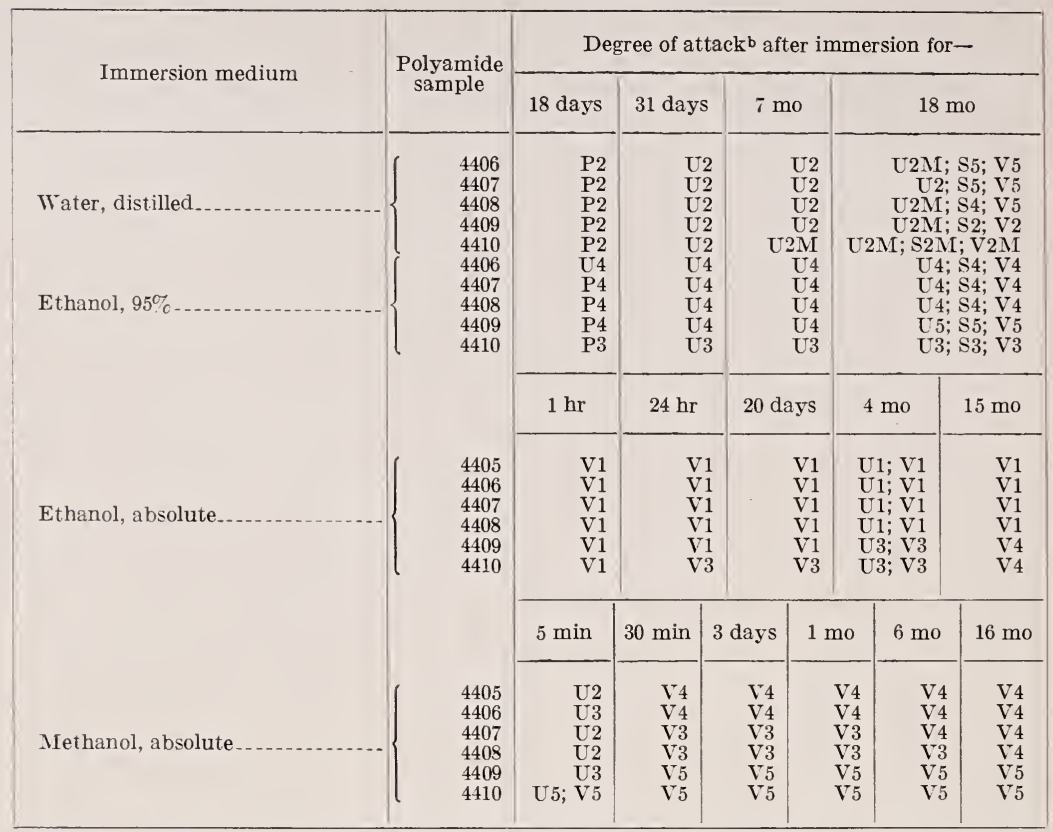

s Pieces of the films approximately $2 \mathrm{sq}$ in. in area were placed in a test tube or in a wide mouthed bottle and covered with the test liquids. The containers were stoppered and allowed to stand at $21^{\circ}$ to $26^{\circ} \mathrm{C}$. The specimens were examined at various time intervals. The test specimens were all conditioned at $25^{\circ} \mathrm{C}$ and 50-percent relative humidity prior to immersion in the liquids. The films were examined by observing the changes in appearance after standing undisturbed, after shaking the container, and after probing the test films with a glass rod.

b Code for conditions of observation:

$\mathrm{U}=$ Undisturbed.

$\mathrm{P}=$ Probed gently with a glass rod.

$\mathrm{S}=$ After gentle shaking.

$V=$ after vigorous shaking.

Code for indicating degree of attack on film: $1=$ Unaffected.

$2=$ Sediment or cloud in liquid; film intact.

$3=$ Cracks through film.

$4=$ Broken into large pieces.

$5=$ Broken into small pieces.

$6=$ Soluble.

$\mathbf{M}=$ Developed a green mold growth.

4410, with more free dipoles and possibly less packing between the polymer chains, ethanol possibly can be absorbed. The size of the ethanol molecule, however, strains the chain configurations of these molecules.

The effects observed with 95-percent ethanol seem to indicate a preferential absorption of the water in samples 4406,4407 , and 4408 . Samples 4409 and 4410 show effects similar to absolute ethanol. In general, cosolvent effects result.

Methanol represents a molecule of intermediate size. The data seem to indicate that the main attack by methanol is at unassociated dipoles. The association of a polar liquid at a free dipole may displace polymer chains, with resultant strain that may make intermolecular bridging between the polymer chains more susceptible to attack by the polar solvent. The effects observed with methanol are certainly greater than the effects observed with water or with ethanol.

Methanol attack is greater after exposure of the films to ultraviolet 
radiant energy (table 17.12). Considering the immediate attack to be at free dipoles in the polymer, it seems logical to assume that exposure to ultraviolet radiant energy releases intermolecularly bridged peptides, thus causing a decrease in the degree of crystallinity. A decrease in molecular weight may also result in increased attack by methanol because of increased association at chain ends.

'The relation between the melting points of the original polyamide samples used in this investigation and the final effects of the liquids are shown in table 17.13 in which the materials are arranged in order of decreasing melting point. If the melting points of these polyamides, which are similar in chemical constittuion, are an indication of the intermolecular forces or the degree of crystallinity, the data in the

TABLE 17.12. Effects of alcohol immersion on polyamides exposed to heat and ultraviolet radiant energy

\begin{tabular}{|c|c|c|c|c|c|}
\hline \multirow{2}{*}{ Immersion medium } & \multirow{2}{*}{$\begin{array}{l}\text { Poly- } \\
\text { amide } \\
\text { sample }\end{array}$} & \multirow{2}{*}{$\begin{array}{l}\text { Exposure prior to } \\
\text { immersion a }\end{array}$} & \multicolumn{3}{|c|}{$\begin{array}{l}\text { Degree of attack b after } \\
\text { immersion for- }\end{array}$} \\
\hline & & & $5 \mathrm{~min}$ & $30 \mathrm{~min}$ & $60 \mathrm{~min}$ \\
\hline \multirow{3}{*}{ Ethanol, 95 percent } & $\begin{array}{l}4406 \\
4407 \\
4408 \\
4409 \\
4410\end{array}$ & \multirow[t]{2}{*}{ Ultraviolet; $168 \mathrm{hr}$} & $\begin{array}{l}\text { V1 } \\
\text { V1 } \\
\text { V1 } \\
\text { V1 } \\
\text { V6 }\end{array}$ & $\begin{array}{l}\text { V5 } \\
\text { V5 } \\
\text { V4 } \\
\text { V5 } \\
\text { V6 }\end{array}$ & $\begin{array}{l}\text { V5 } \\
\text { V5 } \\
\text { V4 } \\
\text { V5 } \\
\text { V6 }\end{array}$ \\
\hline & \multirow[t]{2}{*}{$\begin{array}{l}4406 \\
4407 \\
4408 \\
4409 \\
4410\end{array}$} & & $\begin{array}{l}\text { V1 } \\
\text { V1 } \\
\text { V1 } \\
\text { V1 } \\
\text { V1 }\end{array}$ & $\begin{array}{l}\text { V1 } \\
\text { V1 } \\
\text { V1 } \\
\text { V1 } \\
\text { V1 }\end{array}$ & $\begin{array}{l}\text { V1 } \\
\text { V1 } \\
\text { V1 } \\
\text { V1 } \\
\text { V1 }\end{array}$ \\
\hline & & & $5 \mathrm{~min}$ & \multicolumn{2}{|c|}{$10 \mathrm{mo}$} \\
\hline \multirow{2}{*}{ Methanol, absolute.. } & $\begin{array}{l}4406 \\
4407 \\
4408 \\
4409 \\
4410\end{array}$ & Ultrariolet; $168 \mathrm{hr}$ & $\begin{array}{l}\mathrm{U} 2 ; \mathrm{V} 5 \\
\mathrm{U} 3 ; \mathrm{V} 5 \\
\mathrm{U} 1 ; \mathrm{V} 4 \\
\mathrm{U} 3 ; \mathrm{V} 5 \\
\mathrm{U} 6 ; \mathrm{V} 6\end{array}$ & \multicolumn{2}{|c|}{$\begin{array}{l}\text { V5 } \\
\text { V5 } \\
\text { V5 } \\
\text { V5 } \\
\text { V6 }\end{array}$} \\
\hline & $\begin{array}{l}4406 \\
4407 \\
4408 \\
4409 \\
4410\end{array}$ & Outdoors; 2 mo & $\begin{array}{l}\mathrm{U} 2 ; \mathrm{V} 4 \\
\mathrm{U} 1 ; \mathrm{V} 4 \\
\mathrm{U} 1 ; \mathrm{V} 4 \\
\mathrm{U} 1 ; \mathrm{V} 4 \\
\mathrm{U} 5 ; \mathrm{V} 5\end{array}$ & \multicolumn{2}{|c|}{$\begin{array}{l}\text { V4 } \\
\text { V4 } \\
\text { V4 } \\
\text { V5 } \\
\text { V5 }\end{array}$} \\
\hline
\end{tabular}

s The exposure to ultraviolet radiant energy was made with an S-1 sunlamp. The heating at $87^{\circ} \mathrm{C}$ was done in a vacuum oven.

b See table 17.11 for code indicating degree of attack.

TABLE 17.13. Relation between effects of liquids and the melting points of the polyamides

\begin{tabular}{|c|c|c|c|c|c|c|}
\hline \multirow{3}{*}{ Sample } & \multirow{3}{*}{$\begin{array}{l}\text { Melting } \\
\text { point }\end{array}$} & \multicolumn{5}{|c|}{ Degree of attack \& } \\
\hline & & \multirow{2}{*}{$\begin{array}{l}\text { Water, } \\
18 \mathrm{mo}\end{array}$} & \multirow{2}{*}{$\begin{array}{c}95 \% \text {-eth- } \\
\text { anol, } 18 \mathrm{mo}\end{array}$} & \multirow{2}{*}{$\begin{array}{c}\text { Ethanol, } \\
15 \mathrm{mo}\end{array}$} & \multicolumn{2}{|c|}{ Methanol } \\
\hline & & & & & $30 \mathrm{~min}$ & $16 \mathrm{mo}$ \\
\hline $\begin{array}{l}4407 \\
4408 \\
4405 \\
4406 \\
4409 \\
4410\end{array}$ & $\begin{array}{c}\circ C \\
188 \text { to } 190 \\
186 \text { to } 191 \\
179 \text { to } 180 \\
179 \text { to } 180 \\
165 \text { to } 167 \\
161 \text { to } 163\end{array}$ & $\begin{array}{c}5 \\
5 \\
-5 \\
2 \\
2 \\
\end{array}$ & $\begin{array}{c}4 \\
4 \\
-4 \\
5 \\
3\end{array}$ & $\begin{array}{l}1 \\
1 \\
1 \\
1 \\
4 \\
4\end{array}$ & $\begin{array}{l}3 \\
3 \\
4 \\
4 \\
5 \\
5\end{array}$ & $\begin{array}{l}4 \\
4 \\
4 \\
4 \\
5 \\
5\end{array}$ \\
\hline
\end{tabular}

a See table 17.11 for code indicating degree of attack. 
above table indicate that the more crystalline polymers are more readily attacked by the water and the less crystalline polymers are more readily attacked by the absolute ethanol and methanol. The effect of the 95-percent ethanol is erratic since the mixture exhibits cosolvent effects.

The relation between the effects of absolute methanol and the melting points of the original and exposed samples is shown in table 17.14. The effect of absolute methanol on the original untreated specimens is about the same as that on specimens exposed outdoors, and the melting points are practically the same. The melting points of the specimens exposed to ultraviolet radiant energy are less than those of unexposed specimens, and the effects of the absolute methanol are greater. If this is interpreted in terms of the hypothesis advanced above, it is indicated that there is little or no change in crystallinity in the specimens exposed outdoors and a decrease in crystallinity in the specimens exposed to ultraviolet radiant energy in the laboratory.

TABLE 17.14. Relation between effects of methanol immersion and melting points of original and exposed polyamides

\begin{tabular}{|c|c|c|c|c|c|c|}
\hline \multirow{2}{*}{ Polyamide sample } & \multicolumn{2}{|c|}{ Untreated } & \multicolumn{2}{|c|}{ Exposed to ultraviolet } & \multicolumn{2}{|c|}{$\begin{array}{l}\text { Exposed outdoors for } 2 \\
\text { months }\end{array}$} \\
\hline & $\begin{array}{l}\text { Melting } \\
\text { point }\end{array}$ & $\begin{array}{l}\text { Effect of } \\
\text { metha- } \\
\text { nol a }\end{array}$ & $\begin{array}{l}\text { Melting } \\
\text { point }\end{array}$ & $\begin{array}{c}\text { Effect of } \\
\text { metha- } \\
\text { nol a }\end{array}$ & $\begin{array}{l}\text { Melting } \\
\text { point }\end{array}$ & $\begin{array}{c}\text { Effect of } \\
\text { metha- } \\
\text { nol a }\end{array}$ \\
\hline 4405 & $\begin{array}{l}{ }^{\circ} C \\
179 \text { to } 180\end{array}$ & 4 & ${ }^{\circ} \mathrm{C}$ & & ${ }^{\circ} \mathrm{C}$ & \\
\hline 4106 & 179 to 180 & 4 & 163 to 170 & 5 & 180 to 183 & 4 \\
\hline $4407 \ldots$ & 188 to 190 & 4 & 173 to 178 & 5 & 188 to 189 & 4 \\
\hline & 186 to 191 & 4 & 180 to 182 & 5 & 190 to 191 & 4 \\
\hline $4409 \ldots$ & 165 to 167 & 5 & 152 to 160 & 5 & 162 to 169 & 5 \\
\hline $4410 \ldots \ldots$ & 161 to 163 & 5 & 145 to 151 & 6 & 164 to 166 & 5 \\
\hline
\end{tabular}

a See table 17.11 for code indicating degree of attack.

\section{Relation Between Stability and Chemical Constitution}

The $66 / 610 / 6$ polvamides have compositions which should be favorable to less intermolecular bridging than the $66 / 6$ polyamides. However, factors such as cooling rate from the molten state, which apparently has a considerable effect on the degree of association between chains, must be considered. It has been suggested [35] that a $66 / 610$ $(30: 70)$ polymer is qualitatively less crystalline and consequently has a lower melting point than a 66 polymer. One can expect that the more heterogeneous the chemical composition of a polvamide is, the more resistance it will have to certain types of degradation.

The carbonyl groups in the polymer are a seat of ultraviolet absorption and consequently are detrimental to stability of the molecule. The adjacent, comparably weak, $\mathrm{C}-\mathrm{N}$ bond is an arrangement that is susceptible to chain scission.

A superpolyamide with properties similar to the 66 polymer has been prepared from piperazine and dicarboxylic acids and is thought to contain no hydrogen bridges between molecules [36]. The resistance of polramides to embrittlement on degradation increases when an amino hivdrogen of an aromatic amine having at least two cyclic nuclei (for example, $\beta$-naphthylamine or phenothiazine) is incorporated in the polymer molecule [37]. It is also reported that the 
stability of polyamides to heat and light is increased by adding 0.5 to 4 mole percent of an amide-forming aromatic compound in the monomer mixture prior to condensation [38]. Plasticization of polramides has also been suggested $[39,40]$. Methylation of a fraction of the amide bonds results in polymer molecules with the same strength as the original ones but eliminates the possibility of the formation of hydrogen bridges $[41,42]$. N-substituted polvamides obtained when mixtures of disecondary, secondary-primary, and diprimary amines are polymerized with dibasic acids have less hydrogen bridging and molecular interaction because of the lateral substituents [43]. The amount of $\mathrm{N}$-substitution determines the properties of the polyamides; they can be varied from the hard, tough polymers of the unsubstituted types to the soft, tacky polymers of the 100-percent substituted types. Higher alkyl-N-substituted 610 polymers are less crystalline, have less potential crystallinity, and possess a higher degree of long-range elasticity than methyl-N-substituted 610 polymers. The basic idea involved in modifying these chemical structures is to prevent proximity of dipoles between the molecules.

\section{Improvement of Service Life of Polyamides}

The service life of polvamides could be extended br adding materials that will absorb the ultraviolet radiant energy instead of allowing the short wavelengths to be absorbed by the carbonyl group in the polyamide or by coating or covering with a material that would keep the ultraviolet radiant energy from getting to the carbonyl group. It appears unnecessary to protect from heat, except under extraordinary circumstances. Maintaining the water content at a relatively high value would also extend the service life. Precautions should also be taken to prevent the loss of organic liquids; or; if they are lost, to make certain that they are replaced with water or some other material capable of forming a similar associated complex. The absorption of excess amounts of water should be avoided because they cause a change in the strength properties. Water and organic liquids held in this manner act as volatile plasticizers. The use of less volatile water-insoluble materials that would be bound in a manner similar to the way water and alcohols are held is suggested.

The detrimental effects of changes in crystallinity or order on degradation may be remedied partially by changing the chemical constitution, as mentioned in the previous section. The changes in chemical constitution would need to be carefully controlled to retain the desirable phrsical characteristics. On the basis of our present limited knowledge of such effects, experimentation is the only method of determining how much of the problem can be solved by this method. It appears that as long as the peptide group is present, a definite focal point of attack for degradation exists. The addition of groups, such as phenyl, may only act as radiant energy absorbers and may be capable of transferring this absorbed energy to the weaker $\mathrm{C}-\mathrm{N}$ bond, causing it to break. The resulting smaller polymer molecules would be prevented from crystallizing by the presence of the large side groups. However, it is probable that any appreciable increases in stability by changes in chemical constitution would also produce soft, tacky polymers. 


\section{Summary}

The degradation of polyamides may involve the following: (1) change in chemical structure of the polymer molecule, (2) change in crystallinity (molecular orientation) including effects produced by hydrocarbon packing, dipole rearrangement, and hydrogen bridging, (3) change in the amount of molecularly associated materials, which have a plasticizing effect, such as water and ethanol. The results of examination by various techniques of films degraded under various conditions are summarized in table 17.15 .

TABLE 17.15. Summary of changes detected by various methods of analysis as a result of exposure of polyamides to heat and to ultraviolet radiant energy

\begin{tabular}{|c|c|c|}
\hline Method of analysis & Sumniary of observations & Interpretation of observations \\
\hline $\begin{array}{l}\text { Mechanical nrop- } \\
\text { erties. }\end{array}$ & $\begin{array}{l}\text { Tensile strength increases, elongation } \\
\text { decreases, and tear strength de- } \\
\text { creases as temperature is lowered. } \\
\text { Drying causes a decrease in elonga- } \\
\text { tion and has no effect on tensile } \\
\text { strength. Wetting causes a decrease } \\
\text { in tensile strength and has no effect } \\
\text { on elongation. Out-door exposure } \\
\text { causes films to become weak ane. } \\
\text { brittle. }\end{array}$ & $\begin{array}{l}\text { Water content has an effect on the } \\
\text { strength properties. Outdoor ex- } \\
\text { posure lowers strength properties. }\end{array}$ \\
\hline $\begin{array}{l}\text { Mass spectromet- } \\
\text { ric analysis. }\end{array}$ & $\begin{array}{l}\text { Water, carbon dioxide, ethanol, carbon } \\
\text { monoxide, and hydrocarbons are } \\
\text { given off. }\end{array}$ & $\begin{array}{l}\text { Water, carbon dioxide, carbon mon- } \\
\text { oxide, and hydrocarbons are given } \\
\text { off when polymer molecule breaks } \\
\text { into smaller ones. Water and eth- } \\
\text { anol are sorbed by the polymer; the } \\
\text { loss of these materials causes changes } \\
\text { in properties. The presence of eth- } \\
\text { anol indicates that the firms were } \\
\text { cast from ethanol solutions. }\end{array}$ \\
\hline Pyrolysis ..... & $\begin{array}{l}\text { Carbon monoxide, carbon dioxide, } \\
\text { hydrocarbons, and cyclopentanone } \\
\text { are given off. All the nitrogen is in } \\
\text { the resinous residue. }\end{array}$ & $\begin{array}{l}\text { The } \mathrm{C}-\mathrm{N} \text { bond is the weakest link } \\
\text { in the polymer molccule. When } \\
\text { the bond breaks, the nitrogen remains } \\
\text { attached to the polymer molecule } \\
\text { or is combined in other relatively } \\
\text { nonvolatile compounds. }\end{array}$ \\
\hline $\begin{array}{l}\text { Ultraviolet trans- } \\
\text { mission. }\end{array}$ & $\begin{array}{l}\text { Abserption band between } 250 \text { and } 340 \\
\text { m } \mu \text { is removed by exposure to ultra- } \\
\text { violet radiant energy but not to hest. } \\
\text { Heat lowers over-all absorption. }\end{array}$ & $\begin{array}{l}\text { Water and/or ethanol is lost and/or } \\
\text { changes in structure occur on ex- } \\
\text { posure to ultraviolet radiant energy } \\
\text { but not on exposure to heat. }\end{array}$ \\
\hline $\begin{array}{l}\text { Infrared transmis- } \\
\text { sion. }\end{array}$ & No changes detected & $\begin{array}{l}\text { No appreciable change in the unit of } \\
\text { chemical structure. }\end{array}$ \\
\hline $\begin{array}{l}\text { Viscosity measure- } \\
\text { ments. }\end{array}$ & Molecular weight reduced & $\begin{array}{l}\text { Polymer molecules are broken into } \\
\text { smaller ones. }\end{array}$ \\
\hline Light microscopy -- & $\begin{array}{l}\text { Definite crystalline structure which } \\
\text { changes slightly on exposure to heat } \\
\text { and to ultraviolet radiant energy. } \\
\text { No spherulites were observed. } \\
\text { Spherical inclusions of liquid, rapor, } \\
\text { or air are present. }\end{array}$ & $\begin{array}{l}\text { Changes in crystallinity are insuffi- } \\
\text { cient to cause marked changes in } \\
\text { physical propertics. Changes in } \\
\text { size of spherical inclisions cause in- } \\
\text { ternal stresses. }\end{array}$ \\
\hline $\begin{array}{l}\text { Electron micro- } \\
\text { scopy. }\end{array}$ & $\begin{array}{l}\text { Degree of crystallinity increases on ex- } \\
\text { posure to ultraviolet radiant energy } \\
\text { but not to heat. Polymer with } \\
\text { chemical constitition potentially } \\
\text { more crystalline is affected more. }\end{array}$ & $\begin{array}{l}\text { Some changes in physical properties } \\
\text { may be attributed to changes in } \\
\text { crystallinity. Comparative amount } \\
\text { of change may be predicted from } \\
\text { chemical constitution. }\end{array}$ \\
\hline Electron diffraction. & 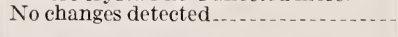 & No appreciable changes in erystal- \\
\hline X-ray diffraction. & $\begin{array}{l}\text { Differcnt polymers can be identified } \\
\text { by differences in patterns. Crystal- } \\
\text { linity of polymer correlates with } \\
\text { chemical constitution. Slight } \\
\text { changes in } 7 \text { A spacing on exposure to } \\
\text { heat and to ultraviolet radiant en- } \\
\text { ergy. }\end{array}$ & $\begin{array}{l}\text { X-ray patterns and erystallinity of } \\
\text { polymers vary from polymier to } \\
\text { polymer. No appreciable changes } \\
\text { on exposure. }\end{array}$ \\
\hline $\begin{array}{l}\text { Electrical measure- } \\
\text { ments. }\end{array}$ & $\begin{array}{l}\text { Samples are electrically nonuniform. } \\
\text { Electrical properties change inder } \\
\text { influence of electrical field. Ex- } \\
\text { posure to ultraviolet radiant energy } \\
\text { redices dielectric constant. }\end{array}$ & $\begin{array}{l}\text { The dipole association varies from } \\
\text { place to place in the samples. Degree } \\
\text { of dipole association changes under } \\
\text { influcnce of an electrical field. }\end{array}$ \\
\hline $\begin{array}{l}\text { Effects of water } \\
\text { and alcohols. }\end{array}$ & $\begin{array}{l}\text { F.ffects of immersion correlate with } \\
\text { chemical constitution and melting } \\
\text { points. Degree of attack increases } \\
\text { when specimens are exposed to illtra- } \\
\text { violet radiant energy. }\end{array}$ & $\begin{array}{l}\text { Lower molecular weight polymer is } \\
\text { produced and/or degrce of crystal- } \\
\text { linity is reduced by exposure to } \\
\text { ultraviolet radiant energy. }\end{array}$ \\
\hline
\end{tabular}


This investigation was conducted under the sponsorship and with the financial assistance of the Office of the Quartermaster General, Department of the Army. The assistance of many of our colleagues at the National Bureau of Standards. who are specialists in the various techniques employed, is gratefully acknowledged.

\section{References}

[1] F. W. Reinhart, Degradation of plastics, SPE News 4, No. 7, 3 (Sept. 1948).

[2] J. Boulton and D. L. C. Jackson, The fluidity of nylon solutions in $m$-cresol: measurement of chemical damage in nylon textiles, J. Soc. Dyers Colourists 59, 21 (1943).

[3] J. Boulton and D. L. C. Jackson, The fluidity of nylon solutions in $m$-cresol: Part II, J. Soc. Drers Colourists 61, 40 (19்4う).

[4] K. Hosino, Studies of synthetic fibers. 1. Decomposition and synthesis of nvlon, J. Chem. Soc. (Japan) 61, 475 (1940).

[5] G. B. Taylor, The relation of the viscosity of nylon solutions in formic acid to molecular weight as determined by end-group measurements, J. Am. Chem. Soc. 69, $635(194 \bar{\tau})$.

[6] W. R. Peterson, Degradation of synthetic linear polyamides, U. S. Patent 2348751 ( Mar 16, 1944).

[7] C. S. Fuller, Heat-oxygen treatment of polyamides, U. S. Patent $236+204$ (Dec. 5, 1944).

[8] B. G. Achhammer, F. IV. Reinhart, and G. M. Kline, Mechanism of the degradation of polyamides, J. Research NBS 46, 391 (1951) RP 2210.

[9] R. A. Stillings and R. J. Van Nostrand. The action of ultraviolet light upon cellulose. I. Irradiation effects. II. Post-irradiation effects, J. Am. Chem. Soc. 66, $753(1944)$.

[10] Plastics, organic: general specifications, test methods, Federal Specificaticn L-P-406a (Government Printing Office, Washington, D. C., Jan. 24, 1944).

[11] S. L. Madorsky and S. Straus, Pyrolytic fractionation of polystyrene in a high vacuum and mass spectrometer analysis of some of the fractions, Ind. Eng. Chem. 40, 848 (1948); J. Research NBS 40, 417 (1948) RP1886.

[12] American Society for Testing Materials tentative method of test for kinematic viscosity, D $445-46 \mathrm{~T}$.

[13] J. IV. M[cElwain, Pump for volatile or toxic liquids in viscometers, Anal. Chem. 21, 194 (Jan. 1949).

[14] G. P. Thomson, Electron diffraction, J. Inst. Metals 69, 191 (1943).

[15] American Society for Testing Materials tentative methods of test for power factor and dielectric constant of electrical insulating materials, D $150-47 \mathrm{~T}$.

[16] T. Alfrey, Jr., Mechanical behavior of high polymers (Interscience Publishers, New York, N. Y., 1948).

[17] H. Leaderman, Proposed nomenclature for elastic and inelastic behavior of high polymers, J. Colloid Sci, 4, 193 (1949).

[18] F. O. Rice and $\mathrm{K}$. Rice, The aliphatic free radicals (The Johns Hopkins University Press, Baltimore, Md., 1935).

[19] G. A. Anslow and S. C. Nassar, The absorption of ultraviolet energy by the peptide linkage, J. Opt. Soc. Am. 31, 118 (1941).

[20] I. M. Klotz, Litraviolet absorption spectroscopy, J. Chem. Education 22, $328(1945)$.

[20a] D. M. Wrinch, On the pattern of proteins, Proc. Roy. Soc. (London) [A] $160,59(1937)$.

[21] A. E. Mirsky and Linus Pauling, On the structure of native, denatured, and coagulated proteins, Proc. Natl. Acad. Sci., U. S. 22, 439 (1936).

[22] H. W. Thompson and P. Torkington, The infrared spectra of compounds of high molecular weight, Trans. Faraday Soc. 41, 246 (1945).

[23] A. M. Buswell, Karl F. Krebs, and W. H. Rodebush, The infrared abscrption of proteins in the $3 \mathrm{~m} \mu$ region, J. Phys. Chem. 44, 1126 (1940).

[24] S. E. Darmon and G. B. B. M. Sutherland, Infrared spectra and structure of natural and synthetic polypeptides, J. Am. Chem. Soc. 69, 207t (1947).

[25] R. B. Barnes, R. C. Gore, U. Liddel, and Y. Z. Williams, Infrared spectroscopy (Reinhold Publishing Corp., New York, X. Y., 1944).

[26] J. E. Field, D. E. Woodford, and S. D. Gehman, Applications of infrared methods in the structural examination of synthetic rubber, J. Applied Phys. 1\%, 386 (1946).

[27] J. B. Nichols, The colloid aspects of nylon. Published in Colloid Chemistry by J. Alexander, vol. 6, p. 107 \% (Reinhold Publishing Corp.. New York, I. Y., 1946). 
[28] W. O. Baker and C. S. Fuller, Thermal evidence of crystallinity in linear polymers, Ind. Eng. Chem. 38, 272 (1946).

[29] D. D. Coffman, G. J. Berchet, W. R. Peterson, and E. W. Spanagel, Polymeric amides from diamines and diabasic acids, J. Polymer Sci. 2, 306 (1947).

[30] W. O. Baker and C. S. Fuller, Macromolecular disorder in linear polyamides. Relation of structure to physical properties of copolyamides, J. Am. Chem. Soc. 64, 2399 (1942).

[31] W. O. Baker, Nature of the solid state of chain polymers, S. B. Twiss, Advancing fronts in chemistry, vol. I, High Polymers, chap. 8 (Reinhold Publishing Corp., New York, N. Y., 1945).

[32] R. Brill, Behavior of polyamides on heating, J. prakt. Chem. 161, 49 (1942).

[33] Leonard Glott and J. W. Ellis, Near infrared absorption of nylon films: dichroism and rupture of NH.O bonds on melting, J. Chem. Phys. 16, $551(1948)$.

[34] W. O. Baker and W. A. Yager, The relation of dielectric properties to structure of crystalline polymers. II. Linear polyamides, J. Am. Chem. Soc. 64, $2171(1942)$.

[35] W. E. Catlin, E. P. Czerwin, and R. H. Wiley, Multi-ingredient polyamides, J. Polymer Sci. 2, 412 (1947).

[36] I. C. Funcke, Uber die zwischen linearen Kettenmolekülen mit Peptidbindungen wirkenden intermolekularen Kräfte, Naturwissenschaften $\mathbf{2 9}, 517$ (1941).

[37] E. I. du Pont de Nemours \& Co. and G. D. Graves, Polymeric materials, Brit. Patent 549370 (Nov. 18, 1942).

[38] G. B. Taylor, Improved polyamides, U. S. Patent 2374145 (April 17, 1945).

[39] C. Coolidge, Plasticizing synthetic linear polyamides for making sheets, filaments, etc., U. S. Patent 2265119 (Dec. 2, 1942).

[40] M. M. Brubaker, Plasticized polyamides, U. S. Patent 2374576 (April 24, 1945).

[41] W. O. Baker and C. S. Fuller, Intermolecular forces and chain configuration in linear polymers. The effect of $\mathrm{N}$-methylation on the X-ray structures and properties of linear polyamides. Ann. N. Y. Acad. Sci. 44, Art. 4, 329 (Nov. 12, 1943).

[42] H. Mark, The mechanical properties of high polymers, Trans. Faraday Soc. 43, 451 (1947).

[43] E. L. Wittbecker, R. C. Houtz, and W. W. Watkins, Elastic N-substituted polyamides, Ind. Eng. Chem. 40, 875 (May 1948).

[44] Rubber goods; general specifications (methods of physical tests and chemical analyses), Federal Specification ZZ-R-601a (Government Printing Office, Washington, D. C., June 25, 1940).

[45] Standard method of test for internal tearing resistance of paper, D 689-44, A. S. T. M. Standards 1946, Part III-B (Am. Soc. Testing Materials, Philadelphia, $\mathrm{Pa}$.).

[46] A. I. Goldberg, W. P. Hohenstein, and H. Mark, Intrinsic viscositymolecular weight relationship for polystyrene, J. Polymer Sci. 2, 503 (1947).

[47] P. J. Flory, Molecular weights and intrinsic viscosities of polyisobutylenes, J. Am. Chem. Soc. 65, 372 (1943).

\section{Discussion}

Dr. Leo Wall, National Bureau of Standards: In our work under high-vacuum conditions the thermal decomposition of nylon or natural proteins did not produce detectable amounts of ammonia or nitrogen. Amino acids, such as glycine, alanine, and cystine, did produce ammonia approximately proportional to the number of $\mathrm{NH}_{2}$ groups in the material decomposed. In the case of glycine the moles of ammonia produced equaled approximately the carbon dioxide and to some extent the hydrocarbons formed. At about atmospheric pressure and/or in the presence of a reducing substance, nylon and proteins can also produce ammonia as a result of secondary reactions. Therefore, it appears clear that the primary steps in the decomposition of peptide links does not produce ammonia or nitrogen, whereas in the case of amino groups ammonia is readily produced. 





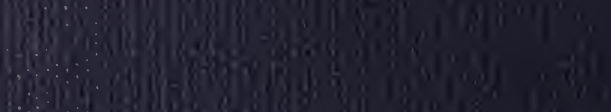

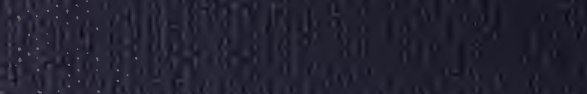

ail $: 3$

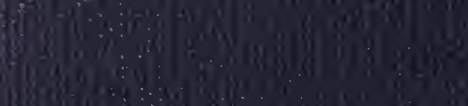

EIf

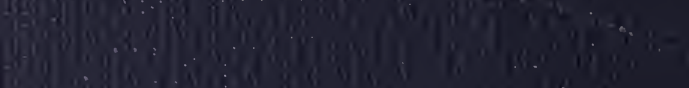

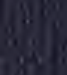

1i.

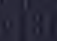

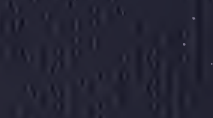

